\title{
Rhodium-Catalyzed Regiodivergent and Enantioselective Hydroboration of Enamides
}

\author{
Xiao-Yan $\mathrm{Bai}^{+}$, Wei Zhao ${ }^{+}$, Xin Sun and Bi-Jie Li* \\ Center of Basic Molecular Science (CBMS), Department of Chemistry, Tsinghua University
}

\section{Table of Contents}

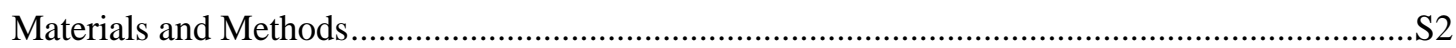

General Procedure for the Synthesis of Racemic Aminoboronic Esters .........................................5

General Procedure for the Synthesis of Chiral Aminoboronic Esters ............................................56

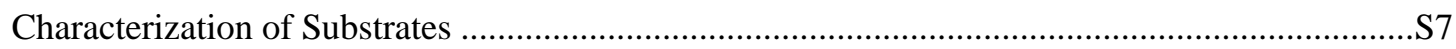

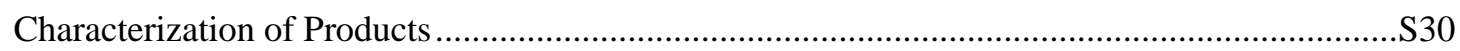

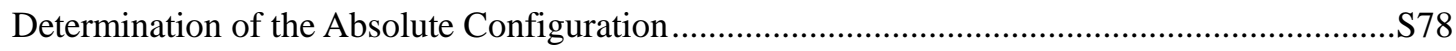

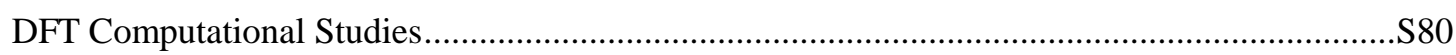

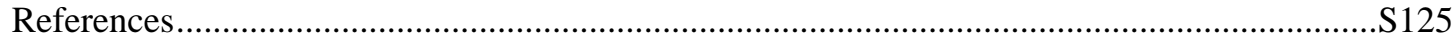

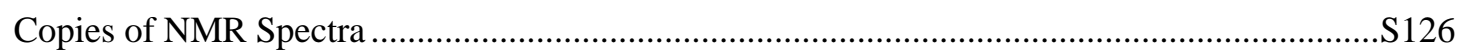




\section{Materials and Methods}

Unless otherwise noted, all reactions were assembled on a Schlenk vacuum line or in a glovebox using oven-dried glassware and were stirred with Teflon-coated magnetic stirring bars. All the ligands were purchased from Strem Chemicals and were used as received. $\left[\mathrm{Rh}(\operatorname{cod})_{2} \mathrm{OTf}\right]^{1}$ was prepared according to literature methods. dcypb (1,4-bis(dicyclohexylphosphino)butane) was purchased from Sigma-Aldrich and was used as received. HBpin (pinacolborane), $\mathrm{Pd}(\mathrm{OAc})_{2}$, $\mathrm{Ad}_{2} \mathrm{P} n \mathrm{Bu}$ (di(1-adamantyl)-n-butylphosphine), DiPrPF (1,1'-bis(diisopropylphosphino)ferrocene) and $\left[\operatorname{Ru}(\operatorname{cod})(\operatorname{met})_{2}\right]$ (bis(2-methallyl)-cycloocta-1,5-diene-ruthenium(II)) were purchased from J\&KScientific and were used as received. Tetrahydrofuran (THF), toluene (PhMe), dichloromethane (DCM), dimethylformamide (DMF) were degassed by purging with nitrogen and then dried with a solvent purification system containing activated alumina. All other solvents and reagents were used as received. The enamide substrates were prepared through $\mathrm{Cu}$-catalyzed amidation of alkenyl halides ${ }^{2}$ or Ru-catalyzed amidation of terminal alkynes ${ }^{3}$ according to literature methods. All work-up and purification procedures were carried out with reagent grade solvents in air. Reaction temperatures above $25{ }^{\circ} \mathrm{C}$ refer to temperatures of an aluminum heating block or a silicon oil bath, which were controlled by an electronic temperature modulator from IKA. NMR spectra were acquired on NMR spectrometer with $400 \mathrm{MHz}$ for ${ }^{1} \mathrm{H}$ NMR and 101 MHz for ${ }^{13} \mathrm{C}$ NMR at the NMR facility at Center of Basic Molecular Science (CBMS). Chemical shifts $(\delta)$ are reported in ppm relative to the residual solvent signal $\left(\delta=7.26\right.$ for ${ }^{1} \mathrm{H}$ NMR and $\delta=$ 77.16 for ${ }^{13} \mathrm{C}$ NMR). Data for ${ }^{1} \mathrm{H}$ NMR spectra are reported as follows: chemical shift (multiplicity, coupling constants, number of hydrogens). Abbreviations are as follows: s (singlet), d (doublet), t (triplet), q (quartet), m (multiplet), br (broad). GC data were obtained on a Shimazu 2010 Plus GC system containing an Rxi ${ }^{\circledR}-5 \mathrm{~ms}$ capillary column. Infrared (IR) spectra were recorded on a Bruker FT-IR alpha (ATR mode) spectrophotometer.High-resolution mass spectral data was performed on a Thermo Scientific Q Exactive (positive mode) at the Mass Spectrometry Facility, CBMS. 
Table S1. Optimization of Rhodium-Catalyzed Hydroboration of Z-enamide ${ }^{a}$

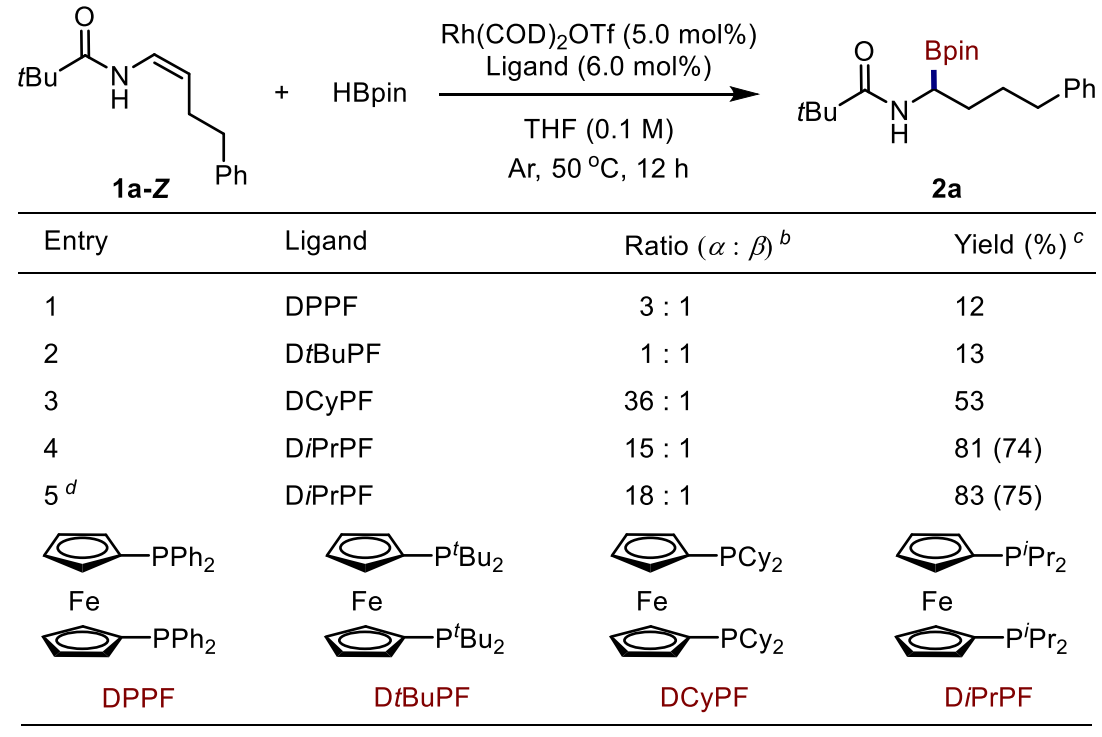

${ }^{a}$ Reaction conditions: $1 \mathrm{a}-\mathrm{Z}$ (1.0 equiv.), HBpin (2.0 equiv.), Rh(COD) ${ }_{2} \mathrm{OTf}$ (5.0 mol\%), Ligand $(6.0 \mathrm{~mol} \%), 50{ }^{\circ} \mathrm{C}, 12 \mathrm{~h} .{ }^{b}$ Ratios were determined by GC. ${ }^{c}$ Yields were determined by GC using $n$-dodecane as an internal standard. Isolated yield in parenthesis. ${ }^{d} \mathrm{Rh}(\mathrm{COD})_{2} \mathrm{OTf}(3.0 \mathrm{~mol} \%)$, Ligand (3.6 mol\%), THF (0.2 M).

Table S2. Optimization of Rhodium-Catalyzed Hydroboration of $E$-enamide ${ }^{\text {a }}$

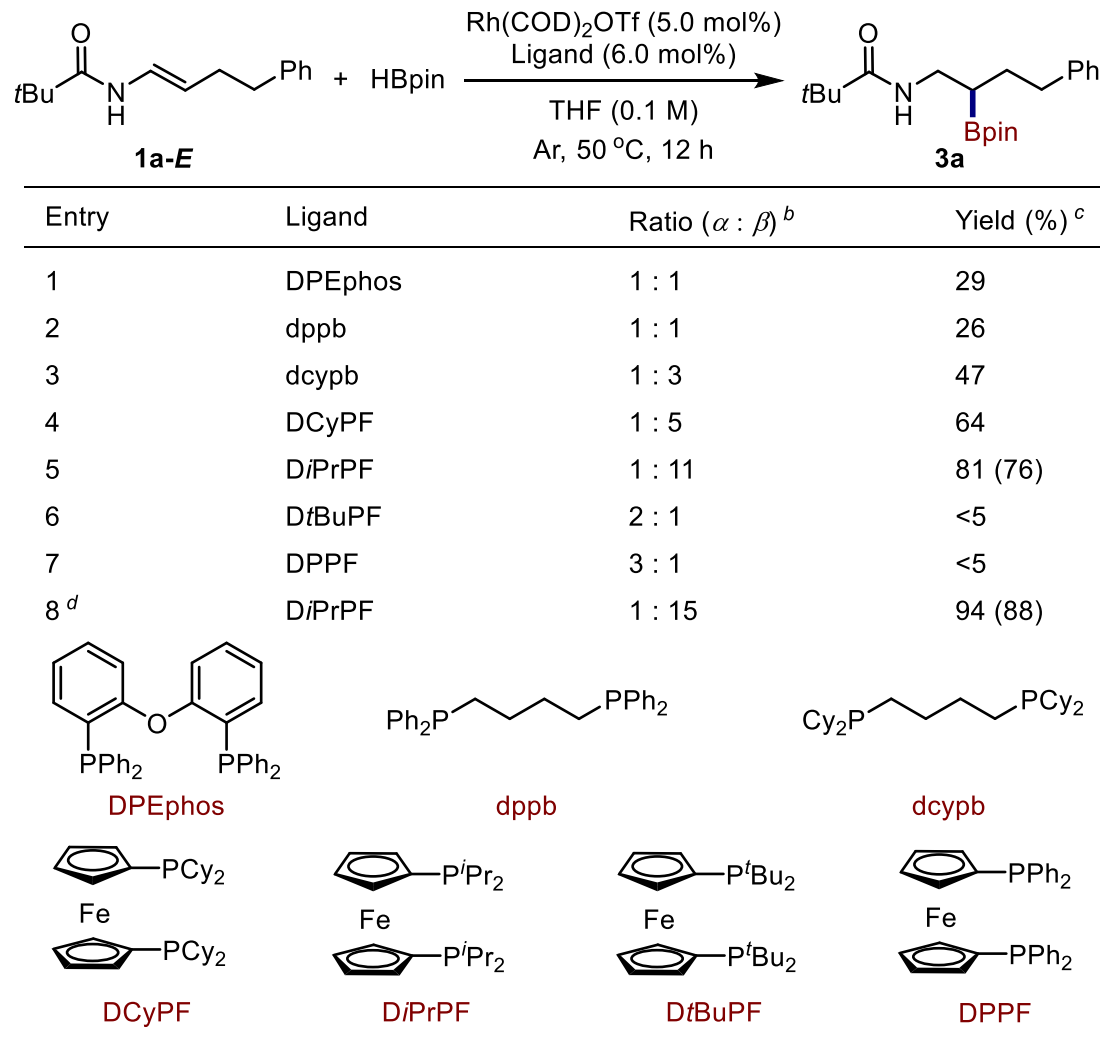

${ }^{a}$ Reaction conditions: $1 \mathrm{a}-\mathrm{E}$ (1.0 equiv.), HBpin (2.0 equiv.), $\mathrm{Rh}(\mathrm{COD})_{2} \mathrm{OTf}(5.0 \mathrm{~mol} \%)$, Ligand $(6.0 \mathrm{~mol} \%), 50{ }^{\circ} \mathrm{C}, 12 \mathrm{~h} .{ }^{b}$ Ratios were determined by GC. ${ }^{c}$ Yields were determined by GC using $n$-dodecane as an internal standard. Isolated yield in parenthesis. ${ }^{d} \mathrm{Rh}(\mathrm{COD})_{2} \mathrm{OTf}(3.0 \mathrm{~mol} \%$ ), Ligand (3.6 mol\%), r.t. 
Table S3. Optimization of Asymmetric Synthsis of $\alpha$-Aminoboronic Esters ${ }^{\mathrm{a}}$
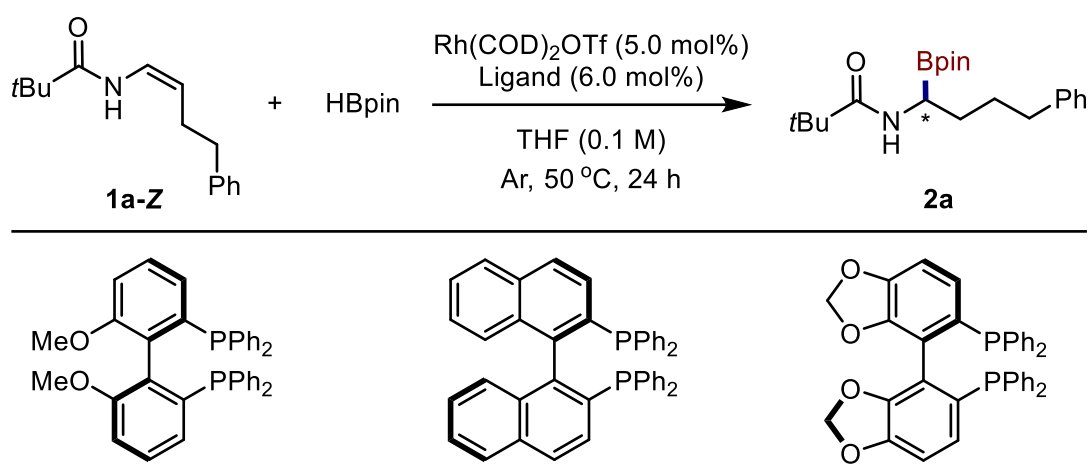

L1, (R)-MeO-BIPHEP $\alpha: \beta=1: 1$

$7 \%, 55 \%$ ee
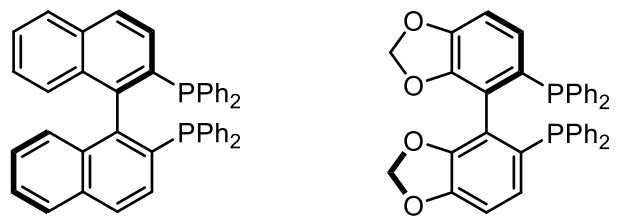

L2, (R)-BINAP

$\alpha: \beta=1: 1$

$9 \%, 43 \%$ ee

L3, (R)-Segphos

$\alpha: \beta=1: 1$

$8 \%, 48 \%$ ee<smiles>CC(C)C1CC[C@@H](C(C)C)P1CCP1[C@H](C(C)C)CC[C@@H]1C(C)C</smiles><smiles>CC(C)C1CCC(C(C)C)P1c1ccccc1P1C(C(C)C)CCC1C(C)C</smiles>

L4, (S,S)-iPr-BPE

$\alpha: \beta=20: 1$ $<5 \%$, N.D.

L5, $(S, S)$-iPr-Duphos $\alpha: \beta=17: 1$ $17 \%, 11 \%$ ee<smiles>CC(C)CP(C)c1nc2ccccc2nc1P(C)C(C)(C)C</smiles>

L6, $(R, R)$-QuinoxP* $\alpha: \beta=3: 1$ $56 \%, 83 \%$ ee<smiles>CC(C)(C)C[PH]1Cc2ccccc2[C@H]1/C=C1/c2ccccc2CP1C(C)(C)C</smiles>

L7, $(R, R, S, S)$-Duanphos

THF $12: 1,72 \%, 94 \%$ ee

DME $5: 1,<5 \%$, N.D.

DCE $2: 1,10 \%, 73 \%$ ee $\mathrm{CHCl}_{3} 10: 1,<5 \%$, N.D.

Toluene $29: 1,76 \%, 97 \%$ ee

${ }^{d}$ Toluene

$35: 1,88 \%, 98 \%$ ee

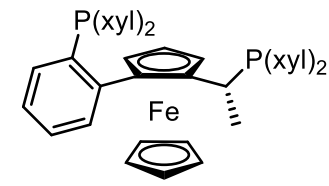

L8, $\left(R, R_{p}\right)$-Walphos $\alpha: \beta=1: 1$ $<5 \%$. N.D.

${ }^{a}$ Reaction conditions: $\mathbf{1 a}-\mathbf{Z}$ (1.0 equiv.), HBpin (2.0 equiv.), $\mathrm{Rh}(\mathrm{COD}){ }_{2} \mathrm{OTf}$ (5.0 mol\%), Ligand $(6.0 \mathrm{~mol} \%), 50{ }^{\circ} \mathrm{C}, 24 \mathrm{~h} .{ }^{b}$ Ratios were determined by GC. ${ }^{c}$ Isolated yield were reported. ${ }^{d} \mathrm{Rh}(\mathrm{COD})_{2} \mathrm{OTf}(3.0 \mathrm{~mol} \%)$, Ligand (3.6 mol\%). 
Table S4. Optimization of Asymmetric Synthsis of $\beta$-Aminoboronic Esters ${ }^{a}$

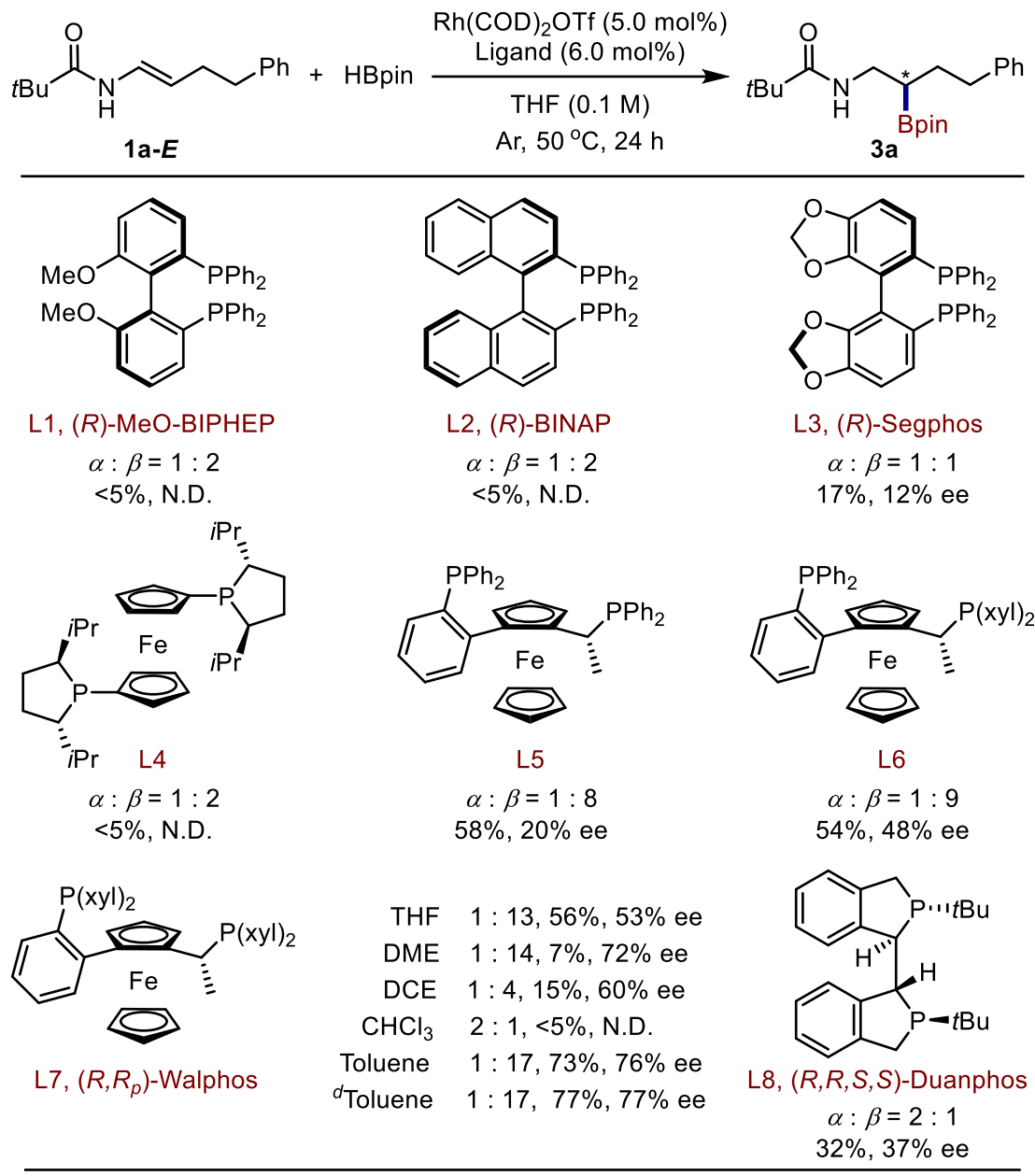

${ }^{a}$ Reaction conditions: $1 \mathrm{a}-\mathrm{E}$ (1.0 equiv.), $\mathrm{HBpin}$ ( 2.0 equiv.), $\mathrm{Rh}(\mathrm{COD})_{2} \mathrm{OTf}(5.0 \mathrm{~mol} \%)$, Ligand $(6.0 \mathrm{~mol} \%), 50{ }^{\circ} \mathrm{C}, 24 \mathrm{~h} .{ }^{b}$ Ratios were determined by GC. ${ }^{c}$ Isolated yield were reported. ${ }^{d} 40^{\circ} \mathrm{C}$.

\section{General Procedure for the Synthesis of Racemic Aminoboronic Esters}

[A] For Z-enamides: In an $\mathrm{N}_{2}$-filled glovebox, the enamide ( $0.10 \mathrm{mmol}, 1.0$ equiv), $\mathrm{Rh}(\operatorname{cod})_{2} \mathrm{OTf}$ (3.0 $\mu \mathrm{mol}, 3.0 \mathrm{~mol} \%), \operatorname{DiPrPF}(3.6 \mu \mathrm{mol}, 3.6 \mathrm{~mol} \%)$ were weighed into a one-dram screw-capped vial. Subsequently, THF $(0.50 \mathrm{~mL})$ and pinacolborane $(0.20 \mathrm{mmol}, 2.0$ equiv $)$ were added via syringe. The vial was capped with a Teflon-lined screw cap, and the resulting solution was then removed from the glovebox, placed in a pre-heated aluminum block at $50^{\circ} \mathrm{C}$ for $12 \mathrm{~h}$. The reaction mixture was concentrated and purified directly by column chromatography on silica gel with EtOAc/hexanes mixture as eluent.

[B] For $\boldsymbol{E}$-enamides: In an $\mathrm{N}_{2}$-filled glovebox, the enamide ( $0.10 \mathrm{mmol}, 1.0$ equiv), $\mathrm{Rh}(\operatorname{cod})_{2} \mathrm{OTf}$ (3.0 $\mu \mathrm{mol}, 3.0 \mathrm{~mol} \%), \operatorname{DiPrPF}(3.6 \mu \mathrm{mol}, 3.6 \mathrm{~mol} \%)$ were weighed into a one-dram screw-capped 
vial. Subsequently, THF $(1.00 \mathrm{~mL})$ and pinacolborane $(0.20 \mathrm{mmol}, 2.0$ equiv) were added via syringe. The vial was capped with a Teflon-lined screw cap, and the resulting solution was then removed from the glovebox, placed in an aluminum block at room temperature for $12 \mathrm{~h}$. The reaction mixture was concentrated and purified directly by column chromatography on silica gel with EtOAc/hexanes mixture as eluent.

[C] For disubstituted enamides: In an $\mathrm{N}_{2}$-filled glovebox, the enamide ( $0.10 \mathrm{mmol}, 1.0$ equiv), $\mathrm{Rh}(\mathrm{cod})_{2} \mathrm{OTf}(3.0 \mu \mathrm{mol}, 3.0 \mathrm{~mol} \%)$, DiPrPF $(3.6 \mu \mathrm{mol}, 3.6 \mathrm{~mol} \%)$ were weighed into a one-dram screw-capped vial. Subsequently, THF $(0.50 \mathrm{~mL})$ and pinacolborane $(0.20 \mathrm{mmol}, 2.0$ equiv $)$ were added via syringe. The vial was capped with a Teflon-lined screw cap, and the resulting solution was then removed from the glovebox, placed in an aluminum block at $30^{\circ} \mathrm{C}$ for $24 \mathrm{~h}$. The reaction mixture was concentrated and purified directly by column chromatography on silica gel with EtOAc/hexanes mixture as eluent.

\section{General Procedure for the Synthesis of Chiral Aminoboronic Esters}

[D] For chiral $\boldsymbol{\alpha}$-aminoboronic Esters: In an $\mathrm{N}_{2}$-filled glovebox, the enamide $(0.10 \mathrm{mmol}, 1.0$ equiv), $\mathrm{Rh}(\operatorname{cod})_{2} \mathrm{OTf}(3.0 \mu \mathrm{mol}, 3.0 \mathrm{~mol} \%)$, Duanphos $(3.6 \mu \mathrm{mol}, 3.6 \mathrm{~mol} \%)$ were weighed into a one-dram screw-capped vial. Subsequently, the corresponding solvent $(1.0 \mathrm{~mL})$ and pinacolborane (2.0 equiv or 2.5 equiv) were added via syringe. The vial was capped with a Teflon-lined screw cap, and the resulting solution was then removed from the glovebox, placed in an aluminum block at $50^{\circ} \mathrm{C}$ for $24 \mathrm{~h}$. The reaction mixture was concentrated and purified directly by column chromatography on silica gel with EtOAc/hexanes mixture as eluent.

[E] For chiral $\boldsymbol{\beta}$-aminoboronic Esters: In an $\mathrm{N}_{2}$-filled glovebox, the enamide $(0.10 \mathrm{mmol}, 1.0$ equiv), $\mathrm{Rh}(\operatorname{cod})_{2} \mathrm{OTf}(5.0 \mu \mathrm{mol}, 5.0 \mathrm{~mol} \%),\left(R, R_{p}\right)$-Walphos $(6.0 \mu \mathrm{mol}, 6.0 \mathrm{~mol} \%)$ were weighed into a one-dram screw-capped vial. Subsequently, toluene $(1.0 \mathrm{~mL})$ and pinacolborane $(0.20 \mathrm{mmol}$, 2.0 equiv) were added via syringe. The vial was capped with a Teflon-lined screw cap, and the resulting solution was then removed from the glovebox, placed in an aluminum block at $40^{\circ} \mathrm{C}$ for $24 \mathrm{~h}$. The reaction mixture was concentrated and purified directly by column chromatography on silica gel with EtOAc/hexanes mixture as eluent. 


\section{Characterization of Substrates}<smiles>CC(C)(C)C(=O)N/C=C\CCc1ccccc1</smiles>

Enamide 1a-Z: ${ }^{1} \mathbf{H}$ NMR (400 MHz, $\left.\mathrm{CDCl}_{3}\right) \delta 7.33-7.26(\mathrm{~m}, 2 \mathrm{H}), 7.23-7.17$ (m, 3H), $6.83(\mathrm{~s}$, 1H), 6.69 (ddt, $J=10.4,8.8,1.5 \mathrm{~Hz}, 1 \mathrm{H}), 4.80(\mathrm{q}, J=8.0 \mathrm{~Hz}, 1 \mathrm{H}), 2.72(\mathrm{t}, J=7.3 \mathrm{~Hz}, 2 \mathrm{H}), 2.30$ $(\mathrm{qd}, J=7.4,1.4 \mathrm{~Hz}, 2 \mathrm{H}), 1.12$ (s, 9H). ${ }^{13} \mathrm{C}$ NMR $\left(101 \mathrm{MHz}, \mathrm{CDCl}_{3}\right) \delta 175.56,141.66,128.71$, 128.65, 126.34, 122.26, 109.89, 38.95, 35.55, 28.35, 27.49. ESI-HR calcd for $\mathrm{C}_{15} \mathrm{H}_{22} \mathrm{NO}^{+}$ $\left([\mathrm{M}+\mathrm{H}]^{+}\right) 232.1696$, found 232.1688. IR $v\left(\mathrm{~cm}^{-1}\right) 3301,1682,1666,897$. M. P. $83-84{ }^{\circ} \mathrm{C}$.<smiles>CC(C)(C)C(=O)N/C=C/CCc1ccccc1</smiles>

Enamide 1a-E: ${ }^{1} \mathbf{H}$ NMR $\left(400 \mathrm{MHz}, \mathrm{CDCl}_{3}\right) \delta 7.28(\mathrm{dd}, J=12.0,4.9 \mathrm{~Hz}, 2 \mathrm{H}), 7.19(\mathrm{t}, J=7.4 \mathrm{~Hz}$ $3 \mathrm{H}), 7.05(\mathrm{~d}, J=9.0 \mathrm{~Hz}, 1 \mathrm{H}), 6.87-6.78(\mathrm{~m}, 1 \mathrm{H}), 5.16(\mathrm{dt}, J=14.2,7.1 \mathrm{~Hz}, 1 \mathrm{H}), 2.73-2.65(\mathrm{~m}$, 2H), $2.41-2.31(\mathrm{~m}, 2 \mathrm{H}), 1.21(\mathrm{~s}, 9 \mathrm{H}) .{ }^{13} \mathrm{C}$ NMR $\left(101 \mathrm{MHz}, \mathrm{CDCl}_{3}\right) \delta$ 175.53, 141.72, 128.56, $128.50,126.04,123.53,111.98,38.78,36.54,31.66,27.58$. ESI-HR calcd for $\mathrm{C}_{15} \mathrm{H}_{22} \mathrm{NO}^{+}$ $\left([\mathrm{M}+\mathrm{H}]^{+}\right)$232.1696, found 232.1688. IR $v\left(\mathrm{~cm}^{-1}\right) 3306,1678,1640,958$. M. P. $122-123{ }^{\circ} \mathrm{C}$.<smiles>CC(=O)N/C=C\CCc1ccccc1</smiles>

Enamide 1b-Z: ${ }^{1} \mathbf{H}$ NMR $\left(400 \mathrm{MHz}, \mathrm{CDCl}_{3}\right) \delta 7.34-7.27(\mathrm{~m}, 2 \mathrm{H}), 7.24-7.17(\mathrm{~m}, 3 \mathrm{H}), 6.83-$ $6.51(\mathrm{~m}, 2 \mathrm{H}), 4.76(\mathrm{q}, J=7.8 \mathrm{~Hz}, 1 \mathrm{H}), 2.70(\mathrm{t}, J=7.3 \mathrm{~Hz}, 2 \mathrm{H}), 2.31(\mathrm{q}, J=7.4 \mathrm{~Hz}, 2 \mathrm{H}), 1.87(\mathrm{~s}$, 3H). ${ }^{13}$ C NMR (101 MHz, $\left.\mathrm{CDCl}_{3}\right) \delta 167.75,142.08,128.98,126.66,122.27,110.02,109.97$, 
35.88, 28.52, 23.56. ESI-HR calcd for $\mathrm{C}_{12} \mathrm{H}_{16} \mathrm{NO}^{+}\left([\mathrm{M}+\mathrm{H}]^{+}\right)$190.1226, found 190.1222. IR $v$ $\left(\mathrm{cm}^{-1}\right) 3289,2653,1604,977$. M. P. $87-88^{\circ} \mathrm{C}$.<smiles>CC(=O)N/C=C/CCc1ccccc1</smiles>

Enamide 1b-E: ${ }^{1} \mathbf{H}$ NMR $\left(400 \mathrm{MHz}, \mathrm{CDCl}_{3}\right) \delta 7.38-7.24(\mathrm{~m}, 3 \mathrm{H}), 7.21-7.13(\mathrm{~m}, 3 \mathrm{H}), 6.83-$ $6.71(\mathrm{~m}, 1 \mathrm{H}), 5.13(\mathrm{td}, J=13.6,6.7 \mathrm{~Hz}, 1 \mathrm{H}), 2.68(\mathrm{t}, J=7.33,2 \mathrm{H}), 2.34(\mathrm{q}, J=7.3 \mathrm{~Hz}, 2 \mathrm{H}), 2.01$ $(\mathrm{d}, J=2.7 \mathrm{~Hz}, 3 \mathrm{H}) .{ }^{13} \mathrm{C}$ NMR $\left(101 \mathrm{MHz}, \mathrm{CDCl}_{3}\right) \delta 167.14,141.64,128.56,128.49,126.05$, 123.16, 111.94, 36.52, 31.59, 23.33. ESI-HR calcd for $\mathrm{C}_{12} \mathrm{H}_{16} \mathrm{NO}^{+}\left([\mathrm{M}+\mathrm{H}]^{+}\right)$190.1226, found 190.1221. IR $v\left(\mathrm{~cm}^{-1}\right) 3271,2924,1682,952$. M. P. $70-72{ }^{\circ} \mathrm{C}$.<smiles>CC(C)C(=O)N/C=C\CCc1ccccc1</smiles>

Enamide 1c-Z: ${ }^{1} \mathbf{H}$ NMR (400 MHz, $\left.\mathrm{CDCl}_{3}\right) \delta 7.34-7.27(\mathrm{~m}, 2 \mathrm{H}), 7.21(\mathrm{ddt}, J=7.2,3.3,1.4 \mathrm{~Hz}$, 3H), $6.68(\mathrm{ddt}, J=10.5,8.9,1.5 \mathrm{~Hz}, 1 \mathrm{H}), 6.50(\mathrm{~s}, 1 \mathrm{H}), 4.79(\mathrm{q}, J=7.8 \mathrm{~Hz}, 1 \mathrm{H}), 2.72(\mathrm{t}, J=7.2 \mathrm{~Hz}$, 2H), $2.31(\mathrm{qd}, J=7.3,1.5 \mathrm{~Hz}, 2 \mathrm{H}), 2.23-2.12(\mathrm{~m}, J=6.9 \mathrm{~Hz}, 1 \mathrm{H}), 1.09(\mathrm{~d}, J=6.9 \mathrm{~Hz}, 6 \mathrm{H}) .{ }^{13} \mathrm{C}$ NMR $\left(101 \mathrm{MHz}, \mathrm{CDCl}_{3}\right) \delta 173.87,141.43,128.37,128.35,126.02,121.75,109.33,35.39,35.25$, 27.98, 19.24. ESI-HR calcd for $\mathrm{C}_{14} \mathrm{H}_{20} \mathrm{NO}^{+}\left([\mathrm{M}+\mathrm{H}]^{+}\right) 218.1539$, found 218.1535. IR $v\left(\mathrm{~cm}^{-1}\right)$ 3301, 1674, 1649, 951. M. P. $107-109^{\circ} \mathrm{C}$.<smiles>CC(C)C(=O)N/C=C/CCc1ccccc1</smiles>

Enamide 1c-E: ${ }^{1} \mathbf{H}$ NMR $\left(400 \mathrm{MHz}, \mathrm{CDCl}_{3}\right) \delta 7.33-7.26(\mathrm{~m}, 2 \mathrm{H}), 7.21-7.14(\mathrm{~m}, 3 \mathrm{H}), 6.99(\mathrm{~s}$, $1 \mathrm{H}), 6.86-6.72(\mathrm{~m}, 1 \mathrm{H}), 5.15(\mathrm{dt}, J=14.2,7.1 \mathrm{~Hz}, 1 \mathrm{H}), 2.74-2.59(\mathrm{~m}, 2 \mathrm{H}), 2.40-2.27(\mathrm{~m}, 3 \mathrm{H})$, $1.15(\mathrm{~d}, J=6.90 \mathrm{~Hz}, 6 \mathrm{H}) .{ }^{13} \mathbf{C}$ NMR $\left(101 \mathrm{MHz}, \mathrm{CDCl}_{3}\right) \delta 174.11,141.69,128.54,128.47,126.03$, 
123.26, 111.92, 36.54, 35.73, 31.63, 19.56. ESI-HR calcd for $\mathrm{C}_{14} \mathrm{H}_{20} \mathrm{NO}^{+}\left([\mathrm{M}+\mathrm{H}]^{+}\right)$218.1539, found 218.1532. IR $v\left(\mathrm{~cm}^{-1}\right) 3276,2931,1658,953$. M. P. $97-99^{\circ} \mathrm{C}$.<smiles>O=C(N/C=C\CCc1ccccc1)c1ccccc1</smiles>

Enamide 1d-Z: ${ }^{1} \mathbf{H}$ NMR $\left(400 \mathrm{MHz}, \mathrm{CDCl}_{3}\right) \delta 7.63-7.58(\mathrm{~m}, 2 \mathrm{H}), 7.54-7.48(\mathrm{~m}, 1 \mathrm{H}), 7.45-$ $7.38(\mathrm{~m}, 2 \mathrm{H}), 7.31-7.20(\mathrm{~m}, 5 \mathrm{H}), 7.18-7.12(\mathrm{~m}, 1 \mathrm{H}), 6.89(\mathrm{ddt}, J=10.6,9.0,1.5 \mathrm{~Hz}, 1 \mathrm{H}), 4.94$ $(\mathrm{dt}, J=9.2,7.8 \mathrm{~Hz}, 1 \mathrm{H}), 2.76(\mathrm{t}, J=7.2 \mathrm{~Hz}, 2 \mathrm{H}), 2.41(\mathrm{qd}, J=7.3,1.5 \mathrm{~Hz}, 2 \mathrm{H}) .{ }^{13} \mathbf{C}$ NMR $(101$ $\left.\mathrm{MHz}, \mathrm{CDCl}_{3}\right) \delta 164.16,141.39,133.56,131.67,128.43,128.41,128.34,126.83,126.08,121.98$, 110.66, 35.27, 28.18. ESI-HR calcd for $\mathrm{C}_{17} \mathrm{H}_{18} \mathrm{NO}^{+}\left([\mathrm{M}+\mathrm{H}]^{+}\right)$252.1383, found 252.1377. IR v $\left(\mathrm{cm}^{-1}\right) 3304,1653,1603,888$. M. P. $98-99^{\circ} \mathrm{C}$.<smiles>O=C(N/C=C/CCc1ccccc1)c1ccccc1</smiles>

Enamide 1d-E: ${ }^{1} \mathbf{H}$ NMR $\left(400 \mathrm{MHz}, \mathrm{CDCl}_{3}\right) \delta 7.84-7.75(\mathrm{~m}, 2 \mathrm{H}), 7.66(\mathrm{~s}, 1 \mathrm{H}), 7.55-7.48(\mathrm{~m}$, 1H), $7.44(\mathrm{t}, J=7.4 \mathrm{~Hz}, 2 \mathrm{H}), 7.33-7.27(\mathrm{~m}, 2 \mathrm{H}), 7.23-7.14(\mathrm{~m}, 3 \mathrm{H}), 7.02(\mathrm{dd}, J=14.2,10.5 \mathrm{~Hz}$, 1H), $5.32(\mathrm{dt}, J=14.2,7.1 \mathrm{~Hz}, 1 \mathrm{H}), 2.73(\mathrm{t}, J=7.7 \mathrm{~Hz}, 2 \mathrm{H}), 2.42(\mathrm{dd}, J=15.1,7.2 \mathrm{~Hz}, 2 \mathrm{H}) .{ }^{13} \mathrm{C}$ NMR $\left(101 \mathrm{MHz}, \mathrm{CDCl}_{3}\right) \delta 164.44,141.62,133.94,131.98,128.82,128.59,128.51,127.14$, 126.08, 123.51, 113.20, 36.51, 31.73. ESI-HR calcd for $\mathrm{C}_{12} \mathrm{H}_{16} \mathrm{NO}^{+}\left([\mathrm{M}+\mathrm{H}]^{+}\right) 252.1383$, found 252.1375. IR $v\left(\mathrm{~cm}^{-1}\right) 3325,2917,1638,949$. M. P. $118-120^{\circ} \mathrm{C}$.<smiles>C/C=C\NC(=O)C(C)(C)C</smiles>

Enamide 1e-Z: ${ }^{1} \mathrm{H}$ NMR $\left(400 \mathrm{MHz}, \mathrm{CDCl}_{3}\right) \delta 7.12(\mathrm{~s}, 1 \mathrm{H}), 6.73(\mathrm{ddq}, J=10.7,8.9,1.8 \mathrm{~Hz}, 1 \mathrm{H})$, $4.87-4.75(\mathrm{~m}, 1 \mathrm{H}), 1.61(\mathrm{dd}, J=7.0,1.8 \mathrm{~Hz}, 3 \mathrm{H}), 1.25(\mathrm{~s}, 9 \mathrm{H}) .{ }^{13} \mathbf{C ~ N M R}\left(101 \mathrm{MHz}, \mathrm{CDCl}_{3}\right) \delta$ 
175.48, 122.44, 105.02, 39.11, 27.62, 10.89. ESI-HR calcd for $\mathrm{C}_{8} \mathrm{H}_{16} \mathrm{NO}^{+}\left([\mathrm{M}+\mathrm{H}]^{+}\right) 142.1226$, found 142.1225. IR $v\left(\mathrm{~cm}^{-1}\right) 3323,2950,1705,1678,1645$, 904. M.P. $64-65^{\circ} \mathrm{C}$.<smiles>C/C=C/NC(=O)C(C)(C)C</smiles>

Enamide 1e-E: ${ }^{1} \mathbf{H}$ NMR $\left(400 \mathrm{MHz}, \mathrm{CDCl}_{3}\right) \delta 7.03(\mathrm{~s}, 1 \mathrm{H}), 6.76(6.81-6.70,1 \mathrm{H}), 5.15(\mathrm{dq}, J=$ 13.5, $6.7 \mathrm{~Hz}, 1 \mathrm{H}), 1.68(\mathrm{dd}, J=6.7,1.5 \mathrm{~Hz}, 3 \mathrm{H}), 1.22(\mathrm{~s}, 9 \mathrm{H}) .{ }^{13} \mathrm{C} \mathrm{NMR}\left(101 \mathrm{MHz}, \mathrm{CDCl}_{3}\right) \delta$ 175.39, 123.82, 107.64, 38.73, 27.59, 14.93. ESI-HR calcd for $\mathrm{C}_{8} \mathrm{H}_{16} \mathrm{NO}^{+}\left([\mathrm{M}+\mathrm{H}]^{+}\right) 142.1226$, found 142.1224. IR $v\left(\mathrm{~cm}^{-1}\right) 3282,1683,1653,733$. M.P. $108-109^{\circ} \mathrm{C}$.<smiles>CCCC/C=C\NC(=O)C(C)(C)C</smiles>

Enamide 1f-Z: ${ }^{1} \mathbf{H}$ NMR $\left(400 \mathrm{MHz}, \mathrm{CDCl}_{3}\right) \delta 7.13(\mathrm{~s}, 1 \mathrm{H}), 6.71(\mathrm{dd}, J=10.6,9.1 \mathrm{~Hz}, 1 \mathrm{H}), 4.74$ $(\mathrm{dd}, J=16.1,7.5 \mathrm{~Hz}, 1 \mathrm{H}), 2.09-1.90(\mathrm{~m}, 2 \mathrm{H}), 1.46-1.30(\mathrm{~m}, 4 \mathrm{H}), 1.24(\mathrm{~s}, 9 \mathrm{H}), 0.92(\mathrm{t}, J=7.1$ $\mathrm{Hz}, 3 \mathrm{H}) .{ }^{13} \mathrm{C}$ NMR $\left(101 \mathrm{MHz}, \mathrm{CDCl}_{3}\right) \delta 175.41,121.40,111.18,39.05,31.52,27.59,25.48$, 22.38, 14.00. ESI-HR calcd for $\mathrm{C}_{11} \mathrm{H}_{22} \mathrm{NO}^{+}\left([\mathrm{M}+\mathrm{H}]^{+}\right)$184.1696, found 184.1692. IR $v\left(\mathrm{~cm}^{-1}\right)$ 3324, 1675, 1653, 734. M. P. $71-72{ }^{\circ} \mathrm{C}$.<smiles>CCCC/C=C/NC(=O)C(C)(C)C</smiles>

Enamide 1f-E: ${ }^{1} \mathbf{H}$ NMR $\left(400 \mathrm{MHz}, \mathrm{CDCl}_{3}\right) \delta 7.07(\mathrm{~s}, 1 \mathrm{H}), 6.75(\mathrm{dd}, J=14.2,10.4 \mathrm{~Hz}, 1 \mathrm{H}), 5.15$ $(\mathrm{dt}, J=14.2,7.1 \mathrm{~Hz}, 1 \mathrm{H}), 2.02(\mathrm{dd}, J=13.2,6.9 \mathrm{~Hz}, 2 \mathrm{H}), 1.43-1.25(\mathrm{~m}, 4 \mathrm{H}), 1.21(\mathrm{~s}, 9 \mathrm{H}), 0.88$ $(\mathrm{t}, J=7.0 \mathrm{~Hz}, 3 \mathrm{H}) .{ }^{13} \mathbf{C}$ NMR $\left(101 \mathrm{MHz}, \mathrm{CDCl}_{3}\right) \delta 175.43,122.95,113.19,38.74,32.20,29.50$, 27.59, 22.24, 14.03. ESI-HR calcd for $\mathrm{C}_{11} \mathrm{H}_{22} \mathrm{NO}^{+}\left([\mathrm{M}+\mathrm{H}]^{+}\right)$184.1696, found 184.1692. IR $v$ $\left(\mathrm{cm}^{-1}\right) 3306,1680,1636,740$. M. P. $92-93^{\circ} \mathrm{C}$. 


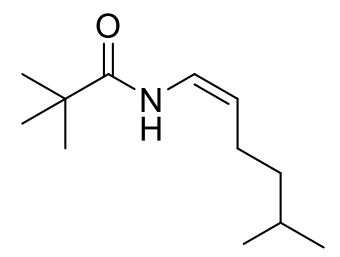

Enamide 1g-Z: ${ }^{1} \mathrm{H}$ NMR $\left(400 \mathrm{MHz}, \mathrm{CDCl}_{3}\right) \delta 7.13(\mathrm{~s}, 1 \mathrm{H}), 6.70(\mathrm{dd}, J=10.6,9.0 \mathrm{~Hz}, 1 \mathrm{H}), 4.74$ $(\mathrm{dd}, J=16.1,7.5 \mathrm{~Hz}, 1 \mathrm{H}), 2.08-1.88(\mathrm{~m}, 2 \mathrm{H}), 1.65-1.53(\mathrm{~m}, 1 \mathrm{H}), 1.30(\mathrm{dt}, J=11.5,5.7 \mathrm{~Hz}$, 2H), 1.25 (s, 9H), $0.91(\mathrm{~d}, J=6.6 \mathrm{~Hz}, 6 \mathrm{H}) .{ }^{13} \mathrm{C} \mathbf{N M R}\left(101 \mathrm{MHz}, \mathrm{CDCl}_{3}\right) \delta 175.43,121.32,111.34$, 39.10, 38.51, 27.63, 27.61, 23.64, 22.59. ESI-HR calcd for $\mathrm{C}_{12} \mathrm{H}_{24} \mathrm{NO}^{+}\left([\mathrm{M}+\mathrm{H}]^{+}\right) 198.1852$, found 198.1850. IR $v\left(\mathrm{~cm}^{-1}\right) 3469,1682,1654,734$. M. P. $87-88^{\circ} \mathrm{C}$.<smiles>CC(C)CC/C=C/NC(=O)C(C)(C)C</smiles>

Enamide 1g-E: ${ }^{1} \mathbf{H}$ NMR $\left(400 \mathrm{MHz}, \mathrm{CDCl}_{3}\right) \delta 7.03(\mathrm{~s}, 1 \mathrm{H}), 6.76(\mathrm{dd}, J=14.1,10.5 \mathrm{~Hz}, 1 \mathrm{H})$, $5.14(\mathrm{dt}, J=14.2,7.1 \mathrm{~Hz}, 1 \mathrm{H}), 2.02(\mathrm{dd}, J=15.1,7.4 \mathrm{~Hz}, 2 \mathrm{H}), 1.56(\mathrm{dp}, J=13.3,6.7 \mathrm{~Hz}, 1 \mathrm{H})$, $1.25(\mathrm{dd}, J=13.2,5.2 \mathrm{~Hz}, 2 \mathrm{H}), 1.22(\mathrm{~s}, 9 \mathrm{H}), 0.88(\mathrm{~d}, J=6.6 \mathrm{~Hz}, 6 \mathrm{H}) .{ }^{13} \mathbf{C}$ NMR $(101 \mathrm{MHz}$, $\left.\mathrm{CDCl}_{3}\right) \delta 175.42,122.86,113.34,39.26,38.75,27.67,27.60,27.52,22.60$. ESI-HR calcd for $\mathrm{C}_{12} \mathrm{H}_{24} \mathrm{NO}^{+}\left([\mathrm{M}+\mathrm{H}]^{+}\right)$198.1852, found 198.1849. IR $\vee\left(\mathrm{cm}^{-1}\right) 3431,1684,1653$, 903. M. P. $108-109^{\circ} \mathrm{C}$.<smiles>CC(C)(C)C(=O)N/C=C\CCCCl</smiles>

Enamide 1h-Z: ${ }^{1} \mathbf{H}$ NMR $\left(400 \mathrm{MHz}, \mathrm{CDCl}_{3}\right) \delta 7.41(\mathrm{~s}, 1 \mathrm{H}), 6.89-6.77(\mathrm{~m}, 1 \mathrm{H}), 4.66(\mathrm{q}, J=8.3$ $\mathrm{Hz}, 1 \mathrm{H}), 3.61(\mathrm{t}, J=5.8 \mathrm{~Hz}, 2 \mathrm{H}), 2.29-2.18(\mathrm{~m}, 2 \mathrm{H}), 1.94-1.84(\mathrm{~m}, 2 \mathrm{H}), 1.25(\mathrm{~s}, 9 \mathrm{H}) .{ }^{13} \mathbf{C}$ NMR $\left(101 \mathrm{MHz}, \mathrm{CDCl}_{3}\right) \delta 175.84,123.50,108.36,44.51,39.08,31.36,27.51,22.22$. ESI-HR calcd for $\mathrm{C}_{10} \mathrm{H}_{19} \mathrm{ClNO}^{+}\left([\mathrm{M}+\mathrm{H}]^{+}\right)$204.1150, found 204.1144. IR $v\left(\mathrm{~cm}^{-1}\right)$ 3316, 1721, 1646, 905.<smiles>CC(C)(C)C(=O)N/C=C/CCCCl</smiles> 
Enamide 1h- $\boldsymbol{E}:{ }^{1} \mathbf{H}$ NMR $\left(400 \mathrm{MHz}, \mathrm{CDCl}_{3}\right) \delta 7.06(\mathrm{~s}, 1 \mathrm{H}), 6.81(\mathrm{dd}, J=14.2,10.4 \mathrm{~Hz}, 1 \mathrm{H})$, $5.13(\mathrm{dt}, J=14.3,7.2 \mathrm{~Hz}, 1 \mathrm{H}), 3.54(\mathrm{t}, J=6.6 \mathrm{~Hz}, 2 \mathrm{H}), 2.19(\mathrm{q}, J=6.9 \mathrm{~Hz}, 2 \mathrm{H}), 1.91-1.77(\mathrm{~m}$, 2H), $1.21(\mathrm{~d}, J=11.7 \mathrm{~Hz}, 9 \mathrm{H}) .{ }^{13} \mathbf{C}$ NMR $\left(101 \mathrm{MHz}, \mathrm{CDCl}_{3}\right) \delta 175.54,124.19,110.82,44.32$, 38.81, 32.83, 27.59, 27.09. ESI-HR calcd for $\mathrm{C}_{10} \mathrm{H}_{19} \mathrm{ClNO}^{+}\left([\mathrm{M}+\mathrm{H}]^{+}\right)$204.1150, found 204.1148. IR $v\left(\mathrm{~cm}^{-1}\right) 3447,1670,1653,732$. M. P. $93-94{ }^{\circ} \mathrm{C}$.

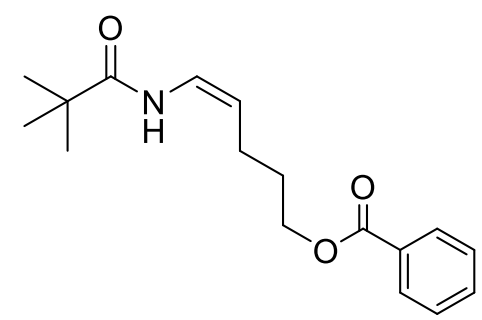

Enamide 1i-Z: ${ }^{1} \mathbf{H}$ NMR $\left(400 \mathrm{MHz}, \mathrm{CDCl}_{3}\right) \delta 8.10-7.99(\mathrm{~m}, 2 \mathrm{H}), 7.57(\mathrm{dd}, J=10.6,4.3 \mathrm{~Hz}$, 1H), $7.45(\mathrm{t}, J=7.7 \mathrm{~Hz}, 2 \mathrm{H}), 7.23(\mathrm{~d}, J=9.0 \mathrm{~Hz}, 1 \mathrm{H}), 6.84-6.71(\mathrm{~m}, 1 \mathrm{H}), 4.76(\mathrm{dd}, J=16.4,7.6$ $\mathrm{Hz}, 1 \mathrm{H}), 4.36(\mathrm{t}, J=6.2 \mathrm{~Hz}, 2 \mathrm{H}), 2.18(\mathrm{qd}, J=7.4,1.4 \mathrm{~Hz}, 2 \mathrm{H}), 1.95-1.85(\mathrm{~m}, 2 \mathrm{H}), 1.19(\mathrm{~s}, 9 \mathrm{H})$. ${ }^{13}$ C NMR $\left(101 \mathrm{MHz}, \mathrm{CDCl}_{3}\right) \delta 175.63,166.75,133.15,130.39,129.66,128.54,122.52,109.51$, 64.19, 39.05, 28.69, 27.51, 22.51. ESI-HR calcd for $\mathrm{C}_{17} \mathrm{H}_{24} \mathrm{NO}^{+}\left([\mathrm{M}+\mathrm{H}]^{+}\right) 290.1751$, found 290.1751. IR $v\left(\mathrm{~cm}^{-1}\right) 3464,1716,1683,1654,733$. M. P. $79-80{ }^{\circ} \mathrm{C}$.<smiles>CC(C)(C)C(=O)N/C=C/CCCOC(=O)c1ccccc1</smiles>

Enamide 1i-E: ${ }^{1} \mathbf{H}$ NMR $\left(400 \mathrm{MHz}, \mathrm{CDCl}_{3}\right) \delta 8.04(\mathrm{~d}, J=7.2 \mathrm{~Hz}, 2 \mathrm{H}), 7.56(\mathrm{t}, J=7.4 \mathrm{~Hz}, 1 \mathrm{H})$, $7.44(\mathrm{t}, J=7.6 \mathrm{~Hz}, 2 \mathrm{H}), 7.07(\mathrm{~d}, J=9.0 \mathrm{~Hz}, 1 \mathrm{H}), 6.83(\mathrm{dd}, J=14.2,10.4 \mathrm{~Hz}, 1 \mathrm{H}), 5.19(\mathrm{dt}, J=$ 14.3, 7.2 Hz, 1H), $4.34(\mathrm{t}, J=6.5 \mathrm{~Hz}, 2 \mathrm{H}), 2.21(\mathrm{q}, J=7.1 \mathrm{~Hz}, 2 \mathrm{H}), 1.91-1.80(\mathrm{~m}, 2 \mathrm{H}), 1.22(\mathrm{~s}$, 9H). ${ }^{13} \mathrm{C}$ NMR $\left(101 \mathrm{MHz}, \mathrm{CDCl}_{3}\right) \delta 175.53,166.74,133.01,130.53,129.69,128.49,123.92$, 111.40, 64.33, 38.78, 29.19, 27.57, 26.45. ESI-HR calcd for $\mathrm{C}_{17} \mathrm{H}_{24} \mathrm{NO}^{+}\left([\mathrm{M}+\mathrm{H}]^{+}\right) 290.1751$, found 290.1745 . IR $v\left(\mathrm{~cm}^{-1}\right) 3447,1716,1683,1653,733$. M. P. $81-82^{\circ} \mathrm{C}$. 
<smiles>CN(C)C(=O)OCCC/C=C\NC(=O)C(C)(C)C</smiles>

Enamide 1j-Z: ${ }^{1} \mathbf{H}$ NMR $\left(400 \mathrm{MHz}, \mathrm{CDCl}_{3}\right) \delta 7.35(\mathrm{~d}, J=10.1 \mathrm{~Hz}, 1 \mathrm{H}), 6.75(\mathrm{ddt}, J=10.7,9.1$, $1.7 \mathrm{~Hz}, 1 \mathrm{H}), 4.73(\mathrm{dt}, J=9.2,7.6 \mathrm{~Hz}, 1 \mathrm{H}), 4.11(\mathrm{t}, J=6.2 \mathrm{~Hz}, 2 \mathrm{H}), 2.92(\mathrm{~s}, 6 \mathrm{H}), 2.10(\mathrm{qd}, J=7.3$, $1.6 \mathrm{~Hz}, 2 \mathrm{H}), 1.80-1.72(\mathrm{~m}, 2 \mathrm{H}), 1.25(\mathrm{~s}, 9 \mathrm{H}) .{ }^{13} \mathbf{C} \mathbf{~ N M R}\left(101 \mathrm{MHz}, \mathrm{CDCl}_{3}\right) \delta 175.71,156.80$, 122.25, 110.13, 64.53, 38.99, $36.21(\mathrm{~d}, J=54.22 \mathrm{~Hz}), 29.13,27.46,22.47$. ESI-HR calcd for $\mathrm{C}_{13} \mathrm{H}_{25} \mathrm{~N}_{2} \mathrm{O}_{3}{ }^{+}\left([\mathrm{M}+\mathrm{H}]^{+}\right)$257.1860, found 257.1852. IR $v\left(\mathrm{~cm}^{-1}\right) 3366,1698,1684,1654,732$.<smiles>CNC(=O)OCCC/C=C/NC(=O)C(C)(C)C</smiles>

Enamide 1j-E: ${ }^{1} \mathbf{H}$ NMR $\left(400 \mathrm{MHz}, \mathrm{CDCl}_{3}\right) \delta 7.14(\mathrm{~s}, 1 \mathrm{H}), 6.88-6.67(\mathrm{~m}, 1 \mathrm{H}), 5.14(\mathrm{dt}, J=$ 14.3, 7.2 Hz, 1H), $4.12-4.00(\mathrm{~m}, 2 \mathrm{H}), 2.89(\mathrm{~s}, 6 \mathrm{H}), 2.10(\mathrm{td}, J=7.3,1.5 \mathrm{~Hz}, 2 \mathrm{H}), 1.77-1.62(\mathrm{~m}$, $2 \mathrm{H}), 1.22(\mathrm{dd}, J=10.7,1.6 \mathrm{~Hz}, 9 \mathrm{H}) .{ }^{13} \mathbf{C}$ NMR $\left(101 \mathrm{MHz}, \mathrm{CDCl}_{3}\right) \delta 175.52,156.83,123.73$, $111.59,64.57,38.75,36.50,35.96,29.50,27.55,26.28$. ESI-HR calcd for $\mathrm{C}_{13} \mathrm{H}_{25} \mathrm{~N}_{2} \mathrm{O}_{3}{ }^{+}\left([\mathrm{M}+\mathrm{H}]^{+}\right)$ 257.1860, found 257.1856. IR $v\left(\mathrm{~cm}^{-1}\right) 3447,1697,1685,1654,731$. M. P. $85-86^{\circ} \mathrm{C}$.

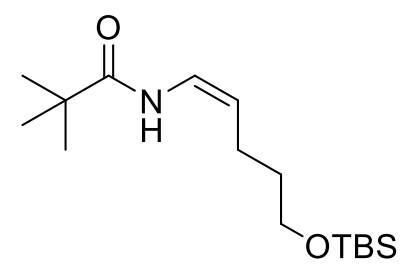

Enamide 1k-Z: ${ }^{1} \mathbf{H}$ NMR $\left(400 \mathrm{MHz}, \mathrm{CDCl}_{3}\right) \delta 6.78-6.71(\mathrm{~m}, 1 \mathrm{H}), 4.74(\mathrm{dd}, J=16.4,7.8 \mathrm{~Hz}$, 1H), $3.64(\mathrm{t}, J=5.9 \mathrm{~Hz}, 2 \mathrm{H}), 2.09(\mathrm{qd}, J=7.4,1.5 \mathrm{~Hz}, 2 \mathrm{H}), 1.60(\mathrm{ddd}, J=6.9,6.5,4.7 \mathrm{~Hz}, 3 \mathrm{H})$, 1.24 (s, 9H), 0.90 (s, 9H), 0.06 (s, 6H). ${ }^{13} \mathbf{C}$ NMR (101 MHz, CDCl $) \delta 175.59,122.12,110.39$, 62.12, 39.07, 32.39, 27.62, 26.18, 21.86, 18.57, -5.02. ESI-HR calcd for $\mathrm{C}_{16} \mathrm{H}_{34} \mathrm{NO}_{2} \mathrm{Si}^{+}\left([\mathrm{M}+\mathrm{H}]^{+}\right)$ 300.2353, found 300.2348. IR $v\left(\mathrm{~cm}^{-1}\right) 3469,1678,1657,908$. M. P. $74-75^{\circ} \mathrm{C}$. 
<smiles>CC(C)(C)C(=O)N/C=C/CCC[SeH2]</smiles>

Enamide 1k-E: ${ }^{1} \mathbf{H}$ NMR (400 MHz, $\left.\mathrm{CDCl}_{3}\right) \delta 7.04(\mathrm{~d}, J=9.0 \mathrm{~Hz}, 1 \mathrm{H}), 6.82-6.72(\mathrm{~m}, 1 \mathrm{H})$, $5.16(\mathrm{dt}, J=14.2,7.1 \mathrm{~Hz}, 1 \mathrm{H}), 3.61(\mathrm{t}, J=6.4 \mathrm{~Hz}, 2 \mathrm{H}), 2.14-2.03(\mathrm{~m}, 2 \mathrm{H}), 1.64-1.53(\mathrm{~m}, 2 \mathrm{H})$, $1.22(\mathrm{~s}, 9 \mathrm{H}), 0.89$ (m, 9H), $0.04(\mathrm{~m}, 6 \mathrm{H}) .{ }^{13} \mathrm{C}$ NMR $\left(101 \mathrm{MHz}, \mathrm{CDCl}_{3}\right) \delta$ 175.42, 123.21, 112.62, $62.62,38.75,33.18,27.59,26.29,26.10,18.47,-5.13$. ESI-HR calcd for $\mathrm{C}_{16} \mathrm{H}_{34} \mathrm{NO}_{2} \mathrm{Si}^{+}\left([\mathrm{M}+\mathrm{H}]^{+}\right)$ 300.2353, found 300.2349. IR $v\left(\mathrm{~cm}^{-1}\right) 3458,1683,1654,904$. M. P. $78-79^{\circ} \mathrm{C}$.

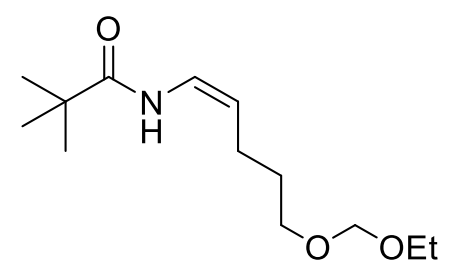

Enamide 1l-Z: ${ }^{1} \mathbf{H}$ NMR $\left(400 \mathrm{MHz}, \mathrm{CDCl}_{3}\right) \delta 7.51(\mathrm{~d}, J=5.6 \mathrm{~Hz}, 1 \mathrm{H}), 6.76(\mathrm{dd}, J=10.4,9.0 \mathrm{~Hz}$, 1H), $4.78-4.69(\mathrm{~m}, 1 \mathrm{H}), 4.68(\mathrm{~s}, 2 \mathrm{H}), 3.63-3.53(\mathrm{~m}, 4 \mathrm{H}), 2.12(\mathrm{td}, J=8.0,1.1 \mathrm{~Hz}, 2 \mathrm{H}), 1.73-$ $1.63(\mathrm{~m}, 2 \mathrm{H}), 1.25-1.18(\mathrm{~m}, 12 \mathrm{H}) .{ }^{13} \mathbf{C}$ NMR $\left(101 \mathrm{MHz}, \mathrm{CDCl}_{3}\right) \delta 175.79,122.95,109.82,94.84$, 65.85, 63.45, 39.02, 28.69, 27.51, 21.91, 15.26. ESI-HR calcd for $\mathrm{C}_{13} \mathrm{H}_{25} \mathrm{NNaO}_{3}{ }^{+}\left([\mathrm{M}+\mathrm{Na}]^{+}\right)$ 266.1727, found 266.1717. IR $v\left(\mathrm{~cm}^{-1}\right) 3366,1684,1653,749$.<smiles>CCOCOCCC/C=C/NC(=O)C(C)(C)C</smiles>

Enamide 1l-E: ${ }^{1} \mathrm{H}$ NMR (400 MHz, $\left.\mathrm{CDCl}_{3}\right) \delta 7.13(\mathrm{~d}, J=8.6 \mathrm{~Hz}, 1 \mathrm{H}), 6.85-6.67(\mathrm{~m}, 1 \mathrm{H}), 5.14$ (dt, $J=14.3,7.2 \mathrm{~Hz}, 1 \mathrm{H}), 4.64(\mathrm{~d}, J=0.9 \mathrm{~Hz}, 2 \mathrm{H}), 3.62-3.47$ (m, 4H), 2.09 (dd, $J=14.5,7.2 \mathrm{~Hz}$, 2H), $1.73-1.56(\mathrm{~m}, 2 \mathrm{H}), 1.24-1.16(\mathrm{~m}, 12 \mathrm{H}) .{ }^{13} \mathbf{C} \mathbf{N M R}\left(101 \mathrm{MHz}, \mathrm{CDCl}_{3}\right) \delta$ 175.44, 123.49, 112.13, 95.20, 67.15, 63.27, 38.73, 30.12, 27.55, 26.59, 15.29. ESI-HR calcd for $\mathrm{C}_{13} \mathrm{H}_{25} \mathrm{NNaO}_{3}{ }^{+}$ $\left([\mathrm{M}+\mathrm{Na}]^{+}\right)$266.1727, found 266.1718. IR $v\left(\mathrm{~cm}^{-1}\right) 3328,1683$, 1653, 733. M. P. 40-41 ${ }^{\circ} \mathrm{C}$. 


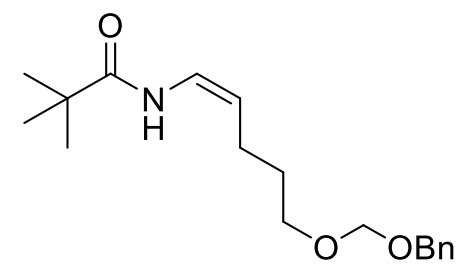

Enamide 1m-Z: ${ }^{1} \mathbf{H}$ NMR $\left(400 \mathrm{MHz}, \mathrm{CDCl}_{3}\right) \delta 7.51(\mathrm{~d}, J=9.2 \mathrm{~Hz}, 1 \mathrm{H}), 7.38-7.28(\mathrm{~m}, 5 \mathrm{H})$, $6.79(\mathrm{ddd}, J=10.5,8.9,1.4 \mathrm{~Hz}, 1 \mathrm{H}), 4.78-4.70(\mathrm{~m}, 3 \mathrm{H}), 4.60(\mathrm{~s}, 2 \mathrm{H}), 3.67-3.59(\mathrm{~m}, 2 \mathrm{H}), 2.19$ $-2.09(\mathrm{~m}, 2 \mathrm{H}), 1.75-1.64(\mathrm{~m}, 6.5 \mathrm{~Hz}, 2 \mathrm{H}), 1.21(\mathrm{~s}, 9 \mathrm{H}) .{ }^{13} \mathrm{C}$ NMR $\left(101 \mathrm{MHz}, \mathrm{CDCl}_{3}\right) \delta 175.80$, $137.78,128.57,128.52,127.91,122.90,109.82,94.33,69.60,66.10,39.02,28.64,27.52,21.94$. ESI-HR calcd for $\mathrm{C}_{18} \mathrm{H}_{28} \mathrm{NO}_{3}{ }^{+}\left([\mathrm{M}+\mathrm{H}]^{+}\right) 306.2064$, found 306.2056. IR $v\left(\mathrm{~cm}^{-1}\right) 3369,1726$, 1663,735 .<smiles>CC(C)(C)C(=O)N/C=C/CCCOCCc1ccccc1</smiles>

Enamide 1m-E: ${ }^{1} \mathbf{H}$ NMR $\left(400 \mathrm{MHz}, \mathrm{CDCl}_{3}\right) \delta 7.35(\mathrm{~d}, J=4.3 \mathrm{~Hz}, 4 \mathrm{H}), 7.29(\mathrm{dd}, J=8.5,4.2 \mathrm{~Hz}$, $1 \mathrm{H}), 7.07(\mathrm{~d}, J=8.0 \mathrm{~Hz}, 1 \mathrm{H}), 6.88-6.70(\mathrm{~m}, 1 \mathrm{H}), 5.15(\mathrm{dt}, J=14.3,7.2 \mathrm{~Hz}, 1 \mathrm{H}), 4.75(\mathrm{~s}, 2 \mathrm{H})$, $4.60(\mathrm{~s}, 2 \mathrm{H}), 3.59(\mathrm{t}, J=6.5 \mathrm{~Hz}, 2 \mathrm{H}), 2.13(\mathrm{dd}, J=14.1,7.0 \mathrm{~Hz}, 2 \mathrm{H}), 1.68(\mathrm{dt}, J=13.7,6.7 \mathrm{~Hz}$, 2H), $1.22(\mathrm{~s}, 9 \mathrm{H}) .{ }^{13} \mathrm{C}$ NMR $\left(101 \mathrm{MHz}, \mathrm{CDCl}_{3}\right) \delta 175.45,138.12,128.54,127.98,127.78,123.52$, 112.09, 94.80, 69.48, 67.39, 38.76, 30.10, 27.57, 26.59. ESI-HR calcd for $\mathrm{C}_{18} \mathrm{H}_{28} \mathrm{NNaO}_{3}{ }^{+}$ $\left([\mathrm{M}+\mathrm{Na}]^{+}\right) 328.1883$, found 328.1869. IR $v\left(\mathrm{~cm}^{-1}\right) 3469,1678,1657,908$. M. P. $44-45^{\circ} \mathrm{C}$.<smiles>CC(C)(C)C(=O)N/C=C\CC1CCCCC1</smiles>

Enamide 1n-Z: ${ }^{1} \mathbf{H}$ NMR $\left(400 \mathrm{MHz}, \mathrm{CDCl}_{3}\right) \delta 7.14(\mathrm{~s}, 1 \mathrm{H}), 6.75(\mathrm{dd}, J=10.5,9.2 \mathrm{~Hz}, 1 \mathrm{H}), 4.77$ $(\mathrm{dd}, J=16.4,8.0 \mathrm{~Hz}, 1 \mathrm{H}), 1.86(\mathrm{dd}, J=10.5,3.8 \mathrm{~Hz}, 2 \mathrm{H}), 1.78-1.62(\mathrm{~m}, 5 \mathrm{H}), 1.41-1.30(\mathrm{~m}$, 1H), $1.24(\mathrm{~s}, 9 \mathrm{H}), 1.23-1.07(\mathrm{~m}, 3 \mathrm{H}), 0.98-0.85(\mathrm{~m}, 2 \mathrm{H}) .{ }^{13} \mathrm{C}$ NMR $\left(101 \mathrm{MHz}, \mathrm{CDCl}_{3}\right) \delta$ 175.41, 121.98, 109.70, 39.06, 38.38, 33.83, 33.37, 27.62, 26.56, 26.43. ESI-HR calcd for $\mathrm{C}_{14} \mathrm{H}_{26} \mathrm{NO}^{+}\left([\mathrm{M}+\mathrm{H}]^{+}\right)$224.2009, found 224.2006. IR $v\left(\mathrm{~cm}^{-1}\right) 3338,1683,1654,733 . \mathbf{M} . \mathbf{P}$. $105-106^{\circ} \mathrm{C}$ 
<smiles>CC(C)(C)C(=O)N/C=C/CC1CCCCC1</smiles>

Enamide 1n-E: ${ }^{1} \mathbf{H}$ NMR (400 MHz, $\left.\mathrm{CDCl}_{3}\right) \delta 7.04(\mathrm{~s}, 1 \mathrm{H}), 6.72(\mathrm{dd}, J=14.1,10.4 \mathrm{~Hz}, 1 \mathrm{H})$, $5.19-5.08(\mathrm{~m}, 1 \mathrm{H}), 1.91(\mathrm{t}, J=7.0 \mathrm{~Hz}, 2 \mathrm{H}), 1.75-1.58(\mathrm{~m}, 5 \mathrm{H}), 1.31-1.28(\mathrm{~m}, 1 \mathrm{H}), 1.22(\mathrm{~s}$, 9H), $1.21-1.05(\mathrm{~m}, 3 \mathrm{H}), 0.88(\mathrm{q}, J=11.5 \mathrm{~Hz}, 2 \mathrm{H}) .{ }^{13} \mathbf{C} \mathbf{N M R}\left(101 \mathrm{MHz}, \mathrm{CDCl}_{3}\right) \delta$ 175.37, 123.60, 111.58, 38.75, 38.58, 37.71, 33.16, 27.60, 26.69, 26.45. ESI-HR calcd for $\mathrm{C}_{14} \mathrm{H}_{26} \mathrm{NO}^{+}$ $\left([\mathrm{M}+\mathrm{H}]^{+}\right) 224.2009$, found 224.2004. IR $v\left(\mathrm{~cm}^{-1}\right) 3285,1653$, 1636, 734. M. P. 136-137 ${ }^{\circ} \mathrm{C}$.<smiles>CC(C)/C=C\NC(=O)C(C)(C)C</smiles>

Enamide 1o-Z: ${ }^{1} \mathbf{H}$ NMR $\left(400 \mathrm{MHz}, \mathrm{CDCl}_{3}\right) \delta 7.14(\mathrm{~s}, 1 \mathrm{H}), 6.65-6.58(\mathrm{~m}, 1 \mathrm{H}), 4.62(\mathrm{t}, J=9.1$ $\mathrm{Hz}, 1 \mathrm{H}), 2.48-2.33(\mathrm{~m}, 1 \mathrm{H}), 1.24(\mathrm{~s}, 9 \mathrm{H}), 1.03(\mathrm{~d}, J=6.7 \mathrm{~Hz}, 6 \mathrm{H}) .{ }^{13} \mathbf{C} \mathbf{~ N M R}\left(101 \mathrm{MHz}, \mathrm{CDCl}_{3}\right)$ $\delta 175.41,119.49,118.73,39.07,27.61,25.95,23.01$. ESI-HR calcd for $\mathrm{C}_{10} \mathrm{H}_{20} \mathrm{NO}^{+}\left([\mathrm{M}+\mathrm{H}]^{+}\right)$ 170.1539, found 170.1536. IR $v\left(\mathrm{~cm}^{-1}\right) 3469,1682,1659,733$. M. P. $103-104{ }^{\circ} \mathrm{C}$.<smiles>CC(C)/C=C/NC(=O)C(C)(C)C</smiles>

Enamide 1o-E: ${ }^{1} \mathbf{H}$ NMR $\left(400 \mathrm{MHz}, \mathrm{CDCl}_{3}\right) \delta 7.01(\mathrm{~s}, 1 \mathrm{H}), 6.80-6.68(\mathrm{~m}, 1 \mathrm{H}), 5.14(\mathrm{dd}, J=$ 14.3, 6.9 Hz, 1H), $2.44-2.25(\mathrm{~m}, 1 \mathrm{H}), 1.22(\mathrm{~s}, 9 \mathrm{H}), 1.01(\mathrm{~d}, J=6.7 \mathrm{~Hz}, 6 \mathrm{H}) .{ }^{13} \mathbf{C}$ NMR $(101$ $\left.\mathrm{MHz}, \mathrm{CDCl}_{3}\right) \delta 175.55,121.03,120.41,38.77,29.08,27.60,23.03$. ESI-HR calcd for $\mathrm{C}_{10} \mathrm{H}_{20} \mathrm{NO}^{+}$ $\left([\mathrm{M}+\mathrm{H}]^{+}\right) 170.1539$, found $170.1536 . \mathbf{I R} v\left(\mathrm{~cm}^{-1}\right) 3284,1683,1636,733$. M. P. $132-133{ }^{\circ} \mathrm{C}$.<smiles>C=CNC(=O)C(C)(C)C</smiles>

Enamide 1p: ${ }^{1} \mathbf{H}$ NMR $\left(400 \mathrm{MHz}, \mathrm{CDCl}_{3}\right) \delta 7.24(\mathrm{~s}, 1 \mathrm{H}), 7.00(\mathrm{ddd}, J=15.8,10.7,8.7 \mathrm{~Hz}, 1 \mathrm{H})$, $4.62(\mathrm{~d}, J=15.8 \mathrm{~Hz}, 1 \mathrm{H}), 4.41(\mathrm{~d}, J=8.7 \mathrm{~Hz}, 1 \mathrm{H}), 1.24(\mathrm{~s}, 9 \mathrm{H}) .{ }^{13} \mathbf{C} \mathbf{N M R}\left(101 \mathrm{MHz}, \mathrm{CDCl}_{3}\right) \delta$ 
175.87, 129.26, 95.08, 38.83, 27.51. ESI-HR calcd for $\mathrm{C}_{7} \mathrm{H}_{14} \mathrm{NO}^{+}\left([\mathrm{M}+\mathrm{H}]^{+}\right) 128.1070$, found 128.1089. IR $v\left(\mathrm{~cm}^{-1}\right) 3284,1660,1631,866$. M. P. $101-102^{\circ} \mathrm{C}$.<smiles>C/C(=C\NC(=O)C(C)(C)C)Cc1ccccc1</smiles>

Enamide 1q- $\boldsymbol{E}:{ }^{1} \mathbf{H}$ NMR $\left(400 \mathrm{MHz}, \mathrm{CDCl}_{3}\right) \delta 7.36-7.26(\mathrm{~m}, 2 \mathrm{H}), 7.22(\mathrm{t}, J=7.6 \mathrm{~Hz}, 3 \mathrm{H}), 7.04$ (d, $J=8.5 \mathrm{~Hz}, 1 \mathrm{H}), 6.77(\mathrm{~d}, J=10.3 \mathrm{~Hz}, 1 \mathrm{H}), 3.34(\mathrm{~s}, 2 \mathrm{H}), 1.56(\mathrm{~s}, 3 \mathrm{H}), 1.27$ (s, 9H). ${ }^{13} \mathbf{C} \mathbf{N M R}$ $\left(101 \mathrm{MHz}, \mathrm{CDCl}_{3}\right) \delta 175.30,139.58,128.82,128.35,126.24,118.76,117.64,43.09,38.90,27.51$, 14.37. ESI-HR calcd for $\mathrm{C}_{15} \mathrm{H}_{23} \mathrm{NO}^{+}\left([\mathrm{M}+\mathrm{H}]^{+}\right) 232.1696$, found 232.1691. IR $v\left(\mathrm{~cm}^{-1}\right) 3469,2984$, 1664, 732. M. P. $75-76^{\circ} \mathrm{C}$.<smiles>C/C(=C\NC(=O)C(C)(C)C)CCc1ccccc1</smiles>

Enamide 1r-E: ${ }^{1} \mathbf{H}$ NMR (400 MHz, $\left.\mathrm{CDCl}_{3}\right) \delta 7.37-7.26(\mathrm{~m}, 2 \mathrm{H}), 7.21(\mathrm{dd}, J=7.0,5.0 \mathrm{~Hz}, 3 \mathrm{H})$, $7.01(\mathrm{~d}, J=9.5 \mathrm{~Hz}, 1 \mathrm{H}), 6.66(\mathrm{dd}, J=10.3,1.2 \mathrm{~Hz}, 1 \mathrm{H}), 2.75(\mathrm{dd}, J=9.6,6.8 \mathrm{~Hz}, 2 \mathrm{H}), 2.41-$ $2.29(\mathrm{~m}, 2 \mathrm{H}), 1.69(\mathrm{~d}, J=1.1 \mathrm{~Hz}, 3 \mathrm{H}), 1.27(\mathrm{~s}, 9 \mathrm{H}) .{ }^{13} \mathbf{C} \mathbf{~ N M R}\left(101 \mathrm{MHz}, \mathrm{CDCl}_{3}\right) \delta$ 175.17, $141.88,128.37,128.31,125.87,117.95,117.83,38.88,38.75,34.84,27.52,14.83$. ESI-HR calcd for $\mathrm{C}_{16} \mathrm{H}_{24} \mathrm{NO}^{+}\left([\mathrm{M}+\mathrm{H}]^{+}\right)$246.1852, found 246.1845. IR $v\left(\mathrm{~cm}^{-1}\right) 3470,2964,1662$, 732 . M. P. $89-90{ }^{\circ} \mathrm{C}$.<smiles>CCCC/C(C)=C/NC(=O)C(C)(C)C</smiles>

Enamide 1s-E: ${ }^{1} \mathbf{H}$ NMR $\left(400 \mathrm{MHz}, \mathrm{CDCl}_{3}\right) \delta 6.99(\mathrm{~d}, J=7.8 \mathrm{~Hz}, 1 \mathrm{H}), 6.58(\mathrm{dd}, J=10.3,1.1 \mathrm{~Hz}$, 1H), $2.02(\mathrm{t}, J=7.4 \mathrm{~Hz}, 2 \mathrm{H}), 1.62(\mathrm{~d}, J=1.1 \mathrm{~Hz}, 3 \mathrm{H}), 1.40(\mathrm{ddd}, J=11.5,8.5,5.7 \mathrm{~Hz}, 2 \mathrm{H}), 1.32$ $(\mathrm{dd}, J=14.5,7.4 \mathrm{~Hz}, 2 \mathrm{H}), 1.27(\mathrm{~d}, J=6.5 \mathrm{~Hz}, 9 \mathrm{H}), 0.91(\mathrm{t}, J=7.2 \mathrm{~Hz}, 3 \mathrm{H}) .{ }^{13} \mathbf{C} \mathbf{N M R}(101 \mathrm{MHz}$, $\left.\mathrm{CDCl}_{3}\right) \delta 175.06,118.77,117.19,38.84,36.29,30.09,27.52,22.28,14.57,13.96$. ESI-HR calcd for $\mathrm{C}_{12} \mathrm{H}_{24} \mathrm{NO}^{+}\left([\mathrm{M}+\mathrm{H}]^{+}\right)$198.1852, found 198.1848. IR $v\left(\mathrm{~cm}^{-1}\right) 3471,2927,1662,733$. 
<smiles>CCCCCCCC/C(C)=C/NC(=O)C(C)(C)C</smiles>

Enamide 1t-E: ${ }^{1} \mathbf{H} \mathbf{N M R}\left(400 \mathrm{MHz}, \mathrm{CDCl}_{3}\right) \delta 6.99(\mathrm{~d}, J=9.4 \mathrm{~Hz}, 1 \mathrm{H}), 6.58(\mathrm{~d}, J=10.3 \mathrm{~Hz}, 1 \mathrm{H})$, $2.01(\mathrm{t}, J=7.5 \mathrm{~Hz}, 2 \mathrm{H}), 1.63(\mathrm{~d}, J=4.1 \mathrm{~Hz}, 3 \mathrm{H}), 1.42(\mathrm{dd}, J=13.6,7.0 \mathrm{~Hz}, 2 \mathrm{H}), 1.35-1.27(\mathrm{~s}$, 9H), $1.26(\mathrm{~m}, 10 \mathrm{H}), 0.90(\mathrm{t}, J=6.6 \mathrm{~Hz}, 3 \mathrm{H}) .{ }^{13} \mathrm{C}$ NMR $\left(101 \mathrm{MHz}, \mathrm{CDCl}_{3}\right) \delta 175.06,118.83$, 117.17, 38.84, 36.64, 31.89, 29.49, 29.27, 27.94, 27.52, 22.68, 14.58, 14.11. ESI-HR calcd for $\mathrm{C}_{16} \mathrm{H}_{32} \mathrm{NO}^{+}\left([\mathrm{M}+\mathrm{H}]^{+}\right)$254.2478, found 254.2471. IR $v\left(\mathrm{~cm}^{-1}\right) 3469,2984,1664,732$.<smiles>CC(C)=CNC(=O)C(C)(C)C</smiles>

Enamide 1u: ${ }^{1} \mathbf{H}$ NMR(400 MHz, $\left.\mathrm{CDCl}_{3}\right) \delta 6.97(\mathrm{~s}, 1 \mathrm{H}), 6.55(\mathrm{dd}, J=10.2,1.2 \mathrm{~Hz}, 1 \mathrm{H}), 1.73(\mathrm{~s}$, 3H), $1.64(\mathrm{~s}, 3 \mathrm{H}), 1.25$ (s, 9H). ${ }^{13} \mathrm{C}$ NMR (101 MHz, $\left.\mathrm{CDCl}_{3}\right) \delta$ 175.07, 117.27, 114.97, 38.81, 27.52, 22.42, 16.39. ESI-HR calcd for $\mathrm{C}_{9} \mathrm{H}_{18} \mathrm{NO}^{+}\left([\mathrm{M}+\mathrm{H}]^{+}\right)$156.1383, found 156.1380. IR v $\left(\mathrm{cm}^{-1}\right) 3471,2966,1658,733$. M. P. $73-74{ }^{\circ} \mathrm{C}$.<smiles>CC(C)(C)C(=O)NC=C1CCOCC1</smiles>

Enamide 1v: ${ }^{1} \mathbf{H}$ NMR $\left(400 \mathrm{MHz}, \mathrm{CDCl}_{3}\right) \delta 7.01(\mathrm{~s}, 1 \mathrm{H}), 6.64(\mathrm{~d}, J=10.2 \mathrm{~Hz}, 1 \mathrm{H}), 3.70(\mathrm{t}, J=$ $5.5 \mathrm{~Hz}, 4 \mathrm{H}), 2.23(\mathrm{dt}, J=17.6,5.2 \mathrm{~Hz}, 4 \mathrm{H}), 1.26(\mathrm{~s}, 9 \mathrm{H}) .{ }^{13} \mathrm{C}$ NMR $\left(101 \mathrm{MHz}, \mathrm{CDCl}_{3}\right) \delta 175.42$, 117.50, 115.99, 69.24, 67.55, 38.92, 33.19, 28.24, 27.47. ESI-HR calcd for $\mathrm{C}_{11} \mathrm{H}_{20} \mathrm{NO}^{+}\left([\mathrm{M}+\mathrm{H}]^{+}\right)$ 198.1489, found 252.1377. IR $v\left(\mathrm{~cm}^{-1}\right) 3468,2962,1654,733$. M. P. $128-129^{\circ} \mathrm{C}$.<smiles>CC/C=C(/C)NC(=O)C(C)(C)C</smiles>

Enamide 1w-Z: ${ }^{1} \mathbf{H}$ NMR $\left(400 \mathrm{MHz}, \mathrm{CDCl}_{3}\right) \delta 6.68(\mathrm{~s}, 1 \mathrm{H}), 4.88(\mathrm{t}, J=6.9 \mathrm{~Hz}, 1 \mathrm{H}), 2.02(\mathrm{~d}, J=$ $0.8 \mathrm{~Hz}, 3 \mathrm{H}), 1.94(\mathrm{dd}, J=14.6,7.3 \mathrm{~Hz}, 2 \mathrm{H}), 1.25(\mathrm{~s}, 9 \mathrm{H}), 0.99(\mathrm{t}, J=7.5 \mathrm{~Hz}, 3 \mathrm{H}) .{ }^{13} \mathbf{C}$ NMR $(101$ $\left.\mathrm{MHz}, \mathrm{CDCl}_{3}\right) \delta 176.42,130.89,120.69,39.32,27.71,21.24,19.96,13.73$. ESI-HR calcd for 
$\mathrm{C}_{10} \mathrm{H}_{20} \mathrm{NO}^{+}\left([\mathrm{M}+\mathrm{H}]^{+}\right) 170.1539$, found 170.1534. IR $v\left(\mathrm{~cm}^{-1}\right) 3450,2962,1651$, 735. M. P. $67-68$ ${ }^{\circ} \mathrm{C}$.<smiles>CCC/C=C(\CC)NC(=O)C(C)(C)C</smiles>

Enamide 1x-E: ${ }^{1} \mathbf{H}$ NMR $\left(400 \mathrm{MHz}, \mathrm{CDCl}_{3}\right) \delta 6.52(\mathrm{~s}, 1 \mathrm{H}), 5.73(\mathrm{t}, J=7.5 \mathrm{~Hz}, 1 \mathrm{H}), 2.30(\mathrm{q}, J=$ $7.5 \mathrm{~Hz}, 2 \mathrm{H}), 2.05(\mathrm{q}, J=7.4 \mathrm{~Hz}, 2 \mathrm{H}), 1.49-1.32(\mathrm{~m}, 2 \mathrm{H}), 1.25(\mathrm{~s}, 9 \mathrm{H}), 1.04(\mathrm{t}, J=7.5 \mathrm{~Hz}, 3 \mathrm{H})$, $0.94(\mathrm{t}, J=7.4 \mathrm{~Hz}, 3 \mathrm{H}) .{ }^{13} \mathrm{C}$ NMR $\left(101 \mathrm{MHz}, \mathrm{CDCl}_{3}\right) \delta 176.80,135.63,117.69,39.37,29.05$, 27.69, 23.15, 22.98, 13.86, 12.57. ESI-HR calcd for $\mathrm{C}_{12} \mathrm{H}_{24} \mathrm{NO}^{+}\left([\mathrm{M}+\mathrm{H}]^{+}\right) 198.1852$, found 198.1850. IR $v\left(\mathrm{~cm}^{-1}\right) 3459,2967,1653,732$.<smiles>C=C(C)NC(=O)C(C)(C)C</smiles>

Enamide 1y': ${ }^{1} \mathrm{H}$ NMR (400 MHz, $\left.\mathrm{CDCl}_{3}\right) \delta 6.63(\mathrm{~s}, 1 \mathrm{H}), 5.44(\mathrm{~s}, 1 \mathrm{H}), 4.47(\mathrm{~s}, 1 \mathrm{H}), 1.95(\mathrm{~s}, 3 \mathrm{H})$, $1.25(\mathrm{~s}, 9 \mathrm{H})$.<smiles>CC(C)(C)C(=O)N/C=C\CCc1ccc(F)cc1</smiles>

Enamide 1z-Z: ${ }^{1}$ H NMR $\left(400 \mathrm{MHz}, \mathrm{CDCl}_{3}\right) \delta 7.23-7.12(\mathrm{~m}, 2 \mathrm{H}), 7.10-6.93(\mathrm{~m}, 3 \mathrm{H}), 6.72(\mathrm{dd}$, $J=10.5,9.2 \mathrm{~Hz}, 1 \mathrm{H}), 4.80(\mathrm{dd}, J=16.4,8.0 \mathrm{~Hz}, 1 \mathrm{H}), 2.75(\mathrm{t}, J=7.5 \mathrm{~Hz}, 2 \mathrm{H}), 2.30(\mathrm{q}, J=7.6 \mathrm{~Hz}$, 2H), $1.18(\mathrm{~s}, 9 \mathrm{H}) .{ }^{13} \mathbf{C}$ NMR $\left(101 \mathrm{MHz}, \mathrm{CDCl}_{3}\right) \delta 175.56,161.35\left(\mathrm{~d}, J_{1}=245.62 \mathrm{~Hz}\right), 131.03(\mathrm{~d}$, $\left.J_{3}=5.23 \mathrm{~Hz}\right), 128.33\left(\mathrm{~d}, J_{2}=15.62 \mathrm{~Hz}\right), 128.12\left(\mathrm{~d}, J_{3}=8.29 \mathrm{~Hz}\right), 124.28\left(\mathrm{~d}, J_{4}=3.6 \mathrm{~Hz}\right), 122.48$, $115.45\left(\mathrm{~d}, J_{2}=21.95 \mathrm{~Hz}\right), 109.45,39.01,29.22\left(\mathrm{~d}, J_{5}=1.75 \mathrm{~Hz}\right), 27.49,26.71$. ESI-HR calcd for $\mathrm{C}_{15} \mathrm{H}_{21} \mathrm{FNO}^{+}\left([\mathrm{M}+\mathrm{H}]^{+}\right)$250.1602, found 250.1593. IR $v\left(\mathrm{~cm}^{-1}\right) 3343,2964,1678,1648$, 735. M. P. $85-87^{\circ} \mathrm{C}$. 
<smiles>CC(C)(C)C(=O)N/C=C/CCc1ccc(F)cc1</smiles>

Enamide 1z-E: ${ }^{1} \mathbf{H}$ NMR $\left(400 \mathrm{MHz}, \mathrm{CDCl}_{3}\right) \delta 7.21-7.08(\mathrm{~m}, 3 \mathrm{H}), 7.08-6.95(\mathrm{~m}, 2 \mathrm{H}), 6.88-$ $6.75(\mathrm{~m}, 1 \mathrm{H}), 5.17(\mathrm{dt}, J=14.2,7.1 \mathrm{~Hz}, 1 \mathrm{H}), 2.71(\mathrm{t}, J=7.7 \mathrm{~Hz}, 2 \mathrm{H}), 2.40-2.27(\mathrm{~m}, 2 \mathrm{H}), 1.21(\mathrm{~s}$, 9H). ${ }^{13}$ C NMR (101 MHz, $\left.\mathrm{CDCl}_{3}\right) \delta 175.57,162.25\left(\mathrm{~d}, J_{1}=246.76 \mathrm{~Hz}\right), 130.73\left(\mathrm{~d}, J_{3}=4.99 \mathrm{~Hz}\right)$, $128.49\left(\mathrm{~d}, J_{2}=16.46 \mathrm{~Hz}\right), 127.76\left(\mathrm{~d}, J_{3}=7.99 \mathrm{~Hz}\right), 124.03\left(\mathrm{~d}, J_{4}=3.64 \mathrm{~Hz}\right), 123.74,115.33\left(\mathrm{~d}, J_{2}\right.$ $=22.13 \mathrm{~Hz}), 111.71,38.75,30.46,29.73\left(\mathrm{~d}, J_{5}=1.83 \mathrm{~Hz}\right), 27.54$. ESI-HR calcd for $\mathrm{C}_{15} \mathrm{H}_{21} \mathrm{FNO}^{+}$ $\left([\mathrm{M}+\mathrm{H}]^{+}\right) 250.1602$, found 250.1592. IR $v\left(\mathrm{~cm}^{-1}\right) 3299,2964,1678,1636,750$. M. P. $117-119^{\circ} \mathrm{C}$.<smiles>CC(C)(C)C(=O)N/C=C\CCc1ccc(Cl)cc1</smiles>

Enamide 1aa-Z: ${ }^{1} \mathbf{H}$ NMR $\left(400 \mathrm{MHz}, \mathrm{CDCl}_{3}\right) \delta 7.29-7.25(\mathrm{~m}, 2 \mathrm{H}), 7.14(\mathrm{~d}, J=8.3 \mathrm{~Hz}, 2 \mathrm{H})$, $6.83(\mathrm{~s}, 1 \mathrm{H}), 6.72(\mathrm{dd}, J=10.4,9.1 \mathrm{~Hz}, 1 \mathrm{H}), 4.77(\mathrm{dd}, J=16.2,7.8 \mathrm{~Hz}, 1 \mathrm{H}), 2.70(\mathrm{t}, J=7.2 \mathrm{~Hz}$, 2H), $2.33-2.23$ (m, 2H), 1.15 (s, 9H). ${ }^{13} \mathbf{C}$ NMR (101 MHz, CDCl $) \delta 175.53,140.03,132.16$, 129.99, 128.77, 122.46, 109.28, 38.95, 34.87, 28.15, 27.45. ESI-HR calcd for $\mathrm{C}_{15} \mathrm{H}_{21} \mathrm{ClNO}^{+}$ $\left([\mathrm{M}+\mathrm{H}]^{+}\right)$266.1306, found 266.1299. IR $v\left(\mathrm{~cm}^{-1}\right) 3463,2965,1679,1658,734$. M. P. $66-68{ }^{\circ} \mathrm{C}$.<smiles>CC(C)(C)C(=O)N/C=C/CCc1ccc(Cl)cc1</smiles>

Enamide 1aa-E: ${ }^{1} \mathbf{H}$ NMR $\left(400 \mathrm{MHz}, \mathrm{CDCl}_{3}\right) \delta 7.30-7.19(\mathrm{~m}, 2 \mathrm{H}), 7.16-6.98(\mathrm{~m}, 3 \mathrm{H}), 6.88-$ $6.72(\mathrm{~m}, 1 \mathrm{H}), 5.13(\mathrm{dt}, J=14.2,7.1 \mathrm{~Hz}, 1 \mathrm{H}), 2.66(\mathrm{t}, J=7.6 \mathrm{~Hz}, 2 \mathrm{H}), 2.33(\mathrm{dd}, J=14.6,7.3 \mathrm{~Hz}$, 2H), 1.21 (s, 9H). ${ }^{13} \mathbf{C}$ NMR (101 MHz, $\left.\mathrm{CDCl}_{3}\right) \delta 175.58,140.10,131.77,129.90,128.58,123.81$, 111.47, 38.78, 35.85, 31.53, 27.56. ESI-HR calcd for $\mathrm{C}_{15} \mathrm{H}_{21} \mathrm{ClNO}^{+}\left([\mathrm{M}+\mathrm{H}]^{+}\right) 266.1306$, found 266.1296. IR $v\left(\mathrm{~cm}^{-1}\right) 3276,2965,1634,732$. M. P. $160-162{ }^{\circ} \mathrm{C}$. 
<smiles>Cc1ccc(CC/C=C\NC(=O)C(C)(C)C)cc1</smiles>

Enamide 1ab-Z: ${ }^{1} \mathrm{H}$ NMR $\left(400 \mathrm{MHz}, \mathrm{CDCl}_{3}\right) \delta 7.14-7.04(\mathrm{~m}, 4 \mathrm{H}), 6.80(\mathrm{~s}, 1 \mathrm{H}), 6.68(\mathrm{t}, J=9.7$ $\mathrm{Hz}, 1 \mathrm{H}), 4.80(\mathrm{q}, J=7.9 \mathrm{~Hz}, 1 \mathrm{H}), 2.68(\mathrm{t}, J=7.2 \mathrm{~Hz}, 2 \mathrm{H}), 2.34-2.23(\mathrm{~m}, 5 \mathrm{H}), 1.11(\mathrm{~s}, 9 \mathrm{H}) .{ }^{13} \mathrm{C}$ NMR $\left(101 \mathrm{MHz}, \mathrm{CDCl}_{3}\right) \delta 175.54,138.61,135.78,129.39,128.54,122.26,110.01,38.89,35.09$, 28.51, 27.42, 21.10. ESI-HR calcd for $\mathrm{C}_{16} \mathrm{H}_{24} \mathrm{NO}^{+}\left([\mathrm{M}+\mathrm{H}]^{+}\right)$246.1852, found 246.1844. IR v $\left(\mathrm{cm}^{-1}\right) 3463,2965,1659,749$. M. P. $48-50{ }^{\circ} \mathrm{C}$.<smiles>Cc1ccc(CC/C=C/NC(=O)C(C)(C)C)cc1</smiles>

Enamide 1ab-E: ${ }^{1} \mathbf{H}$ NMR $\left(400 \mathrm{MHz}, \mathrm{CDCl}_{3}\right) \delta 7.16-6.95(\mathrm{~m}, 5 \mathrm{H}), 6.86-6.77(\mathrm{~m}, 1 \mathrm{H}), 5.16$ $(\mathrm{dt}, J=14.2,7.1 \mathrm{~Hz}, 1 \mathrm{H}), 2.65(\mathrm{t}, J=7.4,2 \mathrm{H}), 2.40-2.27(\mathrm{~m}, 5 \mathrm{H}), 1.21(\mathrm{~s}, 9 \mathrm{H}) .{ }^{13} \mathrm{C}$ NMR $(101$ $\left.\mathrm{MHz}, \mathrm{CDCl}_{3}\right) \delta 175.51,138.64,135.48,129.18,128.42,123.48,112.12,38.77,36.09,31.73$, 27.58, 21.14. ESI-HR calcd for $\mathrm{C}_{16} \mathrm{H}_{24} \mathrm{NO}^{+}\left([\mathrm{M}+\mathrm{H}]^{+}\right)$246.1852, found 246.1844. IR $v\left(\mathrm{~cm}^{-1}\right)$ 3450, 2984, 1659, 732. M.P. $141-143{ }^{\circ} \mathrm{C} . \mathrm{v}$<smiles>COc1ccc(CC/C=C\NC(=O)C(C)(C)C)cc1</smiles>

Enamide 1ac-Z: ${ }^{1} \mathbf{H}$ NMR $\left(400 \mathrm{MHz}, \mathrm{CDCl}_{3}\right) \delta 7.11(\mathrm{~d}, J=8.6 \mathrm{~Hz}, 2 \mathrm{H}), 6.89-6.77(\mathrm{~m}, 3 \mathrm{H})$, $6.69(\mathrm{dd}, J=10.4,9.0 \mathrm{~Hz}, 1 \mathrm{H}), 4.79(\mathrm{dd}, J=16.2,7.8 \mathrm{~Hz}, 1 \mathrm{H}), 3.77$ (s, 3H), 2.66 (t, $J=7.2 \mathrm{~Hz}$, 2H), $2.26(\mathrm{qd}, J=7.4,1.1 \mathrm{~Hz}, 2 \mathrm{H}), 1.12(\mathrm{~s}, 9 \mathrm{H}) .{ }^{13} \mathbf{C}$ NMR $\left(101 \mathrm{MHz}, \mathrm{CDCl}_{3}\right) \delta$ 175.54, 158.19, 133.69, 129.55, 122.25, 114.12, 109.95, 55.34, 38.91, 34.61, 28.58, 27.46. ESI-HR calcd for 
$\mathrm{C}_{16} \mathrm{H}_{24} \mathrm{NO}_{2}{ }^{+}\left([\mathrm{M}+\mathrm{H}]^{+}\right)$262.1802, found 262.1793. IR $v\left(\mathrm{~cm}^{-1}\right) 3455,2961,1656,734$. M. P. 59-61 ${ }^{\circ} \mathrm{C}$.<smiles>COc1ccc(CC/C=C/NC(=O)C(C)(C)C)cc1</smiles>

Enamide 1ac-Z: ${ }^{1} \mathbf{H}$ NMR $\left(400 \mathrm{MHz}, \mathrm{CDCl}_{3}\right) \delta 7.09(\mathrm{~d}, J=8.5 \mathrm{~Hz}, 3 \mathrm{H}), 6.90-6.75(\mathrm{M}, 3 \mathrm{H})$, $5.16(\mathrm{dt}, J=14.2,7.1 \mathrm{~Hz}, 1 \mathrm{H}), 3.79(\mathrm{~s}, 3 \mathrm{H}), 2.63(\mathrm{t}, J=7.7 \mathrm{~Hz}, 2 \mathrm{H}), 2.32(\mathrm{q}, J=7.4 \mathrm{~Hz}, 2 \mathrm{H}), 1.21$ (s, 9H). ${ }^{13} \mathbf{C}$ NMR $\left(101 \mathrm{MHz}, \mathrm{CDCl}_{3}\right) \delta 175.52,157.96,133.80,129.41,123.48,113.91,112.09$, 55.38, 38.75, 35.60, 31.89, 27.56. ESI-HR calcd for $\mathrm{C}_{16} \mathrm{H}_{24} \mathrm{NO}_{2}{ }^{+}\left([\mathrm{M}+\mathrm{H}]^{+}\right)$262.1802, found 262.1792. IR $v\left(\mathrm{~cm}^{-1}\right) 3276,2989,1637,750$. M. P. $127-129^{\circ} \mathrm{C}$.<smiles>CSc1ccc(CC/C=C\NC(=O)C(C)(C)C)cc1</smiles>

Enamide 1ad-Z: ${ }^{1} \mathrm{H}$ NMR $\left(400 \mathrm{MHz}, \mathrm{CDCl}_{3}\right) \delta 7.19(\mathrm{~d}, J=8.2 \mathrm{~Hz}, 2 \mathrm{H}), 7.12(\mathrm{~d}, J=8.2 \mathrm{~Hz}, 2 \mathrm{H})$, $6.82(\mathrm{~s}, 1 \mathrm{H}), 6.69(\mathrm{dd}, J=10.3,9.2 \mathrm{~Hz}, 1 \mathrm{H}), 4.78(\mathrm{dd}, J=16.2,7.9 \mathrm{~Hz}, 1 \mathrm{H}), 2.67(\mathrm{t}, J=7.2 \mathrm{~Hz}$, 2H), $2.45(\mathrm{~s}, 3 \mathrm{H}), 2.27(\mathrm{q}, J=7.2 \mathrm{~Hz}, 2 \mathrm{H}), 1.12(\mathrm{~s}, 9 \mathrm{H}) .{ }^{13} \mathrm{C} \mathrm{NMR}\left(101 \mathrm{MHz}, \mathrm{CDCl}_{3}\right) \delta 175.55$, 138.61, 136.12, 129.17, 127.24, 122.33, 109.69, 38.92, 34.97, 28.29, 27.45, 16.23. ESI-HR calcd for $\mathrm{C}_{16} \mathrm{H}_{24} \mathrm{NOS}^{+}\left([\mathrm{M}+\mathrm{H}]^{+}\right)$278.1573, found 278.1564. IR $v\left(\mathrm{~cm}^{-1}\right)$ 3351, 2921, 1650, 741. M. P. $81-83^{\circ} \mathrm{C}$.<smiles>Cc1ccc(CC/C=C/NC(=O)C(C)(C)C)cc1</smiles>

Enamide 1ad-E: ${ }^{1} \mathbf{H}$ NMR $\left(400 \mathrm{MHz}, \mathrm{CDCl}_{3}\right) \delta 7.20(\mathrm{~d}, J=8.2 \mathrm{~Hz}, 2 \mathrm{H}), 7.10(\mathrm{~d}, J=8.2 \mathrm{~Hz}, 2 \mathrm{H})$, $7.03(\mathrm{~s}, 1 \mathrm{H}), 6.87-6.75(\mathrm{~m}, 1 \mathrm{H}), 5.14(\mathrm{dt}, J=14.2,7.1 \mathrm{~Hz}, 1 \mathrm{H}), 2.65(\mathrm{t}, J=7.3 \mathrm{~Hz}, 2 \mathrm{H}), 2.47(\mathrm{~s}$, 3H), $2.33(\mathrm{dd}, J=14.9,7.4 \mathrm{~Hz}, 2 \mathrm{H}), 1.21(\mathrm{~s}, 9 \mathrm{H}) .{ }^{13} \mathrm{C} \mathbf{N M R}\left(101 \mathrm{MHz}, \mathrm{CDCl}_{3}\right) \delta$ 175.54, 138.85, 
135.58, 129.11, 127.34, 123.64, 111.81, 38.78, 35.97, 31.59, 27.58, 16.49. ESI-HR calcd for $\mathrm{C}_{16} \mathrm{H}_{24} \mathrm{NOS}^{+}\left([\mathrm{M}+\mathrm{H}]^{+}\right)$278.1573, found 278.1563. IR $v\left(\mathrm{~cm}^{-1}\right)$ 3276, 2967, 1680, 1636, 750. M. P. $156-158^{\circ} \mathrm{C}$.

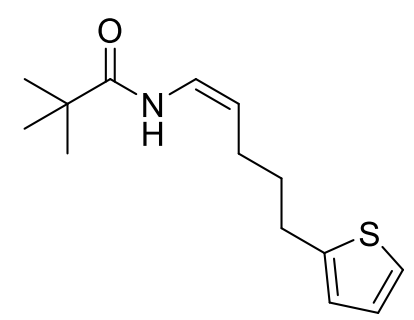

Enamide 1ae-Z: ${ }^{1} \mathbf{H}$ NMR $\left(400 \mathrm{MHz}, \mathrm{CDCl}_{3}\right) \delta 7.13(\mathrm{dd}, J=5.1,1.0 \mathrm{~Hz}, 1 \mathrm{H}), 7.04(\mathrm{~s}, 1 \mathrm{H}), 6.92$ $(\mathrm{dd}, J=5.1,3.4 \mathrm{~Hz}, 1 \mathrm{H}), 6.81-6.71(\mathrm{~m}, 2 \mathrm{H}), 4.74(\mathrm{dd}, J=16.3,7.6 \mathrm{~Hz}, 1 \mathrm{H}), 2.87(\mathrm{t}, J=7.2 \mathrm{~Hz}$, 2H), $2.10-1.98(\mathrm{~m}, 2 \mathrm{H}), 1.81(\mathrm{p}, J=7.2 \mathrm{~Hz}, 2 \mathrm{H}), 1.20(\mathrm{~s}, 9 \mathrm{H}) .{ }^{13} \mathbf{C}$ NMR $\left(101 \mathrm{MHz}, \mathrm{CDCl}_{3}\right) \delta$ 175.56, 144.79, 126.94, 124.60, 123.28, 122.13, 110.16, 39.06, 31.03, 29.06, 27.56, 24.72. ESI-HR calcd for $\mathrm{C}_{14} \mathrm{H}_{22} \mathrm{NOS}^{+}\left([\mathrm{M}+\mathrm{H}]^{+}\right)$252.1417, found 252.1408. IR $v\left(\mathrm{~cm}^{-1}\right) 3464,2934$, $1679,1658,733$. M. P. $86-88^{\circ} \mathrm{C}$.

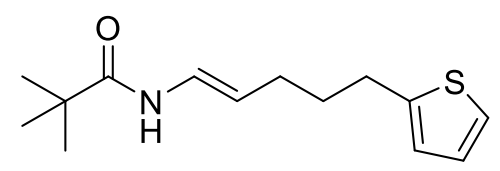

Enamide 1ae-E: ${ }^{1} \mathbf{H}$ NMR $\left(400 \mathrm{MHz}, \mathrm{CDCl}_{3}\right) \delta 7.10(\mathrm{dd}, J=5.1,0.6 \mathrm{~Hz}, 2 \mathrm{H}), 6.91(\mathrm{dd}, J=5.1$, $3.5 \mathrm{~Hz}, 1 \mathrm{H}), 6.84-6.74(\mathrm{~m}, 2 \mathrm{H}), 5.16(\mathrm{dt}, J=14.3,7.2 \mathrm{~Hz}, 1 \mathrm{H}), 2.83(\mathrm{t}, J=7.6 \mathrm{~Hz}, 2 \mathrm{H}), 2.10(\mathrm{q}$, $J=7.1 \mathrm{~Hz}, 2 \mathrm{H}), 1.81-1.70(\mathrm{~m}, 2 \mathrm{H}), 1.22(\mathrm{~s}, 9 \mathrm{H}) .{ }^{13} \mathbf{C} \mathbf{~ N M R}\left(101 \mathrm{MHz}, \mathrm{CDCl}_{3}\right) \delta 175.50,145.27$, 126.83, 124.27, 123.64, 123.02, 112.16, 38.77, 32.00, 29.35, 29.23, 27.58. ESI-HR calcd for $\mathrm{C}_{14} \mathrm{H}_{22} \mathrm{NOS}^{+}\left([\mathrm{M}+\mathrm{H}]^{+}\right)$252.1417, found 252.1408. IR $v\left(\mathrm{~cm}^{-1}\right) 3322,2932,1678,1640,750$. M. P. $85-87^{\circ} \mathrm{C}$.

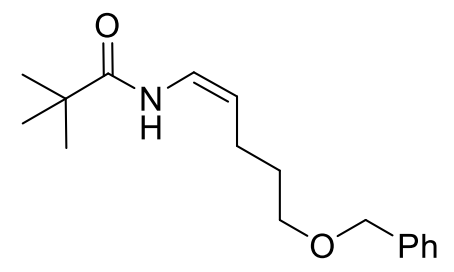


Enamide 1af-Z: ${ }^{1} \mathrm{H}$ NMR $(400 \mathrm{MHz}, \mathrm{CDCl} 3) \delta 7.56(\mathrm{~d}, J=8.4 \mathrm{~Hz}, 1 \mathrm{H}), 7.38-7.28(\mathrm{~m}, 5 \mathrm{H})$, $3.51(\mathrm{t}, J=9.96 \mathrm{~Hz}, 1 \mathrm{H}), 4.73(\mathrm{q}, J=8.2 \mathrm{~Hz}, 1 \mathrm{H}), 4.51(\mathrm{~s}, 2 \mathrm{H}), 3.51(\mathrm{t}, J=5.8 \mathrm{~Hz}, 2 \mathrm{H}), 2.15(\mathrm{q}, J$ $=7.2 \mathrm{~Hz}, 2 \mathrm{H}), 1.71(\mathrm{q}, J=6.3 \mathrm{~Hz}, 2 \mathrm{H}), 1.14(\mathrm{~s}, 9 \mathrm{H}) .{ }^{13} \mathbf{C} \mathbf{~ N M R}\left(101 \mathrm{MHz}, \mathrm{CDCl}_{3}\right) \delta 175.94$, $138.27,128.50,127.90,127.81,122.97,109.80,72.58,68.18,38.91,28.40,27.40,21.78$. ESI-HR calcd for $\mathrm{C}_{17} \mathrm{H}_{26} \mathrm{NO}_{2}{ }^{+}\left([\mathrm{M}+\mathrm{H}]^{+}\right)$276.1958, found 276.1950. IR $v\left(\mathrm{~cm}^{-1}\right) 3054,1670,1654,898$.<smiles>CC(C)(C)C(=O)N/C=C/CCCOCc1ccccc1</smiles>

Enamide 1af-E: ${ }^{1} \mathrm{H}$ NMR $\left(400 \mathrm{MHz}, \mathrm{CDCl}_{3}\right) \delta 7.42-7.19(\mathrm{~m}, 5 \mathrm{H}), 7.08(\mathrm{~s}, 1 \mathrm{H}), 6.84-6.69(\mathrm{~m}$, 1H), 5.15 (dt, $J=14.3,7.2 \mathrm{~Hz}, 1 \mathrm{H}), 4.49$ (s, 2H), 3.48 (t, $J=6.4 \mathrm{~Hz}, 2 \mathrm{H}), 2.13(\mathrm{q}, J=7.3 \mathrm{~Hz}, 2 \mathrm{H}$ ), $1.76-1.60$ (m, 2H), 1.21 (s, 9H). ${ }^{13} \mathbf{C}$ NMR (101 MHz, $\left.\mathrm{CDCl}_{3}\right) \delta$ 175.46, 138.70, 128.48, 127.76, 127.63, 123.37, 112.37, 73.04, 69.76, 38.74, 30.11, 27.56, 26.61. ESI-HR calcd for $\mathrm{C}_{17} \mathrm{H}_{26} \mathrm{NO}_{2}{ }^{+}$ $\left([\mathrm{M}+\mathrm{H}]^{+}\right) 276.1958$, found 276.1947. IR $v\left(\mathrm{~cm}^{-1}\right) 3324,2865,1679,1639,748$. M. P. $80-82^{\circ} \mathrm{C}$.<smiles>C/C(=C/CBr)NC(=O)C(C)(C)C</smiles>

Enamide 1ag-Z: ${ }^{1} \mathbf{H}$ NMR $\left(400 \mathrm{MHz}, \mathrm{CDCl}_{3}\right) \delta 7.29(\mathrm{t}, J=7.3 \mathrm{~Hz}, 2 \mathrm{H}), 7.20(\mathrm{dd}, J=14.5,7.2$ Hz, 3H), 6.29 (s, 1H), 4.97 (t, $J=7.4 \mathrm{~Hz}, 1 \mathrm{H}), 2.67$ (t, $J=7.3 \mathrm{~Hz}, 2 \mathrm{H}), 2.24$ (q, $J=7.3 \mathrm{~Hz}, 2 \mathrm{H}$ ), 1.99 (s, 3H), 1.13 (s, 9H). ${ }^{13} \mathbf{C}$ NMR $\left(101 \mathrm{MHz}, \mathrm{CDCl}_{3}\right) \delta 176.77,141.93,132.55,128.66,128.57$, $J 246.1852$, found 246.1850. IR $v\left(\mathrm{~cm}^{-1}\right) 3308,1646,1496,699$. M. P. $55-57^{\circ} \mathrm{C}$.<smiles>C/C=C(\NC(=O)C(C)(C)C)c1ccc(C(F)(F)F)cc1</smiles>

Enamide 1ah-Z: ${ }^{1} \mathrm{H}$ NMR $\left(400 \mathrm{MHz}, \mathrm{CDCl}_{3}\right) \delta 7.56(\mathrm{~d}, J=8.2 \mathrm{~Hz}, 2 \mathrm{H}), 7.43(\mathrm{~d}, J=8.3 \mathrm{~Hz}, 2 \mathrm{H})$, $6.81(\mathrm{~s}, 1 \mathrm{H}), 5.96(\mathrm{q}, J=7.0 \mathrm{~Hz}, 1 \mathrm{H}), 1.78(\mathrm{~d}, J=7.0 \mathrm{~Hz}, 3 \mathrm{H}), 1.34(\mathrm{~s}, 9 \mathrm{H}) .{ }^{13} \mathbf{C}$ NMR $(101 \mathrm{MHz}$, $\left.\mathrm{CDCl}_{3}\right) \delta 176.79,142.07,129.62\left(\mathrm{q}, J_{2}=32 \mathrm{~Hz}\right), 125.59,125.59,125.53\left(\mathrm{q}, J_{3}=4 \mathrm{~Hz}\right), 124.31(\mathrm{q}$, 
$\left.\mathrm{J}_{1}=273 \mathrm{~Hz}\right), 122.56,39.45,27.87,13.83$. ESI-HR calcd for $\mathrm{C}_{15} \mathrm{H}_{19} \mathrm{~F}_{3} \mathrm{NO}^{+}\left([\mathrm{M}+\mathrm{H}]^{+}\right) 286.1413$, found 286.1411. IR $v\left(\mathrm{~cm}^{-1}\right) 3259,1653,1559,750$. M. P. $86-88^{\circ} \mathrm{C}$.<smiles>C/C=C(\NC(=O)C(C)(C)C)c1ccc(F)cc1</smiles>

Enamide 1ai-Z: ${ }^{1} \mathbf{H}$ NMR $\left(400 \mathrm{MHz}, \mathrm{CDCl}_{3}\right) \delta 7.37-7.23(\mathrm{~m}, 2 \mathrm{H}), 7.05-6.91(\mathrm{~m}, 2 \mathrm{H}), 6.76(\mathrm{~s}$, $1 \mathrm{H}), 5.81(\mathrm{q}, J=6.9 \mathrm{~Hz}, 1 \mathrm{H}), 1.73(\mathrm{~d}, J=6.9 \mathrm{~Hz}, 3 \mathrm{H}), 1.33(\mathrm{~s}, 9 \mathrm{H}) .{ }^{13} \mathbf{C} \mathbf{~ N M R}\left(101 \mathrm{MHz}, \mathrm{CDCl}_{3}\right)$ $\delta 176.56,162.52\left(\mathrm{~d}, J_{1}=245.8 \mathrm{~Hz}\right), 134.79\left(\mathrm{~d}, J_{4}=3.2 \mathrm{~Hz}\right), 133.55,127.08\left(\mathrm{~d}, J_{3}=8.1 \mathrm{~Hz}\right)$, $120.32,115.40\left(\mathrm{~d}, J_{2}=21.9 \mathrm{~Hz}\right), 39.44,27.91,13.83$. ESI-HR calcd for $\mathrm{C}_{14} \mathrm{H}_{19} \mathrm{FNO}^{+}\left([\mathrm{M}+\mathrm{H}]^{+}\right)$ 236.1445, found 236.1445. IR $v\left(\mathrm{~cm}^{-1}\right) 3277,1642,1507,750$. M. P. $115-117^{\circ} \mathrm{C}$.<smiles>C/C=C(\NC(=O)C(C)(C)C)c1cccc(OC)c1</smiles>

Enamide 1aj-Z: ${ }^{1} \mathrm{H}$ NMR $\left(400 \mathrm{MHz}, \mathrm{CDCl}_{3}\right) \delta 7.21(\mathrm{t}, J=8.0 \mathrm{~Hz}, 1 \mathrm{H}), 6.94(\mathrm{~d}, J=7.7 \mathrm{~Hz}, 1 \mathrm{H})$, $6.90-6.85(\mathrm{~m}, 1 \mathrm{H}), 6.80(\mathrm{dd}, J=8.2,2.0 \mathrm{~Hz}, 1 \mathrm{H}), 6.75(\mathrm{~s}, 1 \mathrm{H}), 5.92(\mathrm{q}, J=6.9 \mathrm{~Hz}, 1 \mathrm{H}), 3.79(\mathrm{~s}$, 3H), $1.73(\mathrm{~d}, J=7.0 \mathrm{~Hz}, 3 \mathrm{H}), 1.33(\mathrm{~s}, 9 \mathrm{H}) .{ }^{13} \mathbf{C} \mathbf{N M R}\left(101 \mathrm{MHz}, \mathrm{CDCl}_{3}\right) \delta 176.38,159.79,140.18$ 134.02, 129.51, 120.92, 117.99, 113.29, 111.21, 55.30, 39.42, 27.92, 14.00. ESI-HR calcd for $\mathrm{C}_{15} \mathrm{H}_{22} \mathrm{NO}_{2}{ }^{+}\left([\mathrm{M}+\mathrm{H}]^{+}\right)$266.1306248.1645, found 248.1644IR $v\left(\mathrm{~cm}^{-1}\right) 3290,1646,1488,750 . \mathbf{M}$. P. $83-85^{\circ} \mathrm{C}$<smiles>C/C=C(\NC(=O)C(C)(C)C)c1ccccc1</smiles>

Enamide 1ak-Z: ${ }^{1} \mathbf{H}$ NMR $\left(400 \mathrm{MHz}, \mathrm{CDCl}_{3}\right) \delta 7.43-7.35(\mathrm{~m}, 2 \mathrm{H}), 7.32(\mathrm{t}, J=8.5 \mathrm{~Hz}, 3 \mathrm{H})$, $6.70(\mathrm{~s}, 1 \mathrm{H}), 6.35(\mathrm{q}, J=7.3 \mathrm{~Hz}, 1 \mathrm{H}), 1.72(\mathrm{~d}, J=7.3 \mathrm{~Hz}, 3 \mathrm{H}), 1.24(\mathrm{~s}, 9 \mathrm{H}) .{ }^{13} \mathbf{C} \mathbf{N M R}(101 \mathrm{MHz}$, $\left.\mathrm{CDCl}_{3}\right) \delta 176.89,137.10,133.92,128.62,128.47,127.96,114.88,39.44,27.64,13.87$. ESI-HR 
calcd for $\mathrm{C}_{14} \mathrm{H}_{20} \mathrm{NO}^{+}\left([\mathrm{M}+\mathrm{H}]^{+}\right)$218.1539, found 218.1538. IR $v\left(\mathrm{~cm}^{-1}\right) 3246,1648,1519,751 . \mathbf{M}$. P. $113-115^{\circ} \mathrm{C}$.<smiles>C/C=C(/NC(=O)c1ccccc1)c1ccccc1</smiles>

Enamide 1al-Z: ${ }^{1} \mathbf{H}$ NMR $\left(400 \mathrm{MHz}, \mathrm{CDCl}_{3}\right) \delta 7.90(\mathrm{~d}, J=7.4 \mathrm{~Hz}, 2 \mathrm{H}), 7.54(\mathrm{t}, J=7.1 \mathrm{~Hz}, 1 \mathrm{H})$, $7.50-7.38(\mathrm{~m}, 4 \mathrm{H}), 7.37-7.19(\mathrm{~m}, 4 \mathrm{H}), 6.01(\mathrm{q}, J=6.8 \mathrm{~Hz}, 1 \mathrm{H}), 1.80(\mathrm{t}, J=13.0 \mathrm{~Hz}, 3 \mathrm{H}) .{ }^{13} \mathrm{C}$ NMR $\left(101 \mathrm{MHz}, \mathrm{CDCl}_{3}\right) \delta 165.56,138.24,134.41,134.24,131.96,128.85,128.58,127.93$, 127.43, 125.69, 121.24, 14.31. ESI-HR calcd for $\mathrm{C}_{16} \mathrm{H}_{16} \mathrm{NO}^{+}\left([\mathrm{M}+\mathrm{H}]^{+}\right) 238.1226$, found 238.1224 IR $v\left(\mathrm{~cm}^{-1}\right) 3308,1646,1519,751$. M. P. $158-160^{\circ} \mathrm{C}$.<smiles>CC/C=C(/C)NC(=O)c1ccc(Cl)cc1</smiles>

Enamide 1am-Z: ${ }^{1} \mathbf{H}$ NMR $\left(400 \mathrm{MHz}, \mathrm{CDCl}_{3}\right) \delta 7.73(\mathrm{~d}, J=8.5 \mathrm{~Hz}, 2 \mathrm{H}), 7.41(\mathrm{~d}, J=8.6 \mathrm{~Hz}$, 2H), $7.11(\mathrm{~s}, 1 \mathrm{H}), 5.02(\mathrm{t}, J=7.0 \mathrm{~Hz}, 1 \mathrm{H}), 2.09(\mathrm{~s}, 3 \mathrm{H}), 2.06-1.96(\mathrm{~m}, 2 \mathrm{H}), 1.00(\mathrm{t}, J=7.5 \mathrm{~Hz}$, 3H). ${ }^{13} \mathrm{C}$ NMR $\left(101 \mathrm{MHz}, \mathrm{CDCl}_{3}\right) \delta 164.59,138.02,133.45,130.50,129.04,128.65,122.48$, 21.48, 20.47, 13.89. ESI-HR calcd for $\mathrm{C}_{12} \mathrm{H}_{15} \mathrm{ClNO}^{+}\left([\mathrm{M}+\mathrm{H}]^{+}\right)$224.0837, found 244.0837. IR v $\left(\mathrm{cm}^{-1}\right) 3285,1640,1519,750$. M. P.83-85 ${ }^{\circ} \mathrm{C}$.<smiles>C/C=C(/NC(=O)c1ccc(Cl)cc1)c1ccccc1</smiles>

Enamide 1an-Z: ${ }^{1} \mathbf{H}$ NMR $\left(400 \mathrm{MHz}, \mathrm{CDCl}_{3}\right) \delta 7.83(\mathrm{~d}, J=8.1 \mathrm{~Hz}, 2 \mathrm{H}), 7.50-7.37(\mathrm{~m}, 4 \mathrm{H})$, $7.36-7.22(\mathrm{~m}, 4 \mathrm{H}), 6.02(\mathrm{q}, J=6.8 \mathrm{~Hz}, 1 \mathrm{H}), 1.81(\mathrm{~d}, J=6.9 \mathrm{~Hz}, 3 \mathrm{H}) .{ }^{13} \mathbf{C}$ NMR $(101 \mathrm{MHz}$, $\left.\mathrm{CDCl}_{3}\right) \delta 164.55,138.30,138.07,134.10,132.75,129.12,128.88,128.65,128.06,125.67,121.45$, 14.33. ESI-HR calcd for $\mathrm{C}_{16} \mathrm{H}_{16} \mathrm{ClNO}^{+}\left([\mathrm{M}+\mathrm{H}]^{+}\right)$272.0837, found 272.0837. IR $v\left(\mathrm{~cm}^{-1}\right) 3278$, 1645, 1518, 751. M.P. $197-199^{\circ} \mathrm{C}$ 
<smiles>C/C(=C/NC(=O)C(C)(C)C)Cc1ccc(Cl)cc1</smiles>

Enamide 1ao-Z: ${ }^{1} \mathbf{H}$ NMR $\left(400 \mathrm{MHz}, \mathrm{CDCl}_{3}\right) \delta 7.29(\mathrm{~d}, J=8.3 \mathrm{~Hz}, 2 \mathrm{H}), 7.13(\mathrm{~d}, J=8.1 \mathrm{~Hz}, 2 \mathrm{H})$, $6.94(\mathrm{~s}, 1 \mathrm{H}), 6.71(\mathrm{~d}, J=10.2 \mathrm{~Hz}, 1 \mathrm{H}), 3.32(\mathrm{~s}, 2 \mathrm{H}), 1.73(\mathrm{~s}, 3 \mathrm{H}), 1.11(\mathrm{~s}, 9 \mathrm{H}) .{ }^{13} \mathbf{C}$ NMR $(101$ $\left.\mathrm{MHz}, \mathrm{CDCl}_{3}\right) \delta 175.36,136.94,132.51,129.78,129.06,119.16,116.71,38.86,36.74,27.45$, 21.62. ESI-HR calcd for $\mathrm{C}_{15} \mathrm{H}_{21} \mathrm{ClNO}^{+}\left([\mathrm{M}+\mathrm{H}]^{+}\right)$266.1306, found 266.1306. IR $v\left(\mathrm{~cm}^{-1}\right) 3331$, 1653, 1494, 764. M. P.82-84 C.<smiles>CCCC(=CNC(=O)c1ccccc1)CBr</smiles>

Enamide 1ap- $\boldsymbol{E}:{ }^{\mathbf{1}} \mathbf{H}$ NMR $\left(400 \mathrm{MHz}, \mathrm{CDCl}_{3}\right) \delta 7.77(\mathrm{~d}, J=7.2 \mathrm{~Hz}, 2 \mathrm{H}), 7.59(\mathrm{~d}, J=10.0 \mathrm{~Hz}$, 1H), $7.51(\mathrm{t}, J=7.3 \mathrm{~Hz}, 1 \mathrm{H}), 7.44(\mathrm{t}, J=7.4 \mathrm{~Hz}, 2 \mathrm{H}), 7.28(\mathrm{t}, J=7.5 \mathrm{~Hz}, 2 \mathrm{H}), 7.22-7.15(\mathrm{~m}, 3 \mathrm{H})$ $6.87(\mathrm{~d}, J=10.5 \mathrm{~Hz}, 1 \mathrm{H}), 2.76(\mathrm{dd}, J=9.5,6.9 \mathrm{~Hz}, 2 \mathrm{H}), 2.43-2.31(\mathrm{~m}, 2 \mathrm{H}), 2.11(\mathrm{t}, J=7.6 \mathrm{~Hz}$, 2H), $1.61-1.46(\mathrm{~m}, 2 \mathrm{H}), 0.97(\mathrm{t}, J=7.3 \mathrm{~Hz}, 3 \mathrm{H}) .{ }^{13} \mathbf{C ~ N M R}\left(101 \mathrm{MHz}, \mathrm{CDCl}_{3}\right) \delta 164.18,141.95$, $134.35,131.81,128.81,128.46,128.40,127.00,125.98,123.63,118.16,36.51,35.07,31.51$, 20.93, 14.30. ESI-HR calcd for $\mathrm{C}_{20} \mathrm{H}_{24} \mathrm{NO}^{+}\left([\mathrm{M}+\mathrm{H}]^{+}\right)$294.1852, found 294.1859. IR $v\left(\mathrm{~cm}^{-1}\right)$ 3306, 1639, 1512 , 698. M. P. $88-90^{\circ} \mathrm{C}$.<smiles>C/C(=C\NC(=O)c1ccccc1)CCCCl</smiles>

Enamide 1aq- $\boldsymbol{E}:{ }^{\mathbf{1}} \mathbf{H}$ NMR $\left(400 \mathrm{MHz}, \mathrm{CDCl}_{3}\right) \delta 7.79(\mathrm{~d}, J=7.3 \mathrm{~Hz}, 2 \mathrm{H}), 7.57-7.35(\mathrm{~m}, 4 \mathrm{H})$, $6.82(\mathrm{~d}, J=10.3 \mathrm{~Hz}, 1 \mathrm{H}), 3.54(\mathrm{t}, J=6.6 \mathrm{~Hz}, 2 \mathrm{H}), 2.23(\mathrm{t}, J=7.3 \mathrm{~Hz}, 2 \mathrm{H}), 2.00-1.83(\mathrm{~m}, 2 \mathrm{H})$, $1.72(\mathrm{~s}, 3 \mathrm{H}) .{ }^{13} \mathrm{C}$ NMR $\left(101 \mathrm{MHz}, \mathrm{CDCl}_{3}\right) \delta 164.30,134.26,131.95,128.88,127.07,118.45$, 117.99, 44.47, 34.02, 30.98, 14.91. ESI-HR calcd for $\mathrm{C}_{13} \mathrm{H}_{17} \mathrm{ClNO}^{+}\left([\mathrm{M}+\mathrm{H}]^{+}\right)$238.0993, found 238.0994. IR $v\left(\mathrm{~cm}^{-1}\right) 3307,1640,1511,750$. M. P. $86-88{ }^{\circ} \mathrm{C}$. 
<smiles>CCCC/C(C)=C/NC(=O)c1ccccc1</smiles>

Enamide 1ar- $\boldsymbol{E}:{ }^{1} \mathbf{H}$ NMR $\left(400 \mathrm{MHz}, \mathrm{CDCl}_{3}\right) \delta 7.79(\mathrm{~d}, \boldsymbol{J}=8.0 \mathrm{~Hz}, 2 \mathrm{H}), 7.57-7.38(\mathrm{~m}, 4 \mathrm{H})$, $6.77(\mathrm{~d}, J=10.4 \mathrm{~Hz}, 1 \mathrm{H}), 2.07(\mathrm{t}, J=7.5 \mathrm{~Hz}, 2 \mathrm{H}), 1.70(\mathrm{~s}, 3 \mathrm{H}), 1.48-1.38(\mathrm{~m}, 2 \mathrm{H}), 1.32(\mathrm{dq}, J=$ 14.3, 7.2 Hz, 2H), 0.91 (t, $J=7.3 \mathrm{~Hz}, 3 \mathrm{H}) .{ }^{13} \mathbf{C}$ NMR $\left(101 \mathrm{MHz}, \mathrm{CDCl}_{3}\right) \delta 164.24,134.49,131.80$, 128.84, 127.05, 120.25, 117.41, 36.53, 30.23, 22.41, 14.92, 14.09. ESI-HR calcd for $\mathrm{C}_{14} \mathrm{H}_{20} \mathrm{NO}^{+}$ $\left([\mathrm{M}+\mathrm{H}]^{+}\right)$218.1539, found 218.1541. IR $v\left(\mathrm{~cm}^{-1}\right) 3306,1639,1514,764$. M. P. $48-50^{\circ} \mathrm{C}$.<smiles>C/C(=C/NC(=O)c1ccccc1)C1CCCCC1</smiles>

Enamide 1as-Z: ${ }^{1} \mathbf{H}$ NMR $\left(400 \mathrm{MHz}, \mathrm{CDCl}_{3}\right) \delta 7.78(\mathrm{~d}, J=7.1 \mathrm{~Hz}, 2 \mathrm{H}), 7.60-7.50(\mathrm{~m}, 2 \mathrm{H})$, $7.46(\mathrm{t}, J=7.4 \mathrm{~Hz}, 2 \mathrm{H}), 6.68(\mathrm{~d}, J=10.4 \mathrm{~Hz}, 1 \mathrm{H}), 2.31-2.15(\mathrm{~m}, 1 \mathrm{H}), 1.89-1.77(\mathrm{~m}, 2 \mathrm{H}), 1.69$ $(\mathrm{d}, J=0.9 \mathrm{~Hz}, 3 \mathrm{H}), 1.66-1.49(\mathrm{~m}, 3 \mathrm{H}), 1.44-1.27(\mathrm{~m}, 4 \mathrm{H}), 1.25-1.12(\mathrm{~m}, 1 \mathrm{H}) .{ }^{13} \mathbf{C}$ NMR $(101$ $\left.\mathrm{MHz}, \mathrm{CDCl}_{3}\right) \delta 164.19,134.57,131.79,128.87,127.05,125.24,116.20,39.67,30.64,26.69$, 26.16, 17.06. ESI-HR calcd for $\mathrm{C}_{16} \mathrm{H}_{22} \mathrm{NO}^{+}\left([\mathrm{M}+\mathrm{H}]^{+}\right)$244.1696, found 244.1698. IR $v\left(\mathrm{~cm}^{-1}\right)$ 3463, 2965, 1679, 1658, 734. M. P.136-138º C.<smiles>O=C(NC=C1CCOCC1)c1ccccc1</smiles>

Enamide 1at: ${ }^{1} \mathbf{H}$ NMR $\left(400 \mathrm{MHz}, \mathrm{CDCl}_{3}\right) \delta 7.77(\mathrm{~d}, J=7.5 \mathrm{~Hz}, 2 \mathrm{H}), 7.70(\mathrm{~d}, J=9.5 \mathrm{~Hz}, 1 \mathrm{H})$, 7.49 (t, $J=7.3 \mathrm{~Hz}, 1 \mathrm{H}), 7.41(\mathrm{t}, J=7.5 \mathrm{~Hz}, 2 \mathrm{H}), 6.77(\mathrm{~d}, J=10.2 \mathrm{~Hz}, 1 \mathrm{H}), 3.76-3.59(\mathrm{~m}, 4 \mathrm{H})$, $2.35-2.18(\mathrm{~m}, 4 \mathrm{H}) .{ }^{13} \mathrm{C}$ NMR $\left(101 \mathrm{MHz}, \mathrm{CDCl}_{3}\right) \delta 164.75,134.07,131.92,128.76,127.11$, 119.14, 116.11, 69.36, 67.75, 33.42, 28.53. ESI-HR calcd for $\mathrm{C}_{13} \mathrm{H}_{16} \mathrm{NO}_{2}{ }^{+}\left([\mathrm{M}+\mathrm{H}]^{+}\right)$218.1176, found 218.1177. IR $v\left(\mathrm{~cm}^{-1}\right) 3306,1639,1515,750$. M. P. $122-124^{\circ} \mathrm{C}$. 
<smiles>O=C(NC=C1CCCCC1)c1ccccc1</smiles>

Enamide 1au: ${ }^{1} \mathbf{H}$ NMR $\left(400 \mathrm{MHz}, \mathrm{CDCl}_{3}\right) \delta 7.82-7.74(\mathrm{~m}, 2 \mathrm{H}), 7.56(\mathrm{~s}, 1 \mathrm{H}), 7.53-7.46(\mathrm{~m}$, 1H), $7.46-7.37(\mathrm{~m}, 2 \mathrm{H}), 6.71(\mathrm{~d}, J=10.3 \mathrm{~Hz}, 1 \mathrm{H}), 2.15(\mathrm{~d}, J=4.4 \mathrm{~Hz}, 4 \mathrm{H}), 1.57(\mathrm{~s}, 6 \mathrm{H}) .{ }^{13} \mathrm{C}$ NMR $\left(101 \mathrm{MHz}, \mathrm{CDCl}_{3}\right) \delta 164.45,134.45,131.70,128.73,127.04,124.56,114.41,33.71,28.18$, 27.80, 27.10, 26.68. ESI-HR calcd for $\mathrm{C}_{14} \mathrm{H}_{18} \mathrm{NO}^{+}\left([\mathrm{M}+\mathrm{H}]^{+}\right)$216.1383, found 216.1389. IR v $\left(\mathrm{cm}^{-1}\right) 3305,1637,1515,750$. M. P. $130-132^{\circ} \mathrm{C}$.<smiles>O=C(NC=C1CCCC1)c1ccccc1</smiles>

Enamide 1av: ${ }^{1} \mathbf{H}$ NMR $\left(400 \mathrm{MHz}, \mathrm{CDCl}_{3}\right) \delta 7.84-7.74(\mathrm{~m}, 2 \mathrm{H}), 7.54-7.48(\mathrm{~m}, 1 \mathrm{H}), 7.4-$ $77.40(\mathrm{~m}, 2 \mathrm{H}), 7.31(\mathrm{~s}, 1 \mathrm{H}), 6.94-6.82(\mathrm{~m}, 1 \mathrm{H}), 2.40-2.32(\mathrm{~m}, 2 \mathrm{H}), 2.31-2.22(\mathrm{~m}, 2 \mathrm{H}), 1.80-$ $1.72(\mathrm{~m}, 2 \mathrm{H}), 1.71-1.63(\mathrm{~m}, 2 \mathrm{H}) .{ }^{13} \mathbf{C} \mathbf{~ N M R}\left(101 \mathrm{MHz}, \mathrm{CDCl}_{3}\right) \delta 163.98,134.40,131.74,128.78$, 127.70, 127.05, 114.10, 31.45, 27.80, 26.79, 26.37. ESI-HR calcd for $\mathrm{C}_{13} \mathrm{H}_{16} \mathrm{NO}^{+}\left([\mathrm{M}+\mathrm{H}]^{+}\right)$ 202.1226, found 202.1227. IR $v\left(\mathrm{~cm}^{-1}\right) 3306,1637,1516,750$. M. P. $123-125^{\circ} \mathrm{C}$.<smiles>CC(C)=CNC(=O)c1ccc(Cl)cc1</smiles>

Enamide 1aw: ${ }^{1} \mathrm{H}$ NMR $\left(400 \mathrm{MHz}, \mathrm{CDCl}_{3}\right) \delta 7.76-7.66(\mathrm{~m}, 2 \mathrm{H}), 7.49(\mathrm{~s}, 1 \mathrm{H}), 7.44-7.34(\mathrm{~m}$, 2H), $6.74-6.64(\mathrm{~m}, 1 \mathrm{H}), 1.74(\mathrm{~d}, J=20.4 \mathrm{~Hz}, 6 \mathrm{H}) .{ }^{13} \mathrm{C} \mathbf{N M R}\left(101 \mathrm{MHz}, \mathrm{CDCl}_{3}\right) \delta 163.26$, 138.02, 132.73, 129.01, 128.51, 117.31, 117.13, 22.68, 16.78. ESI-HR calcd for $\mathrm{C}_{11} \mathrm{H}_{13} \mathrm{ClNO}^{+}$ $\left([\mathrm{M}+\mathrm{H}]^{+}\right) 210.0680$, found210.0689. IR $v\left(\mathrm{~cm}^{-1}\right) 3326,1635,1514,751$. M. P. $86-88^{\circ} \mathrm{C}$.<smiles>C/C(=C/NC(=O)C(C)(C)C)CBr</smiles>

Enamide 1ax-Z: ${ }^{1} \mathbf{H}$ NMR (400 MHz, $\left.\mathrm{CDCl}_{3}\right) \delta 7.32-7.27$ (m, 2H), $7.24-7.15$ (m, 3H), 6.47 (s, 2H), $2.73(\mathrm{t}, J=7.0 \mathrm{~Hz}, 2 \mathrm{H}), 2.28(\mathrm{t}, J=7.0 \mathrm{~Hz}, 2 \mathrm{H}), 1.77(\mathrm{~s}, 3 \mathrm{H}), 1.03(\mathrm{~s}, 9 \mathrm{H}) .{ }^{13} \mathbf{C}$ NMR $(101$ 
$\left.\mathrm{MHz}, \mathrm{CDCl}_{3}\right) \delta 175.06,141.58,128.51,128.37,126.11,118.40,117.79,38.39,33.56,33.02$, 27.16, 20.10. ESI-HR calcd for $\mathrm{C}_{16} \mathrm{H}_{24} \mathrm{NO}^{+}\left([\mathrm{M}+\mathrm{H}]^{+}\right)$246.1852, found 246.1853. IR $v\left(\mathrm{~cm}^{-1}\right)$ $3350,1653,1494,764$. M.P. $70-72{ }^{\circ} \mathrm{C}$.

\section{Characterization of Products}<smiles>CC(C)(C)C(=O)NC(CCCc1ccccc1)Cc1ccccc1</smiles>

$\boldsymbol{\alpha}$-Aminoboronic ester 2a: Following the general procedure [A], enamide 1a-Z (23.1 mg, 0.100 mmol) was converted to the $\alpha$-aminoboronic ester. Purification by silica gel chromatography gave $26.9 \mathrm{mg}$ (75\% yield) of $\mathbf{2 a}$ as a white solid. ${ }^{1} \mathbf{H} \mathbf{~ N M R}\left(400 \mathrm{MHz}, \mathrm{CDCl}_{3}\right) \delta 7.29-7.23(\mathrm{~s}, 2 \mathrm{H})$, $7.21-7.14(\mathrm{~m}, 3 \mathrm{H}), 6.53(\mathrm{~s}, 1 \mathrm{H}), 2.64(\mathrm{qd}, J=14.1,6.7 \mathrm{~Hz}, 2 \mathrm{H}), 2.57-2.51(\mathrm{~m}, 1 \mathrm{H}), 1.83-1.60$ (m, 3H), $1.54-1.39$ (m, 1H), 1.19 (s, 21H). ${ }^{13} \mathrm{C}$ NMR (101 MHz, CDCl 3 ) $\delta$ 183.67, 142.64, $128.50,128.42,125.81,80.41,36.03,35.75,31.05,29.88,26.96,25.47,25.25 .{ }^{11}$ B NMR $(128$ MHz, $\left.\mathrm{CDCl}_{3}\right) \delta$ 16.02. ESI-HR calcd for $\mathrm{C}_{21} \mathrm{H}_{35} \mathrm{BNO}_{3}{ }^{+}\left([\mathrm{M}+\mathrm{H}]^{+}\right) 360.2705$, found 360.2695. IR $v\left(\mathrm{~cm}^{-1}\right) 3229,2987,1559,732$. M. P. $129-130^{\circ} \mathrm{C}$.<smiles>CC(C)(C)C(=O)NCC(Br)CCc1ccccc1</smiles>

$\boldsymbol{\beta}$-Aminoboronic ester 3a: Following the general procedure [B], enamide 1a- $\boldsymbol{E}$ (23.1 mg, 0.100 mmol) was converted to the $\beta$-aminoboronic ester. Purification by silica gel chromatography gave $31.6 \mathrm{mg}$ (88\% yield) of $\mathbf{3 a}$ as a colorless oil. ${ }^{1} \mathbf{H}$ NMR $\left(400 \mathrm{MHz}, \mathrm{CDCl}_{3}\right) \delta 7.29-7.23(\mathrm{~m}, 2 \mathrm{H})$, $7.20-7.10(\mathrm{~m}, 3 \mathrm{H}), 6.23(\mathrm{~s}, 1 \mathrm{H}), 3.48-3.38(\mathrm{~m}, 1 \mathrm{H}), 3.36-3.22(\mathrm{~m}, 1 \mathrm{H}), 2.74-2.58(\mathrm{~m}, 2 \mathrm{H})$, $1.85-1.74(\mathrm{~m}, 1 \mathrm{H}), 1.69-1.59(\mathrm{~m}, 1 \mathrm{H}), 1.27(\mathrm{~s}, 12 \mathrm{H}), 1.24-1.20(\mathrm{~m}, 1 \mathrm{H}), 1.17(\mathrm{~d}, J=9.4 \mathrm{~Hz}$, 9H). ${ }^{13} \mathrm{C}$ NMR $\left(101 \mathrm{MHz}, \mathrm{CDCl}_{3}\right) \delta$ 178.23, 142.69, 128.61, 128.42, 125.82, 83.61, 40.03, 38.82, 35.14, 30.81, 27.74, 25.09, 25.00. ${ }^{11} \mathbf{B}$ NMR $\left(128 \mathrm{MHz}, \mathrm{CDCl}_{3}\right) \delta 35.87$. ESI-HR calcd for $\mathrm{C}_{21} \mathrm{H}_{35} \mathrm{BNO}_{3}{ }^{+}\left([\mathrm{M}+\mathrm{H}]^{+}\right)$360.2705, found 360.2698. IR $v\left(\mathrm{~cm}^{-1}\right) 3363,2977,1647,750$. 
<smiles>CC(=O)NC(CCCc1ccccc1)Cc1ccccc1</smiles>

$\boldsymbol{\alpha}$-Aminoboronic ester $\mathbf{2 b}$ : Following the general procedure [A], enamide $\mathbf{1 b}-\boldsymbol{Z}$ (18.9 mg, 0.100 mmol) was converted to the $\alpha$-aminoboronic ester. Purification by silica gel chromatography gave $23.3 \mathrm{mg}$ (73\% yield) of $\mathbf{2 b}$ as a colorless oil. ${ }^{1} \mathbf{H} \mathbf{~ N M R}\left(400 \mathrm{MHz}, \mathrm{CDCl}_{3}\right) \delta 8.49(\mathrm{~s}, 1 \mathrm{H}), 7.25-$ $7.18(\mathrm{~m}, 2 \mathrm{H}), 7.18-7.09(\mathrm{~m}, 3 \mathrm{H}), 2.70-2.51(\mathrm{~m}, 2 \mathrm{H}), 2.46(\mathrm{broad}, 1 \mathrm{H}), 2.02(\mathrm{~s}, 3 \mathrm{H}), 1.83-1.71$ (m, 2H), $1.71-1.56(\mathrm{~m}, 2 \mathrm{H}), 1.54-1.35(\mathrm{~m}, 1 \mathrm{H}), 1.28-1.21(\mathrm{~m}, 1 \mathrm{H}), 1.16(\mathrm{~d}, J=4.8 \mathrm{~Hz}, 12 \mathrm{H})$. ${ }^{13} \mathrm{C}$ NMR $\left(101 \mathrm{MHz}, \mathrm{CDCl}_{3}\right) \delta 174.97,142.45,128.51,128.40,125.82,80.69,36.16,31.05$, 30.21, 25.57, 25.16, 18.03. ${ }^{11} \mathbf{B}$ NMR $\left(128 \mathrm{MHz}, \mathrm{CDCl}_{3}\right) \delta 16.05$. ESI-HR calcd for $\mathrm{C}_{18} \mathrm{H}_{29} \mathrm{BNO}_{3}{ }^{+}\left([\mathrm{M}+\mathrm{H}]^{+}\right)$318.2235, found 318.2225. IR $v\left(\mathrm{~cm}^{-1}\right)$ 2988, 1609, 1275, 749.<smiles>CC(=O)NCC([Pb])CCc1ccccc1</smiles>

$\boldsymbol{\beta}$-Aminoboronic ester 3b:Following the general procedure [B], enamide 1b-E (18.9 mg, 0.100 mmol) was converted to the $\beta$-aminoboronic ester. Purification by silica gel chromatography gave $15.5 \mathrm{mg}$ (49\% yield) of $\mathbf{3 b}$ as a colorless oil. ${ }^{\mathbf{1}} \mathbf{H} \mathbf{~ N M R}\left(400 \mathrm{MHz}, \mathrm{CDCl}_{3}\right) \delta 7.33-7.22(\mathrm{~m}, 2 \mathrm{H})$, $7.23-7.12(\mathrm{~m}, 3 \mathrm{H}), 5.79(\mathrm{~s}, 1 \mathrm{H}), 3.49-3.37(\mathrm{~m}, 1 \mathrm{H}), 3.36-3.24(\mathrm{~m}, 1 \mathrm{H}), 2.74-2.58(\mathrm{~m}, 2 \mathrm{H})$, $1.96(\mathrm{~s}, 3 \mathrm{H}), 1.84-1.72(\mathrm{~m}, 1 \mathrm{H}), 1.71-1.59(\mathrm{~m}, 2 \mathrm{H}), 1.25(\mathrm{~s}, 12 \mathrm{H}) .{ }^{13} \mathbf{C}$ NMR $\left(101 \mathrm{MHz}, \mathrm{CDCl}_{3}\right)$ $\delta 169.91,142.59,128.60,128.45,125.89,83.64,40.46,35.18,30.85,25.04,24.97,23.58 .{ }^{11} \mathbf{B}$ NMR $\left(128 \mathrm{MHz}, \mathrm{CDCl}_{3}\right) \delta$ 34.27. ESI-HR calcd for $\mathrm{C}_{18} \mathrm{H}_{29} \mathrm{BNO}_{3}{ }^{+}\left([\mathrm{M}+\mathrm{H}]^{+}\right) 318.2235$, found 318.2224. IR $v\left(\mathrm{~cm}^{-1}\right) 3293,2980,1650,750$.<smiles>CC(C)C(=O)NC(CCCc1ccccc1)Cc1ccccc1</smiles>

$\boldsymbol{\alpha}$-Aminoboronic ester 2c: Following the general procedure [A], enamide 1c-Z (21.7 mg, 0.100 mmol) was converted to the $\alpha$-aminoboronic ester. Purification by silica gel chromatography gave $22.7 \mathrm{mg}$ (66\% yield) of $\mathbf{2 c}$ as a colorless oil. ${ }^{1} \mathbf{H}$ NMR $\left(400 \mathrm{MHz}, \mathrm{CDCl}_{3}\right) \delta 7.29-7.22(\mathrm{~m}, 2 \mathrm{H})$, $7.20-7.10(\mathrm{~m}, 3 \mathrm{H}), 6.76(\mathrm{~s}, 1 \mathrm{H}), 2.72-2.45(\mathrm{~m}, 4 \mathrm{H}), 1.74-1.61(\mathrm{~m}, 3 \mathrm{H}), 1.54-1.40(\mathrm{~m}, 1 \mathrm{H})$, 
$1.27-1.07(\mathrm{~m}, 18 \mathrm{H}) .{ }^{13} \mathrm{C} \mathbf{~ N M R}\left(101 \mathrm{MHz}, \mathrm{CDCl}_{3}\right) \delta 181.36,142.60,128.51,128.42,125.82$, $80.70,36.07,31.66,31.05,29.92,25.46,25.21,18.89,18.85 .{ }^{11} \mathbf{B}$ NMR $\left(128 \mathrm{MHz}, \mathrm{CDCl}_{3}\right) \delta$ 16.93. ESI-HR calcd for $\mathrm{C}_{20} \mathrm{H}_{33} \mathrm{BNO}_{3}{ }^{+}\left([\mathrm{M}+\mathrm{H}]^{+}\right) 346.2548$, found 346.2538. IR $v\left(\mathrm{~cm}^{-1}\right) 2988$, $1599,1275,749$.<smiles>CC(C)C(=O)NCC(Br)c1ccccc1</smiles>

$\boldsymbol{\beta}$-Aminoboronic ester 3c: Following the general procedure [B], enamide 1c-E (21.7 mg, 0.100 mmol) was converted to the $\beta$-aminoboronic ester. Purification by silica gel chromatography gave $25.5 \mathrm{mg}$ (74\% yield) of $\mathbf{3 c}$ as a colorless oil. ${ }^{1} \mathbf{H}$ NMR $\left(400 \mathrm{MHz}, \mathrm{CDCl}_{3}\right) \delta 7.31-7.22(\mathrm{~m}, 3 \mathrm{H})$, $7.22-7.13(\mathrm{~m}, 3 \mathrm{H}), 5.92(\mathrm{~s}, 1 \mathrm{H}), 3.48-3.37(\mathrm{~m}, 1 \mathrm{H}), 3.37-3.26(\mathrm{~m}, 1 \mathrm{H}), 2.75-2.59(\mathrm{~m}, 2 \mathrm{H})$, $2.40-2.24(\mathrm{~m}, 1 \mathrm{H}), 1.86-1.75(\mathrm{~m}, 1 \mathrm{H}), 1.69-1.59(\mathrm{~m}, 1 \mathrm{H}), 1.26(\mathrm{~s}, 12 \mathrm{H}), 1.15(\mathrm{~d}, J=6.9 \mathrm{~Hz}$, 6H). ${ }^{13} \mathrm{C}$ NMR $\left(101 \mathrm{MHz}, \mathrm{CDCl}_{3}\right) \delta 176.70,142.64,128.59,128.42,125.84,83.61,40.01,35.96$, 35.15, 30.80, 25.05, 24.97, 19.77, 19.76. ${ }^{11} \mathbf{B}$ NMR $\left(128 \mathrm{MHz}, \mathrm{CDCl}_{3}\right) \delta 34.23$. ESI-HR calcd for $\mathrm{C}_{20} \mathrm{H}_{33} \mathrm{BNO}_{3}{ }^{+}\left([\mathrm{M}+\mathrm{H}]^{+}\right)$346.2548, found 346.2541. IR $v\left(\mathrm{~cm}^{-1}\right) 3295,2978,1645,750$.<smiles>O=C(NC(CCCc1ccccc1)Cc1ccccc1)c1ccccc1</smiles>

$\boldsymbol{\alpha}$-Aminoboronic ester 2d: Following the general procedure [A], enamide 1d-Z (25.1 mg, 0.100 mmol) was converted to the $\alpha$-aminoboronic ester. Purification by silica gel chromatography gave $33.2 \mathrm{mg}$ (88\% yield) of $\mathbf{2 d}$ as a colorless oil. ${ }^{\mathbf{1}} \mathbf{H} \mathbf{~ N M R}\left(400 \mathrm{MHz}, \mathrm{CDCl}_{3}\right) \delta 7.78(\mathrm{~d}, J=7.4 \mathrm{~Hz}$, 2H), $7.64-7.54(\mathrm{~m}, 1 \mathrm{H}), 7.52(\mathrm{t}, J=7.4 \mathrm{~Hz}, 1 \mathrm{H}), 7.40(\mathrm{t}, J=7.6 \mathrm{~Hz}, 2 \mathrm{H}), 7.30-7.23(\mathrm{~m}, 2 \mathrm{H})$, $7.20-7.14(\mathrm{~m}, 3 \mathrm{H}), 2.86$ (broad, 1H), $2.73-2.55(\mathrm{~m}, 2 \mathrm{H}), 1.79-1.71(\mathrm{~m}, 2 \mathrm{H}), 1.66-1.55(\mathrm{~m}$, $1 \mathrm{H}), 1.36(\mathrm{~d}, J=9.3 \mathrm{~Hz}, 1 \mathrm{H}), 1.24(\mathrm{~d}, J=6.5 \mathrm{~Hz}, 12 \mathrm{H}) .{ }^{13} \mathbf{C}$ NMR $\left(101 \mathrm{MHz}, \mathrm{CDCl}_{3}\right) \delta 170.79$, $142.61,133.14,128.77,128.55,128.43,128.00,125.83,81.29,36.10,31.15,29.76,25.47,25.23$, 25.00. ${ }^{11} \mathbf{B}$ NMR $\left(128 \mathrm{MHz}, \mathrm{CDCl}_{3}\right) \delta$ 20.13. ESI-HR calcd for $\mathrm{C}_{23} \mathrm{H}_{31} \mathrm{BNO}_{3}{ }^{+}\left([\mathrm{M}+\mathrm{H}]^{+}\right) 380.2392$, found 380.2379. IR $v\left(\mathrm{~cm}^{-1}\right) 2989,1610,1275,749$. 
<smiles>O=C(NCC(CCc1ccccc1)Cc1ccccc1)c1ccccc1</smiles>

$\boldsymbol{\beta}$-Aminoboronic ester 3d: Following the general procedure [B], enamide 1d-E (25.1 mg, 0.100 mmol) was converted to the $\beta$-aminoboronic ester. Purification by silica gel chromatography gave $9.1 \mathrm{mg}$ (24\% yield) of $\mathbf{3 d}$ as a colorless oil. ${ }^{1} \mathbf{H} \mathbf{~ N M R}\left(400 \mathrm{MHz}, \mathrm{CDCl}_{3}\right) \delta 7.80-7.71(\mathrm{~m}, 2 \mathrm{H})$, $7.51-7.46(\mathrm{~m}, 1 \mathrm{H}), 7.46-7.38(\mathrm{~m}, 2 \mathrm{H}), 7.32-7.24(\mathrm{~m}, 2 \mathrm{H}), 7.23-7.14(\mathrm{~m}, 3 \mathrm{H}), 6.72(\mathrm{~s}, 1 \mathrm{H})$, $3.69-3.61(\mathrm{~m}, 1 \mathrm{H}), 3.58-3.50(\mathrm{~m}, 1 \mathrm{H}), 2.76-2.62(\mathrm{~m}, 2 \mathrm{H}), 1.92-1.81(\mathrm{~m}, 1 \mathrm{H}), 1.79-1.63(\mathrm{~m}$, 1H), $1.42-1.33(\mathrm{~m}, 1 \mathrm{H}), 1.28(\mathrm{~s}, 12 \mathrm{H}) .{ }^{13} \mathrm{C} \mathbf{N M R}\left(101 \mathrm{MHz}, \mathrm{CDCl}_{3}\right) \delta 167.18,142.54,135.16$, 131.31, 128.63, 128.46, 126.90, 125.89, 83.75, 40.68, 35.17, 30.90, 29.42, 25.11, 25.01. ${ }^{11} \mathbf{B}$ NMR $\left(128 \mathrm{MHz}, \mathrm{CDCl}_{3}\right) \delta$ 33.91. ESI-HR calcd for $\mathrm{C}_{23} \mathrm{H}_{31} \mathrm{BNO}_{3}{ }^{+}\left([\mathrm{M}+\mathrm{H}]^{+}\right)$380.2392, found 380.2380. IR $v\left(\mathrm{~cm}^{-1}\right) 3294,2989,1633,750$.<smiles>CCC(Cc1ccccc1)NC(=O)C(C)(C)C</smiles>

$\boldsymbol{\alpha}$-Aminoboronic ester 2e: Following the general procedure [A], enamide 1e-Z (28.2 mg, 0.200 mmol) was converted to the $\alpha$-aminoboronic ester. Purification by silica gel chromatography gave $33.0 \mathrm{mg}$ (61\% yield) of $\mathbf{2 b}$ as a white solid. ${ }^{1} \mathbf{H}$ NMR (400 MHz, $\left.\mathrm{CDCl}_{3}\right) \delta 6.59(\mathrm{~s}, 1 \mathrm{H}), 2.52-$ $2.41(\mathrm{~m}, 1 \mathrm{H}), 1.64(\mathrm{tt}, J=14.6,7.4 \mathrm{~Hz}, 1 \mathrm{H}), 1.49-1.35(\mathrm{~m}, 1 \mathrm{H}), 1.21(\mathrm{~s}, 9 \mathrm{H}), 1.19(\mathrm{~s}, 12 \mathrm{H}), 0.95$ (t, $J=7.4 \mathrm{~Hz}, 3 \mathrm{H}) .{ }^{13} \mathrm{C}$ NMR $\left(101 \mathrm{MHz}, \mathrm{CDCl}_{3}\right) \delta 183.52,80.44,35.85,27.03,25.46,25.23$, 24.34, 12.47. ${ }^{11} \mathbf{B}$ NMR $\left(128 \mathrm{MHz}, \mathrm{CDCl}_{3}\right) \delta$ 16.39. ESI-HR calcd for $\mathrm{C}_{14} \mathrm{H}_{29} \mathrm{BNO}_{3}{ }^{+}\left([\mathrm{M}+\mathrm{H}]^{+}\right)$ 270.2235, found 270.2230. IR $v\left(\mathrm{~cm}^{-1}\right) 3212,2971,1577,735$. M. P. $149-150{ }^{\circ} \mathrm{C}$.<smiles>CC([Pb])CNC(=O)C(C)(C)C</smiles>

$\boldsymbol{\beta}$-Aminoboronic ester 3e: Following the general procedure [B], enamide 1e- $\boldsymbol{E}$ (28.2 mg, 0.200 mmol) was converted to the $\beta$-aminoboronic ester. Purification by silica gel chromatography gave $43.6 \mathrm{mg}$ (81\% yield) of $\mathbf{3 b}$ as a colorless oil. ${ }^{1} \mathbf{H} \mathbf{~ N M R}\left(400 \mathrm{MHz}, \mathrm{CDCl}_{3}\right) \delta 6.25(\mathrm{~s}, 1 \mathrm{H}), 3.43-$ $3.32(\mathrm{~m}, 1 \mathrm{H}), 3.10(\mathrm{ddd}, J=13.1,8.2,5.0 \mathrm{~Hz}, 1 \mathrm{H}), 1.23(\mathrm{~s}, 12 \mathrm{H}), 1.21-1.29(\mathrm{~s}, 1 \mathrm{H}), 1.16(\mathrm{~s}, 9 \mathrm{H})$, 
$0.95(\mathrm{~d}, J=7.5 \mathrm{~Hz}, 3 \mathrm{H}) .{ }^{13} \mathbf{C}$ NMR $\left(101 \mathrm{MHz}, \mathrm{CDCl}_{3}\right) \delta 178.19,83.45,42.23,38.77,27.70,24.98$, 24.90, 13.27. ${ }^{11} \mathbf{B}$ NMR $\left(128 \mathrm{MHz}, \mathrm{CDCl}_{3}\right) \delta$ 34.14. ESI-HR calcd for $\mathrm{C}_{14} \mathrm{H}_{29} \mathrm{BNO}_{3}{ }^{+}\left([\mathrm{M}+\mathrm{H}]^{+}\right)$ 270.2235, found 270.2231. IR $v\left(\mathrm{~cm}^{-1}\right) 3357,2976,1638,750$.<smiles>CCCCCC(Br)Cc1ccccc1</smiles>

$\boldsymbol{\alpha}$-Aminoboronic ester 2f: Following the general procedure [A], enamide 1f-Z (18.3 mg, 0.100 mmol) was converted to the $\alpha$-aminoboronic ester. Purification by silica gel chromatography gave $26.5 \mathrm{mg}$ (85\% yield) of $\mathbf{2 f}$ as a white solid. ${ }^{1} \mathbf{H} \mathbf{~ N M R}\left(400 \mathrm{MHz}, \mathrm{CDCl}_{3}\right) \delta 6.55(\mathrm{~s}, 1 \mathrm{H}), 2.58-$ $2.48(\mathrm{~m}, 1 \mathrm{H}), 1.67-1.51(\mathrm{~m}, 1 \mathrm{H}), 1.44-1.26(\mathrm{~m}, 7 \mathrm{H}), 1.21(\mathrm{~s}, 9 \mathrm{H}), 1.19(\mathrm{~s}, 12 \mathrm{H}), 0.92-0.82(\mathrm{~m}$, 3H). ${ }^{13} \mathrm{C}$ NMR $\left(101 \mathrm{MHz}, \mathrm{CDCl}_{3}\right) \delta 183.50,80.41,35.80,32.03,31.26,27.88,27.01,25.44$, 25.25, 22.69, 14.16. ${ }^{11} \mathbf{B}$ NMR $\left(128 \mathrm{MHz}, \mathrm{CDCl}_{3}\right) \delta$ 16.37. ESI-HR calcd for $\mathrm{C}_{17} \mathrm{H}_{35} \mathrm{BNO}_{3}{ }^{+}$ $\left([\mathrm{M}+\mathrm{H}]^{+}\right) 312.2705$, found 312.2701. IR $v\left(\mathrm{~cm}^{-1}\right) 3446,2971,1559,732$. M. P. $120-121^{\circ} \mathrm{C}$.<smiles>CCCCC([Pb])CNC(=O)C(C)(C)C</smiles>

$\boldsymbol{\beta}$-Aminoboronic ester 3f: Following the general procedure [B], enamide 1f- $\boldsymbol{E}(18.3 \mathrm{mg}, 0.100$ mmol) was converted to the $\beta$-aminoboronic ester. Purification by silica gel chromatography gave $27.4 \mathrm{mg}$ (88\% yield) of $\mathbf{3 f}$ as a colorless oil. ${ }^{\mathbf{1}} \mathbf{H} \mathbf{~ N M R}\left(400 \mathrm{MHz}, \mathrm{CDCl}_{3}\right) \delta 6.21(\mathrm{~s}, 1 \mathrm{H}), 3.43-$ $3.33(\mathrm{~m}, 1 \mathrm{H}), 3.23-3.14(\mathrm{~m}, 1 \mathrm{H}), 1.51-1.38(\mathrm{~m}, 1 \mathrm{H}), 1.35-1.26(\mathrm{~m}, 5 \mathrm{H}), 1.24(\mathrm{~s}, 12 \mathrm{H}), 1.21-$ $1.18(\mathrm{~m}, 1 \mathrm{H}), 1.17(\mathrm{~s}, 9 \mathrm{H}), 0.91-0.81(\mathrm{~m}, 3 \mathrm{H}) .{ }^{13} \mathrm{C} \mathbf{N M R}\left(101 \mathrm{MHz}, \mathrm{CDCl}_{3}\right) \delta 178.14,83.46$, 40.37, 38.79, 31.14, 28.46, 27.73, 25.04, 24.94, 22.96, 14.11. ${ }^{11} \mathbf{B}$ NMR $\left(128 \mathrm{MHz}, \mathrm{CDCl}_{3}\right) \delta$ 34.43. ESI-HR calcd for $\mathrm{C}_{17} \mathrm{H}_{35} \mathrm{BNO}_{3}{ }^{+}\left([\mathrm{M}+\mathrm{H}]^{+}\right) 312.2705$, found 312.2702. IR $v\left(\mathrm{~cm}^{-1}\right) 3365$, $2987,1653,750$.<smiles>CC(C)CCCC(Br)c1ccccc1</smiles>

$\boldsymbol{\alpha}$-Aminoboronic ester 2g: Following the general procedure [A], enamide 1g-Z (19.7 mg, 0.100 mmol) was converted to the $\alpha$-aminoboronic ester. Purification by silica gel chromatography gave 
$26.3 \mathrm{mg}(81 \%$ yield $)$ of $\mathbf{2 g}$ as a white solid. ${ }^{1} \mathbf{H} \mathbf{~ N M R}\left(400 \mathrm{MHz}, \mathrm{CDCl}_{3}\right) \delta 6.51(\mathrm{~s}, 1 \mathrm{H}), 2.54(\mathrm{~d}, J$ $=7.0 \mathrm{~Hz}, 1 \mathrm{H}), 1.64-1.48(\mathrm{~m}, 2 \mathrm{H}), 1.44-1.24(\mathrm{~m}, 4 \mathrm{H}), 1.22(\mathrm{~s}, 9 \mathrm{H}), 1.20(\mathrm{~s}, 12 \mathrm{H}), 1.18-1.15(\mathrm{~s}$, 1H), $0.86(\mathrm{dd}, J=6.6,2.5 \mathrm{~Hz}, 6 \mathrm{H}) .{ }^{13} \mathbf{C}$ NMR $\left(101 \mathrm{MHz}, \mathrm{CDCl}_{3}\right) \delta 183.48,80.45,39.18,35.84$, 31.60, 27.96, 27.03, 25.96, 25.47, 25.29, 22.86, 22.64. ${ }^{11} \mathbf{B}$ NMR $\left(128 \mathrm{MHz}, \mathrm{CDCl}_{3}\right) \delta 16.58$. ESI-HR calcd for $\mathrm{C}_{18} \mathrm{H}_{37} \mathrm{BNO}_{3}{ }^{+}\left([\mathrm{M}+\mathrm{H}]^{+}\right) 326.2861$, found 326.2854. IR $v\left(\mathrm{~cm}^{-1}\right) 3421,2969$, 1559, 746. M. P. $81-82^{\circ} \mathrm{C}$.<smiles>CC(C)CCC(CNC(=O)C(C)(C)C)c1ccccc1</smiles>

$\boldsymbol{\beta}$-Aminoboronic ester 3g: Following the general procedure [B], enamide 1g-E (19.7 mg, 0.100 mmol) was converted to the $\beta$-aminoboronic ester. Purification by silica gel chromatography gave $28.6 \mathrm{mg}$ (88\% yield) of $\mathbf{3 g}$ as a colorless oil. ${ }^{1} \mathbf{H}$ NMR $\left(400 \mathrm{MHz}, \mathrm{CDCl}_{3}\right) \delta 6.20(\mathrm{~s}, 1 \mathrm{H}), 3.44-$ $3.32(\mathrm{~m}, 1 \mathrm{H}), 3.19(\mathrm{ddd}, J=13.2,8.1,5.3 \mathrm{~Hz}, 1 \mathrm{H}), 1.55-1.38(\mathrm{~m}, 2 \mathrm{H}), 1.37-1.28(\mathrm{~m}, 1 \mathrm{H}), 1.24$ (s, 12H), $1.22-1.18(\mathrm{~m}, 3 \mathrm{H}), 1.17(\mathrm{~s}, 9 \mathrm{H}), 0.84(\mathrm{~d}, J=6.6 \mathrm{~Hz}, 6 \mathrm{H}) .{ }^{13} \mathbf{C} \mathbf{N M R}\left(101 \mathrm{MHz}, \mathrm{CDCl}_{3}\right)$ $\delta 178.13,83.46,40.38,38.78,38.19,28.30,27.73,26.52,25.05,24.95,22.69 .{ }^{11} \mathbf{B}$ NMR $(128$ $\left.\mathrm{MHz}, \mathrm{CDCl}_{3}\right) \delta$ 33.95. ESI-HR calcd for $\mathrm{C}_{18} \mathrm{H}_{37} \mathrm{BNO}_{3}{ }^{+}\left([\mathrm{M}+\mathrm{H}]^{+}\right) 326.2861$, found 326.2859. IR $v\left(\mathrm{~cm}^{-1}\right) 3446,2983,1653,905$.<smiles>CC(C)(C)C(=O)NC(CCCCCl)Cc1ccccc1</smiles>

$\boldsymbol{\alpha}$-Aminoboronic ester $\mathbf{2 h}$ : Following the general procedure [A], enamide $\mathbf{1 h}-\boldsymbol{Z}$ (40.7 mg, 0.200 mmol) was converted to the $\alpha$-aminoboronic ester. Purification by silica gel chromatography gave $37.1 \mathrm{mg}$ (56\% yield) of $\mathbf{2 h}$ as a white solid. ${ }^{1} \mathbf{H}$ NMR (400 MHz, $\left.\mathrm{CDCl}_{3}\right) \delta 6.59(\mathrm{~s}, 1 \mathrm{H}), 3.63-$ $3.48(\mathrm{~m}, 2 \mathrm{H}), 2.55(\mathrm{t}, J=6.3 \mathrm{~Hz}, 1 \mathrm{H}), 1.88-1.71(\mathrm{~m}, 2 \mathrm{H}), 1.67-1.55(\mathrm{~m}, 1 \mathrm{H}), 1.55-1.37(\mathrm{~m}$, 3H), 1.22 (s, 9H), 1.19 (s, 12H). ${ }^{13} \mathbf{C}$ NMR (101 MHz, $\left.\mathrm{CDCl}_{3}\right) \delta$ 183.83, 80.48, 45.20, 35.85, 32.57, 30.53, 27.00, 25.48, 25.25, 25.17. ${ }^{11} \mathbf{B}$ NMR $\left(128 \mathrm{MHz}, \mathrm{CDCl}_{3}\right) \delta$ 16.15. ESI-HR calcd for $\mathrm{C}_{16} \mathrm{H}_{32} \mathrm{BClNO}_{3}{ }^{+}\left([\mathrm{M}+\mathrm{H}]^{+}\right) 332.2158$, found 332.2151. IR $v\left(\mathrm{~cm}^{-1}\right) 3199,2970,1577,735$. M. P. $117-118^{\circ} \mathrm{C}$. 
<smiles>CC(C)(C)C(=O)NCC(Br)CCCCl</smiles>

$\boldsymbol{\beta}$-Aminoboronic ester 3h: Following the general procedure [B], enamide $\mathbf{1 h}-\boldsymbol{E}$ (20.4 mg, 0.100 mmol) was converted to the $\beta$-aminoboronic ester. Purification by silica gel chromatography gave $22.0 \mathrm{mg}\left(66 \%\right.$ yield) of $\mathbf{3 h}$ as a colorless oil. ${ }^{1} \mathbf{H} \mathbf{~ N M R}\left(400 \mathrm{MHz}, \mathrm{CDCl}_{3}\right) \delta 6.22(\mathrm{~s}, 1 \mathrm{H}), 3.56-$ $3.46(\mathrm{~m}, 2 \mathrm{H}), 3.43-3.34(\mathrm{~m}, 1 \mathrm{H}), 3.28-3.17(\mathrm{~m}, 1 \mathrm{H}), 1.90-1.75(\mathrm{~m}, 2 \mathrm{H}), 1.65-1.52(\mathrm{~m}, 1 \mathrm{H})$, $1.52-1.41(\mathrm{~m}, 1 \mathrm{H}), 1.25(\mathrm{~s}, 12 \mathrm{H}), 1.23-1.20(\mathrm{~m}, 1 \mathrm{H}), 1.18(\mathrm{~s}, 9 \mathrm{H}) .{ }^{13} \mathbf{C} \mathbf{N M R}\left(101 \mathrm{MHz}, \mathrm{CDCl}_{3}\right)$ $\delta 178.27,83.68,45.28,40.06,38.82,31.83,27.73,25.98,25.06,24.98 .{ }^{11} \mathbf{B}$ NMR $(128 \mathrm{MHz}$, $\left.\mathrm{CDCl}_{3}\right) \delta$ 34.13. ESI-HR calcd for $\mathrm{C}_{16} \mathrm{H}_{32} \mathrm{BClNO}_{3}{ }^{+}\left([\mathrm{M}+\mathrm{H}]^{+}\right) 332.2158$, found 332.2154. IR $v$ $\left(\mathrm{cm}^{-1}\right) 3446,2983,1653,907$.<smiles>CC(C)(C)C(=O)NC(CCCCOC(=O)c1ccccc1)Cc1ccccc1</smiles>

$\boldsymbol{\alpha}$-Aminoboronic ester 2i: Following the general procedure [A], enamide 1i-Z (57.9 mg, 0.200 mmol) was converted to the $\alpha$-aminoboronic ester. Purification by silica gel chromatography gave $46.1 \mathrm{mg}(55 \%$ yield $)$ of $\mathbf{2 i}$ as a white solid. ${ }^{1} \mathbf{H} \mathbf{~ N M R}\left(400 \mathrm{MHz}, \mathrm{CDCl}_{3}\right) \delta 8.02(\mathrm{~d}, J=7.5 \mathrm{~Hz}, 2 \mathrm{H})$, $7.54(\mathrm{t}, J=7.4 \mathrm{~Hz}, 1 \mathrm{H}), 7.42(\mathrm{t}, J=7.6 \mathrm{~Hz}, 2 \mathrm{H}), 6.71(\mathrm{~s}, 1 \mathrm{H}), 4.42-4.25(\mathrm{~m}, 2 \mathrm{H}), 2.63-2.47(\mathrm{~m}$, 1H), $1.80(\mathrm{qd}, J=13.6,6.8 \mathrm{~Hz}, 2 \mathrm{H}), 1.73-1.62(\mathrm{~m}, 1 \mathrm{H}), 1.56-1.42(\mathrm{~m}, 3 \mathrm{H}), 1.21(\mathrm{~s}, 9 \mathrm{H}), 1.19$ (s, 12H). ${ }^{13}$ C NMR $\left(101 \mathrm{MHz}, \mathrm{CDCl}_{3}\right) \delta 183.80,166.89,132.99,130.57,129.67,128.45,80.43$, 64.98, 35.81, 31.06, 29.82, 29.02, 26.99, 25.47, 25.26, 24.48. ${ }^{11} \mathbf{B}$ NMR $\left(128 \mathrm{MHz}, \mathrm{CDCl}_{3}\right) \delta$ 16.70. ESI-HR calcd for $\mathrm{C}_{23} \mathrm{H}_{37} \mathrm{BNO}_{5}^{+}\left([\mathrm{M}+\mathrm{H}]^{+}\right) 418.2759$, found 418.2752. IR $v\left(\mathrm{~cm}^{-1}\right) 3275$, 2972, 1716, 732. M. P. $139-140{ }^{\circ} \mathrm{C}$.<smiles>CC(C)(C)C(=O)NCC(Br)c1ccccc1</smiles>

$\boldsymbol{\beta}$-Aminoboronic ester 3i: Following the general procedure [B], enamide 1i- $\boldsymbol{E}(28.9 \mathrm{mg}, 0.100$ mmol) was converted to the $\beta$-aminoboronic ester. Purification by silica gel chromatography gave $30.0 \mathrm{mg}$ (72\% yield) of $\mathbf{3 i}$ as a white solid. ${ }^{1} \mathbf{H} \mathbf{~ N M R}\left(400 \mathrm{MHz}, \mathrm{CDCl}_{3}\right) \delta 8.03(\mathrm{~d}, J=7.3 \mathrm{~Hz}, 2 \mathrm{H})$, 
$7.54(\mathrm{t}, J=7.4 \mathrm{~Hz}, 1 \mathrm{H}), 7.42(\mathrm{t}, J=7.7 \mathrm{~Hz}, 2 \mathrm{H}), 6.24(\mathrm{~s}, 1 \mathrm{H}), 4.33-4.26(\mathrm{~m}, 2 \mathrm{H}), 3.52-3.36(\mathrm{~m}$, $1 \mathrm{H}), 3.25(\mathrm{ddd}, J=13.2,8.0,5.3 \mathrm{~Hz}, 1 \mathrm{H}), 1.90-1.77(\mathrm{~m}, 2 \mathrm{H}), 1.67-1.56(\mathrm{~m}, 1 \mathrm{H}), 1.55-1.44$ $(\mathrm{m}, 1 \mathrm{H}), 1.30-1.26(\mathrm{~m}, 1 \mathrm{H}), 1.25(\mathrm{~s}, 12 \mathrm{H}), 1.17(\mathrm{~s}, 9 \mathrm{H}) .{ }^{13} \mathrm{C}$ NMR $\left(101 \mathrm{MHz}, \mathrm{CDCl}_{3}\right) \delta 178.24$ $166.75,132.88,130.67,129.71,128.41,83.65,65.20,40.21,38.81,28.11,27.73,25.26,25.05$, 24.98. ${ }^{11} \mathbf{B}$ NMR $\left(128 \mathrm{MHz}, \mathrm{CDCl}_{3}\right) \delta$ 33.83. ESI-HR calcd for $\mathrm{C}_{23} \mathrm{H}_{37} \mathrm{BNO}_{5}{ }^{+}\left([\mathrm{M}+\mathrm{H}]^{+}\right) 418.2759$, found 418.2754. IR $v\left(\mathrm{~cm}^{-1}\right) 3363,2976,1717,1647,750$. M. P. $90-91{ }^{\circ} \mathrm{C}$.

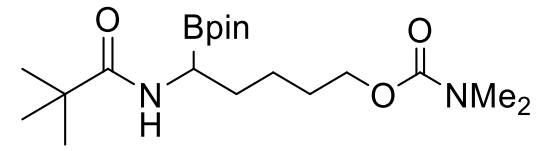

$\boldsymbol{\alpha}$-Aminoboronic ester $\mathbf{2 j}$ : Following the general procedure [A], enamide $\mathbf{1 j}-\mathbf{Z}$ (25.6 mg, 0.100 mmol) was converted to the $\alpha$-aminoboronic ester. Purification by silica gel chromatography gave $25.2 \mathrm{mg}$ (66\% yield) of $\mathbf{2} \mathbf{j}$ as a white solid. ${ }^{\mathbf{1}} \mathbf{H} \mathbf{~ N M R}\left(400 \mathrm{MHz}, \mathrm{CDCl}_{3}\right) \delta 7.07(\mathrm{~s}, 1 \mathrm{H}), 4.11-$ $4.01(\mathrm{~m}, 2 \mathrm{H}), 2.88(\mathrm{~s}, 6 \mathrm{H}), 2.58-2.46(\mathrm{~m}, 1 \mathrm{H}), 1.76-1.53(\mathrm{~m}, 3 \mathrm{H}), 1.48-1.35(\mathrm{~m}, 3 \mathrm{H}), 1.22(\mathrm{~d}$, $J=1.3 \mathrm{~Hz}, 9 \mathrm{H}), 1.18(\mathrm{~d}, J=1.3 \mathrm{~Hz}, 12 \mathrm{H}) .{ }^{13} \mathbf{C} \mathbf{N M R}\left(101 \mathrm{MHz}, \mathrm{CDCl}_{3}\right) \delta 183.87,157.09,80.28$, $65.34,35.73,30.94,29.37,26.99,25.44,25.23,24.74,24.30 .{ }^{11} \mathbf{B} \mathbf{N M R}\left(128 \mathrm{MHz}, \mathrm{CDCl}_{3}\right) \delta$ 15.88. ESI-HR calcd for $\mathrm{C}_{19} \mathrm{H}_{38} \mathrm{BN}_{2} \mathrm{O}_{5}{ }^{+}\left([\mathrm{M}+\mathrm{H}]^{+}\right) 385.2868$, found 385.2862. IR $v\left(\mathrm{~cm}^{-1}\right) 3276$, 2971, 1685, 733. M. P. $114-115^{\circ} \mathrm{C}$.<smiles>CNC(=O)OCCCC(Br)c1ccccc1</smiles>

$\boldsymbol{\beta}$-Aminoboronic ester 3j: Following the general procedure [B], enamide $\mathbf{1 j}$ - $\boldsymbol{E}$ (25.6 mg, 0.100 mmol) was converted to the $\beta$-aminoboronic ester. Purification by silica gel chromatography gave $28.8 \mathrm{mg}(75 \%$ yield $)$ of $\mathbf{3 j}$ as a colorless oil. ${ }^{\mathbf{1}} \mathbf{H} \mathbf{~ N M R}\left(400 \mathrm{MHz}, \mathrm{CDCl}_{3}\right) \delta 6.23(\mathrm{~s}, 1 \mathrm{H}), 4.05-$ $3.98(\mathrm{~m}, 2 \mathrm{H}), 3.39(\mathrm{dt}, J=12.7,5.6 \mathrm{~Hz}, 1 \mathrm{H}), 3.24-3.15(\mathrm{~m}, 1 \mathrm{H}), 2.87(\mathrm{~s}, 6 \mathrm{H}), 1.73-1.61(\mathrm{~m}$, $2 \mathrm{H}), 1.56-1.45(\mathrm{~m}, 1 \mathrm{H}), 1.44-1.34(\mathrm{~m}, 1 \mathrm{H}), 1.23(\mathrm{~s}, 12 \mathrm{H}), 1.21-1.18(\mathrm{~m}, 1 \mathrm{H}), 1.17(\mathrm{~s}, 9 \mathrm{H})$. ${ }^{13} \mathrm{C}$ NMR $\left(101 \mathrm{MHz}, \mathrm{CDCl}_{3}\right) \delta 178.20,156.87,83.57,65.48,40.20,38.77,28.40,27.73,27.69$, 25.15, 25.02, 24.94. ${ }^{11} \mathbf{B}$ NMR $\left(128 \mathrm{MHz}, \mathrm{CDCl}_{3}\right) \delta 33.81$. ESI-HR calcd for $\mathrm{C}_{19} \mathrm{H}_{38} \mathrm{BN}_{2} \mathrm{O}_{5}{ }^{+}$ $\left([\mathrm{M}+\mathrm{H}]^{+}\right)$385.2868, found 385.2864. IR $v\left(\mathrm{~cm}^{-1}\right) 3368,2974,1701,1644,772$. 


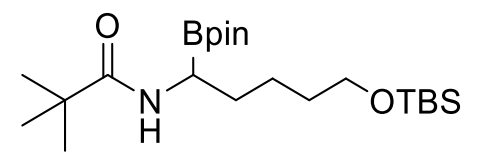

$\boldsymbol{\alpha}$-Aminoboronic ester 2k: Following the general procedure [A], enamide 1k-Z (30.0 mg, 0.100 mmol) was converted to the $\alpha$-aminoboronic ester. Purification by silica gel chromatography gave $34.6 \mathrm{mg}$ (81\% yield) of $\mathbf{2} \mathbf{k}$ as a white solid. ${ }^{1} \mathbf{H} \mathbf{~ N M R}\left(400 \mathrm{MHz}, \mathrm{CDCl}_{3}\right) \delta 6.61(\mathrm{~s}, 1 \mathrm{H}), 3.62$ (t, $J$ $=6.3 \mathrm{~Hz}, 2 \mathrm{H}), 2.59-2.54(\mathrm{~m}, 1 \mathrm{H}), 1.68-1.47(\mathrm{~m}, 4 \mathrm{H}), 1.42-1.38(\mathrm{~m}, 2 \mathrm{H}), 1.22(\mathrm{~s}, 9 \mathrm{H}), 1.20(\mathrm{~s}$, 12H), $0.88(\mathrm{~s}, 9 \mathrm{H}), 0.04(\mathrm{~s}, 6 \mathrm{H}) .{ }^{13} \mathbf{C}$ NMR $\left(101 \mathrm{MHz}, \mathrm{CDCl}_{3}\right) \delta$ 183.65, 80.41, 63.15, 35.81, $32.99,31.02,27.01,26.15,25.47,25.26,24.35,18.53,-5.07 .{ }^{11} \mathbf{B}$ NMR $\left(128 \mathrm{MHz}, \mathrm{CDCl}_{3}\right) \delta$ 15.95. ESI-HR calcd for $\mathrm{C}_{22} \mathrm{H}_{47} \mathrm{BNO}_{4} \mathrm{Si}^{+}\left([\mathrm{M}+\mathrm{H}]^{+}\right) 428.3362$, found 428.3362. IR $v\left(\mathrm{~cm}^{-1}\right) 3178$, 2974, 1653, 722. M.P. $96-97^{\circ} \mathrm{C}$.<smiles>CC(C)(C)C(=O)NCC(Br)CCC[SeH2]</smiles>

$\boldsymbol{\beta}$-Aminoboronic ester 3k: Following the general procedure [B], enamide 1k-E (30.0 mg, 0.100 mmol) was converted to the $\beta$-aminoboronic ester. Purification by silica gel chromatography gave $28.6 \mathrm{mg}(67 \%$ yield $)$ of $\mathbf{3 k}$ as a colorless oil. ${ }^{\mathbf{1}} \mathbf{H} \mathbf{~ N M R}\left(400 \mathrm{MHz}, \mathrm{CDCl}_{3}\right) \delta 6.22(\mathrm{~s}, 1 \mathrm{H}), 3.58(\mathrm{t}, J$ $=6.5 \mathrm{~Hz}, 2 \mathrm{H}), 3.45-3.34(\mathrm{~m}, 1 \mathrm{H}), 3.26-3.15(\mathrm{~m}, 1 \mathrm{H}), 1.59-1.49(\mathrm{~m}, 3 \mathrm{H}), 1.41-1.32(\mathrm{~m}, 1 \mathrm{H})$, $1.24(\mathrm{~s}, 12 \mathrm{H}), 1.21-1.19(\mathrm{~m}, 1 \mathrm{H}), 1.17(\mathrm{~s}, 9 \mathrm{H}), 0.87(\mathrm{~s}, 9 \mathrm{H}), 0.03(\mathrm{~s}, 6 \mathrm{H}) .{ }^{13} \mathbf{C}$ NMR $(101 \mathrm{MHz}$, $\left.\mathrm{CDCl}_{3}\right) \delta 178.17,83.50,63.44,40.47,38.79,32.18,27.73,26.12,25.17,25.05,24.97,18.48$, -5.11. ${ }^{11} \mathbf{B}$ NMR $\left(128 \mathrm{MHz}, \mathrm{CDCl}_{3}\right) \delta$ 34.17. ESI-HR calcd for $\mathrm{C}_{22} \mathrm{H}_{47} \mathrm{BNO}_{4} \mathrm{Si}^{+}\left([\mathrm{M}+\mathrm{H}]^{+}\right)$ 428.3362, found 428.3362. IR $v\left(\mathrm{~cm}^{-1}\right) 3358,2929,1642,775$.<smiles>CCOCCCCC(Br)c1ccccc1</smiles>

$\boldsymbol{\alpha}$-Aminoboronic ester 21: Following the general procedure [A], enamide $\mathbf{1 l}-\boldsymbol{Z}$ (24.3 mg, 0.100 mmol) was converted to the $\alpha$-aminoboronic ester. Purification by silica gel chromatography gave $22.4 \mathrm{mg}\left(60 \%\right.$ yield) of $\mathbf{2 l}$ as a colorless oil. ${ }^{1} \mathbf{H}$ NMR $\left(400 \mathrm{MHz}, \mathrm{CDCl}_{3}\right) \delta 6.73(\mathrm{~s}, 1 \mathrm{H}), 4.65(\mathrm{~s}$, $2 \mathrm{H}), 3.63-3.47(\mathrm{~m}, 4 \mathrm{H}), 2.59-2.48(\mathrm{~m}, 1 \mathrm{H}), 1.69-1.52(\mathrm{~m}, 3 \mathrm{H}), 1.48-1.35(\mathrm{~m}, 3 \mathrm{H}), 1.23-$ $1.19(\mathrm{~m}, 12 \mathrm{H}), 1.18(\mathrm{~s}, 12 \mathrm{H}) .{ }^{13} \mathbf{C}$ NMR $\left(101 \mathrm{MHz}, \mathrm{CDCl}_{3}\right) \delta 183.77,95.23,80.33,67.94,63.30$, 
35.73, 30.98, 29.73, 26.97, 25.42, 25.21, 24.83, 15.29. ${ }^{11} \mathbf{B}$ NMR $\left(128 \mathrm{MHz}, \mathrm{CDCl}_{3}\right) \delta 15.79$.

ESI-HR calcd for $\mathrm{C}_{19} \mathrm{H}_{39} \mathrm{BNO}_{5}{ }^{+}\left([\mathrm{M}+\mathrm{H}]^{+}\right) 372.2916$, found 372.2916. IR $v\left(\mathrm{~cm}^{-1}\right) 3421,2972$, $1559,732$.<smiles>CCOCCCC(CNC(=O)C(C)(C)C)c1ccccc1</smiles>

$\boldsymbol{\beta}$-Aminoboronic ester 31: Following the general procedure [B], enamide 1l- $\boldsymbol{E}$ (24.3 $\mathrm{mg}, 0.100$ mmol) was converted to the $\beta$-aminoboronic ester. Purification by silica gel chromatography gave $32.3 \mathrm{mg}$ (87\% yield) of $\mathbf{3 l}$ as a colorless oil. ${ }^{1} \mathbf{H}$ NMR $\left(400 \mathrm{MHz}, \mathrm{CDCl}_{3}\right) \delta 6.23(\mathrm{~s}, 1 \mathrm{H}), 4.65(\mathrm{~s}$, 2H), $3.58(\mathrm{q}, J=7.1 \mathrm{~Hz}, 2 \mathrm{H}), 3.51(\mathrm{t}, J=6.5 \mathrm{~Hz}, 2 \mathrm{H}), 3.45-3.36(\mathrm{~m}, 1 \mathrm{H}), 3.20(\mathrm{ddd}, J=13.2$, 8.1, $5.2 \mathrm{~Hz}, 1 \mathrm{H}), 1.68-1.59(\mathrm{~m}, 2 \mathrm{H}), 1.57-1.47(\mathrm{~m}, 1 \mathrm{H}), 1.45-1.36(\mathrm{~m}, 1 \mathrm{H}), 1.25(\mathrm{~s}, 12 \mathrm{H})$, $1.21(\mathrm{t}, J=7.1 \mathrm{~Hz}, 4 \mathrm{H}), 1.18(\mathrm{~s}, 9 \mathrm{H}) .{ }^{13} \mathrm{C} \mathbf{N M R}\left(101 \mathrm{MHz}, \mathrm{CDCl}_{3}\right) \delta$ 178.20, 95.11, 83.56, 67.93, $63.23,40.34,38.80,28.98,27.73,25.42,25.05,24.98,15.34 .{ }^{11} \mathbf{B}$ NMR $\left(128 \mathrm{MHz}, \mathrm{CDCl}_{3}\right) \delta$ 34.44. ESI-HR calcd for $\mathrm{C}_{19} \mathrm{H}_{39} \mathrm{BNO}_{5}{ }^{+}\left([\mathrm{M}+\mathrm{H}]^{+}\right) 372.2916$, found 372.2915. IR $v\left(\mathrm{~cm}^{-1}\right) 3365$, $2975,1645,851$.<smiles>CC(C)(C)C(=O)NC(CCCCOCc1ccccc1)Cc1ccccc1</smiles>

$\boldsymbol{\alpha}$-Aminoboronic ester 2m: Following the general procedure [A], enamide $\mathbf{1 m}-\boldsymbol{Z}$ (61.1 mg, 0.200 mmol) was converted to the $\alpha$-aminoboronic ester. Purification by silica gel chromatography gave $54.7 \mathrm{mg}$ (63\% yield) of $\mathbf{2} \mathbf{m}$ as a white solid. ${ }^{1} \mathbf{H} \mathbf{~ N M R}\left(400 \mathrm{MHz}, \mathrm{CDCl}_{3}\right) \delta 7.41-7.31(\mathrm{~m}, 4 \mathrm{H})$, $7.31-7.27(\mathrm{~m}, 1 \mathrm{H}), 6.65(\mathrm{~s}, 1 \mathrm{H}), 4.75(\mathrm{~s}, 2 \mathrm{H}), 4.59(\mathrm{~s}, 2 \mathrm{H}), 3.66-3.54(\mathrm{~m}, 2 \mathrm{H}), 2.60-2.48(\mathrm{~m}$, 1H), $1.72-1.53(\mathrm{~m}, 3 \mathrm{H}), 1.50-1.38(\mathrm{~m}, 3 \mathrm{H}), 1.21(\mathrm{~s}, 9 \mathrm{H}), 1.19$ (s, 12H). ${ }^{13} \mathbf{C}$ NMR (101 MHz, $\left.\mathrm{CDCl}_{3}\right) \delta 183.74,138.10,128.55,127.95,127.80,94.83,80.37,69.50,68.18,35.77,31.05,29.77$, 26.99, 25.47, 25.26, 24.86. ${ }^{11} \mathbf{B}$ NMR (128 MHz, $\left.\mathrm{CDCl}_{3}\right) \delta$ 15.89. ESI-HR calcd for $\mathrm{C}_{24} \mathrm{H}_{41} \mathrm{BNO}_{5}{ }^{+}\left([\mathrm{M}+\mathrm{H}]^{+}\right)$434.3072, found 434.3072. IR $v\left(\mathrm{~cm}^{-1}\right)$ 3276, 2987, 1559, 732. M. P. $92-93^{\circ} \mathrm{C}$. 
<smiles>CC(C)(C)C(=O)NCC(Br)CCCOCOCc1ccccc1</smiles>

$\boldsymbol{\beta}$-Aminoboronic ester 3m: Following the general procedure [B], enamide 1m-E (30.5 mg, 0.100 mmol) was converted to the $\beta$-aminoboronic ester. Purification by silica gel chromatography gave $30.5 \mathrm{mg}$ (70\% yield) of $\mathbf{3 m}$ as a colorless oil. ${ }^{\mathbf{1}} \mathbf{H} \mathbf{~ N M R}\left(400 \mathrm{MHz}, \mathrm{CDCl}_{3}\right) \delta 7.37-7.31(\mathrm{~m}, 4 \mathrm{H})$, $7.31-7.26(\mathrm{~m}, 1 \mathrm{H}), 6.23(\mathrm{~s}, 1 \mathrm{H}), 4.73(\mathrm{~s}, 2 \mathrm{H}), 4.58(\mathrm{~s}, 2 \mathrm{H}), 3.56(\mathrm{t}, J=6.3 \mathrm{~Hz}, 2 \mathrm{H}), 3.47-3.36$ (m, 1H), $3.21(\mathrm{ddd}, J=13.2,8.0,5.3 \mathrm{~Hz}, 1 \mathrm{H}), 1.71-1.61(\mathrm{~m}, 2 \mathrm{H}), 1.59-1.48(\mathrm{~m}, 1 \mathrm{H}), 1.46-$ $1.37(\mathrm{~m}, 1 \mathrm{H}), 1.24(\mathrm{~s}, 12 \mathrm{H}), 1.22-1.19(\mathrm{~m}, 1 \mathrm{H}), 1.18(\mathrm{~s}, 9 \mathrm{H}) .{ }^{13} \mathbf{C}$ NMR $\left(101 \mathrm{MHz}, \mathrm{CDCl}_{3}\right) \delta$ $178.17,138.16,128.50,128.00,127.73,94.62,83.55,69.37,68.13,40.31,38.78,28.93,27.72$, 25.41, 25.04, 24.96. ${ }^{11} \mathbf{B}$ NMR $\left(128 \mathrm{MHz}, \mathrm{CDCl}_{3}\right) \delta$ 34.13. ESI-HR calcd for $\mathrm{C}_{24} \mathrm{H}_{41} \mathrm{BNO}_{5}{ }^{+}$ $\left([\mathrm{M}+\mathrm{H}]^{+}\right)$434.3072, found 434.3070. IR $v\left(\mathrm{~cm}^{-1}\right) 3365,2977,1652,750$.<smiles>CC(C)(C)C(=O)NC(CCC1CCCCC1)Cc1ccccc1</smiles>

$\boldsymbol{\alpha}$-Aminoboronic ester 2n: Following the general procedure [A], enamide 1n- $\boldsymbol{Z}$ (44.7 mg, 0.200 mmol) was converted to the $\alpha$-aminoboronic ester. Purification by silica gel chromatography gave $56.5 \mathrm{mg}$ (80\% yield) of $\mathbf{2 n}$ as a white solid. ${ }^{1} \mathbf{H}$ NMR $\left(400 \mathrm{MHz}, \mathrm{CDCl}_{3}\right) \delta 6.54(\mathrm{~s}, 1 \mathrm{H}), 2.55-$ $2.44(\mathrm{~m}, 1 \mathrm{H}), 1.77-1.53(\mathrm{~m}, 6 \mathrm{H}), 1.45-1.33(\mathrm{~m}, 1 \mathrm{H}), 1.30-1.23(\mathrm{~m}, 6 \mathrm{H}), 1.21(\mathrm{~s}, 9 \mathrm{H}), 1.19(\mathrm{~s}$, 12H), $0.94-0.78(\mathrm{~m}, 2 \mathrm{H}) .{ }^{13} \mathrm{C}$ NMR $\left(101 \mathrm{MHz}, \mathrm{CDCl}_{3}\right) \delta 183.49,80.42,37.96,36.03,35.81$, $33.64,33.45,28.59,27.02,26.85,26.53,26.49,25.48,25.25 .{ }^{11} \mathbf{B}$ NMR $\left(128 \mathrm{MHz}, \mathrm{CDCl}_{3}\right) \delta$ 16.43. ESI-HR calcd for $\mathrm{C}_{20} \mathrm{H}_{39} \mathrm{BNO}_{3}{ }^{+}\left([\mathrm{M}+\mathrm{H}]^{+}\right) 352.3018$, found 352.3017. IR $v\left(\mathrm{~cm}^{-1}\right) 3213$, 2972, 1577, 748. M. P. $151-152^{\circ} \mathrm{C}$.<smiles>CC(C)(C)C(=O)NCC(Br)CC1CCCCC1</smiles>

$\boldsymbol{\beta}$-Aminoboronic ester 3n: Following the general procedure [B], enamide 1n- $\boldsymbol{E}$ (22.3 mg, 0.100 mmol) was converted to the $\beta$-aminoboronic ester. Purification by silica gel chromatography gave $30.6 \mathrm{mg}$ (87\% yield) of $\mathbf{3 n}$ as a colorless oil. ${ }^{1} \mathbf{H} \mathbf{~ N M R}\left(400 \mathrm{MHz}, \mathrm{CDCl}_{3}\right) \delta 6.19(\mathrm{~s}, 1 \mathrm{H}), 3.34(\mathrm{dt}$, 
$J=12.4,5.0 \mathrm{~Hz}, 1 \mathrm{H}), 3.13(\mathrm{ddd}, J=12.9,7.5,5.5 \mathrm{~Hz}, 1 \mathrm{H}), 1.75-1.54(\mathrm{~m}, 5 \mathrm{H}), 1.35-1.25(\mathrm{~m}$, $3 \mathrm{H}), 1.22(\mathrm{~s}, 12 \mathrm{H}), 1.20-1.16(\mathrm{~m}, 2 \mathrm{H}), 1.15(\mathrm{~s}, 9 \mathrm{H}), 1.13-1.02(\mathrm{~m}, 2 \mathrm{H}), 0.89-0.72(\mathrm{~m}, 2 \mathrm{H})$. ${ }^{13} \mathrm{C}$ NMR $\left(101 \mathrm{MHz}, \mathrm{CDCl}_{3}\right) \delta 178.12,83.40,40.54,38.74,36.30,36.23,33.70,33.26,27.69$, 26.74, 26.45, 26.41, 25.00, 24.87. ${ }^{11} \mathbf{B}$ NMR $\left(128 \mathrm{MHz}, \mathrm{CDCl}_{3}\right) \delta 34.71$. ESI-HR calcd for $\mathrm{C}_{20} \mathrm{H}_{39} \mathrm{BNO}_{3}{ }^{+}\left([\mathrm{M}+\mathrm{H}]^{+}\right)$352.3018, found 352.3016. IR $v\left(\mathrm{~cm}^{-1}\right) 3363,2921,1646,750$.<smiles>CC(C)CC(Br)c1ccccc1</smiles>

$\boldsymbol{\alpha}$-Aminoboronic ester 20: Following the general procedure [A], enamide 1o- $\boldsymbol{Z}$ (33.9 mg, 0.200 mmol) was converted to the $\alpha$-aminoboronic ester. Purification by silica gel chromatography gave $51.7 \mathrm{mg}$ (87\% yield) of $\mathbf{2 0}$ as a white solid. ${ }^{1} \mathbf{H}$ NMR $\left(400 \mathrm{MHz}, \mathrm{CDCl}_{3}\right) \delta 6.51(\mathrm{~s}, 1 \mathrm{H}), 2.64(\mathrm{dd}$, $J=9.4,4.7 \mathrm{~Hz}, 1 \mathrm{H}), 1.67-1.52(\mathrm{~m}, 1 \mathrm{H}), 1.48-1.28(\mathrm{~m}, 2 \mathrm{H}), 1.21(\mathrm{~s}, 9 \mathrm{H}), 1.18(\mathrm{~s}, 12 \mathrm{H}), 0.91(\mathrm{t}$, $J=6.4 \mathrm{~Hz}, 6 \mathrm{H}) .{ }^{13} \mathbf{C} \mathbf{N M R}\left(101 \mathrm{MHz}, \mathrm{CDCl}_{3}\right) \delta 183.59,80.36,40.32,35.75,26.97,26.48,25.39$, 25.27, 23.53, 22.12. ${ }^{11} \mathbf{B}$ NMR $\left(128 \mathrm{MHz}, \mathrm{CDCl}_{3}\right) \delta$ 16.34. ESI-HR calcd for $\mathrm{C}_{16} \mathrm{H}_{33} \mathrm{BNO}_{3}{ }^{+}$ $\left([\mathrm{M}+\mathrm{H}]^{+}\right) 298.2548$, found $298.2542 . \mathbf{I R} v\left(\mathrm{~cm}^{-1}\right) 3255,2986,1559,732$. M. P. $149-150^{\circ} \mathrm{C}$.<smiles>CC(C)C([Pb])CNC(=O)C(C)(C)C</smiles>

$\boldsymbol{\beta}$-Aminoboronic ester 3o: Following the general procedure [B], enamide 1o- $\boldsymbol{E}$ (16.9 mg, 0.100 mmol) was converted to the $\beta$-aminoboronic ester. Purification by silica gel chromatography gave $9.00 \mathrm{mg}$ (30\% yield) of $\mathbf{3 o}$ as a colorless oil. ${ }^{1} \mathbf{H}$ NMR $\left(400 \mathrm{MHz}, \mathrm{CDCl}_{3}\right) \delta 6.22(\mathrm{~s}, 1 \mathrm{H}), 3.46-$ $3.38(\mathrm{~m}, 1 \mathrm{H}), 3.28-3.22(\mathrm{~m}, 1 \mathrm{H}), 1.81(\mathrm{td}, J=13.5,6.8 \mathrm{~Hz}, 1 \mathrm{H}), 1.23-1.21(\mathrm{~m}, 1 \mathrm{H}), 1.18(\mathrm{~s}$, 12H), 1.17 (s, 9H), $0.97-0.93$ (m, 6H). ${ }^{13} \mathbf{C}$ NMR (101 MHz, $\left.\mathrm{CDCl}_{3}\right) \delta$ 178.38, 83.46, 38.73, 38.05, 27.89, 27.77, 26.15, 25.17, 24.99, 22.05. ${ }^{11} \mathbf{B}$ NMR $\left(128 \mathrm{MHz}, \mathrm{CDCl}_{3}\right) \delta 34.17$. ESI-HR calcd for $\mathrm{C}_{16} \mathrm{H}_{33} \mathrm{BNO}_{3}{ }^{+}\left([\mathrm{M}+\mathrm{H}]^{+}\right)$298.2548, found 298.2542. IR $v\left(\mathrm{~cm}^{-1}\right) 3347,2957,1637,750$.<smiles>CC(Br)c1ccccc1</smiles> 
$\boldsymbol{\alpha}$-Aminoboronic ester 2p: Following the general procedure [A], enamide 1p (25.4 mg, 0.200 mmol) was converted to a mixture of the $\alpha$-aminoboronic ester and $\beta$-aminoboronic ester. Purification by silica gel chromatography gave $14.6 \mathrm{mg}$ ( $29 \%$ yield) of $\mathbf{2 p}$ as a white solid and $19.6 \mathrm{mg}$ (38\% yield) of $\mathbf{3 p}$ as a colorless oil. ${ }^{\mathbf{1}} \mathbf{H} \mathbf{~ N M R}\left(400 \mathrm{MHz}, \mathrm{CDCl}_{3}\right) \delta 6.72(\mathrm{~s}, 1 \mathrm{H}), 2.64(\mathrm{q}$, $J=7.0 \mathrm{~Hz}, 1 \mathrm{H}), 1.21(\mathrm{~s}, 9 \mathrm{H}), 1.19(\mathrm{~s}, 12 \mathrm{H}), 1.13(\mathrm{~d}, J=7.2 \mathrm{~Hz}, 3 \mathrm{H}) .{ }^{13} \mathbf{C} \mathbf{N M R}\left(101 \mathrm{MHz}, \mathrm{CDCl}_{3}\right)$ $\delta 183.81,80.23,35.61,26.99,25.34,25.06,16.35 .{ }^{11} \mathbf{B}$ NMR $\left(128 \mathrm{MHz}, \mathrm{CDCl}_{3}\right) \delta 15.71$. ESI-HR calcd for $\mathrm{C}_{13} \mathrm{H}_{27} \mathrm{BNO}_{3}{ }^{+}\left([\mathrm{M}+\mathrm{H}]^{+}\right)$256.2079, found 256.2076. IR $v\left(\mathrm{~cm}^{-1}\right) 3198,2971,1583,750$. M. P. $147-148^{\circ} \mathrm{C}$.<smiles>CC(C)(C)C(=O)NCCBr</smiles>

$\boldsymbol{\beta}$-Aminoboronic ester 3p: Following the general procedure [A], enamide 1p (25.4 mg, 0.200 mmol) was converted to a mixture of the $\alpha$-aminoboronic ester and $\beta$-aminoboronic ester. Purification by silica gel chromatography gave $14.6 \mathrm{mg}$ ( $29 \%$ yield) of $\mathbf{2 p}$ as a white solid and $19.6 \mathrm{mg}$ (38\% yield) of $\mathbf{3 p}$ as a colorless oil. ${ }^{\mathbf{1}} \mathbf{H} \mathbf{~ N M R}\left(400 \mathrm{MHz}, \mathrm{CDCl}_{3}\right) \delta 6.16(\mathrm{~s}, 1 \mathrm{H}), 3.36(\mathrm{dd}$, $J=12.6,6.8 \mathrm{~Hz}, 2 \mathrm{H}), 1.26(\mathrm{~s}, 12 \mathrm{H}), 1.18(\mathrm{~s}, 9 \mathrm{H}), 1.04(\mathrm{t}, J=6.9 \mathrm{~Hz}, 2 \mathrm{H}) .{ }^{13} \mathbf{C}$ NMR $(101 \mathrm{MHz}$, $\left.\mathrm{CDCl}_{3}\right) \delta 178.12,83.54,38.71,35.14,27.70,25.03,24.71 .{ }^{11} \mathbf{B} \mathbf{N M R}\left(128 \mathrm{MHz}, \mathrm{CDCl}_{3}\right) \delta 33.49$. ESI-HR calcd for $\mathrm{C}_{13} \mathrm{H}_{27} \mathrm{BNO}_{3}{ }^{+}\left([\mathrm{M}+\mathrm{H}]^{+}\right)$256.2079, found 256.2074. IR $v\left(\mathrm{~cm}^{-1}\right) 3353,2977$, 1638,847

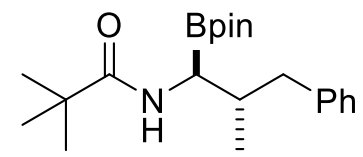

$\boldsymbol{\alpha}$-Aminoboronic ester 2q: Following the general procedure [C], enamide 1q (46.3 mg, 0.200 mmol) was converted to the $\alpha$-aminoboronic ester. Purification by silica gel chromatography gave $51.2 \mathrm{mg}$ (71\% yield) of $\mathbf{2 q}$ as a white solid. ${ }^{1} \mathbf{H}$ NMR $\left(400 \mathrm{MHz}, \mathrm{CDCl}_{3}\right) \delta 7.35-7.17(\mathrm{~m}, 5 \mathrm{H})$, $6.09(\mathrm{~s}, 1 \mathrm{H}), 2.92(\mathrm{dd}, J=13.1,3.8 \mathrm{~Hz}, 1 \mathrm{H}), 2.63(\mathrm{dd}, J=4.9,2.0 \mathrm{~Hz}, 1 \mathrm{H}), 2.47(\mathrm{dd}, J=13.1,8.4$ $\mathrm{Hz}, 1 \mathrm{H}), 2.29-1.99(\mathrm{~m}, 1 \mathrm{H}), 1.24(\mathrm{~d}, J=0.8 \mathrm{~Hz}, 12 \mathrm{H}), 1.14(\mathrm{~s}, 9 \mathrm{H}), 0.88(\mathrm{~d}, J=6.8 \mathrm{~Hz}, 3 \mathrm{H}) .{ }^{13} \mathrm{C}$ NMR $\left(101 \mathrm{MHz}, \mathrm{CDCl}_{3}\right) \delta 183.43,141.09,129.48,128.28,125.98,80.35,42.18,35.69,35.41$, 
26.76, 25.64, 25.22, 15.58. ${ }^{11} \mathbf{B}$ NMR $\left(128 \mathrm{MHz}, \mathrm{CDCl}_{3}\right) \delta$ 15.31. ESI-HR calcd for $\mathrm{C}_{21} \mathrm{H}_{35} \mathrm{BNO}_{3}{ }^{+}$ $\left([\mathrm{M}+\mathrm{H}]^{+}\right) 360.2705$, found $360.2701 . \mathbf{I R} v\left(\mathrm{~cm}^{-1}\right) 3421,2970,1574,736$. M. P. $129-130{ }^{\circ} \mathrm{C}$.<smiles>C[C@H](CCc1ccccc1)C(Cc1ccccc1)NC(=O)C(C)(C)C</smiles>

$\boldsymbol{\alpha}$-Aminoboronic ester 2r: Following the general procedure [C], enamide 1r (49.1 mg, 0.200 mmol) was converted to the $\alpha$-aminoboronic ester. Purification by silica gel chromatography gave $59.9 \mathrm{mg}\left(80 \%\right.$ yield) of $\mathbf{2} \mathbf{r}$ as a white solid. ${ }^{1} \mathbf{H} \mathbf{~ N M R}\left(400 \mathrm{MHz}, \mathrm{CDCl}_{3}\right) \delta 7.33-7.26(\mathrm{~m}, 4 \mathrm{H})$, $7.23-7.17(\mathrm{~m}, 2 \mathrm{H}), 6.35(\mathrm{~s}, 1 \mathrm{H}), 2.72(\mathrm{ddd}, J=15.7,10.2,5.6 \mathrm{~Hz}, 1 \mathrm{H}), 2.68-2.56(\mathrm{~m}, 2 \mathrm{H}), 1.88$ $-1.70(\mathrm{~m}, 2 \mathrm{H}), 1.57-1.44(\mathrm{~m}, 1 \mathrm{H}), 0.98(\mathrm{~d}, J=6.7 \mathrm{~Hz}, 3 \mathrm{H}) .{ }^{13} \mathbf{C} \mathbf{N M R}\left(101 \mathrm{MHz}, \mathrm{CDCl}_{3}\right) \delta$ $142.82,128.39,128.31,125.63,80.57,37.22,36.04,33.77,33.72,26.98,25.54,25.16,16.31,0.01$. ${ }^{11} \mathbf{B}$ NMR $\left(128 \mathrm{MHz}, \mathrm{CDCl}_{3}\right) \delta$ 14.10. ESI-HR calcd for $\mathrm{C}_{22} \mathrm{H}_{37} \mathrm{BNO}_{3}{ }^{+}\left([\mathrm{M}+\mathrm{H}]^{+}\right)$374.2861, found374.2859. IR $v\left(\mathrm{~cm}^{-1}\right) 3258,2970,1579,749$. M. P. $126-127^{\circ} \mathrm{C}$.<smiles>CCCC[C@H](C)C(NC(=O)C(C)(C)C)c1ccccc1</smiles>

$\boldsymbol{\alpha}$-Aminoboronic ester 2s: Following the general procedure [C], enamide 1s (39.5 mg, 0.200 mmol) was converted to the $\alpha$-aminoboronic ester. Purification by silica gel chromatography gave $52.9 \mathrm{mg}\left(81 \%\right.$ yield) of $2 \mathrm{~s}$ as a white solid. ${ }^{1} \mathbf{H} \mathbf{N M R}\left(400 \mathrm{MHz}, \mathrm{CDCl}_{3}\right) \delta 6.39(\mathrm{~s}, 1 \mathrm{H}), 2.59(\mathrm{dd}, J$ $=5.3,2.4 \mathrm{~Hz}, 1 \mathrm{H}), 1.80-1.67(\mathrm{~m}, 1 \mathrm{H}), 1.50-1.39(\mathrm{~m}, 1 \mathrm{H}), 1.39-1.27(\mathrm{~m}, 4 \mathrm{H}), 1.25(\mathrm{~s}, 9 \mathrm{H})$, $1.23(\mathrm{~d}, J=2.2 \mathrm{~Hz}, 12 \mathrm{H}), 1.20-1.01(\mathrm{~m}, 2 \mathrm{H}), 0.91(\mathrm{t}, J=7.5 \mathrm{~Hz}, 6 \mathrm{H}) .{ }^{13} \mathbf{C}$ NMR $(101 \mathrm{MHz}$, $\left.\mathrm{CDCl}_{3}\right) \delta 183.07,80.54,36.05,34.92,33.79,29.63,27.00,25.50,25.17,22.89,16.38,14.19 .{ }^{11} \mathbf{B}$ NMR $\left(128 \mathrm{MHz}, \mathrm{CDCl}_{3}\right) \delta$ 16.44. ESI-HR calcd for $\mathrm{C}_{18} \mathrm{H}_{37} \mathrm{BNO}_{3}{ }^{+}\left([\mathrm{M}+\mathrm{H}]^{+}\right) 326.2861$, found 326.2860. IR $v\left(\mathrm{~cm}^{-1}\right) 3236,2968,1581,750$. M. P. $118-119^{\circ} \mathrm{C}$.<smiles>CCCCCCCC[C@H](C)C(Cc1ccccc1)NC(=O)C(C)(C)C</smiles>

$\boldsymbol{\alpha}$-Aminoboronic ester 2t: Following the general procedure [C], enamide 1t (50.7 mg, 0.200 mmol) was converted to the $\alpha$-aminoboronic ester. Purification by silica gel chromatography gave $56.7 \mathrm{mg}$ (74\% yield) of $\mathbf{2 t}$ as a white solid. ${ }^{1} \mathbf{H} \mathbf{~ N M R}\left(400 \mathrm{MHz}, \mathrm{CDCl}_{3}\right) \delta 6.39(\mathrm{~s}, 1 \mathrm{H}), 2.58(\mathrm{dd}$, 
$J=5.2,2.3 \mathrm{~Hz}, 1 \mathrm{H}), 1.73(\mathrm{dd}, J=11.5,5.0 \mathrm{~Hz}, 1 \mathrm{H}), 1.40(\mathrm{dd}, J=10.7,4.8 \mathrm{~Hz}, 2 \mathrm{H}), 1.35-1.26$ $(\mathrm{m}, 11 \mathrm{H}), 1.25(\mathrm{~s}, 9 \mathrm{H}), 1.23(\mathrm{~d}, J=2.0 \mathrm{~Hz}, 11 \mathrm{H}), 1.16(\mathrm{dd}, J=14.7,6.2 \mathrm{~Hz}, 2 \mathrm{H}), 0.90(\mathrm{dt}, J=6.9$, $3.3 \mathrm{~Hz}, 6 \mathrm{H}) .{ }^{13} \mathrm{C}$ NMR $\left(101 \mathrm{MHz}, \mathrm{CDCl}_{3}\right) \delta$ 183.08, 80.53, 36.05, 35.24, 33.81, 31.92, 29.85, 29.67, 29.33, 27.38, 27.00, 25.51, 25.18, 22.70, 16.38, 14.14. ${ }^{11} \mathbf{B}$ NMR $\left(128 \mathrm{MHz}, \mathrm{CDCl}_{3}\right) \delta$ 16.45. ESI-HR calcd for $\mathrm{C}_{22} \mathrm{H}_{45} \mathrm{BNO}_{3}{ }^{+}\left([\mathrm{M}+\mathrm{H}]^{+}\right) 382.3487$, found 382.3482. IR $v\left(\mathrm{~cm}^{-1}\right) 3235$, 2924, 1580, 750. M. P. $78-79^{\circ} \mathrm{C}$.<smiles>CC(C)C(Cc1ccccc1)NC(=O)C(C)(C)C</smiles>

$\boldsymbol{\alpha}$-Aminoboronic ester 2u: Following the general procedure [C], enamide $1 \mathbf{u}$ (31.0 $\mathrm{mg}, 0.200$ mmol) was converted to the $\alpha$-aminoboronic ester. Purification by silica gel chromatography gave $51.7 \mathrm{mg}$ (91\% yield) of $\mathbf{2 u a s}$ a white solid. ${ }^{1} \mathbf{H}$ NMR $\left(400 \mathrm{MHz}, \mathrm{CDCl}_{3}\right) \delta 6.44(\mathrm{~s}, 1 \mathrm{H}), 2.44(\mathrm{dd}, \mathrm{J}$ = 6.7, $2.2 \mathrm{~Hz}, 1 \mathrm{H}), 1.89(\mathrm{dq}, \mathrm{J}=13.4,6.7 \mathrm{~Hz}, 1 \mathrm{H}), 1.25(\mathrm{~s}, 9 \mathrm{H}), 1.23(\mathrm{~s}, 12 \mathrm{H}), 1.00(\mathrm{~d}, \mathrm{~J}=6.7 \mathrm{~Hz}$, 3H), $0.94(\mathrm{~d}, \mathrm{~J}=6.7 \mathrm{~Hz}, 3 \mathrm{H}) .{ }^{13} \mathrm{C}$ NMR $\left(101 \mathrm{MHz}, \mathrm{CDCl}_{3}\right) \delta 182.89,80.60,36.02,29.31,26.97$, 25.50, 25.31, 20.90, 19.81. ${ }^{11} \mathbf{B}$ NMR $\left(128 \mathrm{MHz}, \mathrm{CDCl}_{3}\right) \delta$ 17.82. ESI-HR calcd for $\mathrm{C}_{15} \mathrm{H}_{31} \mathrm{BNO}_{3}{ }^{+}$ $\left([\mathrm{M}+\mathrm{H}]^{+}\right)$284.2392, found 284.2389. IR $v\left(\mathrm{~cm}^{-1}\right) 3456,2966,1574,731$. M. P. $142-143{ }^{\circ} \mathrm{C}$.<smiles>CC(C)(C)C(=O)NC(Cc1ccccc1)C1CCOCC1</smiles>

$\boldsymbol{\alpha}$-Aminoboronic ester 2v: Following the general procedure [C], enamide $\mathbf{1 v}$ (39.6 mg, 0.200 mmol) was converted to the $\alpha$-aminoboronic ester. Purification by silica gel chromatography gave $60.0 \mathrm{mg}$ (92\% yield) of $\mathbf{2 v}$ as a white solid. ${ }^{1} \mathbf{H} \mathbf{~ N M R}\left(400 \mathrm{MHz}, \mathrm{CDCl}_{3}\right) \delta 6.53(\mathrm{~s}, 1 \mathrm{H}), 3.97(\mathrm{dd}$, $J=11.1,3.2 \mathrm{~Hz}, 2 \mathrm{H}), 3.47-3.33(\mathrm{~m}, 2 \mathrm{H}), 2.46(\mathrm{~d}, J=6.2 \mathrm{~Hz}, 1 \mathrm{H}), 1.90-1.75(\mathrm{~m}, 2 \mathrm{H}), 1.68(\mathrm{~d}$, $J=12.4 \mathrm{~Hz}, 1 \mathrm{H}), 1.49-1.30(\mathrm{~m}, 2 \mathrm{H}), 1.25(\mathrm{~s}, 9 \mathrm{H}), 1.22(\mathrm{~d}, J=2.2 \mathrm{~Hz}, 12 \mathrm{H}) .{ }^{\mathbf{1 3}} \mathbf{C}$ NMR $(101$ $\left.\mathrm{MHz}, \mathrm{CDCl}_{3}\right) \delta 183.45,80.63,68.05,67.97,36.31,36.01,31.25,29.91,26.92,25.50,25.27 .{ }^{11} \mathbf{B}$ NMR $\left(128 \mathrm{MHz}, \mathrm{CDCl}_{3}\right) \delta$ 16.72. ESI-HR calcd for $\mathrm{C}_{17} \mathrm{H}_{33} \mathrm{BNO}_{4}{ }^{+}\left([\mathrm{M}+\mathrm{H}]^{+}\right) 326.2497$, found 326.2493. IR $v\left(\mathrm{~cm}^{-1}\right) 3213,2972,1577,730$. M. P. $169-170{ }^{\circ} \mathrm{C}$. 
<smiles>CCC(Br)C(C)NC(=O)C(C)(C)C</smiles>

$\boldsymbol{\beta}$-Aminoboronic ester 3w: Following the general procedure [C], enamide 1w (33.9 mg, 0.200 mmol) was converted to the $\beta$-aminoboronic ester. Purification by silica gel chromatography gave $47.1 \mathrm{mg}$ (79\% yield) of $\mathbf{3 w}$ as a white solid. ${ }^{1} \mathbf{H} \mathbf{~ N M R}\left(400 \mathrm{MHz}, \mathrm{CDCl}_{3}\right) \delta 6.68(\mathrm{~s}, 1 \mathrm{H}), 4.88(\mathrm{t}, J$ $=6.9 \mathrm{~Hz}, 1 \mathrm{H}), 2.02(\mathrm{~d}, J=0.8 \mathrm{~Hz}, 3 \mathrm{H}), 1.96(\mathrm{dd}, J=14.9,7.4 \mathrm{~Hz}, 2 \mathrm{H}), 1.25(\mathrm{~s}, 9 \mathrm{H}), 0.99(\mathrm{t}, J=$ $7.5 \mathrm{~Hz}, 3 \mathrm{H}) .{ }^{13} \mathrm{C}$ NMR $\left(101 \mathrm{MHz}, \mathrm{CDCl}_{3}\right) \delta 175.11,123.65,116.25,44.69,38.86,31.76,27.52$, 26.59, 26.28, 13.01. ${ }^{11} \mathbf{B}$ NMR $\left(128 \mathrm{MHz}, \mathrm{CDCl}_{3}\right) \delta$ 32.29. ESI-HR calcd for $\mathrm{C}_{16} \mathrm{H}_{33} \mathrm{BNO}_{3}{ }^{+}$ $\left([\mathrm{M}+\mathrm{H}]^{+}\right) 298.2548$, found 298.2544. IR $v\left(\mathrm{~cm}^{-1}\right) 3431,2960,1651,733$. M. P. 69-70 ${ }^{\circ} \mathrm{C}$.<smiles>CCCC(Br)c1ccccc1</smiles>

$\boldsymbol{\beta}$-Aminoboronic ester 3x: Following the general procedure [C], enamide 1x (39.5 mg, 0.200 mmol) was converted to the $\beta$-aminoboronic ester. Purification by silica gel chromatography gave $29.4 \mathrm{mg}\left(45 \%\right.$ yield) of $\mathbf{3 x}$ as a white solid. ${ }^{1} \mathbf{H} \mathbf{~ N M R}\left(400 \mathrm{MHz}, \mathrm{CDCl}_{3}\right) \delta 6.25(\mathrm{~d}, J=9.2 \mathrm{~Hz}$, 1H), $4.02-3.83(\mathrm{~m}, 1 \mathrm{H}), 1.57$ (ddd, $J=12.9,8.3,5.3 \mathrm{~Hz}, 1 \mathrm{H}), 1.45-1.04(\mathrm{~m}, 26 \mathrm{H}), 0.97(\mathrm{~d}, J=$ $7.6 \mathrm{~Hz}, 3 \mathrm{H}), 0.86(\mathrm{t}, J=6.8 \mathrm{~Hz}, 3 \mathrm{H}) .{ }^{13} \mathbf{C} \mathbf{N M R}\left(101 \mathrm{MHz}, \mathrm{CDCl}_{3}\right) \delta 177.44,83.22,51.19,38.69$, 32.56, 28.38, 27.61, 24.96, 24.77, 22.65, 14.03, 12.65. ${ }^{11} \mathbf{B}$ NMR $\left(128 \mathrm{MHz}, \mathrm{CDCl}_{3}\right) \delta 33.61$. ESI-HR calcd for $\mathrm{C}_{18} \mathrm{H}_{37} \mathrm{BNO}_{3}{ }^{+}\left([\mathrm{M}+\mathrm{H}]^{+}\right) 326.2681$, found 326.2856. IR $v\left(\mathrm{~cm}^{-1}\right) 3430,2959$, 1649, 734. M. P. $110-111^{\circ} \mathrm{C}$.<smiles>CC(CBr)NC(=O)C(C)(C)C</smiles>

$\boldsymbol{\beta}$-Aminoboronic ester 3y: Following the general procedure [C], enamide 1y $(28.2 \mathrm{mg}, 0.200$ mmol) was converted to the $\beta$-aminoboronic ester. Purification by silica gel chromatography gave $43.2 \mathrm{mg}$ (80\% yield) of $\mathbf{3 y}$ as a white solid. ${ }^{1} \mathbf{H}$ NMR $\left(400 \mathrm{MHz}, \mathrm{CDCl}_{3}\right) \delta 6.26(\mathrm{~s}, 1 \mathrm{H}), 4.23(\mathrm{dt}, J$ $=12.9,6.5 \mathrm{~Hz}, 1 \mathrm{H}), 1.28(\mathrm{~s}, 12 \mathrm{H}), 1.19(\mathrm{~s}, 9 \mathrm{H}), 1.15(\mathrm{~d}, J=6.6 \mathrm{~Hz}, 3 \mathrm{H}), 1.13-1.00(\mathrm{~m}, 2 \mathrm{H}) .{ }^{13} \mathrm{C}$ NMR $\left(101 \mathrm{MHz}, \mathrm{CDCl}_{3}\right) \delta 177.20,83.35,41.70,38.50,27.51,25.02,24.74,22.79 .{ }^{11} \mathbf{B}$ NMR 
$\left(128 \mathrm{MHz}, \mathrm{CDCl}_{3}\right) \delta$ 32.46. ESI-HR calcd for $\mathrm{C}_{14} \mathrm{H}_{29} \mathrm{BNO}_{3}{ }^{+}\left([\mathrm{M}+\mathrm{H}]^{+}\right)$270.2235, found 270.2228. IR $v\left(\mathrm{~cm}^{-1}\right) 3350,2977,1645,733$. M. P. $97-98^{\circ} \mathrm{C}$.<smiles>CC(C)(C)C(=O)NC(CCCc1ccc(F)cc1)Cc1ccccc1</smiles>

$\boldsymbol{\alpha}$-Aminoboronic ester 4a: Following the general procedure [D], enamide 1z-Z (24.9 mg, 0.100 mmol) was converted to the $\alpha$-aminoboronic ester. Purification by silica gel chromatography gave $29.7 \mathrm{mg}$ (79\% yield) of $\mathbf{4 a}$ as a colorless oil. ${ }^{1} \mathbf{H} \mathbf{~ N M R}\left(400 \mathrm{MHz}, \mathrm{CDCl}_{3}\right) \delta 7.21-7.09(\mathrm{~m}, 2 \mathrm{H})$, $7.07-6.93(\mathrm{~m}, 2 \mathrm{H}), 6.60(\mathrm{~s}, 1 \mathrm{H}), 2.75-2.52(\mathrm{~m}, 3 \mathrm{H}), 1.73-1.60(\mathrm{~m}, 3 \mathrm{H}), 1.55-1.41(\mathrm{~m}, 1 \mathrm{H})$, $1.21(\mathrm{~s}, 9 \mathrm{H}), 1.19(\mathrm{~s}, 12 \mathrm{H}) .{ }^{13} \mathrm{C}$ NMR $\left(101 \mathrm{MHz}, \mathrm{CDCl}_{3}\right) \delta 183.83,161.28\left(\mathrm{~d}, J_{1}=242.9 \mathrm{~Hz}\right)$, $130.79\left(\mathrm{~d}, J_{3}=5.4 \mathrm{~Hz}\right), 129.36\left(\mathrm{~d}, J_{2}=16.8 \mathrm{~Hz}\right), 127.54\left(\mathrm{~d}, J_{3}=8.5 \mathrm{~Hz}\right), 124.05\left(\mathrm{~d}, J_{4}=3.6 \mathrm{~Hz}\right)$, $115.26\left(\mathrm{~d}, J_{2}=22.4 \mathrm{~Hz}\right), 80.41,35.76,30.89,29.17\left(\mathrm{~d}, J_{5}=1.9 \mathrm{~Hz}\right), 28.54,26.95,25.45,25.21$. ${ }^{11} \mathbf{B}$ NMR $\left(128 \mathrm{MHz}, \mathrm{CDCl}_{3}\right) \delta$ 16.20. ESI-HR calcd for $\mathrm{C}_{21} \mathrm{H}_{34} \mathrm{BFNO}_{3}{ }^{+}\left([\mathrm{M}+\mathrm{H}]^{+}\right) 378.2610$, found 378.2599. IR $v\left(\mathrm{~cm}^{-1}\right) 2972,1581,1264,749 .[\alpha]^{20} \mathrm{D}=-142.7\left(c=0.83, \mathrm{CHCl}_{3}\right)$ for a $96 \%$ ee sample. The enantiomeric purity of this compound was determined by HPLC analysis in comparison with authentic racemic material (Chiralpak IC, 97:4 hexanes:i-PrOH, $1.0 \mathrm{~mL} / \mathrm{min}, 220$ $\mathrm{nm}): t_{\text {major }}=6.3 \mathrm{~min}, t_{\mathrm{minor}}=5.0 \mathrm{~min}$.
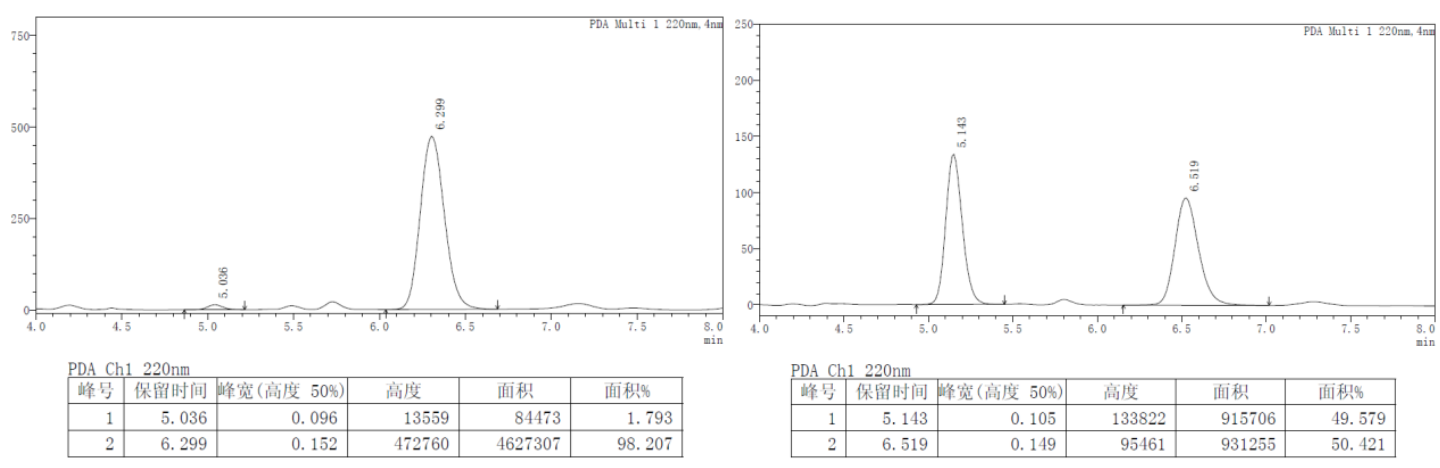
<smiles>CC(C)(C)C(=O)NCC(CCc1ccccc1)Cc1ccccc1</smiles>

$\boldsymbol{\beta}$-Aminoboronic ester 5a: Following the general procedure [E], enamide 1z-E (21.7 mg, 0.100 mmol) was converted to the $\beta$-aminoboronicester. Purification by silica gel chromatography gave $27.7 \mathrm{mg}(73 \%$ yield $)$ of $\mathbf{5 a}$ as a colorless oil. ${ }^{1} \mathbf{H} \mathbf{~ N M R}\left(400 \mathrm{MHz}, \mathrm{CDCl}_{3}\right) \delta 7.22-7.09(\mathrm{~m}, 2 \mathrm{H})$, $7.07-6.93(\mathrm{~m}, 2 \mathrm{H}), 6.22(\mathrm{~s}, 1 \mathrm{H}), 3.49-3.38(\mathrm{~m}, 1 \mathrm{H}), 3.36-3.23(\mathrm{~m}, 1 \mathrm{H}), 2.76-2.61(\mathrm{~m}, 2 \mathrm{H})$, $1.82-1.70(\mathrm{~m}, 1 \mathrm{H}), 1.67-1.56(\mathrm{~m}, 1 \mathrm{H}), 1.26(\mathrm{~s}, 12 \mathrm{H}), 1.23-1.20(\mathrm{~m}, 1 \mathrm{H}), 1.18(\mathrm{~s}, 9 \mathrm{H}) .{ }^{13} \mathrm{C}$ NMR $\left(101 \mathrm{MHz}, \mathrm{CDCl}_{3}\right) \delta 178.27,161.26\left(\mathrm{~d}, J_{1}=246.6 \mathrm{~Hz}\right), 130.86\left(\mathrm{~d}, J_{3}=5.0 \mathrm{~Hz}\right), 129.40(\mathrm{~d}$, $\left.J_{2}=16.5 \mathrm{~Hz}\right), 127.53\left(\mathrm{~d}, J_{3}=7.7 \mathrm{~Hz}\right), 124.03\left(\mathrm{~d}, J_{4}=3.5 \mathrm{~Hz}\right), 115.26\left(\mathrm{~d}, J_{2}=22.3 \mathrm{~Hz}\right), 83.65$, 40.03, 38.81, 29.39, 28.26 (d, $\left.J_{5}=2.3 \mathrm{~Hz}\right), 27.72,25.05,24.98 .{ }^{11} \mathbf{B} \mathbf{N M R}\left(128 \mathrm{MHz}, \mathrm{CDCl}_{3}\right) \delta$ 34.48. ESI-HR calcd for $\mathrm{C}_{21} \mathrm{H}_{34} \mathrm{BFNO}_{3}{ }^{+}\left([\mathrm{M}+\mathrm{H}]^{+}\right) 378.2610$, found 378.2600. IR $v\left(\mathrm{~cm}^{-1}\right) 3364$, $2979,1647,751 .[\alpha]^{20}{ }_{D}=9.4\left(c=1.26, \mathrm{CHCl}_{3}\right)$ for a $76 \%$ ee sample. The enantiomeric purity of this compound was determined by HPLC analysis in comparison with authentic racemic material (Chiralpak IF, 95:5 hexanes:i-PrOH, $1.0 \mathrm{~mL} / \mathrm{min}, 220 \mathrm{~nm}$ ): $t_{\text {major }}=10.2 \mathrm{~min}, t_{\text {minor }}=9.6 \mathrm{~min}$.
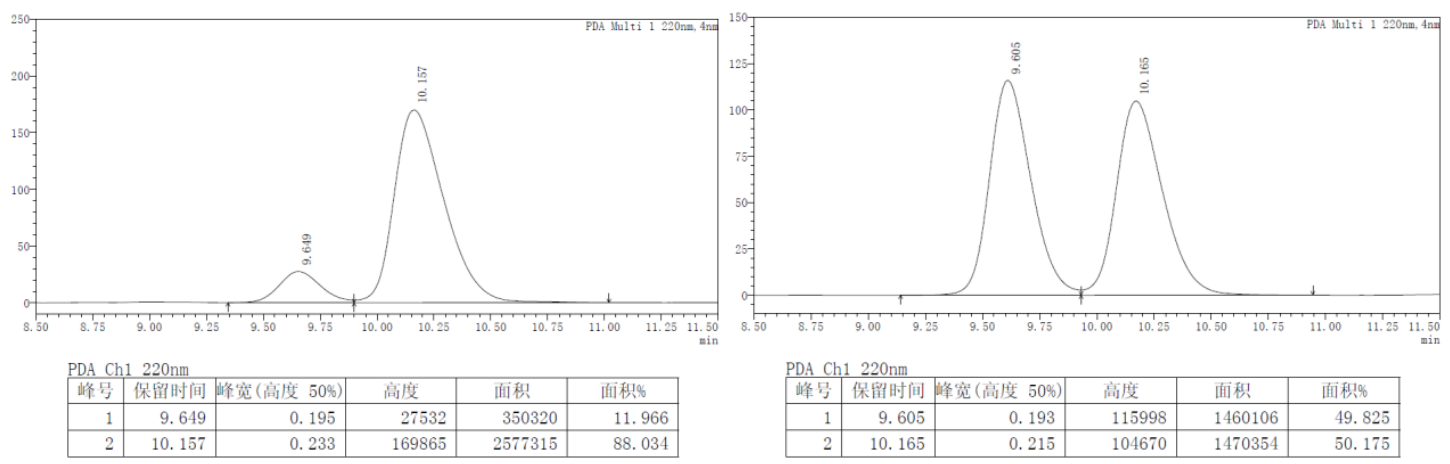<smiles>CC(C)(C)C(=O)NC(Br)c1ccccc1</smiles> 
$\boldsymbol{\alpha}$-Aminoboronic ester 4b: Following the general procedure [D], enamide 1aa- $\boldsymbol{Z}$ (26.6 mg, 0.100 mmol) was converted to the $\alpha$-aminoboronic ester. Purification by silica gel chromatography gave $31.2 \mathrm{mg}(80 \%$ yield $)$ of $\mathbf{4 b}$ as a colorless oil. ${ }^{\mathbf{1}} \mathbf{H} \mathbf{~ N M R}\left(400 \mathrm{MHz}, \mathrm{CDCl}_{3}\right) \delta 7.22(\mathrm{~d}, J=8.3 \mathrm{~Hz}$, 2H), $7.09(\mathrm{~d}, J=8.3 \mathrm{~Hz}, 2 \mathrm{H}), 6.55(\mathrm{~s}, 1 \mathrm{H}), 2.68-2.50(\mathrm{~m}, 3 \mathrm{H}), 1.73-1.57(\mathrm{~m}, 3 \mathrm{H}), 1.50-1.37$ $(\mathrm{m}, 1 \mathrm{H}), 1.19(\mathrm{~s}, 9 \mathrm{H}), 1.18(\mathrm{~d}, J=2.7 \mathrm{~Hz}, 12 \mathrm{H}) .{ }^{13} \mathrm{C} \mathrm{NMR}\left(101 \mathrm{MHz}, \mathrm{CDCl}_{3}\right) \delta 183.80,141.00$ $131.51,129.86,128.48,80.45,35.76,35.38,30.98,29.67,26.95,25.47,25.24 .{ }^{11} \mathbf{B}$ NMR $(128$ $\left.\mathrm{MHz} \mathrm{CDCl}_{3}\right) \delta$ 16.19. ESI-HR calcd for $\mathrm{C}_{21} \mathrm{H}_{34} \mathrm{BCINO}_{3}{ }^{+}\left([\mathrm{M}+\mathrm{H}]^{+}\right)$394.2315, found 394.2306. IR $v\left(\mathrm{~cm}^{-1}\right) 2974,1575,1275,748 .[\alpha]^{20}{ }_{\mathrm{D}}=-94.1\left(c=1.24, \mathrm{CHCl}_{3}\right)$ for a $98 \%$ ee sample. The enantiomeric purity of this compound was determined by HPLC analysis in comparison with authentic racemic material (Chiralpak IC, 97:3 hexanes: $i$-PrOH, $1.0 \mathrm{~mL} / \mathrm{min}, 210 \mathrm{~nm}$ ): $t_{\text {major }}=5.9$ $\min , t_{\text {minor }}=4.8 \mathrm{~min}$.
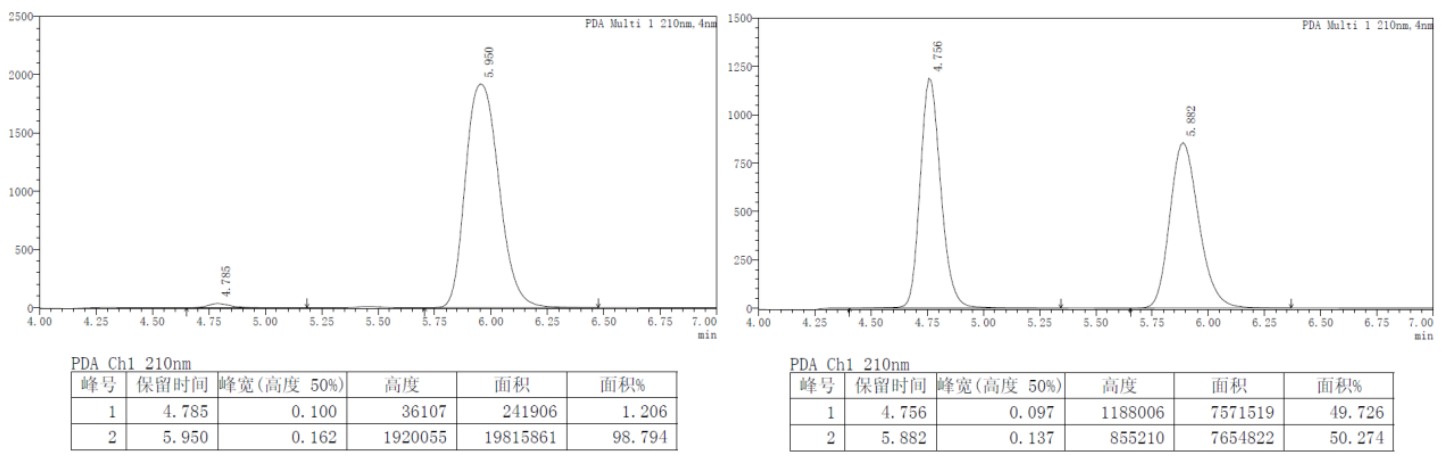<smiles>CC(C)(C)C(=O)NC[C@H](CCc1ccc(Cl)cc1)Cc1ccccc1</smiles>

$\boldsymbol{\beta}$-Aminoboronic ester 5b: Following the general procedure [E], enamide 1aa- $\boldsymbol{E}(26.6 \mathrm{mg}, 0.100$ mmol) was converted to the $\beta$-aminoboronic ester. Purification by silica gel chromatography gave $30.0 \mathrm{mg}\left(76 \%\right.$ yield) of $\mathbf{5 b}$ as a colorless oil. ${ }^{\mathbf{1}} \mathbf{H} \mathbf{~ N M R}\left(400 \mathrm{MHz}, \mathrm{CDCl}_{3}\right) \delta 7.22(\mathrm{~d}, J=8.3 \mathrm{~Hz}$, 2H), $7.10(\mathrm{~d}, J=8.2 \mathrm{~Hz}, 2 \mathrm{H}), 6.24(\mathrm{~s}, 1 \mathrm{H}), 3.45-3.36(\mathrm{~m}, 1 \mathrm{H}), 3.36-3.26(\mathrm{~m}, 1 \mathrm{H}), 2.73-2.55$ $(\mathrm{m}, 2 \mathrm{H}), 1.79-1.68(\mathrm{~m}, 1 \mathrm{H}), 1.63-1.52(\mathrm{~m}, 1 \mathrm{H}), 1.26(\mathrm{~s}, 12 \mathrm{H}), 1.24-1.20(\mathrm{~m}, 1 \mathrm{H}), 1.18(\mathrm{~s}, 9 \mathrm{H})$ ${ }^{13} \mathrm{C}$ NMR $\left(101 \mathrm{MHz}, \mathrm{CDCl}_{3}\right) \delta 178.31,141.10,131.54,129.99,128.51,83.68,39.82,38.83$, 
34.40, 30.61, 27.74, 25.09, 24.99. ${ }^{11} \mathbf{B}$ NMR $\left(128 \mathrm{MHz}, \mathrm{CDCl}_{3}\right) \delta 33.84$. ESI-HR calcd for $\mathrm{C}_{21} \mathrm{H}_{34} \mathrm{BClNO}_{3}{ }^{+}\left([\mathrm{M}+\mathrm{H}]^{+}\right)$394.2315, found 394.2305. IR $v\left(\mathrm{~cm}^{-1}\right) 3362,2978,1647,750 .[\alpha]^{20} \mathrm{D}=$ $7.9\left(c=1.40, \mathrm{CHCl}_{3}\right)$ for a $75 \%$ ee sample. The enantiomeric purity of this compound was determined by HPLC analysis in comparison with authentic racemic material (Chiralpak IF, 95:5 hexanes: $i-\mathrm{PrOH}, 1.0 \mathrm{~mL} / \mathrm{min}, 220 \mathrm{~nm}$ ): $t_{\text {major }}=19.7 \mathrm{~min}, t_{\mathrm{minor}}=18.9 \mathrm{~min}$.
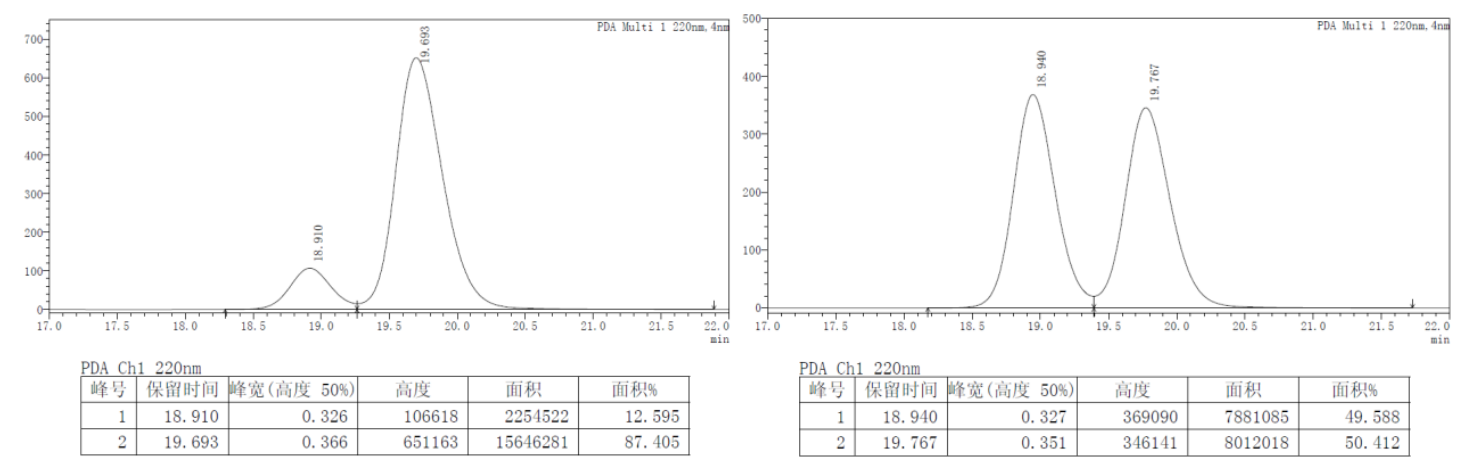<smiles>Cc1ccc(CCCC(Cc2ccccc2)NC(=O)C(C)(C)C)cc1</smiles>

$\boldsymbol{\alpha}$-Aminoboronic ester 4c: Following the general procedure [D], enamide 1ab-Z (24.5 mg, 0.100 mmol) was converted to the $\alpha$-aminoboronicester. Purification by silica gel chromatography gave $25.3 \mathrm{mg}$ (68\% yield) of $\mathbf{4 c}$ as a colorless oil. ${ }^{1} \mathbf{H}$ NMR $\left(400 \mathrm{MHz}, \mathrm{CDCl}_{3}\right) \delta 7.07(\mathrm{~s}, 4 \mathrm{H}), 6.51(\mathrm{~s}$, 1H), $2.70-2.49(\mathrm{~m}, 3 \mathrm{H}), 2.30(\mathrm{~s}, 3 \mathrm{H}), 1.73-1.59(\mathrm{~m}, 3 \mathrm{H}), 1.52-1.40(\mathrm{~m}, 1 \mathrm{H}), 1.21(\mathrm{~s}, 21 \mathrm{H})$. ${ }^{13} \mathrm{C}$ NMR $\left(101 \mathrm{MHz}, \mathrm{CDCl}_{3}\right) \delta 183.72,139.58,135.24,129.12,128.38,80.39,35.72,35.56$, 31.02, 30.05, 26.94, 25.46, 25.23, 21.09. ${ }^{11} \mathbf{B}$ NMR $\left(128 \mathrm{MHz}, \mathrm{CDCl}_{3}\right) \delta$ 15.63. ESI-HR calcd for $\mathrm{C}_{22} \mathrm{H}_{37} \mathrm{BNO}_{3}{ }^{+}\left([\mathrm{M}+\mathrm{H}]^{+}\right)$374.2861, found 374.2850. IR $v\left(\mathrm{~cm}^{-1}\right) 2988,1575,1275,733 .[\alpha]^{20} \mathrm{D}=$ $-136.9\left(c=1.17, \mathrm{CHCl}_{3}\right)$ for a $97 \%$ ee sample. The enantiomeric purity of this compound was determined by HPLC analysis in comparison with authentic racemic material (Chiralpak IC, 97:3 hexanes: $i-\mathrm{PrOH}, 1.0 \mathrm{~mL} / \mathrm{min}, 220 \mathrm{~nm}$ ): $t_{\text {major }}=6.4 \mathrm{~min}, t_{\operatorname{minor}}=5.2 \mathrm{~min}$. 

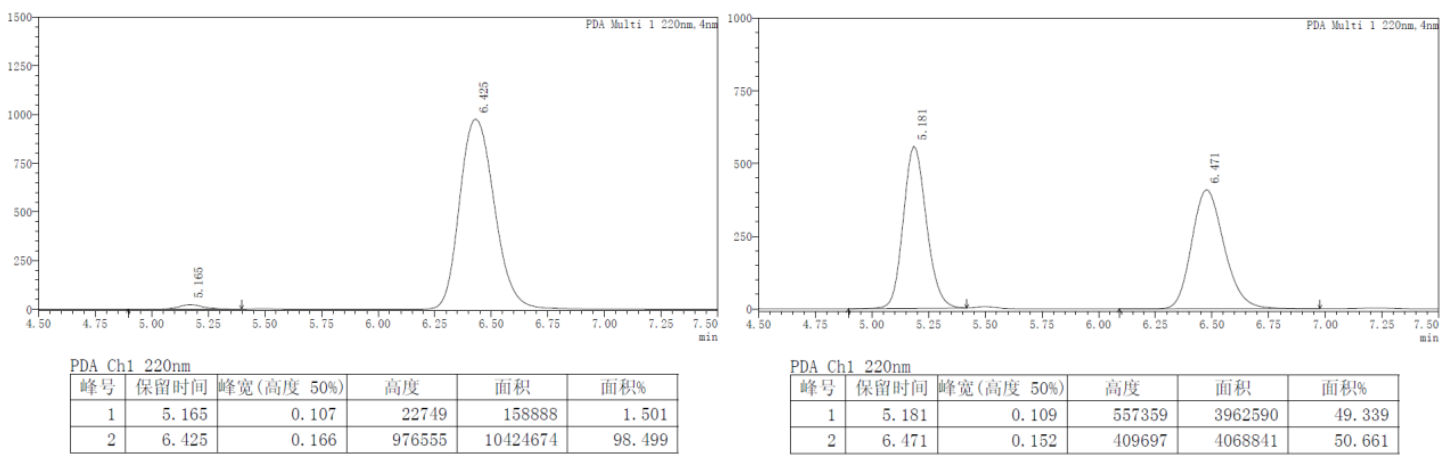<smiles>Cc1ccc(CCC(CNC(=O)C(C)(C)C)Cc2ccccc2)cc1</smiles>

$\boldsymbol{\beta}$-Aminoboronic ester 5c: Following the general procedure [E], enamide 1ab-E (24.5 mg, 0.100 mmol) was converted to the $\beta$-aminoboronic ester. Purification by silica gel chromatography gave $31.0 \mathrm{mg}\left(83 \%\right.$ yield) of $\mathbf{5 c}$ as a colorless oil. ${ }^{1} \mathbf{H}$ NMR $\left(400 \mathrm{MHz}, \mathrm{CDCl}_{3}\right) \delta 7.07(\mathrm{~s}, 4 \mathrm{H}), 6.23(\mathrm{~s}$, $1 \mathrm{H}), 3.49-3.36(\mathrm{~m}, 1 \mathrm{H}), 3.35-3.22(\mathrm{~m}, 1 \mathrm{H}), 2.70-2.52(\mathrm{~m}, 2 \mathrm{H}), 2.30(\mathrm{~s}, 3 \mathrm{H}), 1.81-1.70(\mathrm{~m}$, 1H), $1.66-1.56(\mathrm{~m}, 1 \mathrm{H}), 1.26(\mathrm{~s}, 12 \mathrm{H}), 1.22-1.20(\mathrm{~m}, 1 \mathrm{H}), 1.18(\mathrm{~s}, 9 \mathrm{H}) .{ }^{13} \mathbf{C}$ NMR $(101 \mathrm{MHz}$ $\left.\mathrm{CDCl}_{3}\right) \delta 178.24,139.58,135.22,129.10,128.47,83.59,40.05,38.80,34.65,30.92,27.72,25.07$, 24.97, 21.10. ${ }^{11} \mathbf{B}$ NMR $\left(128 \mathrm{MHz}, \mathrm{CDCl}_{3}\right) \delta 34.19$. ESI-HR calcd for $\mathrm{C}_{22} \mathrm{H}_{37} \mathrm{BNO}_{3}{ }^{+}\left([\mathrm{M}+\mathrm{H}]^{+}\right)$ 374.2861, found 374.2850. IR $v\left(\mathrm{~cm}^{-1}\right) 3362,2978,1645,750 .[\alpha]^{20} \mathrm{D}=8.4\left(c=1.22, \mathrm{CHCl}_{3}\right)$ for a 78\% ee sample. The enantiomeric purity of this compound was determined by HPLC analysis in comparison with authentic racemic material (Chiralpak IF, 95:5 hexanes: $i-\mathrm{PrOH}, 1.0 \mathrm{~mL} / \mathrm{min}, 220$ $\mathrm{nm}): t_{\text {major }}=20.1 \mathrm{~min}, t_{\mathrm{minor}}=19.4 \mathrm{~min}$.
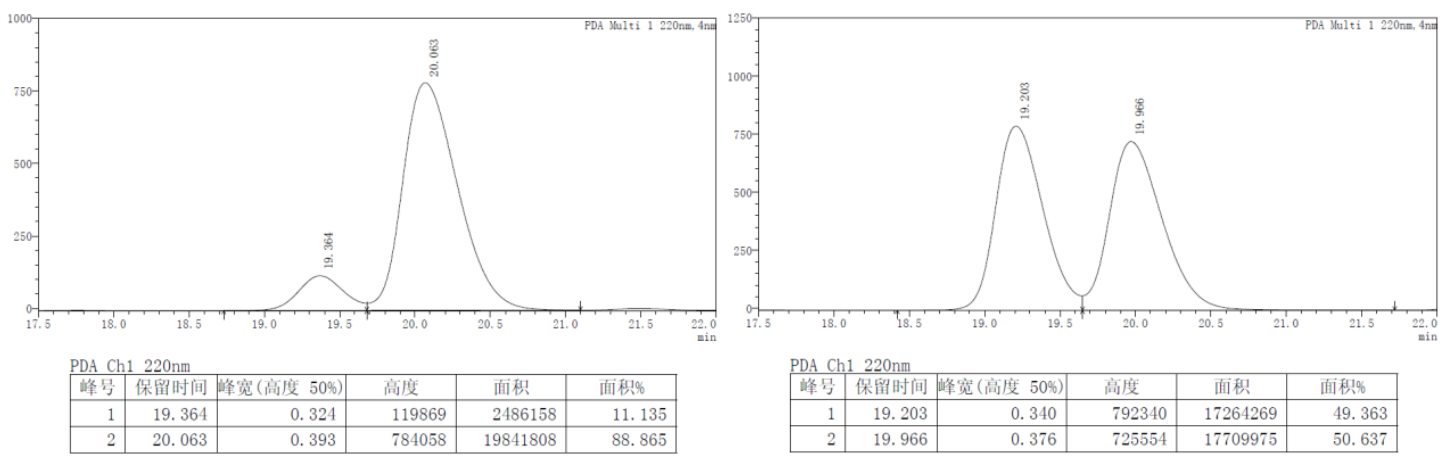
<smiles>COc1ccc(CCCC(Br)c2ccccc2)cc1</smiles>

$\boldsymbol{\alpha}$-Aminoboronic ester 4d: Following the general procedure [D], enamide 1ac- $Z$ (26.1 mg, 0.100 mmol) was converted to the $\alpha$-aminoboronicester. Purification by silica gel chromatography gave $22.5 \mathrm{mg}$ (58\% yield) of $\mathbf{4 d}$ as a colorless oil. ${ }^{1} \mathbf{H} \mathbf{~ N M R}\left(400 \mathrm{MHz}, \mathrm{CDCl}_{3}\right) \delta 7.09(\mathrm{~d}, J=8.2 \mathrm{~Hz}$, 2H), $6.81(\mathrm{~d}, J=8.3 \mathrm{~Hz}, 2 \mathrm{H}), 6.52(\mathrm{~s}, 1 \mathrm{H}), 3.77(\mathrm{~s}, 3 \mathrm{H}), 2.69-2.43(\mathrm{~m}, 3 \mathrm{H}), 1.73-1.55(\mathrm{~d}, J=$ $5.0 \mathrm{~Hz}, 3 \mathrm{H}), 1.52-1.38(\mathrm{~m}, 1 \mathrm{H}), 1.19(\mathrm{~s}, 21 \mathrm{H}) .{ }^{13} \mathbf{C} \mathbf{~ N M R}\left(101 \mathrm{MHz}, \mathrm{CDCl}_{3}\right) \delta 183.73,157.82$, $134.75,129.37,113.89,80.38,55.40,35.73,35.09,30.97,30.15,26.95,25.46,25.23 .{ }^{11} \mathbf{B}$ NMR $\left(128 \mathrm{MHz}, \mathrm{CDCl}_{3}\right) \delta$ 16.20. ESI-HR calcd for $\mathrm{C}_{22} \mathrm{H}_{37} \mathrm{BNO}_{4}{ }^{+}\left([\mathrm{M}+\mathrm{H}]^{+}\right)$390.2810, found 390.2798. IR $v\left(\mathrm{~cm}^{-1}\right) 2972,1582,1275,749 .[\alpha]^{20} \mathrm{D}=-96.2\left(c=1.12, \mathrm{CHCl}_{3}\right)$ for a $97 \%$ ee sample. The enantiomeric purity of this compound was determined by HPLC analysis in comparison with authentic racemic material (Chiralpak IC, 97:3 hexanes:i-PrOH, $1.0 \mathrm{~mL} / \mathrm{min}, 220 \mathrm{~nm}$ ): $t_{\text {major }}=$ $11.6 \mathrm{~min}, t_{\mathrm{minor}}=7.6 \mathrm{~min}$.
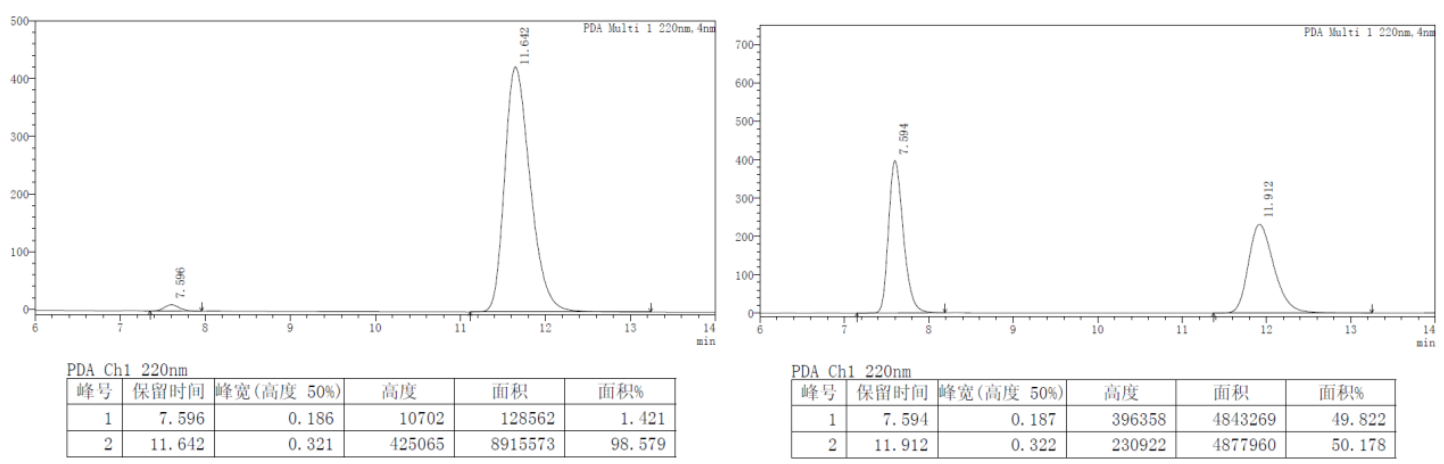<smiles>COc1ccc(CCC(CNC(=O)C(C)(C)C)Cc2ccccc2)cc1</smiles> 
$\boldsymbol{\beta}$-Aminoboronic ester 5d: Following the general procedure [E], enamide 1ac- $\boldsymbol{E}$ (26.1 mg, 0.100 mmol) was converted to the $\beta$-aminoboronic ester. Purification by silica gel chromatography gave $30.8 \mathrm{mg}(79 \%$ yield $)$ of $\mathbf{5 d}$ as a colorless oil. ${ }^{1} \mathbf{H} \mathbf{~ N M R}\left(400 \mathrm{MHz}, \mathrm{CDCl}_{3}\right) \delta 7.09(\mathrm{~d}, J=8.4 \mathrm{~Hz}$, $2 \mathrm{H}), 6.81(\mathrm{~d}, J=8.5 \mathrm{~Hz}, 2 \mathrm{H}), 6.23(\mathrm{~s}, 1 \mathrm{H}), 3.77(\mathrm{~s}, 3 \mathrm{H}), 3.46-3.36(\mathrm{~m}, 1 \mathrm{H}), 3.35-3.25(\mathrm{~m}, 1 \mathrm{H})$, $2.69-2.52(\mathrm{~m}, 2 \mathrm{H}), 1.80-1.74(\mathrm{~m}, 1 \mathrm{H}), 1.63-1.52(\mathrm{~m}, 1 \mathrm{H}), 1.26(\mathrm{~s}, 12 \mathrm{H}), 1.24-1.21(\mathrm{~m}, 1 \mathrm{H})$, 1.18 (s, 9H). ${ }^{13} \mathbf{C}$ NMR $\left(101 \mathrm{MHz}, \mathrm{CDCl}_{3}\right) \delta 178.25,157.86,134.78,129.49,113.89,83.60,55.40$, 40.04, 38.82, 34.18, 31.02, 27.74, 25.09, 25.00. ${ }^{11} \mathbf{B}$ NMR $\left(128 \mathrm{MHz}, \mathrm{CDCl}_{3}\right) \delta 34.17$. ESI-HR calcd for $\mathrm{C}_{22} \mathrm{H}_{37} \mathrm{BNO}_{4}^{+}\left([\mathrm{M}+\mathrm{H}]^{+}\right) 390.2810$, found 390.2800. IR $v\left(\mathrm{~cm}^{-1}\right) 3363,2978,1647,750$. $[\alpha]^{20} \mathrm{D}=9.2\left(c=1.45, \mathrm{CHCl}_{3}\right)$ for a $79 \%$ ee sample. The enantiomeric purity of this compound was determined by HPLC analysis in comparison with authentic racemic material (Chiralpak IF, 95:5 hexanes: $i-\mathrm{PrOH}, 0.5 \mathrm{~mL} / \mathrm{min}, 220 \mathrm{~nm}$ ): $t_{\text {major }}=26.0 \mathrm{~min}, t_{\text {minor }}=25.2 \mathrm{~min}$.
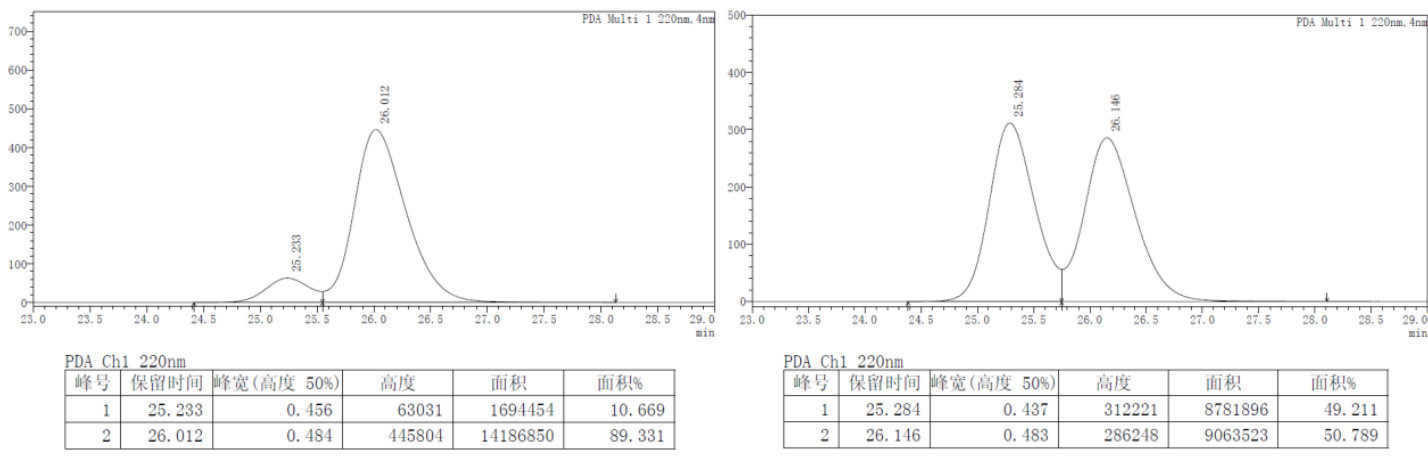<smiles></smiles>

$\boldsymbol{\alpha}$-Aminoboronic ester 4e: Following the general procedure [D], enamide 1ad-Z (27.7 mg, 0.100 mmol) was converted to the $\alpha$-aminoboronic ester. Purification by silica gel chromatography gave $37.8 \mathrm{mg}\left(90 \%\right.$ yield) of $\mathbf{4 e}$ as a colorless oil. ${ }^{1} \mathbf{H} \mathbf{~ N M R}\left(400 \mathrm{MHz}, \mathrm{CDCl}_{3}\right) \delta 7.18(\mathrm{~d}, J=8.1 \mathrm{~Hz}$, 2H), $7.09(\mathrm{~d}, J=8.1 \mathrm{~Hz}, 2 \mathrm{H}), 6.54(\mathrm{~s}, 1 \mathrm{H}), 2.70-2.50(\mathrm{~m}, 3 \mathrm{H}), 2.45(\mathrm{~s}, 3 \mathrm{H}), 1.76-1.56(\mathrm{~m}, 3 \mathrm{H})$, $1.50-1.38(\mathrm{~m}, 1 \mathrm{H}), 1.19(\mathrm{~s}, 21 \mathrm{H}) .{ }^{13} \mathbf{C}$ NMR $\left(101 \mathrm{MHz}, \mathrm{CDCl}_{3}\right) \delta 183.74,139.82,135.23$, $129.06,127.38,80.39,35.73,35.46,30.99,29.78,26.94,25.46,25.23,16.55 .{ }^{11}$ B NMR (128 
MHz, $\left.\mathrm{CDCl}_{3}\right) \delta$ 15.75. ESI-HR calcd for $\mathrm{C}_{22} \mathrm{H}_{37} \mathrm{BNO}_{3} \mathrm{~S}^{+}\left([\mathrm{M}+\mathrm{H}]^{+}\right) 406.2582$, found 406.2569. IR $v\left(\mathrm{~cm}^{-1}\right) 2988,1572,1275,732 .[\alpha]^{20} \mathrm{D}=-101.3\left(c=1.73, \mathrm{CHCl}_{3}\right)$ for a $98 \%$ ee sample. The enantiomeric purity of this compound was determined by HPLC analysis in comparison with authentic racemic material (Chiralpak IC, 97:3 hexanes: $i-\mathrm{PrOH}, 1.0 \mathrm{~mL} / \mathrm{min}, 220 \mathrm{~nm}$ ): $t_{\text {major }}=9.7$ $\min , t_{\operatorname{minor}}=6.6 \mathrm{~min}$.
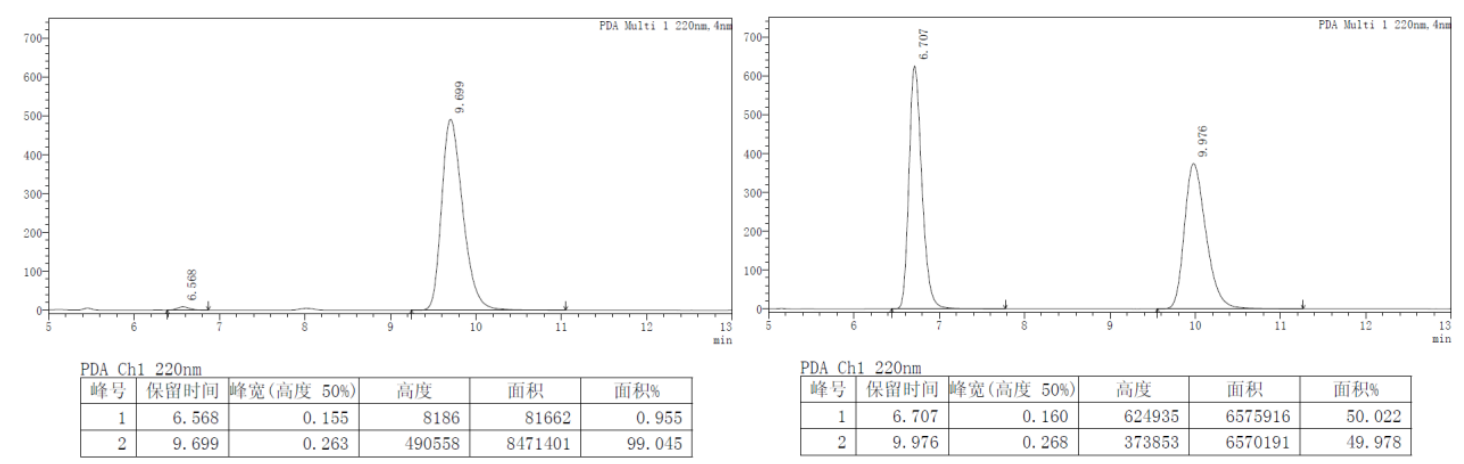<smiles>COc1ccc(CCC(CNC(=O)C(C)(C)C)Cc2ccccc2)cc1</smiles>

$\boldsymbol{\beta}$-Aminoboronic ester 5e: Following the general procedure [E], enamide 1ad-E (27.7 mg, 0.100 mmol) was converted to the $\beta$-aminoboronic ester. Purification by silica gel chromatography gave $29.0 \mathrm{mg}(72 \%$ yield $)$ of $\mathbf{5 e}$ as a colorless oil. ${ }^{\mathbf{1}} \mathbf{H} \mathbf{N M R}\left(400 \mathrm{MHz}, \mathrm{CDCl}_{3}\right) \delta 7.18(\mathrm{~d}, J=8.2 \mathrm{~Hz}$, 2H), $7.10(\mathrm{~d}, J=8.1 \mathrm{~Hz}, 2 \mathrm{H}), 6.23(\mathrm{~s}, 1 \mathrm{H}), 3.47-3.36(\mathrm{~m}, 1 \mathrm{H}), 3.35-3.22(\mathrm{~m}, 1 \mathrm{H}), 2.70-2.55$ (m, 2H), $2.45(\mathrm{~s}, 3 \mathrm{H}), 1.80-1.69(\mathrm{~m}, 1 \mathrm{H}), 1.69-1.55(\mathrm{~m}, 2 \mathrm{H}), 1.26(\mathrm{~s}, 12 \mathrm{H}), 1.24-1.20(\mathrm{~m}, 1 \mathrm{H})$, 1.18 (s, 9H). ${ }^{13}$ C NMR $\left(101 \mathrm{MHz}, \mathrm{CDCl}_{3}\right) \delta 178.26,139.89,135.25,129.18,127.42,83.63,39.94$, 38.82, 34.53, 30.71, 27.74, 25.09, 24.99, 16.58. ${ }^{11} \mathbf{B}$ NMR $\left(128 \mathrm{MHz}, \mathrm{CDCl}_{3}\right) \delta 34.48$. ESI-HR calcd for $\mathrm{C}_{22} \mathrm{H}_{37} \mathrm{BNO}_{3} \mathrm{~S}^{+}\left([\mathrm{M}+\mathrm{H}]^{+}\right)$406.2582, found 406.2571. IR $v\left(\mathrm{~cm}^{-1}\right) 3362,2978,1646,750$. $[\alpha]^{20} \mathrm{D}=9.4\left(c=1.50, \mathrm{CHCl}_{3}\right)$ for a $78 \%$ ee sample. The enantiomeric purity of this compound was determined by HPLC analysis in comparison with authentic racemic material (Chiralpak IF, 95:5 hexanes: $i-\mathrm{PrOH}, 0.5 \mathrm{~mL} / \mathrm{min}, 220 \mathrm{~nm}$ ): $t_{\text {major }}=25.6 \mathrm{~min}, t_{\text {minor }}=24.8 \mathrm{~min}$. 

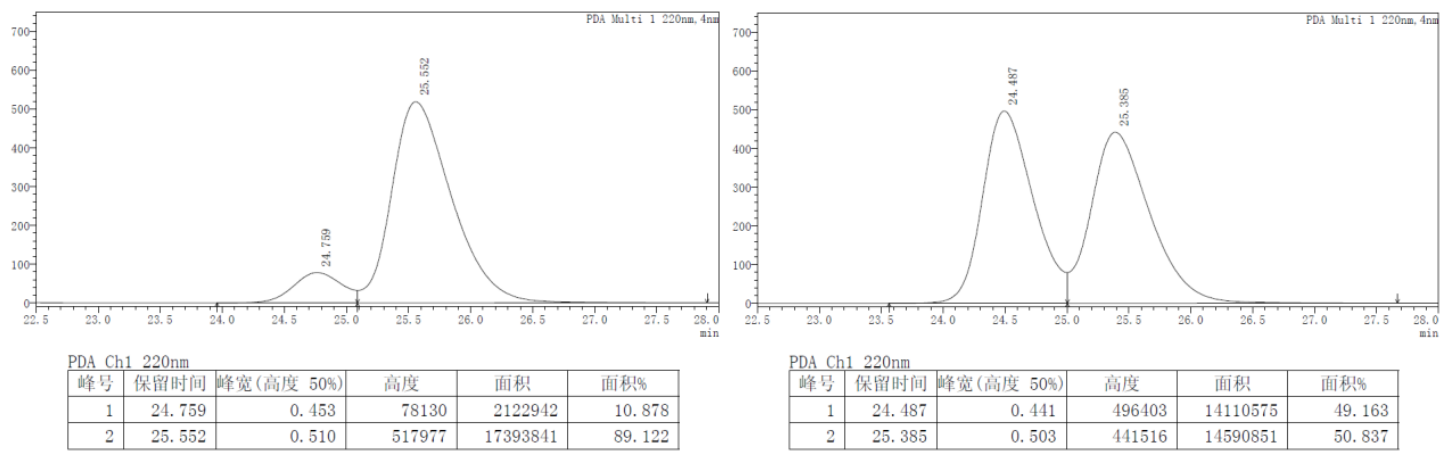<smiles>CC(C)(C)C(=O)NC(Br)CCCCc1cccs1</smiles>

$\boldsymbol{\alpha}$-Aminoboronic ester 4f: Following the general procedure [D], enamide 1ae- $\boldsymbol{Z}$ (25.1 mg, 0.100 mmol) was converted to the $\alpha$-aminoboronic ester. Purification by silica gel chromatography gave $30.3 \mathrm{mg}$ (80\% yield) of $\mathbf{4} \mathbf{f}$ as a white solid. ${ }^{1} \mathbf{H} \mathbf{~ N M R}\left(400 \mathrm{MHz}, \mathrm{CDCl}_{3}\right) \delta 7.12-7.05(\mathrm{~m}, 1 \mathrm{H})$, $6.90(\mathrm{dd}, J=5.0,3.5 \mathrm{~Hz}, 1 \mathrm{H}), 6.76(\mathrm{~d}, J=3.2 \mathrm{~Hz}, 1 \mathrm{H}), 6.53(\mathrm{~s}, 1 \mathrm{H}), 2.93-2.75(\mathrm{~m}, 2 \mathrm{H}), 2.59-$ $2.47(\mathrm{~m}, 1 \mathrm{H}), 1.78-1.66(\mathrm{~m}, 2 \mathrm{H}), 1.66-1.55(\mathrm{~m}, 1 \mathrm{H}), 1.50-1.33(\mathrm{~m}, 3 \mathrm{H}), 1.21(\mathrm{~s}, 9 \mathrm{H}), 1.19(\mathrm{~s}$, 12H). ${ }^{13} \mathbf{C}$ NMR $\left(101 \mathrm{MHz}, \mathrm{CDCl}_{3}\right) \delta 183.75,145.65,126.81,124.21,122.92,80.39,35.76,31.67$, 30.96, 29.79, 27.30, 26.97, 25.45, 25.23. ${ }^{11} \mathbf{B}$ NMR $\left(128 \mathrm{MHz}, \mathrm{CDCl}_{3}\right) \delta$ 16.36. ESI-HR calcd for $\mathrm{C}_{20} \mathrm{H}_{35} \mathrm{BNO}_{3} \mathrm{~S}^{+}\left([\mathrm{M}+\mathrm{H}]^{+}\right) 380.2425$, found 380.2414. IR $v\left(\mathrm{~cm}^{-1}\right) 2989,1422,1275$, 749. M. P. $52-55{ }^{\circ} \mathrm{C} .[\alpha]^{20} \mathrm{D}=-98.5\left(c=0.72, \mathrm{CHCl}_{3}\right)$ for a $93 \%$ ee sample. The enantiomeric purity of this compound was determined by HPLC analysis in comparison with authentic racemic material (Chiralpak IC, 97:3 hexanes:i-PrOH, $1.0 \mathrm{~mL} / \mathrm{min}, 220 \mathrm{~nm}$ ): $t_{\text {major }}=6.5 \mathrm{~min}, t_{\text {minor }}=5.3 \mathrm{~min}$.
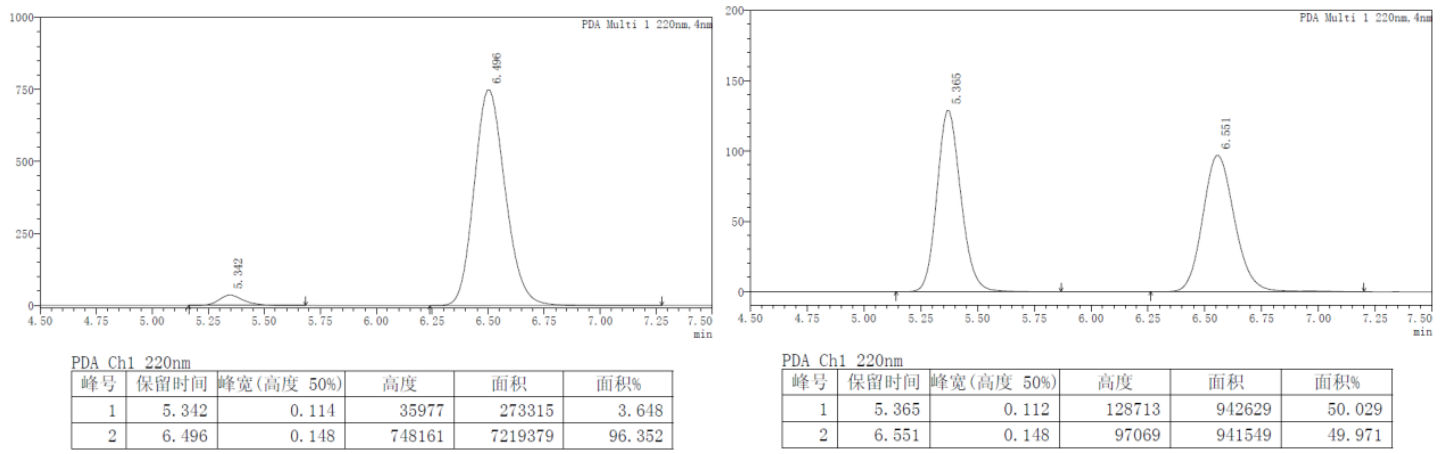
<smiles>CC(C)(C)C(=O)NC[C@@H]([B]c1ccccc1)CCCc1cccs1</smiles>

$\boldsymbol{\beta}$-Aminoboronic ester 5f: Following the general procedure [E], enamide 1ae- $\boldsymbol{E}(25.1 \mathrm{mg}, 0.100$ mmol) was converted to the $\beta$-aminoboronic ester. Purification by silica gel chromatography gave $31.1 \mathrm{mg}\left(82 \%\right.$ yield) of $\mathbf{5 f}$ as a colorless oil. ${ }^{\mathbf{1}} \mathbf{H} \mathbf{N M R}\left(400 \mathrm{MHz}, \mathrm{CDCl}_{3}\right) \delta 7.08(\mathrm{~d}, J=5.5 \mathrm{~Hz}$, 1H), $6.89(\mathrm{t}, J=4.9 \mathrm{~Hz}, 1 \mathrm{H}), 6.76(\mathrm{~d}, J=2.5 \mathrm{~Hz}, 1 \mathrm{H}), 6.20(\mathrm{~s}, 1 \mathrm{H}), 3.45-3.33(\mathrm{~m}, 1 \mathrm{H}), 3.25-$ $3.15(\mathrm{~m}, 1 \mathrm{H}), 2.81(\mathrm{t}, J=7.4 \mathrm{~Hz}, 2 \mathrm{H}), 1.86-1.61(\mathrm{~m}, 3 \mathrm{H}), 1.58-1.47(\mathrm{~m}, 1 \mathrm{H}), 1.46-1.35(\mathrm{~m}$, 1H), 1.25 (s, 12H), $1.17(\mathrm{~s}, 9 \mathrm{H}) .{ }^{13} \mathrm{C}$ NMR $\left(101 \mathrm{MHz}, \mathrm{CDCl}_{3}\right) \delta 178.22,145.53,126.75,124.12$, 122.87, 83.57, 40.37, 38.79, 30.99, 30.18, 28.29, 27.71, 25.05, 24.96. ${ }^{11} \mathbf{B}$ NMR (128 MHz, $\left.\mathrm{CDCl}_{3}\right)$ $\delta$ 34.77. ESI-HR calcd for $\mathrm{C}_{20} \mathrm{H}_{35} \mathrm{BNO}_{3} \mathrm{~S}^{+}\left([\mathrm{M}+\mathrm{H}]^{+}\right) 380.2425$, found 380.2414. IR $v\left(\mathrm{~cm}^{-1}\right) 3352$, 2977, 1641, 750. $[\alpha]^{20} \mathrm{D}=2.8\left(c=1.45, \mathrm{CHCl}_{3}\right)$ for a $78 \%$ ee sample. The enantiomeric purity of this compound was determined by HPLC analysis in comparison with authentic racemic material (Chiralpak IF, 95:5 hexanes: $i-\mathrm{PrOH}, 1.0 \mathrm{~mL} / \mathrm{min}, 220 \mathrm{~nm}$ ): $t_{\text {major }}=11.2 \mathrm{~min}, t_{\mathrm{minor}}=10.7 \mathrm{~min}$.
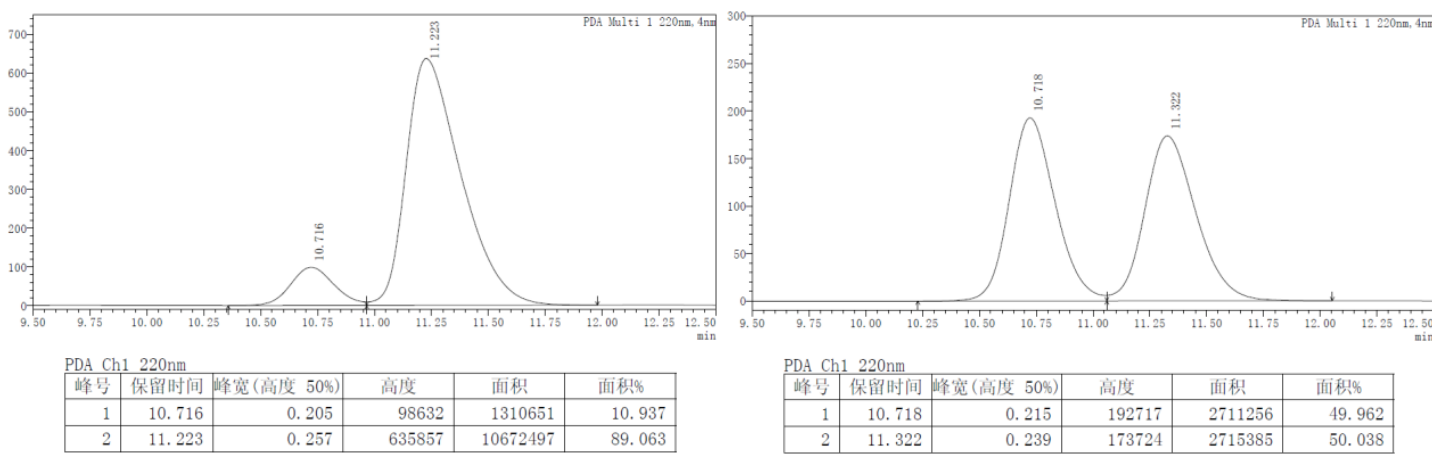<smiles>CC(C)(C)C(=O)NC(CCCCOCc1ccccc1)c1ccccc1</smiles>

$\boldsymbol{\alpha}$-Aminoboronic ester 4g: Following the general procedure [D], enamide 1af- $Z$ (27.5 mg, 0.100 mmol) was converted to the $\alpha$-aminoboronicester. Purification by silica gel chromatography gave $26.9 \mathrm{mg}\left(67 \%\right.$ yield) of $\mathbf{4 g}$ as a colorless oil. ${ }^{\mathbf{1}} \mathbf{H} \mathbf{N M R}\left(400 \mathrm{MHz}, \mathrm{CDCl}_{3}\right) \delta 7.36-7.29(\mathrm{~m}, 4 \mathrm{H})$, $7.29-7.24(\mathrm{~m}, 1 \mathrm{H}), 6.80(\mathrm{~s}, 1 \mathrm{H}), 4.49(\mathrm{~s}, 2 \mathrm{H}), 3.49(\mathrm{t}, J=6.3 \mathrm{~Hz}, 2 \mathrm{H}), 2.59-2.49(\mathrm{~m}, 1 \mathrm{H}), 1.74$ 
$-1.54(\mathrm{~m}, 3 \mathrm{H}), 1.51-1.36(\mathrm{~m}, 3 \mathrm{H}), 1.18(\mathrm{~s}, 21 \mathrm{H}) .{ }^{13} \mathbf{C} \mathbf{~ N M R}\left(101 \mathrm{MHz}, \mathrm{CDCl}_{3}\right) \delta 183.79,138.63$, $128.50,127.80,127.69,80.26,73.07,70.48,35.67,30.88,29.64,26.94,25.44,25.21,24.79 .{ }^{11} \mathbf{B}$ NMR $\left(128 \mathrm{MHz}, \mathrm{CDCl}_{3}\right) \delta$ 15.48. ESI-HR calcd for $\mathrm{C}_{23} \mathrm{H}_{39} \mathrm{BNO}_{4}{ }^{+}\left([\mathrm{M}+\mathrm{H}]^{+}\right) 404.2967$, found 404.2957. IR $\vee\left(\mathrm{cm}^{-1}\right) 2987,1652,1275,732 .[\alpha]^{20} \mathrm{D}=-77.7\left(c=0.82, \mathrm{CHCl}_{3}\right)$ for a $98 \%$ ee sample. The enantiomeric purity of this compound was determined by HPLC analysis in comparison with authentic racemic material (Chiralpak IE, 95:5 hexanes: $i-\mathrm{PrOH}, 1.0 \mathrm{~mL} / \mathrm{min}, 220$ $\mathrm{nm}): t_{\text {major }}=7.9 \min , t_{\text {minor }}=10.3 \mathrm{~min}$.
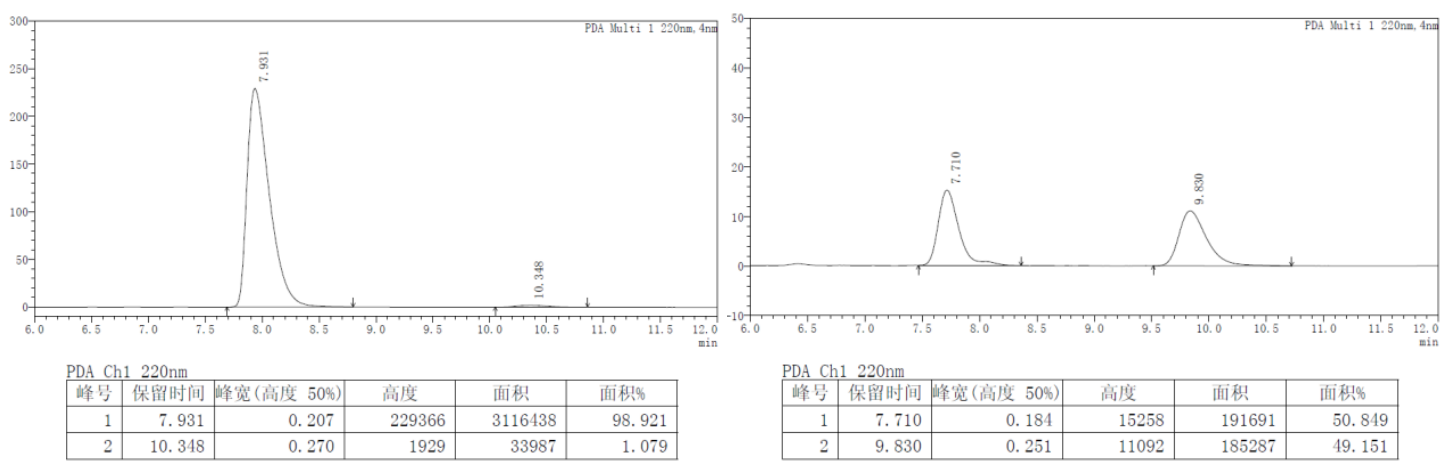<smiles>CC(C)(C)C(=O)NC[C@H](CCCOCc1ccccc1)Cc1ccccc1</smiles>

$\boldsymbol{\beta}$-Aminoboronic ester 5g: Following the general procedure [E], enamide 1af-E (27.5 mg, 0.100 mmol) was converted to the $\beta$-aminoboronic ester. Purification by silica gel chromatography gave $31.7 \mathrm{mg}$ (79\% yield) of $\mathbf{5 g}$ as a colorless oil. ${ }^{\mathbf{1}} \mathbf{H} \mathbf{~ N M R}\left(400 \mathrm{MHz}, \mathrm{CDCl}_{3}\right) \delta 7.37-7.29(\mathrm{~m}, 4 \mathrm{H})$, $7.29-7.23(\mathrm{~m}, 1 \mathrm{H}), 6.23(\mathrm{~s}, 1 \mathrm{H}), 4.48(\mathrm{~s}, 2 \mathrm{H}), 3.49-3.43(\mathrm{~m}, 2 \mathrm{H}), 3.43-3.34(\mathrm{~m}, 1 \mathrm{H}), 3.26-$ $3.16(\mathrm{~m}, 1 \mathrm{H}), 1.72-1.61(\mathrm{~m}, 2 \mathrm{H}), 1.59-1.48(\mathrm{~m}, 1 \mathrm{H}), 1.46-1.35(\mathrm{~m}, 1 \mathrm{H}), 1.24(\mathrm{~s}, 12 \mathrm{H}), 1.22-$ $1.18(\mathrm{~m}, 1 \mathrm{H}), 1.17(\mathrm{~s}, 9 \mathrm{H}),{ }^{13} \mathbf{C}$ NMR $\left(101 \mathrm{MHz}, \mathrm{CDCl}_{3}\right) \delta 178.22,138.82,128.42,127.71$, $127.53,83.53,72.83,70.56,40.35,38.77,28.92,27.70,25.36,25.03,24.96 .{ }^{11} \mathbf{B}$ NMR $(128 \mathrm{MHz}$, $\left.\mathrm{CDCl}_{3}\right) \delta$ 34.33. ESI-HR calcd for $\mathrm{C}_{23} \mathrm{H}_{39} \mathrm{BNO}_{4}{ }^{+}\left([\mathrm{M}+\mathrm{H}]^{+}\right)$404.2967, found 404.2954. IR $v\left(\mathrm{~cm}^{-1}\right)$ $3364,2983,1651,750 .[\alpha]^{20} \mathrm{D}=-1.6\left(c=1.50, \mathrm{CHCl}_{3}\right)$ for a $80 \%$ ee sample. The enantiomeric purity of this compound was determined by HPLC analysis in comparison with authentic racemic material (Chiralpak IF, 95:5 hexanes: $i-\mathrm{PrOH}, 1.0 \mathrm{~mL} / \mathrm{min}, 210 \mathrm{~nm}$ ): $t_{\text {major }}=13.6 \mathrm{~min}, t_{\text {minor }}=12.8$ $\min$. 

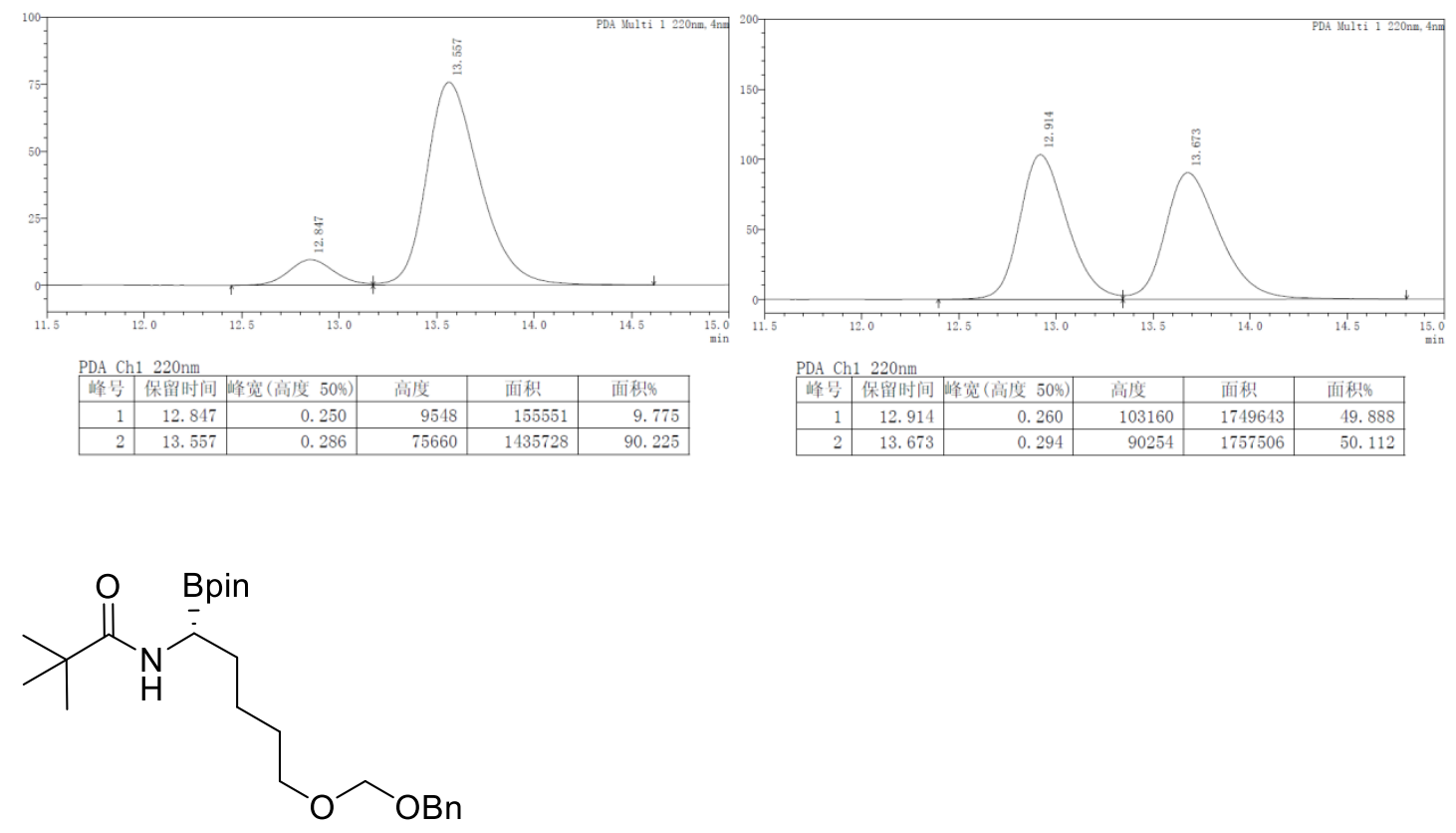

$\boldsymbol{\alpha}$-Aminoboronic ester $\mathbf{4 h}$ : Following the general procedure [D], enamide $\mathbf{1 m}-\mathbf{Z}$ (30.5 mg, 0.100 mmol) was converted to the $\alpha$-aminoboronicester. Purification by silica gel chromatography gave $19.4 \mathrm{mg}(45 \%$ yield $)$ of $\mathbf{4 h}$ as a white solid. $[\alpha]^{20} \mathrm{D}=-138.7\left(c=0.70, \mathrm{CHCl}_{3}\right)$ for a $98 \%$ ee sample. The enantiomeric purity of this compound was determined by HPLC analysis in comparison with authentic racemic material (Chiralpak IE, 95:5 hexanes:i-PrOH, $1.0 \mathrm{~mL} / \mathrm{min}, 220 \mathrm{~nm}$ ): $t_{\text {major }}=9.0$ $\min , t_{\text {minor }}=11.4 \mathrm{~min}$.
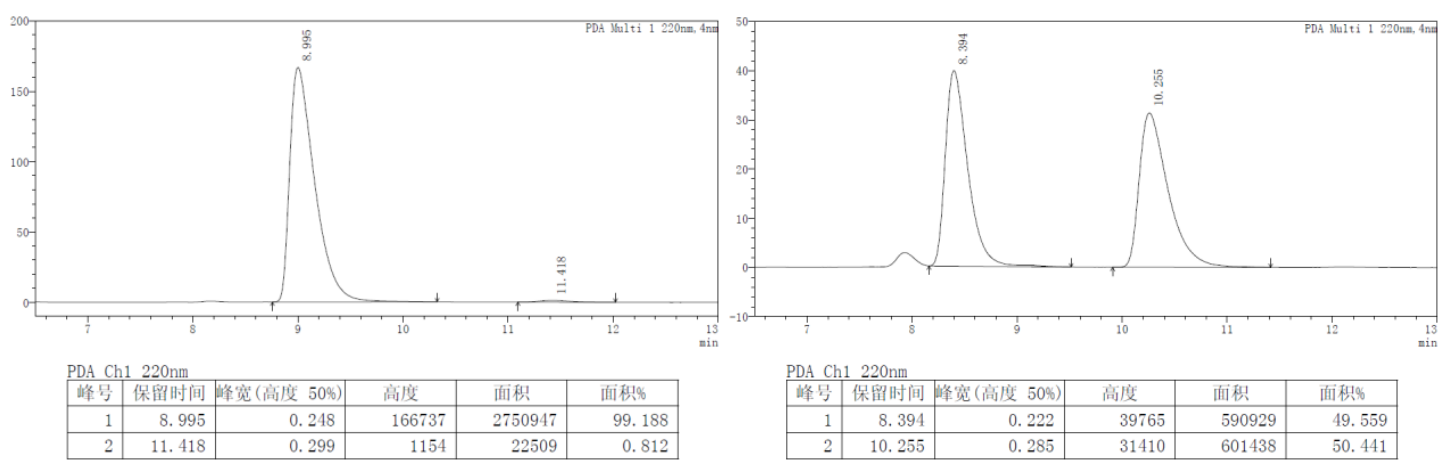<smiles>CC(C)(C)C(=O)NC[C@H](CCCOCc1ccccc1)Cc1ccccc1</smiles>

$\boldsymbol{\beta}$-Aminoboronic ester 5h: Following the general procedure [E], enamide 1m-E (30.5 mg, 0.100 mmol) was converted to the $\beta$-aminoboronic ester. Purification by silica gel chromatography gave $34.9 \mathrm{mg}(81 \%$ yield $)$ of $\mathbf{5 h}$ as a colorless oil. $[\alpha]^{20} \mathrm{D}=-4.9\left(c=1.69, \mathrm{CHCl}_{3}\right)$ for a $79 \%$ ee sample. 
The enantiomeric purity of this compound was determined by HPLC analysis in comparison with authentic racemic material (Chiralpak ID, 95:5 hexanes:i-PrOH, $0.6 \mathrm{~mL} / \mathrm{min}, 220 \mathrm{~nm}$ ): $t_{\text {major }}=$ $31.2 \mathrm{~min}, t_{\mathrm{minor}}=30.1 \mathrm{~min}$.
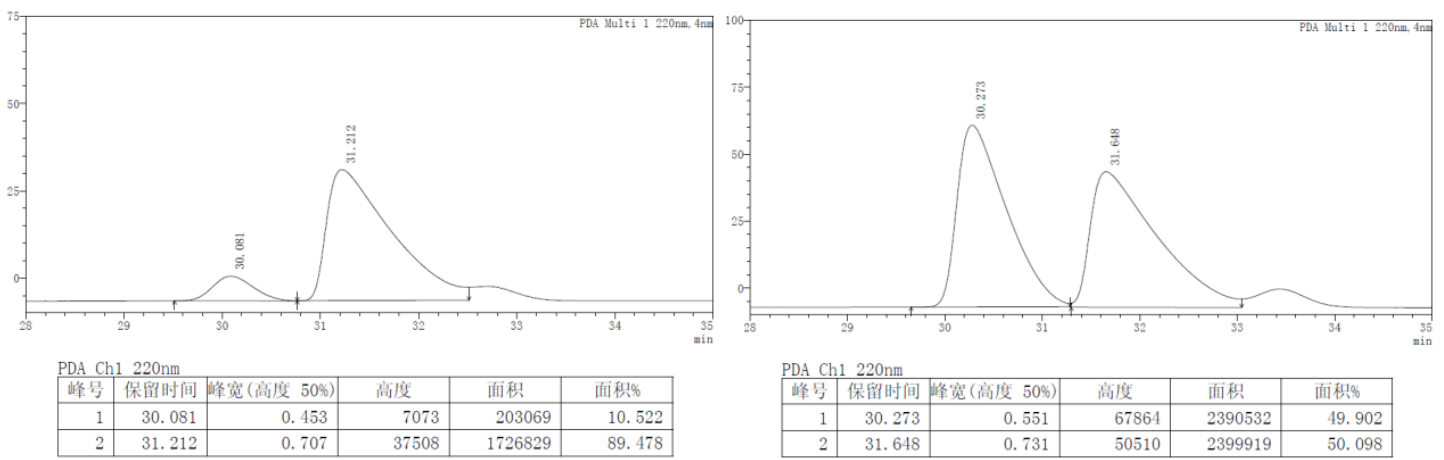<smiles>CC(C)(C)C(=O)NC(CCCCOC(=O)c1ccccc1)[Pb]c1ccccc1</smiles>

$\boldsymbol{\alpha}$-Aminoboronic ester 4i: Following the general procedure [D], enamide 1i-Z (28.9 mg, 0.100 mmol) was converted to the $\alpha$-aminoboronicester. Purification by silica gel chromatography gave $29.8 \mathrm{mg}(71 \%$ yield $)$ of $\mathbf{4 i}$ as a white solid. $[\alpha]^{20} \mathrm{D}=-89.9\left(c=1.45, \mathrm{CHCl}_{3}\right)$ for a $85 \%$ ee sample. The enantiomeric purity of this compound was determined by HPLC analysis in comparison with authentic racemic material (Chiralpak IE, 95:5 hexanes:i-PrOH, $1.0 \mathrm{~mL} / \mathrm{min}, 220 \mathrm{~nm}$ ): $t_{\text {major }}=$ $12.8 \mathrm{~min}, t_{\mathrm{minor}}=15.9 \mathrm{~min}$.
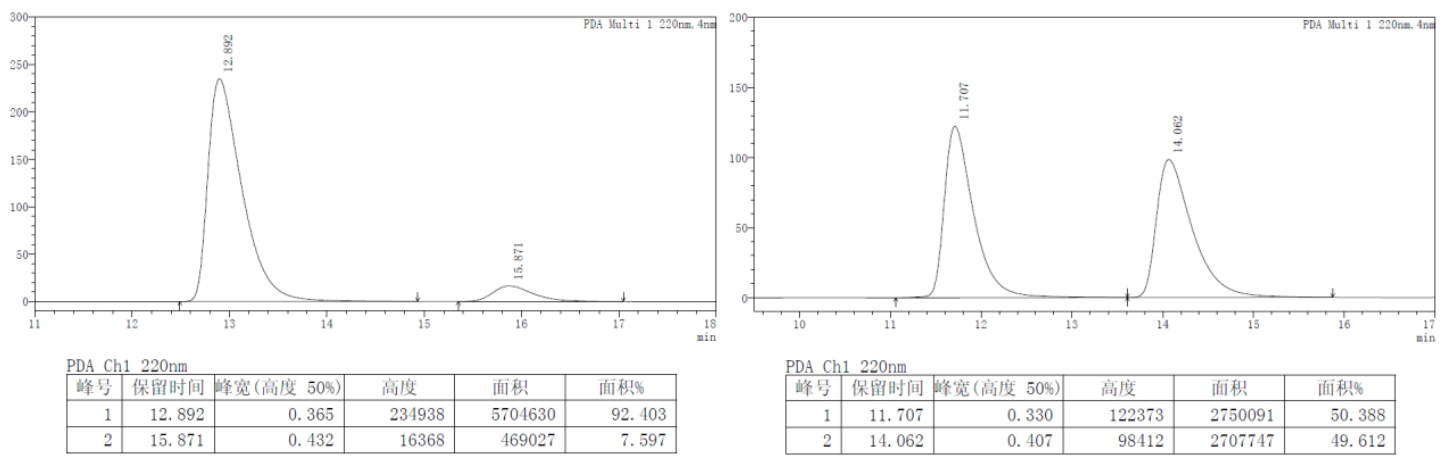
<smiles>CC(C)(C)C(=O)NC[C@H](CCCOC(=O)c1ccccc1)Cc1ccccc1</smiles>

$\boldsymbol{\beta}$-Aminoboronic ester 5i: Following the general procedure [E], enamide 1i- $\boldsymbol{E}$ (28.9 mg, 0.100 mmol) was converted to the $\beta$-aminoboronic ester. Purification by silica gel chromatography gave $37.3 \mathrm{mg}(89 \%$ yield $)$ of $\mathbf{5 i}$ as a colorless oil. $[\alpha]^{20} \mathrm{D}=-11.1\left(c=1.76, \mathrm{CHCl}_{3}\right)$ for a $82 \%$ ee sample. The enantiomeric purity of this compound was determined by HPLC analysis in comparison with authentic racemic material (Chiralpak IF, 95:5 hexanes: $i-\mathrm{PrOH}, 1.0 \mathrm{~mL} / \mathrm{min}, 220 \mathrm{~nm}$ ): $t_{\text {major }}=21.8$ $\min , t_{\text {minor }}=20.7 \mathrm{~min}$.
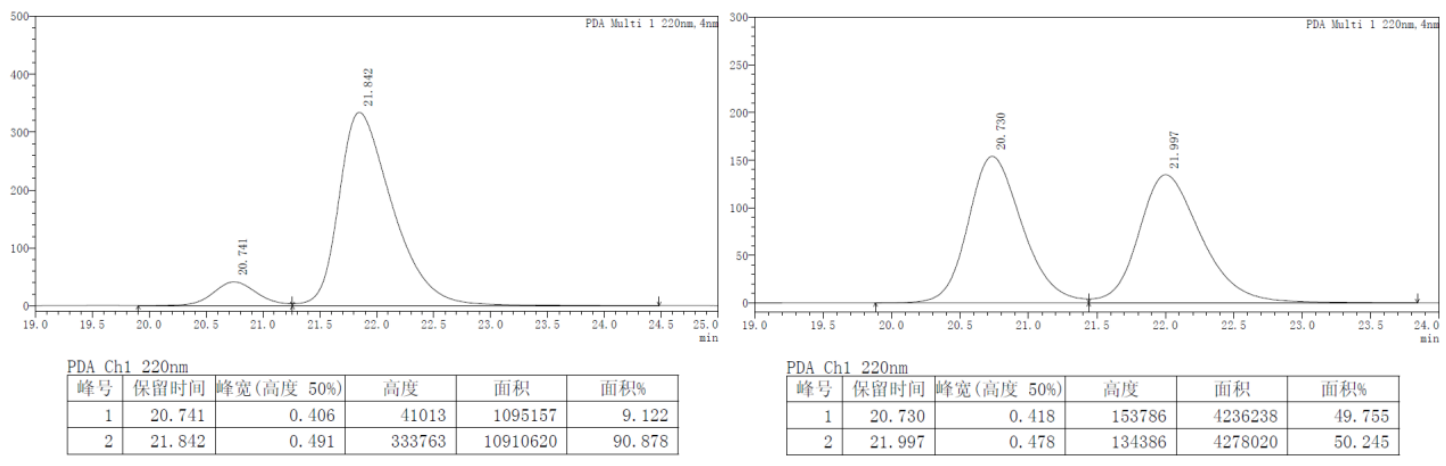<smiles>CC(CBr)(CCBr)NC(=O)C(C)(C)C</smiles>

$\boldsymbol{\alpha}$-Aminoboronic ester $\mathbf{4 j}$ : Following the general procedure [D], enamide 1ag- $\boldsymbol{Z}$ (51.2 mg, 0.200 mmol) was converted to the $\alpha$-aminoboronic ester. Purification by silica gel chromatography gave $53.9 \mathrm{mg}\left(72 \%\right.$ yield) of $\mathbf{4 j}$ as a white solid. ${ }^{\mathbf{1}} \mathbf{H} \mathbf{~ N M R}\left(400 \mathrm{MHz}, \mathrm{CDCl}_{3}\right) \delta 7.29(\mathrm{dd}, J=8.8,6.1$ $\mathrm{Hz}, 2 \mathrm{H}), 7.24-7.15(\mathrm{~m}, 3 \mathrm{H}), 6.28(\mathrm{~s}, 1 \mathrm{H}), 2.68(\mathrm{dt}, J=14.0,7.0 \mathrm{~Hz}, 1 \mathrm{H}), 2.63-2.52(\mathrm{~m}, 1 \mathrm{H})$, $1.83-1.72(\mathrm{~m}, 1 \mathrm{H}), 1.67-1.53(\mathrm{~m}, 3 \mathrm{H}), 1.22(\mathrm{~s}, 9 \mathrm{H}), 1.20(\mathrm{~d}, J=3.1 \mathrm{~Hz}, 12 \mathrm{H}), 1.13(\mathrm{~s}, 3 \mathrm{H})$. ${ }^{13} \mathrm{C}$ NMR $\left(101 \mathrm{MHz}, \mathrm{CDCl}_{3}\right) \delta 183.20,142.85,128.53,128.38,125.76,80.13,38.04,36.65$, 35.47, 26.90, 26.63, 25.38, 25.32, 22.03. ${ }^{11} \mathbf{B}$ NMR $\left(128 \mathrm{MHz}, \mathrm{CDCl}_{3}\right) \delta$ 15.26. ESI-HR calcd for $\mathrm{C}_{22} \mathrm{H}_{37} \mathrm{BNO}_{3}{ }^{+}\left([\mathrm{M}+\mathrm{H}]^{+}\right) 374.2861$, found 374.2868. IR $v\left(\mathrm{~cm}^{-1}\right)$ 2989, 1610, 1275, 749. M. P. $66-68{ }^{\circ} \mathrm{C} .[\alpha]^{20} \mathrm{D}=-37.8\left(c=1.54, \mathrm{CHCl}_{3}\right)$ for a $97 \%$ ee sample. The enantiomeric purity of this 
compound was determined by HPLC analysis in comparison with authentic racemic material (Chiralpak IE, 99:1 hexanes: $i-\mathrm{PrOH}, 1.0 \mathrm{~mL} / \mathrm{min}, 210 \mathrm{~nm}$ ): $t_{\text {major }}=20.0 \mathrm{~min}, t_{\text {minor }}=25.1 \mathrm{~min}$.
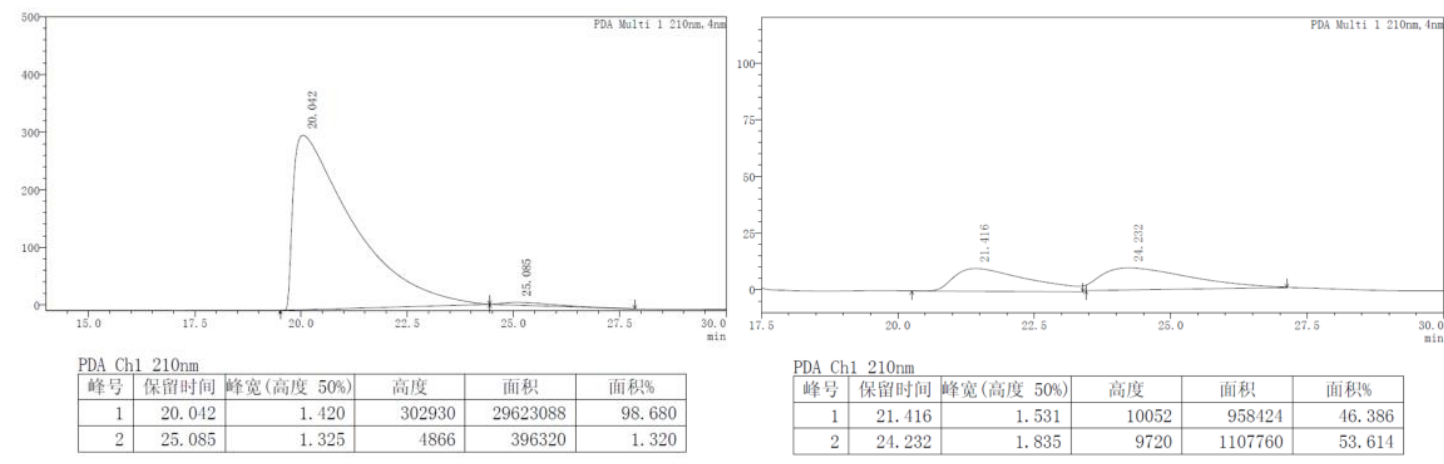<smiles>CCC(Br)(Br)c1ccc(C(F)(F)F)cc1</smiles>

$\boldsymbol{\alpha}$-Aminoboronic ester 4k: Following the general procedure [D], enamide 1ah-Z (57.2 mg, 0.200 mmol) was converted to the $\alpha$-aminoboronic ester. Purification by silica gel chromatography gave $72.9 \mathrm{mg}$ ( $88 \%$ yield) of $\mathbf{4 k}$ as a white solid. ${ }^{1} \mathbf{H} \mathbf{~ N M R}\left(400 \mathrm{MHz}, \mathrm{CDCl}_{3}\right) \delta 7.52(\mathrm{~d}, J=8.3 \mathrm{~Hz}$, 2H), $7.21(\mathrm{~d}, J=8.2 \mathrm{~Hz}, 2 \mathrm{H}), 7.03-6.82(\mathrm{~m}, 1 \mathrm{H}), 2.19(\mathrm{dq}, J=15.0,7.5 \mathrm{~Hz}, 1 \mathrm{H}), 2.00(\mathrm{dq}, \mathrm{J}=$ 14.7, $7.3 \mathrm{~Hz}, 1 \mathrm{H}), 1.36(\mathrm{~s}, 9 \mathrm{H}), 1.11(\mathrm{~s}, 6 \mathrm{H}), 1.03(\mathrm{~s}, 6 \mathrm{H}), 0.74(\mathrm{t}, J=7.4 \mathrm{~Hz}, 3 \mathrm{H}) .{ }^{13} \mathbf{C}$ NMR $(101$ $\mathrm{MHz}, \mathrm{CDCl} 3) \delta 184.86,148.15,127.12\left(J_{2}=33 \mathrm{~Hz}\right), 125.38,124.82\left(J_{3}=4 \mathrm{~Hz}\right), 124.48\left(J_{1}=\right.$ 272Hz), 80.41, 35.78, 28.37, 26.85, 25.20, 24.97, 8.44. ${ }^{11} \mathbf{B}$ NMR $\left(128 \mathrm{MHz}, \mathrm{CDCl}_{3}\right) \delta 14.93$. ESI-HR calcd for $\mathrm{C}_{21} \mathrm{H}_{32} \mathrm{BF}_{4} \mathrm{NO}_{3}{ }^{+}\left([\mathrm{M}+\mathrm{H}]^{+}\right)$414.2422, found 414.2434. IR $v\left(\mathrm{~cm}^{-1}\right) 2973,1558$, 1214, 744. M. P. $88-90{ }^{\circ} \mathrm{C} .[\alpha]^{20} \mathrm{D}=-69.2\left(c=1.40, \mathrm{CHCl}_{3}\right)$ for a $99 \%$ ee sample. The enantiomeric purity of this compound was determined by HPLC analysis in comparison with authentic racemic material (Chiralpak IE 99:1 hexanes:i-PrOH, $1.0 \mathrm{~mL} / \mathrm{min}, 230 \mathrm{~nm}$ ): $t_{\text {major }}=$ $8.8 \min$.
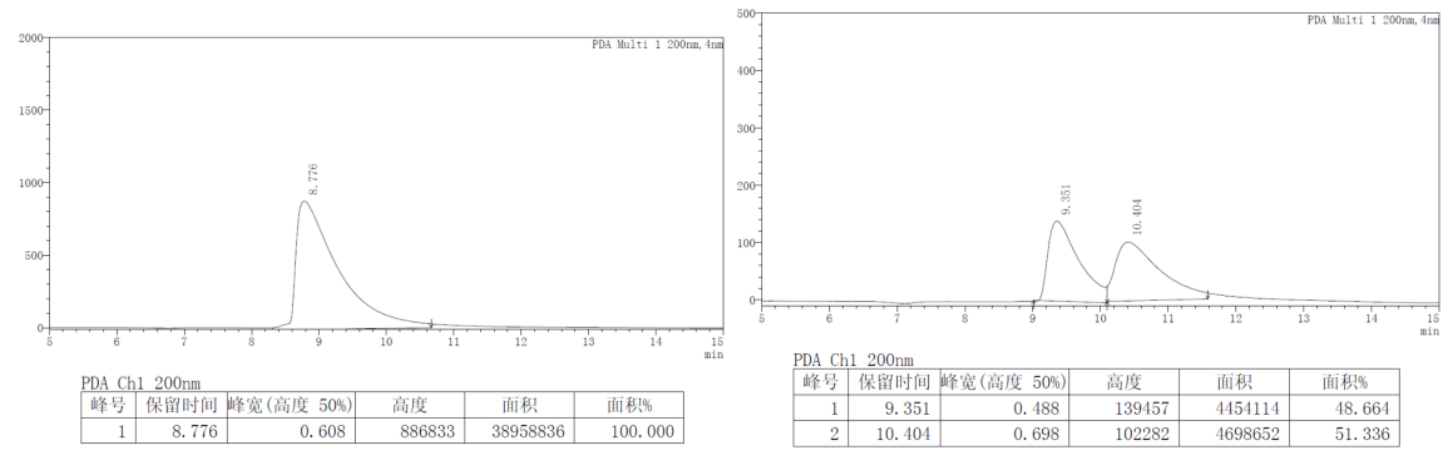
<smiles>CCC(C)(C)C(=O)N[C@](Br)(c1ccccc1)c1ccc(F)cc1</smiles>

$\boldsymbol{\alpha}$-Aminoboronic ester 41: Following the general procedure [D], enamide 1ai- $Z$ (47.2 mg, 0.200 mmol) was converted to the $\alpha$-aminoboronic ester. Purification by silica gel chromatography gave $64.1 \mathrm{mg}(88 \%$ yield $)$ of $\mathbf{4} \mathbf{l}$ as a white solid. ${ }^{1} \mathbf{H}$ NMR $\left(400 \mathrm{MHz}, \mathrm{CDCl}_{3}\right) \delta 7.05(\mathrm{tt}, J=5.2,2.6 \mathrm{~Hz}$, 2H), $7.01-6.92(\mathrm{~m}, 2 \mathrm{H}), 6.82(\mathrm{~s}, 1 \mathrm{H}), 2.12(\mathrm{dq}, J=15.0,7.5 \mathrm{~Hz}, 1 \mathrm{H}), 2.03-1.90(\mathrm{~m}, 1 \mathrm{H}), 1.34$ (s, 9H), $1.11(\mathrm{~s}, 6 \mathrm{H}), 1.03(\mathrm{~s}, 6 \mathrm{H}), 0.74(\mathrm{t}, J=7.4 \mathrm{~Hz}, 3 \mathrm{H}) .{ }^{13} \mathbf{C}$ NMR $\left(101 \mathrm{MHz}, \mathrm{CDCl}_{3}\right) \delta 184.43$, $160.70\left(J_{1}=243 \mathrm{~Hz}\right), 139.20,126.61\left(J_{3}=8 \mathrm{~Hz}\right), 114.57\left(J_{2}=21 \mathrm{~Hz}\right), 80.32,35.75,28.39,26.87$, 25.17, 24.99, 8.37. ${ }^{11} \mathbf{B}$ NMR $\left(128 \mathrm{MHz}, \mathrm{CDCl}_{3}\right) \delta$ 14.50. ESI-HR calcd for $\mathrm{C}_{20} \mathrm{H}_{32} \mathrm{BFNO}_{3}{ }^{+}$ $\left([\mathrm{M}+\mathrm{H}]^{+}\right) 364.2454$, found $364.2458 . \mathbf{I R} v\left(\mathrm{~cm}^{-1}\right) 3261,2967,1533,731$. M. P. $83-85{ }^{\circ} \mathrm{C} .[\alpha]^{20} \mathrm{D}=$ $-24.4\left(c=1.24, \mathrm{CHCl}_{3}\right)$ for a $98 \%$ ee sample. The enantiomeric purity of this compound was determined by HPLC analysis in comparison with authentic racemic material (Chiralpak OD, 99.5:0.5 hexanes: $i-\mathrm{PrOH}, 1.0 \mathrm{~mL} / \mathrm{min}, 220 \mathrm{~nm}): t_{\text {major }}=20.8 \mathrm{~min}, t_{\text {minor }}=37.3 \mathrm{~min}$.

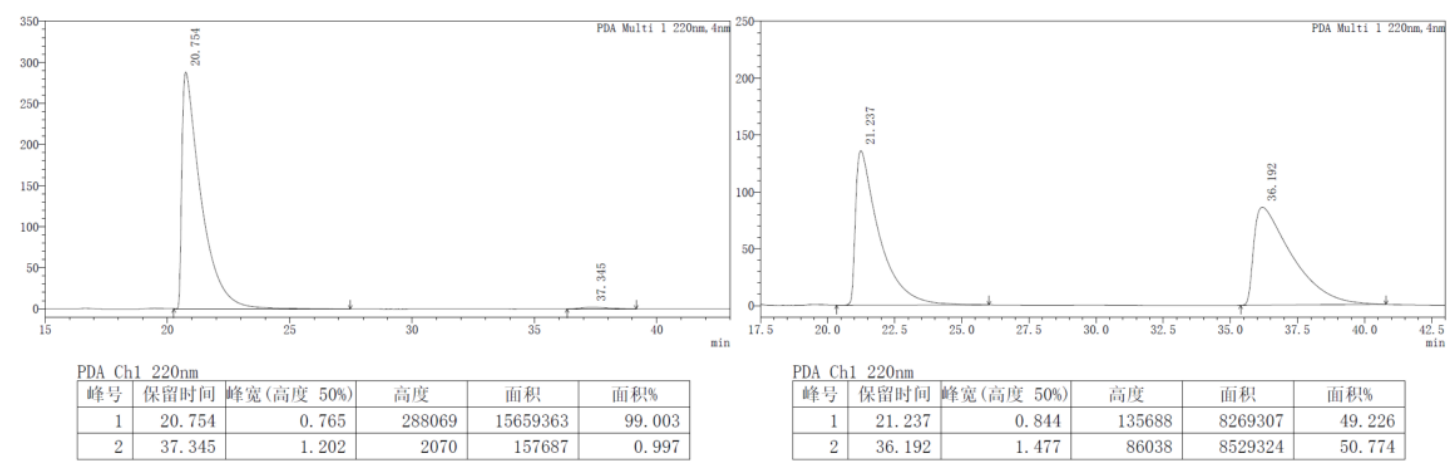<smiles>CCC(=O)[C@H](NC(=O)C(C)(C)C)c1cccc(OC)c1</smiles>

$\boldsymbol{\alpha}$-Aminoboronic ester 4m: Following the general procedure [D], enamide 1aj-Z (49.6 mg, 0.200 mmol) was converted to the $\alpha$-aminoboronic ester. Purification by silica gel chromatography gave $64.6 \mathrm{mg}$ (86\% yield) of $\mathbf{4 m}$ as a colorless oil. ${ }^{1} \mathbf{H} \mathbf{~ N M R}\left(400 \mathrm{MHz}, \mathrm{CDCl}_{3}\right) \delta 7.21(\mathrm{t}, J=8.1 \mathrm{~Hz}$, 1H), $6.99-6.83(\mathrm{~m}, 1 \mathrm{H}), 6.79-6.60(\mathrm{~m}, 3 \mathrm{H}), 3.87-3.74(\mathrm{~m}, 3 \mathrm{H}), 2.16(\mathrm{dq}, J=15.0,7.4 \mathrm{~Hz}$, 1H), $1.97(\mathrm{dq}, J=14.7,7.2 \mathrm{~Hz}, 1 \mathrm{H}), 1.36(\mathrm{~s}, 9 \mathrm{H}), 1.16(\mathrm{~s}, 6 \mathrm{H}), 1.09$ (s, 6H), 0.74 (t, $J=7.4 \mathrm{~Hz}$, 
3H) ${ }^{13} \mathrm{C}$ NMR $\left(101 \mathrm{MHz}, \mathrm{CDCl}_{3}\right) \delta 184.19,159.36,145.45,128.78,117.70,111.84,109.71$, 80.27, 55.09, 35.71, 28.68, 26.86, 25.24, 25.07, 8.46. ${ }^{11} \mathbf{B}$ NMR $\left(128 \mathrm{MHz}, \mathrm{CDCl}_{3}\right) \delta 13.82$. ESI-HR calcd for $\mathrm{C}_{21} \mathrm{H}_{35} \mathrm{BNO}_{4}{ }^{+}\left([\mathrm{M}+\mathrm{H}]^{+}\right) 376.2654$, found 374.2690. IR $v\left(\mathrm{~cm}^{-1}\right) 3215,2969$, $1565,750 \ldots[\alpha]^{20} \mathrm{D}=8.3\left(c=1.34, \mathrm{CHCl}_{3}\right)$ for a $97 \%$ ee sample. The enantiomeric purity of this compound was determined by HPLC analysis in comparison with authentic racemic material (Chiralpak IC, 99:1 hexanes:i-PrOH, $1.0 \mathrm{~mL} / \mathrm{min}, 210 \mathrm{~nm}$ ): $t_{\text {major }}=18.3, t_{\text {minor }}=17.4 \mathrm{~min}$.
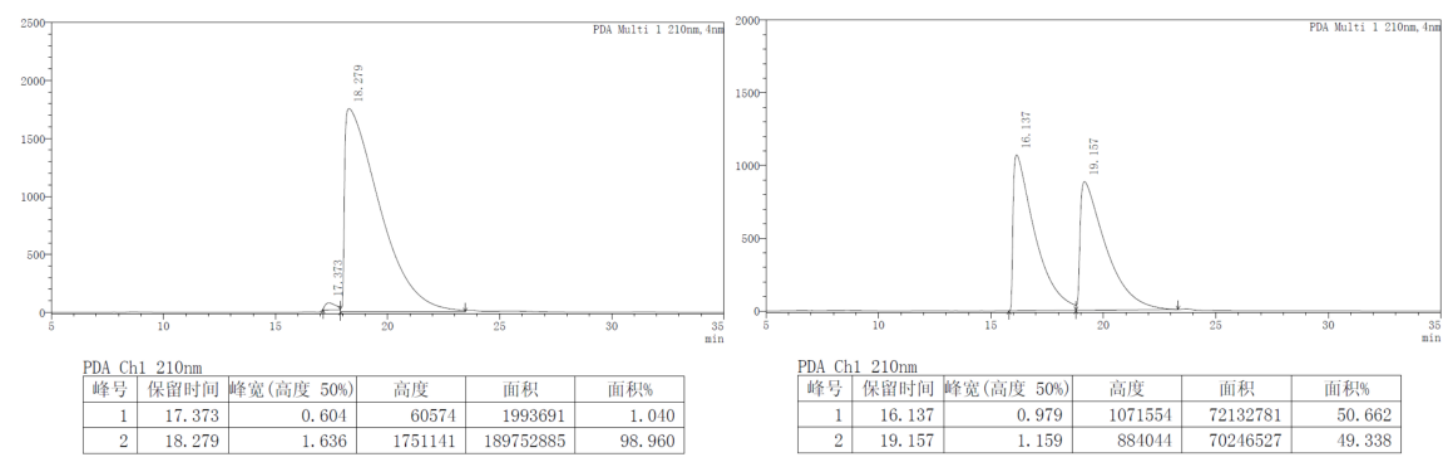<smiles>CCC(Br)(Br)c1ccccc1NC(=O)CC(C)(C)C</smiles>

$\boldsymbol{\alpha}$-Aminoboronic ester 4n: Following the general procedure [D], enamide 1ak-Z (43.6 mg, 0.200 mmol) was converted to the $\alpha$-aminoboronic ester. Purification by silica gel chromatography gave $62.3 \mathrm{mg}$ (90\% yield) of $\mathbf{4 n}$ as a white solid. ${ }^{1} \mathbf{H} \mathbf{~ N M R}\left(400 \mathrm{MHz}, \mathrm{CDCl}_{3}\right) \delta 7.29(\mathrm{dd}, J=10.3,5.1$ $\mathrm{Hz}, 2 \mathrm{H}), 7.14(\mathrm{t}, J=7.0 \mathrm{~Hz}, 3 \mathrm{H}), 6.85(\mathrm{~s}, 1 \mathrm{H}), 2.21(\mathrm{dq}, J=14.9,7.4 \mathrm{~Hz}, 1 \mathrm{H}), 2.07-1.93(\mathrm{~m}$, 1H), 1.37 (s, 9H), 1.09 (d, $J=35.0 \mathrm{~Hz}, 12 \mathrm{H}), 0.77$ (t, $J=7.4 \mathrm{~Hz}, 3 \mathrm{H}) \cdot{ }^{13} \mathbf{C}$ NMR (101 MHz, $\left.\mathrm{CDCl}_{3}\right) \delta 184.25,143.46,127.88,125.18,124.85,80.23,35.73,28.27,26.89,25.18,24.99,8.44$. ${ }^{11} \mathbf{B}$ NMR $\left(128 \mathrm{MHz}, \mathrm{CDCl}_{3}\right) \delta$ 14.79. ESI-HR calcd for $\mathrm{C}_{20} \mathrm{H}_{33} \mathrm{BNO}_{3}{ }^{+}\left([\mathrm{M}+\mathrm{H}]^{+}\right) 346.2548$, found 346.2566. IR $v\left(\mathrm{~cm}^{-1}\right) 3211,2968,1559,697$. M. P. $95-97{ }^{\circ} \mathrm{C} .[\alpha]^{20} \mathrm{D}=12.6(c=1.04$, $\mathrm{CHCl}_{3}$ ) for a $99 \%$ ee sample. The enantiomeric purity of this compound was determined by HPLC analysis in comparison with authentic racemic material (Chiralpak AD, 99.2:0.8 hexanes: $i$-PrOH, $1.0 \mathrm{~mL} / \mathrm{min}, 210 \mathrm{~nm}$ ): $t_{\text {major }}=8.3 \mathrm{~min}, t_{\text {minor }}=7.5 \mathrm{~min}$. 

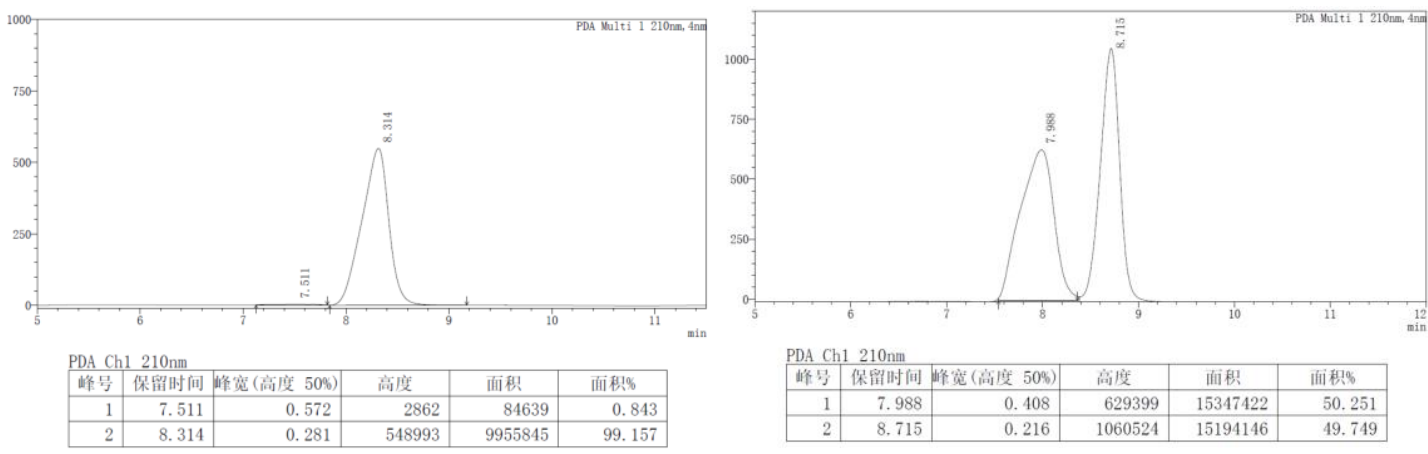<smiles>CCC(Br)(Br)c1ccccc1NC(=O)c1ccccc1</smiles>

$\boldsymbol{\alpha}$-Aminoboronic ester 4o: Following the general procedure [D], enamide 1al- $Z$ (47.6 mg, 0.200 mmol) was converted to the $\alpha$-aminoboronic ester. Purification by silica gel chromatography gave $65.9 \mathrm{mg}\left(90 \%\right.$ yield) of $\mathbf{4 o}$ as a white solid. ${ }^{1} \mathbf{H} \mathbf{~ N M R}\left(400 \mathrm{MHz}, \mathrm{CDCl}_{3}\right) \delta 7.95(\mathrm{~d}, J=8.0 \mathrm{~Hz}$, 2H), $7.67(\mathrm{t}, J=7.5 \mathrm{~Hz}, 1 \mathrm{H}), 7.55(\mathrm{t}, J=7.7 \mathrm{~Hz}, 2 \mathrm{H}), 7.31(\mathrm{dd}, J=14.9,7.5 \mathrm{~Hz}, 3 \mathrm{H}), 7.25(\mathrm{~d}, J=$ $8.1 \mathrm{~Hz}, 2 \mathrm{H}), 7.17(\mathrm{t}, J=7.2 \mathrm{~Hz}, 1 \mathrm{H}), 2.32(\mathrm{dq}, J=14.9,7.4 \mathrm{~Hz}, 1 \mathrm{H}), 2.16(\mathrm{dq}, J=14.7,7.3 \mathrm{~Hz}$, 1H), $1.20(\mathrm{~s}, 6 \mathrm{H}), 1.10(\mathrm{~s}, 6 \mathrm{H}), 0.86(\mathrm{t}, J=7.4 \mathrm{~Hz}, 3 \mathrm{H}) .{ }^{13} \mathbf{C}$ NMR $\left(101 \mathrm{MHz}, \mathrm{CDCl}_{3}\right) \delta 171.39$, $143.30,133.83,129.03,127.96,127.93,127.42,125.46,125.06,80.77,28.29,25.14,25.03,8.59$. ${ }^{11} \mathbf{B}$ NMR $\left(128 \mathrm{MHz}, \mathrm{CDCl}_{3}\right) \delta$ 17.21. ESI-HR calcd for $\mathrm{C}_{22} \mathrm{H}_{29} \mathrm{BNO}_{3}{ }^{+}\left([\mathrm{M}+\mathrm{H}]^{+}\right) 366.2235$, found 366.2243. IR $v\left(\mathrm{~cm}^{-1}\right) 3204,2968,1602$, 752. M. P. $158-160{ }^{\circ} \mathrm{C} .[\alpha]^{20} \mathrm{D}=21.8(c=1.1$, $\mathrm{CHCl}_{3}$ ) for a $97 \%$ ee sample. The enantiomeric purity of this compound was determined by HPLC analysis in comparison with authentic racemic material (Chiralpak IE, 97:3 hexanes:i-PrOH, 1.0 $\mathrm{mL} / \mathrm{min}, 210 \mathrm{~nm}): t_{\text {major }}=16.6 \mathrm{~min}, t_{\text {minor }}=14.9 \mathrm{~min}$.

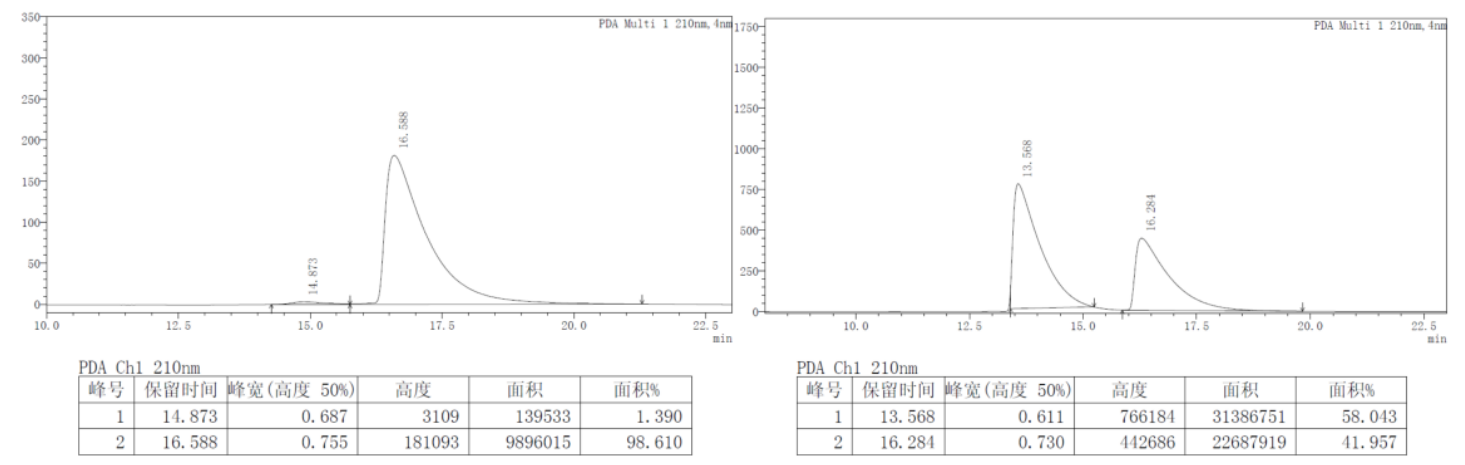


<smiles>CCCC(C)([Pb])NC(=O)c1ccc(Cl)cc1</smiles>

$\boldsymbol{\alpha}$-Aminoboronic ester 4p: Following the general procedure [D], enamide 1am- $Z$ (44.8 mg, 0.200 mmol) was converted to the $\alpha$-aminoboronic ester. Purification by silica gel chromatography gave $50.0 \mathrm{mg}\left(71 \%\right.$ yield) of $\mathbf{4 p}$ as a white solid. ${ }^{1} \mathbf{H} \mathbf{~ N M R}\left(400 \mathrm{MHz}, \mathrm{CDCl}_{3}\right) \delta 7.76(\mathrm{~d}, J=8.5 \mathrm{~Hz}$, 2H), $7.42(\mathrm{~d}, J=8.4 \mathrm{~Hz}, 2 \mathrm{H}), 6.95(\mathrm{~s}, 1 \mathrm{H}), 1.67-1.53(\mathrm{~m}, 2 \mathrm{H}), 1.43$ (dddd, $J=16.4,12.9,8.2$, $5.4 \mathrm{~Hz}, 2 \mathrm{H}), 1.25(\mathrm{~d}, J=7.6 \mathrm{~Hz}, 15 \mathrm{H}), 0.97-0.88(\mathrm{~m}, 3 \mathrm{H}) .{ }^{13} \mathbf{C} \mathbf{N M R}\left(101 \mathrm{MHz}, \mathrm{CDCl}_{3}\right) \delta$ 168.80, 139.47, 129.13, 129.06, 127.04, 81.04, 40.52, 25.15, 25.14, 22.06, 17.78, 14.76. ${ }^{11} \mathbf{B}$ NMR $\left(128 \mathrm{MHz}, \mathrm{CDCl}_{3}\right) \delta$ 17.54. ESI-HR calcd for $\mathrm{C}_{18} \mathrm{H}_{28} \mathrm{BClNO}_{3}{ }^{+}\left([\mathrm{M}+\mathrm{H}]^{+}\right)$352.1845, found 352.1852. IR $v\left(\mathrm{~cm}^{-1}\right) 3219,2964,1604,751$. M. P. $103-105{ }^{\circ} \mathrm{C} .[\alpha]^{20} \mathrm{D}=-14.8\left(c=1.13, \mathrm{CHCl}_{3}\right)$ for a $92 \%$ ee sample. The enantiomeric purity of this compound was determined by HPLC analysis in comparison with authentic racemic material (Chiralpak IC, 99:1 hexanes: $i-\mathrm{PrOH}, 1.0$ $\mathrm{mL} / \mathrm{min}, 254 \mathrm{~nm}): t_{\text {major }}=32.9 \mathrm{~min}, t_{\text {minor }}=20.1 \mathrm{~min}$.

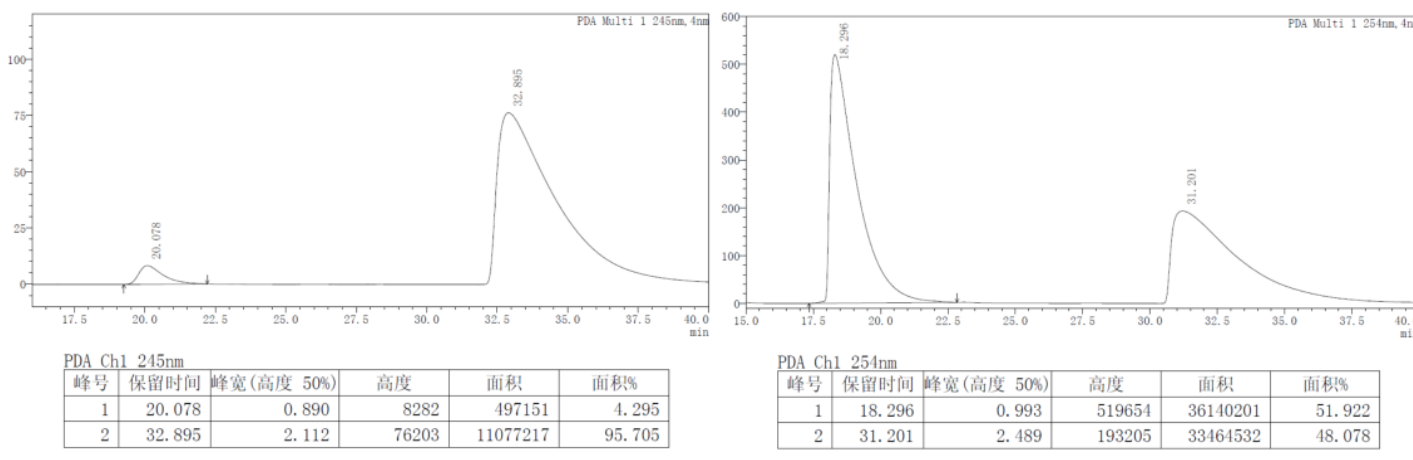<smiles>CCC(Br)(Br)c1ccccc1</smiles>

$\boldsymbol{\alpha}$-Aminoboronic ester 4q: Following the general procedure [D], enamide 1an- $\boldsymbol{Z}$ (54.4 mg, 0.200 mmol) was converted to the $\alpha$-aminoboronic ester. Purification by silica gel chromatography gave $60.8 \mathrm{mg}$ ( $76 \%$ yield) of $\mathbf{4 q}$ as a white solid. ${ }^{1} \mathbf{H} \mathbf{~ N M R}\left(400 \mathrm{MHz}, \mathrm{CDCl}_{3}\right) \delta$ $7.88(\mathrm{~d}, J=8.6 \mathrm{~Hz}, 2 \mathrm{H}), 7.52(\mathrm{~d}, J=8.6 \mathrm{~Hz}, 2 \mathrm{H}), 7.32(\mathrm{t}, J=7.6 \mathrm{~Hz}, 2 \mathrm{H}), 7.27-7.14(\mathrm{~m}, 4 \mathrm{H})$, $2.31(\mathrm{dq}, J=14.9,7.4 \mathrm{~Hz}, 1 \mathrm{H}), 2.17(\mathrm{dq}, J=14.7,7.3 \mathrm{~Hz}, 1 \mathrm{H}), 1.19(\mathrm{~s}, 6 \mathrm{H}), 1.11(\mathrm{~s}, 6 \mathrm{H}), 0.85$ (t, $J=7.4 \mathrm{~Hz}, 3 \mathrm{H}) .{ }^{13} \mathbf{C}$ NMR $(101 \mathrm{MHz}, \mathrm{CDCl} 3) \delta 169.96,142.92,140.11,129.38,129.19,128.04$, 
126.58, 125.44, 125.28, 81.20, 28.16, 25.05, 24.96, 8.55. ${ }^{11} \mathbf{B}$ NMR $\left(128 \mathrm{MHz}, \mathrm{CDCl}_{3}\right) \delta 17.32$. ESI-HR calcd for $\mathrm{C}_{22} \mathrm{H}_{28} \mathrm{BClNO}_{3}{ }^{+}\left([\mathrm{M}+\mathrm{H}]^{+}\right) 400.1845$, found 400.1854. IR $v\left(\mathrm{~cm}^{-1}\right) 3235,2970$, 1602, 699. M. P. $180-182{ }^{\circ} \mathrm{C} .[\alpha]^{20} \mathrm{D}=-17.5\left(c=1.13, \mathrm{CHCl}_{3}\right)$ for a $98 \%$ ee sample. The enantiomeric purity of this compound was determined by HPLC analysis in comparison with authentic racemic material (Chiralpak IE, 98:2 hexanes:i-PrOH, $1.0 \mathrm{~mL} / \mathrm{min}, 254 \mathrm{~nm}$ ): $t_{\text {major }}=$ $25.3 \mathrm{~min}, t_{\text {minor }}=21.9 \mathrm{~min}$. Single crystal suitable for X-ray analysis was obtained through slow diffusion of hexane into a solution of $4 \mathrm{q}$ in ethyl acetate (CCDC number 1956587)
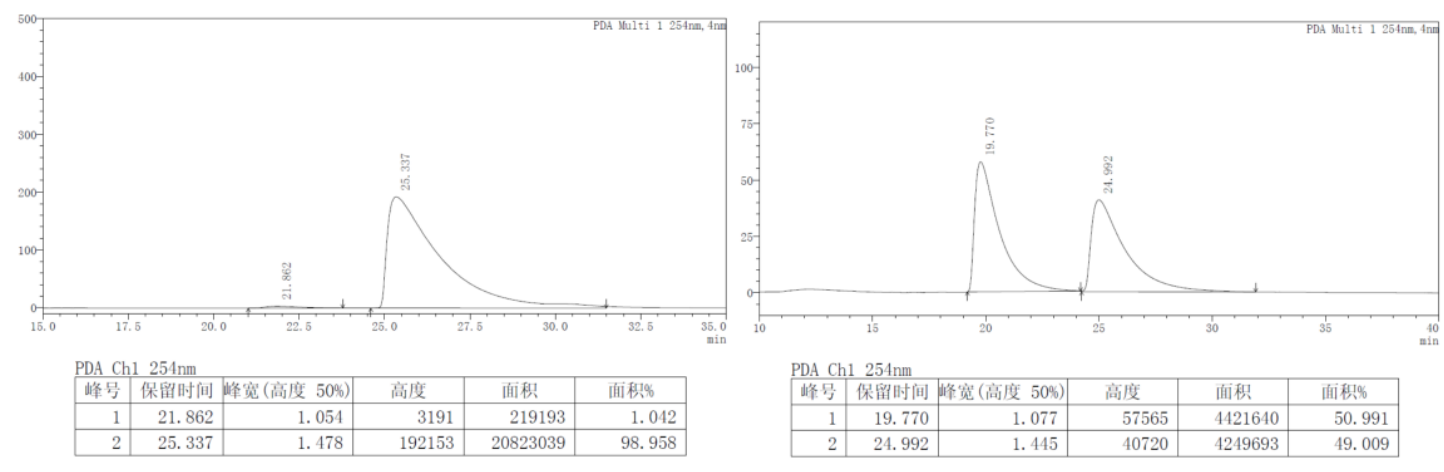<smiles>CC(Cc1ccccc1)C(Cc1ccccc1)NC(=O)C(C)(C)C</smiles>

$\boldsymbol{\alpha}$-Aminoboronic ester 4r: Following the general procedure [D], enamide 1q- $\boldsymbol{E}$ (46.4 mg, 0.200 mmol) was converted to the $\alpha$-aminoboronic ester. Purification by silica gel chromatography gave $57.6 \mathrm{mg}$ (80\% yield) of $\mathbf{4 r}$ as a white solid. ${ }^{1} \mathbf{H} \mathbf{~ N M R}\left(400 \mathrm{MHz}, \mathrm{CDCl}_{3}\right) \delta 7.35-7.17(\mathrm{~m}, 5 \mathrm{H})$, 6.09 (s, 1H), $2.92(\mathrm{dd}, J=13.1,3.8 \mathrm{~Hz}, 1 \mathrm{H}), 2.63(\mathrm{dd}, J=4.9,2.0 \mathrm{~Hz}, 1 \mathrm{H}), 2.47(\mathrm{dd}, J=13.1,8.4$ $\mathrm{Hz}, 1 \mathrm{H}), 2.29-1.99(\mathrm{~m}, 1 \mathrm{H}), 1.24(\mathrm{~d}, J=0.8 \mathrm{~Hz}, 12 \mathrm{H}), 1.14(\mathrm{~s}, 9 \mathrm{H}), 0.88(\mathrm{~d}, J=6.8 \mathrm{~Hz}, 3 \mathrm{H}) .13 \mathrm{C}$ NMR $(101 \mathrm{MHz}, \mathrm{CDCl} 3) \delta 183.43,141.09,129.48,128.28,125.98,80.35,42.18,35.69,35.41$, 26.76, 25.64, 25.22, 15.58. ${ }^{11} \mathbf{B}$ NMR $\left(128 \mathrm{MHz}, \mathrm{CDCl}_{3}\right) \delta$ 15.31. ESI-HR calcd for $\mathrm{C}_{21} \mathrm{H}_{35} \mathrm{BNO}_{3}{ }^{+}$ $\left([\mathrm{M}+\mathrm{H}]^{+}\right) 360.270592$, found $360.2709 . \mathbf{I R} v\left(\mathrm{~cm}^{-1}\right) 3227,2969,1578,1155$. M. P. $128-132{ }^{\circ} \mathrm{C}$. $[\alpha]^{20} \mathrm{D}=-117.1\left(c=1.06, \mathrm{CHCl}_{3}\right)$ for a $95 \%$ ee sample. The enantiomeric purity of this compound was determined by HPLC analysis in comparison with authentic racemic material (Chiralpak IA, 98:2 hexane: $i-\mathrm{PrOH}, 1.0 \mathrm{~mL} / \mathrm{min}, 220 \mathrm{~nm}$ ): $t_{\text {major }}=7.0 \mathrm{~min}, t_{\mathrm{minor}}=5.8 \mathrm{~min}$. 

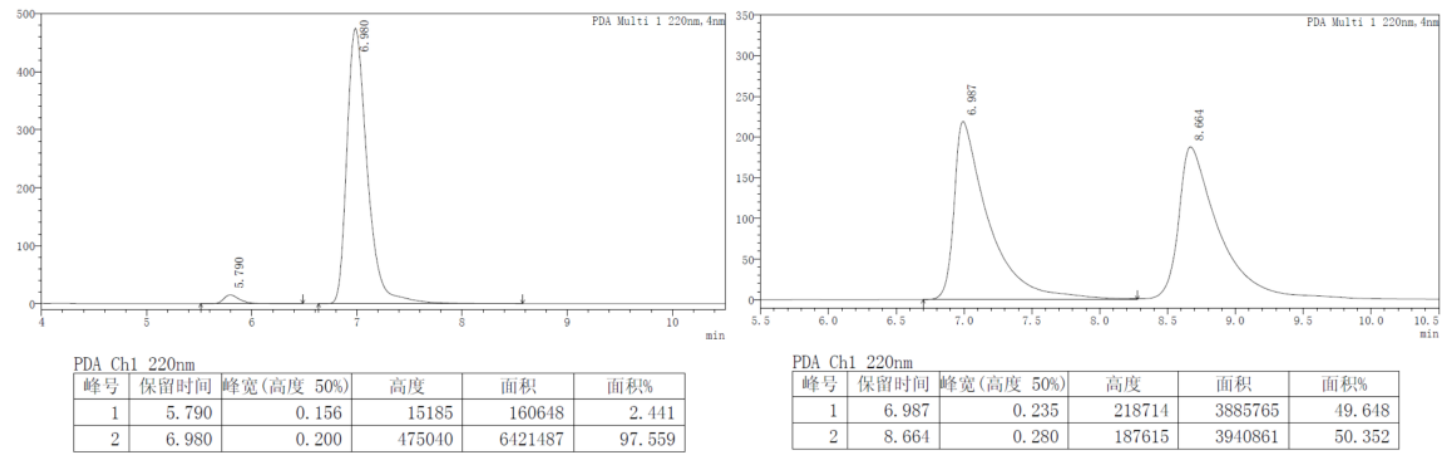<smiles>CC(Cc1ccccc1)C(Cc1ccc(Cl)cc1)NC(=O)CC(C)(C)C</smiles>

$\boldsymbol{\alpha}$-Aminoboronic ester 4s: Following the general procedure [D], enamide 1ao- $Z$ (53.2 mg, 0.200 mmol) was converted to the $\alpha$-aminoboronic ester. Purification by silica gel chromatography gave $57.5 \mathrm{mg}$ (53\% yield) of $4 \mathbf{s}$ as a white solid. ${ }^{1} \mathbf{H} \mathbf{~ N M R}\left(400 \mathrm{MHz}, \mathrm{CDCl}_{3}\right) \delta 7.31-7.21(\mathrm{~m}, 2 \mathrm{H})$, $7.16(\mathrm{~d}, J=8.3 \mathrm{~Hz}, 2 \mathrm{H}), 6.40(\mathrm{~s}, 1 \mathrm{H}), 2.85(\mathrm{dd}, J=13.3,5.3 \mathrm{~Hz}, 1 \mathrm{H}), 2.71-2.59(\mathrm{~m}, 1 \mathrm{H}), 2.32$ $(\mathrm{dd}, J=13.2,8.9 \mathrm{~Hz}, 1 \mathrm{H}), 2.03(\mathrm{tq}, J=12.9,6.6 \mathrm{~Hz}, 1 \mathrm{H}), 1.25(\mathrm{~s}, 12 \mathrm{H}), 1.18(\mathrm{~s}, 9 \mathrm{H}), 0.95(\mathrm{~d}, J=$ $6.8 \mathrm{~Hz}, 3 \mathrm{H}) .{ }^{13} \mathrm{C}$ NMR $\left(101 \mathrm{MHz}, \mathrm{CDCl}_{3}\right) \delta 183.05,140.31,131.57,130.41,128.40,80.73,40.52$, 36.86, 35.96, 26.82, 25.60, 25.40, 17.80. ${ }^{11} \mathbf{B}$ NMR $\left(128 \mathrm{MHz}, \mathrm{CDCl}_{3}\right) \delta$ 17.78. ESI-HR calcd for $\mathrm{C}_{21} \mathrm{H}_{34} \mathrm{BClNO}_{3}{ }^{+}\left([\mathrm{M}+\mathrm{H}]^{+}\right) 394.2315$, found 394.2321. IR $v\left(\mathrm{~cm}^{-1}\right) 3219,2969,1577$, 754. M. P. $194-196{ }^{\circ} \mathrm{C} .[\alpha]^{20}{ }_{\mathrm{D}}=-63.5\left(c=1.03, \mathrm{CHCl}_{3}\right)$ for a $96 \%$ ee sample. The enantiomeric purity of this compound was determined by HPLC analysis in comparison with authentic racemic material (Chiralpak IE, 95:5 hexanes:i-PrOH, $1.0 \mathrm{~mL} / \mathrm{min}, 254 \mathrm{~nm}$ ): $t_{\text {major }}=4.4 \mathrm{~min}, t_{\text {minor }}=4.8 \mathrm{~min}$. Single crystal suitable for X-ray analysis was obtained through slow diffusion of hexane into a solution of $\mathbf{4 q}$ in ethyl acetate (CCDC number 1956588)

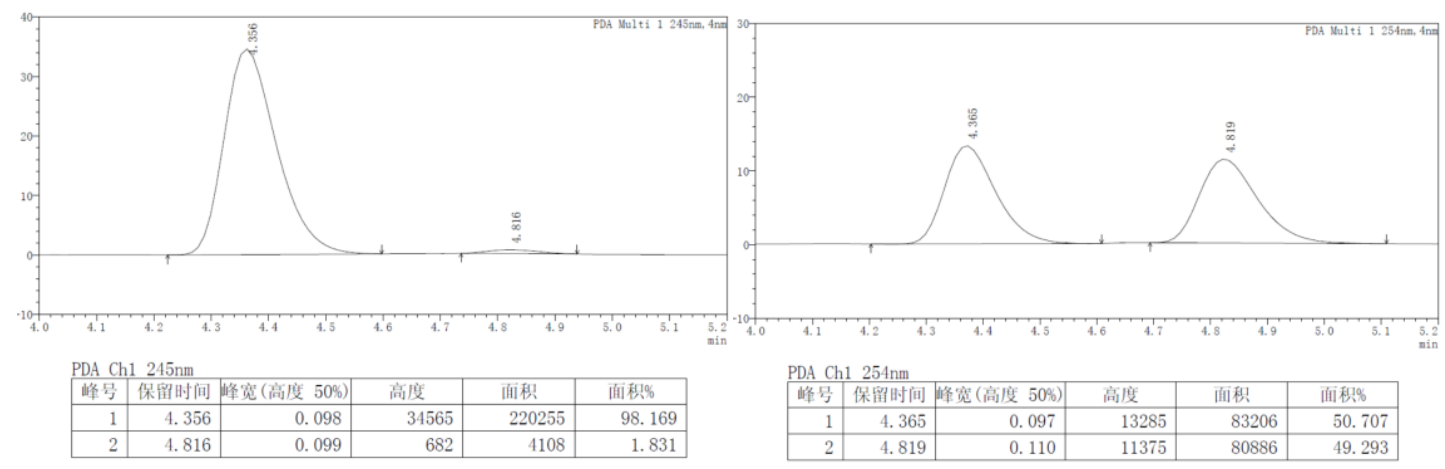


$\overbrace{\mathrm{Be}}^{\mathrm{Bpin}}$

$\boldsymbol{\alpha}$-Aminoboronic ester 4t: Following the general procedure [D], enamide 1r- $\boldsymbol{E}$ (49.2 $\mathrm{mg}, 0.200$ mmol) was converted to the $\alpha$-aminoboronic ester. Purification by silica gel chromatography gave $65.6 \mathrm{mg}$ (88\% yield) of $\mathbf{4 t}$ as a white solid. ${ }^{1} \mathbf{H}$ NMR $\left(400 \mathrm{MHz}, \mathrm{CDCl}_{3}\right) \delta 7.28(\mathrm{t}, J=7.4 \mathrm{~Hz}, 3 \mathrm{H})$, $7.25-7.15$ (m, 3H), 6.34 (s, 1H), 2.67 (qdd, $J=13.6,10.1,5.8 \mathrm{~Hz}, 3 \mathrm{H}), 1.78$ (dddd, $J=19.0$, 14.7, 7.7, $2.9 \mathrm{~Hz}, 2 \mathrm{H}), 1.60-1.44(\mathrm{~m}, 1 \mathrm{H}), 1.24(\mathrm{~s}, 9 \mathrm{H}), 1.22(\mathrm{~d}, J=3.3 \mathrm{~Hz}, 12 \mathrm{H}), 0.98(\mathrm{~d}, J=$ $6.7 \mathrm{~Hz}, 3 \mathrm{H}) .{ }^{13} \mathbf{C}$ NMR $\left(101 \mathrm{MHz}, \mathrm{CDCl}_{3}\right) \delta 183.11,142.81,128.37,128.29,125.61,80.67,37.15$, $36.11,33.75,33.74,26.99,25.50,25.16,24.87,16.33 .{ }^{11} \mathbf{B}$ NMR $\left(128 \mathrm{MHz}, \mathrm{CDCl}_{3}\right) \delta 14.10 . \mathbf{I R} v$ $\left(\mathrm{cm}^{-1}\right) 3253,2970,1578,749$. M. P. $124-126^{\circ} \mathrm{C} .[\alpha]^{20} \mathrm{D}=-46.5\left(c=1.54, \mathrm{CHCl}_{3}\right)$ for a $92 \%$ ee sample. The enantiomeric purity of this compound was determined by HPLC analysis in comparison with authentic racemic material (Chiralpak IE, 98:2 hexanes:i-PrOH, $1.0 \mathrm{~mL} / \mathrm{min}, 254$ $\mathrm{nm}): t_{\text {major }}=9.1 \mathrm{~min}, t_{\text {minor }}=11.2 \mathrm{~min}$.
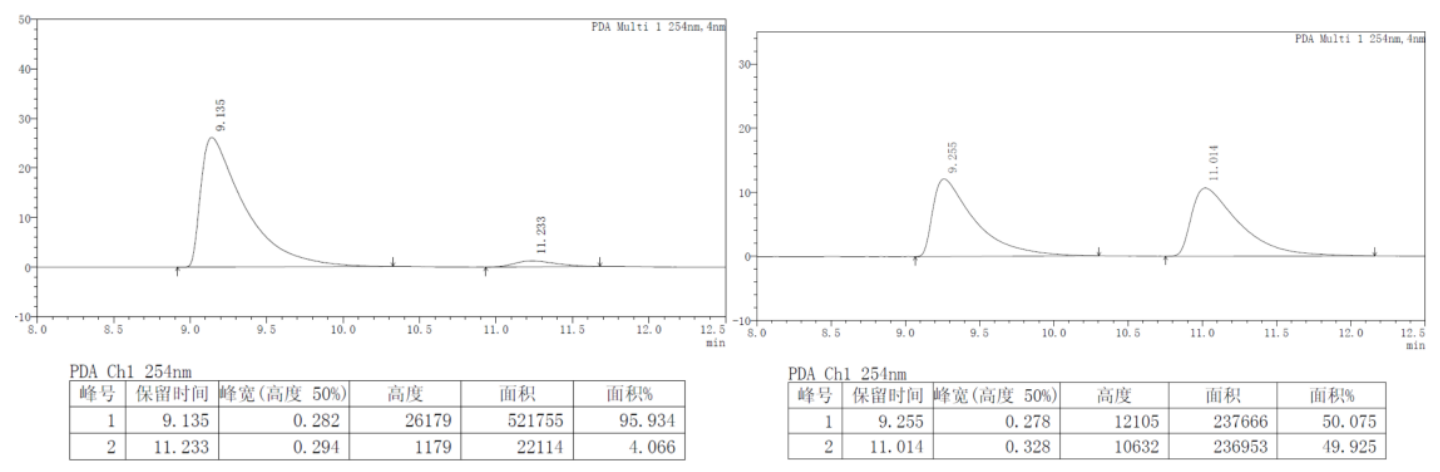<smiles>O=C(NC(Cc1ccccc1)C(Br)CBr)c1ccccc1</smiles>

$\boldsymbol{\alpha}$-Aminoboronic ester 4u: Following the general procedure [D], enamide 1ap- $\boldsymbol{E}$ (58.8 mg, 0.200 mmol) was converted to the $\alpha$-aminoboronic ester. Purification by silica gel chromatography gave $60.1 \mathrm{mg}\left(68 \%\right.$ yield) of $\mathbf{4 u}$ as a white solid. ${ }^{1} \mathbf{H}$ NMR $\left(400 \mathrm{MHz}, \mathrm{CDCl}_{3}\right) \delta 7.79-7.73(\mathrm{~m}, 2 \mathrm{H})$, $7.53(\mathrm{t}, J=7.4 \mathrm{~Hz}, 1 \mathrm{H}), 7.42(\mathrm{t}, J=7.6 \mathrm{~Hz}, 2 \mathrm{H}), 7.32-7.24(\mathrm{~m}, 3 \mathrm{H}), 7.18(\mathrm{dd}, J=15.9,7.3 \mathrm{~Hz}$, 3H), $6.94(\mathrm{~s}, 1 \mathrm{H}), 3.41(\mathrm{dd}, J=5.8,3.0 \mathrm{~Hz}, 1 \mathrm{H}), 2.80-2.65(\mathrm{~m}, 2 \mathrm{H}), 1.82(\mathrm{qd}, J=11.2,5.0 \mathrm{~Hz}$, 1H), $1.78-1.65(\mathrm{~m}, 2 \mathrm{H}), 1.54(\mathrm{ddd}, J=14.6,12.2,6.3 \mathrm{~Hz}, 1 \mathrm{H}), 1.50-1.38(\mathrm{~m}, 2 \mathrm{H}), 1.36-1.25$ 
(m, 13H), $0.94(\mathrm{t}, J=7.1 \mathrm{~Hz}, 3 \mathrm{H}) .{ }^{13} \mathbf{C}$ NMR $\left(101 \mathrm{MHz}, \mathrm{CDCl}_{3}\right) \delta 169.57,142.91,132.21,131.11$, $128.54,128.47,128.27,127.48,125.58,82.23,39.20,33.88,33.85,33.78,25.27,25.14,20.54$, 14.37. ${ }^{11} \mathbf{B}$ NMR $\left(128 \mathrm{MHz}, \mathrm{CDCl}_{3}\right) \delta$ 20.17. ESI-HR calcd for $\mathrm{C}_{23} \mathrm{H}_{31} \mathrm{BNO}_{3}{ }^{+}\left([\mathrm{M}+\mathrm{H}]^{+}\right) 422.2861$, found 422.2895. IR $v\left(\mathrm{~cm}^{-1}\right) 3195,2967,1641,750$. M. P. $138-140{ }^{\circ} \mathrm{C} .[\alpha]^{20} \mathrm{D}=-13.4(c=0.89$, $\mathrm{CHCl}_{3}$ ) for a $98 \%$ ee sample. The enantiomeric purity of this compound was determined by HPLC analysis in comparison with authentic racemic material (Chiralpak IE, 95:5 hexanes: $i-\mathrm{PrOH}, 1.0$ $\mathrm{mL} / \mathrm{min}, 254 \mathrm{~nm}): t_{\text {major }}=6.1 \mathrm{~min}, t_{\text {minor }}=6.9 \mathrm{~min}$.

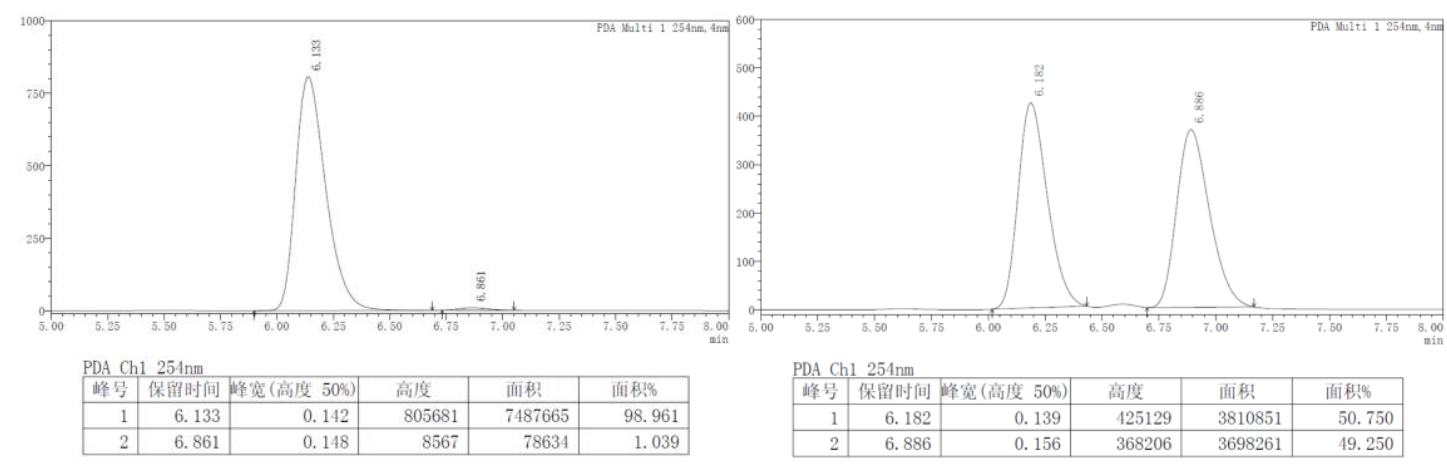<smiles>CC(CCCCl)C(Cc1ccccc1)NC(=O)c1ccccc1</smiles>

$\boldsymbol{\alpha}$-Aminoboronic ester 4v: Following the general procedure [D], enamide 1aq- $\boldsymbol{E}$ (47.6 mg, 0.200 mmol) was converted to the $\alpha$-aminoboronic ester. Purification by silica gel chromatography gave $64.4 \mathrm{mg}$ (88\% yield) of $\mathbf{4 v}$ as a white solid. ${ }^{1} \mathbf{H} \mathbf{~ N M R}\left(400 \mathrm{MHz}, \mathrm{CDCl}_{3}\right) \delta 7.84-7.73(\mathrm{~m}, 2 \mathrm{H})$, $7.54(\mathrm{t}, J=7.4 \mathrm{~Hz}, 1 \mathrm{H}), 7.45(\mathrm{q}, J=7.7 \mathrm{~Hz}, 2 \mathrm{H}), 7.12(\mathrm{~s}, 1 \mathrm{H}), 3.62-3.48(\mathrm{~m}, 2 \mathrm{H}), 3.13(\mathrm{dd}, J=$ 5.9, $3.2 \mathrm{~Hz}, 1 \mathrm{H}), 1.98-1.82(\mathrm{~m}, 3 \mathrm{H}), 1.66(\mathrm{dtd}, J=15.5,10.5,5.3 \mathrm{~Hz}, 1 \mathrm{H}), 1.47-1.20(\mathrm{~m}, 14 \mathrm{H})$, $1.02(\mathrm{~d}, J=6.8 \mathrm{~Hz}, 3 \mathrm{H}) .{ }^{13} \mathbf{C} \mathbf{~ N M R}\left(101 \mathrm{MHz}, \mathrm{CDCl}_{3}\right) \delta 169.94,132.44,130.66,128.62,127.52$, 82.15, 45.39, 34.38, 32.11, 30.69, 25.30, 25.15, 16.81. ${ }^{11} \mathbf{B}$ NMR (128 MHz, $\left.\mathrm{CDCl}_{3}\right) \delta 22.49$. ESI-HR calcd for $\mathrm{C}_{19} \mathrm{H}_{30} \mathrm{BClNO}_{3}{ }^{+}\left([\mathrm{M}+\mathrm{H}]^{+}\right) 366.2002$, found 366.2032. IR $v\left(\mathrm{~cm}^{-1}\right) 3206,2971$, 1610, 709. M. P. $83-85{ }^{\circ} \mathrm{C} .[\alpha]^{20} \mathrm{D}=-49.5\left(c=1.26, \mathrm{CHCl}_{3}\right)$ for a $97 \%$ ee sample. The enantiomeric purity of this compound was determined by HPLC analysis in comparison with authentic racemic material (Chiralpak IE, 97:3 hexanes:i-PrOH, $1.0 \mathrm{~mL} / \mathrm{min}, 220 \mathrm{~nm}$ ): $t_{\text {major }}=$ $12.1 \mathrm{~min}, t_{\mathrm{minor}}=14.5 \mathrm{~min}$. 

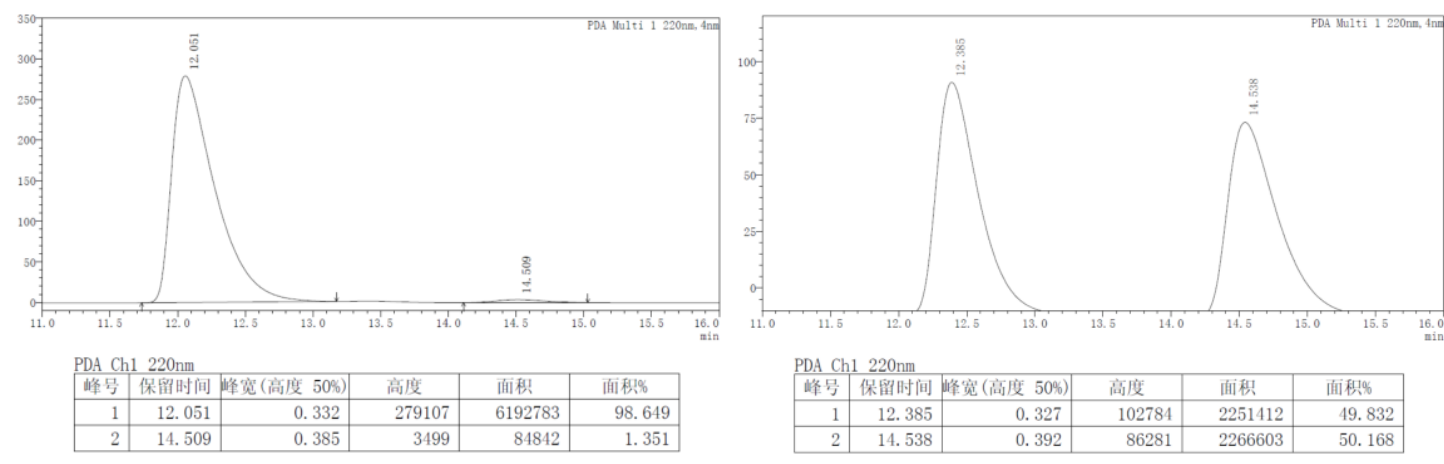<smiles>CCCCC(CNC(=O)c1ccccc1)Cc1ccccc1</smiles>

$\boldsymbol{\alpha}$-Aminoboronic ester 4w: Following the general procedure [D], enamide 1ar- $\boldsymbol{E}$ (43.6 mg, 0.200 mmol) was converted to the $\alpha$-aminoboronic ester. Purification by silica gel chromatography gave $62.3 \mathrm{mg}\left(90 \%\right.$ yield) of $\mathbf{4 w}$ as a white solid. ${ }^{1} \mathbf{H}$ NMR $\left(400 \mathrm{MHz}, \mathrm{CDCl}_{3}\right) \delta 7.77(\mathrm{~d}, J=7.3 \mathrm{~Hz}$, 2H), $7.51(\mathrm{t}, J=7.4 \mathrm{~Hz}, 1 \mathrm{H}), 7.40(\mathrm{t}, J=7.6 \mathrm{~Hz}, 2 \mathrm{H}), 7.11(\mathrm{~s}, 1 \mathrm{H}), 3.09(\mathrm{t}, J=4.4 \mathrm{~Hz}, 1 \mathrm{H}), 1.94-$ $1.80(\mathrm{~m}, 2 \mathrm{H}), 1.56-1.45(\mathrm{~m}, 1 \mathrm{H}), 1.43-1.14(\mathrm{~m}, 16 \mathrm{H}), 0.97(\mathrm{~d}, J=6.8 \mathrm{~Hz}, 3 \mathrm{H}), 0.89(\mathrm{t}, J=6.9$ $\mathrm{Hz}, 3 \mathrm{H}) .{ }^{13} \mathrm{C}$ NMR $\left(101 \mathrm{MHz}, \mathrm{CDCl}_{3}\right) \delta 169.82,132.31,130.74,128.54,127.53,81.96,34.79$, 34.61, 29.75, 25.28, 25.11, 22.88, 16.72, 14.14. ${ }^{11} \mathbf{B}$ NMR $\left(128 \mathrm{MHz}, \mathrm{CDCl}_{3}\right) \delta 22.53$. ESI-HR calcd for $\mathrm{C}_{20} \mathrm{H}_{33} \mathrm{BNO}_{3}{ }^{+}\left([\mathrm{M}+\mathrm{H}]^{+}\right)$346.2548, found 346.2561. IR $v\left(\mathrm{~cm}^{-1}\right) 3204,2960,1603,745$. M. P. $135-137{ }^{\circ} \mathrm{C} .[\alpha]^{20} \mathrm{D}=-55.5\left(c=1.12, \mathrm{CHCl}_{3}\right)$ for a $99 \%$ ee sample. The enantiomeric purity of this compound was determined by HPLC analysis in comparison with authentic racemic material (Chiralpak IE, 97:3 hexanes: $i-\mathrm{PrOH}, 1.0 \mathrm{~mL} / \mathrm{min}, 220 \mathrm{~nm}$ ): $t_{\text {major }}=9.4 \mathrm{~min}, t_{\text {minor }}=11.0$ $\min$.

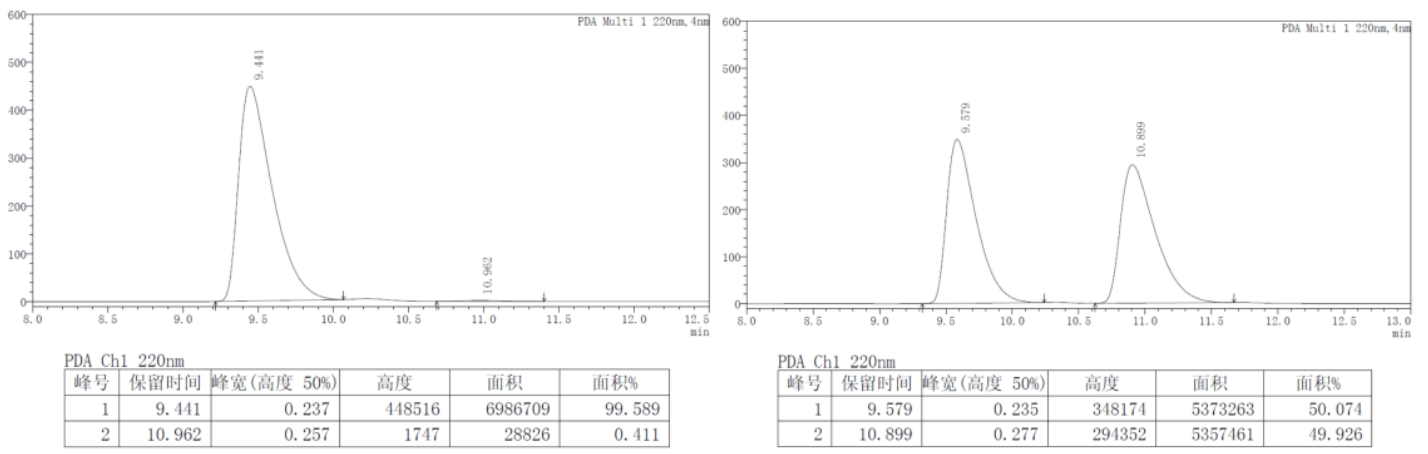


<smiles>CC(C(=O)NC(=O)c1ccccc1)C1CCCCC1</smiles>

$\boldsymbol{\alpha}$-Aminoboronic ester 4x: Following the general procedure [D], enamide 1as-Z (48.8 mg, 0.200 mmol) was converted to the $\alpha$-aminoboronic ester. Purification by silica gel chromatography gave $66.3 \mathrm{mg}$ (89\% yield) of $\mathbf{2 d}$ as a white solid. ${ }^{1} \mathbf{H} \mathbf{~ N M R}\left(400 \mathrm{MHz}, \mathrm{CDCl}_{3}\right) \delta 7.83-7.76(\mathrm{~m}, 2 \mathrm{H})$, $7.54(\mathrm{t}, J=7.4 \mathrm{~Hz}, 1 \mathrm{H}), 7.45(\mathrm{t}, J=7.5 \mathrm{~Hz}, 2 \mathrm{H}), 6.81(\mathrm{~s}, 1 \mathrm{H}), 3.25(\mathrm{dd}, J=7.7,4.3 \mathrm{~Hz}, 1 \mathrm{H}), 1.90$ $-1.68(\mathrm{~m}, 6 \mathrm{H}), 1.51-1.36(\mathrm{~m}, 1 \mathrm{H}), 1.30(\mathrm{~d}, J=2.1 \mathrm{~Hz}, 12 \mathrm{H}), 1.28-1.12(\mathrm{~m}, 4 \mathrm{H}), 1.08-0.91$ (m, 4H). ${ }^{13} \mathrm{C}$ NMR $\left(101 \mathrm{MHz}, \mathrm{CDCl}_{3}\right) \delta 169.04,132.11,131.56,128.59,127.34,82.29,40.10$, 39.81, 31.65, 28.26, 26.75, 26.70, 25.42, 25.30, 13.62. ${ }^{11} \mathbf{B}$ NMR $\left(128 \mathrm{MHz}, \mathrm{CDCl}_{3}\right) \delta 22.68$. ESI-HR calcd for $\mathrm{C}_{22} \mathrm{H}_{35} \mathrm{BNO}_{3}{ }^{+}\left([\mathrm{M}+\mathrm{H}]^{+}\right) 372.2705$, found 372.2711. IR $v\left(\mathrm{~cm}^{-1}\right) 3196,2920$, 1611, 744. M. P. $106-108{ }^{\circ} \mathrm{C} .[\alpha]^{20}{ }_{\mathrm{D}}=-26.2\left(c=1.02, \mathrm{CHCl}_{3}\right)$ for a $99 \%$ ee sample. The enantiomeric purity of this compound was determined by HPLC analysis in comparison with authentic racemic material (Chiralpak IE, 97:3 hexanes:i-PrOH, $1.0 \mathrm{~mL} / \mathrm{min}, 220 \mathrm{~nm}$ ): $t_{\text {major }}=$ $12.5 \mathrm{~min}, t_{\mathrm{minor}}=16.4 \mathrm{~min}$.
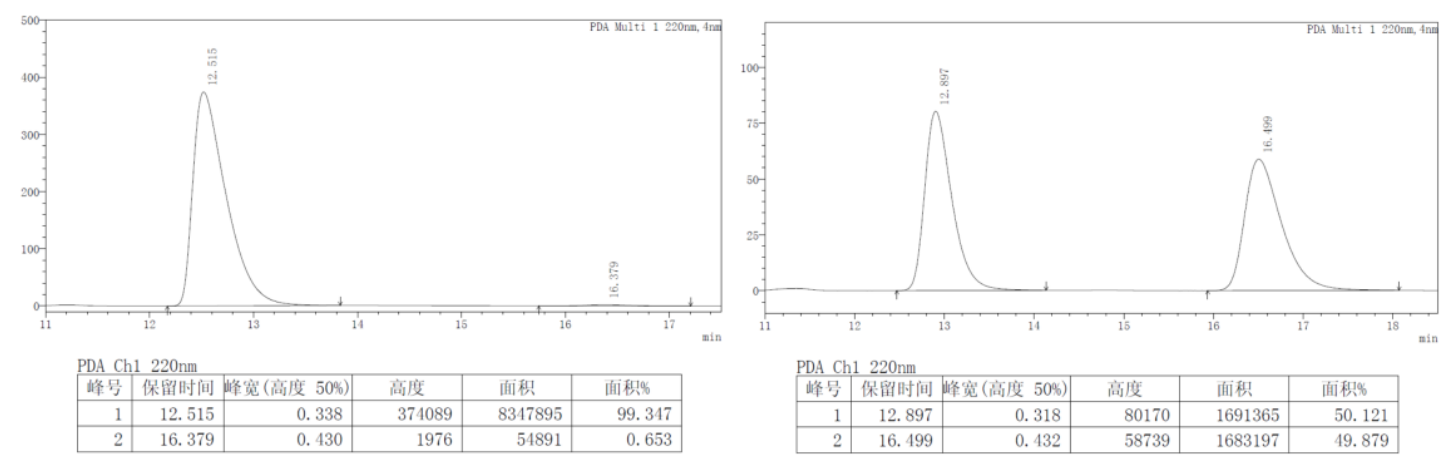<smiles>O=C(NC(Cc1ccccc1)C1CCOCC1)c1ccccc1</smiles>

$\boldsymbol{\alpha}$-Aminoboronic ester 4y: Following the general procedure [D], enamide 1at (43.6 mg, 0.200 mmol) was converted to the $\alpha$-aminoboronic ester. Purification by silica gel chromatography gave $62.3 \mathrm{mg}$ (90\% yield) of $\mathbf{2 d}$ as a white solid. ${ }^{1} \mathbf{H} \mathbf{~ N M R}\left(400 \mathrm{MHz}, \mathrm{CDCl}_{3}\right) \delta 7.82(\mathrm{~d}, J=7.6 \mathrm{~Hz}$, 2H), $7.56(\mathrm{t}, J=7.4 \mathrm{~Hz}, 1 \mathrm{H}), 7.44(\mathrm{t}, J=7.6 \mathrm{~Hz}, 3 \mathrm{H}), 3.98(\mathrm{dd}, J=11.1,3.5 \mathrm{~Hz}, 2 \mathrm{H}), 3.41(\mathrm{dd}, J$ $=19.1,10.2 \mathrm{~Hz}, 2 \mathrm{H}), 2.87(\mathrm{~d}, J=3.4 \mathrm{~Hz}, 1 \mathrm{H}), 2.03-1.89(\mathrm{~m}, 1 \mathrm{H}), 1.83-1.69(\mathrm{~m}, 2 \mathrm{H}), 1.49(\mathrm{ddd}$, 
$J=14.8,12.2,4.1 \mathrm{~Hz}, 2 \mathrm{H}), 1.40(\mathrm{t}, J=5.8 \mathrm{~Hz}, 1 \mathrm{H}), 1.27(\mathrm{~d}, J=14.7 \mathrm{~Hz}, 12 \mathrm{H}) .{ }^{13} \mathbf{C}$ NMR $(101$ $\left.\mathrm{MHz}, \mathrm{CDCl}_{3}\right) \delta 170.38,132.83,129.56,128.66,127.71,81.77,68.07,68.04,36.84,31.16,30.00$, 25.37, 25.23. ${ }^{11} \mathbf{B}$ NMR $\left(128 \mathrm{MHz}, \mathrm{CDCl}_{3}\right) \delta$ 17.93. ESI-HR calcd for $\mathrm{C}_{19} \mathrm{H}_{29} \mathrm{BNO}_{4}{ }^{+}\left([\mathrm{M}+\mathrm{H}]^{+}\right)$ 346.2184, found 346.2209. IR $v\left(\mathrm{~cm}^{-1}\right) 3206,2969,1610,745$. M. P. $164-166^{\circ} \mathrm{C} .[\alpha]^{20} \mathrm{D}=-19.9(c$ $=1.26, \mathrm{CHCl}_{3}$ ) for a $99 \%$ ee sample. The enantiomeric purity of this compound was determined by HPLC analysis in comparison with authentic racemic material (Chiralpak IE, 95:5 hexanes: $i-\operatorname{PrOH}, 1.0 \mathrm{~mL} / \mathrm{min}, 230 \mathrm{~nm}): t_{\text {major }}=35.9 \mathrm{~min}$.

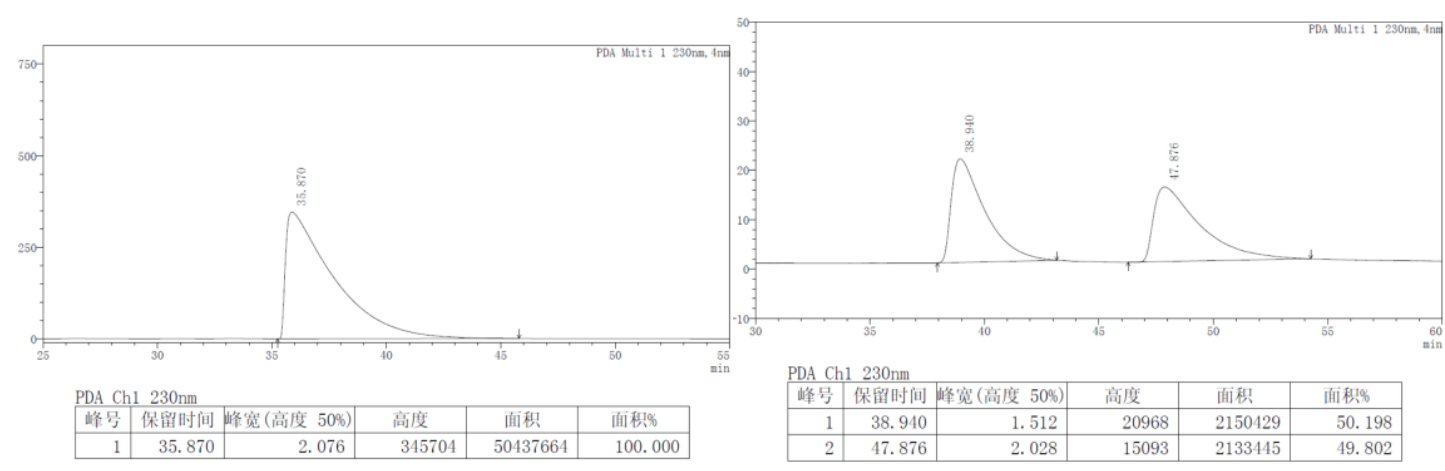<smiles>O=C(NC(Cc1ccccc1)C1CCCCC1)c1ccccc1</smiles>

$\boldsymbol{\alpha}$-Aminoboronic ester 4z: Following the general procedure [D], enamide 1au (43.2 mg, 0.200 mmol) was converted to the $\alpha$-aminoboronic ester. Purification by silica gel chromatography gave $62.0 \mathrm{mg}$ (90\% yield) of $\mathbf{2 d}$ as a white solid. ${ }^{1} \mathbf{H} \mathbf{~ N M R}\left(400 \mathrm{MHz}, \mathrm{CDCl}_{3}\right) \delta 7.83-7.76(\mathrm{~m}, 2 \mathrm{H})$, $7.52(\mathrm{t}, J=7.4 \mathrm{~Hz}, 1 \mathrm{H}), 7.43(\mathrm{t}, J=7.5 \mathrm{~Hz}, 2 \mathrm{H}), 6.83(\mathrm{~s}, 1 \mathrm{H}), 3.07-2.99(\mathrm{~m}, 1 \mathrm{H}), 1.94-1.79(\mathrm{~m}$, 2H), $1.77-1.70(\mathrm{~m}, 2 \mathrm{H}), 1.67(\mathrm{t}, J=7.4 \mathrm{~Hz}, 1 \mathrm{H}), 1.28(\mathrm{~d}, J=1.9 \mathrm{~Hz}, 12 \mathrm{H}), 1.26-1.22(\mathrm{~m}, 2 \mathrm{H})$, $1.22-1.00(\mathrm{~m}, 4 \mathrm{H}) .{ }^{13} \mathbf{C}$ NMR $\left(101 \mathrm{MHz}, \mathrm{CDCl}_{3}\right) \delta 169.37,132.21,131.31,128.59,127.40$, 82.23, 39.68, 31.26, 30.33, 26.49, 26.31, 25.24. ${ }^{11} \mathbf{B}$ NMR $\left(128 \mathrm{MHz}, \mathrm{CDCl}_{3}\right) \delta 22.72$. ESI-HR calcd for $\mathrm{C}_{20} \mathrm{H}_{31} \mathrm{BNO}_{3}{ }^{+}\left([\mathrm{M}+\mathrm{H}]^{+}\right)$344.2392, found 344.2410. IR $v\left(\mathrm{~cm}^{-1}\right) 3204,2923,1611,749$. M. P. $172-174^{\circ} \mathrm{C} .[\alpha]^{20} \mathrm{D}=-16.1\left(c=1.18, \mathrm{CHCl}_{3}\right)$ for a $99.9 \%$ ee sample. The enantiomeric purity of this compound was determined by HPLC analysis in comparison with authentic racemic material (Chiralpak IE, 95:5 hexanes: $i-\mathrm{PrOH}, 1.0 \mathrm{~mL} / \mathrm{min}, 220 \mathrm{~nm}$ ): $t_{\text {major }}=7.7 \mathrm{~min}$. 

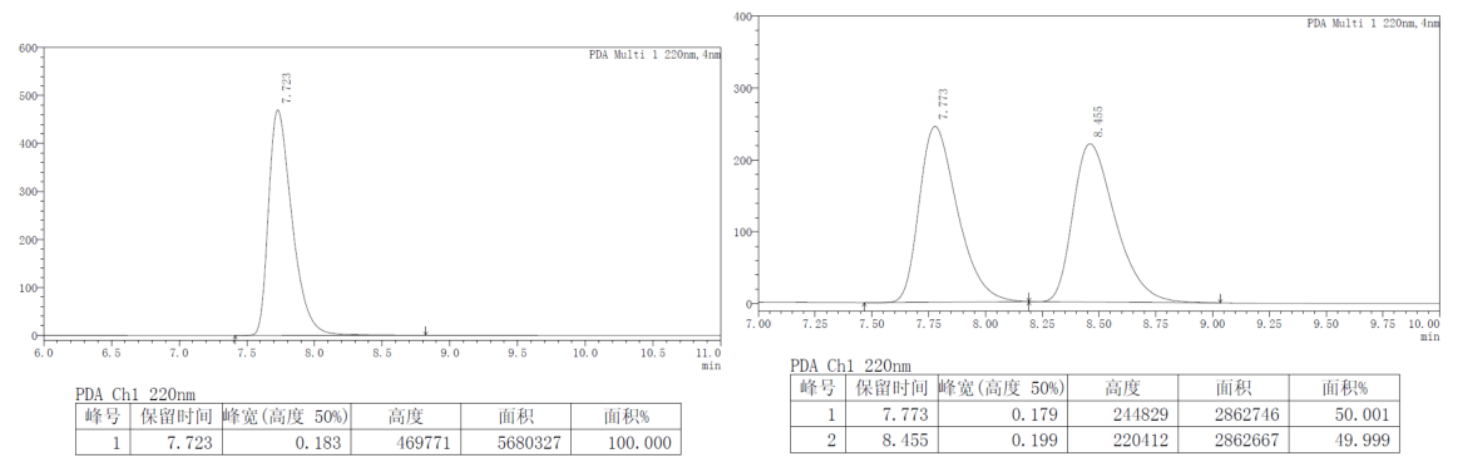<smiles>O=C(NC(Cc1ccccc1)C1CCCC1)c1ccccc1</smiles>

$\boldsymbol{\alpha}$-Aminoboronic ester 4aa: Following the general procedure [A], enamide 1av (40.4 mg, 0.200 mmol) was converted to the $\alpha$-aminoboronic ester. Purification by silica gel chromatography gave $40.3 \mathrm{mg}$ (61\% yield) of $\mathbf{4 a a}$ as a white solid. ${ }^{1} \mathbf{H}$ NMR $\left(400 \mathrm{MHz}, \mathrm{CDCl}_{3}\right) \delta 7.85-7.77(\mathrm{~m}, 2 \mathrm{H})$, $7.55(\mathrm{t}, J=7.4 \mathrm{~Hz}, 1 \mathrm{H}), 7.50-7.41(\mathrm{~m}, 2 \mathrm{H}), 7.03(\mathrm{~s}, 1 \mathrm{H}), 2.88(\mathrm{dd}, J=8.8,2.9 \mathrm{~Hz}, 1 \mathrm{H}), 2.26-$ $2.10(\mathrm{~m}, 1 \mathrm{H}), 2.06-1.83(\mathrm{~m}, 2 \mathrm{H}), 1.76-1.49(\mathrm{~m}, 4 \mathrm{H}), 1.30(\mathrm{~s}, 12 \mathrm{H}) .{ }^{13} \mathrm{C}$ NMR $(101 \mathrm{MHz}$, $\left.\mathrm{CDCl}_{3}\right) \delta 169.91,132.57,129.92,128.61,127.59,81.65,41.64,31.09,30.48,25.35,25.22,24.86$, 24.58. IR $v\left(\mathrm{~cm}^{-1}\right) 3233,2969,1610,750 .{ }^{11} \mathbf{B}$ NMR $\left(128 \mathrm{MHz}, \mathrm{CDCl}_{3}\right) \delta 22.68$. M. P. $154-158$ ${ }^{\circ} \mathrm{C} .[\alpha]^{20} \mathrm{D}=-48.1\left(c=0.87, \mathrm{CHCl}_{3}\right)$ for a $91 \%$ ee sample. The enantiomeric purity of this compound was determined by HPLC analysis in comparison with authentic racemic material (Chiralpak IE, 97:3 hexanes:i-PrOH, $1.0 \mathrm{~mL} / \mathrm{min}, 254 \mathrm{~nm}$ ): $t_{\text {major }}=15.0 \mathrm{~min}, t_{\text {minor }}=16.1 \mathrm{~min}$.
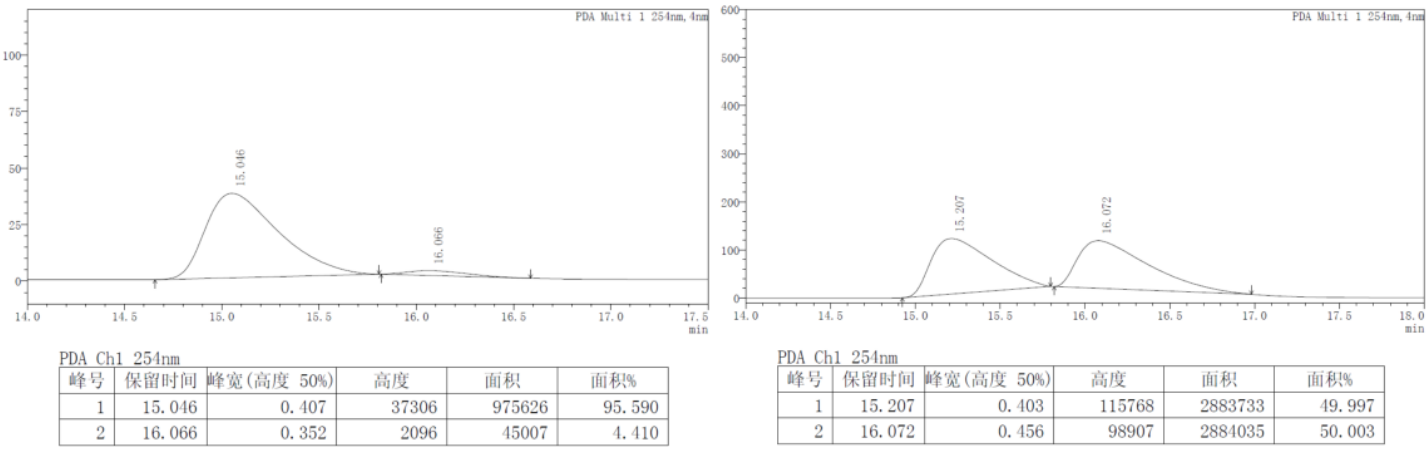<smiles>CC(C)C(Cc1ccccc1)NC(=O)c1ccc(Cl)cc1</smiles> 
$\boldsymbol{\alpha}$-Aminoboronic ester 4ab: Following the general procedure [D], enamide 1aw (42.0 mg, 0.200 mmol) was converted to the $\alpha$-aminoboronic ester. Purification by silica gel chromatography gave $58.2 \mathrm{mg}\left(86 \%\right.$ yield) of $\mathbf{2 d}$ as a white solid. ${ }^{1} \mathbf{H} \mathbf{N M R}\left(400 \mathrm{MHz}, \mathrm{CDCl}_{3}\right) \delta 7.72(\mathrm{~d}, J=8.5 \mathrm{~Hz}$, 2H), $7.38(\mathrm{~d}, J=8.5 \mathrm{~Hz}, 2 \mathrm{H}), 6.99(\mathrm{~s}, 1 \mathrm{H}), 3.05(\mathrm{t}, J=5.3 \mathrm{~Hz}, 1 \mathrm{H}), 2.10-2.01(\mathrm{~m}, 1 \mathrm{H}), 1.28(\mathrm{~d}, J$ $=2.7 \mathrm{~Hz}, 12 \mathrm{H}), 1.01(\mathrm{dd}, J=6.5,5.5 \mathrm{~Hz}, 6 \mathrm{H}) .{ }^{13} \mathbf{C ~ N M R}\left(101 \mathrm{MHz}, \mathrm{CDCl}_{3}\right) \delta 168.21,138.46$, 129.94, 128.83 (overlapping), 82.53, 29.98, 25.18, 20.60, 19.95. ${ }^{11} \mathbf{B} \mathbf{~ N M R}\left(128 \mathrm{MHz}, \mathrm{CDCl}_{3}\right) \delta$ 20.32. ESI-HR calcd for $\mathrm{C}_{17} \mathrm{H}_{26} \mathrm{BCINO}_{3}{ }^{+}\left([\mathrm{M}+\mathrm{H}]^{+}\right) 338.1689$, found 338.1695. IR $v\left(\mathrm{~cm}^{-1}\right) 3194$, 2970, 1605, 752. M. P.166-168 ${ }^{\circ} \mathrm{C} .[\alpha]^{20}{ }_{\mathrm{D}}=-29.7\left(c=1.06, \mathrm{CHCl}_{3}\right)$ for a $99 \%$ ee sample. The enantiomeric purity of this compound was determined by HPLC analysis in comparison with authentic racemic material (Chiralpak IE, 98:2 hexanes:i-PrOH, $1.0 \mathrm{~mL} / \mathrm{min}, 220 \mathrm{~nm}$ ): $t_{\text {major }}=$ $19.3 \mathrm{~min}, t_{\operatorname{minor}}=18.6 \mathrm{~min}$.

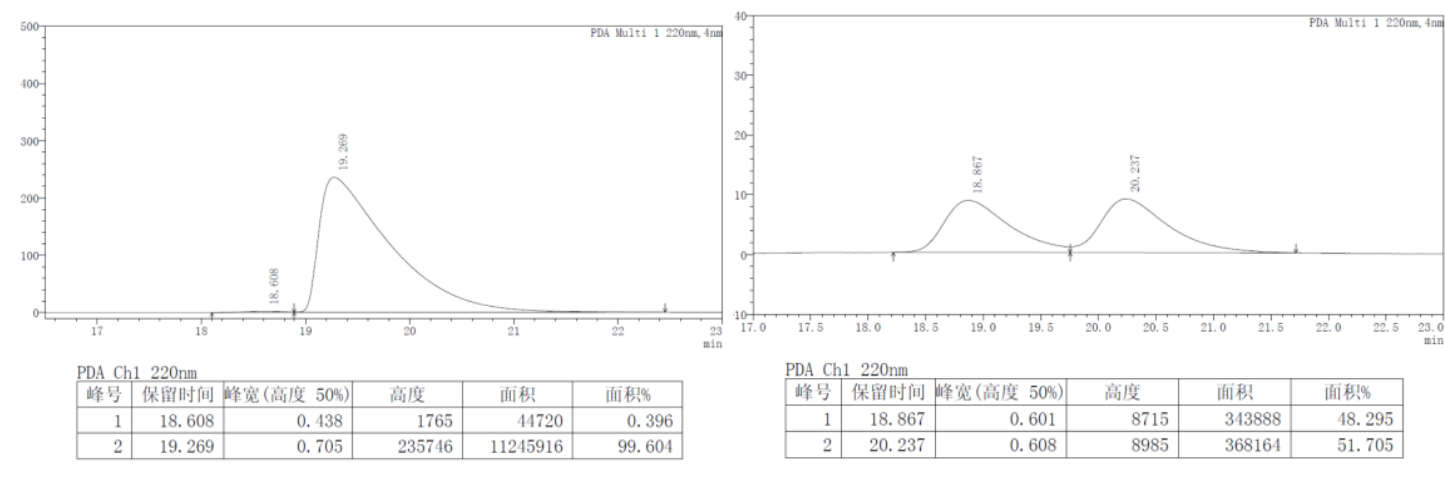<smiles>CC(C)C(=O)NC([Ga]c1ccccc1)[C@H](C)Cc1ccccc1</smiles>

$\boldsymbol{\alpha}$-Aminoboronic ester 4t': Following the general procedure [D], enamide $\mathbf{1 r}-\boldsymbol{Z}$ (49.2 mg, 0.200 mmol) was converted to the $\alpha$-aminoboronic ester. Purification by silica gel chromatography gave $64.4 \mathrm{mg}\left(85 \%\right.$ yield) of $\mathbf{4 t}{ }^{\prime}$ as a white solid. ${ }^{1} \mathbf{H}$ NMR $\left(400 \mathrm{MHz}, \mathrm{CDCl}_{3}\right) \delta 7.29(\mathrm{dd}, J=8.5,6.3$ Hz, 2H), 7.19 (dd, $J=16.2,7.5 \mathrm{~Hz}, 3 \mathrm{H}), 6.34$ (s, 1H), 2.75 (ddd, $J=14.5,9.4,5.5 \mathrm{~Hz}, 1 \mathrm{H}), 2.59$ (ddd, $J=16.1,8.0,5.8 \mathrm{~Hz}, 2 \mathrm{H}), 1.93-1.82(\mathrm{~m}, 1 \mathrm{H}), 1.81-1.71(\mathrm{~m}, 1 \mathrm{H}), 1.54-1.40(\mathrm{~m}, 1 \mathrm{H})$, $1.26-1.17(\mathrm{~m}, 21 \mathrm{H}), 1.05(\mathrm{t}, J=6.7 \mathrm{~Hz}, 3 \mathrm{H}) .{ }^{13} \mathrm{C}$ NMR $(101 \mathrm{MHz}, \mathrm{CDCl} 3) \delta 182.58,142.78$, $128.45,128.31,125.62,80.82,36.13,35.40,34.02,33.18,26.97,25.49,25.33,17.17 .{ }^{11} \mathbf{B}$ NMR $\left(128 \mathrm{MHz} \mathrm{CDCl}_{3}\right) \delta$ 14.21. ESI-HR calcd for $\mathrm{C}_{22} \mathrm{H}_{37} \mathrm{BNO}_{3}{ }^{+}\left([\mathrm{M}+\mathrm{H}]^{+}\right)$374.2861, found 374.2866. IR $v\left(\mathrm{~cm}^{-1}\right) 3244,2969,1577,747$. M. P. $125-127{ }^{\circ} \mathrm{C} .[\alpha]^{20} \mathrm{D}=-109.4\left(c=1.72, \mathrm{CHCl}_{3}\right)$ for a $96 \%$ 
ee sample. The enantiomeric purity of this compound was determined by HPLC analysis in comparison with authentic racemic material (Chiralpak IE, 98:2 hexanes:i-PrOH, $1.0 \mathrm{~mL} / \mathrm{min}, 254$ $\mathrm{nm}): t_{\text {major }}=9.5 \mathrm{~min}, t_{\text {minor }}=11.9 \mathrm{~min}$.
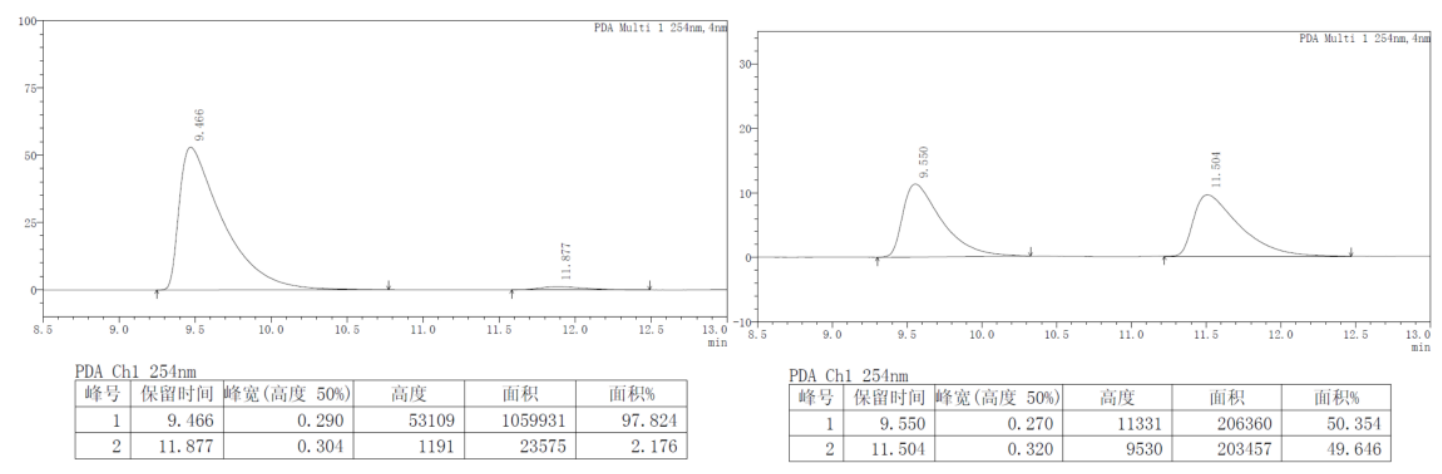<smiles>CC(C)/C=C\NC(=O)C(Cc1ccccc1)NC(=O)OC(C)(C)C</smiles>

Enamide 6-Z: ${ }^{1} \mathbf{H}$ NMR $\left(400 \mathrm{MHz}, \mathrm{CDCl}_{3}\right) \delta 7.38(\mathrm{~s}, 1 \mathrm{H}), 7.35-7.29(\mathrm{~m}, 2 \mathrm{H}), 7.27-7.21(\mathrm{~m}$, 4H), $6.50(\mathrm{t}, J=9.9 \mathrm{~Hz}, 1 \mathrm{H}), 5.09(\mathrm{~s}, 1 \mathrm{H}), 4.58(\mathrm{t}, J=9.3 \mathrm{~Hz}, 1 \mathrm{H}), 4.43-4.25(\mathrm{~m}, 1 \mathrm{H}), 3.14(\mathrm{dd}$, $J=13.9,6.6 \mathrm{~Hz}, 1 \mathrm{H}), 3.05(\mathrm{dd}, J=13.8,7.7 \mathrm{~Hz}, 1 \mathrm{H}), 2.16-2.02(\mathrm{~m}, 1 \mathrm{H}), 1.43(\mathrm{~s}, 9 \mathrm{H}), 0.89(\mathrm{dd}$, $J=8.0,6.7 \mathrm{~Hz}, 6 \mathrm{H}) .{ }^{13} \mathrm{C}$ NMR $\left(101 \mathrm{MHz}, \mathrm{CDCl}_{3}\right) \delta 168.65,155.88,136.88,129.37,128.92$, $127.15,120.27,118.26,80.60,56.15,38.24,28.39,25.65,22.97,22.92$. ESI-HR calcd for $\mathrm{C}_{19} \mathrm{H}_{29} \mathrm{~N}_{2} \mathrm{O}_{3}{ }^{+}\left([\mathrm{M}+\mathrm{H}]^{+}\right)$333.2173, found 333.2180. IR $v\left(\mathrm{~cm}^{-1}\right) 3297,1650$, 1522, 698. M. P. $118-120^{\circ} \mathrm{C}$<smiles>CC(C)C[C@H](Cc1ccccc1)NC(=O)[C@H](Cc1ccccc1)NC(=O)O</smiles>

$\boldsymbol{\alpha}$-Aminoboronic ester 7 : Following the general procedure [D], enamide 1ax- $Z$ (66.6 mg, 0.200 mmol) was converted to the $\alpha$-aminoboronic ester. Purification by silica gel chromatography gave $56.3 \mathrm{mg}\left(61 \%\right.$ yield) of 7 as a colorless oil. ${ }^{1} \mathbf{H}$ NMR $\left(400 \mathrm{MHz}, \mathrm{CDCl}_{3}\right) \delta 7.33-7.27(\mathrm{~m}, 3 \mathrm{H})$, $7.27-7.19(\mathrm{~m}, 3 \mathrm{H}), 6.29(\mathrm{~s}, 1 \mathrm{H}), 5.07(\mathrm{~s}, 1 \mathrm{H}), 4.37(\mathrm{dd}, J=14.8,7.0 \mathrm{~Hz}, 1 \mathrm{H}), 3.14-2.90(\mathrm{~m}$, 3H), $1.52-1.33(\mathrm{~m}, 13 \mathrm{H}), 1.25(\mathrm{~d}, J=2.8 \mathrm{~Hz}, 12 \mathrm{H}), 0.90-0.82(\mathrm{~m}, 6 \mathrm{H}) .{ }^{13} \mathbf{C}$ NMR $(101 \mathrm{MHz}$, $\left.\mathrm{CDCl}_{3}\right) \delta 172.48,170.92,155.33,136.50,129.45,128.62,82.77,39.76,38.19,28.23,25.48,24.97$, 
24.87, 23.15, 21.85. ${ }^{11} \mathbf{B}$ NMR $\left(128 \mathrm{MHz}, \mathrm{CDCl}_{3}\right) \delta$ 21.54. ESI-HR calcd for $\mathrm{C}_{25} \mathrm{H}_{42} \mathrm{BN}_{2} \mathrm{O}_{5}{ }^{+}$ $\left([\mathrm{M}+\mathrm{H}]^{+}\right)$461.3181, found 461.3158. IR $v\left(\mathrm{~cm}^{-1}\right) 3279,2973,1689,753 .[\alpha]^{20} \mathrm{D}=-109.4(c=2.28$, $\mathrm{CHCl}_{3}$ ). The dr value was determined by HPLC (Chiralpak IE, 97:3 hexanes:i-PrOH, $1.0 \mathrm{~mL} / \mathrm{min}$, $210 \mathrm{~nm}$ ). When ent-Duanphos was used, reverse dr of 7 was obtained, showing that the new stereocenter was generated under catalyst control.
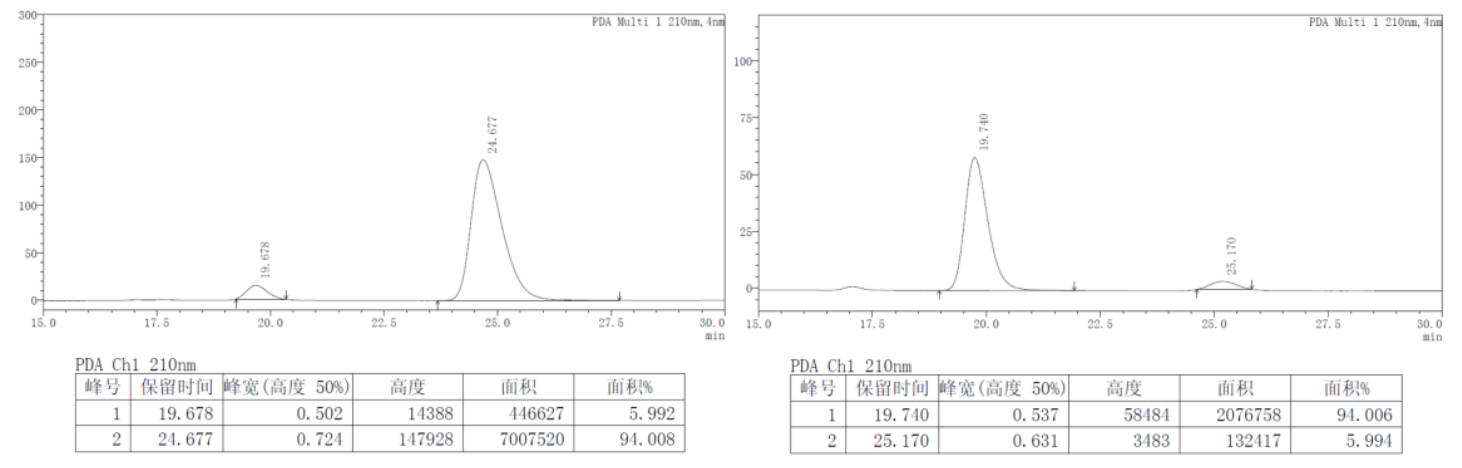<smiles>CCCCC(Br)CNC(=O)C(C)(C)C</smiles>

Amide 8: To solution of boronic ester $\mathbf{3 f}(62.3 \mathrm{mg}, 0.200 \mathrm{mmol}, 1.0$ equiv) in methanol (1.0 mL) was added water $(0.4 \mathrm{~mL}), \mathrm{KHF}_{2}(140.6 \mathrm{mg}, 1.800 \mathrm{mmol}$, 9.0 equiv). The mixture was stirred at $\mathrm{rt}$ for $5 \mathrm{~h}$ and then concentrated. To the residue was added $\mathrm{MeOH}(5 \mathrm{~mL})$ and the resulting mixture was further evaporated to dryness. Evaporation-dissolution cycles were repeated for 3 times. The solid residue was then triturated with dry acetone $(5 \mathrm{~mL})$. The liquid phase was carefully decanted, and the residual inorganic salts were washed with acetone 3 times. The combined acetone was concentrated to give the product 4 as colorless solid (48.3 mg, 83\% yield). ${ }^{1} \mathbf{H}$ NMR (400 MHz, Acetone) $\delta 6.72(\mathrm{~s}, 1 \mathrm{H}), 3.20-3.03(\mathrm{~m}, 2 \mathrm{H}), 2.89(\mathrm{~s}, 1 \mathrm{H}), 1.45-1.30(\mathrm{~m}, 2 \mathrm{H}), 1.29-1.17(\mathrm{~m}$, 3H), 1.10 (s, 9H), 0.85 (t, $J=7.0 \mathrm{~Hz}, 3 \mathrm{H}), 0.35$ (d, $J=4.2 \mathrm{~Hz}, 1 \mathrm{H}) .{ }^{13} \mathrm{C}$ NMR (101 MHz, Acetone) $\delta 177.46,42.21,38.89,32.28,29.53,28.01,24.11,14.59 .{ }^{11} \mathbf{B}$ NMR (128 MHz, Acetone) $\delta 5.43$. ${ }^{19} \mathbf{F}$ NMR (376 MHz, Acetone) $\delta$-143.75. ESI-HR calcd for $\mathrm{C}_{11} \mathrm{H}_{22} \mathrm{BF}_{3} \mathrm{NO}^{-}\left([\mathrm{M}-\mathrm{K}]^{-}\right)$252.1752, found 252.1755. IR $v\left(\mathrm{~cm}^{-1}\right) 3164,2944,1647,750$.<smiles>CCCCC(O)CNC(=O)C(C)(C)C</smiles> 
Amide 9: To a solution of $\mathbf{3 f}\left(62.3 \mathrm{mg}, 0.200 \mathrm{mmol}, 1.0\right.$ equiv) in $\mathrm{THF} / \mathrm{H}_{2} \mathrm{O}(1: 1,4.0 \mathrm{~mL})$ was added $\mathrm{NaBO}_{3} \bullet 4 \mathrm{H}_{2} \mathrm{O}(154 \mathrm{mg}, 1.00 \mathrm{mmol}, 5.0$ equiv) and the reaction mixture was stirred at room temperature for $2 \mathrm{~h}$. Then it was diluted with $\mathrm{H}_{2} \mathrm{O}$ and extracted with ethyl acetate. The combined organic layers were dried over anhydrous $\mathrm{MgSO}_{4}$ and concentrated under reduced pressure. The residue was purified by flash column chromatography to give $35.4 \mathrm{mg}$ (90\% yield) of $\mathbf{5}$ as a colorless oil. ${ }^{1} \mathbf{H}$ NMR $\left(400 \mathrm{MHz}, \mathrm{CDCl}_{3}\right) \delta 6.10(\mathrm{~s}, 1 \mathrm{H}), 3.69(\mathrm{~s}, 1 \mathrm{H}), 3.46$ (ddd, $J=14.0,6.3$, $2.8 \mathrm{~Hz}, 1 \mathrm{H}), 3.18-3.06(\mathrm{~m}, 1 \mathrm{H}), 2.69(\mathrm{~d}, J=3.3 \mathrm{~Hz}, 1 \mathrm{H}), 1.50-1.37(\mathrm{~m}, 3 \mathrm{H}), 1.37-1.27(\mathrm{~m}$, 3H), $1.21(\mathrm{~s}, 9 \mathrm{H}), 0.90(\mathrm{t}, J=6.9 \mathrm{~Hz}, 3 \mathrm{H}) .{ }^{13} \mathrm{C} \mathbf{~ N M R}\left(101 \mathrm{MHz}, \mathrm{CDCl}_{3}\right) \delta$ 179.83, 71.84, 45.97, 38.88, 34.96, 27.75, 25.01, 22.79, 14.11. ESI-HR calcd for $\mathrm{C}_{11} \mathrm{H}_{24} \mathrm{NO}_{2}{ }^{+}\left([\mathrm{M}+\mathrm{H}]^{+}\right)$202.1802, found 202.1797. IR $v\left(\mathrm{~cm}^{-1}\right) 3447,3365,2964,1636,749$.<smiles>C=CC(CCCC)CNC(=O)C(C)(C)C</smiles>

Amide 10: Vinylmagnesium bromide solution (1.0 M in THF, $1.0 \mathrm{mmol}$, 5.0 equiv) was added to a solution of $\mathbf{3 f}(62.3 \mathrm{mg}, 0.200 \mathrm{mmol}, 1.0$ equiv) in THF $(2 \mathrm{~mL})$ and the reaction mixture was stirred for $30 \mathrm{~min}$ at $-78{ }^{\circ} \mathrm{C}$. A solution of iodine $(203 \mathrm{mg}, 0.800 \mathrm{mmol}, 4.0$ equiv) in $\mathrm{MeOH}(2$ $\mathrm{mL}$ ) was added. The mixture was stirred for another $30 \mathrm{~min}$, and allowed to warm to $0{ }^{\circ} \mathrm{C}$. Upon completion of the reaction, the reaction mixture was quenched with sat. $\mathrm{Na}_{2} \mathrm{~S}_{2} \mathrm{O}_{3}$ (aq) and extracted with ethyl acetate. The combined organic layers were dried over $\mathrm{MgSO}_{4}$ and concentrated in vacuo. The crude product was purified by flash chromatography to give $36.3 \mathrm{mg}$ ( $86 \%$ yield) of $\mathbf{6}$ as a colorless oil. ${ }^{1} \mathbf{H}$ NMR $\left(400 \mathrm{MHz}, \mathrm{CDCl}_{3}\right) \delta 5.66(\mathrm{~s}, 1 \mathrm{H}), 5.54$ (ddd, $J=17.1$, 10.1, $9.2 \mathrm{~Hz}, 1 \mathrm{H}), 5.15-4.99(\mathrm{~m}, 2 \mathrm{H}), 3.45$ (ddd, $J=13.1,6.7,4.8 \mathrm{~Hz}, 1 \mathrm{H}), 2.95-2.77(\mathrm{~m}, 1 \mathrm{H})$, $2.24-2.06(\mathrm{~m}, 1 \mathrm{H}), 1.45-1.34(\mathrm{~m}, 1 \mathrm{H}), 1.34-1.21(\mathrm{~m}, 5 \mathrm{H}), 1.16(\mathrm{~s}, 9 \mathrm{H}), 0.87(\mathrm{t}, J=6.6 \mathrm{~Hz}$, 3H). ${ }^{13} \mathrm{C}$ NMR $\left(101 \mathrm{MHz}, \mathrm{CDCl}_{3}\right) \delta 178.25,141.06,116.59,44.63,42.93,38.79,32.10,29.29$, 27.70, 22.76, 14.09. ESI-HR calcd for $\mathrm{C}_{13} \mathrm{H}_{26} \mathrm{NO}^{+}\left([\mathrm{M}+\mathrm{H}]^{+}\right)$212.2009, found 212.2006. IR $v$ $\left(\mathrm{cm}^{-1}\right) 3349,2959,1637,1533,750$. 
<smiles>CCCCC(CNC(=O)C(C)(C)C)c1cccs1</smiles>

Amide 11: A solution of thiophene (42.1 mg, $0.500 \mathrm{mmol}, 5.0$ equiv) in THF (1.0 mL) was cooled to $-78{ }^{\circ} \mathrm{C}$ and treated with $n \mathrm{BuLi}(1.6 \mathrm{M}$ in hexanes, $0.600 \mathrm{mmol}, 6.0$ equiv). The cooling bath was removed and the mixture was stirred at room temperature for $1 \mathrm{~h}$. The mixture was cooled to $-78{ }^{\circ} \mathrm{C}$ and a solution of $\mathbf{3 f}(31.1 \mathrm{mg}, 0.1 \mathrm{mmol}, 1.0$ equiv) in THF ( $0.5 \mathrm{~mL})$ was added. The mixture was stirred at $-78{ }^{\circ} \mathrm{C}$ for $1 \mathrm{~h}$ and then, a solution of NBS $(89.0 \mathrm{mg}, 0.500 \mathrm{mmol}, 5.0$ equiv) in THF (1.0 mL) was added. After $10 \mathrm{~h}$ at room temperature, sat. $\mathrm{Na}_{2} \mathrm{~S}_{2} \mathrm{O}_{3}(\mathrm{aq})(2 \mathrm{~mL})$ was added. The reaction mixture was diluted with water and extracted with ethyl acetate. The combined organic layers were dried over $\mathrm{MgSO}_{4}$ and concentrated in vacuo. The crude product was purified by column chromatography to give $11.2 \mathrm{mg}$ (42\% yield) of $\mathbf{7}$ as a colorless oil. ${ }^{\mathbf{1}} \mathbf{H}$ NMR $\left(400 \mathrm{MHz}, \mathrm{CDCl}_{3}\right) \delta 7.19(\mathrm{~d}, J=5.0 \mathrm{~Hz}, 1 \mathrm{H}), 6.96(\mathrm{dd}, J=4.8,3.6 \mathrm{~Hz}, 1 \mathrm{H}), 6.81(\mathrm{~d}, J=$ $3.0 \mathrm{~Hz}, 1 \mathrm{H}), 5.58(\mathrm{~s}, 1 \mathrm{H}), 3.72(\mathrm{p}, J=9.1 \mathrm{~Hz}, 1 \mathrm{H}), 3.20-3.00(\mathrm{~m}, 2 \mathrm{H}), 1.77-1.64(\mathrm{~m}, 1 \mathrm{H}), 1.64$ - $1.51(\mathrm{~m}, 1 \mathrm{H}), 1.38-1.21(\mathrm{~m}, 4 \mathrm{H}), 1.10(\mathrm{~s}, 9 \mathrm{H}), 0.85(\mathrm{t}, J=6.8 \mathrm{~Hz}, 3 \mathrm{H}) .{ }^{13} \mathbf{C}$ NMR (101 MHz, $\left.\mathrm{CDCl}_{3}\right) \delta 178.41,147.20,126.90,124.59,123.69,45.82,41.44,38.80,34.64,29.55,27.64,22.69$, 14.04. ESI-HR calcd for $\mathrm{C}_{15} \mathrm{H}_{26} \mathrm{NOS}^{+}\left([\mathrm{M}+\mathrm{H}]^{+}\right)$268.1730, found 268.1723. IR $v\left(\mathrm{~cm}^{-1}\right) 3447$, $2987,1654,1508,740$.<smiles>CC(Cc1ccccc1)NC(=O)C(C)(C)C</smiles>

Amide 12: To solution of boronic ester $\mathbf{3 y}$ ( $67.3 \mathrm{mg}, 0.250 \mathrm{mmol}, 1.0$ equiv) in methanol (1.5 mL) was added water $(0.5 \mathrm{~mL}), \mathrm{KHF}_{2}(175.7 \mathrm{mg}, 2.250 \mathrm{mmol}, 9.0$ equiv). The mixture was stirred at $\mathrm{rt}$ for $5 \mathrm{~h}$ and then concentrated. To the residue was added $\mathrm{MeOH}(15 \mathrm{~mL})$ and the resulting mixture was further evaporated to dryness. Evaporation-dissolution cycles were repeated for 3 times. The solid residue was then triturated with dry acetone $(15 \mathrm{~mL})$. The liquid phase was carefully decanted, and the residual inorganic salts were washed with acetone 3 times. The combined acetone was concentrated to give the potassium trifluoroborate salts in quantitative yield. Then the potassium trifluoroborate salts $\left(12.5 \mathrm{mg}, 0.050 \mathrm{mmol}, 1.0\right.$ equiv), $\mathrm{Pd}(\mathrm{OAc})_{2}(1.3 \mathrm{mg}, 0.0050 \mathrm{mmol}$, $10 \mathrm{~mol} \%), \mathrm{Ad}_{2} \mathrm{P} n \mathrm{Bu}$ (3.6 mg, $\left.0.010 \mathrm{mmol}, 20 \mathrm{~mol} \%\right), \mathrm{Cs}_{2} \mathrm{CO}_{3}$ (48.9 mg, $0.150 \mathrm{mmol}, 3.0$ equiv), 
toluene $(0.45 \mathrm{~mL})$, water $(0.050 \mathrm{~mL})$ and $\mathrm{PhBr}(11.8 \mathrm{mg}, 0.075 \mathrm{mmol}, 1.5$ equiv) were added. The reaction was stirred under nitrogen at $100{ }^{\circ} \mathrm{C}$ for $24 \mathrm{~h}$. The reaction mixture was concentrated and purified directly by column chromatography on silica gel to give $6.8 \mathrm{mg}$ (62\% yield) of $\mathbf{8}$ as a white solid. ${ }^{1} \mathbf{H}$ NMR $\left(400 \mathrm{MHz}, \mathrm{CDCl}_{3}\right) \delta 7.29(\mathrm{t}, J=7.3 \mathrm{~Hz}, 2 \mathrm{H}), 7.22(\mathrm{t}, J=7.3 \mathrm{~Hz}, 1 \mathrm{H}), 7.17$ $(\mathrm{d}, J=7.0 \mathrm{~Hz}, 2 \mathrm{H}), 5.36(\mathrm{~s}, 1 \mathrm{H}), 4.31-4.19(\mathrm{~m}, 1 \mathrm{H}), 2.85-2.69(\mathrm{~m}, 2 \mathrm{H}), 1.16-1.06(\mathrm{~m}, 12 \mathrm{H})$. ${ }^{13} \mathrm{C}$ NMR $\left(101 \mathrm{MHz}, \mathrm{CDCl}_{3}\right) \delta 177.71,138.14,129.64,128.47,126.57,45.85,42.54,38.70$, 27.66, 20.22. ESI-HR calcd for $\mathrm{C}_{14} \mathrm{H}_{22} \mathrm{NO}^{+}\left([\mathrm{M}+\mathrm{H}]^{+}\right)$220.1696, found 220.1692. IR $v\left(\mathrm{~cm}^{-1}\right)$ 3339, 2966, 1632, 742. M.P. $99-100{ }^{\circ} \mathrm{C}$.

\section{Determination of the Absolute Configuration}<smiles>CC(C)(C)C(=O)N/C=C\Cc1ccccc1</smiles>

Enamide S1-Z: ${ }^{1} \mathbf{H}$ NMR $\left(400 \mathrm{MHz}, \mathrm{CDCl}_{3}\right) \delta 7.33(\mathrm{t}, J=7.1 \mathrm{~Hz}, 2 \mathrm{H}), 7.28-7.20(\mathrm{~m}, 4 \mathrm{H}), 7.05$ (s, 1H), $6.84(\mathrm{dd}, J=10.6,9.2 \mathrm{~Hz}, 1 \mathrm{H}), 5.02(\mathrm{dd}, J=15.8,7.0 \mathrm{~Hz}, 1 \mathrm{H}), 3.40(\mathrm{~d}, J=6.9 \mathrm{~Hz}, 2 \mathrm{H})$, 1.07 (s, 9H). ${ }^{13} \mathrm{C}$ NMR $\left(101 \mathrm{MHz}, \mathrm{CDCl}_{3}\right) \delta 175.57,139.72,129.04,128.54,126.73,123.06$, 108.26, 38.91, 32.41, 27.37. ESI-HR calcd for $\mathrm{C}_{14} \mathrm{H}_{20} \mathrm{NO}^{+}\left([\mathrm{M}+\mathrm{H}]^{+}\right)$218.1539, found 218.1531. IR $v\left(\mathrm{~cm}^{-1}\right) 3434,2988,1655,749$.<smiles>CC(C)(C)C(=O)NC(CCc1ccccc1)c1ccccc1</smiles>

$\boldsymbol{\alpha}$-Aminoboronic ester S2: Following the general procedure [D], enamide S1-Z (10.9 mg, 0.050 mmol) was converted to the $\alpha$-aminoboronic ester. Purification by silica gel chromatography gave $8.9 \mathrm{mg}\left(52 \%\right.$ yield) of $\mathbf{S 2}$ as a white solid. ${ }^{1} \mathbf{H} \mathbf{~ N M R}\left(400 \mathrm{MHz}, \mathrm{CDCl}_{3}\right) \delta 7.30(\mathrm{t}, J=7.4 \mathrm{~Hz}, 2 \mathrm{H})$, $7.26-7.17(\mathrm{~m}, 3 \mathrm{H}), 6.02(\mathrm{~s}, 1 \mathrm{H}), 3.00-2.89(\mathrm{~m}, 1 \mathrm{H}), 2.65-2.53(\mathrm{~m}, 2 \mathrm{H}), 2.11-1.98(\mathrm{~m}, 1 \mathrm{H})$, $1.79-1.70(\mathrm{~m}, 1 \mathrm{H}), 1.20(\mathrm{~s}, 12 \mathrm{H}), 0.99(\mathrm{~s}, 9 \mathrm{H}) .{ }^{13} \mathbf{C}$ NMR (101 MHz, $\left.\mathrm{CDCl}_{3}\right) \delta$ 183.52, 143.19, 128.93, 128.77, 126.33, 80.18, 36.15, 35.33, 32.79, 26.62, 25.50, 25.27. ${ }^{11}$ B NMR (128 MHz, $\left.\mathrm{CDCl}_{3}\right) \delta$ 15.30. ESI-HR calcd for $\mathrm{C}_{20} \mathrm{H}_{33} \mathrm{BNO}_{3}{ }^{+}\left([\mathrm{M}+\mathrm{H}]^{+}\right) 346.2548$, found 346.2537. IR $v\left(\mathrm{~cm}^{-1}\right)$ 
2989, $1582,1275,749$. M. P. $165-166^{\circ} \mathrm{C} \cdot[\alpha]^{20}{ }_{\mathrm{D}}=-149.4\left(c=0.415, \mathrm{CHCl}_{3}\right)$ for a $97 \%$ ee sample. The enantiomeric purity of this compound was determined by HPLC analysis in comparison with authentic racemic material (Chiralpak IC, 98:2 hexanes:i-PrOH, $1.0 \mathrm{~mL} / \mathrm{min}, 220 \mathrm{~nm}$ ): $t_{\text {major }}=$ $12.5 \mathrm{~min}, t_{\mathrm{minor}}=8.0 \mathrm{~min}$.
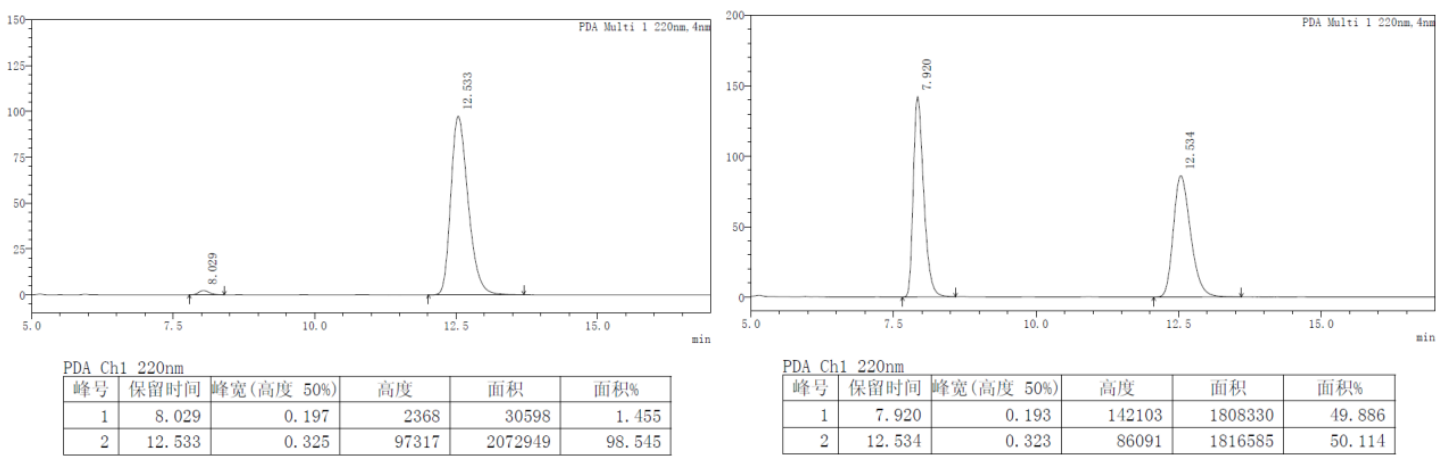

The specific rotation data $\left([\alpha]^{20} \mathrm{D}=-149.4\left(c=0.415, \mathrm{CHCl}_{3}\right)\right.$ for a $97 \%$ ee sample $)$ of $\mathbf{S 2}$ derived from S1-Z, was compared to the specific rotation data $\left([\alpha]^{31} \mathrm{D}=+80.5\left(c=1.00, \mathrm{CHCl}_{3}\right)\right.$ for a $98 \%$ ee sample)

(S)-(+)-N-[3-Phenyl-1-(4,4,5,5-tetramethyl-1,3,2-dioxaborolan-2-yl)propyl]pivalamide. ${ }^{5}$

It indicates that the absolute configuration of the product $\mathbf{S 2}$ formed from the hydroboration reaction is $(R)$. The absolute configurations of other amide products were assigned by analogy.

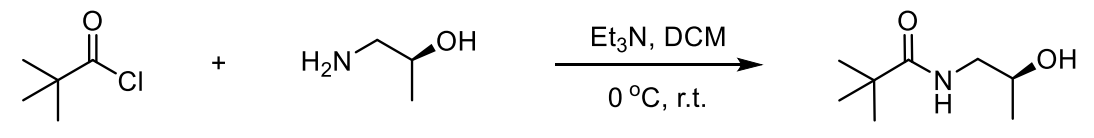

(S)-1-aminopropan-2-ol

(S)-N-(2-hydroxypropyl)pivalamide

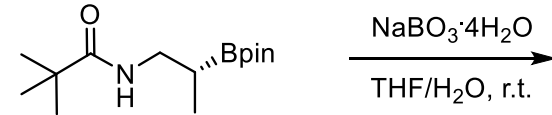

S3<smiles>C[C@@H](O)CNC(=O)C(C)(C)C</smiles>

S4<smiles>CC(CNC(=O)C(C)(C)C)Cc1ccccc1</smiles>

$\boldsymbol{\beta}$-Aminoboronic ester S3: Following the general procedure [E], enamide 1e- $\boldsymbol{E}$ (28.2 mg, 0.200 mmol) was converted to the $\beta$-aminoboronic ester. Purification by silica gel chromatography gave $18.7 \mathrm{mg}$ (69\% yield) of $\mathbf{S 3}$ as a colorless oil. $[\alpha]^{20} \mathrm{D}=-22.1\left(c=0.68, \mathrm{CHCl}_{3}\right)$ for a $71 \%$ ee sample. 
The enantiomeric purity of this compound was determined by HPLC analysis in comparison with authentic racemic material (Chiralpak ID, 97:3 hexanes:i-PrOH, $1.0 \mathrm{~mL} / \mathrm{min}, 205 \mathrm{~nm}$ ): $t_{\text {major }}=$ $14.3 \mathrm{~min}, t_{\operatorname{minor}}=13.6 \mathrm{~min}$.
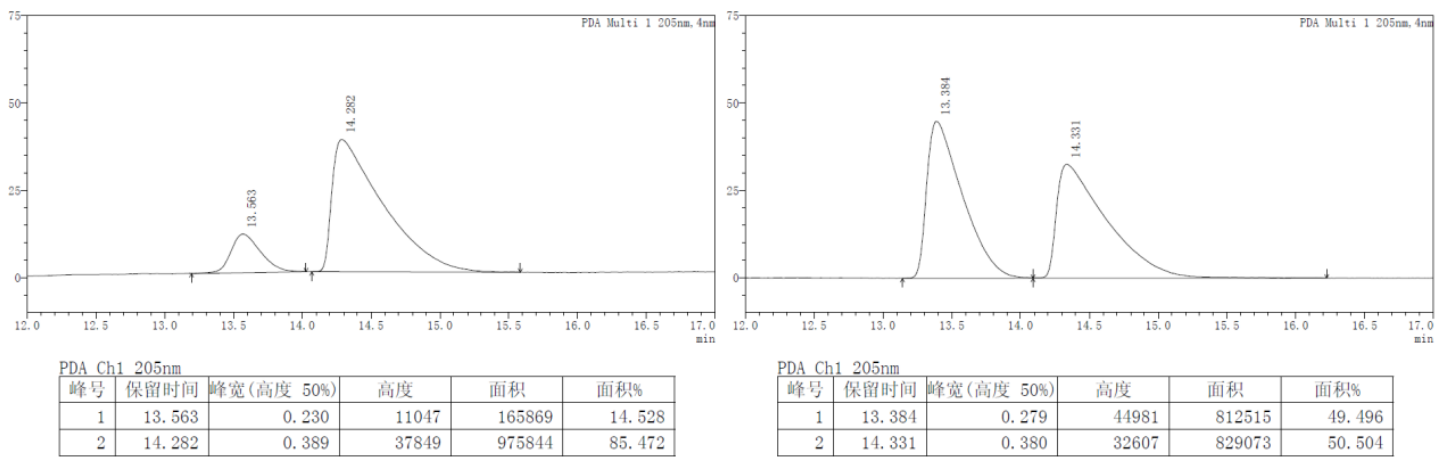<smiles>C[C@H](O)CNC(=O)C(C)(C)C</smiles>

ק-Hydroxyl amide S4: ${ }^{1} \mathbf{H}$ NMR $\left(400 \mathrm{MHz}, \mathrm{CDCl}_{3}\right) \delta 6.17(\mathrm{~s}, 1 \mathrm{H}), 3.98-3.82(\mathrm{~m}, 1 \mathrm{H}), 3.49-$ $3.31(\mathrm{~m}, 1 \mathrm{H}), 3.20-3.04(\mathrm{~m}, 1 \mathrm{H}), 2.59$ (broad, 1H), $1.20(\mathrm{~s}, 9 \mathrm{H}), 1.17(\mathrm{~d}, J=6.2 \mathrm{~Hz}, 3 \mathrm{H}) .{ }^{13} \mathrm{C}$ NMR $\left(101 \mathrm{MHz}, \mathrm{CDCl}_{3}\right) \delta 179.94,67.55,47.18,38.85,27.69$, 21.01. ESI-HR calcd for $\mathrm{C}_{8} \mathrm{H}_{18} \mathrm{NO}_{2}{ }^{+}\left([\mathrm{M}+\mathrm{H}]^{+}\right)$160.1332, found 160.1326. IR $v\left(\mathrm{~cm}^{-1}\right) 3346,2969,1636,750 .[\alpha]^{20}{ }_{\mathrm{D}}=$ $-25.3\left(c=0.70, \mathrm{CHCl}_{3}\right)$ for a $71 \%$ ee sample.

The specific rotation data $\left([\alpha]^{20} \mathrm{D}=-25.3\left(c=0.70, \mathrm{CHCl}_{3}\right)\right.$ for a $71 \%$ ee sample $)$ of $\mathbf{S} \mathbf{4}$ derived from $\mathbf{S 2}$ was compared to the specific rotation data $\left([\alpha]^{20} \mathrm{D}=+38.6\left(c=1.65, \mathrm{CHCl}_{3}\right)\right.$ for a $>99 \%$ ee sample) of (S)-N-(2-hydroxypropyl)pivalamide. It indicates that the absolute configuration of the product $\mathbf{S 4}$ formed from the hydroboration reaction is $(R)$. The absolute configurations of other amide products were assigned by analogy.

\section{DFT Computational Studies}

\section{General information for computation}

All calculations were performed with the Gaussian 09 program ${ }^{6}$.Density functional theory calculations using the M06 functional ${ }^{7}$.Geometry optimizations were conducted with the Gaussian 09 software package, B3LYP functional (with gd3 dispersion correction), and LANL2DZ basis set for $\mathrm{Rh}$ and $6-3 \lg (\mathrm{d}, \mathrm{p})$ basis set for all other atoms. Single-point energy calculations were 
conducted with the M06 functional and SDD basis set for Rh and the 6-311++g(d,p) basis set for all other atoms, along with the SMD THF solvent correction. Thermal corrections were applied to the optimized geometries to provide Gibbs free energies.

The energy profiles of catalytic hydroboration of trans-enamide were computed. Extensive conformational searches were conducted for all intermediates and transition states, and the lowest energy conformers are shown in Fig S1. The energy profiles indicate that migratory insertion of the alkene into the rhodium hydride is the rate-limiting and selectivity-determining step.

In addition, many possible transition state structures of migratory insertion were considered during the computation for the activation energies of catalytic hydroboration of four enamide substrates. For each substrate, four possible structures were computed. The structures and energies were summarized in Fig S2. The computational results are in agreement with experimental observation. 


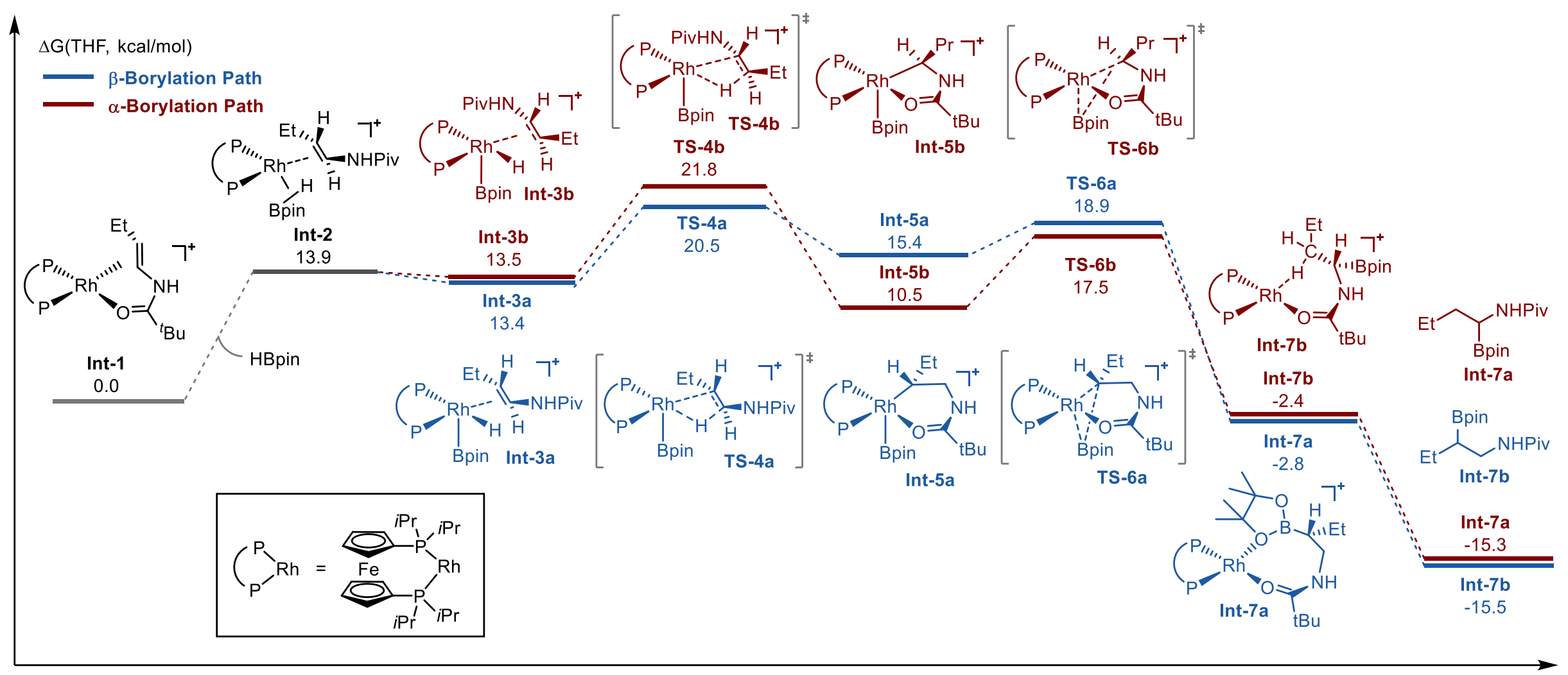

reaction coordinate

Figure S1. Energy profile for Rh-catalyzed hydroboration of trans-enamide. Gibbs free energies in $\operatorname{THF}\left(\Delta G_{\text {solv298K }}\right)$ are reported. 


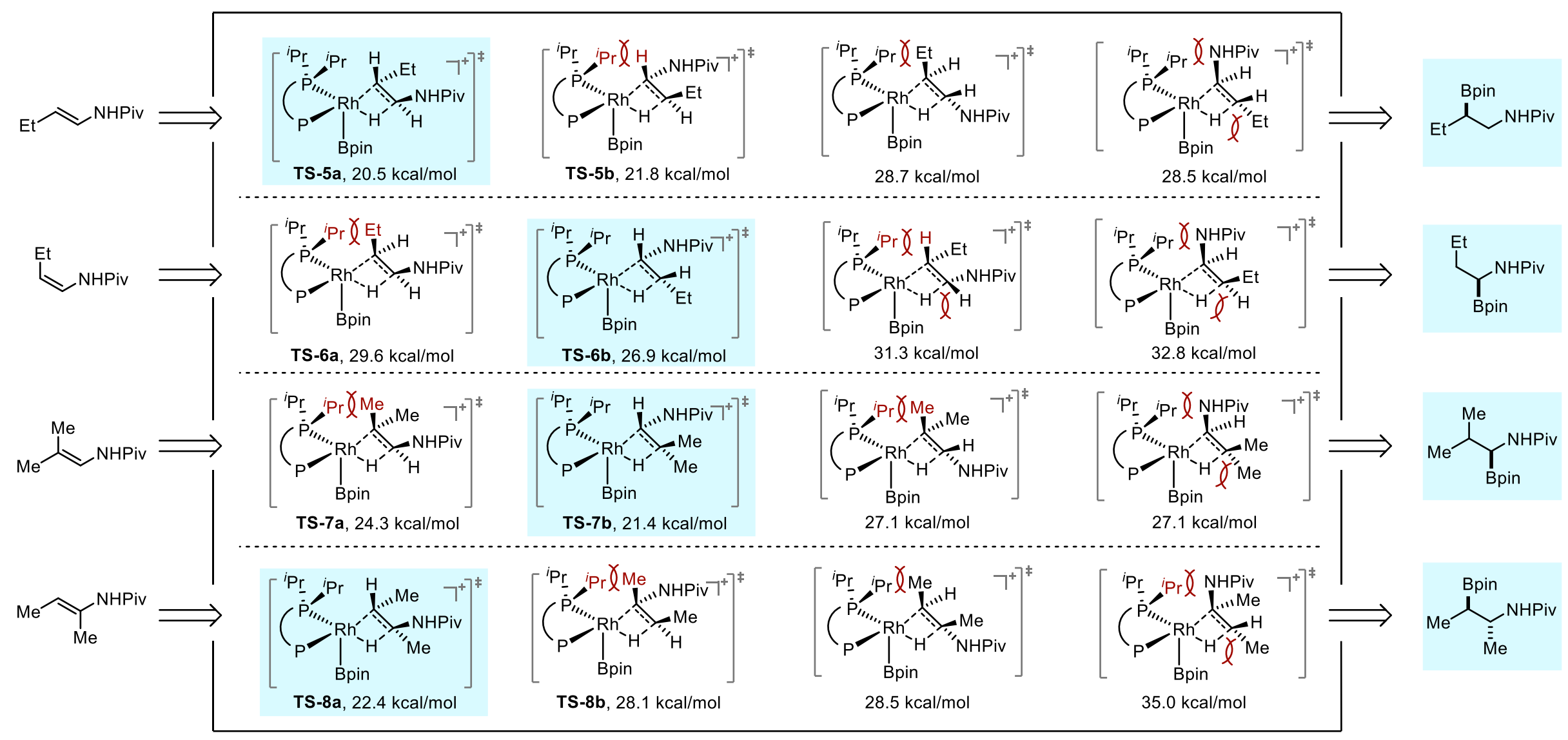

Figure S2. Overview of transition states of migratory insertion leading to $\alpha$ and $\beta$-hydroboration. 


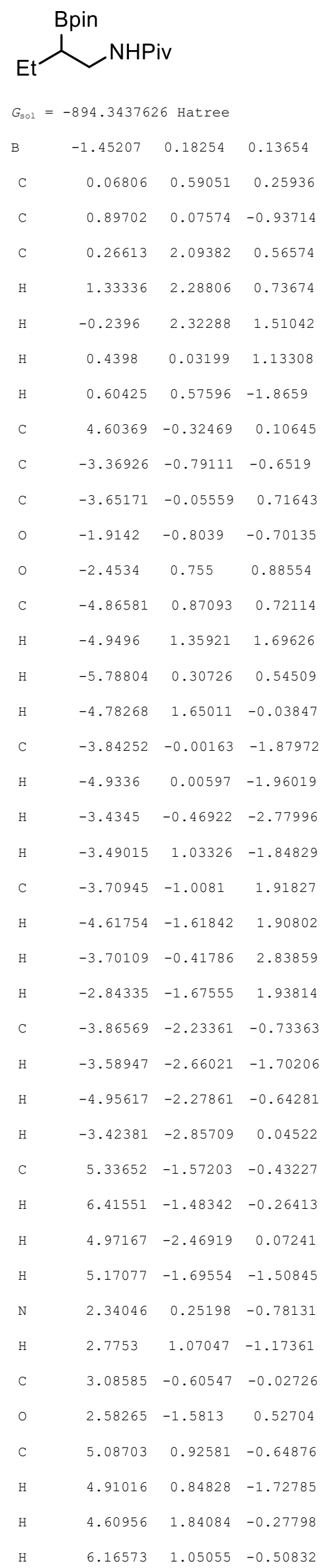

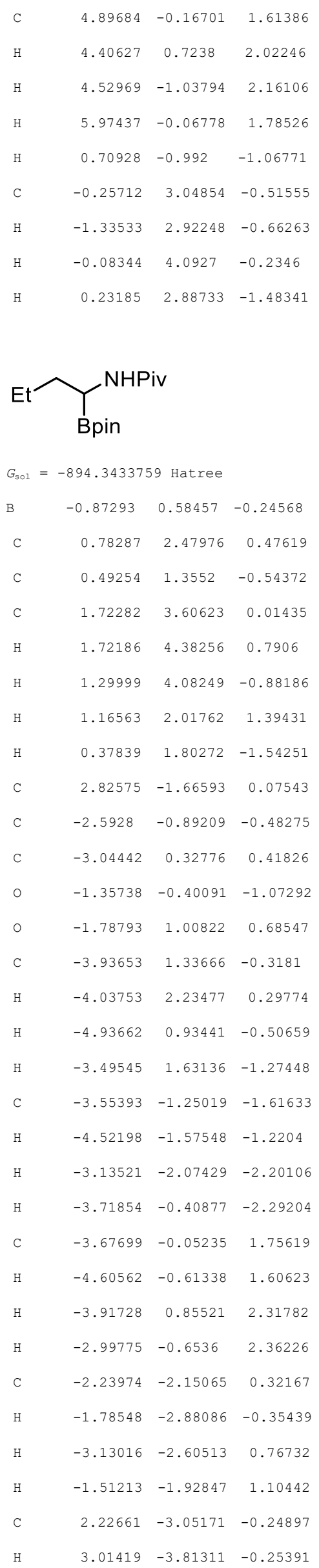




\begin{tabular}{|c|c|c|c|}
\hline $\mathrm{H}$ & 1.47731 & -3.32411 & 0.49739 \\
\hline $\mathrm{H}$ & 1.7442 & -3.05329 & -1.23245 \\
\hline N & 1.58769 & 0.37798 & -0.65798 \\
\hline $\mathrm{H}$ & 2.34709 & 0.55041 & -1.29583 \\
\hline C & 1.64125 & -0.68245 & 0.18289 \\
\hline 0 & 0.74691 & -0.86697 & 1.01751 \\
\hline C & 3.85943 & -1.28452 & -0.99872 \\
\hline $\mathrm{H}$ & 3.41978 & -1.26628 & -2.00257 \\
\hline $\mathrm{H}$ & 4.32181 & -0.31109 & -0.79748 \\
\hline $\mathrm{H}$ & 4.66594 & -2.02461 & -1.01476 \\
\hline C & 3.50588 & -1.70932 & 1.46076 \\
\hline $\mathrm{H}$ & 3.96243 & -0.74479 & 1.70989 \\
\hline $\mathrm{H}$ & 2.77334 & -1.94815 & 2.23478 \\
\hline $\mathrm{H}$ & 4.29445 & -2.4695 & 1.47216 \\
\hline C & 3.17778 & 3.20859 & -0.26673 \\
\hline $\mathrm{H}$ & 3.27062 & 2.5705 & -1.15246 \\
\hline $\mathrm{H}$ & 3.79568 & 4.09426 & -0.44984 \\
\hline $\mathrm{H}$ & 3.61415 & 2.66742 & 0.58037 \\
\hline & -0.17832 & 2.92978 & 0.7486 \\
\hline
\end{tabular}

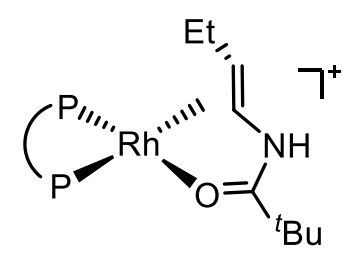

$$
\begin{array}{llll}
G_{\text {sol }}= & -2670.545887 & \text { Hatree } & \\
\mathrm{Fe} & -3.43514 & -0.70787 & -0.10338 \\
\mathrm{P} & -0.19179 & -1.72866 & 0.50308 \\
\mathrm{P} & -0.89122 & 1.72393 & -0.5338 \\
\mathrm{C} & -1.93889 & -2.10548 & 0.10958 \\
\mathrm{C} & -3.00868 & -2.39292 & 1.02762 \\
\mathrm{H} & -2.9422 & -2.40203 & 2.10539 \\
\mathrm{C} & -4.19957 & -2.62135 & 0.28097 \\
\mathrm{H} & -5.17708 & -2.81873 & 0.69904 \\
\mathrm{C} & -3.88783 & -2.49201 & -1.10162 \\
\mathrm{H} & -4.58576 & -2.57924 & -1.92293 \\
\mathrm{C} & -2.5047 & -2.17388 & -1.21454 \\
\mathrm{H} & -1.98006 & -1.99764 & -2.14021 \\
\mathrm{C} & -2.61888 & 1.18526 & -0.24026 \\
\mathrm{C} & -3.20399 & 0.97005 & 1.05915 \\
\mathrm{H} & -2.67336 & 0.98956 & 1.99894 \\
\mathrm{C} & -4.59473 & 0.70932 & 0.89342 \\
\mathrm{H} & -5.29308 & 0.47491 & 1.68488 \\
\mathrm{C} & -4.88884 & 0.74948 & -0.49962 \\
\mathrm{H} & -5.84847 & 0.54707 & -0.95504
\end{array}
$$

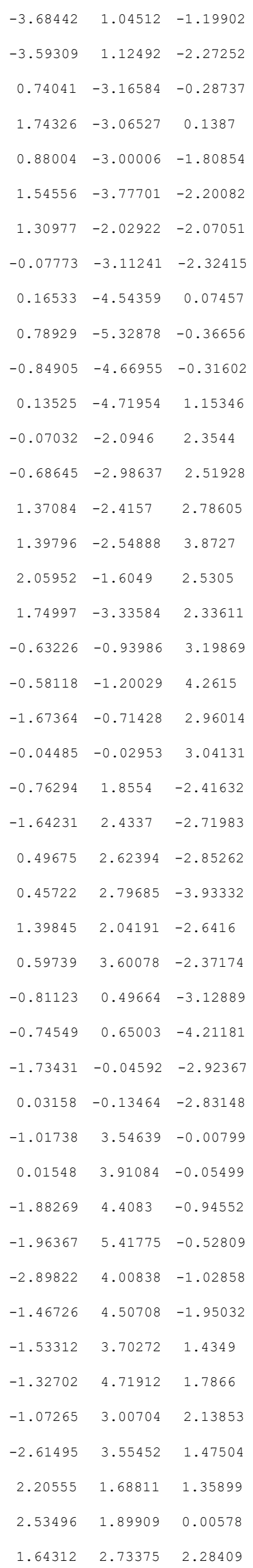




\begin{tabular}{|c|c|c|c|}
\hline $\mathrm{H}$ & 0.87671 & 2.28912 & 2.92835 \\
\hline $\mathrm{H}$ & 1.16516 & 3.53394 & 1.7155 \\
\hline $\mathrm{H}$ & 2.77842 & 0.91941 & 1.87885 \\
\hline $\mathrm{H}$ & 2.32557 & 2.84094 & -0.48973 \\
\hline C & 5.086 & -0.84138 & -0.81529 \\
\hline C & 4.77097 & -1.89084 & -1.9051 \\
\hline $\mathrm{H}$ & 5.67509 & -2.46139 & -2.1368 \\
\hline $\mathrm{H}$ & 4.00022 & -2.58668 & -1.56816 \\
\hline $\mathrm{H}$ & 4.4272 & -1.41522 & -2.82979 \\
\hline $\mathrm{N}$ & 3.67722 & 1.2046 & -0.55705 \\
\hline $\mathrm{H}$ & 4.4863 & 1.74419 & -0.83222 \\
\hline C & 3.77915 & -0.1279 & -0.45888 \\
\hline 0 & 2.78234 & -0.79249 & -0.06076 \\
\hline C & 6.17028 & 0.12573 & -1.32632 \\
\hline $\mathrm{H}$ & 5.85883 & 0.65358 & -2.23484 \\
\hline $\mathrm{H}$ & 6.46314 & 0.8593 & -0.56626 \\
\hline $\mathrm{H}$ & 7.06979 & -0.44132 & -1.58019 \\
\hline C & 5.58409 & -1.55095 & 0.46651 \\
\hline $\mathrm{H}$ & 5.81804 & -0.83065 & 1.25793 \\
\hline $\mathrm{H}$ & 4.83418 & -2.24891 & 0.84529 \\
\hline $\mathrm{H}$ & 6.49693 & -2.11091 & 0.24344 \\
\hline $\mathrm{Rh}$ & 0.95066 & 0.40446 & 0.08859 \\
\hline C & 2.75391 & 3.33234 & 3.17037 \\
\hline $\mathrm{H}$ & 3.52694 & 3.81712 & 2.5656 \\
\hline $\mathrm{H}$ & 2.33644 & 4.08242 & 3.84842 \\
\hline H & 3.23612 & 2.56177 & 3.78084 \\
\hline
\end{tabular}

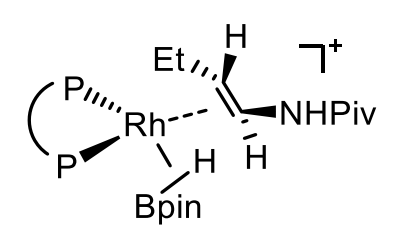

$\begin{array}{llll}G_{\text {sol }}= & -2670.523717 & \text { Hatree } & \\ \mathrm{Fe} & -3.96946 & -0.10339 & -0.47053 \\ \mathrm{P} & -1.35224 & -0.14222 & 1.91109 \\ \mathrm{P} & -0.78723 & 0.68884 & -1.70108 \\ \mathrm{C} & -3.01404 & -0.59193 & 1.28765 \\ \mathrm{C} & -4.27916 & 0.02926 & 1.57888 \\ \mathrm{H} & -4.42813 & 0.95404 & 2.11737 \\ \mathrm{C} & -5.31449 & -0.75534 & 0.99466 \\ \mathrm{H} & -6.36896 & -0.51608 & 1.00571 \\ \mathrm{C} & -4.71384 & -1.87214 & 0.34565 \\ \mathrm{H} & -5.2329 & -2.63404 & -0.21935 \\ \mathrm{C} & -3.30256 & -1.7758 & 0.51717 \\ \mathrm{H} & -2.56976 & -2.46574 & 0.12381\end{array}$

\begin{tabular}{|c|c|c|c|}
\hline C & -2.62231 & 0.71444 & -1.78967 \\
\hline & -3.48857 & 1.73688 & -1.25794 \\
\hline & -3.18055 & 2.59278 & -0.68114 \\
\hline & -4.83233 & 1.42679 & -1.60933 \\
\hline & -5.70706 & 1.99315 & -1.32037 \\
\hline & -4.82459 & 0.2171 & -2.35808 \\
\hline & -5.69203 & -0.30448 & -2.73854 \\
\hline & -3.47602 & -0.22069 & -2.47838 \\
\hline & -3.16552 & -1.12255 & -2.98285 \\
\hline & -1.21451 & -1.38719 & 3.34369 \\
\hline & -0.24666 & -1.16589 & 3.80563 \\
\hline & -1.19302 & -2.84103 & 2.8384 \\
\hline & -0.86761 & -3.50765 & 3.64393 \\
\hline & -0.52175 & -2.98958 & 1.98599 \\
\hline & -2.1904 & -3.16244 & 2.52847 \\
\hline & -2.30133 & -1.23057 & 4.42094 \\
\hline & -2.17171 & -2.01009 & 5.17973 \\
\hline & -3.3047 & -1.34558 & 4.00029 \\
\hline & -2.25161 & -0.26884 & 4.93653 \\
\hline & -1.63451 & 1.48584 & 2.82957 \\
\hline & -2.53508 & 1.31533 & 3.42963 \\
\hline & -0.47859 & 1.82409 & 3.78344 \\
\hline & -0.74164 & 2.71663 & 4.36102 \\
\hline & 0.43425 & 2.04991 & 3.22535 \\
\hline & -0.25917 & 1.02835 & 4.49974 \\
\hline & -1.89262 & 2.66945 & 1.8901 \\
\hline & -2.02296 & 3.58226 & 2.4818 \\
\hline & -2.79489 & 2.52744 & 1.29601 \\
\hline & -1.04742 & 2.82355 & 1.21284 \\
\hline & -0.36228 & -0.68961 & -2.92665 \\
\hline & -1.0594 & -0.54265 & -3.75889 \\
\hline & 1.07157 & -0.59197 & -3.47632 \\
\hline & 1.19697 & -1.33616 & -4.27045 \\
\hline & 1.82001 & -0.81072 & -2.71082 \\
\hline & 1.29734 & 0.38389 & -3.9122 \\
\hline & -0.60139 & -2.08446 & -2.3262 \\
\hline & -0.40158 & -2.84919 & -3.0852 \\
\hline & -1.62458 & -2.22445 & -1.97213 \\
\hline & 0.07396 & -2.25751 & -1.48283 \\
\hline & -0.35049 & 2.26148 & -2.66769 \\
\hline & 0.74302 & 2.2428 & -2.70052 \\
\hline & -0.91236 & 2.2592 & -4.10001 \\
\hline & -0.62912 & 3.19446 & -4.59527 \\
\hline & -2.00551 & 2.20905 & -4.09835 \\
\hline & -0.53319 & 1.44054 & -4.71495 \\
\hline
\end{tabular}




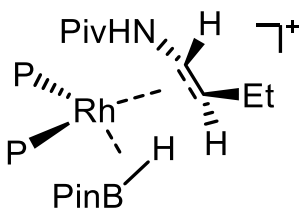

$G_{\text {sol }}=-2670.52444$ Hatree$$
\mathrm{F}
$$$$
\text { P }
$$

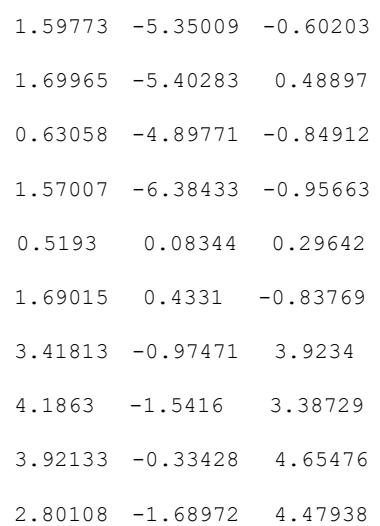

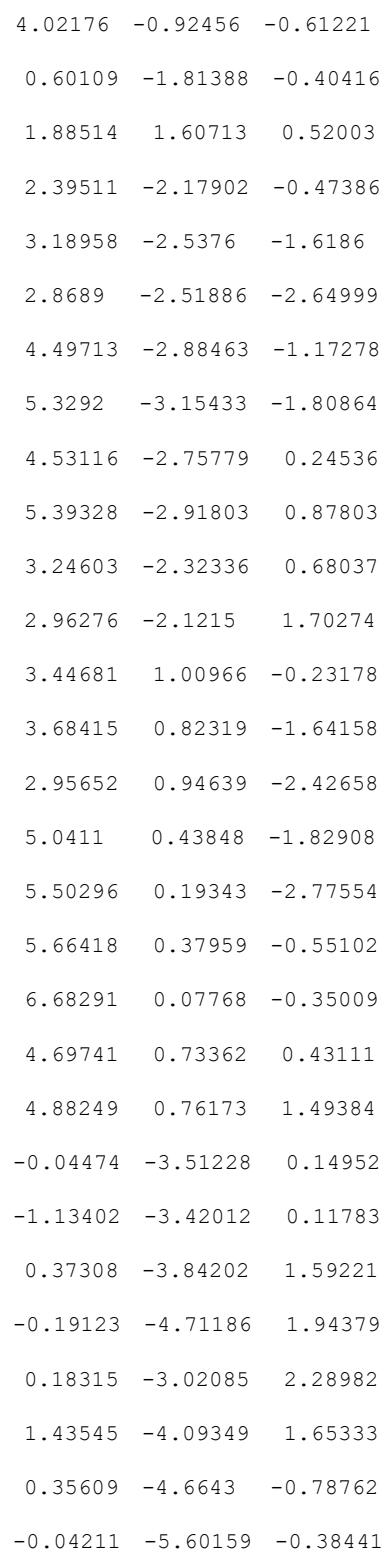




\begin{tabular}{|c|c|c|c|}
\hline & 1.44225 & -4.77156 & -0.86253 \\
\hline & -0.05131 & -4.55333 & -1.79544 \\
\hline & 0.06122 & -1.75304 & -2.21154 \\
\hline & 0.50403 & -2.64226 & -2.6732 \\
\hline & -1.46689 & -1.85563 & -2.33027 \\
\hline & -1.74462 & -1.85321 & -3.38993 \\
\hline & -1.95143 & -0.99649 & -1.85626 \\
\hline & -1.87402 & -2.76438 & -1.88311 \\
\hline & 0.56545 & -0.515 & -2.96246 \\
\hline & 0.18341 & -0.5333 & -3.98926 \\
\hline & 1.65364 & -0.48385 & -3.01548 \\
\hline & 0.21149 & 0.40489 & -2.48714 \\
\hline & 2.37251 & 1.86815 & 2.32968 \\
\hline & 3.34194 & 2.37584 & 2.2792 \\
\hline & 1.39114 & 2.78995 & 3.07472 \\
\hline & 1.7801 & 2.99144 & 4.07849 \\
\hline & 0.41041 & 2.3201 & 3.18757 \\
\hline & 1.24724 & 3.75364 & 2.58051 \\
\hline & 2.5532 & 0.54951 & 3.09904 \\
\hline & 2.83916 & 0.76701 & 4.1341 \\
\hline & 3.32585 & -0.08729 & 2.66726 \\
\hline & 1.62281 & -0.02363 & 3.12617 \\
\hline & 1.81719 & 3.37946 & -0.1567 \\
\hline & 0.88108 & 3.77587 & 0.24627 \\
\hline & 2.99813 & 4.24794 & 0.31294 \\
\hline & 2.92138 & 5.23421 & -0.15746 \\
\hline & 3.95729 & 3.81658 & 0.00958 \\
\hline & 3.01965 & 4.40694 & 1.39294 \\
\hline & 1.72649 & 3.43294 & -1.6916 \\
\hline & 1.42632 & 4.44292 & -1.99002 \\
\hline & 0.98841 & 2.73989 & -2.09584 \\
\hline & 2.69845 & 3.23536 & -2.15007 \\
\hline & -1.84797 & -0.19525 & 2.30744 \\
\hline & -2.28608 & -1.18848 & 1.45819 \\
\hline & -1.10117 & -0.52331 & 3.58349 \\
\hline & -0.46072 & 0.31669 & 3.86705 \\
\hline & -0.44564 & -1.38732 & 3.43203 \\
\hline & -1.47879 & 1.82736 & 0.05417 \\
\hline & -2.43619 & 0.71762 & 2.33784 \\
\hline & -1.90638 & -2.19716 & 1.55268 \\
\hline & -5.35669 & -2.05619 & -0.58642 \\
\hline & -3.4376 & 2.95046 & -0.40662 \\
\hline & -2.33386 & 3.34256 & -1.45354 \\
\hline & -2.86114 & 1.73304 & 0.19099 \\
\hline & -1.12186 & 2.83265 & -0.80997 \\
\hline
\end{tabular}

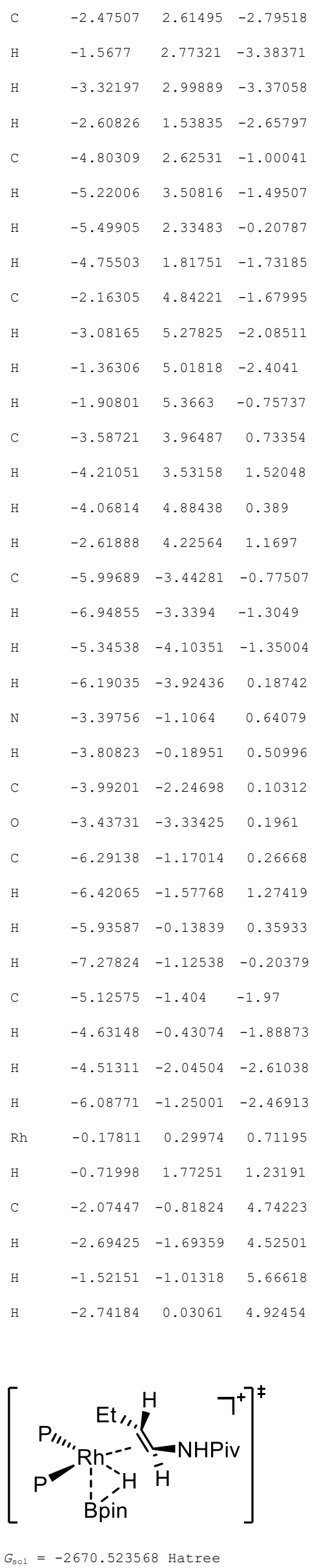




\begin{tabular}{|c|c|c|c|}
\hline $\mathrm{H}$ & -1.63965 & -0.19453 & -0.91827 \\
\hline $\mathrm{Rh}$ & -0.56267 & -0.07961 & 0.22672 \\
\hline B & -1.70698 & -1.74176 & -0.08207 \\
\hline C & 2.96553 & 0.67768 & 1.39564 \\
\hline C & 4.29103 & 0.14228 & 1.55034 \\
\hline $\mathrm{H}$ & 4.54365 & -0.83225 & 1.94261 \\
\hline C & 5.22709 & 1.0951 & 1.0555 \\
\hline $\mathrm{H}$ & 6.29787 & 0.95563 & 0.99775 \\
\hline C & 4.50292 & 2.23307 & 0.59768 \\
\hline $\mathrm{H}$ & 4.9273 & 3.11308 & 0.13445 \\
\hline C & 3.11496 & 1.98121 & 0.7977 \\
\hline $\mathrm{H}$ & 2.30901 & 2.64801 & 0.52873 \\
\hline $\mathrm{C}$ & 2.57199 & -0.13726 & -1.89588 \\
\hline C & 3.48313 & -1.18291 & -1.50508 \\
\hline $\mathrm{H}$ & 3.20749 & -2.12616 & -1.06453 \\
\hline $\mathrm{C}$ & 4.81181 & -0.76382 & -1.79501 \\
\hline $\mathrm{H}$ & 5.71183 & -1.32377 & -1.5814 \\
\hline C & 4.74914 & 0.54129 & -2.35928 \\
\hline $\mathrm{H}$ & 5.59267 & 1.15364 & -2.64712 \\
\hline C & 3.38145 & 0.93005 & -2.42692 \\
\hline $\mathrm{H}$ & 3.02863 & 1.88129 & -2.79564 \\
\hline C & 1.10923 & 0.97806 & 3.55208 \\
\hline $\mathrm{H}$ & 0.16091 & 0.60018 & 3.95135 \\
\hline C & 0.94769 & 2.4814 & 3.2631 \\
\hline $\mathrm{H}$ & 0.5578 & 2.98963 & 4.15132 \\
\hline $\mathrm{H}$ & 0.26477 & 2.68817 & 2.43184 \\
\hline $\mathrm{H}$ & 1.90945 & 2.93931 & 3.01885 \\
\hline C & 2.19843 & 0.77075 & 4.61765 \\
\hline $\mathrm{H}$ & 1.98958 & 1.41119 & 5.48157 \\
\hline $\mathrm{H}$ & 3.18694 & 1.04535 & 4.23684 \\
\hline $\mathrm{H}$ & 2.24535 & -0.25777 & 4.98291 \\
\hline C & 1.81724 & -1.72856 & 2.62211 \\
\hline $\mathrm{H}$ & 2.69684 & -1.56529 & 3.25459 \\
\hline C & 0.69222 & -2.30903 & 3.49251 \\
\hline $\mathrm{H}$ & 1.01977 & -3.26545 & 3.91353 \\
\hline $\mathrm{H}$ & -0.19918 & -2.50227 & 2.88979 \\
\hline $\mathrm{H}$ & 0.4133 & -1.66492 & 4.33027 \\
\hline C & 2.18464 & -2.73543 & 1.52407 \\
\hline $\mathrm{H}$ & 2.43433 & -3.69672 & 1.98721 \\
\hline $\mathrm{H}$ & 3.05094 & -2.4141 & 0.94626 \\
\hline $\mathrm{H}$ & 1.34471 & -2.90148 & 0.84277 \\
\hline C & 0.22124 & 1.3046 & -2.806 \\
\hline $\mathrm{H}$ & 0.92277 & 1.36225 & -3.64577 \\
\hline C & -1.20596 & 1.21332 & -3.37304 \\
\hline $\mathrm{H}$ & -1.39812 & 2.09833 & -3.98959 \\
\hline
\end{tabular}

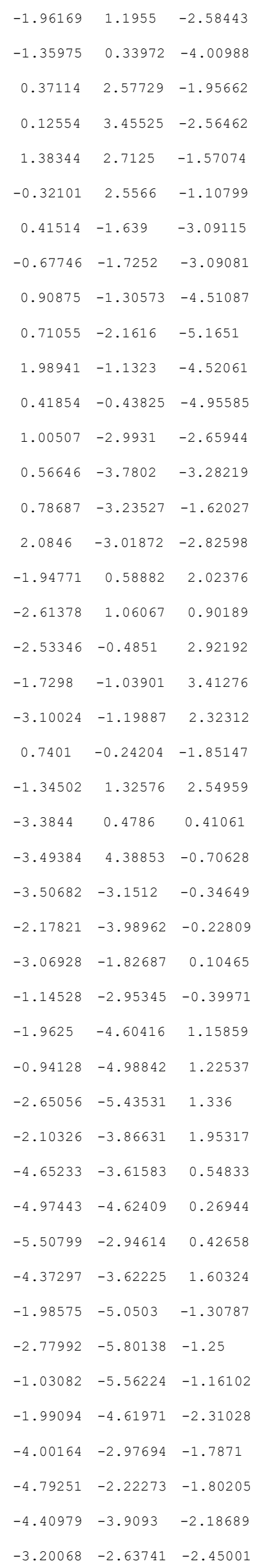




\begin{tabular}{|c|c|c|c|}
\hline C & -4.92484 & 4.73123 & -0.22175 \\
\hline $\mathrm{H}$ & -5.11324 & 5.80131 & -0.35308 \\
\hline $\mathrm{H}$ & -5.66915 & 4.17396 & -0.79544 \\
\hline $\mathrm{H}$ & -5.05771 & 4.49293 & 0.83921 \\
\hline N & -2.56967 & 2.3859 & 0.49772 \\
\hline $\mathrm{H}$ & -2.08483 & 3.05087 & 1.08342 \\
\hline C & -3.35248 & 2.86124 & -0.55951 \\
\hline 0 & -3.93756 & 2.07608 & -1.28737 \\
\hline C & -2.46511 & 5.19536 & 0.10832 \\
\hline $\mathrm{H}$ & -2.59505 & 5.07149 & 1.19061 \\
\hline $\mathrm{H}$ & -1.43293 & 4.94163 & -0.15972 \\
\hline $\mathrm{H}$ & -2.59639 & 6.26172 & -0.09594 \\
\hline C & -3.36169 & 4.73315 & -2.20406 \\
\hline $\mathrm{H}$ & -2.34389 & 4.55319 & -2.56634 \\
\hline $\mathrm{H}$ & -4.04816 & 4.12813 & -2.799 \\
\hline $\mathrm{H}$ & -3.59408 & 5.79016 & -2.36414 \\
\hline P & 1.36677 & -0.0195 & 1.95461 \\
\hline $\mathrm{Fe}$ & 3.88419 & 0.5418 & -0.45168 \\
\hline C & -3.44501 & 0.1322 & 3.99902 \\
\hline $\mathrm{H}$ & -4.30094 & 0.64487 & 3.54806 \\
\hline $\mathrm{H}$ & -3.83195 & -0.64569 & 4.66474 \\
\hline H & -2.90644 & 0.85918 & 4.61747 \\
\hline
\end{tabular}

\section{Bpin}

$\begin{array}{llll}G_{\text {sol }}= & -2670.523226 & \text { Hatree } & \\ \text { Fe } & -3.7413 & -0.93337 & -0.33567 \\ \text { P } & -1.31362 & 0.13157 & 1.96739 \\ \text { P } & -0.67239 & -0.19348 & -1.91479 \\ \text { C } & -2.82632 & -0.79836 & 1.51514 \\ \text { C } & -4.20649 & -0.41154 & 1.62614 \\ \text { H } & -4.57581 & 0.55696 & 1.92968 \\ \text { C } & -5.02065 & -1.50748 & 1.21911 \\ \text { H } & -6.09991 & -1.50061 & 1.15195 \\ \text { C } & -4.16485 & -2.58881 & 0.863 \\ \text { H } & -4.47886 & -3.55124 & 0.48288 \\ \text { C } & -2.81881 & -2.15734 & 1.03628 \\ \text { H } & -1.93704 & -2.74378 & 0.82612 \\ \text { C } & -2.49697 & -0.3488 & -1.88117 \\ \text { C } & -3.4091 & 0.71113 & -1.53888 \\ \text { H } & -3.12927 & 1.68741 & -1.17772 \\ \text { C } & -4.74002 & 0.24949 & -1.7413\end{array}$

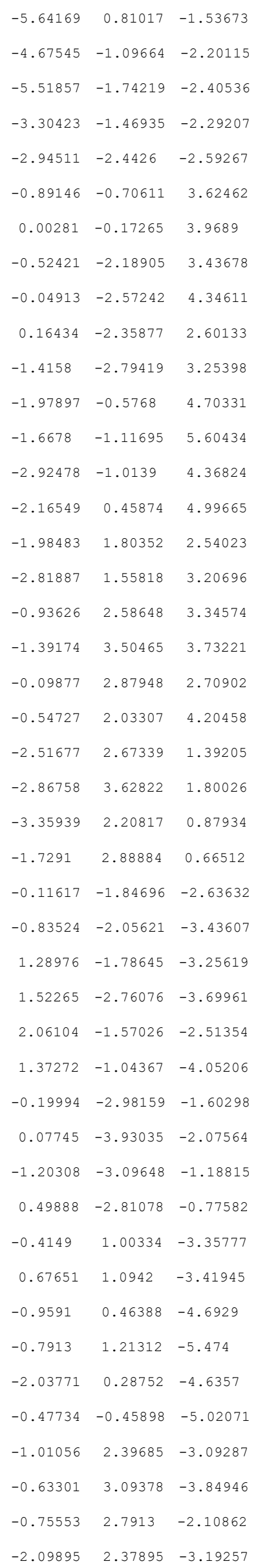




\begin{tabular}{|c|c|c|c|}
\hline$\gamma$ & 2.12128 & -0.0709 & 1.93683 \\
\hline & 2.809 & -0.64882 & 0.87887 \\
\hline 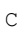 & 2.60299 & 1.21118 & 2.58903 \\
\hline $\mathrm{H}$ & 1.75691 & 1.76321 & 3.00743 \\
\hline $\mathrm{H}$ & 3.06912 & 1.84952 & 1.83538 \\
\hline & 1.30967 & 1.94274 & -0.26643 \\
\hline 4 & 1.62916 & -0.76212 & 2.61835 \\
\hline 1 & 3.49682 & -0.07812 & 0.26582 \\
\hline$C$ & 4.00322 & -4.09415 & -0.19924 \\
\hline$C$ & 2.72679 & 3.64216 & -0.91511 \\
\hline$C$ & 1.41392 & 4.25025 & -0.27578 \\
\hline D & 2.61956 & 2.22158 & -0.56781 \\
\hline O & 0.52972 & 3.07128 & -0.18212 \\
\hline$C$ & 1.60446 & 4.78007 & 1.14883 \\
\hline $\mathrm{H}$ & 0.62869 & 5.03434 & 1.57067 \\
\hline $\mathrm{H}$ & 2.2193 & 5.6843 & 1.15392 \\
\hline $\mathrm{H}$ & 2.07612 & 4.04176 & 1.80106 \\
\hline 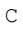 & 4.03662 & 4.1778 & -0.34085 \\
\hline H & 4.14629 & 5.24521 & -0.55677 \\
\hline $\mathrm{H}$ & 4.87761 & 3.65455 & -0.80363 \\
\hline 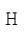 & 4.10287 & 4.03618 & 0.73919 \\
\hline $\mathcal{C}$ & 0.71134 & 5.31031 & -1.12111 \\
\hline $\mathrm{H}$ & 1.34589 & 6.19589 & -1.22678 \\
\hline $\mathrm{H}$ & -0.21344 & 5.62016 & -0.62667 \\
\hline $\mathrm{H}$ & 0.4589 & 4.94733 & -2.11755 \\
\hline C & 2.76557 & 3.71422 & -2.44553 \\
\hline H & 3.60934 & 3.12084 & -2.80705 \\
\hline $\mathrm{H}$ & 2.89569 & 4.74254 & -2.7944 \\
\hline $\mathrm{H}$ & 1.85281 & 3.31179 & -2.89196 \\
\hline C & 5.47374 & -4.20713 & 0.2752 \\
\hline $\mathrm{H}$ & 5.77061 & -5.25982 & 0.31204 \\
\hline $\mathrm{H}$ & 6.1413 & -3.68162 & -0.41181 \\
\hline $\mathrm{H}$ & 5.60758 & -3.78427 & 1.27694 \\
\hline N & 2.90848 & -2.02278 & 0.70689 \\
\hline $\mathrm{H}$ & 2.52869 & -2.62401 & 1.42408 \\
\hline$C$ & 3.70381 & -2.58583 & -0.29604 \\
\hline o & 4.1753 & -1.87712 & -1.16989 \\
\hline C & 3.08449 & -4.8545 & 0.77588 \\
\hline $\mathrm{H}$ & 3.22251 & -4.53942 & 1.81767 \\
\hline $\mathrm{H}$ & 2.0256 & -4.75636 & 0.50986 \\
\hline $\mathrm{H}$ & 3.32558 & -5.92086 & 0.7446 \\
\hline $\mathrm{C}$ & 3.87204 & -4.69208 & -1.61494 \\
\hline $\mathrm{H}$ & 2.83266 & -4.67451 & -1.95991 \\
\hline 1 & 4.47751 & -4.12873 & -2.32711 \\
\hline & 4.2082 & -5.73311 & -1.6118 \\
\hline
\end{tabular}

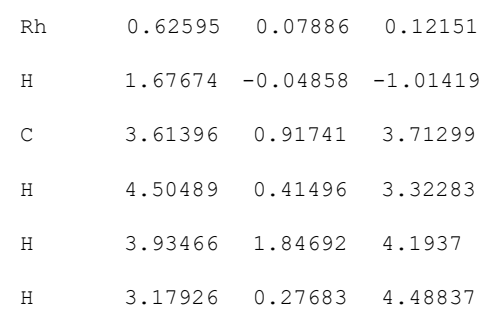

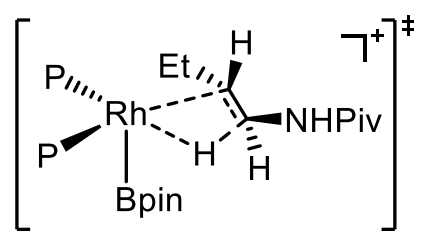

$G_{\text {sol }}=-2670.513244$ Hatree

\begin{tabular}{|c|c|c|c|}
\hline $\mathrm{Rh}$ & 0.50299 & 0.10815 & 0.29558 \\
\hline $\mathrm{H}$ & 1.92284 & -0.20193 & -0.46087 \\
\hline C & 2.78687 & -0.25 & 0.8077 \\
\hline C & 1.98441 & 0.24387 & 1.89434 \\
\hline C & -4.28166 & -0.52879 & 1.51235 \\
\hline $\mathrm{H}$ & -4.74245 & 0.43983 & 1.63726 \\
\hline C & -4.98437 & -1.74343 & 1.26984 \\
\hline $\mathrm{H}$ & -6.05632 & -1.84076 & 1.16585 \\
\hline C & -4.02912 & -2.79009 & 1.13236 \\
\hline $\mathrm{H}$ & -4.24605 & -3.82597 & 0.91082 \\
\hline C & -2.73156 & -2.2221 & 1.27795 \\
\hline $\mathrm{H}$ & -1.80113 & -2.76484 & 1.2117 \\
\hline C & -2.39868 & -0.88947 & -1.88318 \\
\hline C & -3.4137 & 0.1243 & -1.7517 \\
\hline $\mathrm{H}$ & -3.23981 & 1.16694 & -1.53727 \\
\hline C & -4.68918 & -0.48239 & -1.93163 \\
\hline $\mathrm{H}$ & -5.64447 & 0.01895 & -1.85828 \\
\hline C & -4.48757 & -1.87298 & -2.16289 \\
\hline $\mathrm{H}$ & -5.26158 & -2.61714 & -2.2917 \\
\hline C & -3.08664 & -2.12806 & -2.13775 \\
\hline $\mathrm{H}$ & -2.63145 & -3.10004 & -2.26019 \\
\hline C & -0.99621 & -0.23728 & 3.61288 \\
\hline $\mathrm{H}$ & -0.1426 & 0.40069 & 3.86728 \\
\hline C & -0.52678 & -1.70347 & 3.6394 \\
\hline $\mathrm{H}$ & -0.00305 & -1.90585 & 4.57985 \\
\hline $\mathrm{H}$ & 0.1527 & -1.95404 & 2.81805 \\
\hline $\mathrm{H}$ & -1.37673 & -2.38799 & 3.5851 \\
\hline C & -2.09706 & -0.03745 & 4.66732 \\
\hline $\mathrm{H}$ & -1.75745 & -0.43745 & 5.62906 \\
\hline $\mathrm{H}$ & -3.01093 & -0.57408 & 4.39353 \\
\hline $\mathrm{H}$ & -2.35056 & 1.01265 & 4.82694 \\
\hline C & -2.31121 & 1.98524 & 2.18938 \\
\hline
\end{tabular}




\begin{tabular}{|c|c|c|c|}
\hline $\mathrm{H}$ & -3.12951 & 1.74593 & 2.87794 \\
\hline C & -1.35094 & 2.95928 & 2.89125 \\
\hline $\mathrm{H}$ & -1.87918 & 3.89497 & 3.10399 \\
\hline $\mathrm{H}$ & -0.50108 & 3.19735 & 2.24901 \\
\hline $\mathrm{H}$ & -0.97299 & 2.57839 & 3.84358 \\
\hline C & -2.90642 & 2.63577 & 0.93148 \\
\hline $\mathrm{H}$ & -3.3967 & 3.57418 & 1.21474 \\
\hline $\mathrm{H}$ & -3.65742 & 2.00451 & 0.4546 \\
\hline $\mathrm{H}$ & -2.12453 & 2.86953 & 0.20616 \\
\hline C & 0.14463 & -2.25772 & -2.27212 \\
\hline $\mathrm{H}$ & -0.50326 & -2.66677 & -3.05519 \\
\hline C & 1.57041 & -2.14125 & -2.83441 \\
\hline $\mathrm{H}$ & 1.95258 & -3.14454 & -3.05229 \\
\hline $\mathrm{H}$ & 2.26062 & -1.67448 & -2.12787 \\
\hline $\mathrm{H}$ & 1.61584 & -1.57291 & -3.76537 \\
\hline C & 0.11942 & -3.21837 & -1.07234 \\
\hline $\mathrm{H}$ & 0.50291 & -4.19908 & -1.37514 \\
\hline $\mathrm{H}$ & -0.8864 & -3.36305 & -0.67325 \\
\hline $\mathrm{H}$ & 0.76429 & -2.85238 & -0.26396 \\
\hline C & -0.34538 & 0.41016 & -3.43285 \\
\hline $\mathrm{H}$ & 0.73775 & 0.58273 & -3.45948 \\
\hline C & -0.77176 & -0.35519 & -4.69784 \\
\hline $\mathrm{H}$ & -0.60342 & 0.27981 & -5.57444 \\
\hline $\mathrm{H}$ & -1.8386 & -0.59766 & -4.66976 \\
\hline $\mathrm{H}$ & -0.21541 & -1.28082 & -4.85704 \\
\hline C & -1.05877 & 1.77255 & -3.39971 \\
\hline $\mathrm{H}$ & -0.69862 & 2.38729 & -4.23218 \\
\hline $\mathrm{H}$ & -0.89131 & 2.32076 & -2.47115 \\
\hline $\mathrm{H}$ & -2.13597 & 1.65033 & -3.5386 \\
\hline C & -2.87013 & -0.80685 & 1.51788 \\
\hline P & -0.59257 & -0.57483 & -1.8341 \\
\hline C & 2.34736 & 1.56776 & 2.55467 \\
\hline $\mathrm{H}$ & 1.46507 & 1.99618 & 3.041 \\
\hline $\mathrm{H}$ & 2.66789 & 2.28134 & 1.79139 \\
\hline B & 0.96646 & 1.96934 & -0.34323 \\
\hline $\mathrm{H}$ & 1.71274 & -0.52324 & 2.62252 \\
\hline $\mathrm{H}$ & 3.46764 & 0.42784 & 0.29956 \\
\hline C & 4.82228 & -3.41697 & 0.17985 \\
\hline C & 2.27683 & 3.64402 & -1.24555 \\
\hline C & 0.92272 & 4.25112 & -0.69097 \\
\hline 0 & 2.25122 & 2.27789 & -0.71319 \\
\hline 0 & 0.1157 & 3.04364 & -0.4124 \\
\hline C & 1.08002 & 5.0001 & 0.63591 \\
\hline $\mathrm{H}$ & 0.09221 & 5.26648 & 1.01978 \\
\hline $\mathrm{H}$ & 1.64855 & 5.92389 & 0.49879 \\
\hline
\end{tabular}

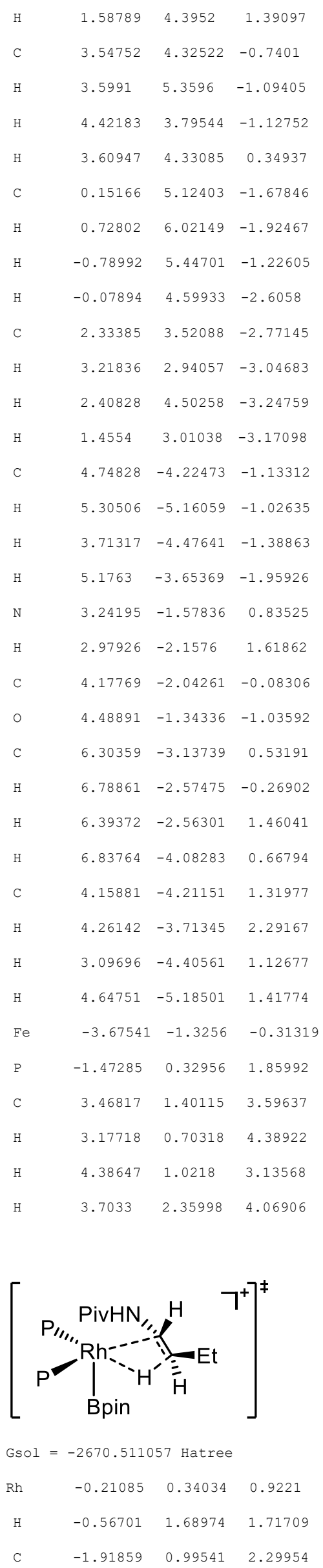




\begin{tabular}{|c|c|c|c|}
\hline 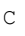 & -2.28827 & -0.20673 & 1.62882 \\
\hline & 5.22074 & -1.23591 & 0.05969 \\
\hline & 5.7985 & -2.13962 & 0.19582 \\
\hline & 4.09408 & -0.84552 & 0.83532 \\
\hline & 3.69729 & -1.40072 & 1.66709 \\
\hline & 1.62307 & -1.93113 & -1.33256 \\
\hline & 1.82577 & -0.9012 & -2.32075 \\
\hline & 1.24413 & 0.00673 & -2.40517 \\
\hline & 2.93549 & -1.27173 & -3.13231 \\
\hline & 3.35599 & -0.68371 & -3.93663 \\
\hline & 3.43683 & -2.5191 & -2.65923 \\
\hline & 4.30436 & -3.04245 & -3.03736 \\
\hline & 2.63138 & -2.93212 & -1.55987 \\
\hline $\mathrm{H}$ & 2.77943 & -3.83411 & -0.98362 \\
\hline & 2.48684 & 1.42771 & 2.83564 \\
\hline & 1.76012 & 2.18373 & 3.15541 \\
\hline r & 2.16982 & 0.1457 & 3.62164 \\
\hline & 2.14848 & 0.36836 & 4.69389 \\
\hline & 1.19588 & -0.27984 & 3.35748 \\
\hline & 2.93113 & -0.62272 & 3.47064 \\
\hline & 3.9022 & 1.92539 & 3.16765 \\
\hline & 4.01297 & 2.0118 & 4.25431 \\
\hline & 4.66384 & 1.22601 & 2.81128 \\
\hline & 4.11938 & 2.90769 & 2.74096 \\
\hline & 2.48166 & 3.0684 & 0.42786 \\
\hline & 3.5591 & 3.17451 & 0.59769 \\
\hline & 1.74533 & 4.11287 & 1.28338 \\
\hline & 1.90824 & 5.10816 & 0.85647 \\
\hline & 0.66424 & 3.93528 & 1.2983 \\
\hline J & 2.09911 & 4.14492 & 2.31604 \\
\hline & 2.19697 & 3.32113 & -1.05915 \\
\hline & 2.62466 & 4.28705 & -1.34952 \\
\hline & 2.60337 & 2.55834 & -1.72391 \\
\hline & 1.12613 & 3.35075 & -1.24246 \\
\hline & 0.53847 & -3.338 & 1.05866 \\
\hline & 0.58545 & -4.22173 & 0.41496 \\
\hline & -0.64926 & -3.50331 & 2.02373 \\
\hline & -0.54427 & -4.44978 & 2.56469 \\
\hline & -0.64553 & -2.70477 & 2.77516 \\
\hline 11 & -1.62802 & -3.50016 & 1.54097 \\
\hline & 1.84401 & -3.28634 & 1.8577 \\
\hline & 1.90644 & -4.18041 & 2.48807 \\
\hline & 2.72795 & -3.26597 & 1.22082 \\
\hline & 1.87155 & -2.42125 & 2.5252 \\
\hline & -1.17466 & -2.6731 & -1.19378 \\
\hline
\end{tabular}

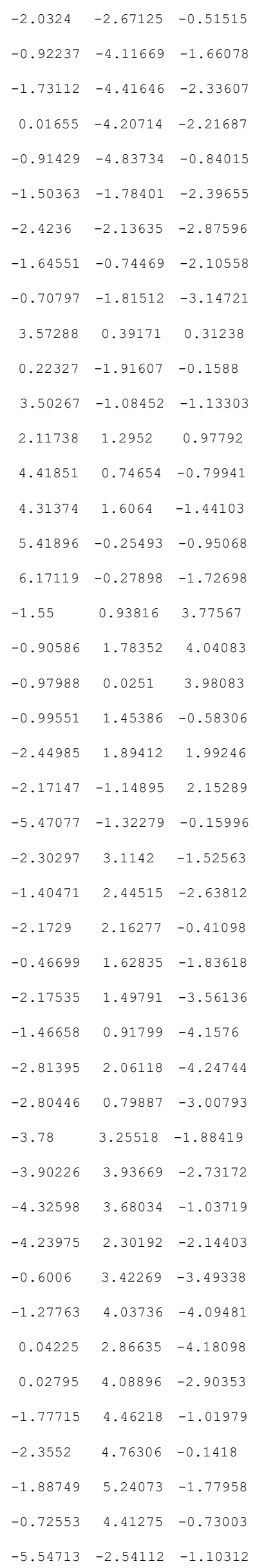




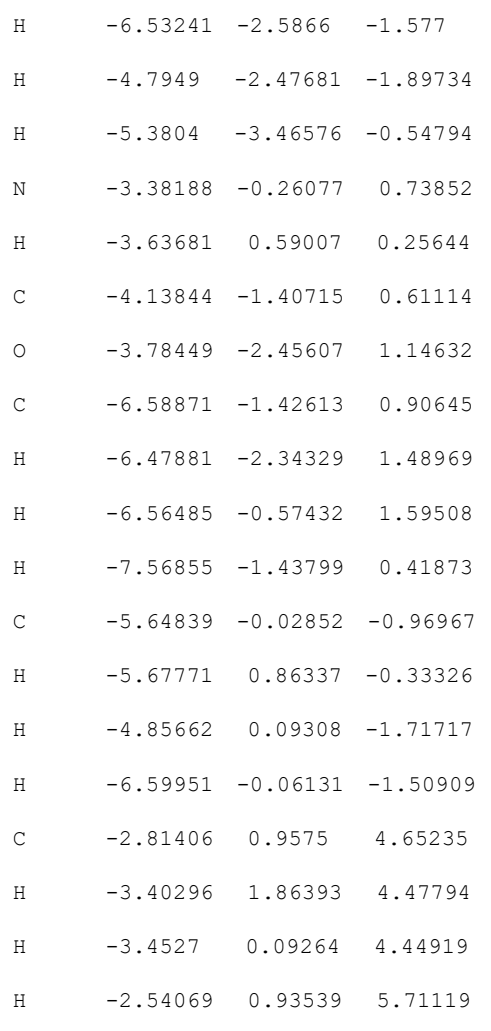

$$
\begin{aligned}
& \begin{array}{llll}
\text { C } & -3.30916 & 3.03838 & 0.06806
\end{array} \\
& \begin{array}{llll}
-3.23957 & 3.66849 & -0.80709
\end{array} \\
& \begin{array}{lll}
-2.03504 & -2.92281 & -1.95542
\end{array} \\
& \begin{array}{lll}
-1.15013 & -3.55853 & -1.86097
\end{array} \\
& \begin{array}{lll}
-1.95404 & -2.17638 & -3.29868
\end{array} \\
& \begin{array}{lll}
-1.72661 & -2.88483 & -4.10186
\end{array} \\
& \begin{array}{lll}
-1.18034 & -1.40287 & -3.31387
\end{array} \\
& \begin{array}{lll}
-2.90897 & -1.70385 & -3.54054
\end{array} \\
& \begin{array}{lll}
-3.28392 & -3.81988 & -1.93869
\end{array} \\
& \begin{array}{lll}
-3.3116 & -4.41527 & -2.85803
\end{array} \\
& \begin{array}{lll}
-4.20304 & -3.22698 & -1.90386
\end{array} \\
& \begin{array}{lll}
-3.29569 & -4.52176 & -1.10172
\end{array} \\
& \begin{array}{lll}
-2.29828 & -2.8942 & 0.96057
\end{array} \\
& \begin{array}{lll}
-3.27381 & -3.31495 & 0.69282
\end{array} \\
& \begin{array}{lll}
-1.28519 & -4.04896 & 1.03422
\end{array} \\
& \begin{array}{lll}
-1.53602 & -4.70075 & 1.87781
\end{array} \\
& \begin{array}{lll}
-0.2688 & -3.68128 & 1.19299
\end{array} \\
& \begin{array}{lll}
-1.2853 & -4.66961 & 0.13441
\end{array} \\
& \begin{array}{lll}
-2.44239 & -2.19273 & 2.32016
\end{array} \\
& \begin{array}{lll}
-2.73095 & -2.93533 & 3.07323
\end{array} \\
& \begin{array}{lll}
-3.21436 & -1.42154 & 2.30866
\end{array} \\
& \begin{array}{lll}
-1.5054 & -1.7314 & 2.63214
\end{array} \\
& \begin{array}{llll}
-0.40663 & 3.01614 & -1.53618
\end{array} \\
& \begin{array}{llll}
-0.88845 & 3.96321 & -1.26883
\end{array} \\
& \begin{array}{llll}
1.06183 & 3.30406 & -1.89463
\end{array} \\
& \begin{array}{lll}
1.09101 & 4.02976 & -2.71442
\end{array} \\
& \begin{array}{llll}
1.59195 & 2.41202 & -2.23483
\end{array} \\
& \begin{array}{llll}
1.63558 & 3.72864 & -1.06819
\end{array} \\
& \begin{array}{llll}
-1.13796 & 2.43937 & -2.75693
\end{array} \\
& \begin{array}{llll}
-0.96987 & 3.08896 & -3.62263
\end{array} \\
& \begin{array}{llll}
-2.21453 & 2.36744 & -2.59889
\end{array} \\
& \begin{array}{lll}
-0.75766 & 1.44526 & -3.02389
\end{array} \\
& \begin{array}{lll}
0.37827 & 2.97483 & 1.29973
\end{array} \\
& \begin{array}{lll}
1.43391 & 2.7973 \quad 1.05936
\end{array} \\
& 0.08438 \quad 4.48318 \quad 1.21796 \\
& 0.62014 \quad 4.99594 \quad 2.02425 \\
& \begin{array}{lll}
-0.9826 & 4.68454 & 1.35781
\end{array} \\
& \begin{array}{lll}
0.4005 & 4.93965 & 0.27839
\end{array} \\
& \begin{array}{lll}
0.11275 & 2.48935 & 2.73407
\end{array} \\
& \begin{array}{lll}
0.83563 & 2.95662 & 3.41227
\end{array} \\
& \begin{array}{lll}
0.18891 & 1.40657 & 2.83702
\end{array} \\
& \begin{array}{lll}
-0.88376 & 2.79093 \quad 3.06798
\end{array} \\
& \begin{array}{lll}
-3.45444 & -0.79928 & -0.69669
\end{array} \\
& \begin{array}{lll}
-0.56028 & 1.94011 & 0.01124
\end{array} \\
& \begin{array}{lll}
1.39744 & -2.59561 & -2.6836
\end{array}
\end{aligned}
$$




\begin{tabular}{|c|c|c|c|}
\hline $\mathrm{H}$ & 0.93598 & -1.94878 & -3.43785 \\
\hline $\mathrm{H}$ & 0.66738 & -3.36957 & -2.43884 \\
\hline B & 1.19146 & -0.72015 & 1.2075 \\
\hline $\mathrm{H}$ & 2.16976 & -2.40875 & -0.64226 \\
\hline $\mathrm{H}$ & 2.30802 & -0.07806 & -2.63516 \\
\hline C & 5.86271 & 1.03086 & -1.10265 \\
\hline C & 2.88667 & -0.97009 & 2.76974 \\
\hline C & 1.50364 & -1.38897 & 3.40176 \\
\hline o & 2.55782 & -0.8863 & 1.34022 \\
\hline 0 & 0.54032 & -0.88738 & 2.4044 \\
\hline C & 1.31525 & -2.90586 & 3.49819 \\
\hline $\mathrm{H}$ & 0.28231 & -3.12721 & 3.77574 \\
\hline $\mathrm{H}$ & 1.96827 & -3.33377 & 4.26368 \\
\hline $\mathrm{H}$ & 1.5292 & -3.40445 & 2.54963 \\
\hline C & 4.00918 & -1.99084 & 2.94637 \\
\hline $\mathrm{H}$ & 4.22811 & -2.14114 & 4.00801 \\
\hline $\mathrm{H}$ & 4.9247 & -1.62675 & 2.47196 \\
\hline $\mathrm{H}$ & 3.75903 & -2.95675 & 2.50495 \\
\hline C & 1.18436 & -0.74569 & 4.74883 \\
\hline $\mathrm{H}$ & 1.90495 & -1.07099 & 5.50586 \\
\hline $\mathrm{H}$ & 0.19055 & -1.05838 & 5.08071 \\
\hline $\mathrm{H}$ & 1.20071 & 0.34351 & 4.70365 \\
\hline C & 3.37025 & 0.41792 & 3.20134 \\
\hline $\mathrm{H}$ & 4.23566 & 0.70384 & 2.5974 \\
\hline $\mathrm{H}$ & 3.67815 & 0.42111 & 4.25051 \\
\hline $\mathrm{H}$ & 2.59712 & 1.17696 & 3.0661 \\
\hline C & 5.90783 & 2.41915 & -0.42503 \\
\hline $\mathrm{H}$ & 6.93232 & 2.6514 & -0.1183 \\
\hline $\mathrm{H}$ & 5.56641 & 3.19379 & -1.11523 \\
\hline $\mathrm{H}$ & 5.2747 & 2.45066 & 0.46938 \\
\hline N & 3.71301 & -0.27134 & -1.15834 \\
\hline $\mathrm{H}$ & 3.99263 & -0.75167 & -0.31442 \\
\hline C & 4.42844 & 0.8292 & -1.62369 \\
\hline o & 3.93126 & 1.57886 & -2.45266 \\
\hline C & 6.32586 & -0.05312 & -0.11423 \\
\hline $\mathrm{H}$ & 5.7222 & -0.06273 & 0.80082 \\
\hline $\mathrm{H}$ & 6.30766 & -1.05318 & -0.56053 \\
\hline $\mathrm{H}$ & 7.35715 & 0.14754 & 0.18999 \\
\hline C & 6.78701 & 1.03075 & -2.34228 \\
\hline $\mathrm{H}$ & 6.79016 & 0.05316 & -2.83603 \\
\hline $\mathrm{H}$ & 6.4591 & 1.77979 & -3.06575 \\
\hline $\mathrm{H}$ & 7.81341 & 1.25913 & -2.03929 \\
\hline $\mathrm{Fe}$ & -3.94315 & 1.05852 & 0.04742 \\
\hline P & -1.89202 & -1.73891 & -0.47491 \\
\hline $\mathrm{C}$ & 2.64061 & -3.27023 & -3.29437 \\
\hline
\end{tabular}
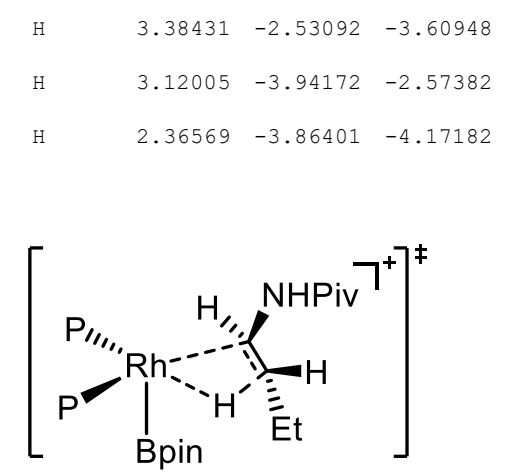

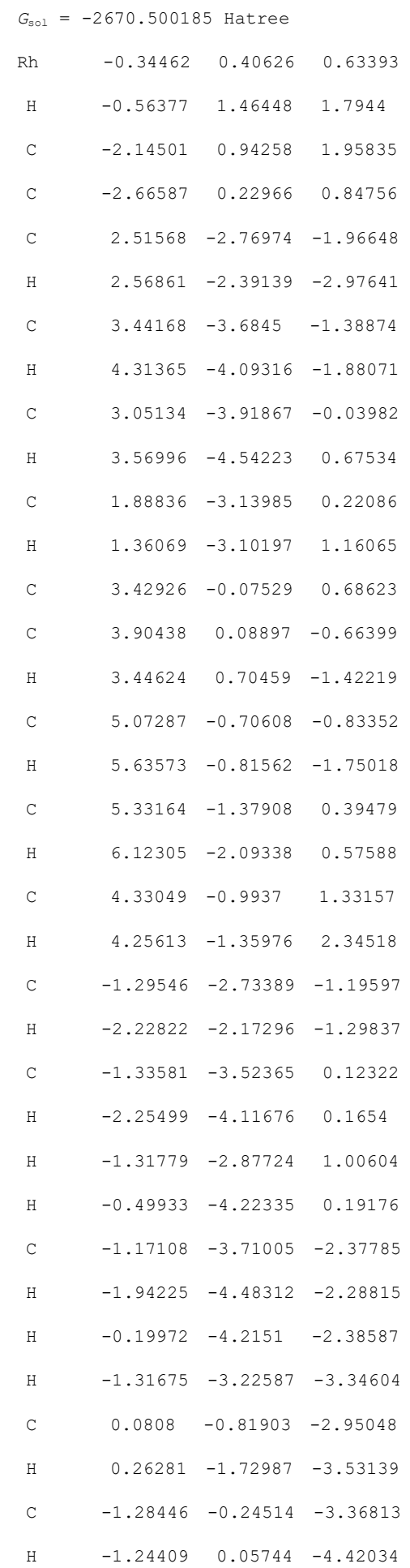




\begin{tabular}{|c|c|c|c|}
\hline $\mathrm{H}$ & -1.55499 & 0.6313 & -2.77319 \\
\hline $\mathrm{H}$ & -2.09883 & -0.96581 & -3.26424 \\
\hline C & 1.22115 & 0.16886 & -3.23723 \\
\hline $\mathrm{H}$ & 1.1716 & 0.48527 & -4.28513 \\
\hline $\mathrm{H}$ & 2.20296 & -0.2775 & -3.07411 \\
\hline $\mathrm{H}$ & 1.14717 & 1.06011 & -2.61192 \\
\hline $\mathrm{C}$ & 1.95323 & 0.24715 & 3.19111 \\
\hline $\mathrm{H}$ & 2.99421 & 0.25264 & 3.53104 \\
\hline C & 1.15013 & 1.19316 & 4.10147 \\
\hline $\mathrm{H}$ & 1.26273 & 0.8708 & 5.14215 \\
\hline $\mathrm{H}$ & 0.08255 & 1.16556 & 3.86562 \\
\hline $\mathrm{H}$ & 1.47692 & 2.23321 & 4.04437 \\
\hline C & 1.40991 & -1.18449 & 3.3208 \\
\hline $\mathrm{H}$ & 1.37741 & -1.47603 & 4.37646 \\
\hline $\mathrm{H}$ & 2.02577 & -1.91139 & 2.7908 \\
\hline $\mathrm{H}$ & 0.38474 & -1.26093 & 2.93357 \\
\hline C & 2.73359 & 2.58619 & 1.55915 \\
\hline $\mathrm{H}$ & 1.88306 & 3.18671 & 1.90414 \\
\hline C & 3.87949 & 2.67383 & 2.58375 \\
\hline $\mathrm{H}$ & 4.27313 & 3.69589 & 2.58907 \\
\hline $\mathrm{H}$ & 4.70485 & 2.00996 & 2.30661 \\
\hline $\mathrm{H}$ & 3.57917 & 2.43868 & 3.60631 \\
\hline C & 3.22456 & 3.1624 & 0.21987 \\
\hline $\mathrm{H}$ & 3.42093 & 4.23245 & 0.34899 \\
\hline $\mathrm{H}$ & 2.49384 & 3.04544 & -0.57816 \\
\hline $\mathrm{H}$ & 4.1657 & 2.69476 & -0.08088 \\
\hline P & 2.00734 & 0.83442 & 1.39201 \\
\hline C & 1.53824 & -2.4122 & -0.97302 \\
\hline C & -2.60602 & 2.35637 & 2.29859 \\
\hline $\mathrm{H}$ & -2.66943 & 2.9423 & 1.38312 \\
\hline $\mathrm{H}$ & -1.87572 & 2.84298 & 2.95593 \\
\hline B & -0.62244 & 2.02791 & -0.55735 \\
\hline $\mathrm{H}$ & -1.98842 & 0.33014 & 2.85358 \\
\hline $\mathrm{H}$ & -3.06807 & 0.7668 & -0.00224 \\
\hline C & -5.25938 & -2.53195 & 0.65023 \\
\hline C & -1.77493 & 3.80405 & -1.50685 \\
\hline C & -0.23976 & 3.87241 & -1.91735 \\
\hline 0 & -1.87935 & 2.50101 & -0.83932 \\
\hline o & 0.36507 & 2.78945 & -1.1207 \\
\hline C & 0.02798 & 3.5693 & -3.39371 \\
\hline $\mathrm{H}$ & 1.10637 & 3.49933 & -3.5586 \\
\hline $\mathrm{H}$ & -0.35794 & 4.37176 & -4.02862 \\
\hline $\mathrm{H}$ & -0.42636 & 2.63098 & -3.71316 \\
\hline C & -2.76366 & 3.80593 & -2.67215 \\
\hline $\mathrm{H}$ & -2.71845 & 4.7527 & -3.21892 \\
\hline
\end{tabular}

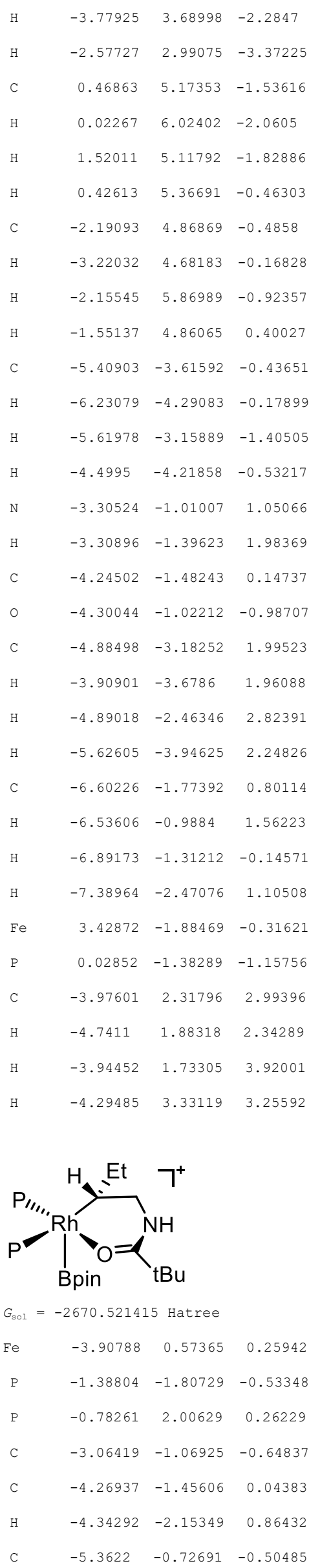




\begin{tabular}{|c|c|c|c|}
\hline $\mathrm{H}$ & -6.3857 & -0.77153 & -0.15874 \\
\hline C & -4.86234 & 0.10985 & -1.54284 \\
\hline $\mathrm{H}$ & -5.43926 & 0.81125 & -2.12975 \\
\hline C & -3.45722 & -0.0986 & -1.63513 \\
\hline $\mathrm{H}$ & -2.7915 & 0.40128 & -2.31953 \\
\hline C & -2.49288 & 1.91952 & 0.91304 \\
\hline $\mathrm{C}$ & -2.9 & 1.04952 & 1.98712 \\
\hline $\mathrm{H}$ & -2.26043 & 0.36009 & 2.51609 \\
\hline C & -4.2957 & 1.22119 & 2.20935 \\
\hline $\mathrm{H}$ & -4.89418 & 0.67918 & 2.92867 \\
\hline C & -4.77399 & 2.18741 & 1.27915 \\
\hline $\mathrm{H}$ & -5.80035 & 2.50789 & 1.16346 \\
\hline C & -3.67286 & 2.62446 & 0.48798 \\
\hline $\mathrm{H}$ & -3.73733 & 3.33706 & -0.32114 \\
\hline C & -1.45722 & -2.91272 & -2.08797 \\
\hline $\mathrm{H}$ & -0.50184 & -3.44695 & -2.08046 \\
\hline C & -1.55001 & -2.08786 & -3.38597 \\
\hline $\mathrm{H}$ & -1.29351 & -2.72179 & -4.24137 \\
\hline $\mathrm{H}$ & -0.88064 & -1.22232 & -3.40286 \\
\hline $\mathrm{H}$ & -2.56829 & -1.72417 & -3.54239 \\
\hline C & -2.58381 & -3.9605 & -2.07461 \\
\hline $\mathrm{H}$ & -2.58613 & -4.48995 & -3.03382 \\
\hline $\mathrm{H}$ & -3.56705 & -3.49595 & -1.95558 \\
\hline $\mathrm{H}$ & -2.4598 & -4.7145 & -1.29449 \\
\hline C & -1.61074 & -3.09536 & 0.8309 \\
\hline $\mathrm{H}$ & -2.53177 & -3.60351 & 0.52608 \\
\hline C & -0.47554 & -4.12939 & 0.82165 \\
\hline $\mathrm{H}$ & -0.68245 & -4.89846 & 1.57393 \\
\hline $\mathrm{H}$ & 0.47766 & -3.66672 & 1.07473 \\
\hline $\mathrm{H}$ & -0.37177 & -4.63881 & -0.14003 \\
\hline C & -1.83981 & -2.52325 & 2.23802 \\
\hline $\mathrm{H}$ & -2.02631 & -3.35455 & 2.92791 \\
\hline $\mathrm{H}$ & -2.70691 & -1.8628 & 2.27803 \\
\hline $\mathrm{H}$ & -0.96477 & -1.97817 & 2.58862 \\
\hline C & -0.93132 & 3.35096 & -1.07425 \\
\hline $\mathrm{H}$ & -1.57644 & 4.11872 & -0.632 \\
\hline C & 0.41273 & 4.00766 & -1.42684 \\
\hline $\mathrm{H}$ & 0.23442 & 4.82831 & -2.13045 \\
\hline $\mathrm{H}$ & 1.08597 & 3.29113 & -1.89947 \\
\hline $\mathrm{H}$ & 0.92303 & 4.42737 & -0.55798 \\
\hline C & -1.60132 & 2.84572 & -2.36104 \\
\hline $\mathrm{H}$ & -1.64187 & 3.65681 & -3.09654 \\
\hline $\mathrm{H}$ & -2.62171 & 2.50029 & -2.1933 \\
\hline $\mathrm{H}$ & -1.02756 & 2.02756 & -2.814 \\
\hline $\mathrm{C}$ & 0.10463 & 2.92482 & 1.66259 \\
\hline
\end{tabular}

\begin{tabular}{|c|c|c|c|}
\hline $\mathrm{H}$ & 1.13664 & 3.00036 & 1.29949 \\
\hline C & -0.44871 & 4.32831 & 1.95631 \\
\hline $\mathrm{H}$ & 0.10293 & 4.77159 & 2.79308 \\
\hline $\mathrm{H}$ & -1.50272 & 4.28067 & 2.24869 \\
\hline $\mathrm{H}$ & -0.36188 & 5.01508 & 1.11173 \\
\hline C & 0.11013 & 2.09233 & 2.95534 \\
\hline $\mathrm{H}$ & 0.82499 & 2.52337 & 3.6652 \\
\hline $\mathrm{H}$ & 0.38151 & 1.04818 & 2.78315 \\
\hline $\mathrm{H}$ & -0.87092 & 2.11139 & 3.43795 \\
\hline C & 1.58896 & -1.54761 & -1.90289 \\
\hline C & 2.17475 & -0.64413 & -2.99126 \\
\hline C & 2.60799 & -2.54119 & -1.32713 \\
\hline $\mathrm{H}$ & 2.11621 & -3.12357 & -0.53991 \\
\hline $\mathrm{H}$ & 3.42736 & -2.00441 & -0.84204 \\
\hline B & 1.54962 & -0.76644 & 0.95635 \\
\hline $\mathrm{H}$ & 0.82438 & -2.12679 & -2.41824 \\
\hline $\mathrm{H}$ & 1.38867 & -0.01285 & -3.43081 \\
\hline C & 4.30108 & 2.10912 & -1.35289 \\
\hline C & 3.40676 & -0.81159 & 2.3509 \\
\hline C & 2.23232 & -1.66807 & 2.98488 \\
\hline O & 2.9007 & -0.50235 & 1.01382 \\
\hline 0 & 1.09189 & -1.35204 & 2.11182 \\
\hline $\mathrm{C}$ & 2.46196 & -3.18129 & 2.90914 \\
\hline $\mathrm{H}$ & 1.56344 & -3.70236 & 3.24827 \\
\hline $\mathrm{H}$ & 3.28927 & -3.48533 & 3.55626 \\
\hline $\mathrm{H}$ & 2.68524 & -3.51143 & 1.89185 \\
\hline C & 4.73223 & -1.55892 & 2.19435 \\
\hline $\mathrm{H}$ & 5.13382 & -1.84703 & 3.17081 \\
\hline $\mathrm{H}$ & 5.46685 & -0.9048 & 1.7165 \\
\hline $\mathrm{H}$ & 4.63063 & -2.45624 & 1.58247 \\
\hline C & 1.85029 & -1.28894 & 4.41545 \\
\hline $\mathrm{H}$ & 2.67926 & -1.48978 & 5.10133 \\
\hline $\mathrm{H}$ & 0.99809 & -1.89302 & 4.73858 \\
\hline $\mathrm{H}$ & 1.57459 & -0.23769 & 4.50663 \\
\hline C & 3.66203 & 0.52063 & 3.06394 \\
\hline $\mathrm{H}$ & 4.41606 & 1.08629 & 2.51079 \\
\hline $\mathrm{H}$ & 4.04204 & 0.36219 & 4.07708 \\
\hline $\mathrm{H}$ & 2.75846 & 1.12938 & 3.12489 \\
\hline C & 4.0033 & 3.01328 & -0.14671 \\
\hline $\mathrm{H}$ & 4.86734 & 3.65595 & 0.04655 \\
\hline $\mathrm{H}$ & 3.13974 & 3.65438 & -0.33265 \\
\hline $\mathrm{H}$ & 3.80206 & 2.42238 & 0.74855 \\
\hline $\mathrm{N}$ & 3.26858 & 0.21828 & -2.54228 \\
\hline $\mathrm{H}$ & 4.15433 & 0.161 & -3.02135 \\
\hline & 3.1175 & 1.15475 & -1.60083 \\
\hline
\end{tabular}



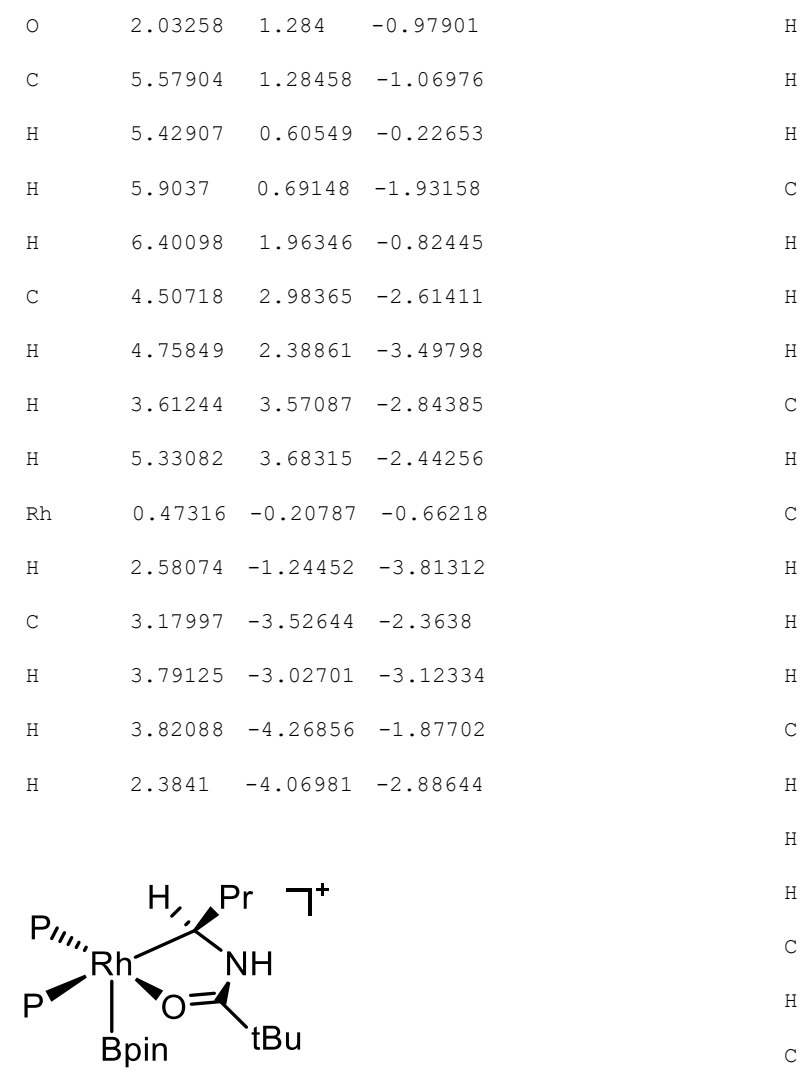

$G_{\text {sol }}=-2670.529178$ Hatree

\begin{tabular}{|c|c|c|c|}
\hline $\mathrm{Fe}$ & 3.88281 & -0.42571 & -0.04512 \\
\hline $\mathrm{P}$ & 1.1703 & 1.67412 & 0.86195 \\
\hline $\mathrm{P}$ & 0.88564 & -1.40254 & -1.47721 \\
\hline $\mathrm{C}$ & 2.94392 & 1.32191 & 0.51432 \\
\hline $\mathrm{C}$ & 3.99677 & 1.01026 & 1.45023 \\
\hline $\mathrm{H}$ & 3.88492 & 0.81615 & 2.50519 \\
\hline $\mathrm{C}$ & 5.23495 & 0.96746 & 0.74946 \\
\hline $\mathrm{H}$ & 6.19296 & 0.71405 & 1.18217 \\
\hline $\mathrm{C}$ & 4.98034 & 1.25875 & -0.61953 \\
\hline $\mathrm{H}$ & 5.71055 & 1.27154 & -1.41684 \\
\hline $\mathrm{C}$ & 3.58216 & 1.47547 & -0.7689 \\
\hline $\mathrm{H}$ & 3.08613 & 1.69868 & -1.69646 \\
\hline $\mathrm{C}$ & 2.55791 & -1.78367 & -0.85615 \\
\hline $\mathrm{C}$ & 2.80993 & -2.07796 & 0.53292 \\
\hline $\mathrm{H}$ & 2.06569 & -2.06928 & 1.31633 \\
\hline $\mathrm{C}$ & 4.19764 & -2.35348 & 0.69048 \\
\hline $\mathrm{H}$ & 4.69893 & -2.57443 & 1.62282 \\
\hline $\mathrm{C}$ & 4.82186 & -2.2211 & -0.58377 \\
\hline $\mathrm{H}$ & 5.87908 & -2.32107 & -0.7884 \\
\hline $\mathrm{C}$ & 3.8188 & -1.88046 & -1.53712 \\
\hline $\mathrm{H}$ & 3.9941 & -1.69279 & -2.58684 \\
\hline $\mathrm{C}$ & 1.12986 & 3.53591 & 0.47616 \\
\hline $\mathrm{H}$ & 0.10576 & 3.82669 & 0.73275 \\
\hline $\mathrm{C}$ & 1.36872 & 3.83881 & -1.01492 \\
\hline
\end{tabular}

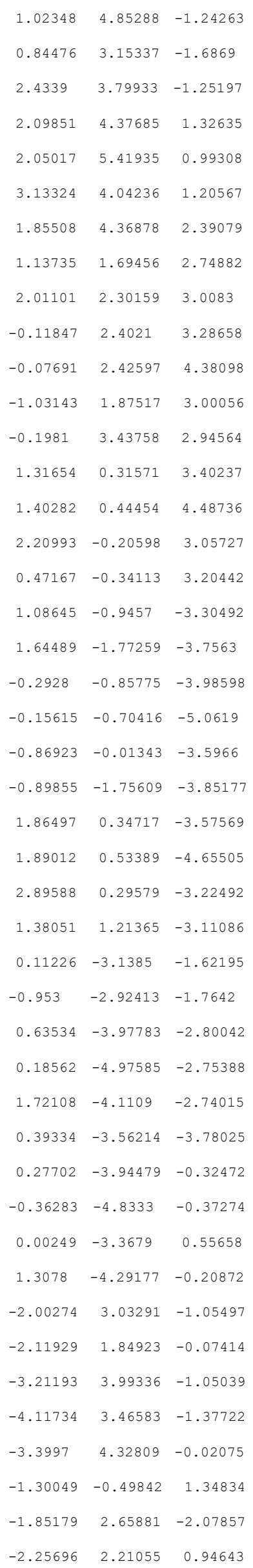



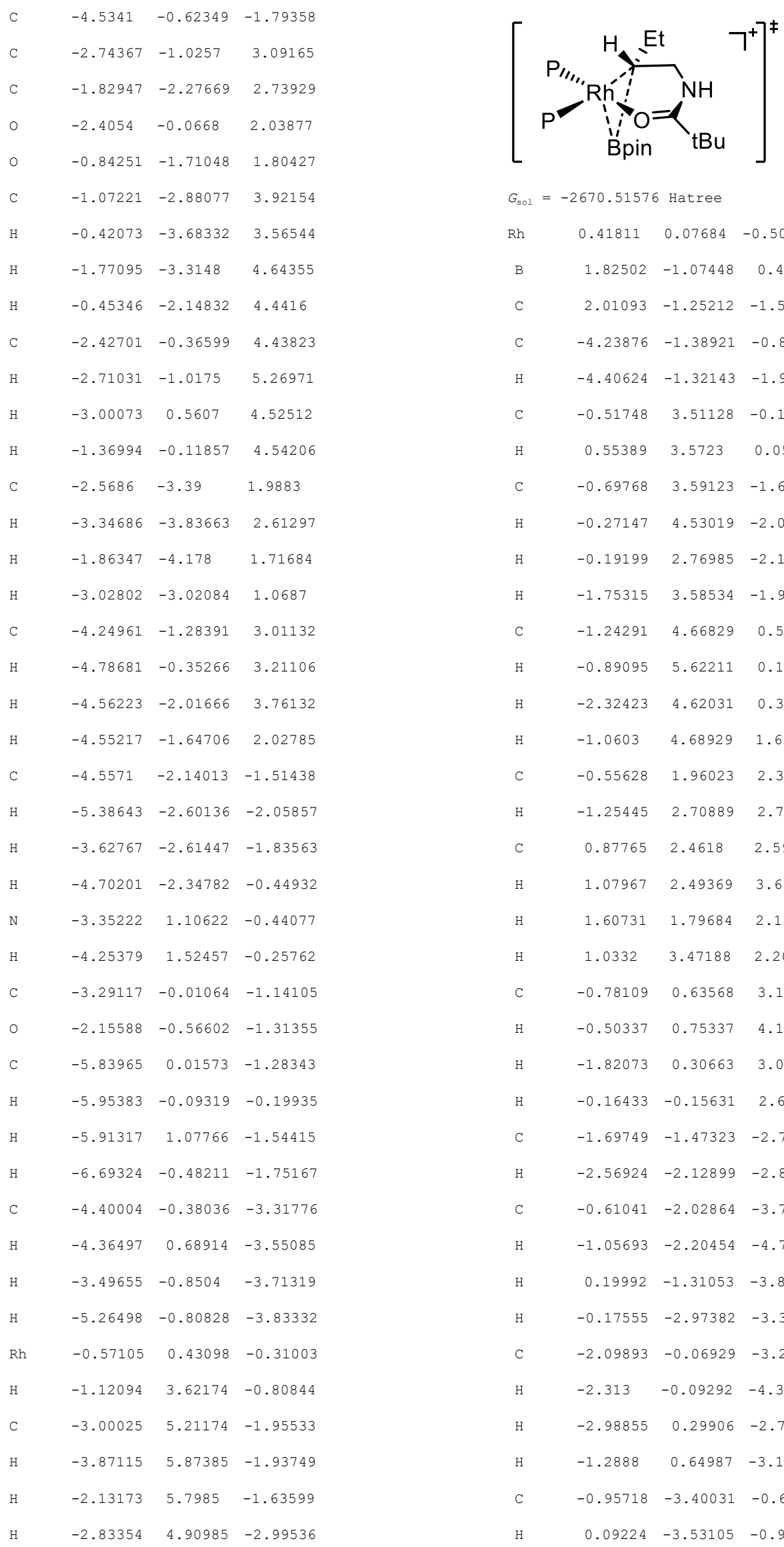

$G_{\text {sol }}=-2670.51576$ Hatree

$$
\begin{aligned}
& \begin{array}{llll}
0.41811 & 0.07684 & -0.50471
\end{array} \\
& \begin{array}{lll}
1.82502 & -1.07448 & 0.49336
\end{array} \\
& 2.01093-1.25212-1.5786 \\
& \begin{array}{llll}
-4.23876 & -1.38921 & -0.85592
\end{array} \\
& \begin{array}{lll}
-4.40624 & -1.32143 & -1.92042
\end{array} \\
& \begin{array}{llll}
-0.51748 & 3.51128 & -0.15195
\end{array} \\
& \begin{array}{llll}
0.55389 & 3.5723 & 0.05223
\end{array} \\
& \begin{array}{llll}
-0.69768 & 3.59123 & -1.67596
\end{array} \\
& \begin{array}{llll}
-0.27147 \quad 4.53019 & -2.04606
\end{array} \\
& \begin{array}{llll}
-0.19199 & 2.76985 & -2.19237
\end{array} \\
& \begin{array}{lll}
-1.75315 & 3.58534 & -1.96197
\end{array} \\
& \begin{array}{lll}
-1.24291 & 4.66829 & 0.55148
\end{array} \\
& \begin{array}{lll}
-0.89095 & 5.62211 & 0.14286
\end{array} \\
& \begin{array}{llll}
-2.32423 & 4.62031 & 0.39163
\end{array} \\
& \begin{array}{lll}
-1.0603 & 4.68929 & 1.62932
\end{array} \\
& \begin{array}{lll}
-0.55628 & 1.96023 & 2.36987
\end{array} \\
& \begin{array}{lll}
-1.25445 & 2.70889 & 2.76184
\end{array} \\
& \begin{array}{lll}
0.87765 & 2.4618 & 2.59699
\end{array} \\
& \begin{array}{lll}
1.07967 & 2.49369 & 3.67325
\end{array} \\
& \begin{array}{lll}
1.60731 & 1.79684 & 2.12995
\end{array} \\
& \begin{array}{lll}
1.0332 & 3.47188 & 2.20994
\end{array} \\
& \begin{array}{lll}
-0.78109 & 0.63568 & 3.11339
\end{array} \\
& \begin{array}{lll}
-0.50337 & 0.75337 & 4.16669
\end{array} \\
& \begin{array}{lll}
-1.82073 & 0.30663 & 3.08052
\end{array} \\
& \begin{array}{lll}
-0.16433 & -0.15631 & 2.68095
\end{array} \\
& \begin{array}{llll}
-1.69749 & -1.47323 & -2.79188
\end{array} \\
& \begin{array}{llll}
-2.56924 & -2.12899 & -2.88313
\end{array} \\
& \begin{array}{lll}
-0.61041 & -2.02864 & -3.73028
\end{array} \\
& -1.05693 \quad-2.20454 \quad-4.71504 \\
& \begin{array}{llll}
0.19992 & -1.31053 & -3.87425
\end{array} \\
& \begin{array}{lll}
-0.17555 & -2.97382 & -3.39876
\end{array} \\
& \begin{array}{lll}
-2.09893 & -0.06929 & -3.26585
\end{array} \\
& \begin{array}{lll}
-2.313 & -0.09292 & -4.34026
\end{array} \\
& \begin{array}{llll}
-2.98855 & 0.29906 & -2.75694
\end{array} \\
& \begin{array}{llll}
-1.2888 & 0.64987 & -3.10539
\end{array} \\
& \begin{array}{llll}
-0.95718 & -3.40031 & -0.6525
\end{array} \\
& \begin{array}{llll}
0.09224 & -3.53105 & -0.93547
\end{array} \\
& \begin{array}{llll}
-1.84737 & -4.32495 & -1.50412
\end{array} \\
& \begin{array}{lll}
-1.62929 & -5.36464 & -1.23714
\end{array}
\end{aligned}
$$




\begin{tabular}{|c|c|c|c|}
\hline $\mathrm{H}$ & -2.90845 & -4.15482 & -1.29455 \\
\hline $\mathrm{H}$ & -1.69034 & -4.22924 & -2.57952 \\
\hline C & -1.10187 & -3.81096 & 0.82401 \\
\hline $\mathrm{H}$ & -0.66137 & -4.80562 & 0.95326 \\
\hline $\mathrm{H}$ & -0.58574 & -3.13326 & 1.50079 \\
\hline $\mathrm{H}$ & -2.15467 & -3.88003 & 1.1087 \\
\hline C & -2.79464 & 1.86642 & 0.52645 \\
\hline $\mathrm{P}$ & -1.28882 & -1.54586 & -0.93393 \\
\hline $\mathrm{Fe}$ & -4.04143 & 0.24515 & 0.40934 \\
\hline P & -0.96442 & 1.80972 & 0.53301 \\
\hline C & -3.68167 & 1.85423 & 1.66125 \\
\hline $\mathrm{H}$ & -5.9096 & 1.89109 & 1.81024 \\
\hline C & -5.02332 & 1.92651 & 1.19179 \\
\hline $\mathrm{H}$ & -3.39454 & 1.76935 & 2.69839 \\
\hline C & -4.99249 & 2.00123 & -0.22892 \\
\hline $\mathrm{H}$ & -5.85183 & 2.03568 & -0.88437 \\
\hline C & -3.63121 & 1.96834 & -0.64322 \\
\hline $\mathrm{H}$ & -3.29169 & 2.00032 & -1.66572 \\
\hline C & -2.96165 & -1.40657 & -0.18724 \\
\hline C & -3.24191 & -1.46261 & 1.22484 \\
\hline $\mathrm{H}$ & -2.50465 & -1.48326 & 2.01218 \\
\hline C & -4.65395 & -1.48004 & 1.40998 \\
\hline $\mathrm{H}$ & -5.16718 & -1.4846 & 2.36174 \\
\hline $\mathrm{H}$ & -6.33035 & -1.38316 & -0.07186 \\
\hline C & -5.2684 & -1.42952 & 0.12648 \\
\hline C & 2.34057 & -0.14252 & -2.59072 \\
\hline C & 3.2519 & -2.17348 & -1.39782 \\
\hline $\mathrm{H}$ & 3.04777 & -2.90987 & -0.62162 \\
\hline $\mathrm{H}$ & 4.10705 & -1.58171 & -1.05792 \\
\hline $\mathrm{H}$ & 1.25467 & -1.88608 & -2.03496 \\
\hline $\mathrm{H}$ & 1.43533 & 0.38039 & -2.92104 \\
\hline C & 4.03819 & 2.83907 & -0.82227 \\
\hline C & 3.43238 & -1.23907 & 2.18143 \\
\hline C & 2.44583 & -2.4968 & 2.24138 \\
\hline 0 & 2.91451 & -0.44544 & 1.07151 \\
\hline o & 1.54027 & -2.27341 & 1.11618 \\
\hline C & 3.12079 & -3.85894 & 2.03875 \\
\hline H & 2.3517 & -4.63454 & 1.98929 \\
\hline $\mathrm{H}$ & 3.78071 & -4.09523 & 2.87886 \\
\hline $\mathrm{H}$ & 3.70905 & -3.91013 & 1.12205 \\
\hline C & 4.89221 & -1.58768 & 1.86522 \\
\hline $\mathrm{H}$ & 5.34497 & -2.15655 & 2.6827 \\
\hline $\mathrm{H}$ & 5.46395 & -0.66277 & 1.74725 \\
\hline H & 4.99625 & -2.16695 & 0.94736 \\
\hline C & 1.59493 & -2.57674 & 3.51502 \\
\hline
\end{tabular}

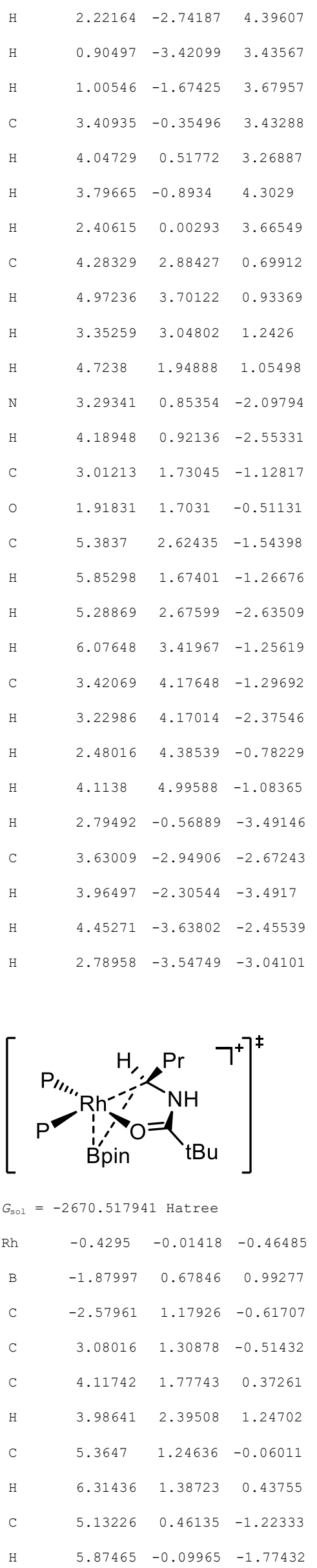




\begin{tabular}{|c|c|c|c|}
\hline C & 3.73886 & 0.5025 & -1.50943 \\
\hline H & 3.25747 & -0.00858 & -2.32435 \\
\hline C & 2.59814 & -1.73004 & 0.75492 \\
\hline C & 2.84679 & -0.9471 & 1.93957 \\
\hline $\mathrm{H}$ & 2.12294 & -0.33144 & 2.45287 \\
\hline C & 4.21928 & -1.08605 & 2.29225 \\
\hline H & 4.71441 & -0.58644 & 3.11348 \\
\hline C & 4.83673 & -1.94888 & 1.34237 \\
\hline $\mathrm{H}$ & 5.88323 & -2.21943 & 1.31268 \\
\hline $\mathrm{C}$ & 3.84874 & -2.35299 & 0.40062 \\
\hline H & 4.02607 & -2.99625 & -0.44862 \\
\hline C & 1.14626 & 2.63182 & -2.16383 \\
\hline $\mathrm{H}$ & 0.18234 & 3.14001 & -2.098 \\
\hline C & 1.07349 & 1.66702 & -3.36038 \\
\hline $\mathrm{H}$ & 0.75142 & 2.2146 & -4.25295 \\
\hline H & 0.36548 & 0.84852 & -3.19397 \\
\hline $\mathrm{H}$ & 2.04986 & 1.23247 & -3.58896 \\
\hline C & 2.22997 & 3.69864 & -2.38961 \\
\hline $\mathrm{H}$ & 2.0486 & 4.19808 & -3.34784 \\
\hline $\mathrm{H}$ & 3.22919 & 3.25694 & -2.4306 \\
\hline $\mathrm{H}$ & 2.22933 & 4.47074 & -1.6163 \\
\hline C & 1.30855 & 3.16538 & 0.7406 \\
\hline $\mathrm{H}$ & 2.22477 & 3.70696 & 0.48051 \\
\hline C & 0.12945 & 4.14155 & 0.60027 \\
\hline $\mathrm{H}$ & 0.27459 & 4.96682 & 1.30641 \\
\hline $\mathrm{H}$ & -0.81727 & 3.66007 & 0.85179 \\
\hline $\mathrm{H}$ & 0.06203 & 4.58577 & -0.39663 \\
\hline C & 1.42262 & 2.66869 & 2.1898 \\
\hline $\mathrm{H}$ & 1.51891 & 3.52889 & 2.86224 \\
\hline $\mathrm{H}$ & 2.28671 & 2.02263 & 2.35345 \\
\hline H & 0.52394 & 2.12014 & 2.46372 \\
\hline C & 1.3271 & -3.01857 & -1.54774 \\
\hline $\mathrm{H}$ & 1.94969 & -3.81511 & -1.12415 \\
\hline C & 0.05432 & -3.65949 & -2.1225 \\
\hline $\mathrm{H}$ & 0.34251 & -4.38922 & -2.88677 \\
\hline $\mathrm{H}$ & -0.57929 & -2.90431 & -2.589 \\
\hline $\mathrm{H}$ & -0.54362 & -4.18083 & -1.37344 \\
\hline C & 2.11358 & -2.35381 & -2.68483 \\
\hline $\mathrm{H}$ & 2.24016 & -3.07411 & -3.50043 \\
\hline H & 3.10723 & -2.03267 & -2.3739 \\
\hline $\mathrm{H}$ & 1.57409 & -1.49254 & -3.09207 \\
\hline C & 0.06664 & -3.08251 & 1.11203 \\
\hline $\mathrm{H}$ & -0.91836 & -3.19166 & 0.64807 \\
\hline C & 0.74467 & -4.45789 & 1.23985 \\
\hline $\mathrm{H}$ & 0.18465 & -5.06314 & 1.9609 \\
\hline
\end{tabular}

\begin{tabular}{|c|c|c|c|}
\hline I & 1.76813 & -4.3659 & 1.61759 \\
\hline H & 0.77332 & -5.01841 & 0.30311 \\
\hline & -0.13285 & -2.46544 & 2.50217 \\
\hline $\mathrm{H}$ & -0.76806 & -3.12896 & 3.09905 \\
\hline 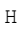 & -0.62922 & -1.49791 & 2.43813 \\
\hline $\mathrm{H}$ & 0.81625 & -2.35564 & 3.03451 \\
\hline C & -2.24035 & 2.20055 & -1.72175 \\
\hline ? & 0.97356 & -1.90093 & -0.05641 \\
\hline 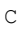 & -3.48758 & 2.82534 & -2.37606 \\
\hline & -4.08053 & 2.04456 & -2.86963 \\
\hline $\mathrm{H}$ & -4.12693 & 3.25854 & -1.5935 \\
\hline ? & 1.28586 & 1.73204 & -0.49565 \\
\hline $\mathrm{H}$ & -1.61976 & 1.74576 & -2.49933 \\
\hline $\mathrm{H}$ & -3.2433 & 1.74703 & 0.04811 \\
\hline 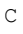 & -4.05398 & -2.23796 & -1.70026 \\
\hline 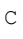 & -3.30174 & 0.22733 & 2.76765 \\
\hline$C$ & -2.34349 & 1.42677 & 3.15424 \\
\hline J & -2.67085 & -0.31424 & 1.57117 \\
\hline J & -1.67693 & 1.72702 & 1.88606 \\
\hline 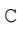 & -3.0573 & 2.6993 & 3.6146 \\
\hline $\mathrm{H}$ & -2.31927 & 3.47966 & 3.81898 \\
\hline & -3.61748 & 2.51953 & 4.53773 \\
\hline & -3.74769 & 3.08061 & 2.86021 \\
\hline 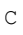 & -4.72093 & 0.66868 & 2.3748 \\
\hline H & -5.27332 & 1.05174 & 3.23706 \\
\hline 1 & -5.26611 & -0.19445 & 1.98262 \\
\hline $\mathrm{f}$ & -4.72123 & 1.44879 & 1.60825 \\
\hline C & -1.27755 & 1.04781 & 4.18973 \\
\hline & -1.736 & 0.80841 & 5.15315 \\
\hline $\mathrm{H}$ & -0.60291 & 1.89253 & 4.34657 \\
\hline I & -0.68267 & 0.18948 & 3.86977 \\
\hline C & -3.40624 & -0.88385 & 3.81202 \\
\hline $\mathrm{H}$ & -4.04359 & -1.68546 & 3.42823 \\
\hline H & -3.86207 & -0.50793 & 4.73346 \\
\hline $\mathrm{f}$ & -2.43511 & -1.31361 & 4.05663 \\
\hline C & -3.92591 & -3.47082 & -0.78109 \\
\hline $\mathrm{H}$ & -4.61978 & -4.24861 & -1.11323 \\
\hline H & -2.91424 & -3.87855 & -0.80836 \\
\hline $\mathrm{H}$ & -4.16636 & -3.21854 & 0.25627 \\
\hline J & -3.42154 & 0.09296 & -1.13972 \\
\hline $\mathrm{H}$ & -4.39609 & 0.29835 & -1.30222 \\
\hline C & -3.0429 & -1.17943 & -1.23034 \\
\hline D & -1.85739 & -1.5217 & -0.96587 \\
\hline C & -5.50723 & -1.72469 & -1.65894 \\
\hline & -5.79993 & -1.3981 & -0.65523 \\
\hline
\end{tabular}



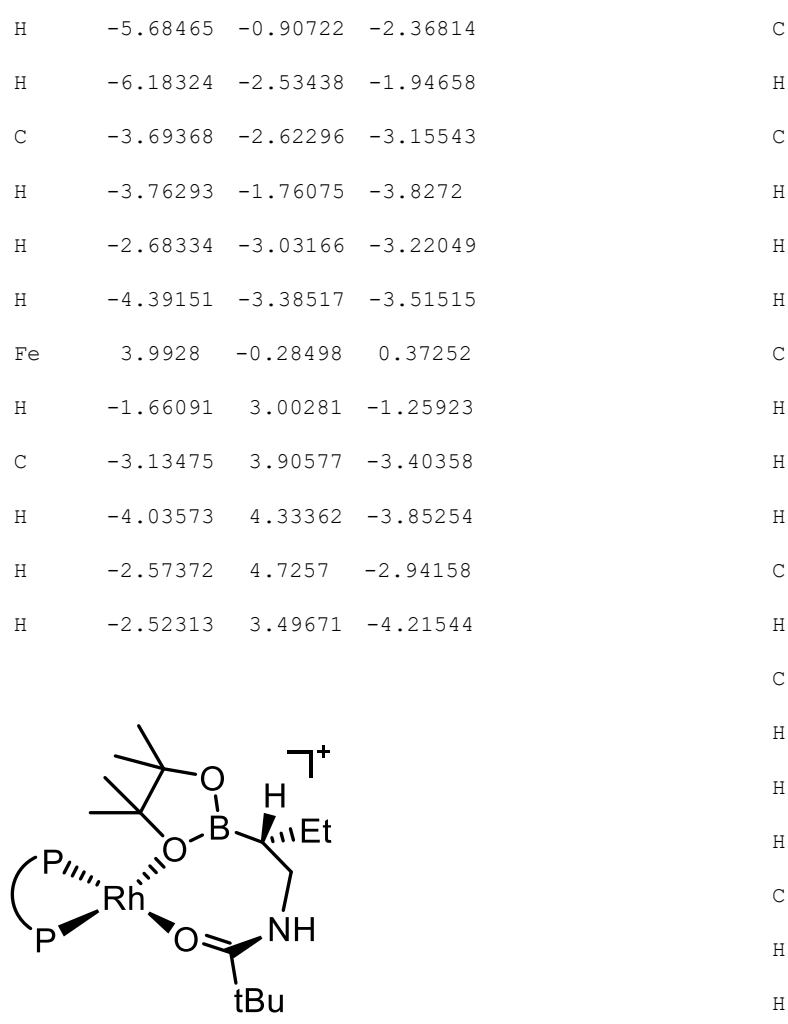

$G_{\text {sol }}=-2670.550295$ Hatree

\begin{tabular}{|c|c|c|c|}
\hline $\mathrm{Rh}$ & 0.49662 & 1.12755 & -0.41903 \\
\hline B & -4.37336 & -1.25659 & -0.0649 \\
\hline C & -4.48506 & 0.28846 & -0.40212 \\
\hline C & 3.87001 & -0.83663 & 2.36843 \\
\hline $\mathrm{H}$ & 4.45802 & 0.01198 & 2.685 \\
\hline C & 2.31972 & 1.14476 & -3.24649 \\
\hline $\mathrm{H}$ & 1.34913 & 1.47176 & -3.64184 \\
\hline C & 3.04692 & 2.39992 & -2.73874 \\
\hline $\mathrm{H}$ & 3.16261 & 3.11415 & -3.56125 \\
\hline $\mathrm{H}$ & 2.4905 & 2.89047 & -1.93457 \\
\hline $\mathrm{H}$ & 4.05046 & 2.16539 & -2.3723 \\
\hline C & 3.10587 & 0.46208 & -4.37779 \\
\hline $\mathrm{H}$ & 3.29904 & 1.18487 & -5.17785 \\
\hline $\mathrm{H}$ & 4.07334 & 0.0952 & -4.02161 \\
\hline $\mathrm{H}$ & 2.56624 & -0.37968 & -4.82001 \\
\hline $\mathrm{C}$ & 0.84718 & -1.36967 & -2.74084 \\
\hline $\mathrm{H}$ & 1.56059 & -1.88522 & -3.39493 \\
\hline C & -0.26288 & -0.7592 & -3.61464 \\
\hline $\mathrm{H}$ & -0.86043 & -1.56319 & -4.0571 \\
\hline $\mathrm{H}$ & -0.94543 & -0.14346 & -3.01674 \\
\hline $\mathrm{H}$ & 0.12105 & -0.15115 & -4.43684 \\
\hline C & 0.23301 & -2.39063 & -1.76943 \\
\hline $\mathrm{H}$ & -0.23815 & -3.19677 & -2.34351 \\
\hline $\mathrm{H}$ & 0.97623 & -2.84427 & -1.11118 \\
\hline $\mathrm{H}$ & -0.53768 & -1.92464 & -1.14851 \\
\hline
\end{tabular}

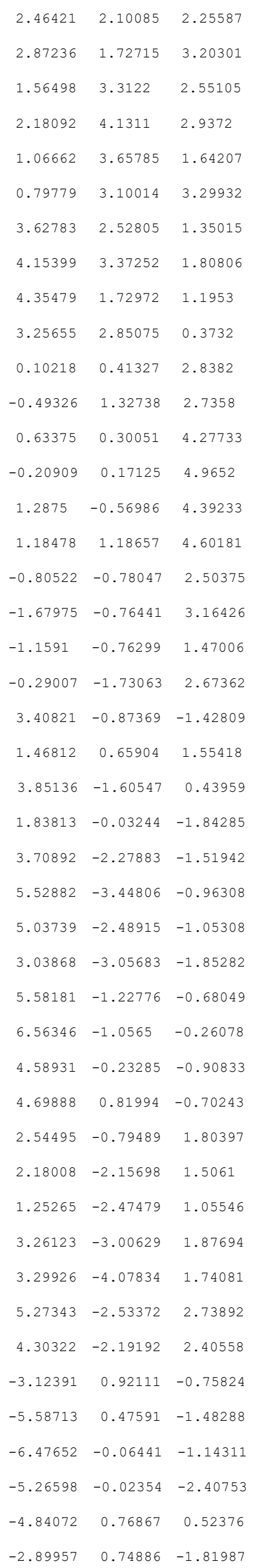



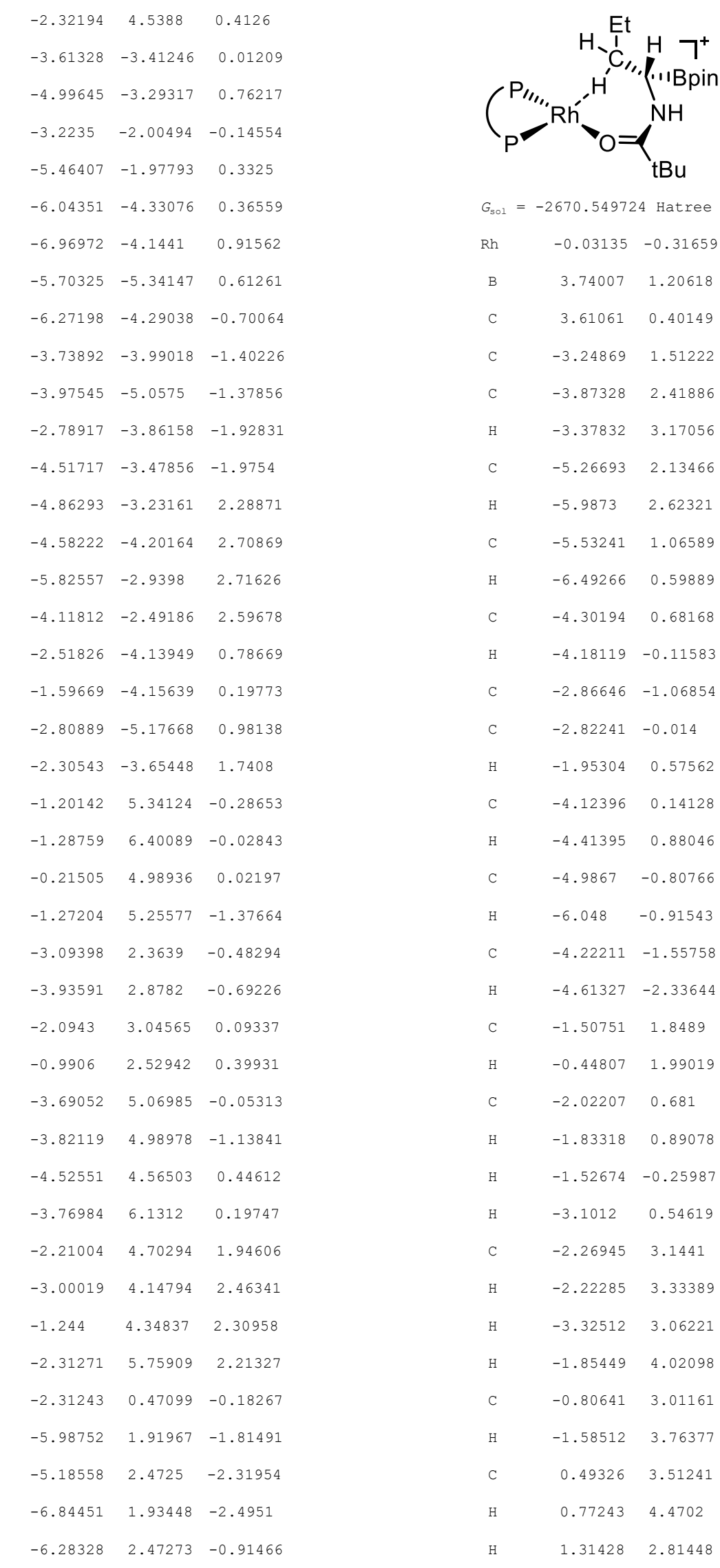

$G_{\text {sol }}=-2670.549724$ Hatree

$$
\begin{aligned}
& \begin{array}{llll}
-0.03135 & -0.31659 & 0.61875
\end{array} \\
& \begin{array}{lll}
3.74007 & 1.20618 & 0.01974
\end{array} \\
& \begin{array}{lll}
3.61061 & 0.40149 & 1.39615
\end{array} \\
& \begin{array}{lll}
-3.24869 & 1.51222 & 0.55983
\end{array} \\
& \begin{array}{llll}
-3.87328 & 2.41886 & -0.37083
\end{array} \\
& \begin{array}{llll}
-3.37832 & 3.17056 & -0.96591
\end{array} \\
& \begin{array}{llll}
-5.26693 & 2.13466 & -0.41836
\end{array} \\
& \begin{array}{llll}
-5.9873 & 2.62321 & -1.05986
\end{array} \\
& \begin{array}{lll}
-5.53241 & 1.06589 & 0.48272
\end{array} \\
& \begin{array}{lll}
-6.49266 & 0.59889 & 0.6531
\end{array} \\
& \begin{array}{lll}
-4.30194 & 0.68168 & 1.08726
\end{array} \\
& \begin{array}{lll}
-4.18119 & -0.11583 & 1.80188
\end{array} \\
& \begin{array}{llll}
-2.86646 & -1.06854 & -1.48433
\end{array} \\
& \begin{array}{lll}
-2.82241 & -0.014 & -2.46497
\end{array} \\
& \begin{array}{lll}
-1.95304 & 0.57562 & -2.713
\end{array} \\
& \begin{array}{llll}
-4.12396 & 0.14128 & -3.02169
\end{array} \\
& \begin{array}{llll}
-4.41395 & 0.88046 & -3.75565
\end{array} \\
& \begin{array}{llll}
-4.9867 & -0.80766 & -2.40241
\end{array} \\
& \begin{array}{lll}
-6.048 & -0.91543 & -2.57933
\end{array} \\
& \begin{array}{lll}
-4.22211 & -1.55758 & -1.46496
\end{array} \\
& \begin{array}{lll}
-4.61327 & -2.33644 & -0.82748
\end{array} \\
& \begin{array}{lll}
-1.50751 & 1.8489 & 2.85891
\end{array} \\
& \begin{array}{lll}
-0.44807 & 1.99019 & 3.10373
\end{array} \\
& \begin{array}{lll}
-2.02207 & 0.681 & 3.71552
\end{array} \\
& \begin{array}{lll}
-1.83318 & 0.89078 & 4.77373
\end{array} \\
& \begin{array}{lll}
-1.52674 & -0.25987 & 3.45886
\end{array} \\
& \begin{array}{lll}
-3.1012 & 0.54619 & 3.60382
\end{array} \\
& \begin{array}{lll}
-2.26945 & 3.1441 & 3.18362
\end{array} \\
& \begin{array}{lll}
-2.22285 & 3.33389 & 4.26157
\end{array} \\
& \begin{array}{lll}
-3.32512 \quad 3.06221 & 2.90835
\end{array} \\
& \begin{array}{lll}
-1.85449 & 4.02098 \quad 2.6801
\end{array} \\
& \begin{array}{lll}
-0.80641 & 3.01161 & 0.2242
\end{array} \\
& \begin{array}{llll}
-1.58512 & 3.76377 & 0.39537
\end{array} \\
& \begin{array}{lll}
0.49326 & 3.51241 & 0.87314
\end{array} \\
& \begin{array}{lll}
0.77243 \quad 4.4702 & 0.42014
\end{array} \\
& \begin{array}{lll}
1.31428 & 2.81448 & 0.68934
\end{array} \\
& \begin{array}{lll}
0.40731 & 3.67829 & 1.94965
\end{array} \\
& \begin{array}{llll}
-0.58901 & 2.84497 & -1.28839
\end{array} \\
& \begin{array}{llll}
-0.27442 & 3.80186 & -1.72107
\end{array}
\end{aligned}
$$




\begin{tabular}{|c|c|c|c|}
\hline $\mathrm{H}$ & -1.49126 & 2.52699 & -1.81283 \\
\hline $\mathrm{H}$ & 0.19511 & 2.10763 & -1.48112 \\
\hline C & -2.14297 & -3.12508 & 0.45459 \\
\hline $\mathrm{H}$ & -2.76291 & -3.67428 & -0.26404 \\
\hline C & -1.03471 & -4.06184 & 0.95664 \\
\hline $\mathrm{H}$ & -1.48402 & -4.87919 & 1.53072 \\
\hline $\mathrm{H}$ & -0.34161 & -3.52949 & 1.61421 \\
\hline $\mathrm{H}$ & -0.46251 & -4.51146 & 0.14203 \\
\hline C & -3.02752 & -2.69087 & 1.63084 \\
\hline $\mathrm{H}$ & -3.41322 & -3.57993 & 2.14156 \\
\hline $\mathrm{H}$ & -3.8828 & -2.09496 & 1.3118 \\
\hline $\mathrm{H}$ & -2.45264 & -2.11071 & 2.35854 \\
\hline C & -0.40037 & -2.54033 & -1.85854 \\
\hline $\mathrm{H}$ & 0.36702 & -3.04457 & -1.26523 \\
\hline C & -1.18247 & -3.57666 & -2.68188 \\
\hline $\mathrm{H}$ & -0.5094 & -4.04268 & -3.41007 \\
\hline $\mathrm{H}$ & -1.99917 & -3.11023 & -3.24116 \\
\hline $\mathrm{H}$ & -1.60399 & -4.37951 & -2.07065 \\
\hline C & 0.31606 & -1.53055 & -2.76943 \\
\hline $\mathrm{H}$ & 1.01557 & -2.06631 & -3.42155 \\
\hline $\mathrm{H}$ & 0.87939 & -0.79443 & -2.18925 \\
\hline $\mathrm{H}$ & -0.38982 & -1.00446 & -3.41926 \\
\hline C & 2.47235 & 0.70118 & 2.38603 \\
\hline P & -1.43114 & -1.68265 & -0.5353 \\
\hline C & 2.67941 & 0.02621 & 3.7506 \\
\hline $\mathrm{H}$ & 2.70228 & -1.06154 & 3.61796 \\
\hline $\mathrm{H}$ & 3.67337 & 0.3118 & 4.12064 \\
\hline P & -1.47119 & 1.42511 & 1.01598 \\
\hline $\mathrm{H}$ & 1.48356 & 0.40112 & 1.98094 \\
\hline $\mathrm{H}$ & 4.52724 & 0.67996 & 1.93081 \\
\hline C & 3.53049 & -3.37545 & 0.39162 \\
\hline C & 3.68559 & 1.67676 & -2.21006 \\
\hline C & 4.72802 & 2.61545 & -1.48712 \\
\hline O & 2.92368 & 1.12226 & -1.07595 \\
\hline 0 & 4.84711 & 1.98056 & -0.17463 \\
\hline C & 6.11405 & 2.65865 & -2.1259 \\
\hline $\mathrm{H}$ & 6.76897 & 3.30574 & -1.53645 \\
\hline $\mathrm{H}$ & 6.06097 & 3.06754 & -3.1398 \\
\hline $\mathrm{H}$ & 6.57221 & 1.66941 & -2.17318 \\
\hline C & 4.33171 & 0.47793 & -2.91455 \\
\hline $\mathrm{H}$ & 5.02344 & -0.05077 & -2.25223 \\
\hline $\mathrm{H}$ & 4.88147 & 0.78741 & -3.80745 \\
\hline $\mathrm{H}$ & 3.54873 & -0.22124 & -3.21988 \\
\hline$c$ & 4.21422 & 4.03851 & -1.24189 \\
\hline H & 4.15965 & 4.61067 & -2.17213 \\
\hline
\end{tabular}

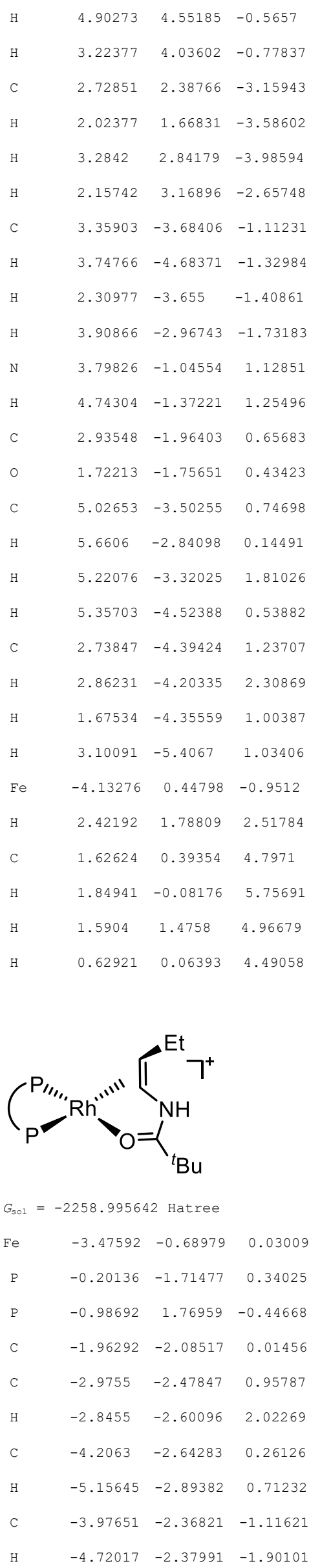




\begin{tabular}{|c|c|c|c|}
\hline C & -2.60403 & -2.02364 & -1.27457 \\
\hline $\mathrm{H}$ & -2.13122 & -1.74815 & -2.20381 \\
\hline C & -2.68247 & 1.21248 & -0.02885 \\
\hline C & -3.13848 & 0.89104 & 1.30004 \\
\hline $\mathrm{H}$ & -2.52143 & 0.84481 & 2.18423 \\
\hline $\mathrm{C}$ & -4.53775 & 0.63046 & 1.25025 \\
\hline $\mathrm{H}$ & -5.15489 & 0.3274 & 2.08481 \\
\hline C & -4.96672 & 0.77892 & -0.09934 \\
\hline $\mathrm{H}$ & -5.96628 & 0.60531 & -0.47367 \\
\hline C & -3.83707 & 1.13992 & -0.88735 \\
\hline $\mathrm{H}$ & -3.85185 & 1.29797 & -1.95555 \\
\hline C & 0.70381 & -3.05832 & -0.62626 \\
\hline $\mathrm{H}$ & 1.72805 & -2.97042 & -0.25225 \\
\hline C & 0.74542 & -2.75959 & -2.13322 \\
\hline $\mathrm{H}$ & 1.40929 & -3.47604 & -2.62954 \\
\hline $\mathrm{H}$ & 1.12563 & -1.75427 & -2.33788 \\
\hline $\mathrm{H}$ & -0.23894 & -2.86265 & -2.59871 \\
\hline C & 0.17733 & -4.47511 & -0.35223 \\
\hline $\mathrm{H}$ & 0.78929 & -5.20483 & -0.89391 \\
\hline $\mathrm{H}$ & -0.85535 & -4.59171 & -0.69468 \\
\hline $\mathrm{H}$ & 0.21408 & -4.74199 & 0.70757 \\
\hline C & 0.02876 & -2.24126 & 2.141 \\
\hline $\mathrm{H}$ & -0.54659 & -3.16854 & 2.24795 \\
\hline C & 1.50047 & -2.54422 & 2.47004 \\
\hline $\mathrm{H}$ & 1.58626 & -2.78683 & 3.53457 \\
\hline $\mathrm{H}$ & 2.14298 & -1.68333 & 2.26627 \\
\hline $\mathrm{H}$ & 1.88935 & -3.39778 & 1.91001 \\
\hline C & -0.53004 & -1.19905 & 3.12296 \\
\hline $\mathrm{H}$ & -0.39087 & -1.55118 & 4.15078 \\
\hline $\mathrm{H}$ & -1.59557 & -1.01378 & 2.97697 \\
\hline $\mathrm{H}$ & -0.00381 & -0.24507 & 3.01761 \\
\hline C & -1.08461 & 2.11991 & -2.30179 \\
\hline $\mathrm{H}$ & -2.01668 & 2.68117 & -2.43142 \\
\hline C & 0.07546 & 2.99699 & -2.80114 \\
\hline $\mathrm{H}$ & -0.11531 & 3.28614 & -3.8399 \\
\hline $\mathrm{H}$ & 1.01754 & 2.44197 & -2.78451 \\
\hline $\mathrm{H}$ & 0.20304 & 3.919 & -2.22757 \\
\hline C & -1.16265 & 0.84052 & -3.14862 \\
\hline $\mathrm{H}$ & -1.2041 & 1.10661 & -4.21058 \\
\hline $\mathrm{H}$ & -2.04745 & 0.24533 & -2.92092 \\
\hline $\mathrm{H}$ & -0.27766 & 0.21449 & -2.99591 \\
\hline C & -0.99751 & 3.50864 & 0.30947 \\
\hline $\mathrm{H}$ & -0.00361 & 3.90917 & 0.07432 \\
\hline C & -2.05058 & 4.44917 & -0.30198 \\
\hline H & -2.01787 & 5.41338 & 0.21667 \\
\hline
\end{tabular}

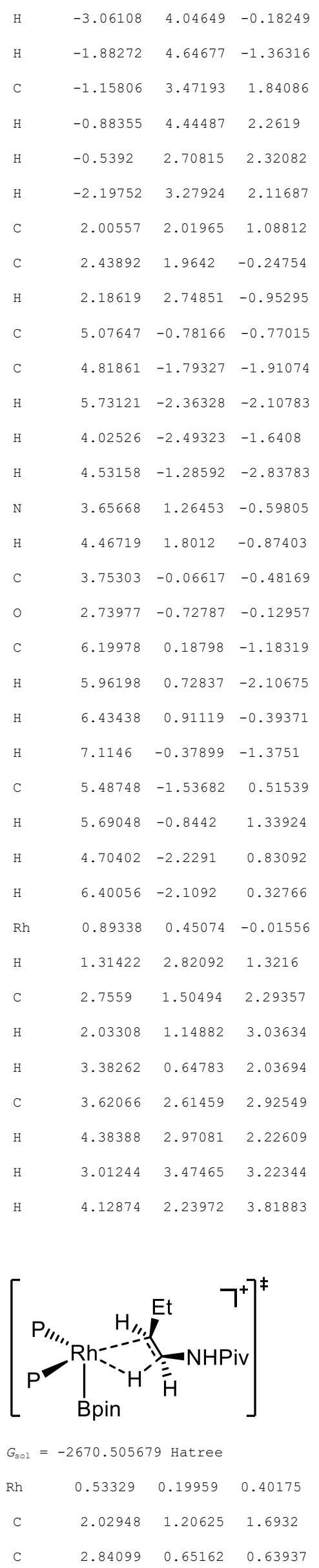




\begin{tabular}{|c|c|c|c|}
\hline $\mathrm{H}$ & 1.91825 & 0.10456 & -0.44808 \\
\hline C & -3.58945 & -3.47841 & -0.86954 \\
\hline $\mathrm{H}$ & -4.17985 & -4.18395 & -0.30134 \\
\hline C & -2.26897 & -3.05424 & -0.5551 \\
\hline $\mathrm{H}$ & -1.68681 & -3.40549 & 0.27809 \\
\hline C & -3.09511 & -0.07829 & 1.02023 \\
\hline C & -3.44795 & 0.63183 & -0.18147 \\
\hline $\mathrm{H}$ & -2.80209 & 1.3223 & -0.70626 \\
\hline C & -4.77399 & 0.26441 & -0.54756 \\
\hline H & -5.30628 & 0.60573 & -1.42482 \\
\hline $\mathrm{C}$ & -5.25505 & -0.67688 & 0.40845 \\
\hline $\mathrm{H}$ & -6.21223 & -1.17959 & 0.38274 \\
\hline C & -4.23112 & -0.88824 & 1.37557 \\
\hline $\mathrm{H}$ & -4.29869 & -1.56748 & 2.21327 \\
\hline $\mathrm{C}$ & 1.02708 & -2.62818 & -1.56218 \\
\hline $\mathrm{H}$ & 1.95655 & -2.10467 & -1.81273 \\
\hline C & 1.23945 & -3.2804 & -0.18567 \\
\hline H & 2.11387 & -3.93948 & -0.22263 \\
\hline $\mathrm{H}$ & 1.42006 & -2.53778 & 0.60116 \\
\hline $\mathrm{H}$ & 0.38905 & -3.89864 & 0.11339 \\
\hline $\mathrm{C}$ & 0.73876 & -3.68986 & -2.63311 \\
\hline H & 1.52606 & -4.45157 & -2.61633 \\
\hline H & -0.21555 & -4.19314 & -2.45161 \\
\hline $\mathrm{H}$ & 0.71494 & -3.27215 & -3.64284 \\
\hline C & -0.13063 & -0.54216 & -3.26778 \\
\hline $\mathrm{H}$ & -0.49367 & -1.37022 & -3.88795 \\
\hline C & 1.31202 & -0.21188 & -3.68555 \\
\hline $\mathrm{H}$ & 1.30187 & 0.22651 & -4.68947 \\
\hline $\mathrm{H}$ & 1.77723 & 0.51218 & -3.01077 \\
\hline $\mathrm{H}$ & 1.95593 & -1.0926 & -3.72494 \\
\hline C & -1.04479 & 0.67135 & -3.49915 \\
\hline H & -1.05846 & 0.91472 & -4.56748 \\
\hline $\mathrm{H}$ & -2.0748 & 0.51145 & -3.17817 \\
\hline $\mathrm{H}$ & -0.68117 & 1.54293 & -2.95815 \\
\hline C & -1.5484 & -1.16221 & 3.25838 \\
\hline $\mathrm{H}$ & -2.53593 & -1.03884 & 3.71439 \\
\hline C & -0.49296 & -0.93692 & 4.35296 \\
\hline $\mathrm{H}$ & -0.71302 & -1.59464 & 5.20066 \\
\hline H & 0.50593 & -1.19677 & 3.9943 \\
\hline H & -0.46347 & 0.08588 & 4.73407 \\
\hline $\mathrm{C}$ & -1.45168 & -2.60636 & 2.75664 \\
\hline $\mathrm{H}$ & -1.44608 & -3.28595 & 3.61623 \\
\hline $\mathrm{H}$ & -2.29724 & -2.87587 & 2.12603 \\
\hline $\mathrm{H}$ & -0.52631 & -2.78244 & 2.19826 \\
\hline C & -1.90908 & 1.71963 & 2.96761 \\
\hline
\end{tabular}

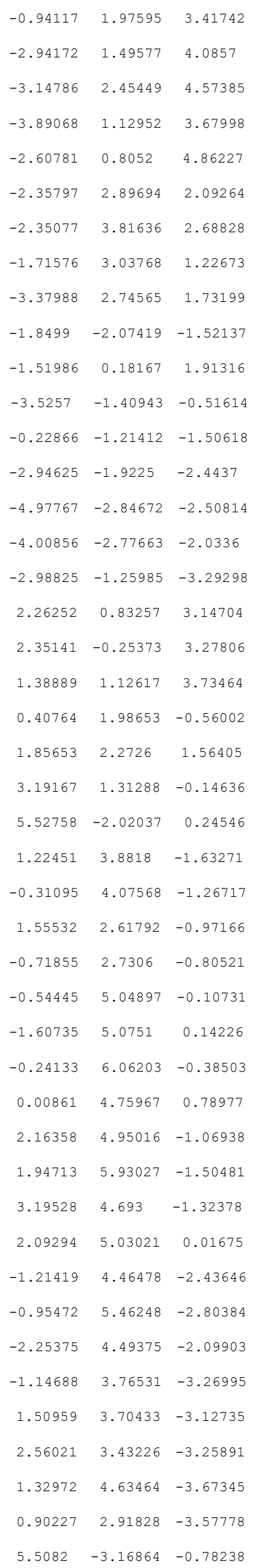




\begin{tabular}{|c|c|c|c|}
\hline $\mathrm{H}$ & 6.36115 & -3.83282 & -0.61494 \\
\hline$H$ & 4.59471 & -3.76701 & -0.69487 \\
\hline $\mathrm{H}$ & 5.56286 & -2.77607 & -1.79925 \\
\hline N & 3.70386 & -0.42484 & 0.89042 \\
\hline $\mathrm{H}$ & 3.77691 & -0.78834 & 1.82795 \\
\hline $\mathrm{C}$ & 4.4271 & -1.01462 & -0.13826 \\
\hline 0 & 4.21143 & -0.67847 & -1.29534 \\
\hline C & 6.86614 & -1.24758 & 0.13197 \\
\hline H & 6.99849 & -0.85097 & -0.87776 \\
\hline $\mathrm{H}$ & 6.90921 & -0.41244 & 0.8394 \\
\hline H & 7.70086 & -1.92009 & 0.35265 \\
\hline $\mathrm{C}$ & 5.37682 & -2.58727 & 1.66989 \\
\hline H & 5.49171 & -1.81773 & 2.44274 \\
\hline $\mathrm{H}$ & 4.41663 & -3.0966 & 1.8129 \\
\hline $\mathrm{H}$ & 6.16242 & -3.32597 & 1.85259 \\
\hline C & 3.50785 & 1.52725 & 3.73371 \\
\hline $\mathrm{H}$ & 4.42018 & 1.25242 & 3.19393 \\
\hline $\mathrm{H}$ & 3.40854 & 2.61586 & 3.67697 \\
\hline & 3.64356 & 1.25765 & 4.78568 \\
\hline
\end{tabular}

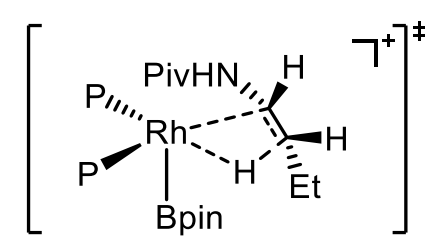

$\begin{array}{llll}G_{\text {sol }}= & -2670.51258 & \text { Hatree } & \\ \text { Rh } & -0.18975 & 0.25125 & 0.82022 \\ \text { H } & -0.73448 & 1.39026 & 1.81431 \\ \text { C } & -1.81021 & 0.40475 & 2.47561 \\ \text { C } & -2.16248 & -0.65825 & 1.59994 \\ \mathrm{C} & 3.14113 & -2.68491 & -1.50624 \\ \mathrm{H} & 2.86543 & -2.70319 & -2.54976 \\ \mathrm{C} & 4.43902 & -2.98399 & -1.00221 \\ \mathrm{H} & 5.30145 & -3.24622 & -1.59957 \\ \mathrm{C} & 4.41307 & -2.82561 & 0.4119 \\ \mathrm{H} & 5.25118 & -2.95008 & 1.08371 \\ \mathrm{C} & 3.10082 & -2.41798 & 0.78271 \\ \mathrm{H} & 2.77541 & -2.21262 & 1.7894 \\ \mathrm{C} & 3.37075 & 0.92657 & -0.04604 \\ \mathrm{C} & 3.42797 & 0.73655 & -1.47312 \\ \mathrm{H} & 2.59919 & 0.85349 & -2.15499 \\ \mathrm{C} & 4.75295 & 0.35258 & -1.8245 \\ \mathrm{H} & 5.09757 & 0.10665 & -2.81948 \\ \mathrm{C} & 5.52636 & 0.28631 & -0.62981 \\ \mathrm{H} & 6.56021 & -0.02223 & -0.5564\end{array}$

\begin{tabular}{|c|c|c|c|}
\hline & 4.68599 & 0.64062 & 0.46417 \\
\hline & 4.98865 & 0.65985 & 1.50137 \\
\hline & -0.2274 & -3.42849 & 0.43813 \\
\hline & -1.30863 & -3.2691 & 0.38519 \\
\hline & 0.15444 & -3.49591 & 1.92783 \\
\hline & -0.50339 & -4.20579 & 2.43957 \\
\hline & 0.05693 & -2.53071 & 2.43951 \\
\hline & 1.18193 & -3.8425 & 2.06574 \\
\hline & 0.11291 & -4.75802 & -0.25313 \\
\hline & -0.33222 & -5.5827 & 0.31409 \\
\hline & 1.19327 & -4.92997 & -0.29433 \\
\hline & -0.28371 & -4.81849 & -1.26972 \\
\hline & 0.00172 & -2.1519 & -2.21166 \\
\hline & 0.42784 & -3.12241 & -2.49046 \\
\hline & -1.52783 & -2.23922 & -2.33954 \\
\hline & -1.79566 & -2.38957 & -3.39099 \\
\hline & -2.00351 & -1.31213 & -2.00631 \\
\hline & -1.95907 & -3.06271 & -1.76645 \\
\hline & 0.56705 & -1.07999 & -3.15645 \\
\hline & 0.24507 & -1.29981 & -4.18097 \\
\hline & 1.6579 & -1.05518 & -3.15406 \\
\hline & 0.20403 & -0.08608 & -2.88893 \\
\hline & 2.40083 & 1.58277 & 2.65477 \\
\hline & 3.4014 & 2.02849 & 2.67254 \\
\hline & 1.46656 & 2.4686 & 3.49803 \\
\hline & 1.86985 & 2.55204 & 4.51271 \\
\hline & 0.46759 & 2.03016 & 3.5777 \\
\hline & 1.35605 & 3.48151 & 3.10548 \\
\hline & 2.49045 & 0.18038 & 3.27443 \\
\hline & 2.75258 & 0.26073 & 4.33506 \\
\hline & 3.2459 & -0.43748 & 2.78882 \\
\hline & 1.52928 & -0.34591 & 3.21778 \\
\hline & 1.95358 & 3.39869 & 0.3837 \\
\hline & 1.03295 & 3.80192 & 0.82405 \\
\hline & 3.16675 & 4.13962 & 0.97482 \\
\hline & 3.15505 & 5.17734 & 0.62421 \\
\hline & 4.10595 & 3.69247 & 0.6338 \\
\hline & 3.17677 & 4.16807 & 2.06608 \\
\hline & 1.91516 & 3.62963 & -1.13578 \\
\hline & 1.72627 & 4.6911 & -1.3318 \\
\hline & 1.14306 & 3.04284 & -1.63235 \\
\hline & 2.87939 & 3.38416 & -1.5887 \\
\hline & 1.89744 & 1.55335 & 0.83177 \\
\hline & 2.29021 & -2.3204 & -0.40501 \\
\hline & -1.15193 & 1.40906 & -0.5168 \\
\hline
\end{tabular}




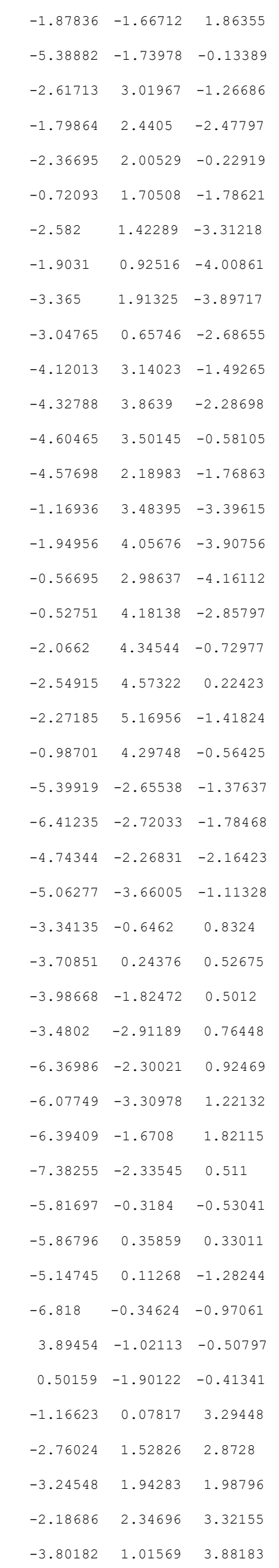




\begin{tabular}{|c|c|c|c|}
\hline $\mathrm{H}$ & 0.09471 & 3.59764 & 1.23881 \\
\hline $\mathrm{H}$ & 0.86659 & 4.61102 & 0.00583 \\
\hline C & 2.42386 & 2.32336 & 2.14634 \\
\hline $\mathrm{H}$ & 2.68316 & 3.10103 & 2.8739 \\
\hline $\mathrm{H}$ & 3.26176 & 1.62616 & 2.09988 \\
\hline $\mathrm{H}$ & 1.54573 & 1.79132 & 2.51672 \\
\hline C & 0.50397 & -3.1806 & -1.08157 \\
\hline $\mathrm{H}$ & 1.12446 & -4.04131 & -0.80765 \\
\hline C & -0.95448 & -3.65647 & -1.19478 \\
\hline $\mathrm{H}$ & -1.00441 & -4.46385 & -1.93334 \\
\hline $\mathrm{H}$ & -1.62422 & -2.86712 & -1.54318 \\
\hline $\mathrm{H}$ & -1.35339 & -4.05243 & -0.25817 \\
\hline C & 0.98911 & -2.65029 & -2.44053 \\
\hline $\mathrm{H}$ & 0.80591 & -3.40171 & -3.21601 \\
\hline $\mathrm{H}$ & 2.05543 & -2.41959 & -2.44291 \\
\hline $\mathrm{H}$ & 0.44033 & -1.74686 & -2.73558 \\
\hline C & 0.05824 & -2.82599 & 1.81795 \\
\hline $\mathrm{H}$ & -1.02652 & -2.78338 & 1.65924 \\
\hline C & 0.50122 & -4.29568 & 1.91932 \\
\hline $\mathrm{H}$ & 0.08145 & -4.73622 & 2.83026 \\
\hline $\mathrm{H}$ & 1.59078 & -4.37333 & 1.99083 \\
\hline $\mathrm{H}$ & 0.16847 & -4.90813 & 1.07944 \\
\hline C & 0.38961 & -2.10101 & 3.13359 \\
\hline $\mathrm{H}$ & -0.22573 & -2.5168 & 3.93939 \\
\hline $\mathrm{H}$ & 0.2114 & -1.02561 & 3.08287 \\
\hline $\mathrm{H}$ & 1.4336 & -2.26324 & 3.41349 \\
\hline C & 3.34458 & 0.94748 & -0.91534 \\
\hline P & 0.75994 & -1.92348 & 0.30767 \\
\hline C & -2.24059 & 2.74814 & -1.1738 \\
\hline $\mathrm{H}$ & -1.47266 & 3.46109 & -0.85458 \\
\hline $\mathrm{H}$ & -2.82688 & 2.52077 & -0.27864 \\
\hline B & -1.23321 & 0.64245 & 1.18247 \\
\hline $\mathrm{H}$ & -0.94318 & 1.69369 & -2.57648 \\
\hline $\mathrm{H}$ & -2.03597 & -0.39131 & -2.67936 \\
\hline C & -5.76234 & -1.22407 & -1.42753 \\
\hline C & -2.9914 & 0.7405 & 2.68165 \\
\hline C & -1.7374 & 1.49788 & 3.26883 \\
\hline 0 & -2.60787 & 0.54765 & 1.27551 \\
\hline 0 & -0.66215 & 1.08575 & 2.34929 \\
\hline C & -1.84925 & 3.02319 & 3.18165 \\
\hline $\mathrm{H}$ & -0.89169 & 3.47124 & 3.45693 \\
\hline $\mathrm{H}$ & -2.60791 & 3.40276 & 3.87139 \\
\hline $\mathrm{H}$ & -2.10579 & 3.3568 & 2.17269 \\
\hline$c$ & -4.29496 & 1.53499 & 2.73037 \\
\hline H & -4.56083 & 1.77066 & 3.76556 \\
\hline
\end{tabular}

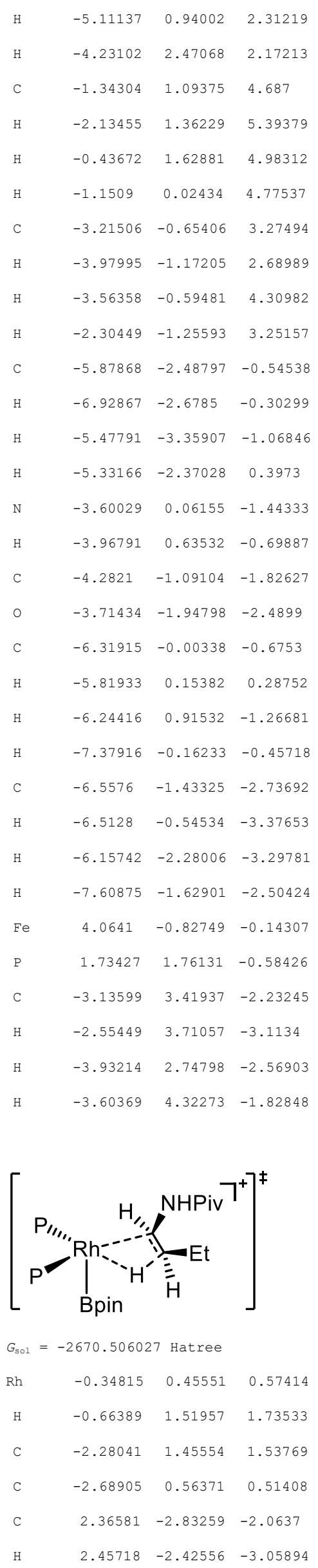




\begin{tabular}{|c|c|c|c|}
\hline & 3.24207 & -3.80324 & -1.50067 \\
\hline & 4.1079 & -4.22796 & -1.98985 \\
\hline 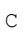 & 2.81169 & -4.07192 & -0.17106 \\
\hline $\mathrm{H}$ & 3.28822 & -4.74217 & 0.53104 \\
\hline & 1.67314 & -3.25988 & 0.09169 \\
\hline 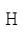 & 1.12775 & -3.23306 & 1.01979 \\
\hline C & 3.30719 & -0.31297 & 0.77191 \\
\hline C & 3.73751 & -0.04658 & -0.57647 \\
\hline & 3.24548 & 0.62149 & -1.26778 \\
\hline$C$ & 4.89859 & -0.82619 & -0.84161 \\
\hline $\mathrm{H}$ & 5.43297 & -0.86723 & -1.78078 \\
\hline & 5.19538 & -1.58949 & 0.32446 \\
\hline 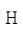 & 5.9914 & -2.31452 & 0.42552 \\
\hline & 4.2251 & -1.27652 & 1.31954 \\
\hline & 4.17855 & -1.7172 & 2.30522 \\
\hline 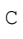 & -1.47806 & -2.5783 & -1.24763 \\
\hline $\mathrm{H}$ & -2.36088 & -1.95085 & -1.39239 \\
\hline & -1.61547 & -3.30947 & 0.09809 \\
\hline $\mathrm{H}$ & -2.5738 & -3.83807 & 0.13096 \\
\hline $\mathrm{H}$ & -1.58477 & -2.62841 & 0.95467 \\
\hline & -0.83258 & -4.0609 & 0.22571 \\
\hline$C$ & -1.4068 & -3.59948 & -2.39451 \\
\hline$H$ & -2.2427 & -4.30227 & -2.30509 \\
\hline & -0.48147 & -4.18355 & -2.36167 \\
\hline & -1.48658 & -3.13405 & -3.37966 \\
\hline & 0.01226 & -0.77178 & -3.02217 \\
\hline $\mathcal{H}$ & 0.10334 & -1.69722 & -3.60141 \\
\hline & -1.30302 & -0.07859 & -3.4202 \\
\hline $\mathrm{d}$ & -1.2586 & 0.20001 & -4.47876 \\
\hline $\mathcal{H}$ & -1.47037 & 0.83413 & -2.84044 \\
\hline 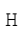 & -2.18149 & -0.71324 & -3.2831 \\
\hline C & 1.22697 & 0.11319 & -3.33909 \\
\hline $\mathrm{H}$ & 1.19131 & 0.41033 & -4.39323 \\
\hline H & 2.17425 & -0.40057 & -3.17257 \\
\hline \& & 1.23155 & 1.01962 & -2.73399 \\
\hline 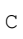 & 1.75968 & -0.23321 & 3.27677 \\
\hline H & 2.78055 & -0.27571 & 3.67118 \\
\hline & 0.91081 & 0.62508 & 4.23082 \\
\hline H & 0.99119 & 0.22292 & 5.24636 \\
\hline H & -0.14462 & 0.59404 & 3.94966 \\
\hline H & 1.21744 & 1.67255 & 4.26785 \\
\hline $\mathrm{C}$ & 1.2052 & -1.66368 & 3.24162 \\
\hline $\mathrm{H}$ & 1.08622 & -2.03676 & 4.26491 \\
\hline & 1.86769 & -2.34783 & 2.71206 \\
\hline & 0.21696 & -1.70242 & 2.76561 \\
\hline
\end{tabular}

\begin{tabular}{|c|c|c|c|}
\hline & 2.74052 & 2.23105 & 2.00841 \\
\hline & 1.90375 & 2.85124 & 2.35343 \\
\hline & 3.79514 & 2.12377 & 3.12498 \\
\hline & 4.25995 & 3.10542 & 3.26652 \\
\hline & 4.59237 & 1.42701 & 2.84594 \\
\hline & 3.39157 & 1.81657 & 4.09139 \\
\hline & 3.38535 & 2.9123 & 0.78991 \\
\hline & 3.6428 & 3.94333 & 1.05649 \\
\hline & 2.72624 & 2.93498 & -0.07503 \\
\hline & 4.3142 & 2.40673 & 0.51153 \\
\hline & 1.92116 & 0.56665 & 1.56891 \\
\hline & 1.38118 & -2.47074 & -1.07776 \\
\hline & -2.66686 & 1.26415 & 2.99609 \\
\hline & -1.99223 & 1.84514 & 3.63199 \\
\hline & -2.54432 & 0.21914 & 3.30808 \\
\hline & -0.27124 & 2.11316 & -0.59601 \\
\hline & -2.23695 & 2.4891 & 1.21371 \\
\hline & -2.87142 & 0.94825 & -0.48008 \\
\hline & -5.50993 & -1.98603 & 0.41019 \\
\hline & -1.08696 & 4.13232 & -1.36232 \\
\hline & 0.39025 & 3.92493 & -1.88392 \\
\hline & -1.43564 & 2.79623 & -0.86982 \\
\hline & 0.82983 & 2.7333 & -1.12637 \\
\hline & 0.47551 & 3.59146 & -3.37512 \\
\hline & 1.49954 & 3.30155 & -3.62345 \\
\hline & 0.21807 & 4.46532 & -3.98012 \\
\hline & -0.18956 & 2.77297 & -3.65528 \\
\hline & -2.10639 & 4.52844 & -2.42708 \\
\hline & -1.86104 & 5.50626 & -2.85311 \\
\hline & -3.09886 & 4.60041 & -1.97413 \\
\hline & -2.15786 & 3.79918 & -3.23661 \\
\hline & 1.3505 & 5.06946 & -1.56517 \\
\hline & 1.01978 & 5.99056 & -2.05527 \\
\hline & 2.34842 & 4.83199 & -1.94343 \\
\hline & 1.42945 & 5.25706 & -0.49366 \\
\hline & -1.19085 & 5.08065 & -0.16137 \\
\hline & -2.20951 & 5.04584 & 0.23513 \\
\hline & -0.97902 & 6.11436 & -0.44732 \\
\hline & -0.50103 & 4.7987 & 0.63959 \\
\hline & -5.67372 & -3.14729 & -0.59151 \\
\hline & -6.55463 & -3.74233 & -0.33222 \\
\hline & -5.79485 & -2.76261 & -1.60569 \\
\hline & -4.80549 & -3.81484 & -0.58013 \\
\hline & -3.48182 & -0.56337 & 0.80718 \\
\hline & -3.58342 & -0.85048 & 1.76864 \\
\hline
\end{tabular}



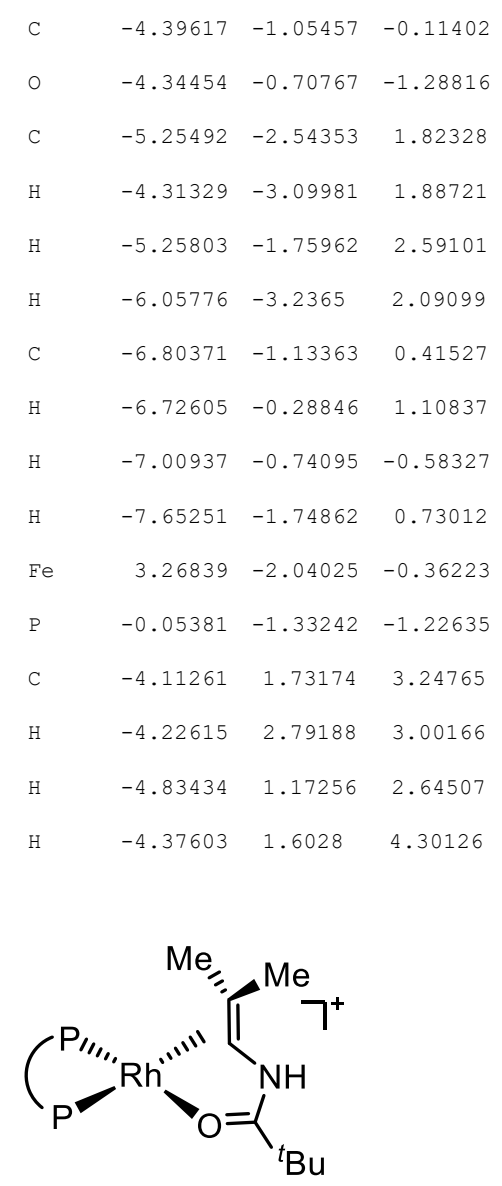

$$
\begin{aligned}
& G_{\text {sol }}=-2258.985205 \text { Hatree }
\end{aligned}
$$

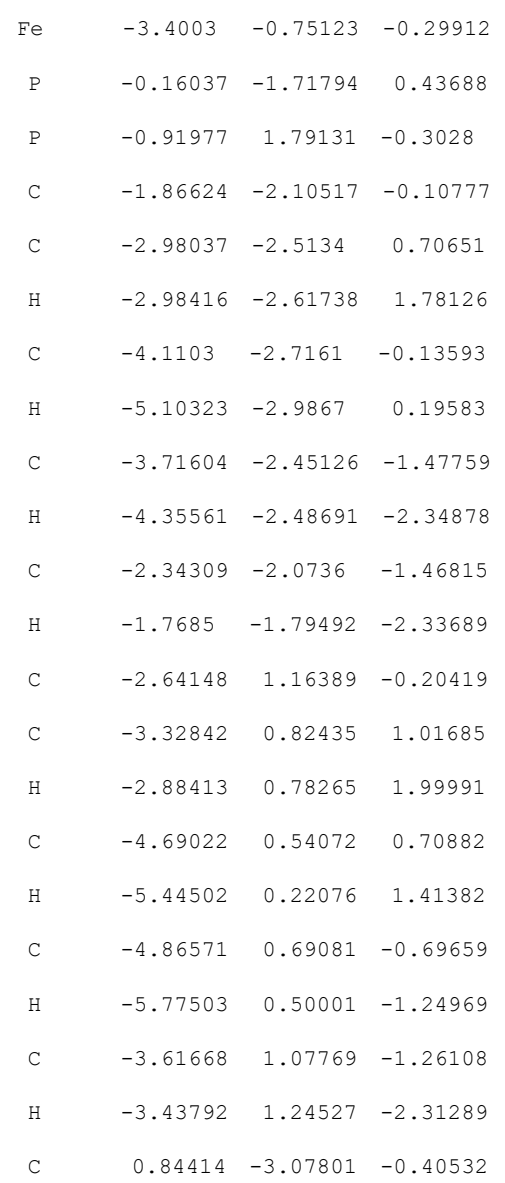

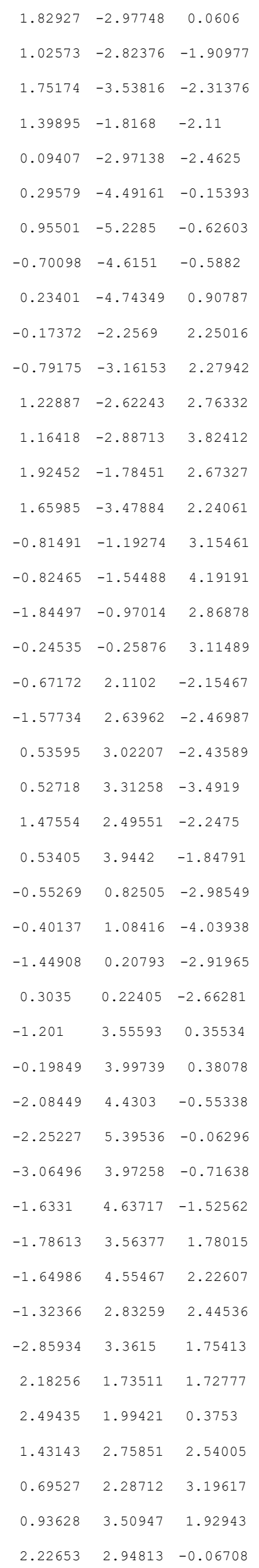



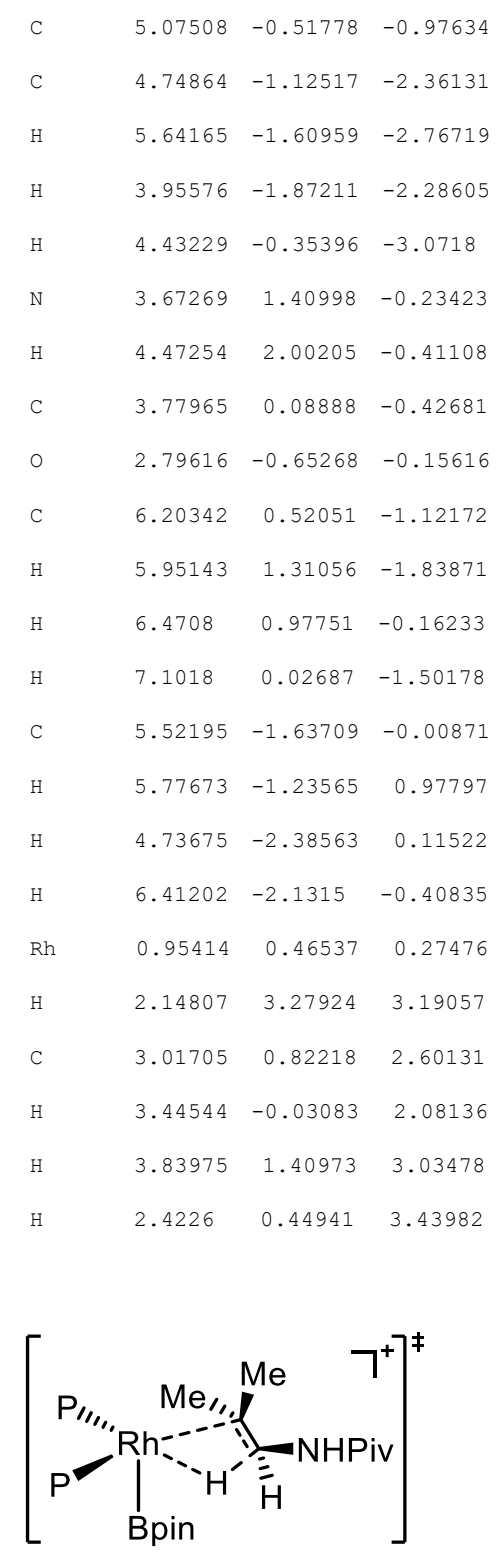

$\begin{array}{llll}G_{\text {sol }}= & -2670.506417 & \text { Hatree } & \\ \text { Rh } & 0.46611 & 0.05406 & 0.57454 \\ \mathrm{H} & 1.96585 & -0.15094 & -0.01971 \\ \mathrm{C} & 2.77762 & 0.09746 & 1.2589 \\ \mathrm{C} & 1.84836 & 0.31367 & 2.33966 \\ \mathrm{C} & -4.5863 & -0.20819 & 0.78851 \\ \mathrm{H} & -4.95621 & 0.80447 & 0.74988 \\ \mathrm{C} & -5.34564 & -1.36091 & 0.44044 \\ \mathrm{H} & -6.3651 & -1.35735 & 0.07984 \\ \mathrm{C} & -4.51426 & -2.50486 & 0.59961 \\ \mathrm{H} & -4.78971 & -3.52883 & 0.3877 \\ \mathrm{C} & -3.23603 & -2.05838 & 1.03624 \\ \mathrm{H} & -2.38848 & -2.69108 & 1.23456 \\ \mathrm{C} & -1.99074 & -1.08748 & -2.01774 \\ \mathrm{C} & -2.80598 & 0.0915 & -2.15859 \\ \mathrm{H} & -2.49091 & 1.09949 & -1.93242\end{array}$

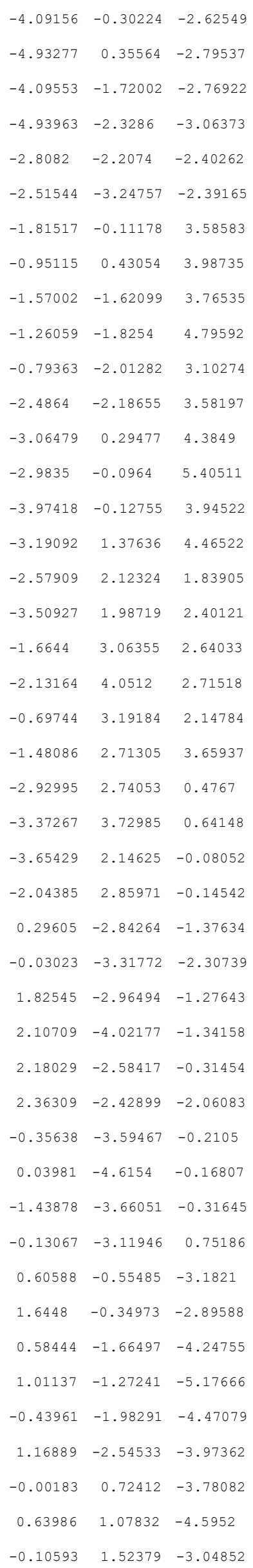




\begin{tabular}{|c|c|c|c|}
\hline t & -0.98894 & 0.52365 & -4.20697 \\
\hline & -3.25688 & -0.62313 & 1.15532 \\
\hline & -0.22907 & -1.03195 & -1.54104 \\
\hline & 1.876 & 1.6847 & 3.001 \\
\hline & 0.96702 & 1.8674 & 3.57875 \\
\hline & 1.99961 & 2.48702 & 2.27401 \\
\hline & 0.82114 & 1.87275 & -0.26657 \\
\hline & 3.21753 & 0.96634 & 0.78188 \\
\hline & 5.63421 & -2.26984 & 0.403 \\
\hline & 2.13559 & 3.59929 & -1.05959 \\
\hline & 0.6146 & 3.94439 & -1.27835 \\
\hline & 2.05262 & 2.47146 & -0.12737 \\
\hline & -0.03845 & 2.64069 & -1.0158 \\
\hline & 0.06852 & 4.94765 & -0.25861 \\
\hline & -1.01741 & 5.01536 & -0.35392 \\
\hline & 0.48381 & 5.94353 & -0.43508 \\
\hline & 0.30481 & 4.65687 & 0.76737 \\
\hline & 2.96797 & 4.69932 & -0.40574 \\
\hline & 3.00671 & 5.58574 & -1.04662 \\
\hline & 3.99302 & 4.3464 & -0.26375 \\
\hline & 2.5717 & 4.9927 & 0.56773 \\
\hline & 0.24666 & 4.39818 & -2.68834 \\
\hline & 0.72818 & 5.35534 & -2.91265 \\
\hline & -0.83462 & 4.5454 & -2.75952 \\
\hline & 0.54437 & 3.679 & -3.45111 \\
\hline & 2.84604 & 3.08386 & -2.31568 \\
\hline & 3.81569 & 2.66751 & -2.03173 \\
\hline & 3.01401 & 3.8906 & -3.03476 \\
\hline & 2.27435 & 2.29498 & -2.81009 \\
\hline & 5.77472 & -3.03803 & -0.92688 \\
\hline & 6.62749 & -3.72144 & -0.87364 \\
\hline & 4.88024 & -3.63273 & -1.14076 \\
\hline & 5.93154 & -2.34511 & -1.75565 \\
\hline & 3.62188 & -1.02184 & 1.23766 \\
\hline & 3.59465 & -1.67785 & 2.00306 \\
\hline & 4.55037 & -1.19158 & 0.2155 \\
\hline & 4.51301 & -0.45935 & -0.76292 \\
\hline & 6.948 & -1.50492 & 0.69989 \\
\hline & 7.17711 & -0.80604 & -0.10818 \\
\hline & 6.88102 & -0.94019 & 1.63619 \\
\hline & 7.77673 & -2.21366 & 0.79265 \\
\hline & 5.339 & -3.25534 & 1.54936 \\
\hline & 5.33063 & -2.76563 & 2.53055 \\
\hline & 4.3903 & -3.78605 & 1.40607 \\
\hline & 6.12588 & -4.0139 & 1.58951 \\
\hline
\end{tabular}
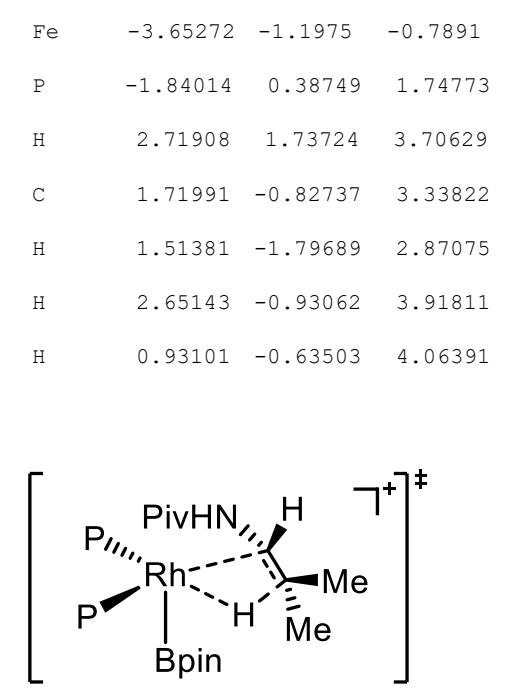

$G_{\text {sol }}=-2670.510965$ Hatree

\begin{tabular}{|c|c|c|c|}
\hline $\mathrm{Rh}$ & -0.21883 & 0.29598 & 1.00649 \\
\hline $\mathrm{H}$ & -0.5961 & 1.61828 & 1.85248 \\
\hline $\mathrm{C}$ & -1.92176 & 0.89277 & 2.52695 \\
\hline C & -2.3117 & -0.23323 & 1.73833 \\
\hline C & 5.19967 & -1.19208 & -0.18261 \\
\hline $\mathrm{H}$ & 5.82162 & -2.07572 & -0.14302 \\
\hline C & 4.11737 & -0.89765 & 0.69329 \\
\hline $\mathrm{H}$ & 3.80074 & -1.51746 & 1.51486 \\
\hline C & 1.51608 & -1.90093 & -1.40119 \\
\hline C & 1.69332 & -0.85499 & -2.37756 \\
\hline $\mathrm{H}$ & 1.12891 & 0.06494 & -2.41525 \\
\hline C & 2.78034 & -1.21105 & -3.22489 \\
\hline $\mathrm{H}$ & 3.18224 & -0.60741 & -4.02711 \\
\hline C & 3.29181 & -2.46716 & -2.78826 \\
\hline $\mathrm{H}$ & 4.14816 & -2.98526 & -3.19772 \\
\hline C & 2.51537 & -2.89932 & -1.67644 \\
\hline $\mathrm{H}$ & 2.67328 & -3.81675 & -1.12902 \\
\hline C & 2.55514 & 1.05345 & 2.97743 \\
\hline $\mathrm{H}$ & 1.84165 & 1.75905 & 3.41766 \\
\hline C & 2.27775 & -0.31257 & 3.62292 \\
\hline $\mathrm{H}$ & 2.2451 & -0.2014 & 4.71216 \\
\hline $\mathrm{H}$ & 1.32449 & -0.74702 & 3.30941 \\
\hline $\mathrm{H}$ & 3.06756 & -1.03356 & 3.40202 \\
\hline C & 3.98184 & 1.51734 & 3.315 \\
\hline $\mathrm{H}$ & 4.13615 & 1.45653 & 4.39812 \\
\hline $\mathrm{H}$ & 4.73104 & 0.88001 & 2.83716 \\
\hline $\mathrm{H}$ & 4.17669 & 2.5497 & 3.017 \\
\hline C & 2.49866 & 3.01555 & 0.85514 \\
\hline $\mathrm{H}$ & 3.5841 & 3.06639 & 0.99569 \\
\hline C & 1.81872 & 3.92511 & 1.89342 \\
\hline H & 1.992 & 4.97118 & 1.61982 \\
\hline
\end{tabular}




\begin{tabular}{|c|c|c|c|}
\hline H & 0.73391 & 3.76766 & 1.91026 \\
\hline H & 2.20132 & 3.79182 & 2.90699 \\
\hline $\mathrm{C}$ & 2.1678 & 3.53989 & -0.54816 \\
\hline $\mathrm{H}$ & 2.68527 & 4.49255 & -0.70566 \\
\hline $\mathrm{H}$ & 2.44076 & 2.8635 & -1.3575 \\
\hline H & 1.09768 & 3.71779 & -0.63661 \\
\hline C & 0.47878 & -3.39256 & 0.96055 \\
\hline $\mathrm{H}$ & 0.41812 & -4.24601 & 0.27845 \\
\hline C & -0.64167 & -3.53629 & 2.00681 \\
\hline $\mathrm{H}$ & -0.5361 & -4.50002 & 2.51624 \\
\hline $\mathrm{H}$ & -0.55463 & -2.75888 & 2.77541 \\
\hline $\mathrm{H}$ & -1.65105 & -3.48778 & 1.59384 \\
\hline C & 1.84186 & -3.46451 & 1.65793 \\
\hline $\mathrm{H}$ & 1.91377 & -4.42107 & 2.18759 \\
\hline $\mathrm{H}$ & 2.68204 & -3.40199 & 0.96759 \\
\hline $\mathrm{H}$ & 1.95051 & -2.67735 & 2.40514 \\
\hline C & -1.29326 & -2.60221 & -1.18089 \\
\hline $\mathrm{H}$ & -2.06578 & -2.79184 & -0.42883 \\
\hline C & -0.97828 & -3.91339 & -1.92121 \\
\hline H & -1.86161 & -4.22003 & -2.49151 \\
\hline $\mathrm{H}$ & -0.15357 & -3.78925 & -2.62934 \\
\hline $\mathrm{H}$ & -0.73475 & -4.7418 & -1.25221 \\
\hline C & -1.83627 & -1.56713 & -2.16925 \\
\hline $\mathrm{H}$ & -2.70205 & -1.98192 & -2.69714 \\
\hline $\mathrm{H}$ & -2.15963 & -0.65504 & -1.66316 \\
\hline H & -1.0886 & -1.30733 & -2.92385 \\
\hline C & 3.51479 & 0.34758 & 0.2861 \\
\hline $\mathrm{P}$ & 0.15883 & -1.90362 & -0.17682 \\
\hline $\mathrm{Fe}$ & 3.39944 & -1.0553 & -1.24047 \\
\hline P & 2.10869 & 1.17916 & 1.13067 \\
\hline C & 4.25483 & 0.79766 & -0.86367 \\
\hline $\mathrm{H}$ & 4.06201 & 1.68512 & -1.44524 \\
\hline C & 5.28241 & -0.14767 & -1.14497 \\
\hline $\mathrm{H}$ & 5.97462 & -0.09571 & -1.97386 \\
\hline C & -1.33643 & 0.5695 & 3.89487 \\
\hline $\mathrm{H}$ & -0.67946 & 1.3623 & 4.26205 \\
\hline H & -0.79004 & -0.37681 & 3.90587 \\
\hline B & -0.87615 & 1.39994 & -0.56117 \\
\hline H & -2.17105 & -1.22012 & 2.1627 \\
\hline C & -5.59125 & -1.18818 & 0.03933 \\
\hline C & -2.21769 & 2.93113 & -1.66729 \\
\hline C & -1.00276 & 2.52707 & -2.59015 \\
\hline o & -2.16445 & 1.90526 & -0.6125 \\
\hline o & -0.14028 & 1.77585 & -1.65762 \\
\hline C & -1.37849 & 1.58807 & -3.7405 \\
\hline
\end{tabular}

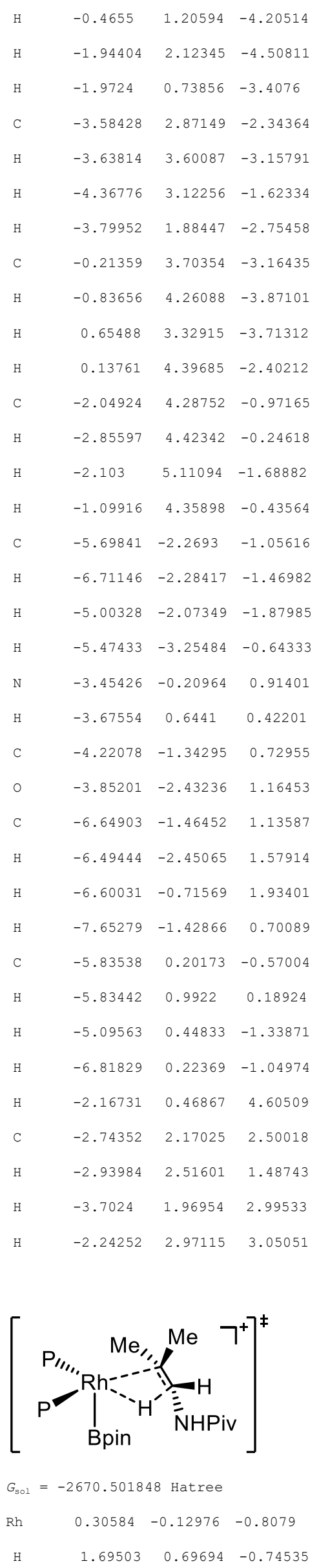




\begin{tabular}{|c|c|c|c|}
\hline C & 2.39659 & 0.00701 & -1.92134 \\
\hline C & 1.63338 & -1.16695 & -2.27548 \\
\hline C & -3.20716 & -0.94448 & 0.07295 \\
\hline C & -2.89094 & -0.90408 & 1.47687 \\
\hline $\mathrm{H}$ & -1.90137 & -1.02949 & 1.89297 \\
\hline C & -4.09133 & -0.65934 & 2.20258 \\
\hline $\mathrm{H}$ & -4.17034 & -0.54224 & 3.27476 \\
\hline $\mathrm{C}$ & -5.15932 & -0.53508 & 1.2673 \\
\hline H & -6.18914 & -0.30276 & 1.50183 \\
\hline $\mathrm{C}$ & -4.62406 & -0.71002 & -0.04083 \\
\hline $\mathrm{H}$ & -5.1934 & -0.64487 & -0.95674 \\
\hline $\mathrm{C}$ & 0.18356 & 3.30953 & -0.69109 \\
\hline $\mathrm{H}$ & 1.27063 & 3.1802 & -0.63891 \\
\hline C & -0.21414 & 3.10334 & -2.16298 \\
\hline $\mathrm{H}$ & 0.36401 & 3.78328 & -2.79757 \\
\hline $\mathrm{H}$ & -0.01069 & 2.08283 & -2.51086 \\
\hline $\mathrm{H}$ & -1.27162 & 3.3177 & -2.33817 \\
\hline C & -0.18375 & 4.72325 & -0.21872 \\
\hline $\mathrm{H}$ & 0.25427 & 5.4624 & -0.89847 \\
\hline $\mathrm{H}$ & -1.26741 & 4.87525 & -0.21497 \\
\hline $\mathrm{H}$ & 0.1929 & 4.94249 & 0.78382 \\
\hline C & 0.31424 & 2.38362 & 2.09691 \\
\hline H & -0.16052 & 3.33622 & 2.35986 \\
\hline $\mathrm{C}$ & 1.8319 & 2.60826 & 1.99716 \\
\hline $\mathrm{H}$ & 2.22804 & 2.82979 & 2.99422 \\
\hline $\mathrm{H}$ & 2.35036 & 1.72129 & 1.62266 \\
\hline H & 2.0956 & 3.44603 & 1.34882 \\
\hline $\mathrm{C}$ & -0.00907 & 1.36141 & 3.19775 \\
\hline $\mathrm{H}$ & 0.40259 & 1.71091 & 4.15133 \\
\hline $\mathrm{H}$ & -1.07825 & 1.19844 & 3.3365 \\
\hline $\mathrm{H}$ & 0.42633 & 0.38954 & 2.97088 \\
\hline C & -2.96339 & -1.03147 & -2.82627 \\
\hline $\mathrm{H}$ & -3.91186 & -1.5319 & -2.60666 \\
\hline $\mathrm{C}$ & -2.3781 & -1.69779 & -4.08462 \\
\hline H & -3.16427 & -1.75919 & -4.84482 \\
\hline H & -1.57018 & -1.10063 & -4.51022 \\
\hline H & -2.00699 & -2.71206 & -3.92284 \\
\hline $\mathrm{C}$ & -3.28301 & 0.43466 & -3.13863 \\
\hline H & -3.83248 & 0.4865 & -4.0853 \\
\hline H & -3.90666 & 0.88832 & -2.37019 \\
\hline $\mathrm{H}$ & -2.37655 & 1.0374 & -3.26055 \\
\hline C & -2.00038 & -3.25113 & -1.22472 \\
\hline $\mathrm{H}$ & -1.2547 & -3.51315 & -1.98657 \\
\hline C & -3.35063 & -3.88492 & -1.60138 \\
\hline $\mathrm{H}$ & -3.27245 & -4.97288 & -1.49992 \\
\hline
\end{tabular}

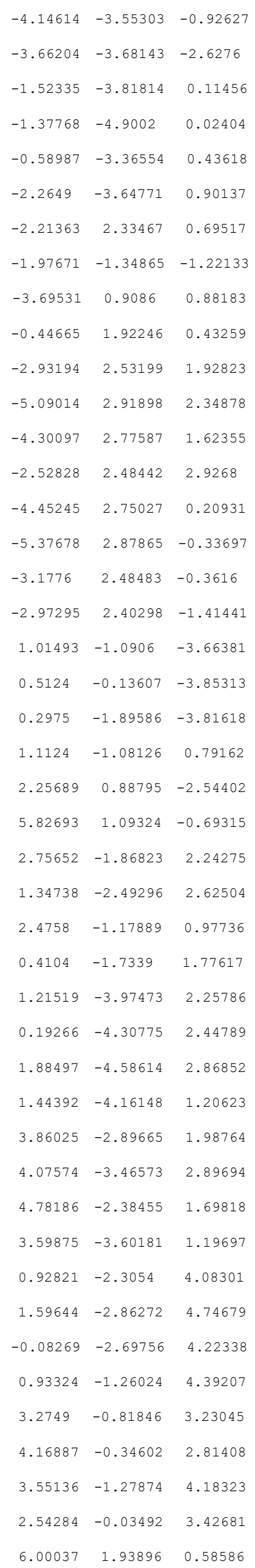




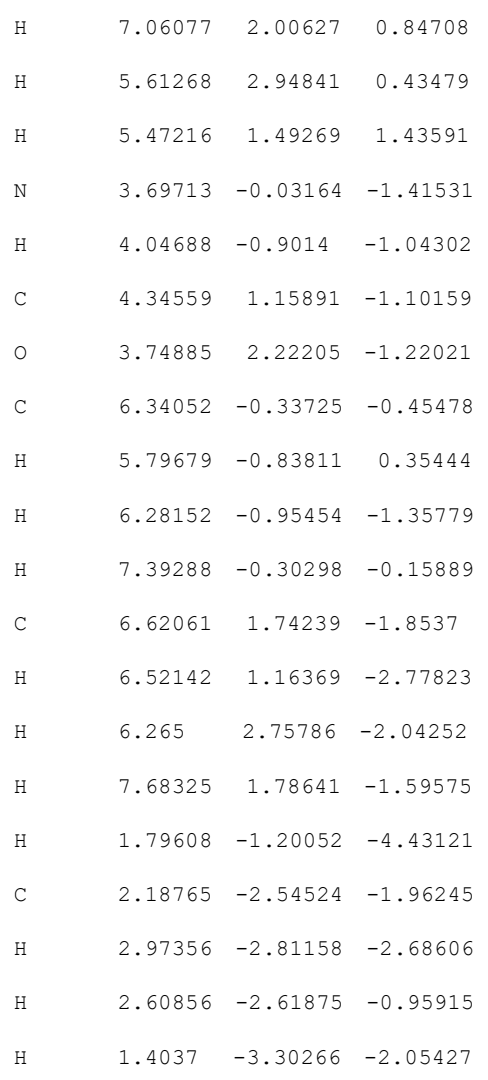

$G_{\text {sol }}=-2670.501886$ Hatree

\begin{tabular}{|c|c|c|c|}
\hline $\mathrm{Rh}$ & -0.27801 & 0.41335 & 0.87439 \\
\hline $\mathrm{H}$ & -0.39372 & 1.58172 & 1.94988 \\
\hline C & -1.94039 & 1.1105 & 2.36395 \\
\hline $\mathrm{C}$ & -2.55527 & 0.528 & 1.21098 \\
\hline $\mathrm{H}$ & 3.9658 & -0.87918 & -3.57129 \\
\hline C & 3.46979 & -2.75305 & -2.45211 \\
\hline $\mathrm{H}$ & 4.28895 & -3.41297 & -2.70289 \\
\hline C & 2.40203 & -3.05492 & -1.55871 \\
\hline $\mathrm{H}$ & 2.27451 & -3.99279 & -1.03719 \\
\hline C & 2.41869 & 1.26827 & 3.04747 \\
\hline $\mathrm{H}$ & 1.69445 & 2.05297 & 3.29719 \\
\hline C & 2.05003 & 0.0202 & 3.86591 \\
\hline $\mathrm{H}$ & 2.00129 & 0.27866 & 4.92928 \\
\hline $\mathrm{H}$ & 1.07897 & -0.39692 & 3.5818 \\
\hline $\mathrm{H}$ & 2.80406 & -0.76385 & 3.76115 \\
\hline C & 3.83013 & 1.74974 & 3.41614 \\
\hline $\mathrm{H}$ & 3.90488 & 1.86868 & 4.5027 \\
\hline $\mathrm{H}$ & 4.58943 & 1.02345 & 3.11044 \\
\hline
\end{tabular}

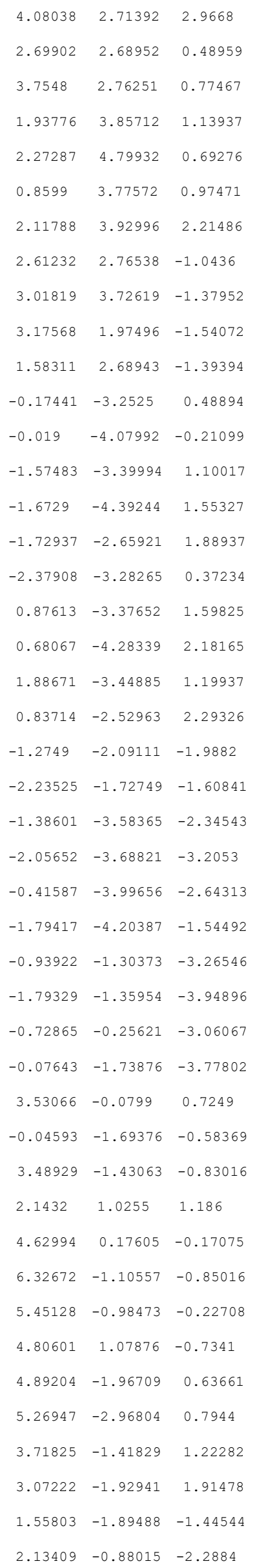




\begin{tabular}{|c|c|c|c|}
\hline H & 1.73773 & 0.11592 & -2.42304 \\
\hline C & 3.29976 & -1.41366 & -2.90789 \\
\hline C & -2.24235 & 2.56894 & 2.69408 \\
\hline $\mathrm{H}$ & -2.25766 & 3.18685 & 1.79917 \\
\hline $\mathrm{H}$ & -1.51839 & 2.97974 & 3.4044 \\
\hline B & -0.72483 & 1.78844 & -0.54641 \\
\hline H & -2.92597 & 1.20395 & 0.45123 \\
\hline C & -5.42506 & -1.89537 & 0.60696 \\
\hline C & -1.64111 & 3.71798 & -1.45821 \\
\hline C & -1.34603 & 2.6535 & -2.59717 \\
\hline O & -1.37901 & 2.95294 & -0.23178 \\
\hline O & -0.52617 & 1.64179 & -1.89383 \\
\hline C & -2.60324 & 1.94095 & -3.10694 \\
\hline $\mathrm{H}$ & -2.32238 & 1.19194 & -3.84979 \\
\hline H & -3.27873 & 2.65029 & -3.59289 \\
\hline $\mathrm{H}$ & -3.14454 & 1.43027 & -2.30665 \\
\hline C & -3.08798 & 4.20743 & -1.39207 \\
\hline H & -3.35378 & 4.75889 & -2.2991 \\
\hline $\mathrm{H}$ & -3.2032 & 4.88868 & -0.5443 \\
\hline $\mathrm{H}$ & -3.79388 & 3.38456 & -1.26919 \\
\hline C & -0.53023 & 3.1789 & -3.77594 \\
\hline $\mathrm{H}$ & -1.09422 & 3.94237 & -4.321 \\
\hline H & -0.32286 & 2.36042 & -4.47029 \\
\hline $\mathrm{H}$ & 0.4222 & 3.61144 & -3.46566 \\
\hline $\mathrm{C}$ & -0.69688 & 4.92348 & -1.45453 \\
\hline $\mathrm{H}$ & -0.87141 & 5.51171 & -0.5496 \\
\hline H & -0.88397 & 5.5683 & -2.31767 \\
\hline $\mathrm{H}$ & 0.3531 & 4.62761 & -1.46867 \\
\hline C & -5.46789 & -2.94762 & -0.52143 \\
\hline $\mathrm{H}$ & -6.35396 & -3.57978 & -0.40898 \\
\hline $\mathrm{H}$ & -5.50827 & -2.4594 & -1.49714 \\
\hline $\mathrm{H}$ & -4.58842 & -3.60076 & -0.49902 \\
\hline $\mathrm{N}$ & -3.38698 & -0.60856 & 1.3072 \\
\hline $\mathrm{H}$ & -3.4829 & -1.07882 & 2.19406 \\
\hline C & -4.27867 & -0.91034 & 0.29627 \\
\hline O & -4.18727 & -0.35956 & -0.79772 \\
\hline $\mathrm{C}$ & -5.30978 & -2.59475 & 1.97351 \\
\hline $\mathrm{H}$ & -4.39393 & -3.18921 & 2.06386 \\
\hline H & -5.35979 & -1.88602 & 2.80919 \\
\hline $\mathrm{H}$ & -6.14973 & -3.28362 & 2.1016 \\
\hline C & -6.7249 & -1.05459 & 0.568 \\
\hline $\mathrm{H}$ & -6.73347 & -0.29471 & 1.35723 \\
\hline $\mathrm{H}$ & -6.82675 & -0.55096 & -0.39575 \\
\hline $\mathrm{H}$ & -7.59271 & -1.70447 & 0.71752 \\
\hline $\mathrm{H}$ & -3.23279 & 2.60524 & 3.1665 \\
\hline
\end{tabular}
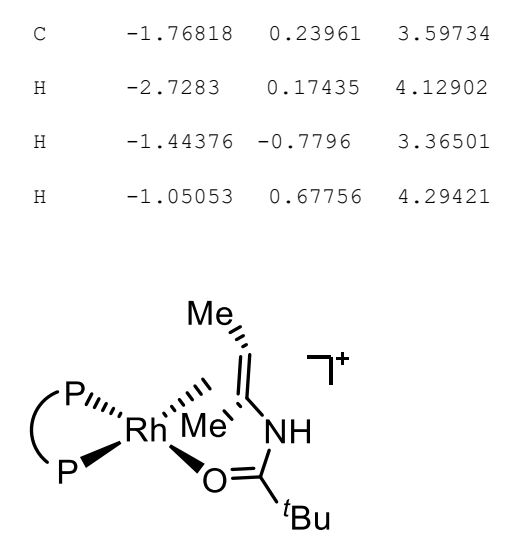

$$
\begin{aligned}
& G_{\text {sol }}=-2258.985518 \text { Hatree } \\
& \text { Fe } \quad-3.40144 \quad-0.83076-0.14506 \\
& \begin{array}{lllll}
\mathrm{P} & & -0.10886 & -1.73904 & 0.31329
\end{array} \\
& \begin{array}{llll}
\mathrm{P} & -0.97545 & 1.77212 & -0.303
\end{array} \\
& \begin{array}{llll}
\text { C } & -1.83849 & -2.16319 & -0.1088
\end{array} \\
& \begin{array}{lllll}
\text { C } & -2.88008 & -2.61144 & 0.77733
\end{array} \\
& \begin{array}{llll}
\mathrm{H} & -2.79838 & -2.74571 & 1.84552
\end{array} \\
& \begin{array}{lllll}
\text { C } & -4.06769 & -2.81261 & 0.01813
\end{array} \\
& \text { H } \quad \begin{array}{llll}
-5.0279 & -3.10898 & 0.41735
\end{array} \\
& \begin{array}{llll}
\text { C } & -3.78164 & -2.50757 & -1.3422
\end{array} \\
& \text { H } \quad \begin{array}{llll} 
& -4.48475 & -2.53556 & -2.16323
\end{array} \\
& \text { C } \quad \begin{array}{llll}
-2.41795 & -2.10775 & -1.42772
\end{array} \\
& \text { H } \quad \begin{array}{llll}
-1.91285 & -1.80343 & -2.33049
\end{array} \\
& \text { C } \quad-2.66692 \quad 1.09601 \quad-0.06715 \\
& \text { C } \quad \begin{array}{llll}
-3.23442 & 0.70989 & 1.20014
\end{array} \\
& \begin{array}{llll}
\mathrm{H} & -2.70129 & 0.65234 & 2.13705
\end{array} \\
& \begin{array}{llll}
\text { C } & -4.61394 & 0.40933 & 1.00997
\end{array} \\
& \begin{array}{llll}
\mathrm{H} & -5.2951 & 0.0558 & 1.77175
\end{array} \\
& \begin{array}{lllll}
\text { C } & -4.9197 & 0.59431 & -0.36851
\end{array} \\
& \text { H } \quad \begin{array}{llll}
-5.87255 & 0.40175 & -0.84205
\end{array} \\
& \begin{array}{lllll}
\text { C } & -3.73397 & 1.01961 & -1.03251
\end{array} \\
& \mathrm{H} \quad \begin{array}{llll}
-3.65493 & 1.21752 & -2.09122
\end{array} \\
& \begin{array}{lllll}
\text { C } & 0.87576 & -3.03536 & -0.6451
\end{array} \\
& \text { H } \quad \begin{array}{llll}
1.87681 & -2.94226 & -0.21509
\end{array} \\
& \begin{array}{llll}
\text { C } & 0.99377 & -2.69231 & -2.13794
\end{array} \\
& \text { H } \quad \begin{array}{llll}
1.68975 & -3.38891 & -2.61852
\end{array} \\
& \text { H } \quad \begin{array}{llll}
1.37602 & -1.67962 & -2.29225
\end{array} \\
& \text { H } \quad \begin{array}{llll}
0.03724 & -2.79022 & -2.65904
\end{array} \\
& \begin{array}{llll}
\text { C } & 0.36212 & -4.46923 & -0.44378
\end{array} \\
& \text { H } \quad 1.01937 \quad-5.17031 \quad-0.97011 \\
& \text { H } \quad-0.64616 \quad-4.59435 \quad-0.84953 \\
& \begin{array}{llll}
\mathrm{H} & 0.3421 & -4.76874 & 0.60769
\end{array} \\
& \begin{array}{llll}
\text { C } & 0.04498 & -2.33081 & 2.10522
\end{array} \\
& \begin{array}{llll}
\mathrm{H} & -0.51943 & -3.26957 & 2.14881
\end{array} \\
& \begin{array}{llll}
\text { C } & 1.50317 & -2.6278 & 2.49273
\end{array}
\end{aligned}
$$




\begin{tabular}{|c|c|c|c|}
\hline & 1.54747 & -2.88574 & 3.55612 \\
\hline & 2.14823 & -1.76053 & 2.3277 \\
\hline & 1.92146 & -3.46954 & 1.93621 \\
\hline & -0.5789 & -1.33859 & 3.09864 \\
\hline & -0.50447 & -1.73998 & 4.11515 \\
\hline & -1.63352 & -1.14708 & 2.89292 \\
\hline & -0.05104 & -0.38091 & 3.07269 \\
\hline & -0.90801 & 2.12162 & -2.16154 \\
\hline & -1.84516 & 2.64307 & -2.38336 \\
\hline & 0.26026 & 3.04656 & -2.53798 \\
\hline & 0.1707 & 3.32895 & -3.59244 \\
\hline & 1.21607 & 2.53027 & -2.41374 \\
\hline & 0.29065 & 3.9693 & -1.95383 \\
\hline & -0.84983 & 0.84856 & -3.01755 \\
\hline & -0.82948 & 1.12379 & -4.07791 \\
\hline & -1.71251 & 0.20302 & -2.856 \\
\hline & 0.05591 & 0.27077 & -2.80806 \\
\hline & -1.23936 & 3.50802 & 0.43727 \\
\hline & -0.24167 & 3.95884 & 0.43487 \\
\hline & -2.17784 & 4.40279 & -0.39305 \\
\hline & -2.32811 & 5.34954 & 0.1371 \\
\hline & -3.16173 & 3.9395 & -0.51651 \\
\hline & -1.78328 & 4.64577 & -1.38151 \\
\hline & -1.75101 & 3.46203 & 1.88976 \\
\hline & -1.60966 & 4.44386 & 2.35417 \\
\hline & -1.23979 & 2.72437 & 2.51035 \\
\hline & -2.8202 & 3.23994 & 1.91555 \\
\hline & 2.10198 & 1.54314 & 1.68752 \\
\hline & 2.61288 & 1.9992 & 0.45013 \\
\hline & 1.45509 & 2.4162 & 2.72549 \\
\hline & 0.72376 & 1.86066 & 3.3185 \\
\hline & 0.97035 & 3.2993 & 2.31273 \\
\hline & 5.14992 & -0.73246 & -0.53612 \\
\hline & 4.92731 & -1.54565 & -1.8316 \\
\hline & 5.8347 & -2.10745 & -2.07159 \\
\hline & 4.10389 & -2.25313 & -1.71634 \\
\hline & 4.70302 & -0.89052 & -2.68005 \\
\hline & 3.7866 & 1.30135 & -0.06121 \\
\hline & 4.63521 & 1.83885 & -0.17969 \\
\hline & 3.83493 & -0.02568 & -0.19463 \\
\hline & 2.77847 & -0.69843 & -0.03557 \\
\hline & 6.31749 & 0.25108 & -0.73946 \\
\hline & 6.13223 & 0.94691 & -1.56574 \\
\hline & 6.54252 & 0.82224 & 0.16866 \\
\hline & 7.22191 & -0.30848 & -0.99237 \\
\hline
\end{tabular}

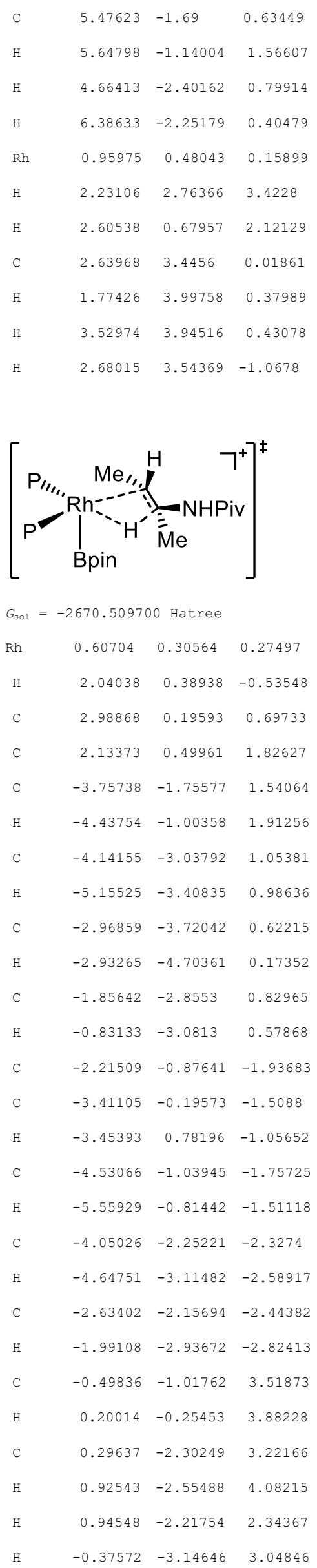




\begin{tabular}{|c|c|c|c|}
\hline C & -1.52786 & -1.30794 & 4.62386 \\
\hline $\mathrm{H}$ & -1.03043 & -1.80495 & 5.46411 \\
\hline $\mathrm{H}$ & -2.31499 & -1.97889 & 4.26555 \\
\hline $\mathrm{H}$ & -1.99945 & -0.40521 & 5.01767 \\
\hline C & -2.41822 & 1.03616 & 2.65149 \\
\hline $\mathrm{H}$ & -3.08579 & 0.47044 & 3.31117 \\
\hline C & -1.64814 & 2.062 & 3.49864 \\
\hline $\mathrm{H}$ & -2.35559 & 2.77817 & 3.93046 \\
\hline H & -0.94238 & 2.62121 & 2.88229 \\
\hline H & -1.10422 & 1.60461 & 4.32919 \\
\hline C & -3.27153 & 1.74158 & 1.58834 \\
\hline H & -3.921 & 2.47466 & 2.08019 \\
\hline $\mathrm{H}$ & -3.91316 & 1.04392 & 1.04814 \\
\hline $\mathrm{H}$ & -2.63864 & 2.2743 & 0.87615 \\
\hline $\mathrm{C}$ & 0.5026 & -1.45226 & -2.81394 \\
\hline $\mathrm{H}$ & -0.12285 & -1.79064 & -3.64768 \\
\hline C & 1.80219 & -0.86354 & -3.38743 \\
\hline $\mathrm{H}$ & 2.34542 & -1.65069 & -3.92097 \\
\hline $\mathrm{H}$ & 2.46797 & -0.49751 & -2.60387 \\
\hline $\mathrm{H}$ & 1.62432 & -0.05337 & -4.09757 \\
\hline C & 0.80789 & -2.66244 & -1.91713 \\
\hline H & 1.40353 & -3.39249 & -2.4756 \\
\hline H & -0.0962 & -3.16584 & -1.56815 \\
\hline H & 1.3941 & -2.36255 & -1.04171 \\
\hline $\mathrm{C}$ & -0.70766 & 1.20374 & -3.22485 \\
\hline H & 0.28554 & 1.66771 & -3.25367 \\
\hline C & -1.0619 & 0.66255 & -4.62112 \\
\hline H & -1.17915 & 1.50427 & -5.31239 \\
\hline $\mathrm{H}$ & -2.01227 & 0.11999 & -4.60208 \\
\hline $\mathrm{H}$ & -0.29995 & 0.00348 & -5.04063 \\
\hline C & -1.73686 & 2.27438 & -2.82589 \\
\hline $\mathrm{H}$ & -1.62812 & 3.14215 & -3.48583 \\
\hline $\mathrm{H}$ & -1.62399 & 2.61176 & -1.7943 \\
\hline $\mathrm{H}$ & -2.75584 & 1.90304 & -2.95787 \\
\hline C & -2.33132 & -1.62206 & 1.40661 \\
\hline $\mathrm{P}$ & -0.52668 & -0.15425 & -1.91131 \\
\hline C & 2.30418 & 1.78252 & 2.61605 \\
\hline $\mathrm{H}$ & 1.46093 & 1.93541 & 3.29405 \\
\hline $\mathrm{H}$ & 2.39034 & 2.66046 & 1.97633 \\
\hline B & 0.52563 & 2.30881 & 0.01547 \\
\hline H & 1.9706 & -0.35653 & 2.47879 \\
\hline C & 4.71485 & -3.18458 & 0.01375 \\
\hline C & 1.23352 & 4.43143 & -0.60167 \\
\hline $\mathrm{C}$ & -0.10193 & 4.52783 & 0.25105 \\
\hline 0 & 1.59509 & 3.0158 & -0.4749 \\
\hline
\end{tabular}

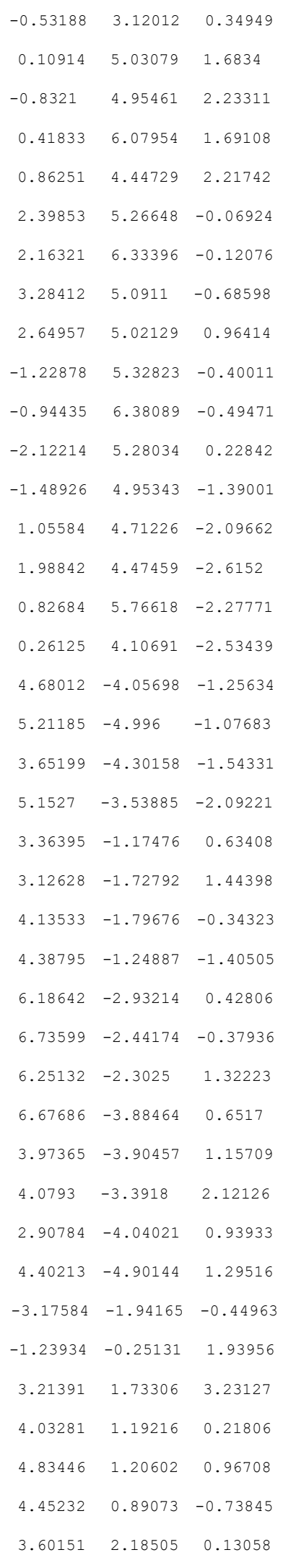




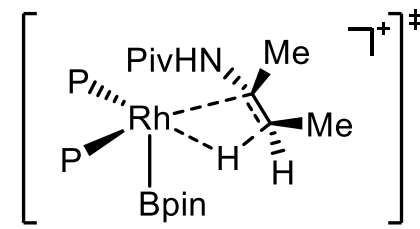

$G_{\text {sol }}=-2670.500574$ Hatree

$\begin{array}{llll}\mathrm{H} & -0.48147 & 1.6648 & 1.76779\end{array}$

C $\quad \begin{array}{llll}-1.89633 & 1.13294 & 2.37752\end{array}$

$\begin{array}{llll}\text { C } & -2.33837 & -0.16288 & 1.961\end{array}$

$\begin{array}{llll}\text { C } & 5.21446 & -1.169 & -0.17417\end{array}$

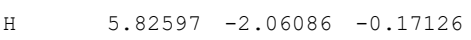

$\begin{array}{llll}\text { C } & 4.12166 & -0.90714 & 0.69876\end{array}$

H

$\begin{array}{llll}0.73928 & -4.21767 & -0.27338\end{array}$

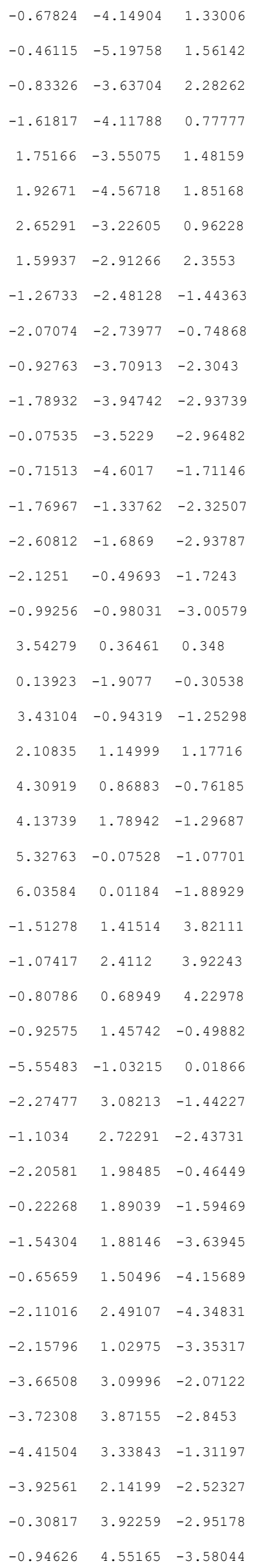




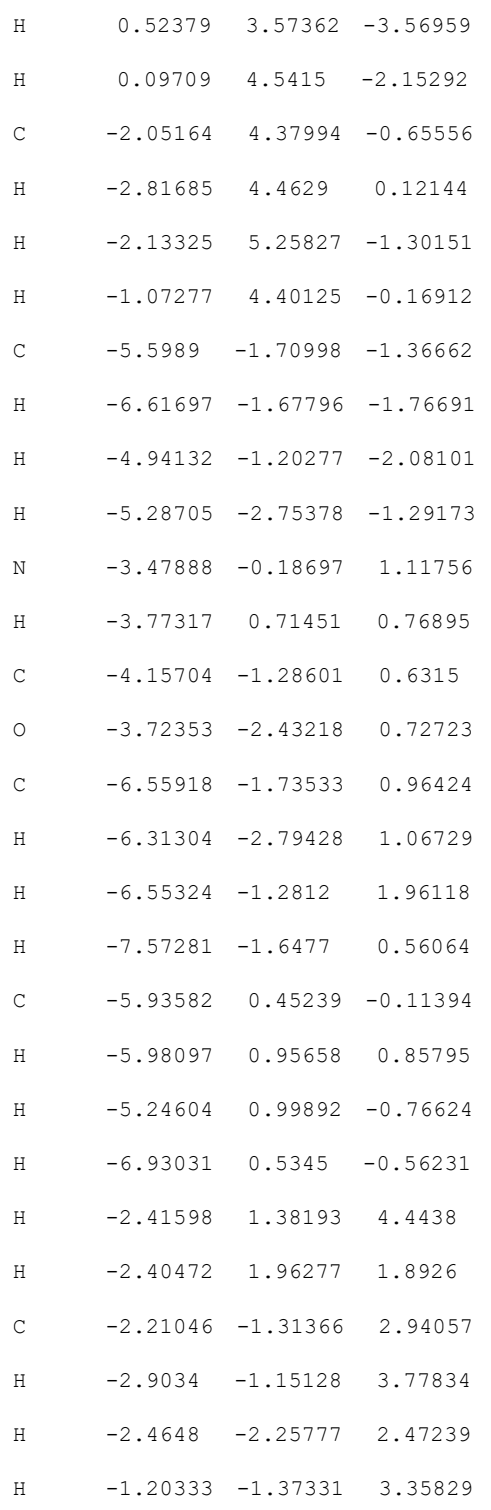

\begin{tabular}{|c|c|c|c|}
\hline H & -6.30309 & 0.2591 & -1.21093 \\
\hline & -4.63633 & 0.5997 & 0.23904 \\
\hline & -5.12664 & 0.43254 & 1.18737 \\
\hline & 0.26464 & -3.39563 & 0.25007 \\
\hline & 1.34058 & -3.19374 & 0.28877 \\
\hline & -0.2231 & -3.54708 & 1.70061 \\
\hline & 0.38371 & -4.30206 & 2.21159 \\
\hline & -0.13668 & -2.61808 & 2.27273 \\
\hline & -1.26198 & -3.88391 & 1.74518 \\
\hline & 0.01071 & -4.6935 & -0.53169 \\
\hline & 0.44223 & -5.53814 & 0.01663 \\
\hline & -1.06031 & -4.88799 & -0.64794 \\
\hline & 0.46658 & -4.6881 & -1.5247 \\
\hline & 0.29117 & -1.98643 & -2.33128 \\
\hline & -0.14309 & -2.91501 & -2.71936 \\
\hline & 1.82072 & -2.13551 & -2.30929 \\
\hline & 2.18941 & -2.21287 & -3.33813 \\
\hline & 2.30126 & -1.27165 & -1.8438 \\
\hline & 2.15227 & -3.02698 & -1.77393 \\
\hline & -0.12909 & -0.83717 & -3.26133 \\
\hline & 0.24109 & -1.03916 & -4.27297 \\
\hline & -1.20965 & -0.70559 & -3.32319 \\
\hline & 0.28496 & 0.1151 & -2.93202 \\
\hline & -2.73966 & 0.77179 & 2.91879 \\
\hline & -3.70738 & 1.28239 & 2.89474 \\
\hline & -1.96523 & 1.28782 & 4.14173 \\
\hline & -2.60338 & 1.21058 & 5.02843 \\
\hline & -1.0811 & 0.67376 & 4.32846 \\
\hline & -1.64385 & 2.32755 & 4.05758 \\
\hline & -3.01478 & -0.72513 & 3.09259 \\
\hline & -3.44142 & -0.89952 & 4.08678 \\
\hline & -3.72489 & -1.09639 & 2.35574 \\
\hline & -2.0969 & -1.31952 & 3.02649 \\
\hline & -2.07258 & 3.17453 & 1.39167 \\
\hline & -1.19172 & 3.46245 & 1.97918 \\
\hline & -3.33747 & 3.6976 & 2.09435 \\
\hline & -3.34829 & 4.79121 & 2.0309 \\
\hline & -4.24411 & 3.33764 & 1.5966 \\
\hline & -3.3958 & 3.43714 & 3.15271 \\
\hline & -1.96485 & 3.82702 & 0.00593 \\
\hline & -1.79797 & 4.90338 & 0.12415 \\
\hline & -1.15204 & 3.4079 & -0.58281 \\
\hline H & -2.89461 & 3.69737 & -0.5557 \\
\hline & -2.1858 & -2.26785 & -0.86389 \\
\hline & -1.94035 & 1.27534 & 1.26856 \\
\hline
\end{tabular}



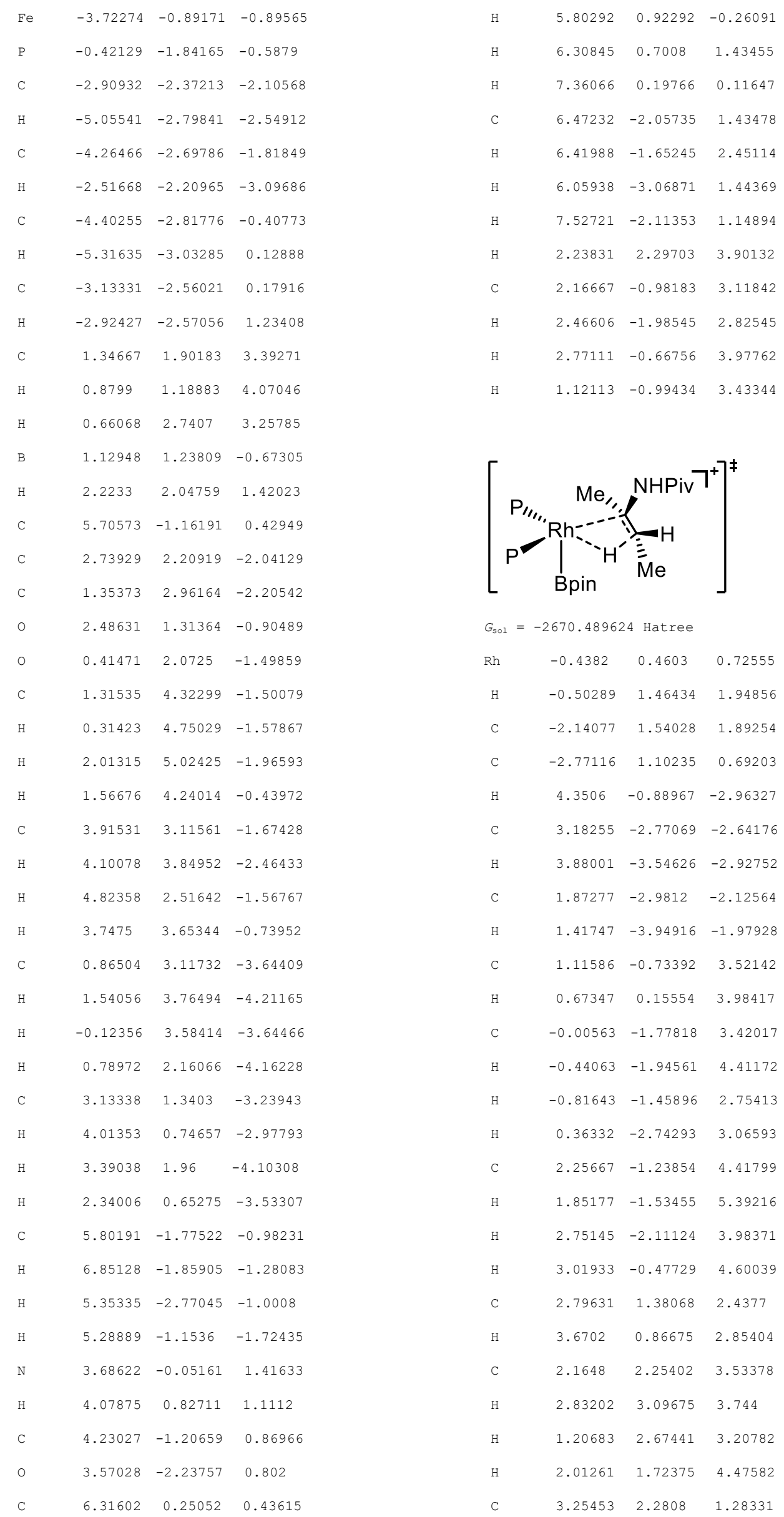


\begin{tabular}{|c|c|c|c|}
\hline $\mathrm{H}$ & 4.10254 & 2.89388 & 1.60782 \\
\hline $\mathrm{H}$ & 3.55308 & 1.73336 & 0.38889 \\
\hline $\mathrm{H}$ & 2.44488 & 2.95578 & 1.00489 \\
\hline C & -1.07097 & -3.076 & -0.86577 \\
\hline $\mathrm{H}$ & -0.82866 & -3.60801 & -1.7913 \\
\hline C & -2.59404 & -3.11291 & -0.69094 \\
\hline $\mathrm{H}$ & -2.92053 & -4.15839 & -0.65682 \\
\hline $\mathrm{H}$ & -2.88958 & -2.64852 & 0.25456 \\
\hline $\mathrm{H}$ & -3.13999 & -2.61915 & -1.49424 \\
\hline C & -0.41091 & -3.83806 & 0.28678 \\
\hline $\mathrm{H}$ & -0.82852 & -4.85043 & 0.33132 \\
\hline $\mathrm{H}$ & 0.66676 & -3.9276 & 0.16286 \\
\hline H & -0.61582 & -3.36269 & 1.24882 \\
\hline C & -1.35856 & -0.92154 & -2.78812 \\
\hline $\mathrm{H}$ & -2.39382 & -0.84179 & -2.4393 \\
\hline C & -1.26639 & -2.00345 & -3.87644 \\
\hline $\mathrm{H}$ & -1.81953 & -1.66729 & -4.76009 \\
\hline $\mathrm{H}$ & -0.2309 & -2.1805 & -4.18458 \\
\hline $\mathrm{H}$ & -1.70329 & -2.95801 & -3.57407 \\
\hline C & -0.96326 & 0.44095 & -3.36068 \\
\hline $\mathrm{H}$ & -1.597 & 0.66986 & -4.22467 \\
\hline $\mathrm{H}$ & -1.09084 & 1.23479 & -2.62543 \\
\hline $\mathrm{H}$ & 0.07758 & 0.45565 & -3.69557 \\
\hline C & 2.91705 & -1.23484 & 1.22728 \\
\hline $\mathrm{P}$ & -0.39855 & -1.3247 & -1.1987 \\
\hline $\mathrm{Fe}$ & 3.03886 & -1.98621 & -0.70653 \\
\hline $\mathrm{P}$ & 1.68103 & -0.03298 & 1.85215 \\
\hline $\mathrm{C}$ & 4.2111 & -0.95488 & 0.65909 \\
\hline H & 5.80747 & -2.29688 & -0.13893 \\
\hline C & 4.84195 & -2.1896 & 0.33583 \\
\hline $\mathrm{H}$ & 4.63893 & 0.01823 & 0.47661 \\
\hline C & 3.9597 & -3.24636 & 0.69484 \\
\hline $\mathrm{H}$ & 4.13657 & -4.30326 & 0.55013 \\
\hline C & 2.77893 & -2.66794 & 1.23868 \\
\hline $\mathrm{H}$ & 1.92718 & -3.21918 & 1.60079 \\
\hline C & 1.29159 & -1.70156 & -1.81151 \\
\hline C & 2.27706 & -0.70788 & -2.14604 \\
\hline $\mathrm{H}$ & 2.15197 & 0.35385 & -2.00005 \\
\hline C & 3.42959 & -1.36784 & -2.65861 \\
\hline C & -2.14288 & 2.98945 & 2.35054 \\
\hline H & -1.76617 & 3.66679 & 1.58865 \\
\hline $\mathrm{H}$ & -1.53107 & 3.11288 & 3.24841 \\
\hline B & 0.26301 & 2.10775 & -0.2592 \\
\hline $\mathrm{H}$ & -2.27079 & 0.84604 & 2.72846 \\
\hline & -5.79927 & -1.23095 & 0.49925 \\
\hline
\end{tabular}

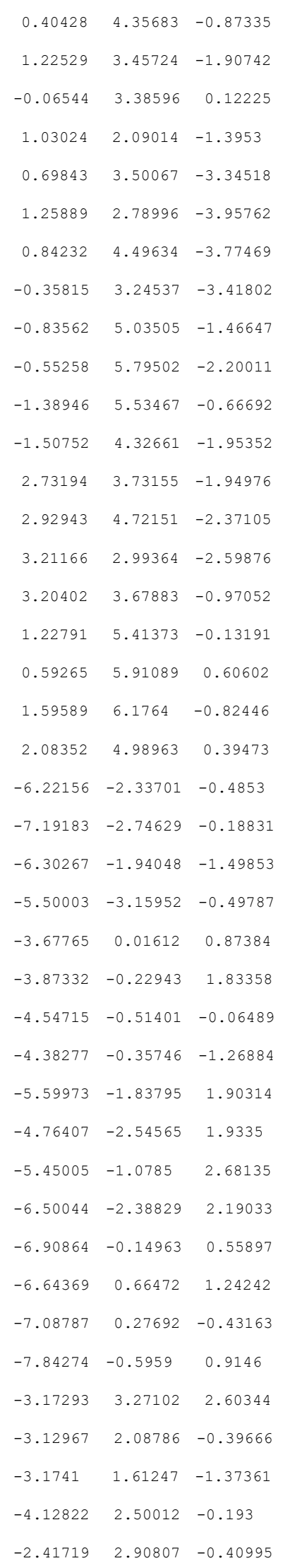




\section{References}

1 Tsuchikama, K.; Kasagawa, M.; Endo, K.; Shibata, T. Org. Lett.2009, 11, 1821.

2 Jiang, L.; Job, G. E.; Klapars, A.; Buchwald, S. L. Org. Lett.2003, 5, 3667.

3 Gooßen, L. J.; Salih, K. S. M.; Blanchot, M. Angew. Chem. Int. Ed.2008, 47, 8492.

4 Coussanes, G.; Gaus, K.; O’Sullivan, A. C.; Eur. J. Org. Chem. 2016, 4176.

5 Ohmura, T.; Miwa, K.; Awano, T.; Suginome, M. Chem. Asian J. 2018, 13, 2414.

6 Full citation of Gaussian 09: Frisch, M. J.; Trucks, G. W.; Schlegel, H. B.; Scuseria, G. E.; Robb, M. A.; Cheeseman, J. R.; Scalmani, G.; Barone, V.; Mennucci, B.; Petersson, G. A.; Nakatsuji, H.; Caricato, M.; Li, X.; Hratchian, H. P.; Izmaylov, A. F.; Bloino, J.; Zheng, G.; Sonnenberg, J. L.; Hada, M.; Ehara, M.; Toyota, K.; Fukuda, R.; Hasegawa, J.; Ishida, M.; Nakajima, T.; Honda, Y.; Kitao, O.; Nakai, H.; Vreven, T.; Montgomery, J. A., Jr.; Peralta, J. E.; Ogliaro, F.; Bearpark, M.; Heyd, J. J.; Brothers, E.; Kudin, K. N.; Staroverov, V. N.; Keith, T.; Kobayashi, R.; Normand, J.; Raghavachari, K.; Rendell, A.; Burant, J. C.; Iyengar, S. S.; Tomasi, J.; Cossi, M.; Rega, N.; Millam, J. M.; Klene, M.; Knox, J. E.; Cross, J. B.; Bakken, V.; Adamo, C.; Jaramillo, J.; Gomperts, R.; Stratmann, R. E.; Yazyev, O.; Austin, A. J.; Cammi, R.; Pomelli, C.; Ochterski, J. W.; Martin, R. L.; Morokuma, K.; Zakrzewski, V. G.; Voth, G. A.; Salvador, P.; Dannenberg, J. J.; Dapprich, S.; Daniels, A. D.; Farkas, O.; Foresman, J. B.; Ortiz, J. V.; Cioslowski, J.; Fox, D. J.; Gaussian 09, revision B.01; Gaussian Inc.: Wallingford, CT, 2010.)

7 (a) Zhao, Y.; Truhlar, D. G. Theor. Chem. Acc. 2008, 120, 215-241. (b) Zhao, Y.; Truhlar, D. G. Acc. Chem. Res. 2008, 41, 157-167. 


\section{Copies of NMR Spectra}<smiles>CC(C)(C)C(=O)N/C=C\CCP</smiles>

$\mathrm{Ph}$

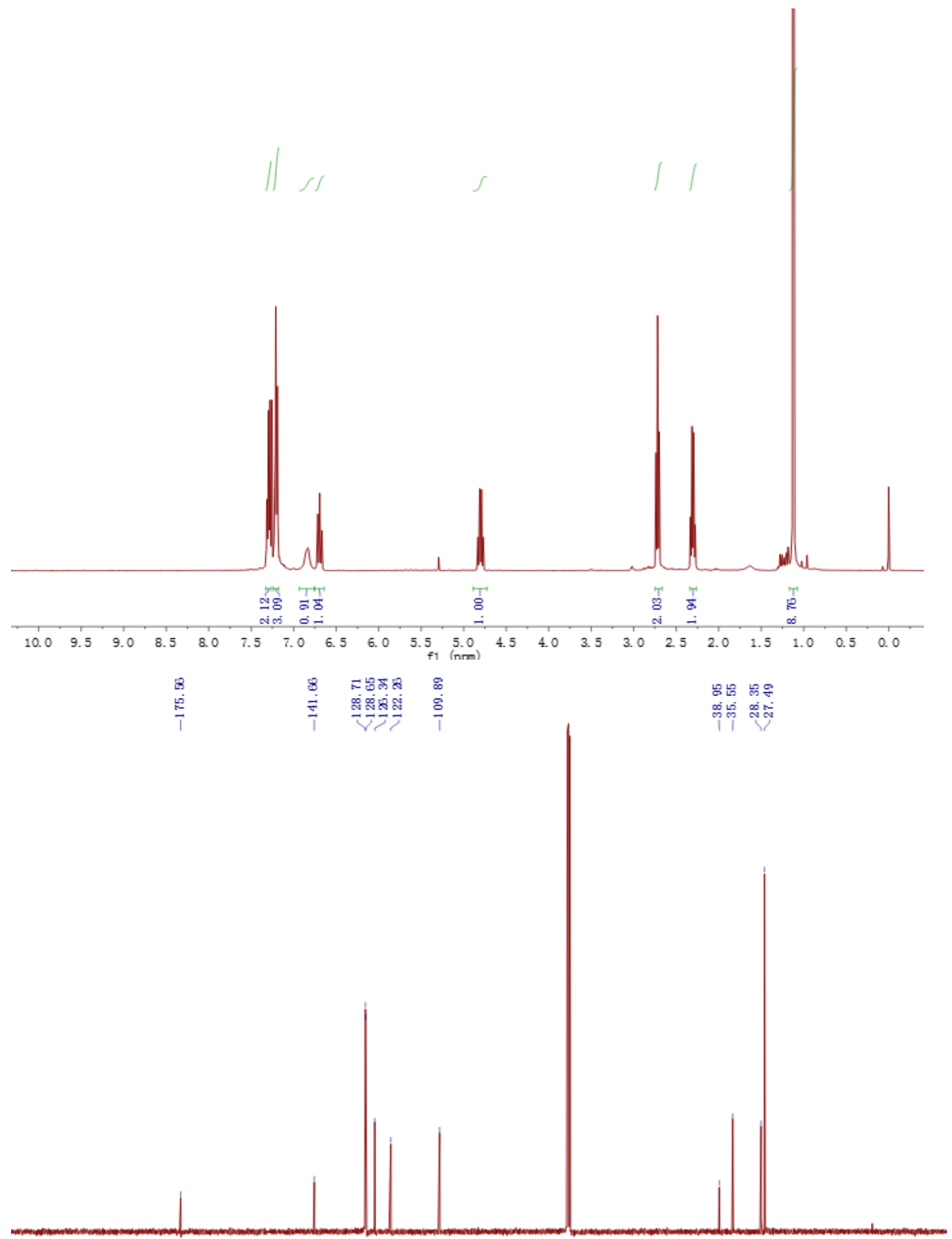

$\begin{array}{lllllllllllllllllllllll}210 & 200 & 190 & 180 & 170 & 160 & 150 & 140 & 130 & 120 & 110 \underset{\mathrm{f} 1}{1000}(\mathrm{pm}) & 90 & 80 & 70 & 60 & 50 & 40 & 30 & 20 & 10 & 0 & -10\end{array}$ 
<smiles>CC(C)(C)C(=O)N/C=C/CCc1ccccc1</smiles>

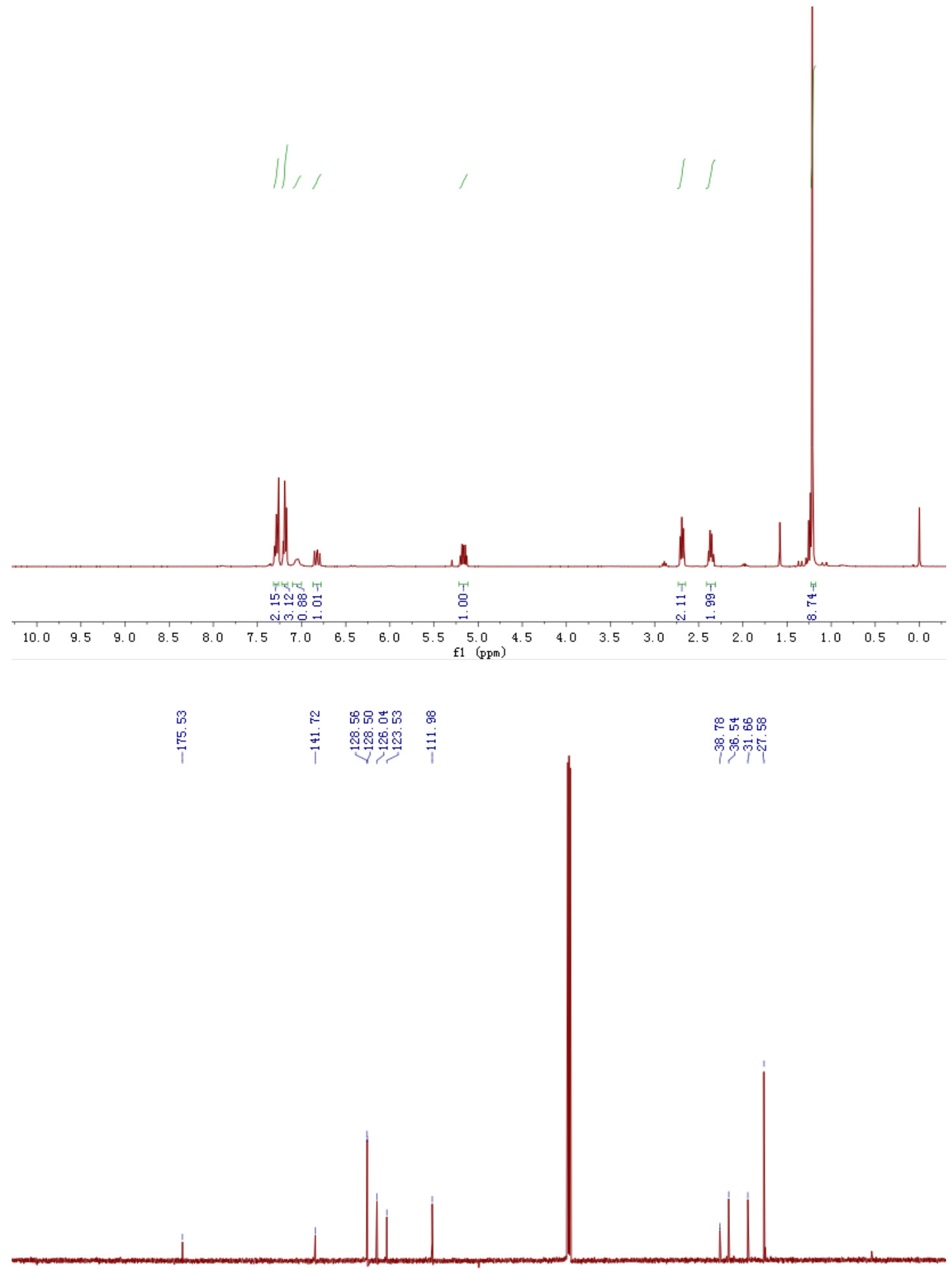

$\begin{array}{llllllllllllllllllllllllll}210 & 200 & 190 & 180 & 170 & 160 & 150 & 140 & 130 & 120 & 110 & 100 & 90 & 80 & 70 & 60 & 50 & 40 & 30 & 20 & 10 & 0 & -10\end{array}$ f1 $(\mathrm{ppm})$ 
<smiles>CC(=O)N/C=C\CCc1ccccc1</smiles>
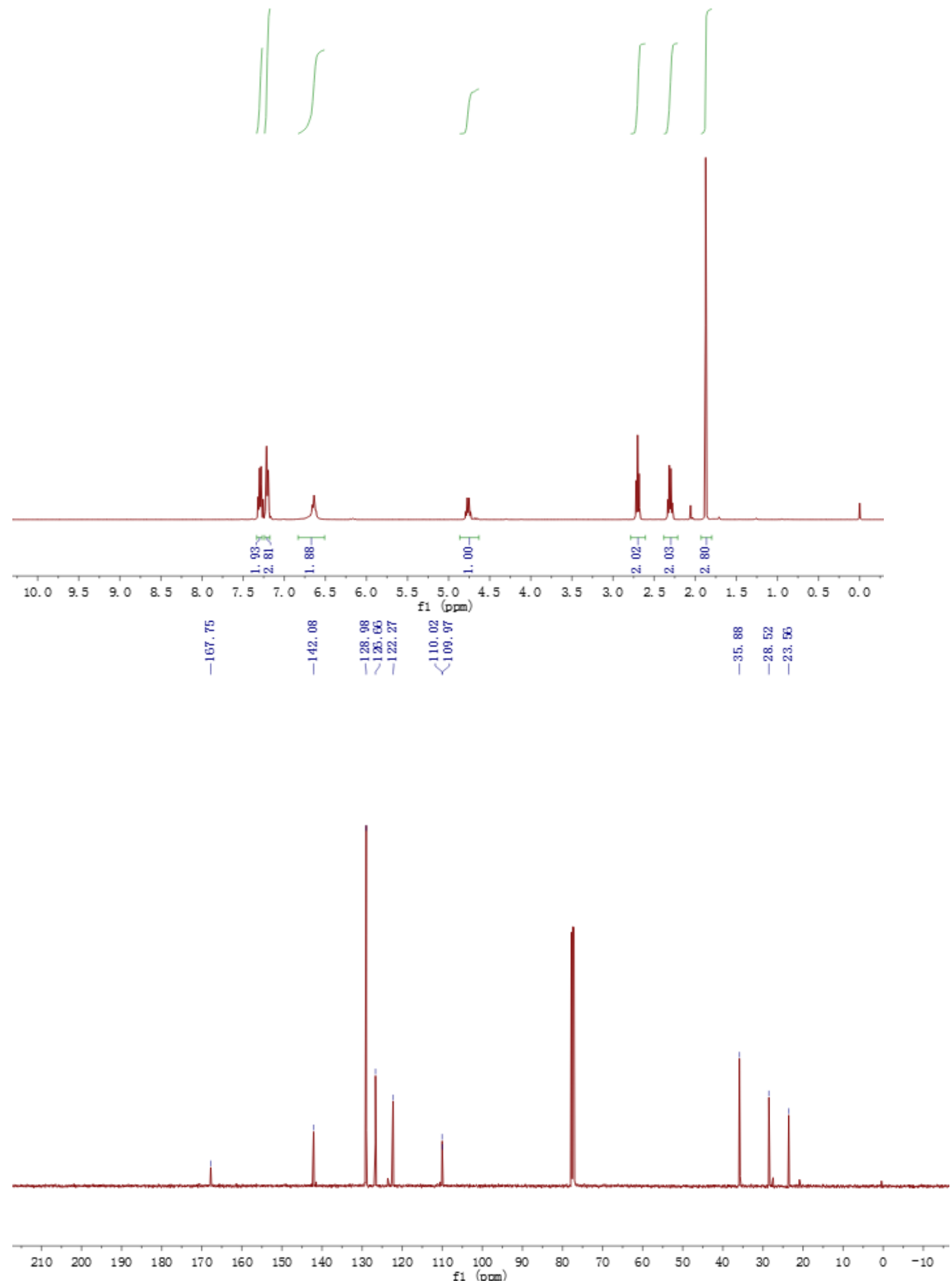
<smiles>CC(=O)N/C=C/CCc1ccccc1</smiles>
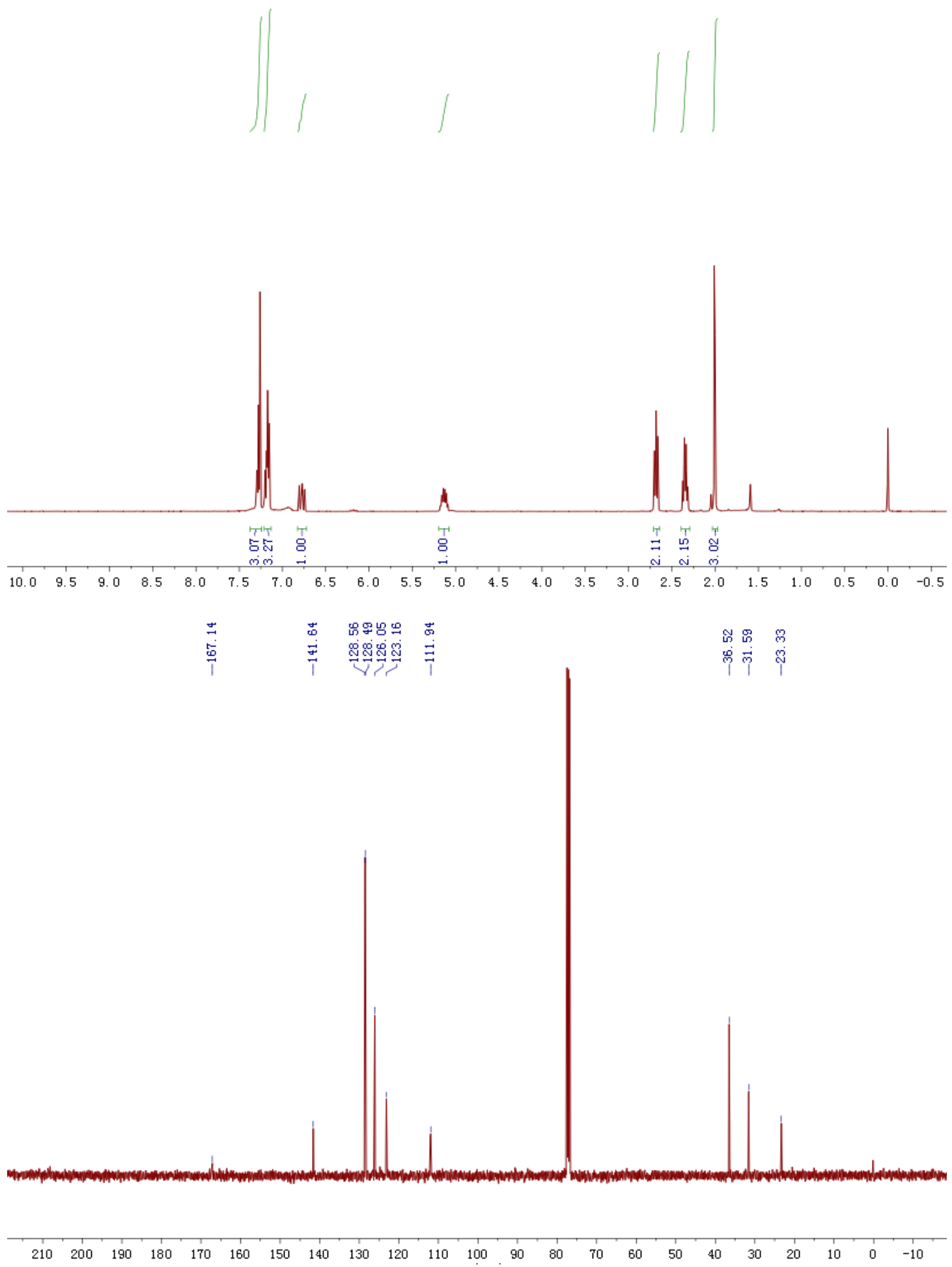

S129 
<smiles>CC(C)C(=O)N/C=C\CCc1ccccc1</smiles>
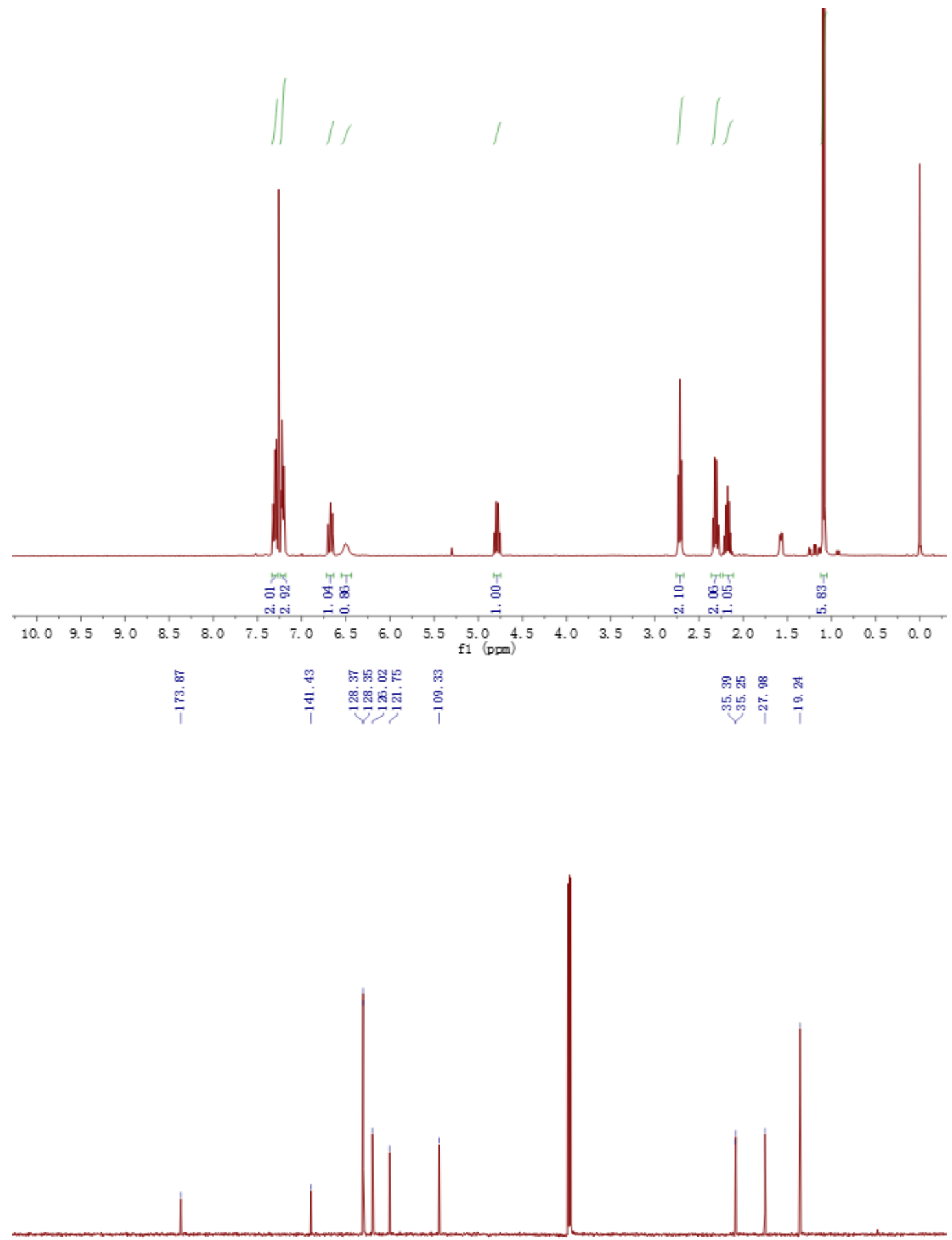

$\begin{array}{llllllllllllllllllllllllllll}210 & 200 & 190 & 180 & 170 & 160 & 150 & 140 & 130 & 120 & 110 & 100 & 90 & 80 & 70 & 60 & 50 & 40 & 30 & 20 & 10 & 0 & -10\end{array}$ 
<smiles>CC(C)C(=O)N/C=C/CCc1ccccc1</smiles>
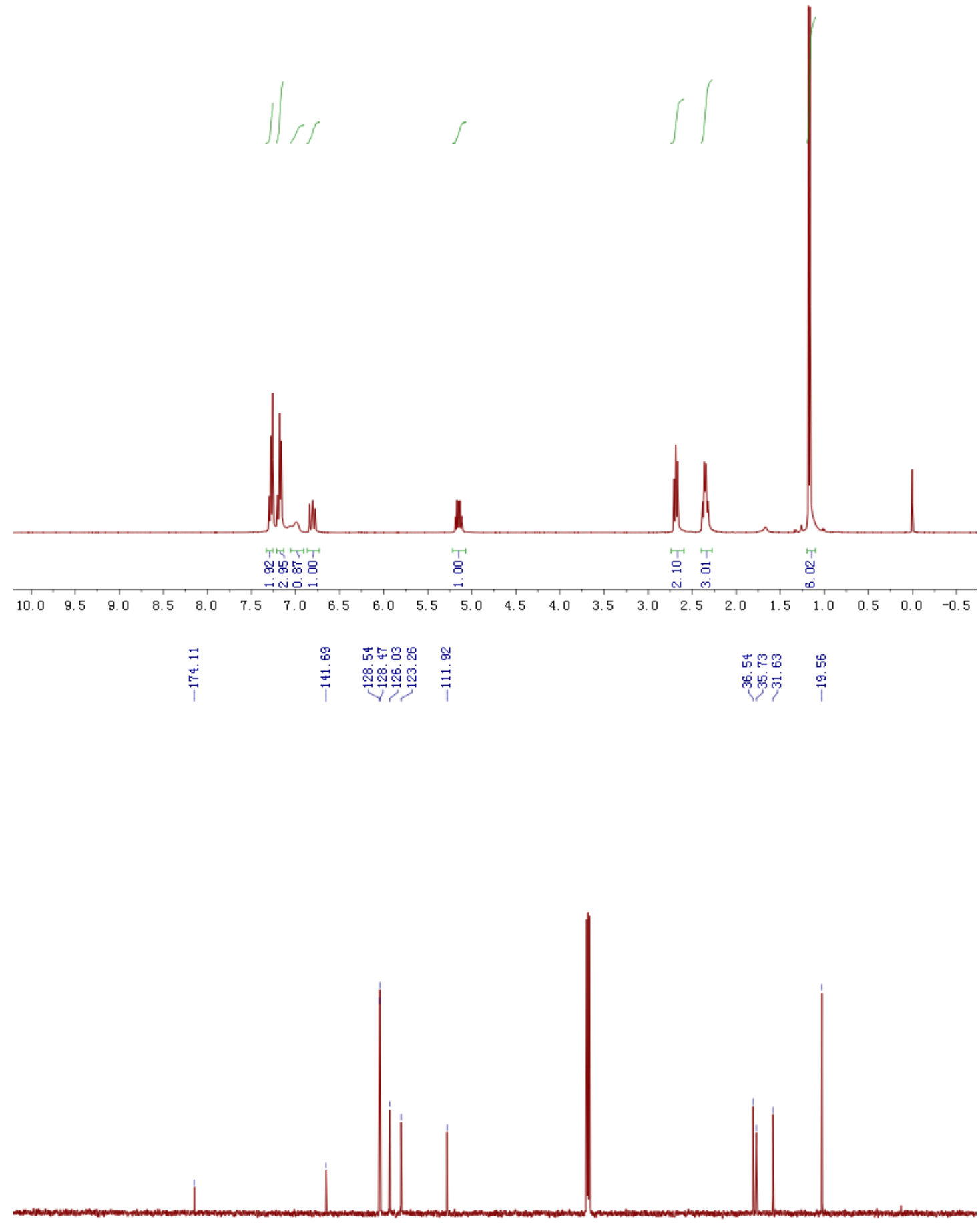

$\begin{array}{lllllllllllllllllllllll}210 & 200 & 190 & 180 & 170 & 160 & 150 & 140 & 130 & 120 & 110 & 100 & 90 & 80 & 70 & 60 & 50 & 40 & 30 & 20 & 10 & 0 & -10\end{array}$ 


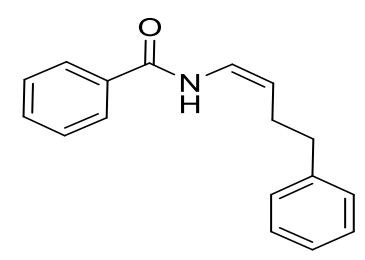

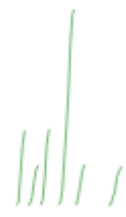
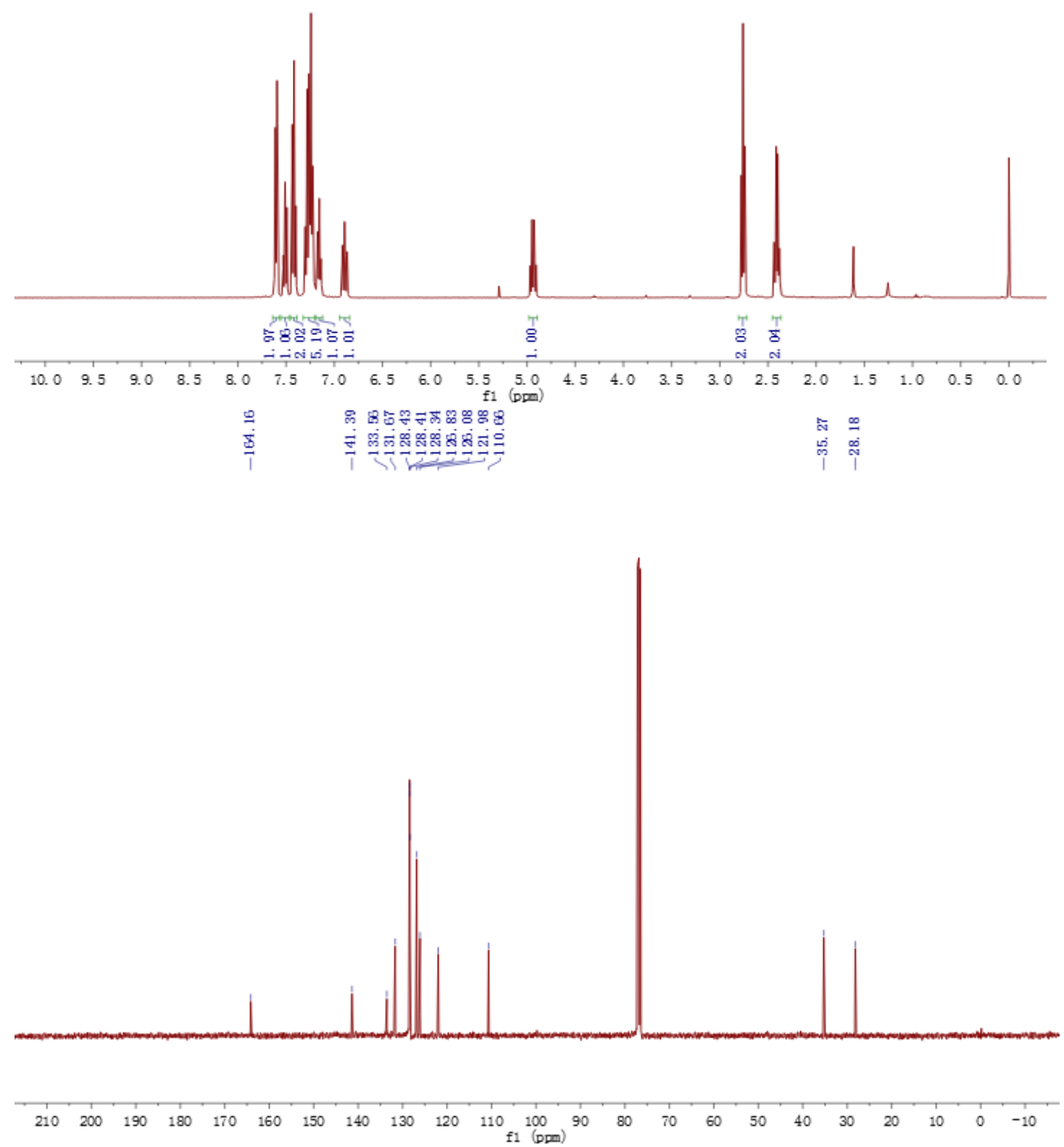

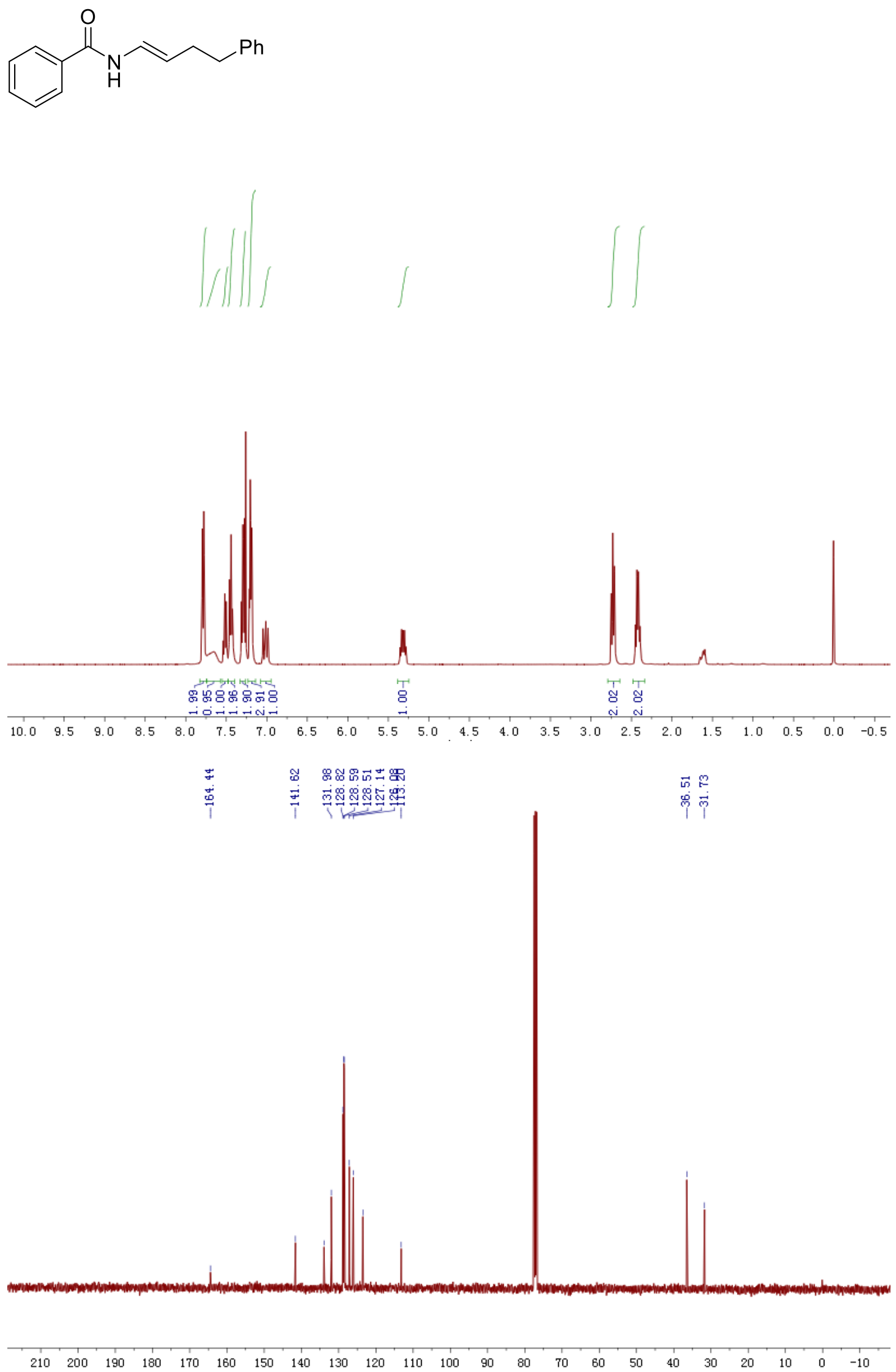

S133 
<smiles>C/C=C\NC(=O)C(C)(C)C</smiles>

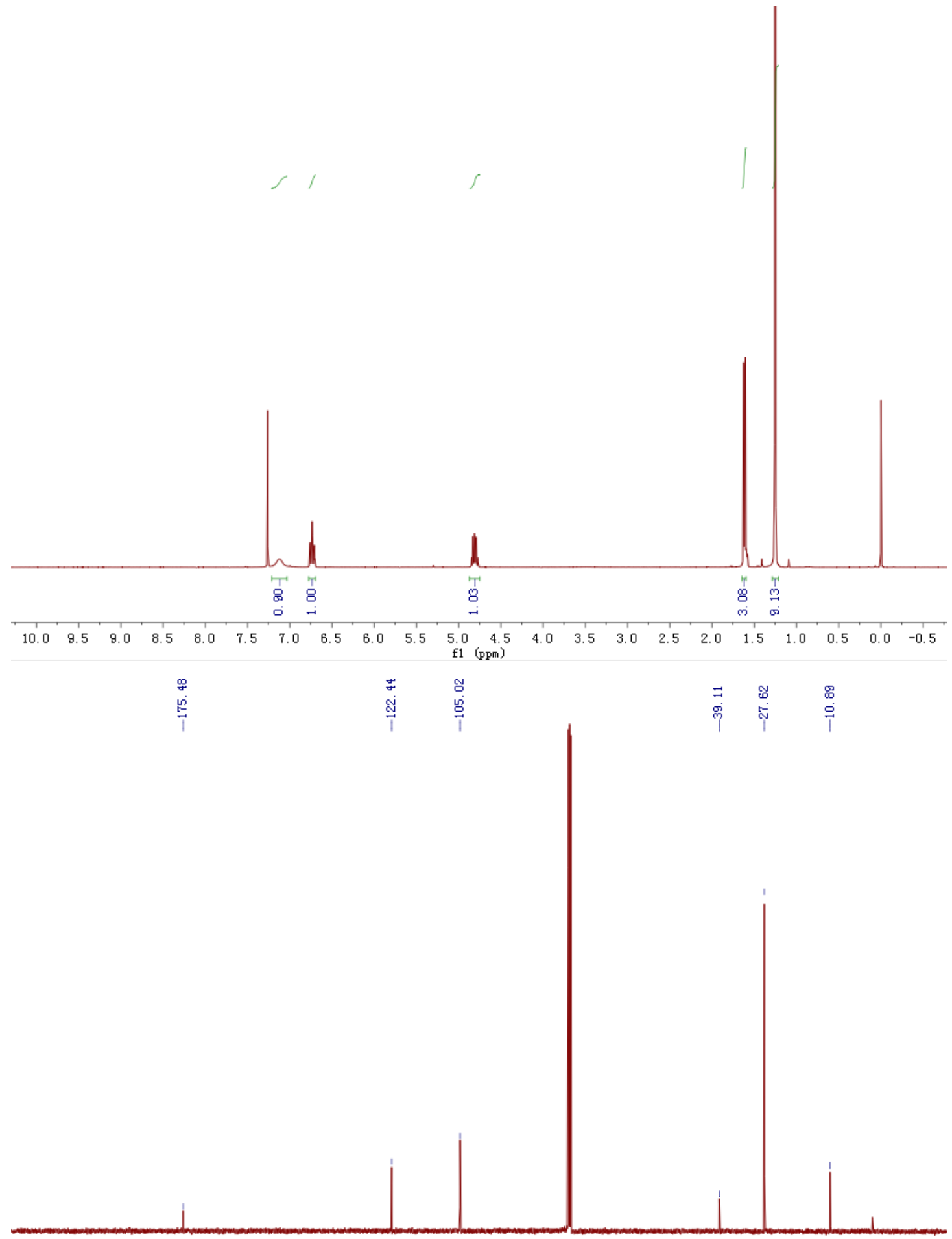

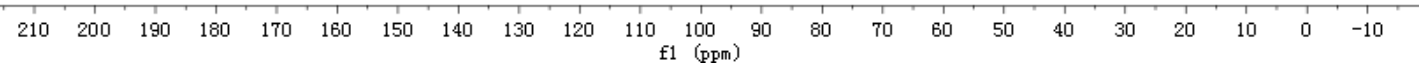


<smiles>CC=CNC(=O)C(C)(C)C</smiles>
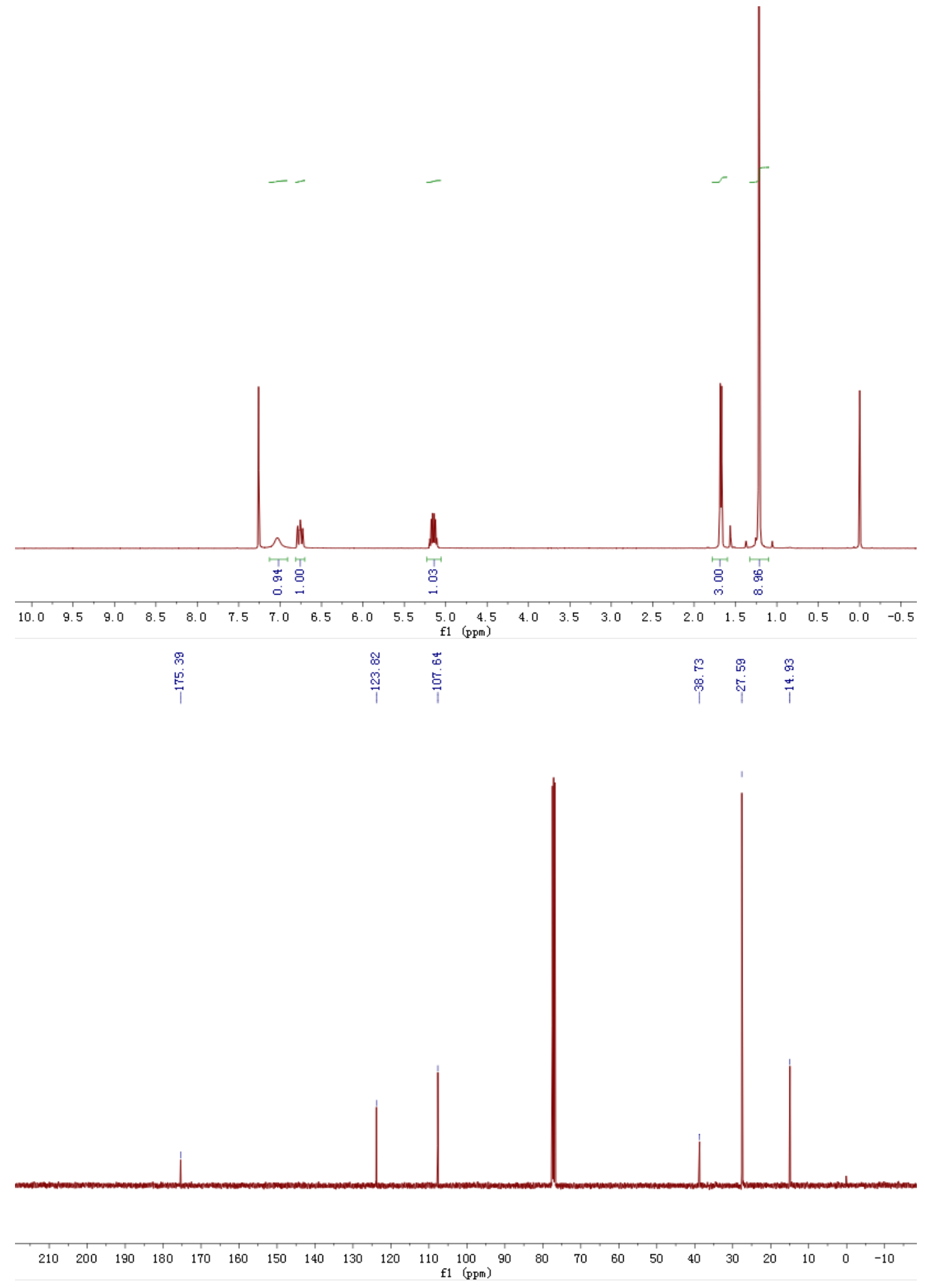

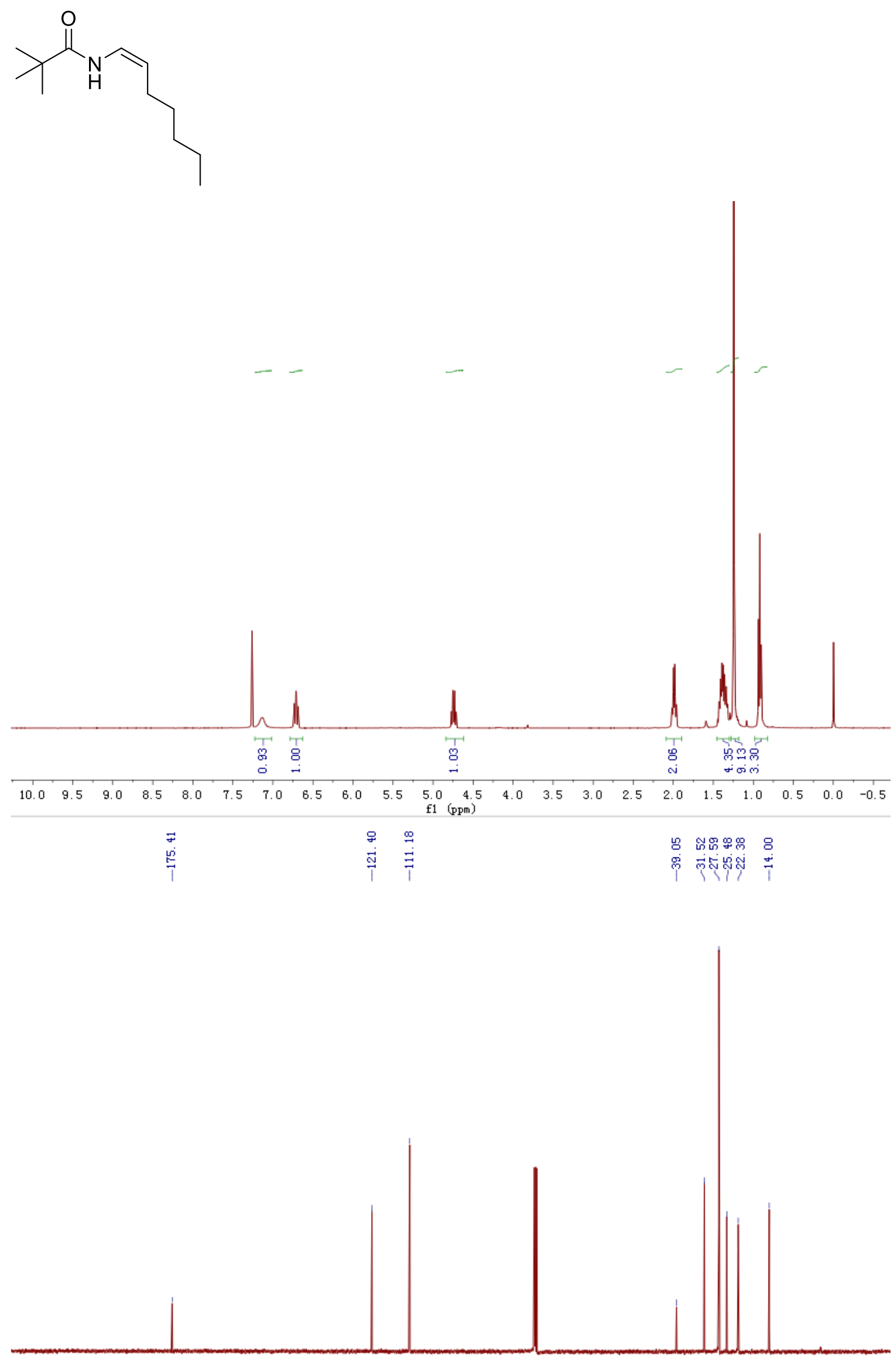

$\begin{array}{lllllllllllllllllllllll}210 & 200 & 190 & 180 & 170 & 160 & 150 & 140 & 130 & 120 & 110 & 100 & 90 & 80 & 70 & 60 & 50 & 40 & 30 & 20 & 10 & 0 & -10\end{array}$ f1 (ppm) 
<smiles>CCCC/C=C/NC(=O)C(C)(C)C</smiles>
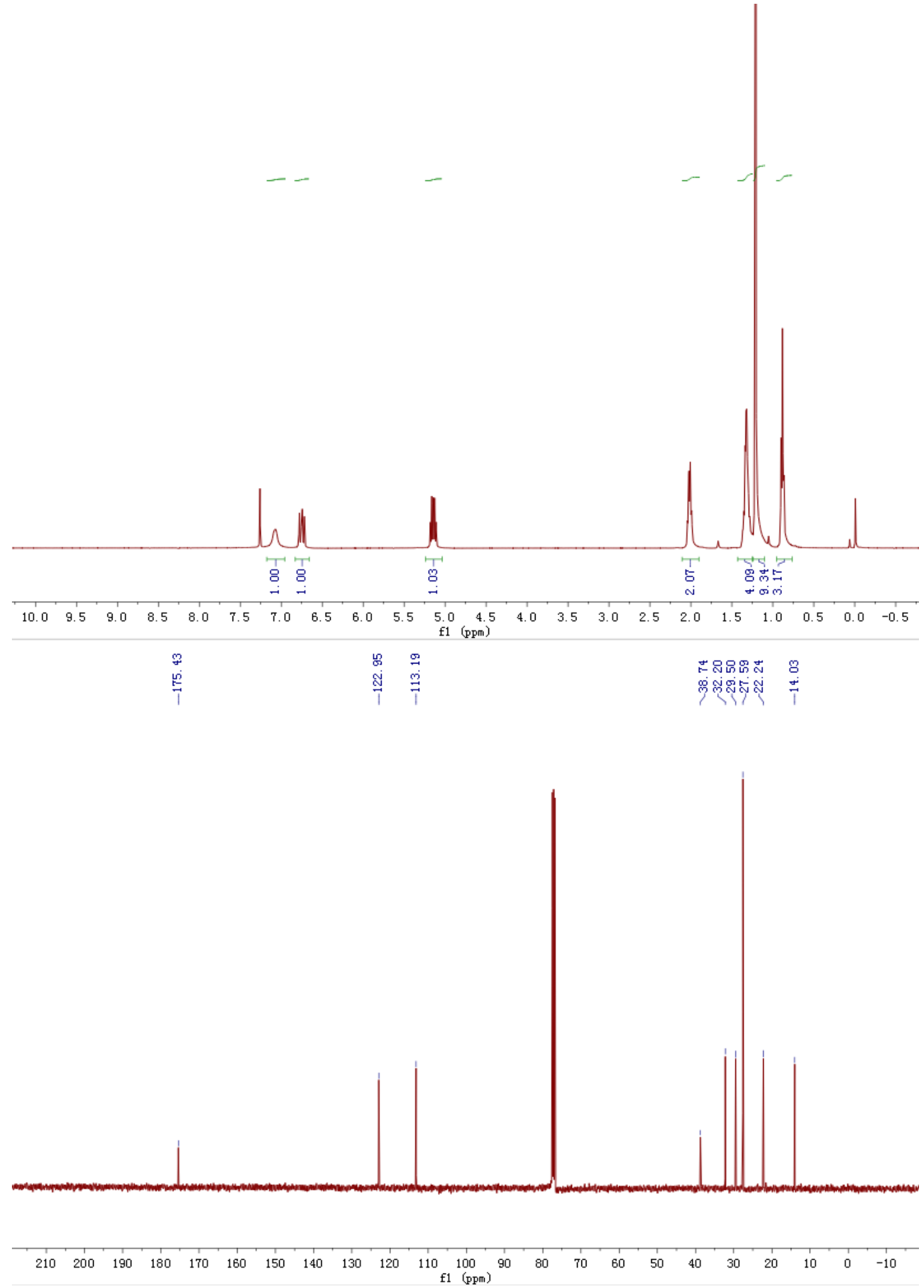

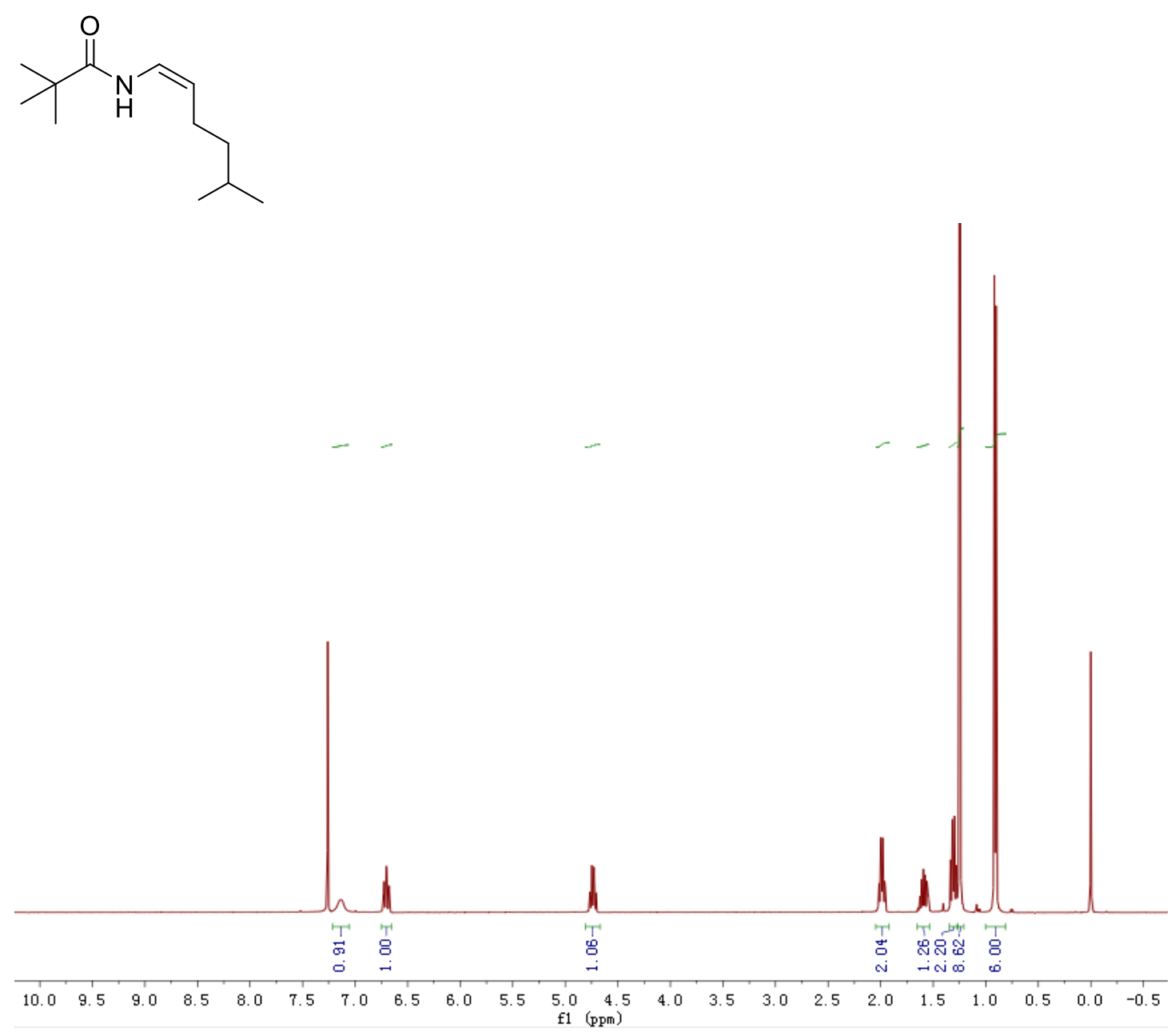

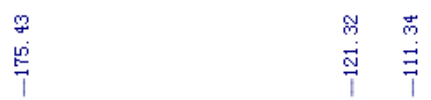

으

กับ

$\begin{array}{lllllllllllllllllllllll}210 & 200 & 190 & 180 & 170 & 160 & 150 & 140 & 130 & 120 & 110 & 100 & 90 & 80 & 70 & 60 & 50 & 40 & 30 & 20 & 10 & 0 & -10\end{array}$ 
<smiles>CC(C)CC/C=C/NC(=O)C(C)(C)C</smiles>
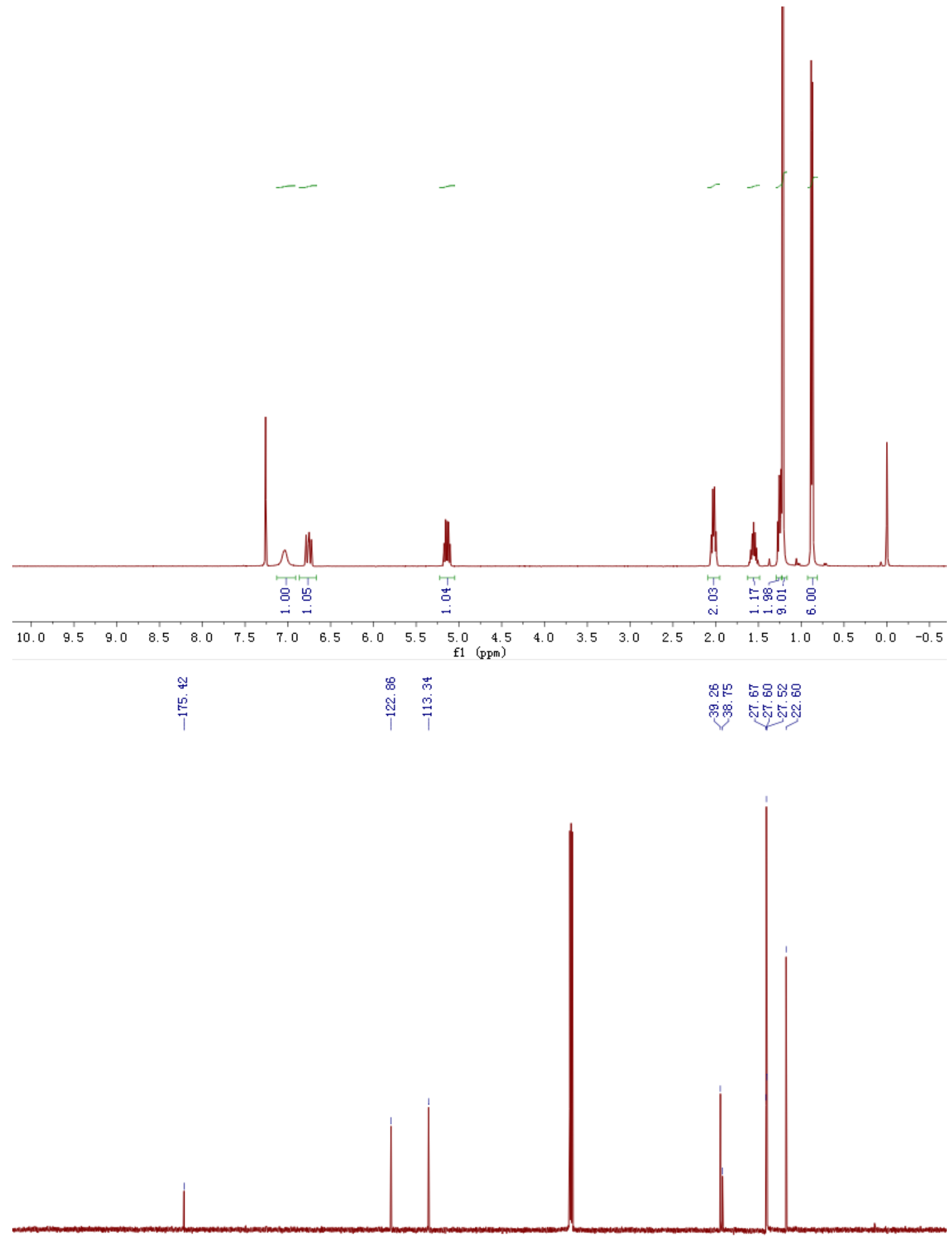

$\begin{array}{lllllllllllllllllllllll}210 & 200 & 190 & 180 & 170 & 160 & 150 & 140 & 130 & 120 & 110 & 100 & 90 & 80 & 70 & 60 & 50 & 40 & 30 & 20 & 10 & 0 & -10\end{array}$ 
<smiles>CC(C)(C)C(=O)N/C=C\CCCCl</smiles>
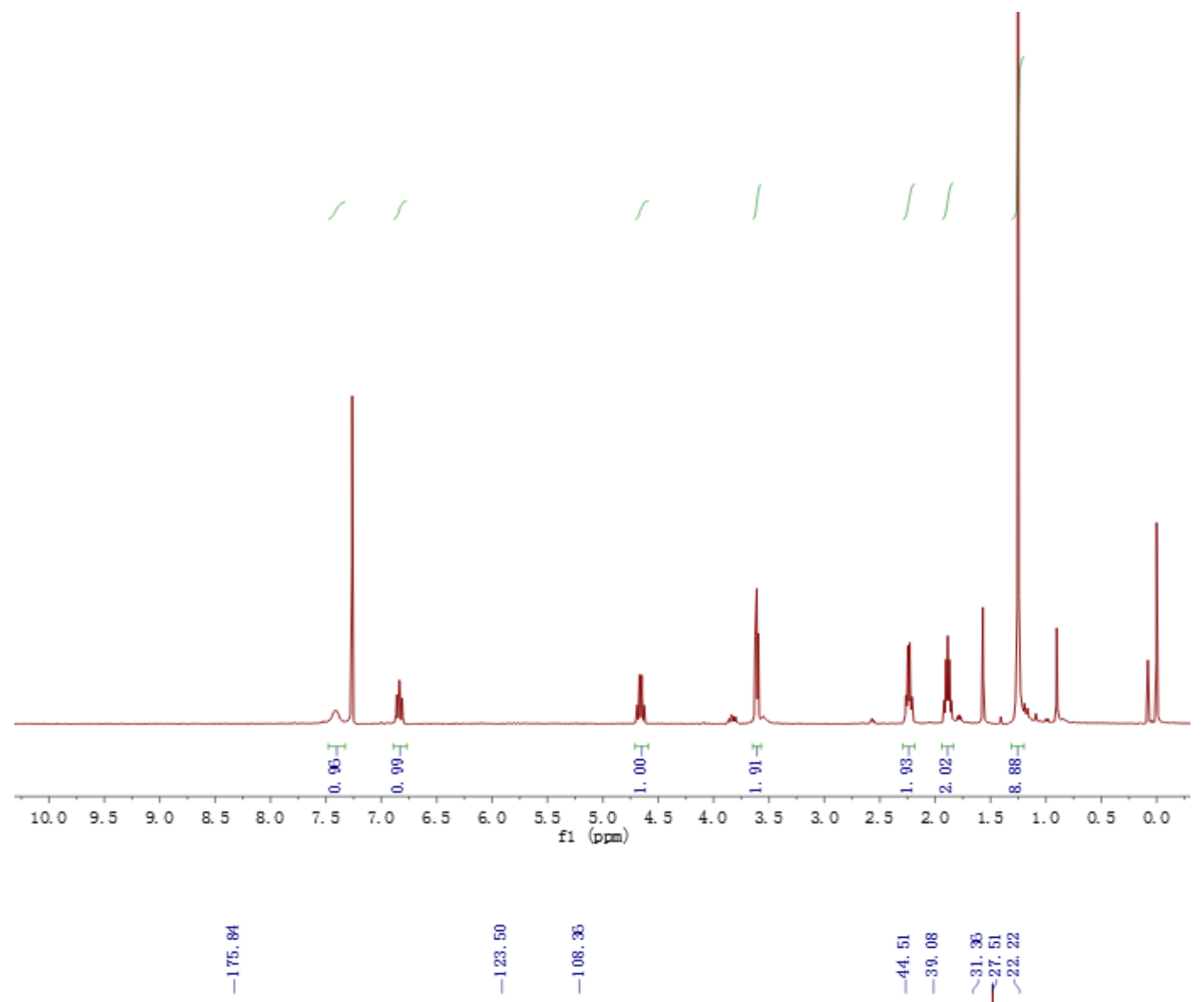

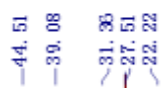

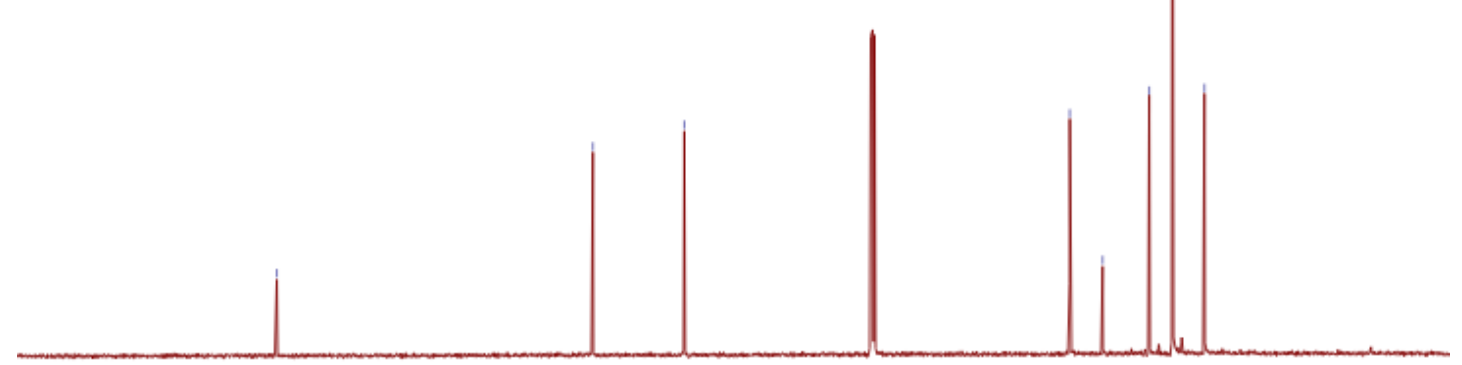

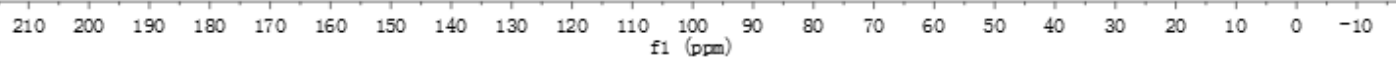


<smiles>CC(C)(C)C(=O)N/C=C/CCCCl</smiles>

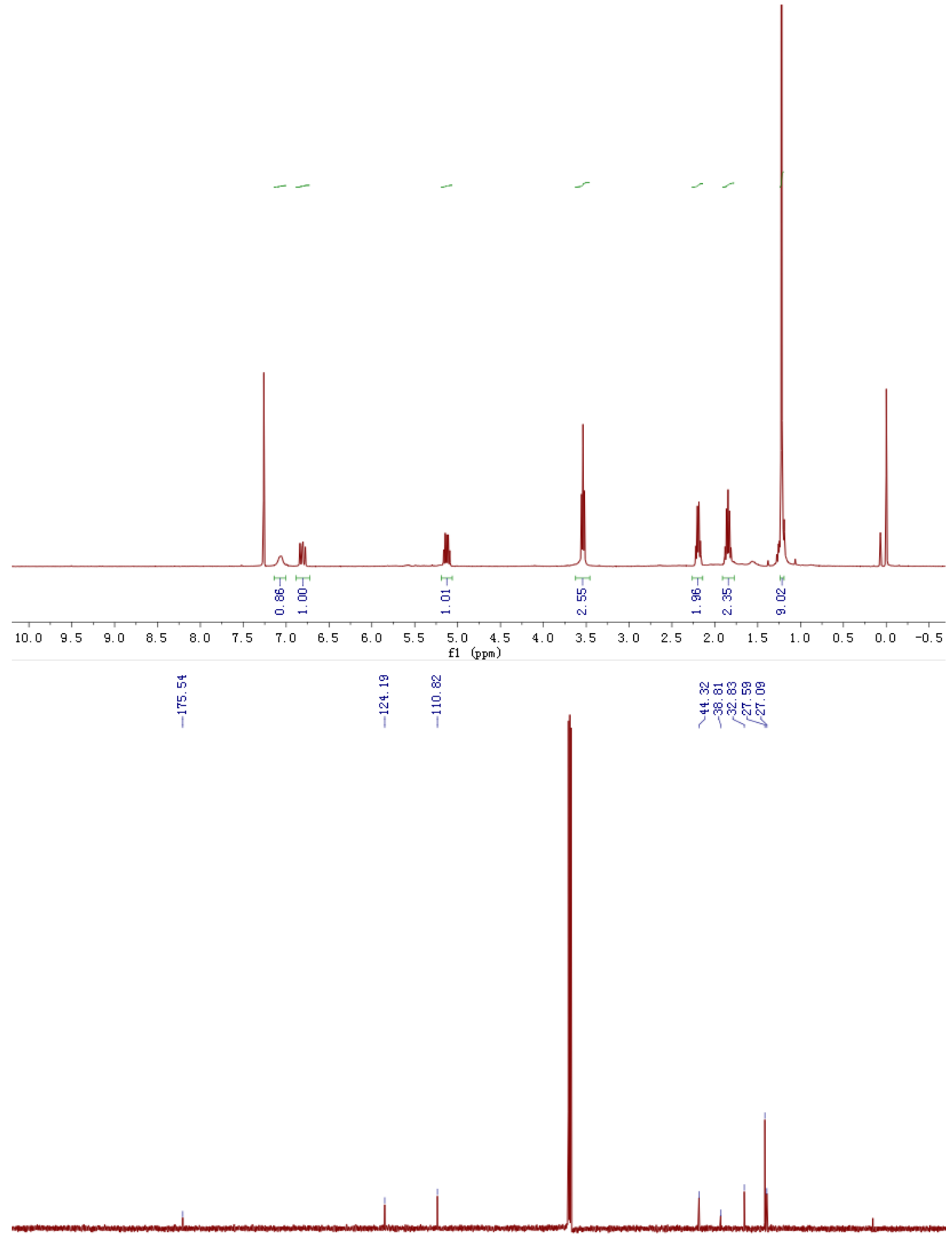

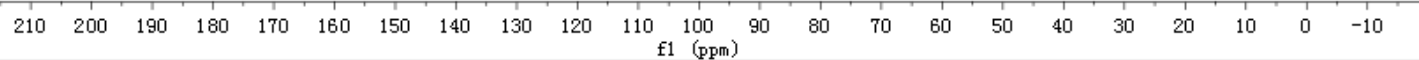


<smiles>CC(C)(C)C(=O)N/C=C\CCCOC(=O)c1ccccc1</smiles>
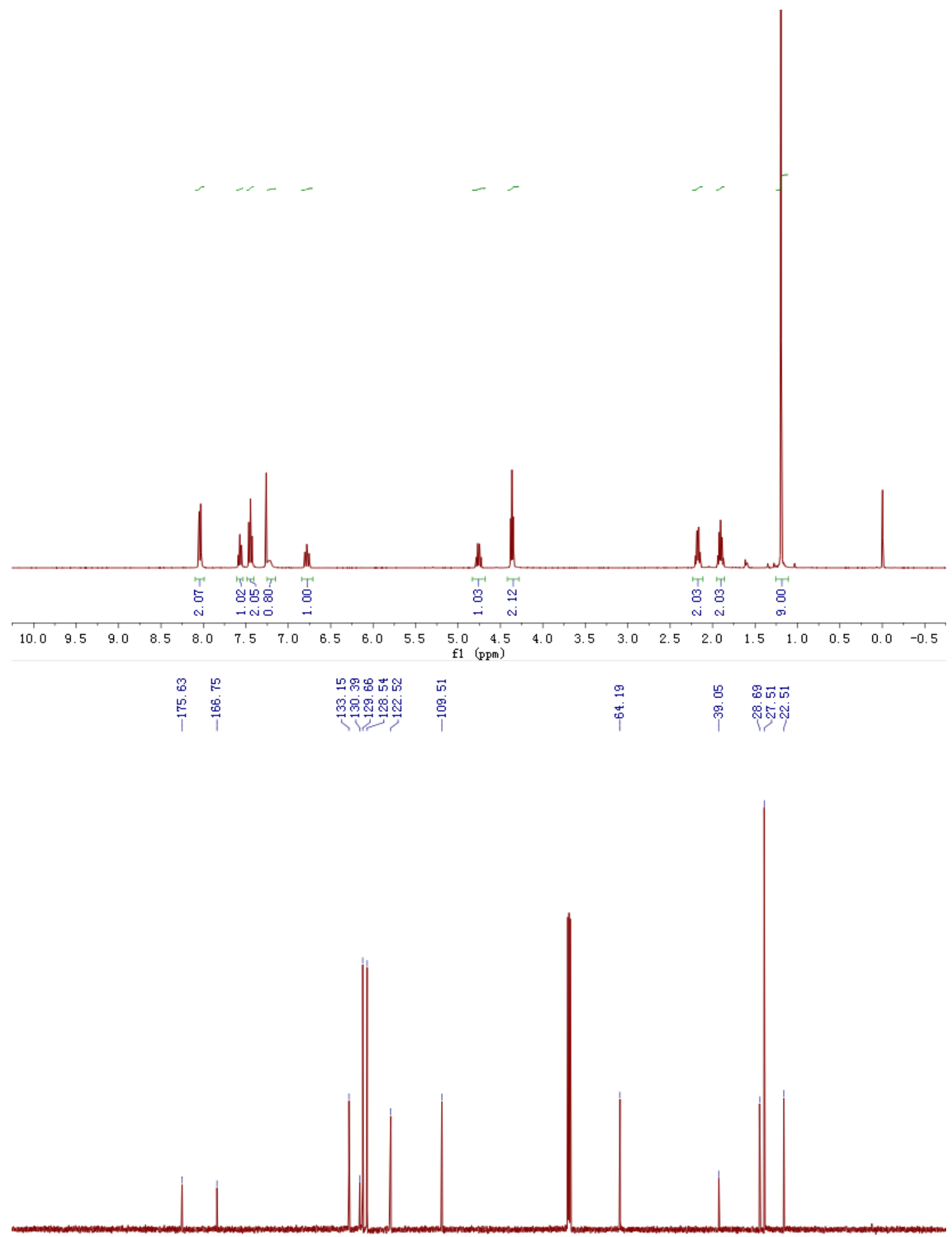

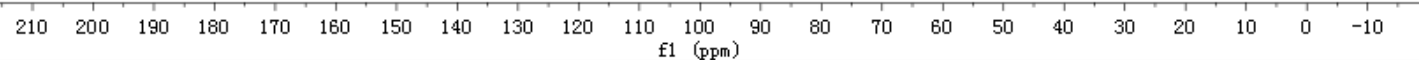


<smiles>CC(C)(C)C(=O)N/C=C/CCCOC(=O)c1ccccc1</smiles>
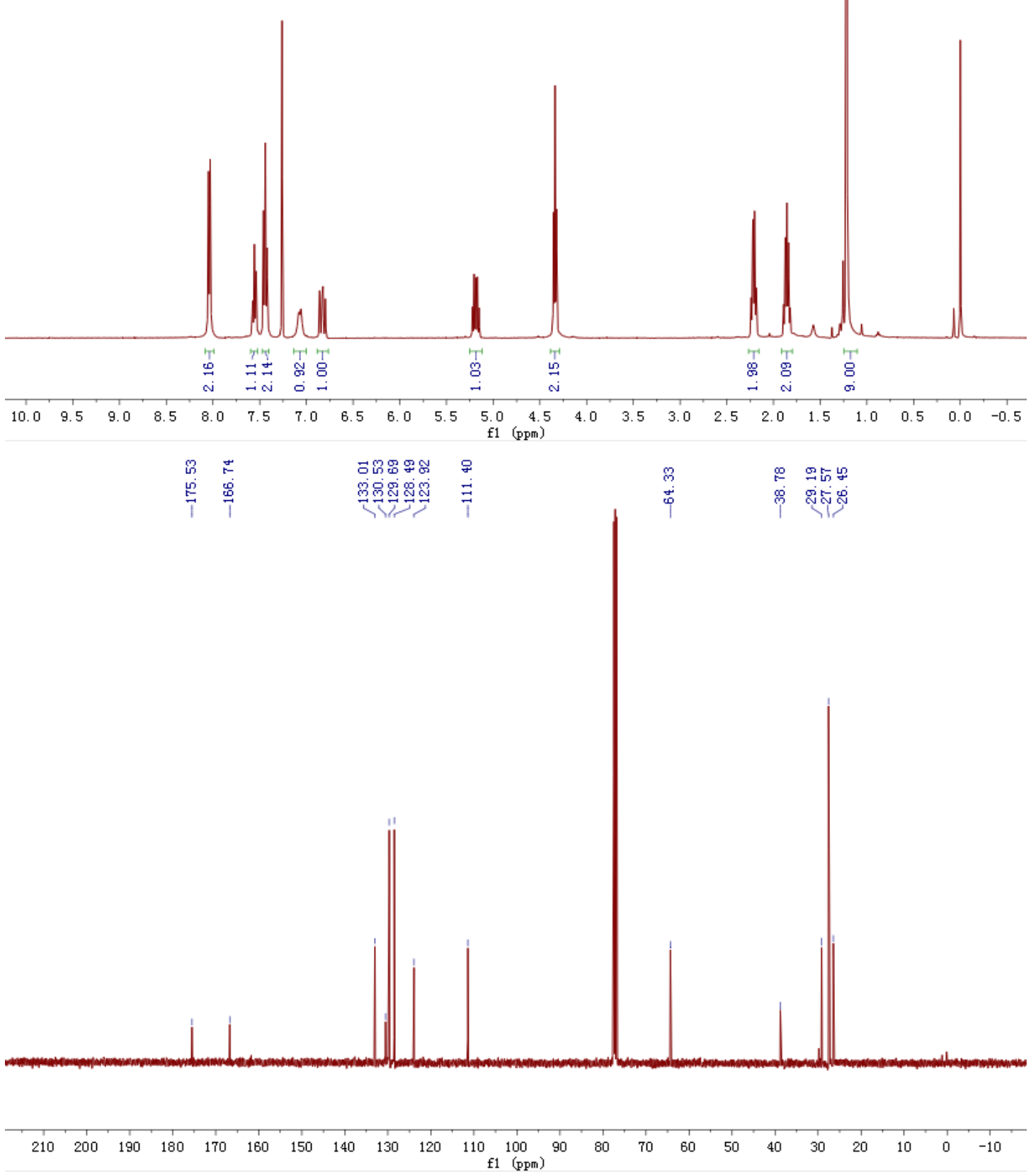

S143 
<smiles>CNC(=O)OCCC/C=C\NC(=O)C(C)(C)C</smiles>
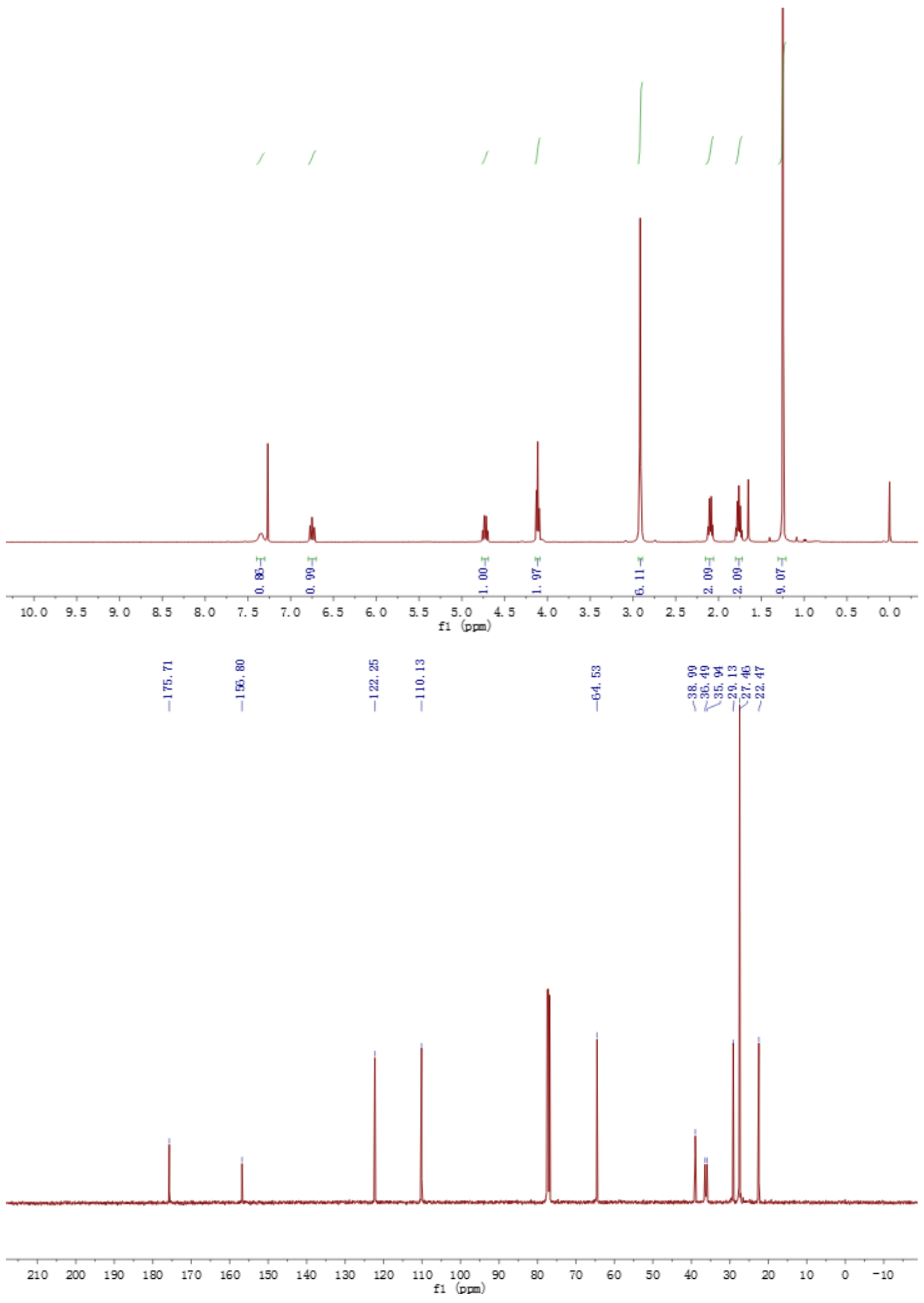

S144 
<smiles>CC(=O)OCCC/C=C/NC(=O)C(C)(C)C</smiles>
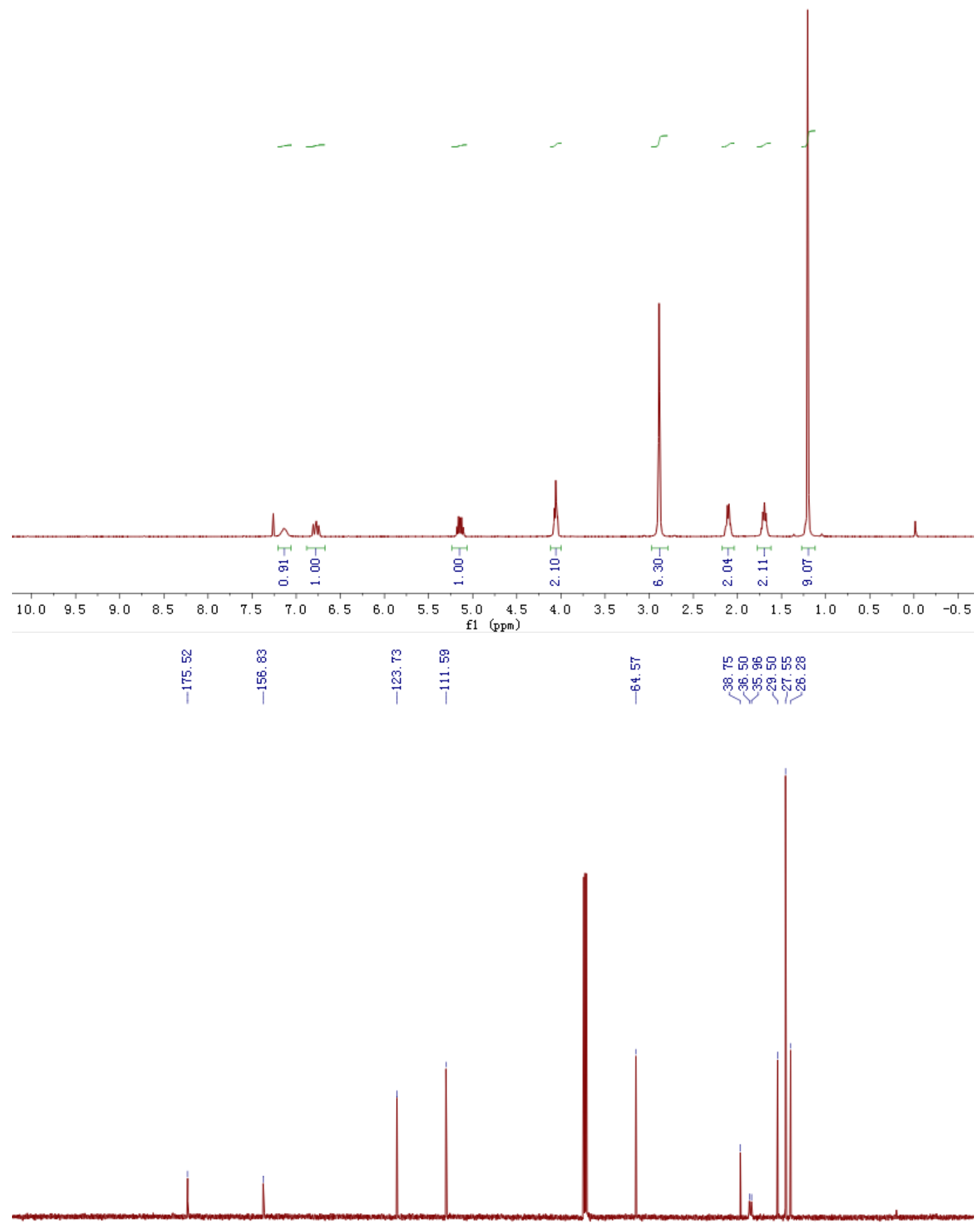

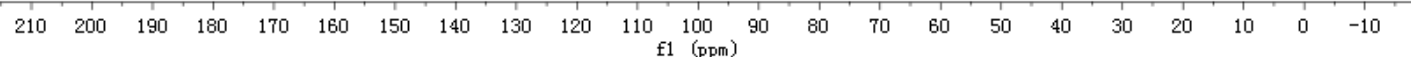



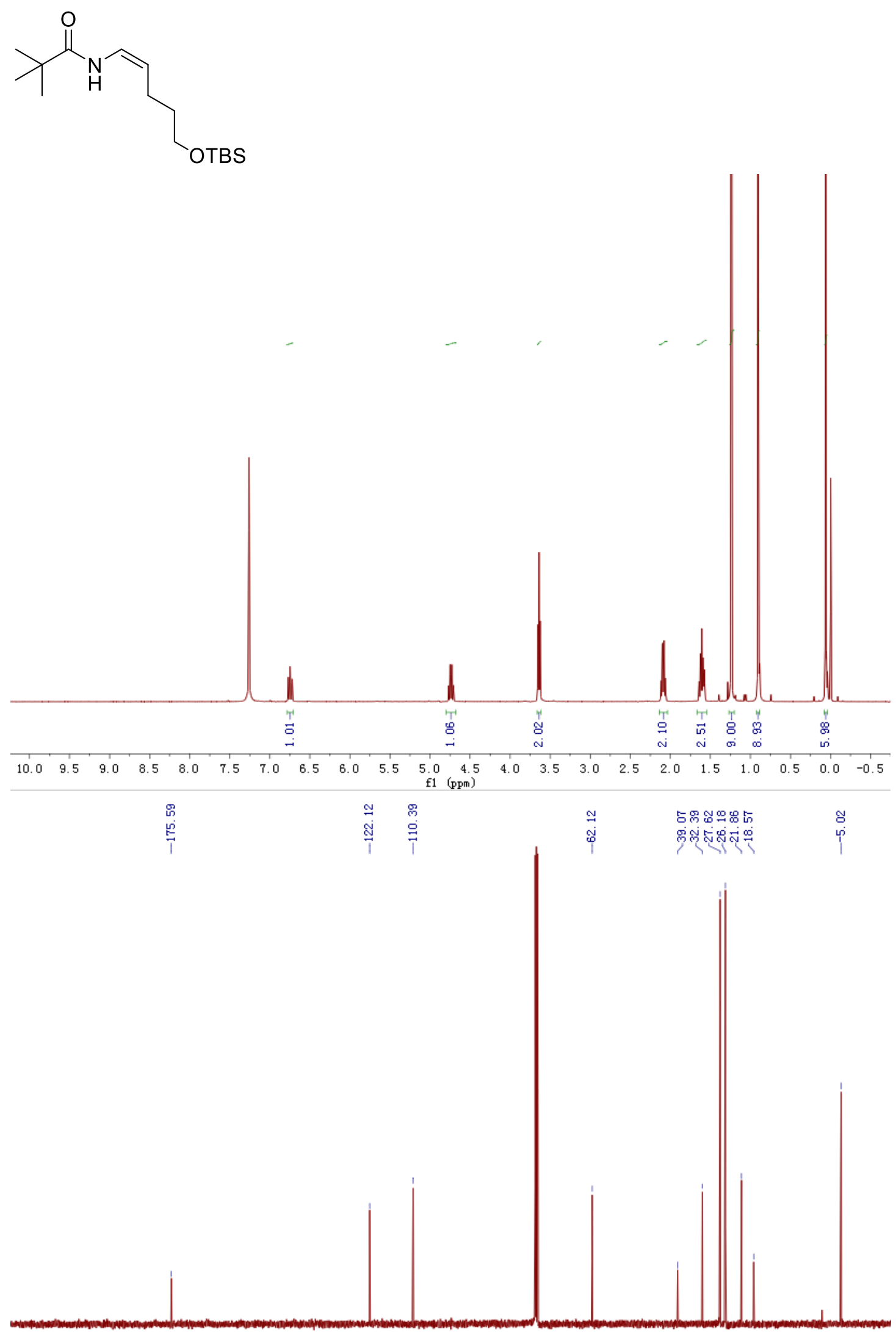

$\begin{array}{lllllllllllllllllllllll}210 & 200 & 190 & 180 & 170 & 160 & 150 & 140 & 130 & 120 & 110 & 100 & 90 & 80 & 70 & 60 & 50 & 40 & 30 & 20 & 10 & 0 & -10\end{array}$ 
<smiles>CC(C)(C)C(=O)N/C=C/CCC[O+]S(C)(=O)=O</smiles>
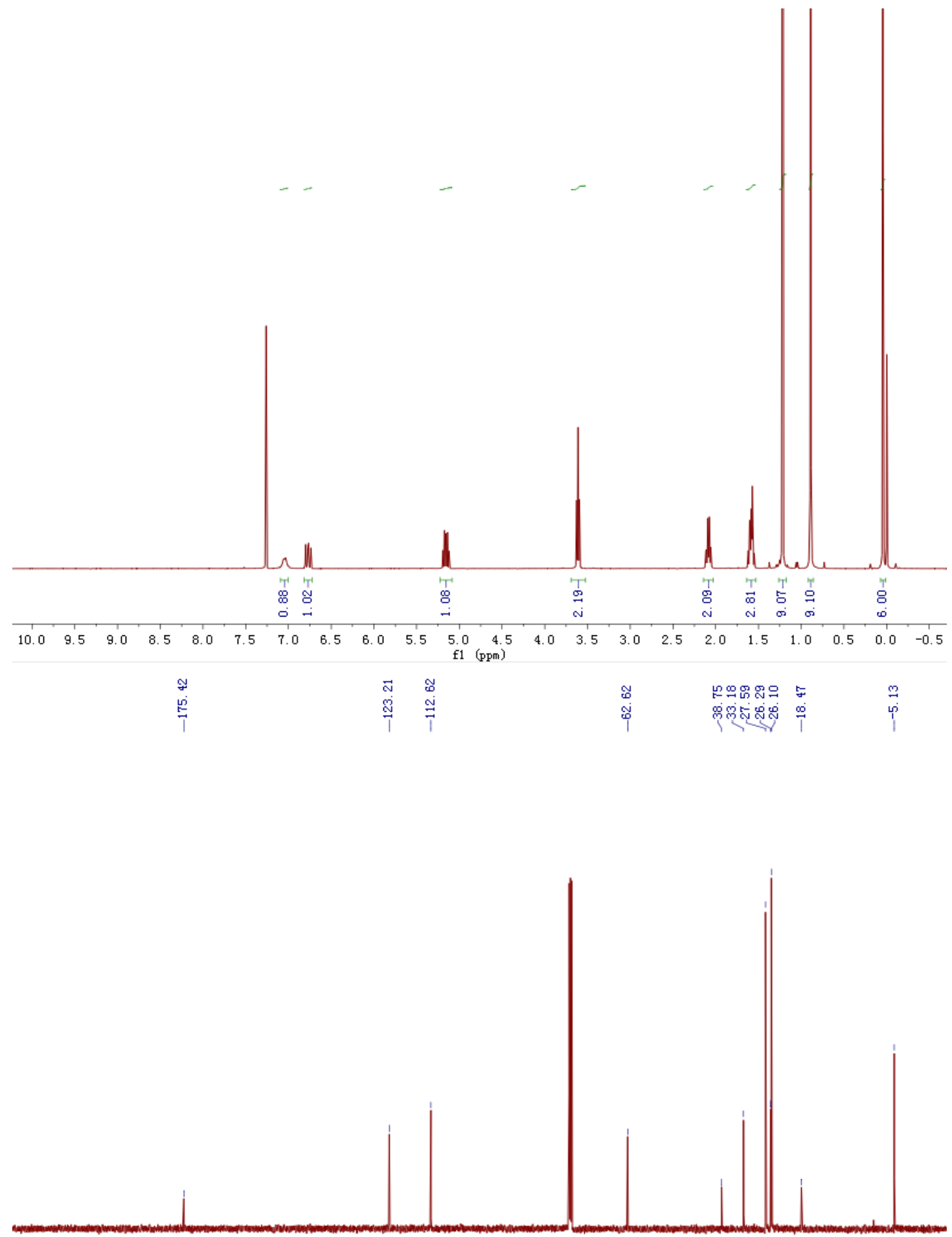

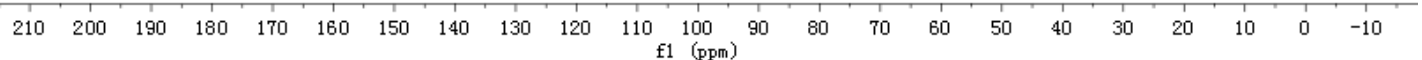



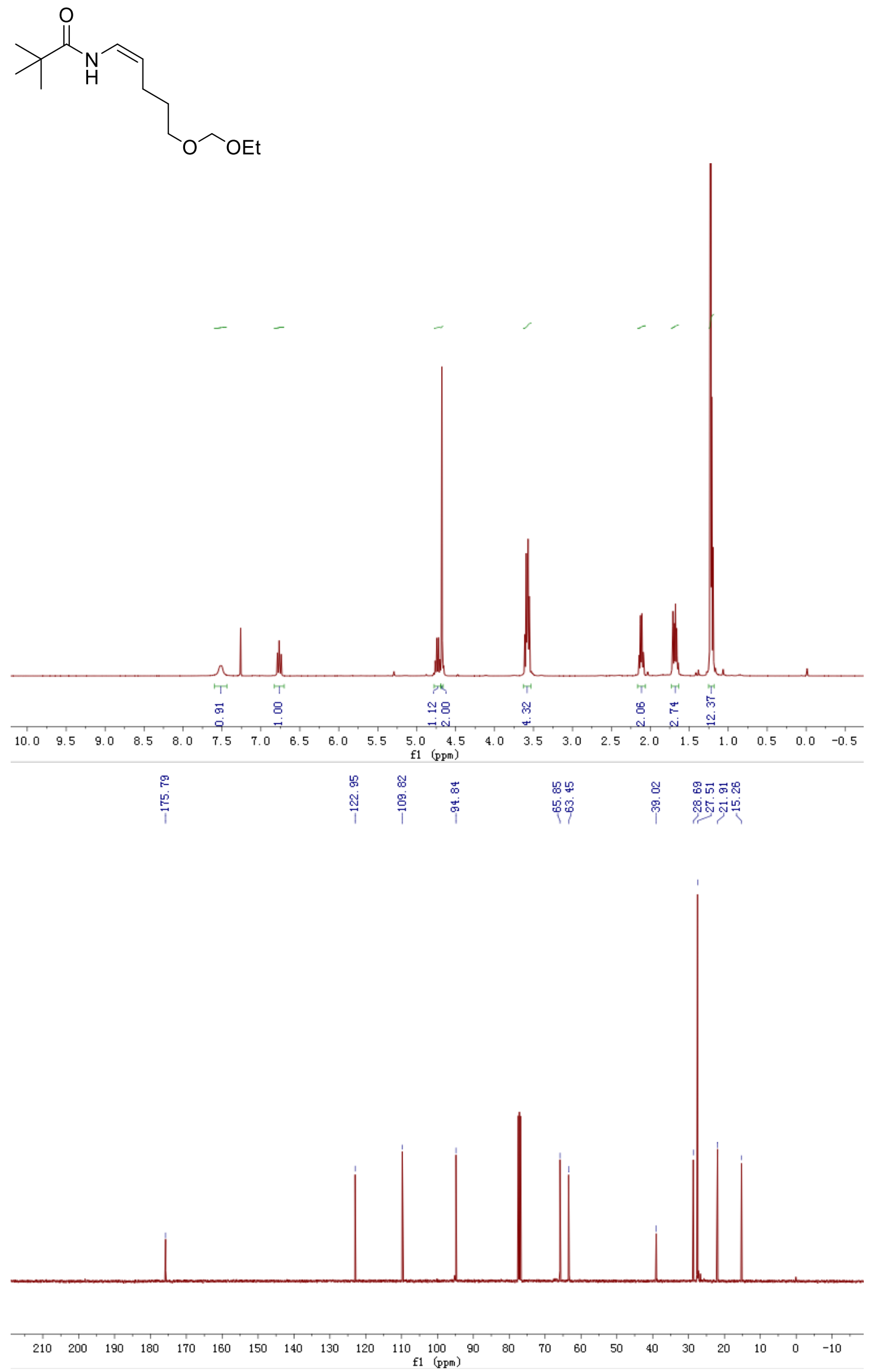
<smiles>CCOCOCCC/C=C/NC(=O)C(C)(C)C</smiles>
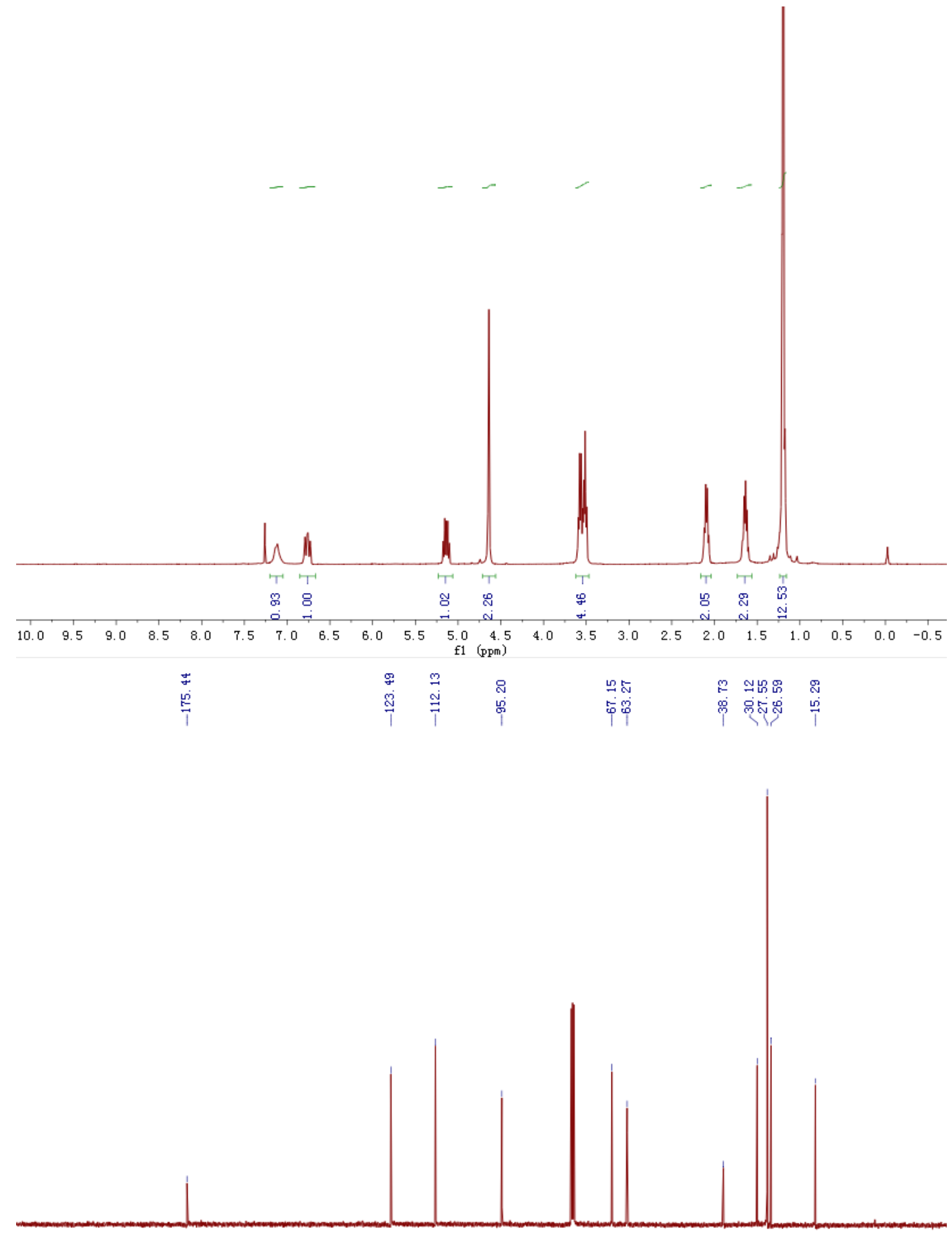

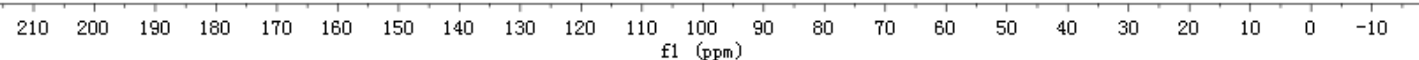



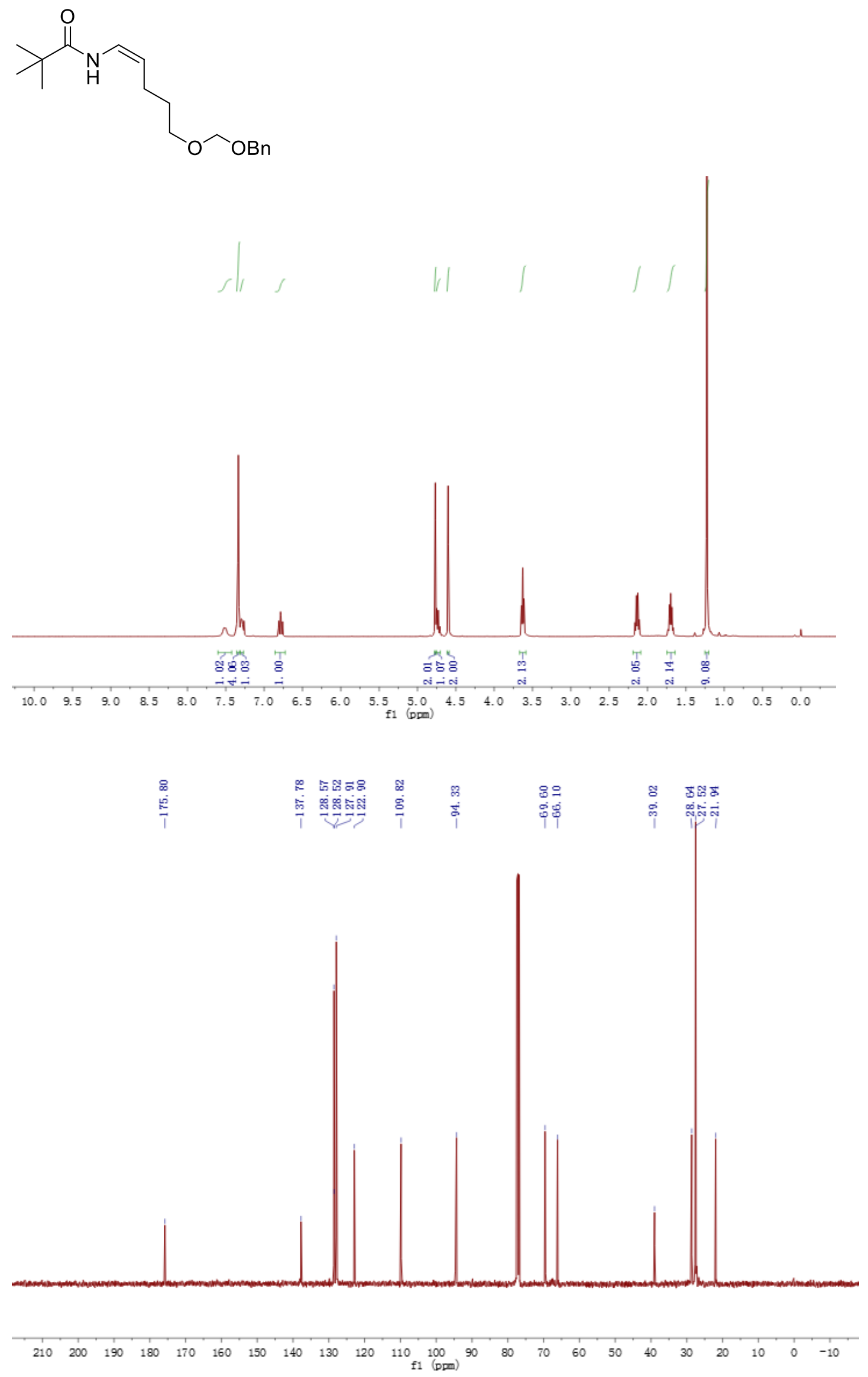
<smiles>CC(C)(C)C(=O)N/C=C/CCCOCCc1ccccc1</smiles>
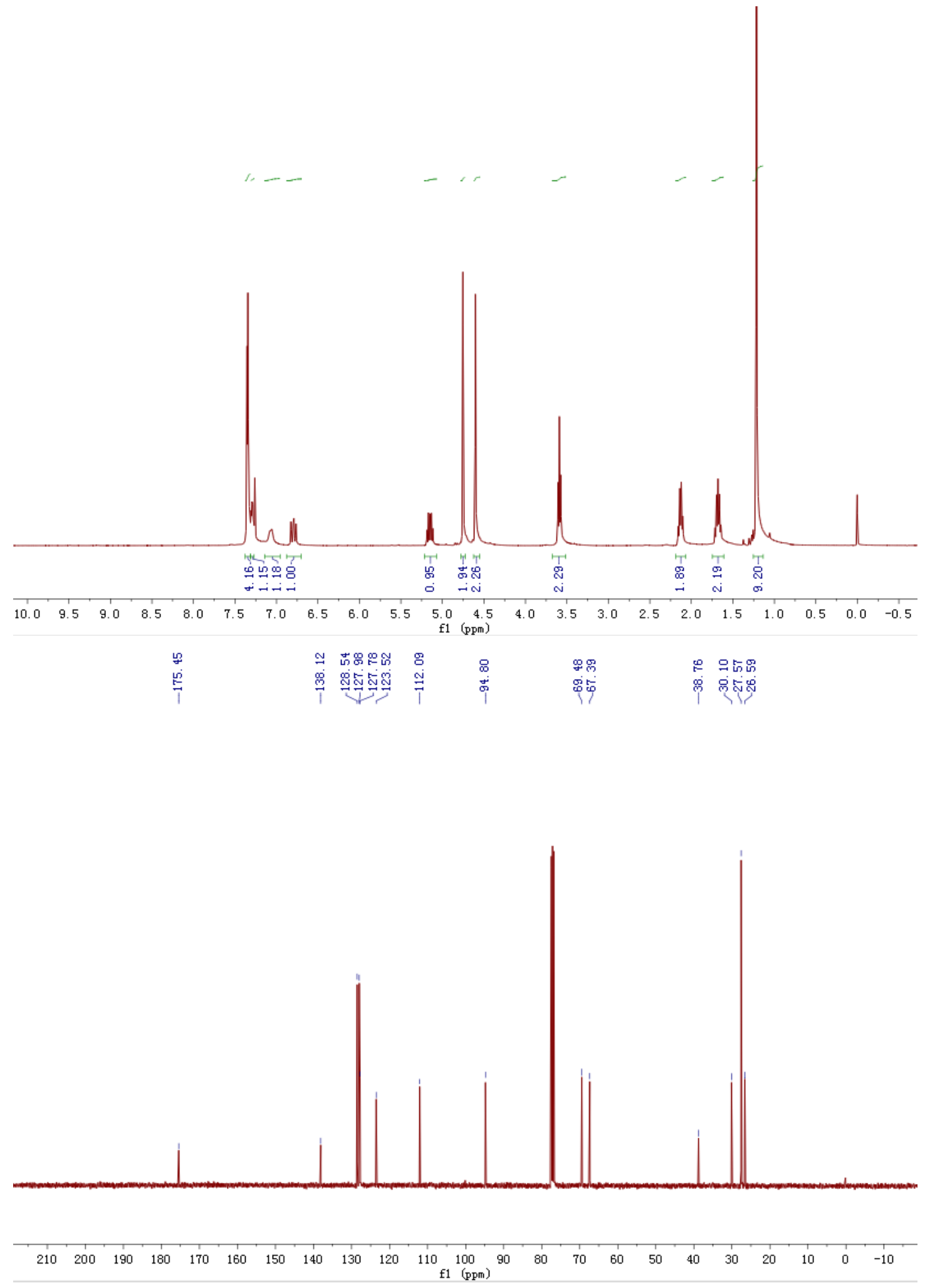
<smiles>CC(C)(C)C(=O)N/C=C\CC1CCCCC1</smiles>
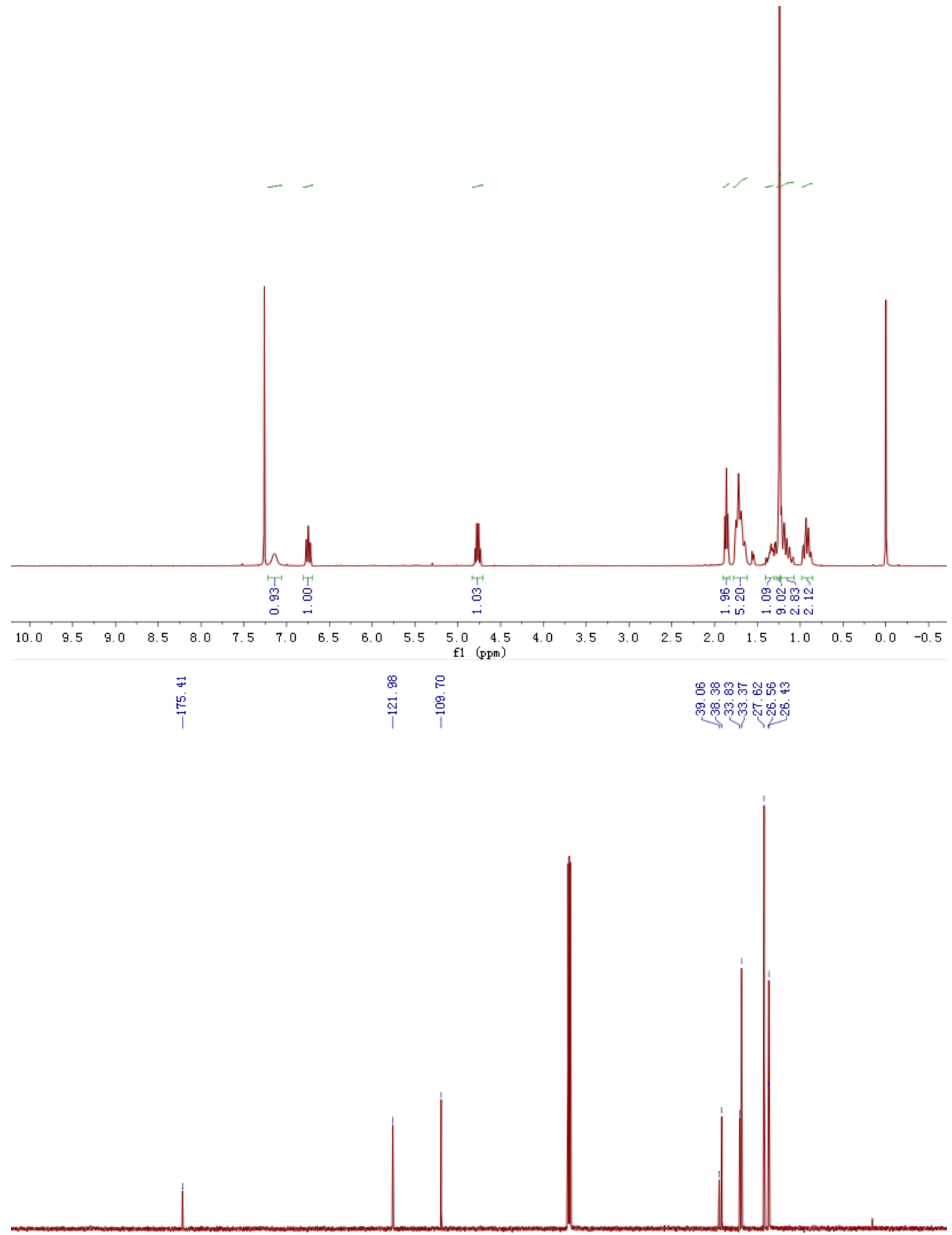

$\begin{array}{lllllllllllllllllllllll}210 & 200 & 190 & 180 & 170 & 160 & 150 & 140 & 130 & 120 & 110 & 100 & 90 & 80 & 70 & 60 & 50 & 40 & 30 & 20 & 10 & 0 & -10\end{array}$ 
<smiles>CC(C)(C)C(=O)N/C=C/CC1CCCCC1</smiles>
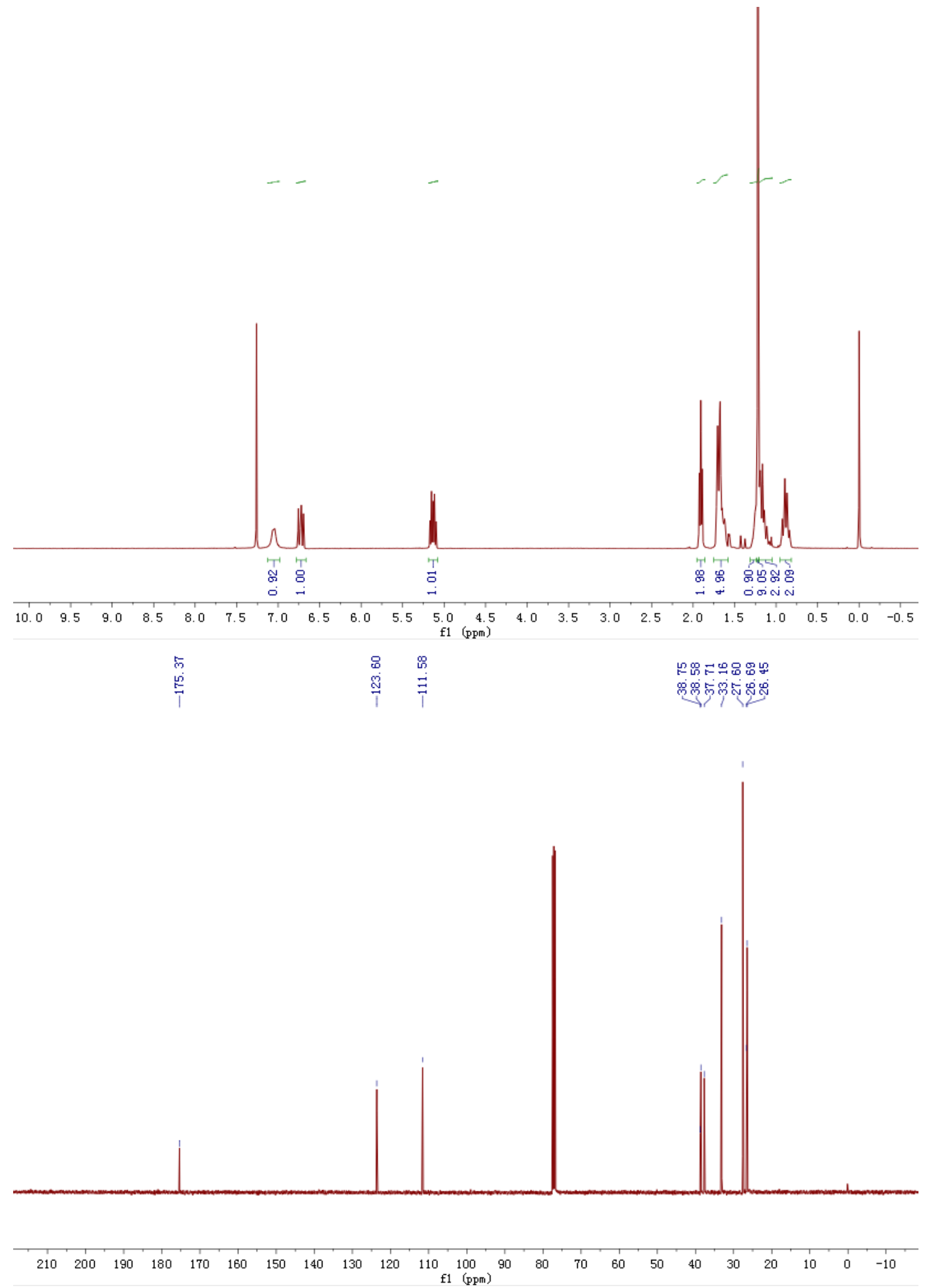

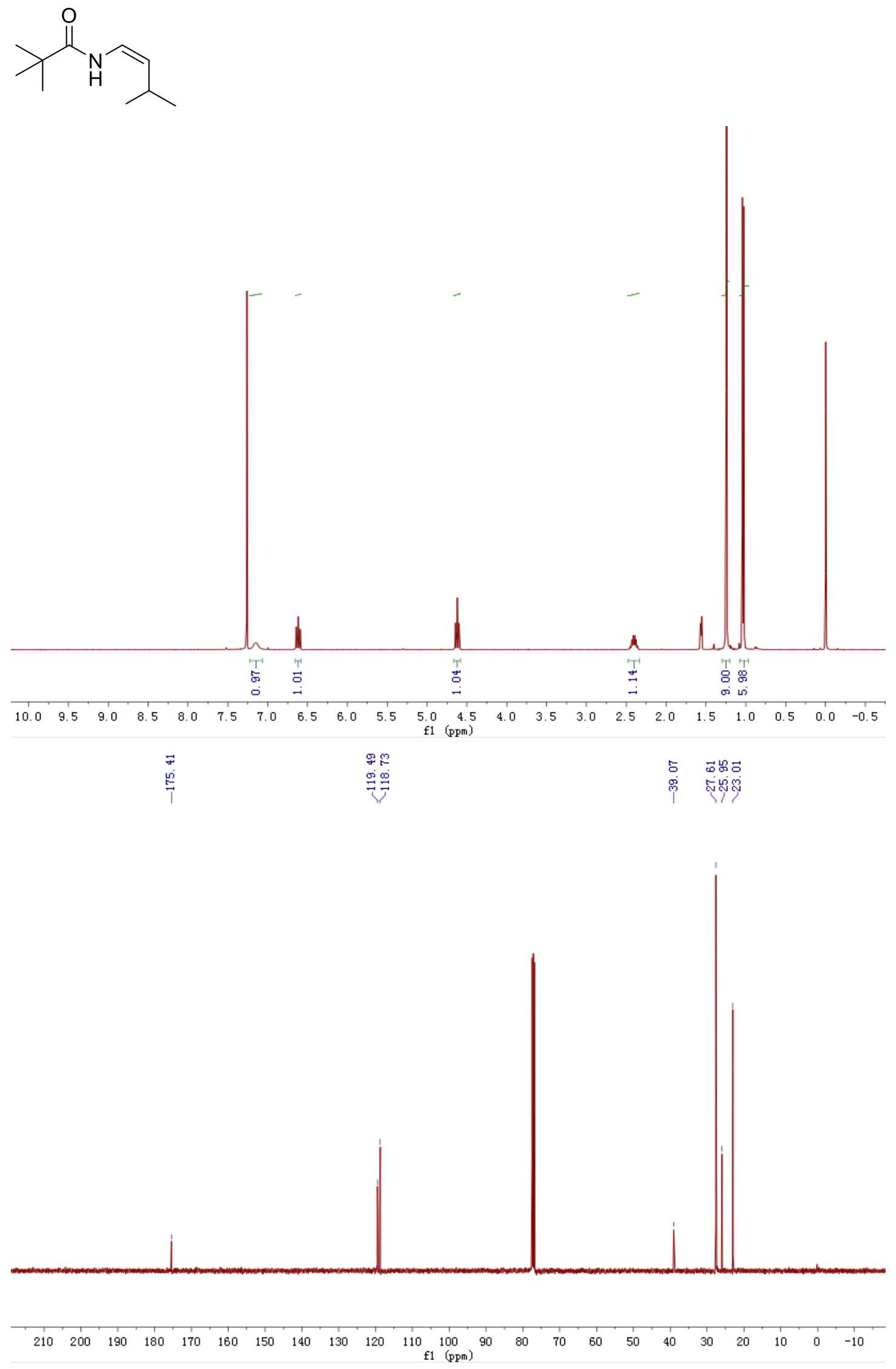

S154 
<smiles>CC(C)/C=C/NC(=O)C(C)(C)C</smiles>

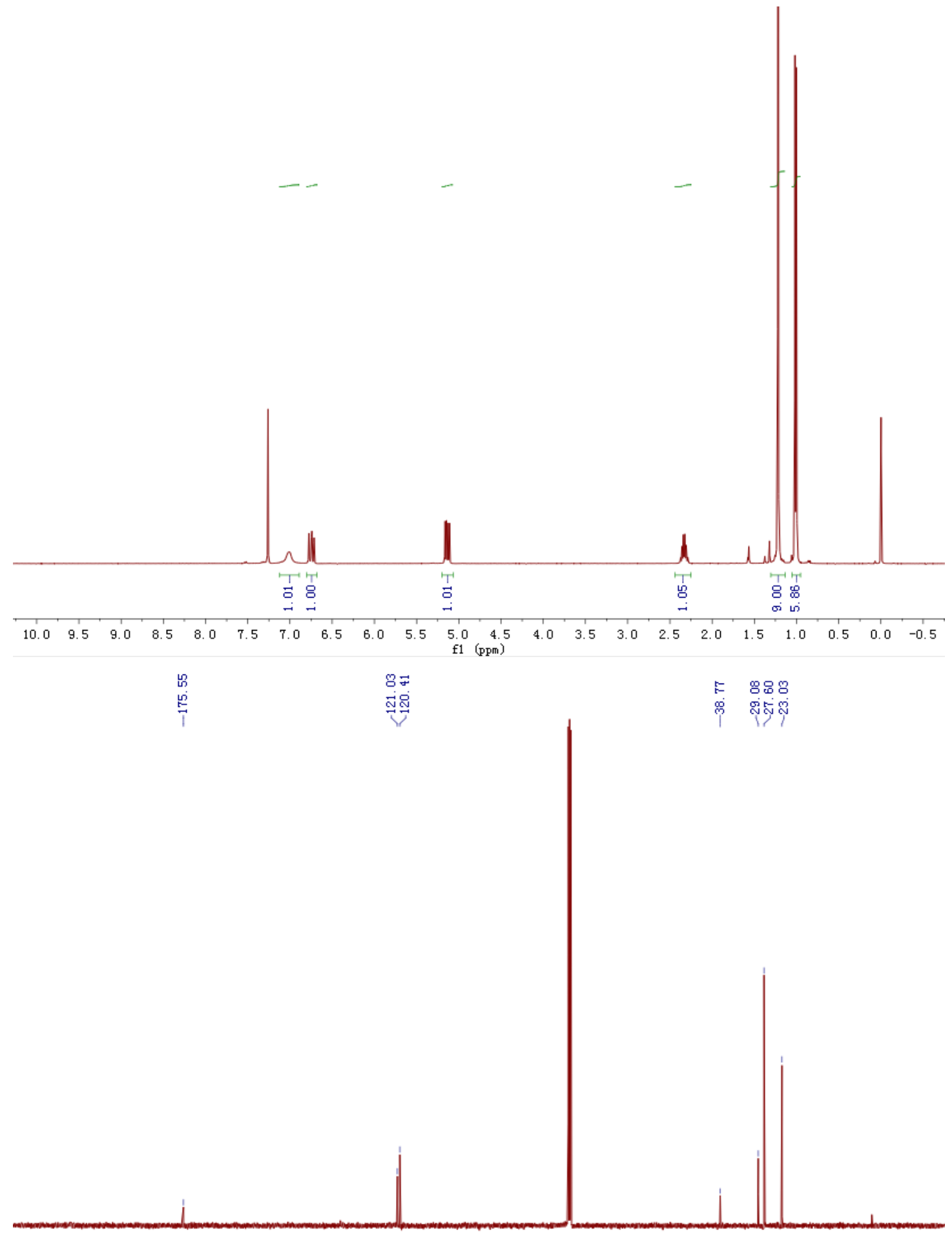

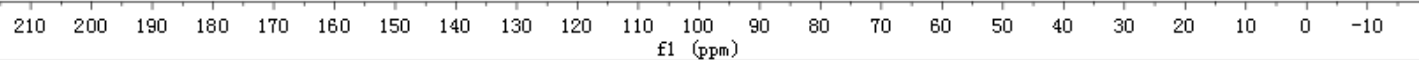


$>_{\mathrm{H}}^{\mathrm{O}}$
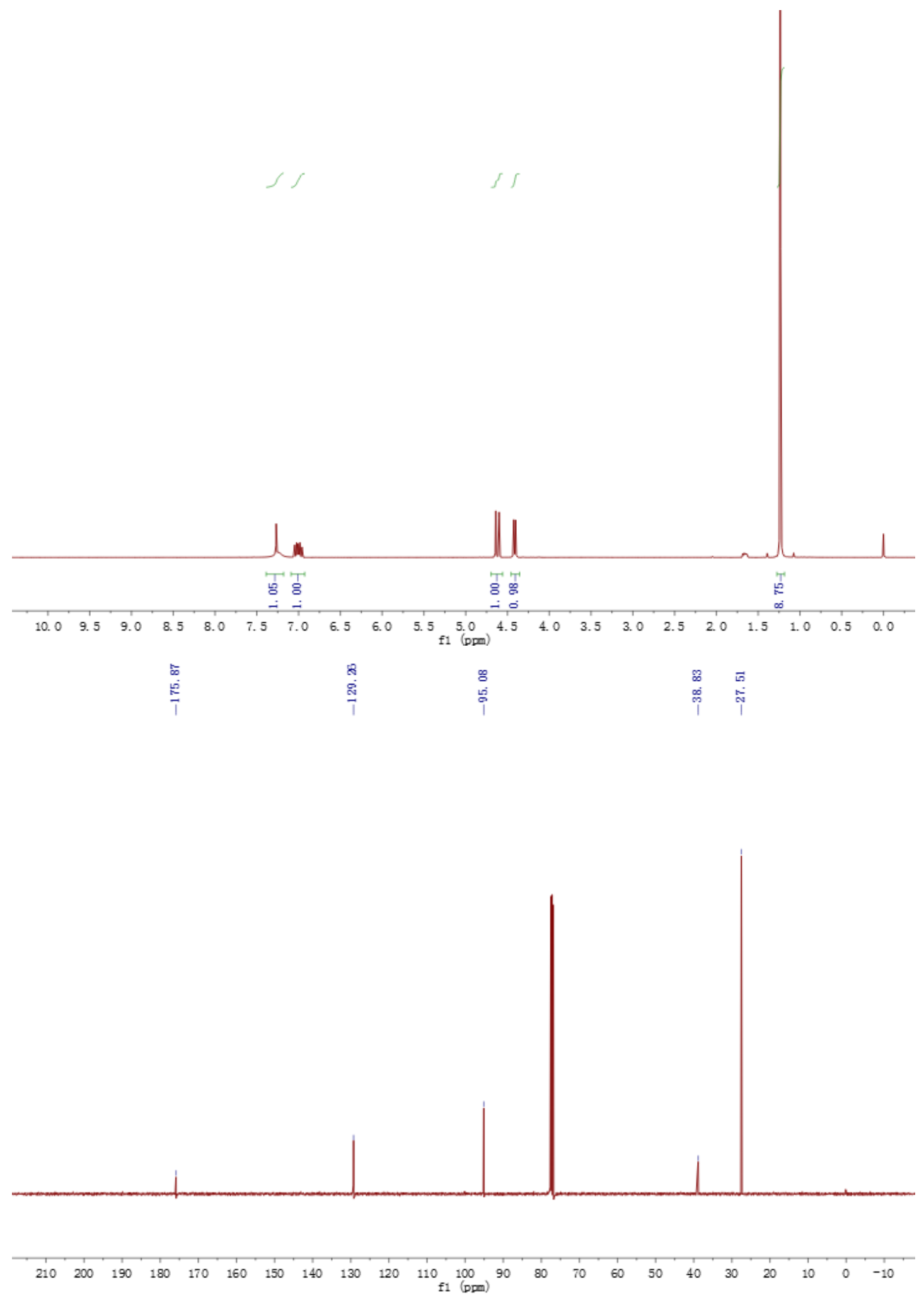
<smiles>C/C(=C\NC(=O)C(C)(C)C)Cc1ccccc1</smiles>
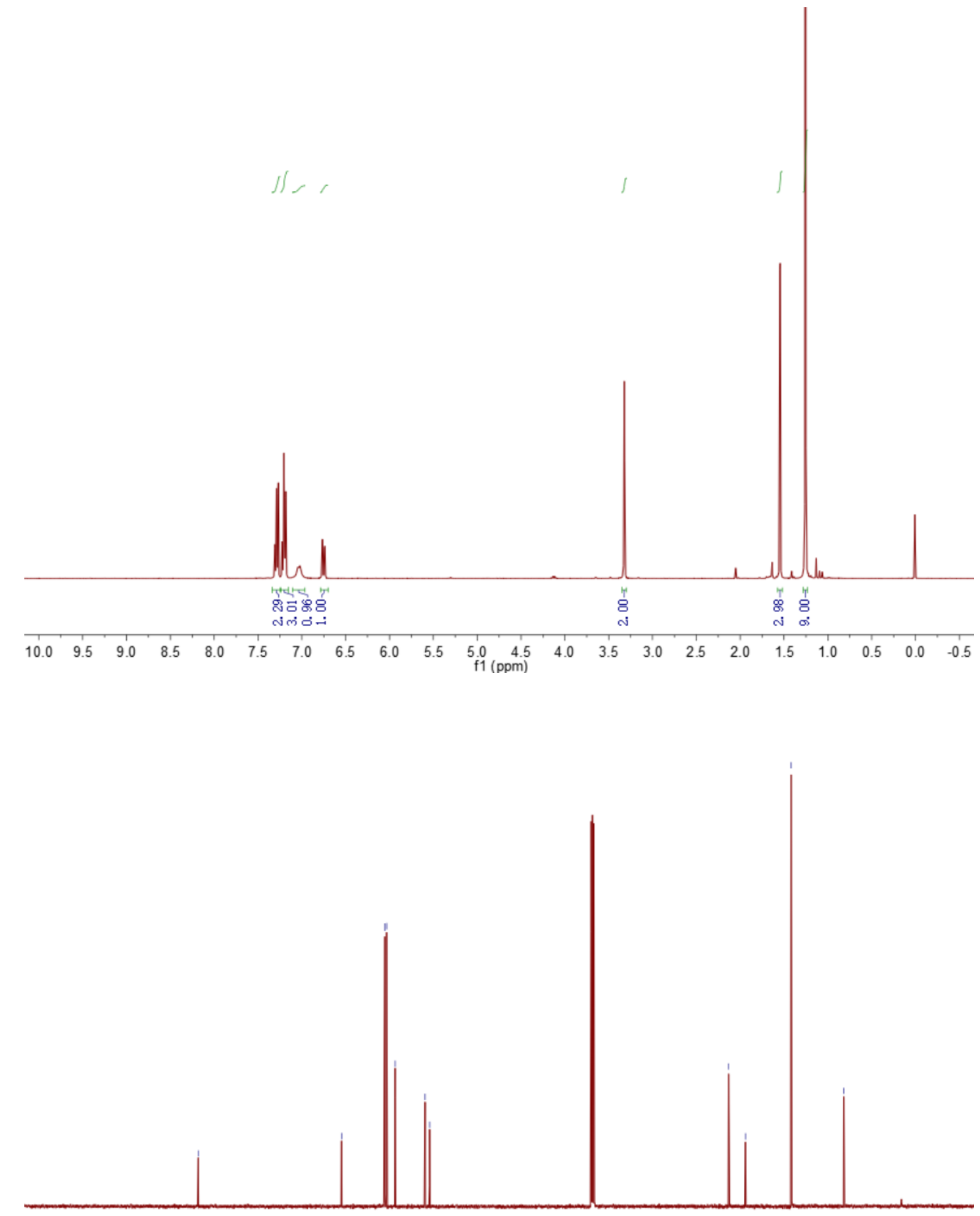

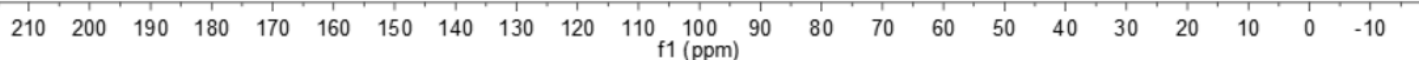


<smiles>C/C(=C\NC(=O)C(C)(C)C)CCc1ccccc1</smiles>

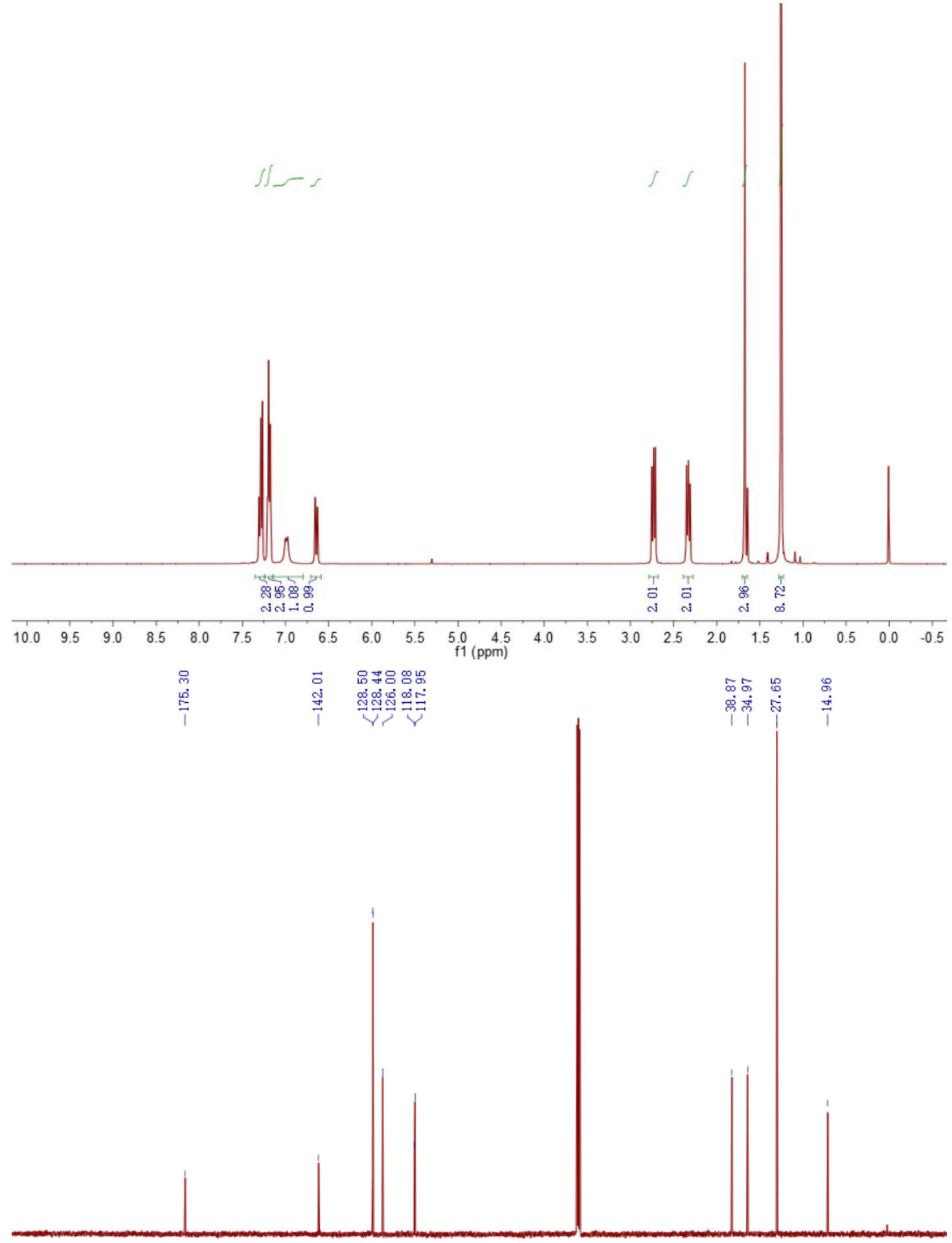

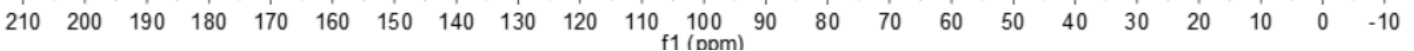


<smiles>CCCC/C(C)=C/NC(=O)C(C)(C)C</smiles>
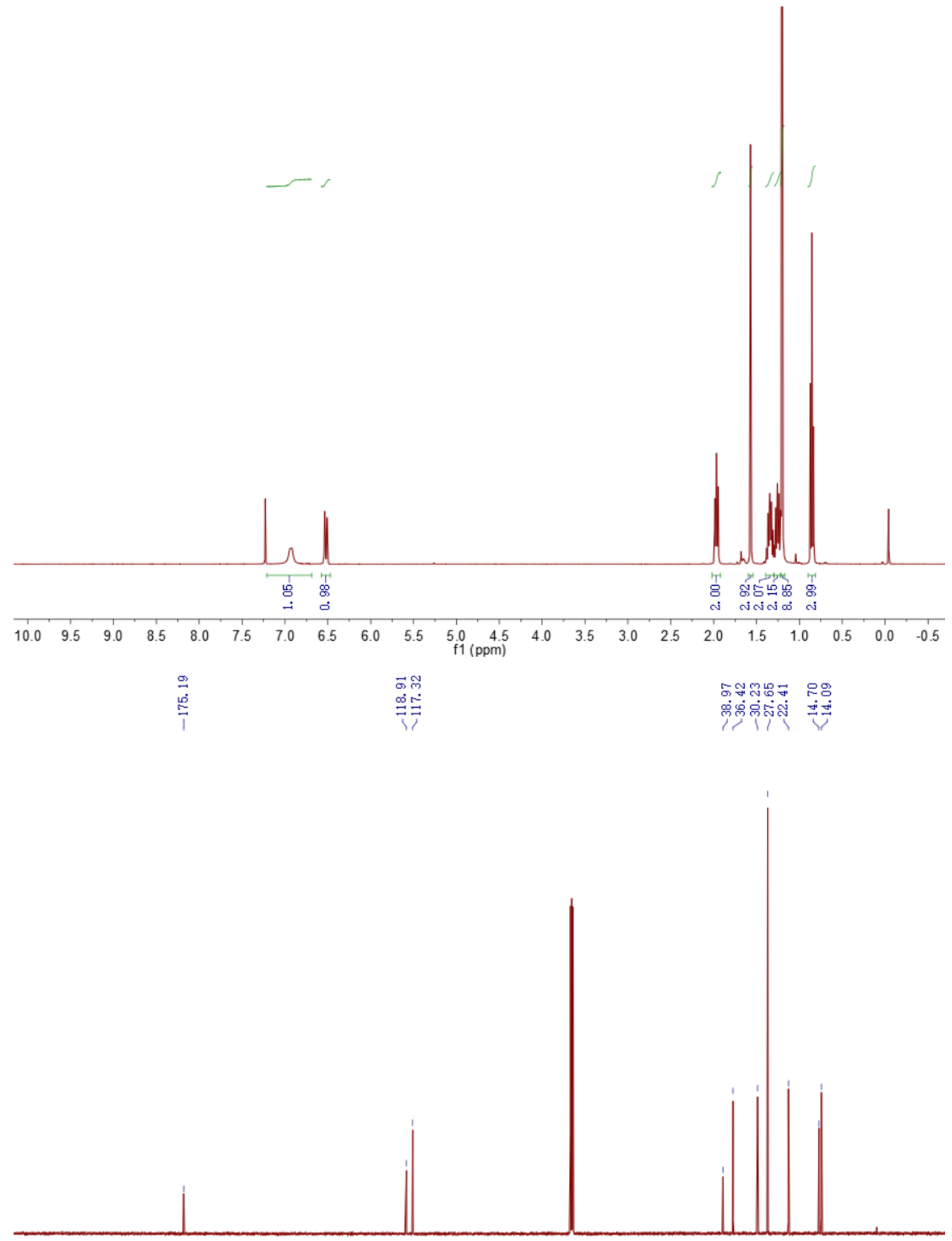

$\begin{array}{lllllllllllllllllllllll}210 & 200 & 190 & 180 & 170 & 160 & 150 & 140 & 130 & 120 & 110 & 100 & 90 & 80 & 70 & 60 & 50 & 40 & 30 & 20 & 10 & 0 & -10\end{array}$ 
$\mathrm{N}_{\mathrm{H}}^{\mathrm{O}}$

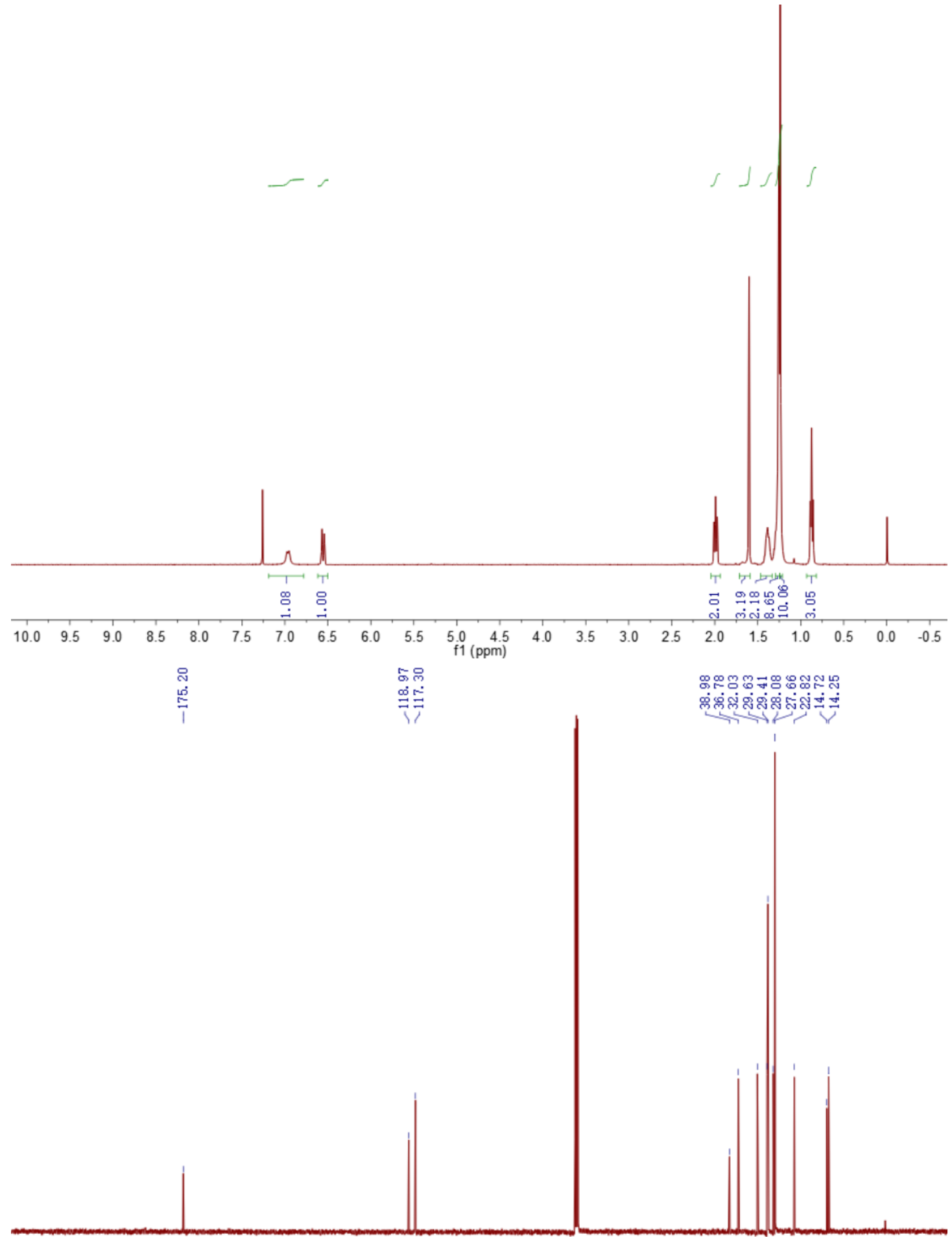

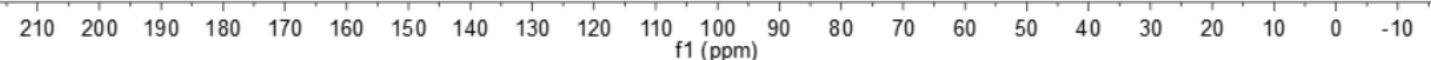


<smiles>CC(C)=CNC(=O)C(C)(C)C</smiles>
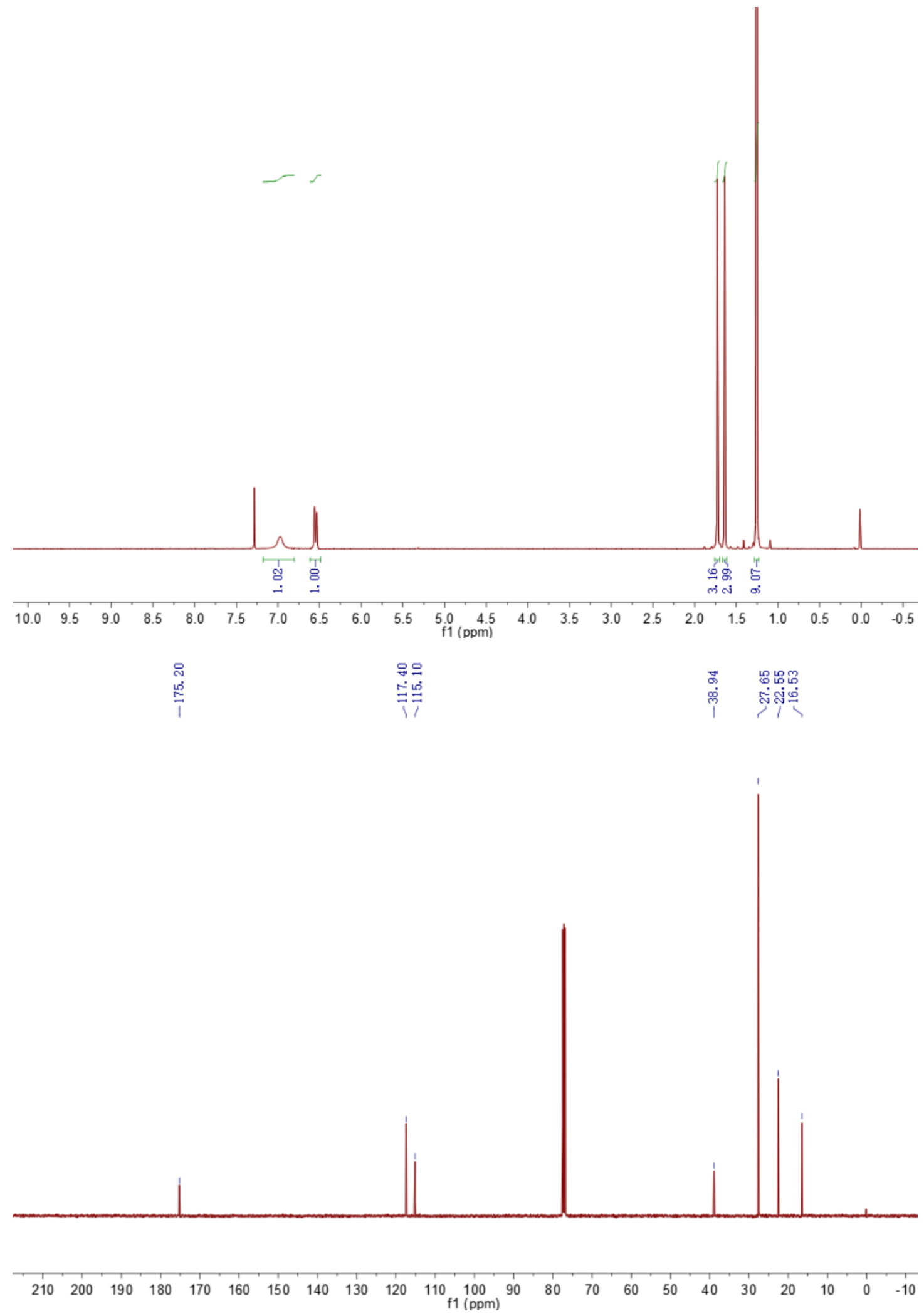
<smiles>CC(C)(C)C(=O)NC=C1CCOCC1</smiles>
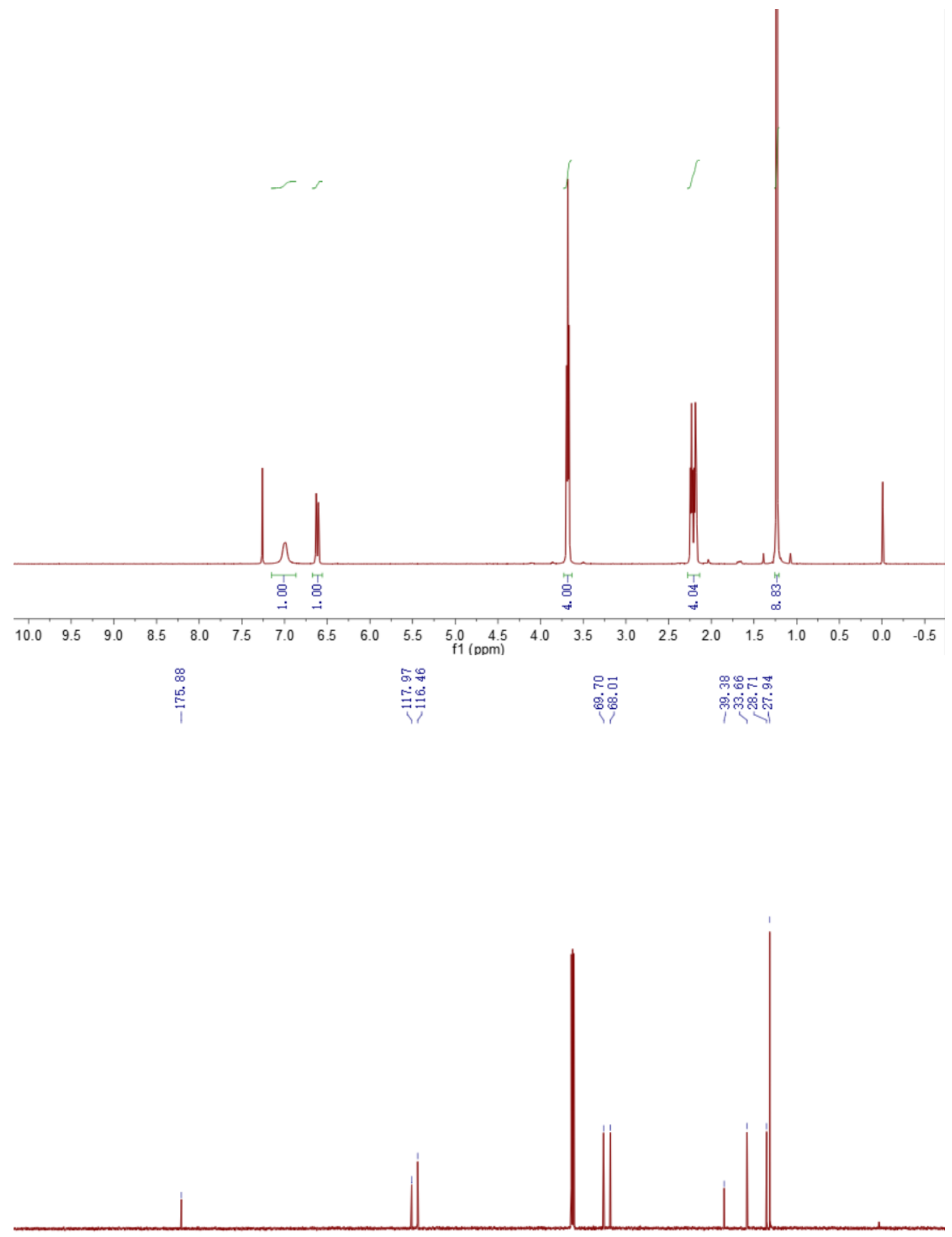

$\begin{array}{llllllllllllllllllllll}210 & 200 & 190 & 180 & 170 & 160 & 150 & 140 & 130 & 120 & 110 \begin{array}{c}100 \\ \mathrm{f} 1(\mathrm{ppm})\end{array} & 90 & 80 & 70 & 60 & 50 & 40 & 30 & 20 & 10 & 0 & -10\end{array}$ 
<smiles>CC/C=C(/C)NC(=O)C(C)(C)C</smiles>
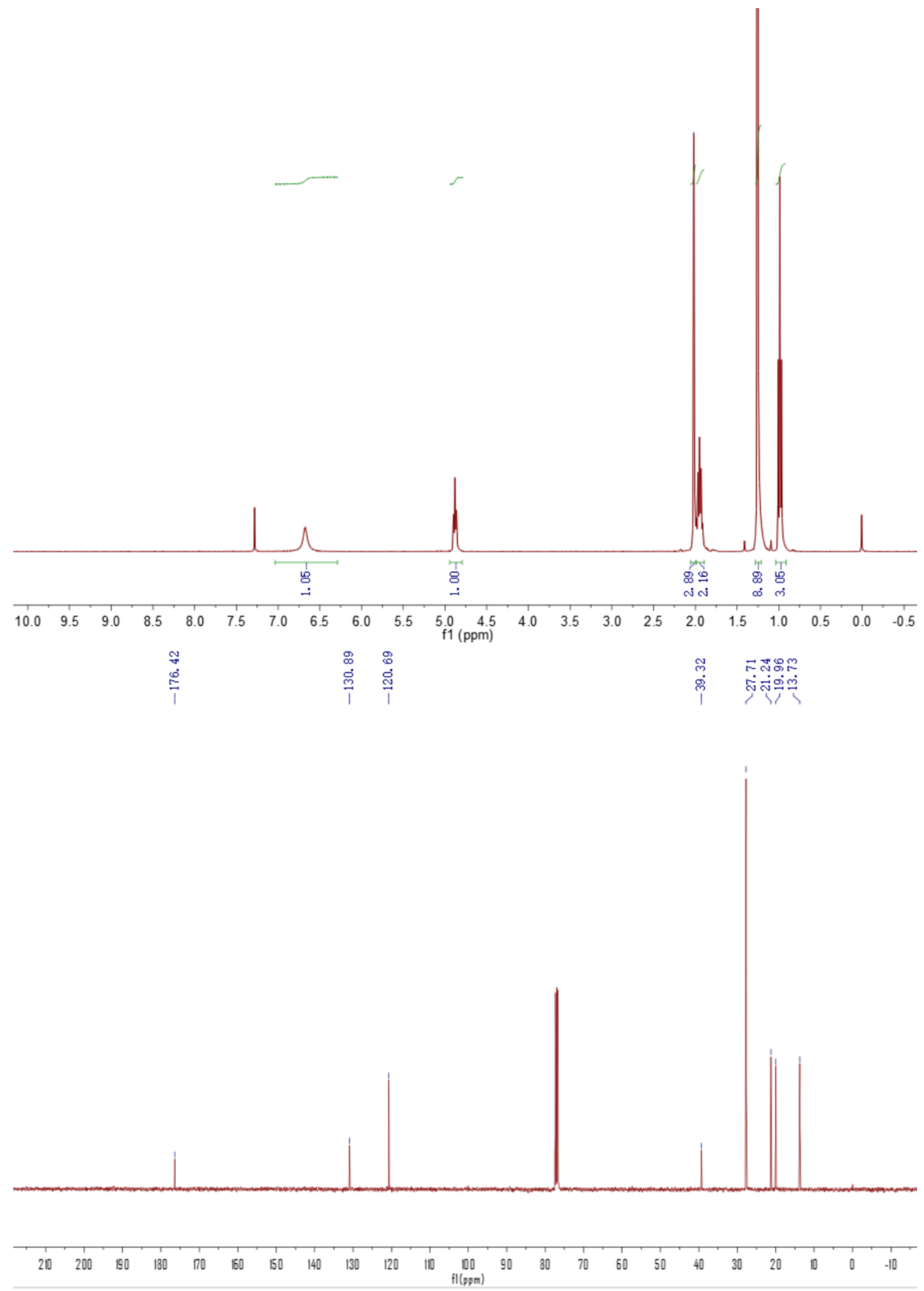
<smiles>CCC/C=C(\CC)NC(=O)C(C)(C)C</smiles>
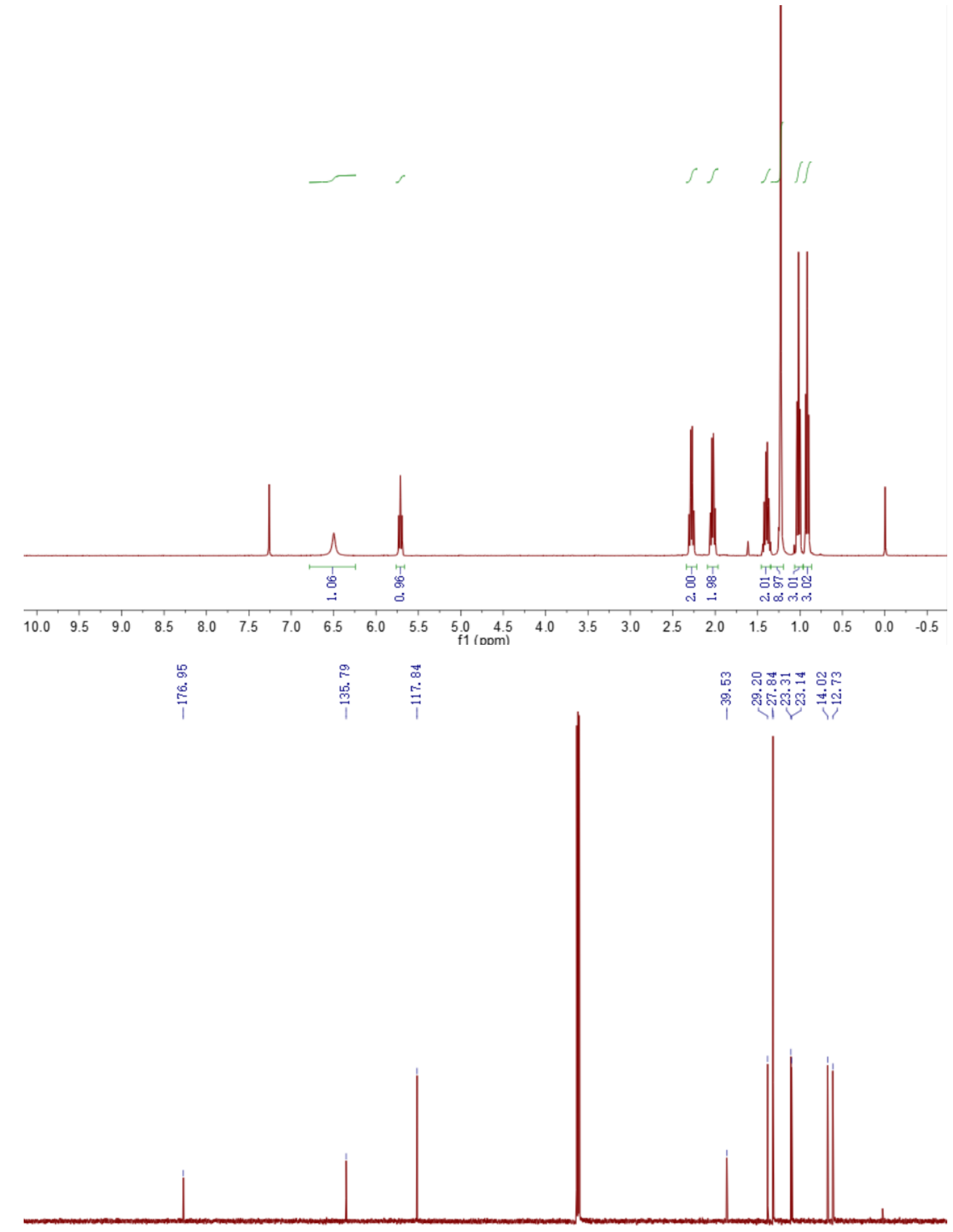

$\begin{array}{llllllllllllllllllllllll}210 & 200 & 190 & 180 & 170 & 160 & 150 & 140 & 130 & 120 & 110 & 100 & 90 & 80 & 70 & 60 & 50 & 40 & 30 & 20 & 10 & 0 & -10\end{array}$ 
<smiles>CC(C)(C)C(=O)N/C=C\CCc1ccc(F)cc1</smiles>
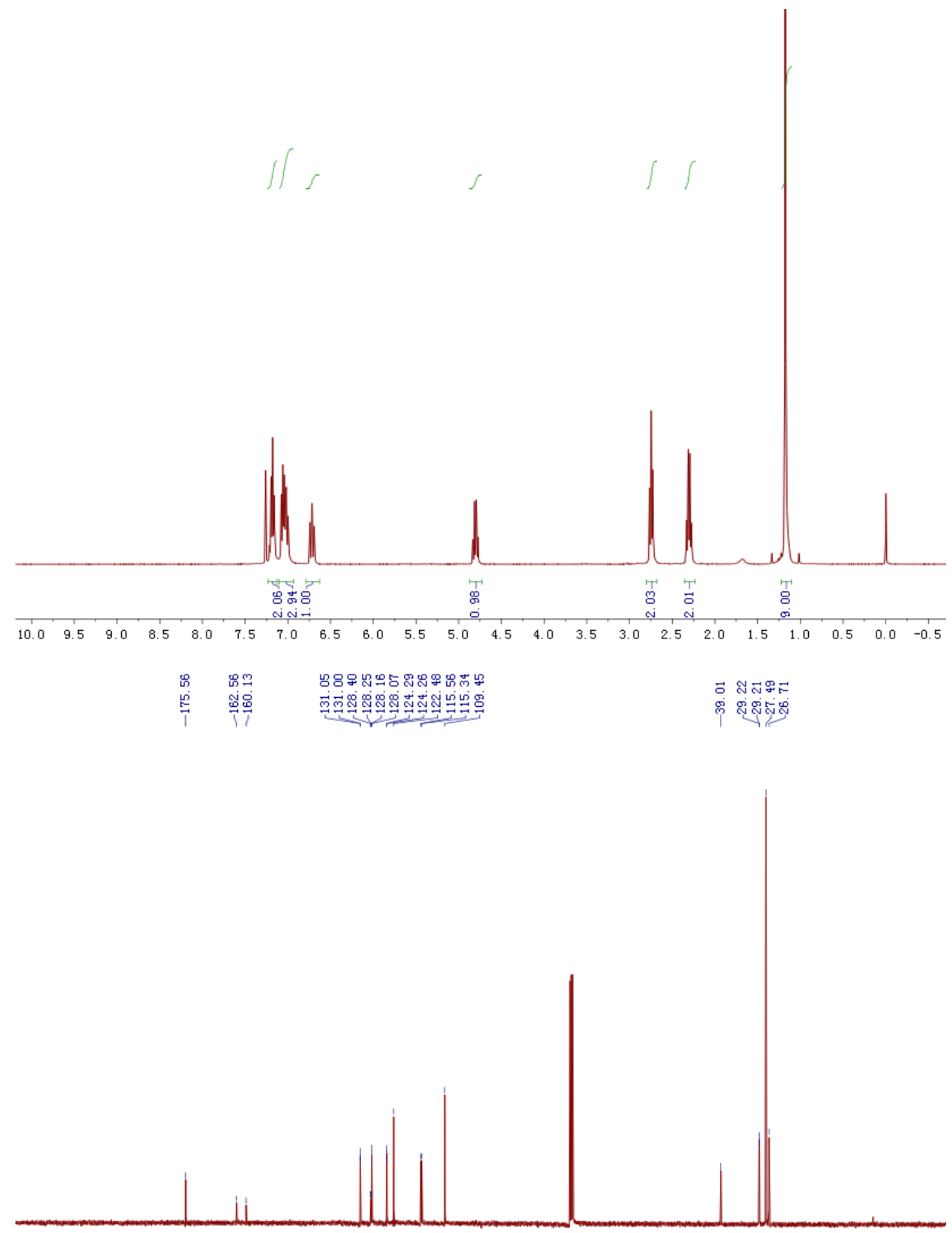

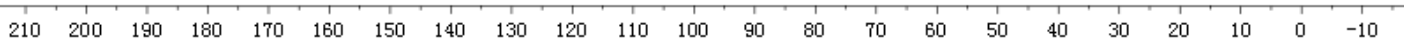


<smiles>CC(C)(C)C(=O)N/C=C/CCc1ccc(F)cc1</smiles>

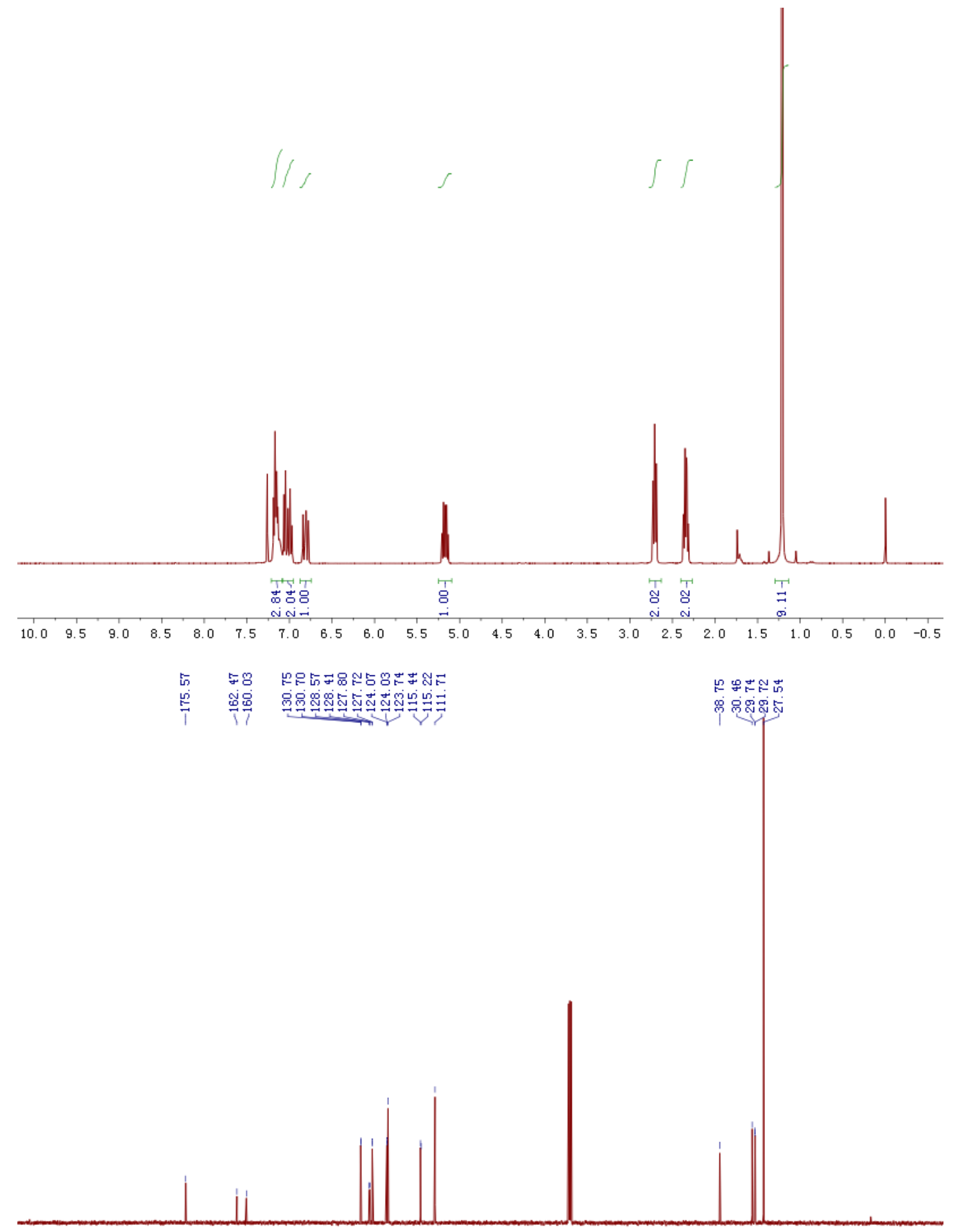

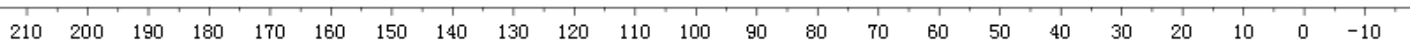


<smiles>CC(C)(C)C(=O)N/C=C\CCc1ccc(Cl)cc1</smiles>
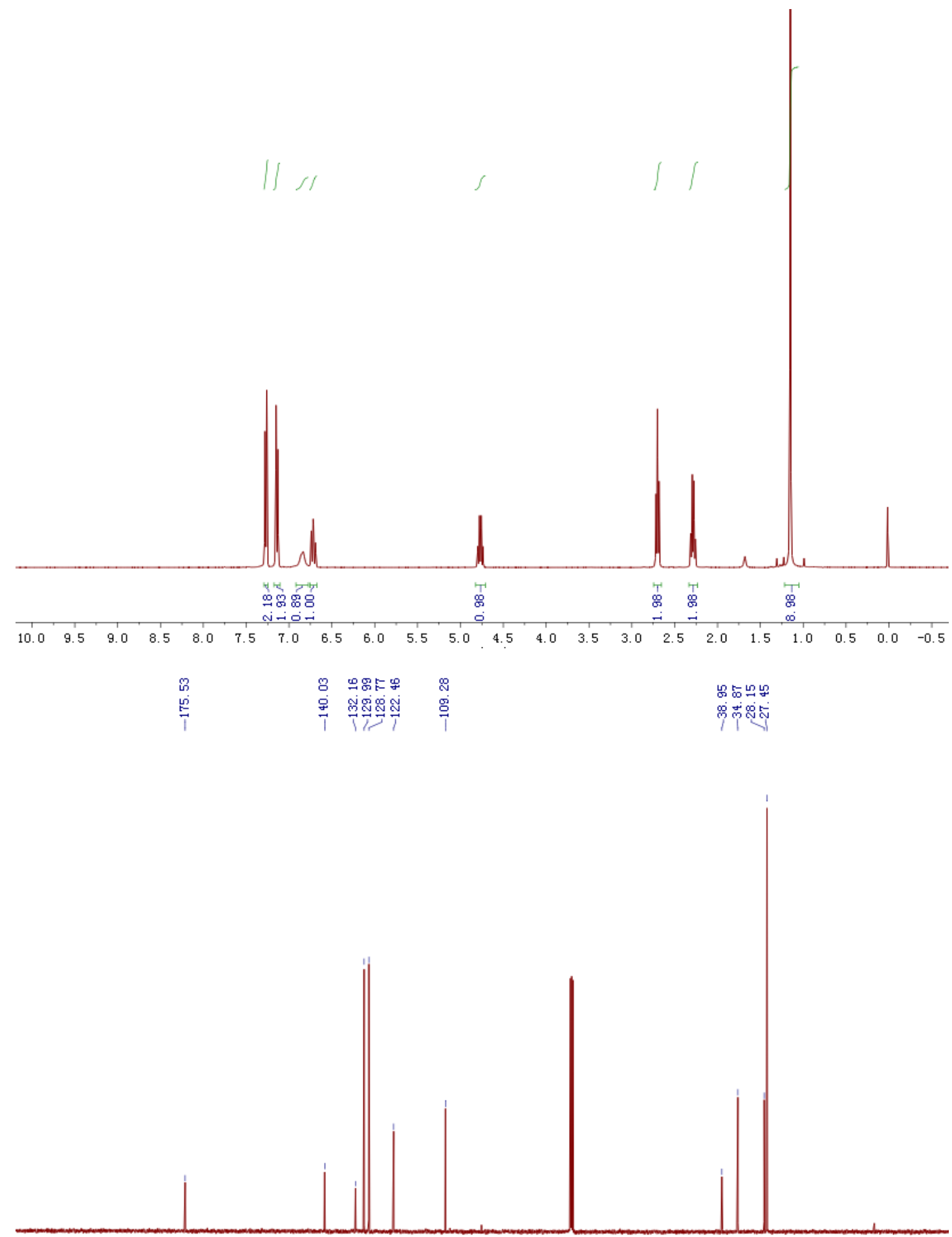

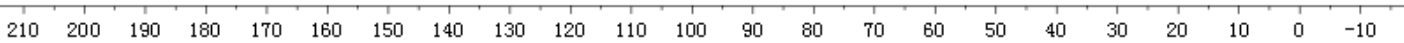


<smiles>CC(C)(C)C(=O)N/C=C/CCc1ccc(Cl)cc1</smiles>
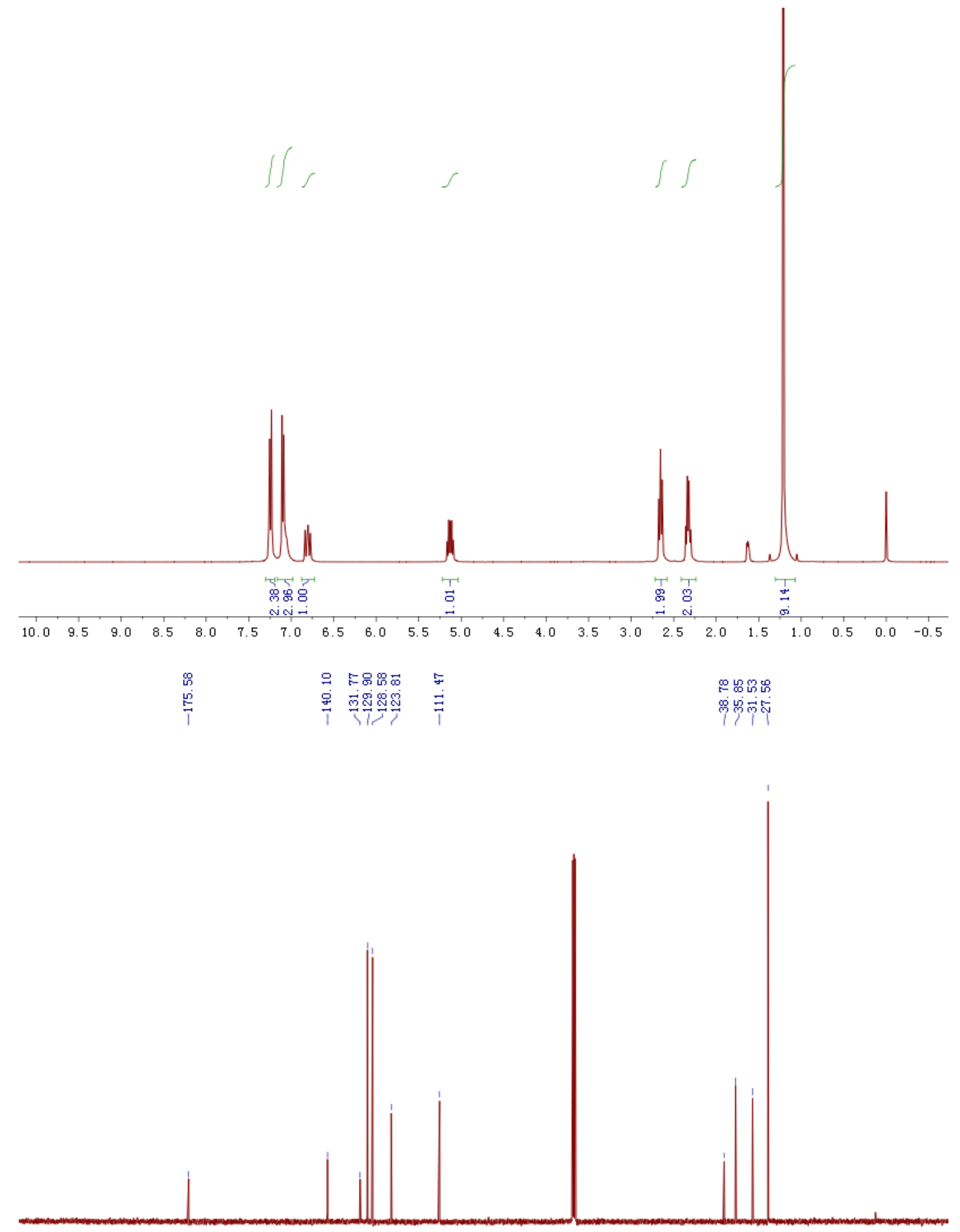

$\begin{array}{llllllllllllllllllllllll}210 & 200 & 190 & 180 & 170 & 160 & 150 & 140 & 130 & 120 & 110 & 100 & 90 & 80 & 70 & 60 & 50 & 40 & 30 & 20 & 10 & 0 & -10\end{array}$ 
<smiles>Cc1ccc(CC/C=C\NC(=O)C(C)(C)C)cc1</smiles>
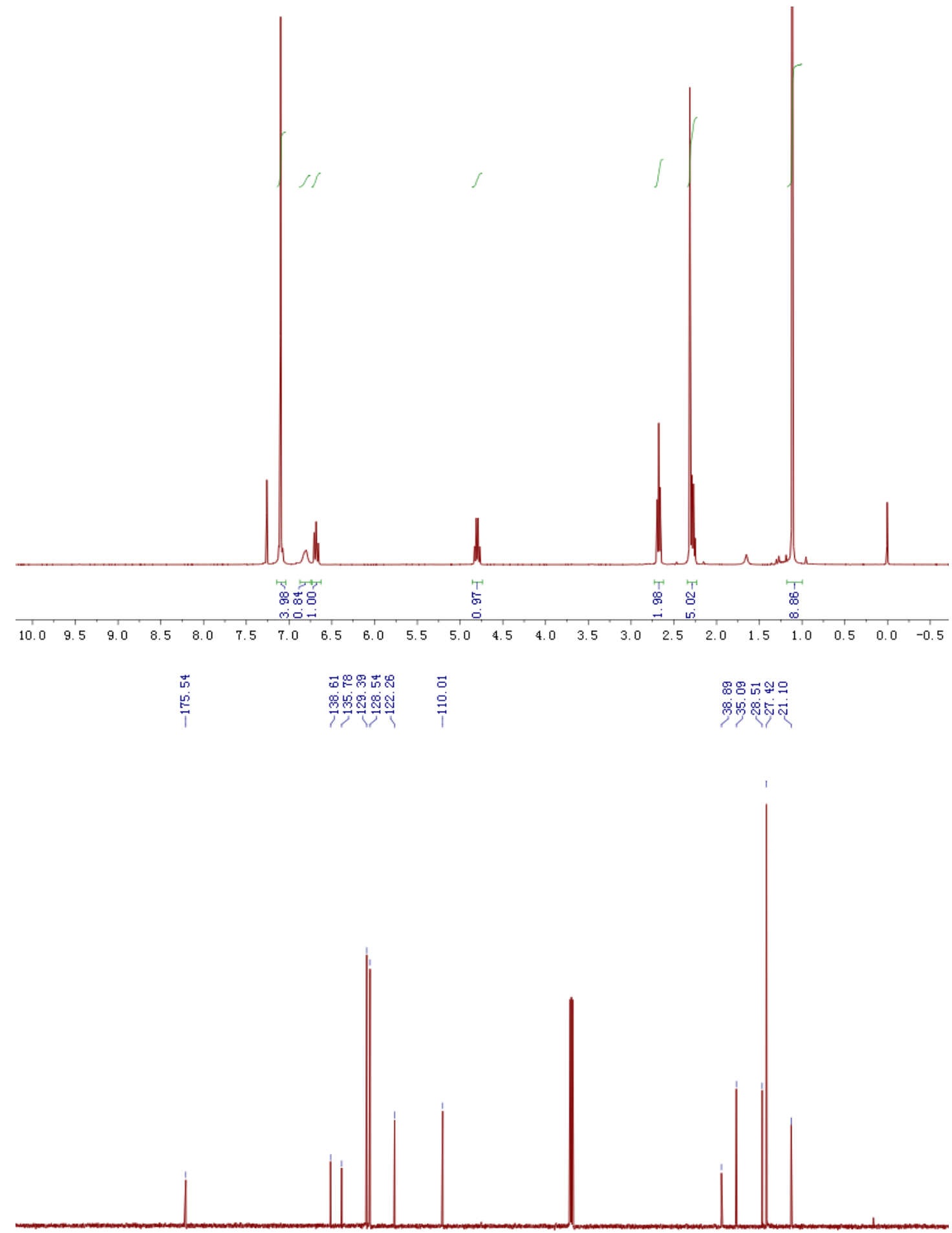

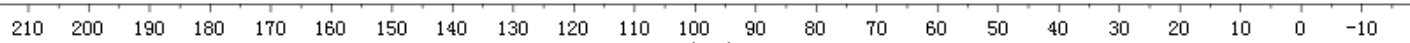


${ }_{\mathrm{H}}^{\mathrm{Me}}$

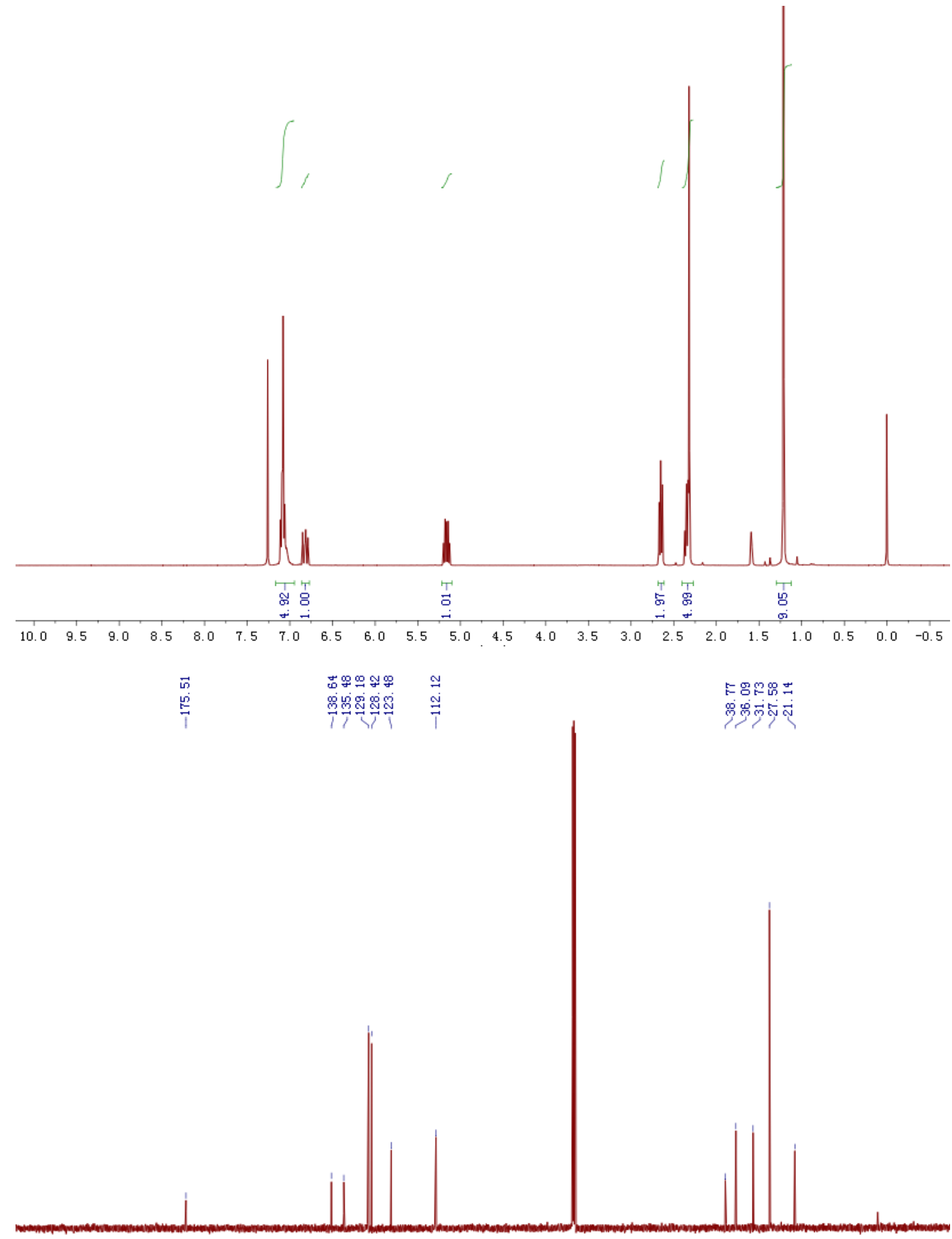

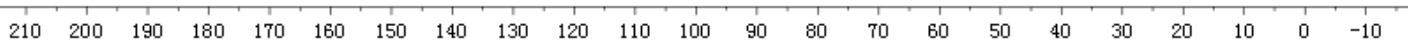


<smiles>COc1ccc(CC/C=C\NC(=O)C(C)(C)C)cc1</smiles>

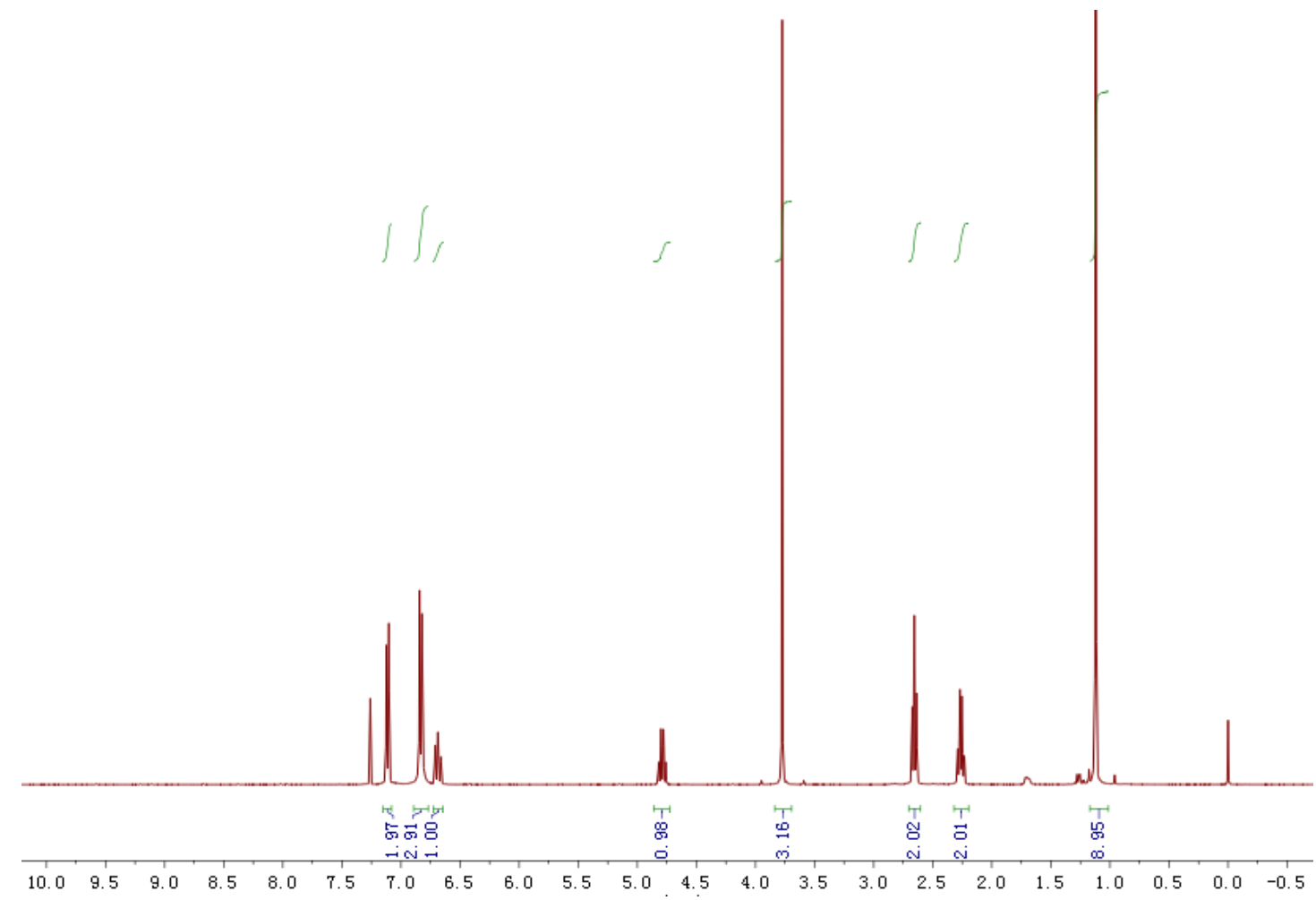

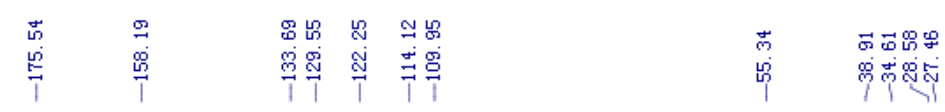

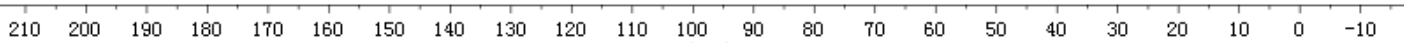


<smiles>COc1ccc(CC/C=C/NC(=O)C(C)(C)C)cc1</smiles>
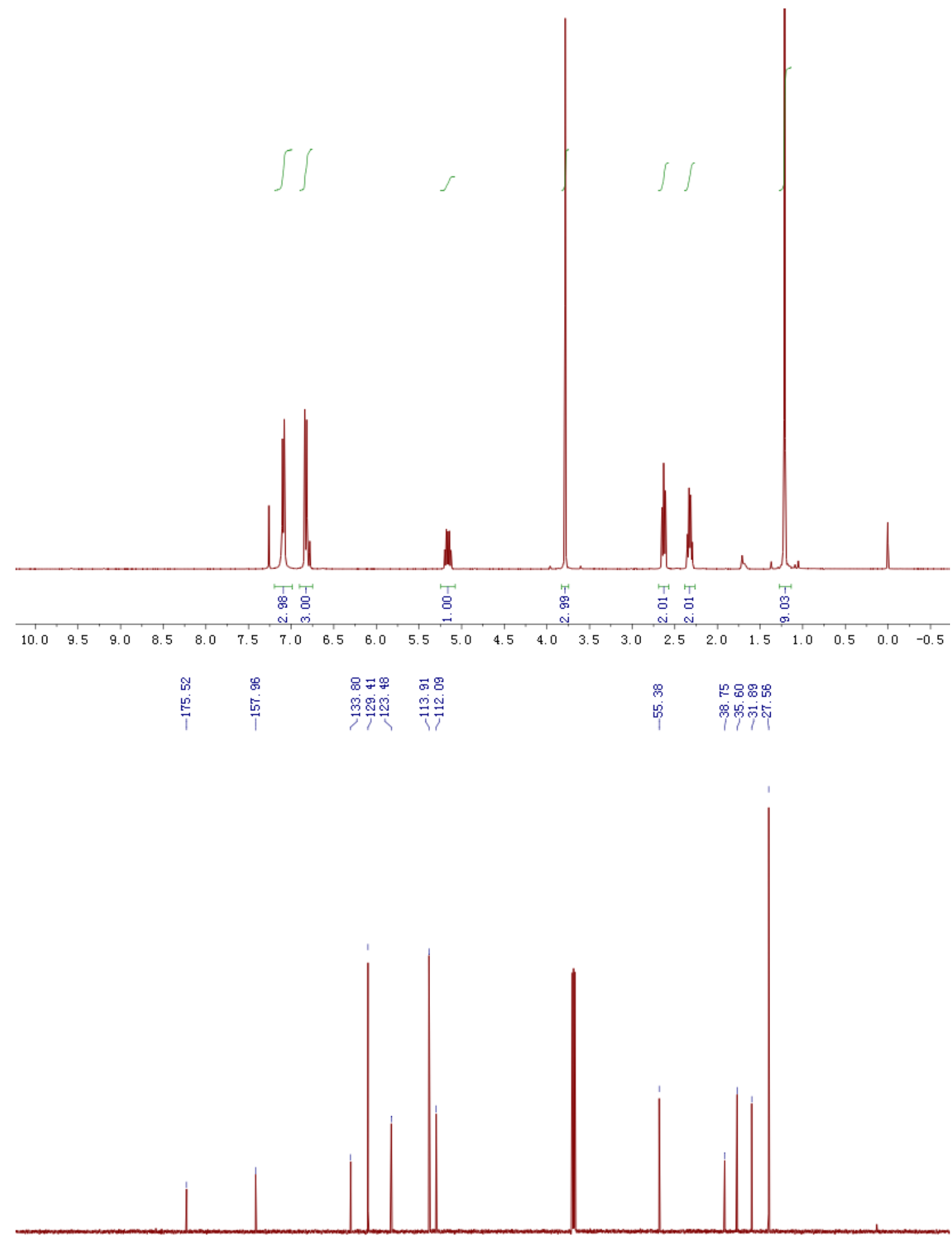

$\begin{array}{lllllllllllllllllllllll}210 & 200 & 190 & 180 & 170 & 160 & 150 & 140 & 130 & 120 & 110 & 100 & 90 & 80 & 70 & 60 & 50 & 40 & 30 & 20 & 10 & 0 & -10\end{array}$ 
<smiles>CSc1ccc(CCC/C=C\NC(=O)C(C)(C)C)cc1</smiles>
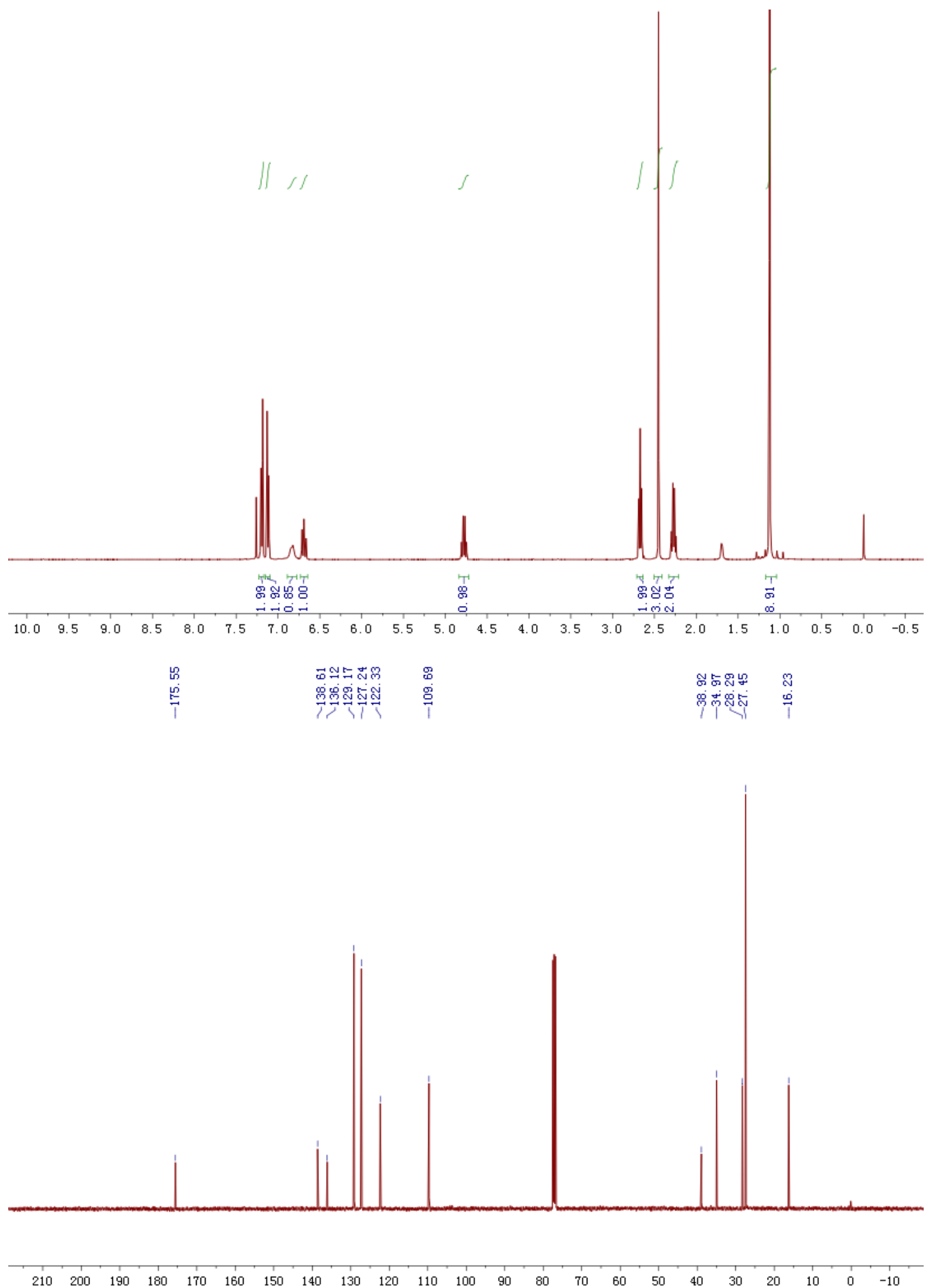

S173 
<smiles>C[As]c1ccc(CC/C=C/NC(=O)C(C)(C)C)cc1</smiles>

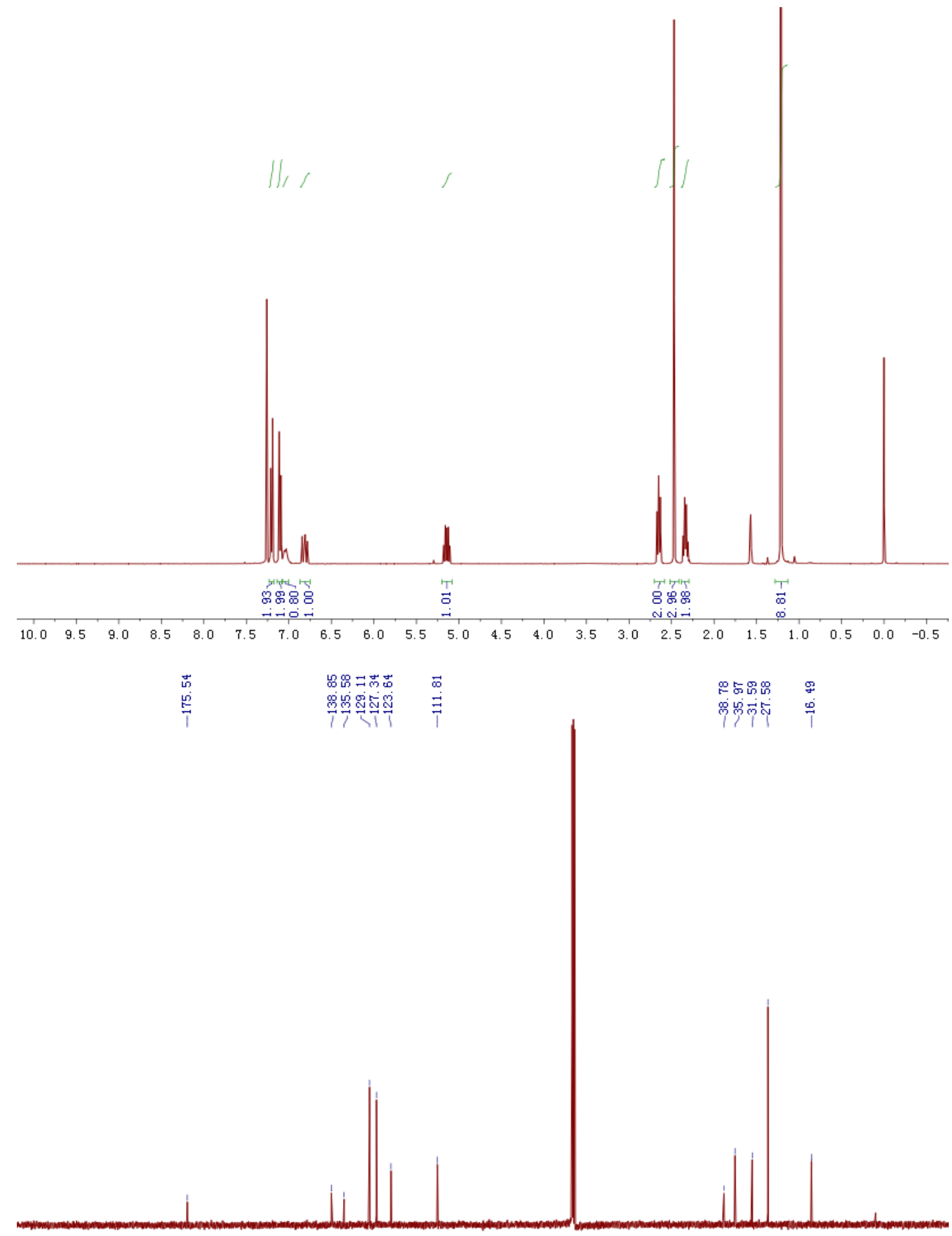

$\begin{array}{llllllllllllllllllllllll}210 & 200 & 190 & 180 & 170 & 160 & 150 & 140 & 130 & 120 & 110 & 100 & 90 & 80 & 70 & 60 & 50 & 40 & 30 & 20 & 10 & 0 & -10\end{array}$ 

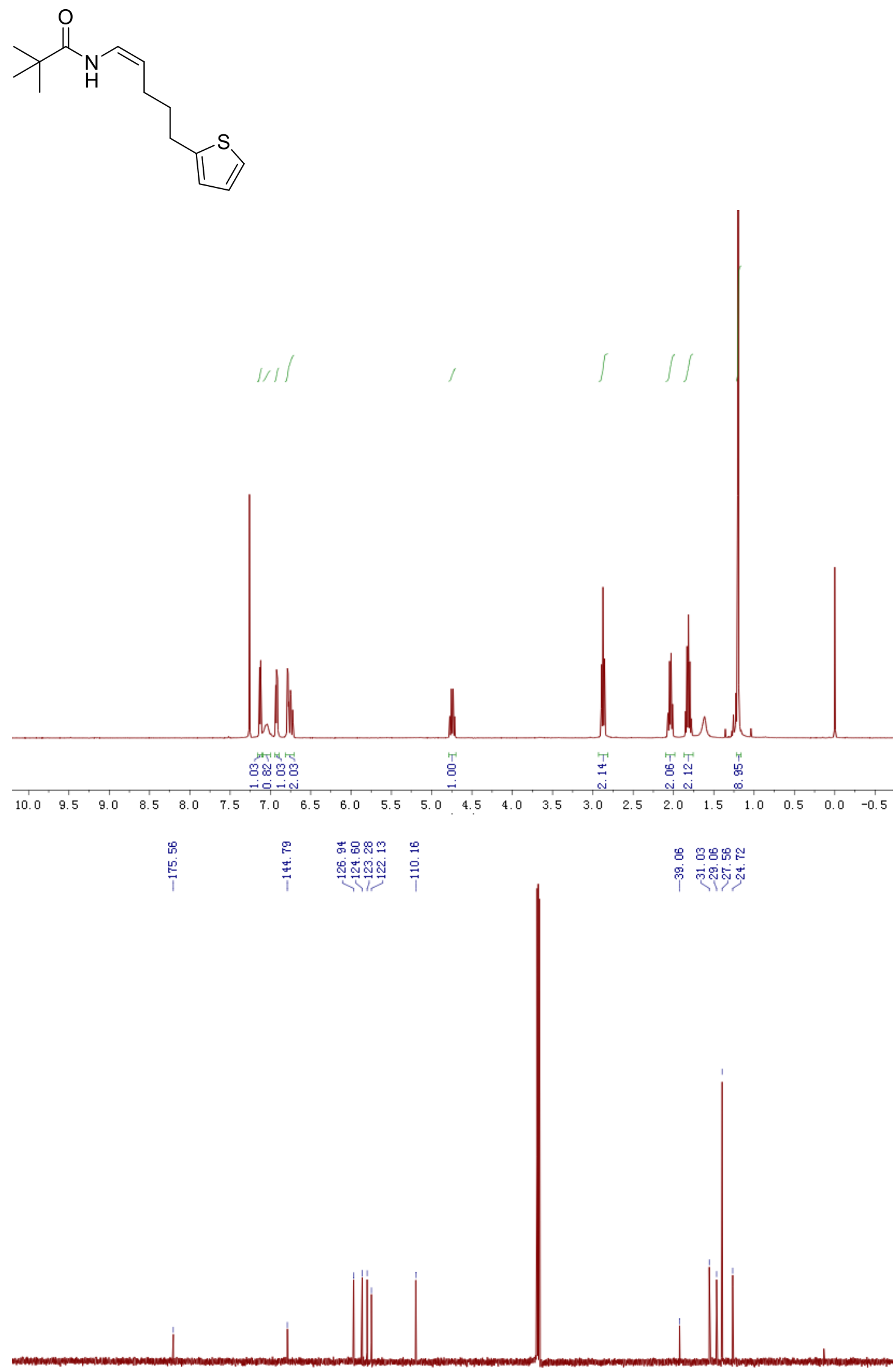

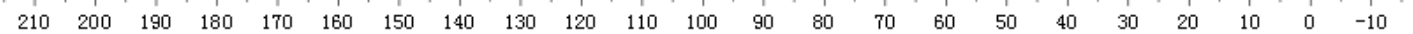


<smiles>CC(C)(C)C(=O)N/C=C/CCCc1cccs1</smiles>
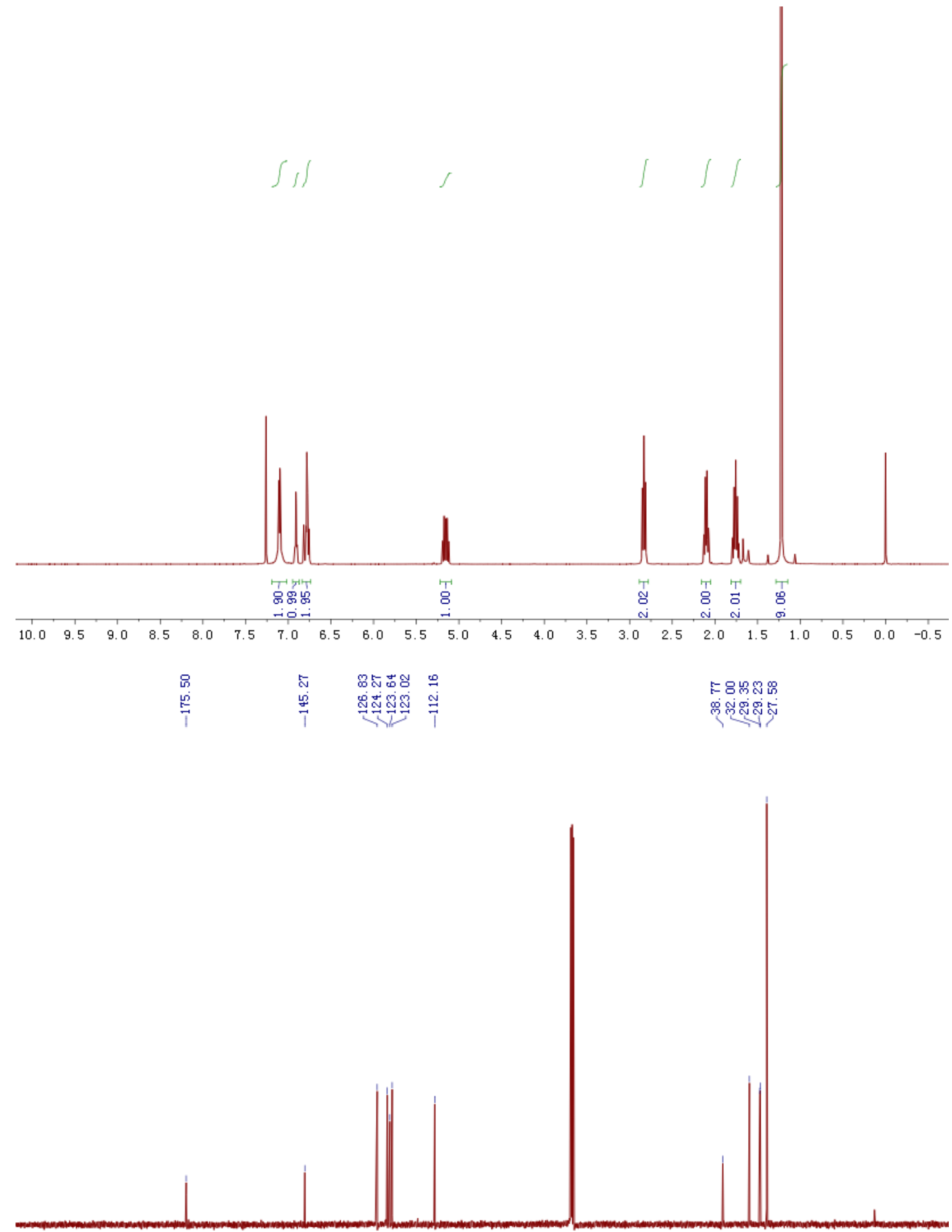

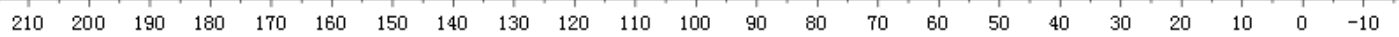



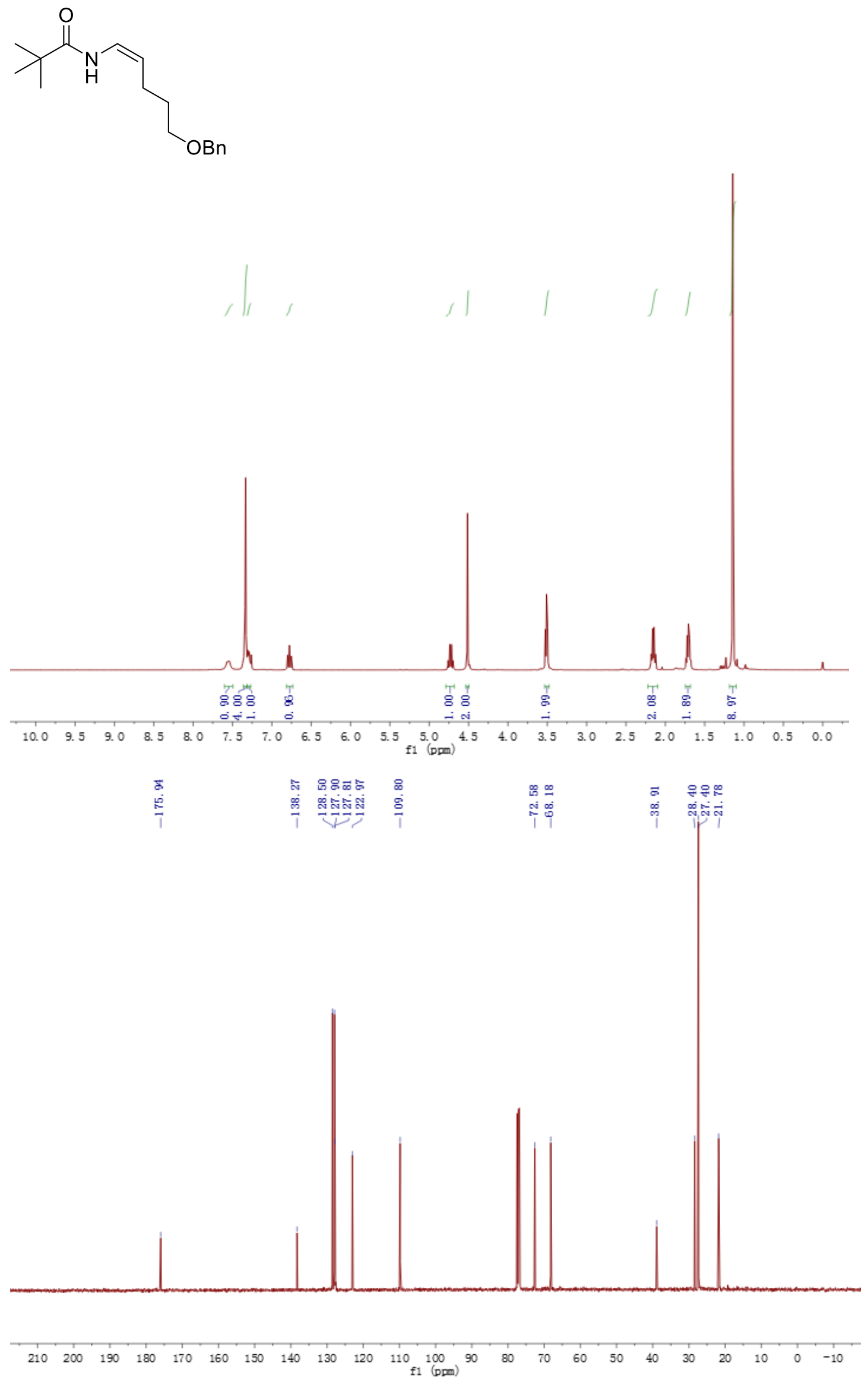
$>_{\mathrm{H}}^{\mathrm{O}} \sim \sim_{\mathrm{OBn}}$
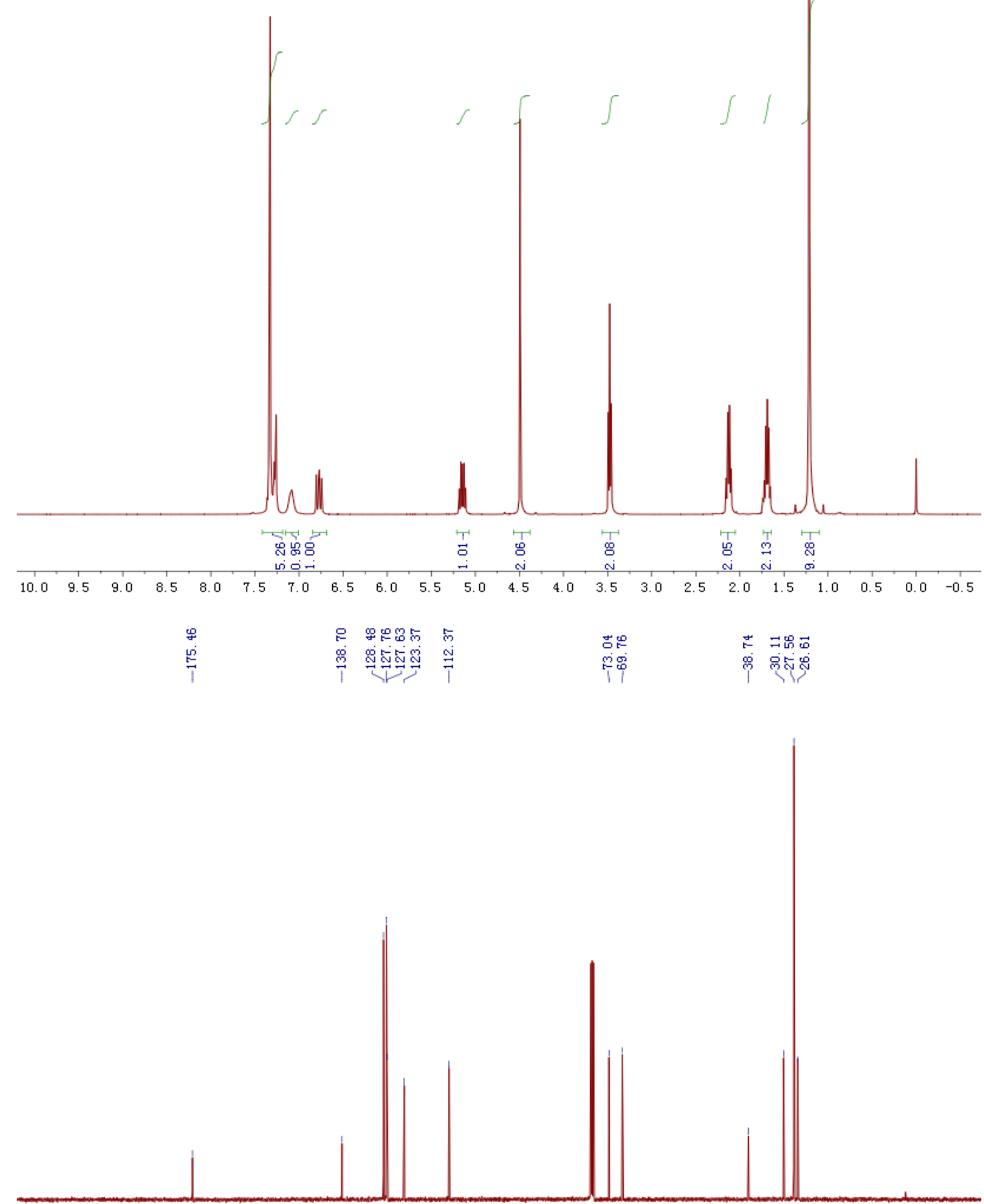

$\begin{array}{lllllllllllllllllllllll}210 & 200 & 190 & 180 & 170 & 160 & 150 & 140 & 130 & 120 & 110 & 100 & 90 & 80 & 70 & 60 & 50 & 40 & 30 & 20 & 10 & 0 & -10\end{array}$ 

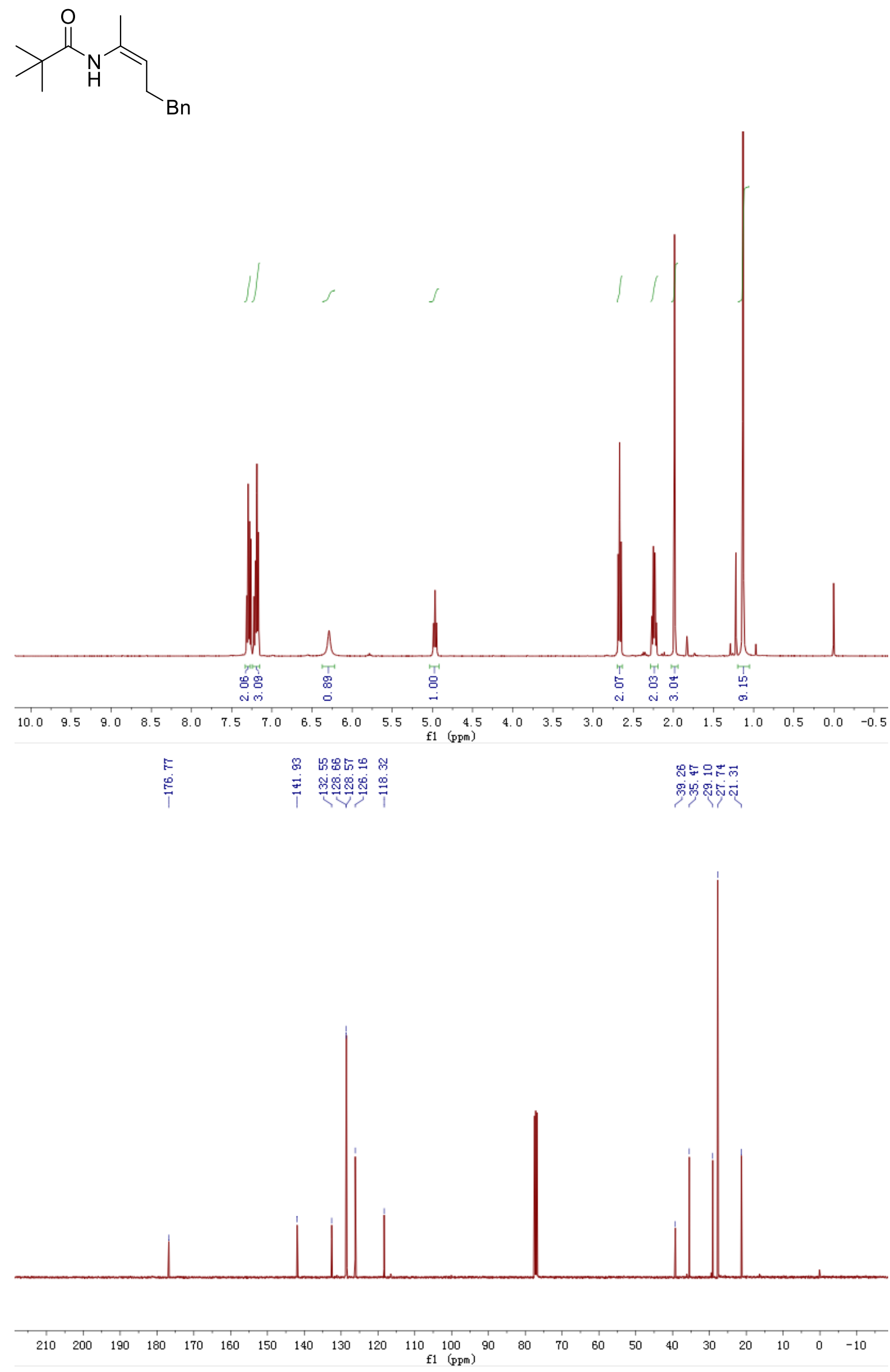
<smiles>C/C=C(\NC(=O)C(C)(C)C)c1ccc(C(F)(F)F)cc1</smiles>

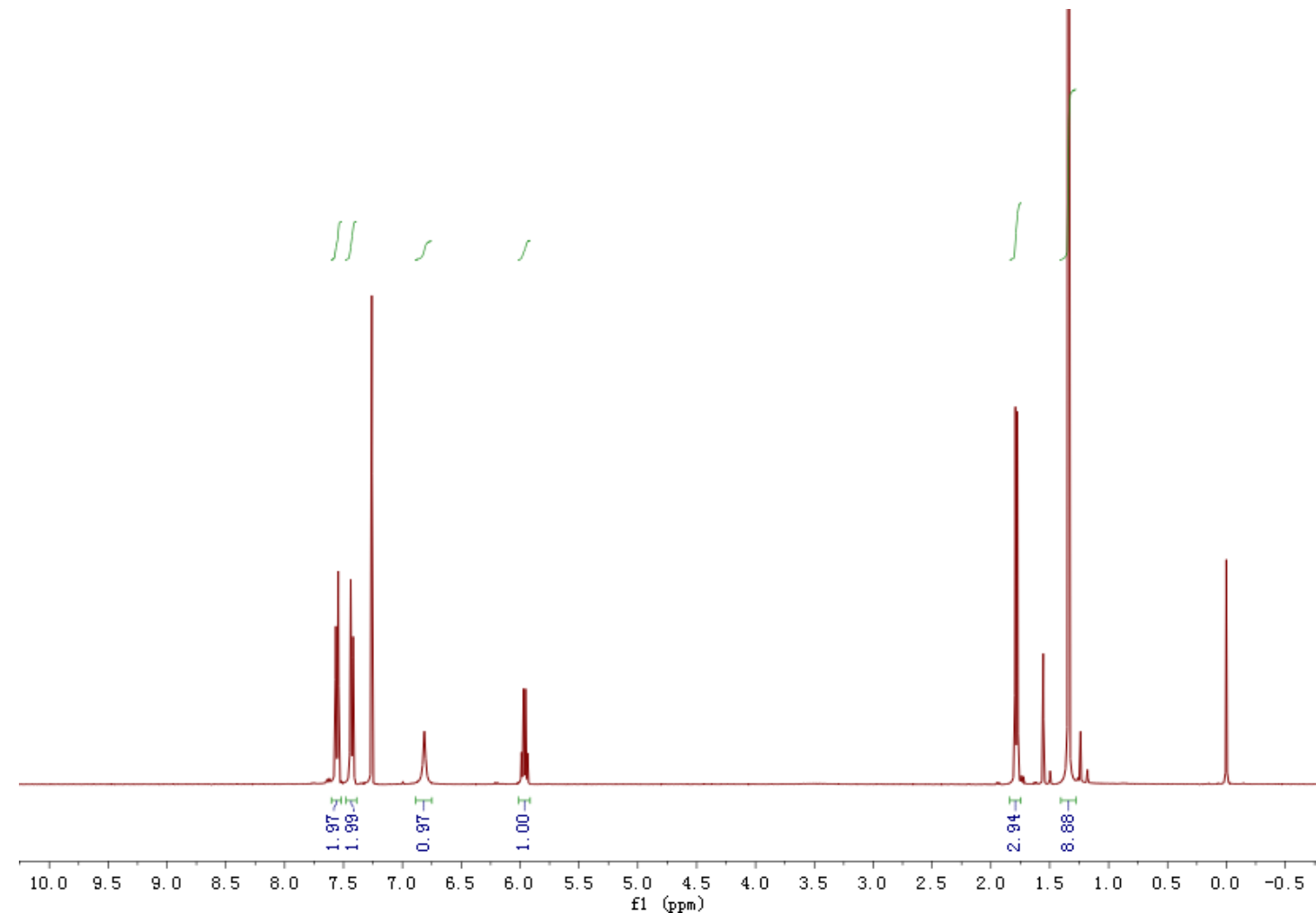


<smiles>C/C=C(\NC(=O)C(C)(C)C)c1ccc(F)cc1</smiles>

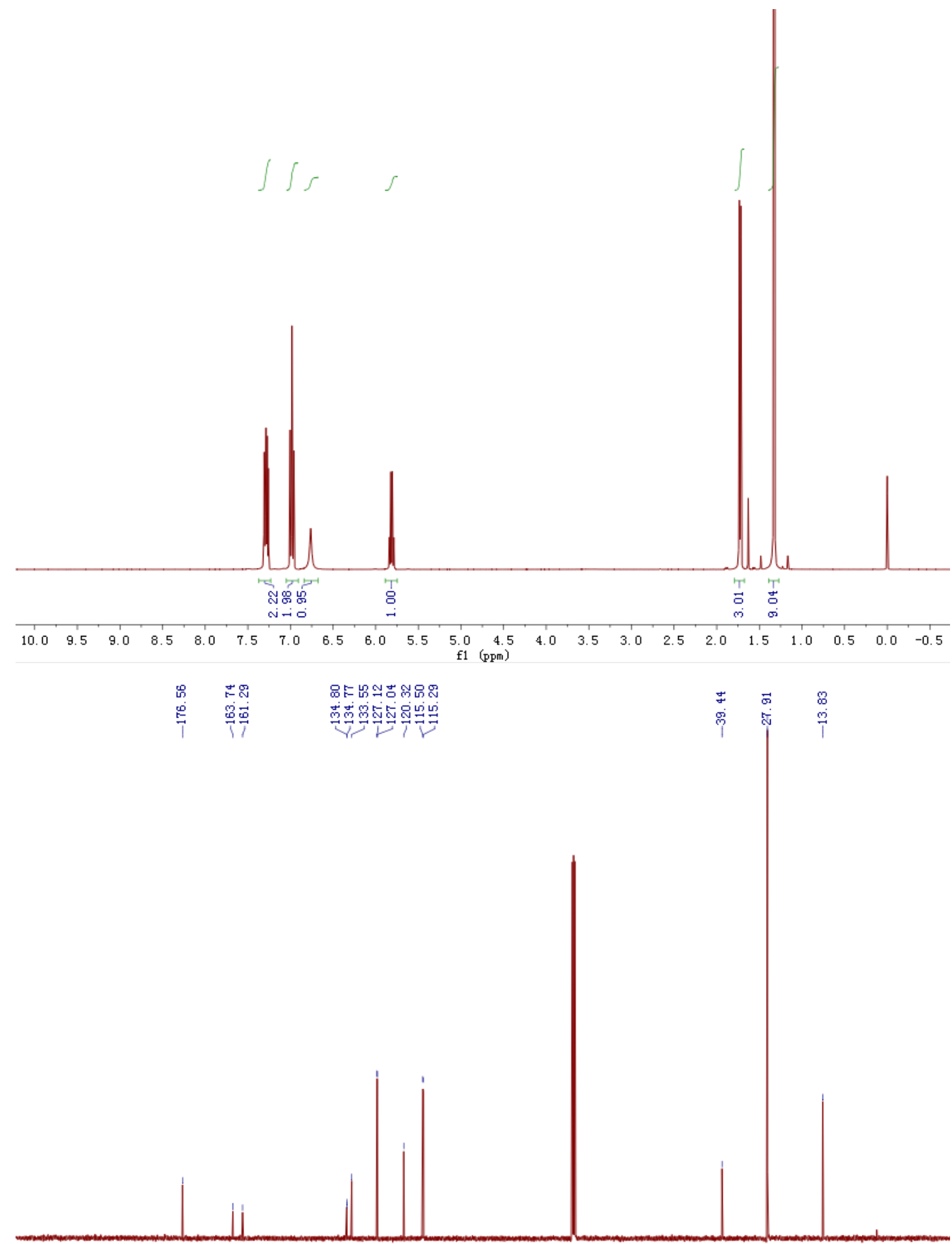

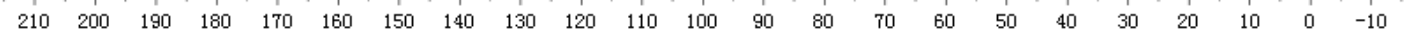


<smiles>C/C=C(\NC(=O)C(C)(C)C)c1cccc(OC)c1</smiles>
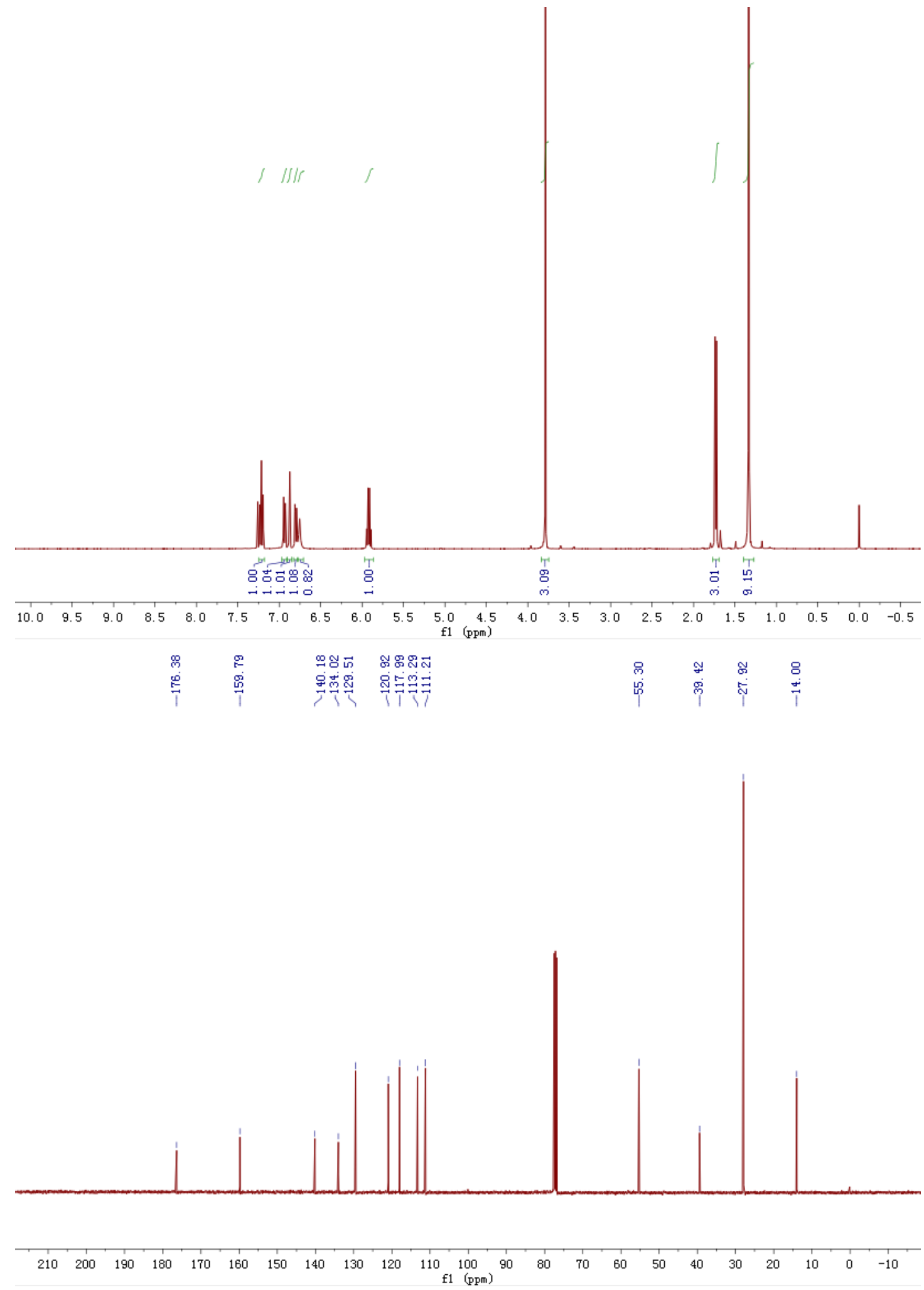
<smiles>C/C=C(\NC(=O)C(C)(C)C)c1ccccc1</smiles>

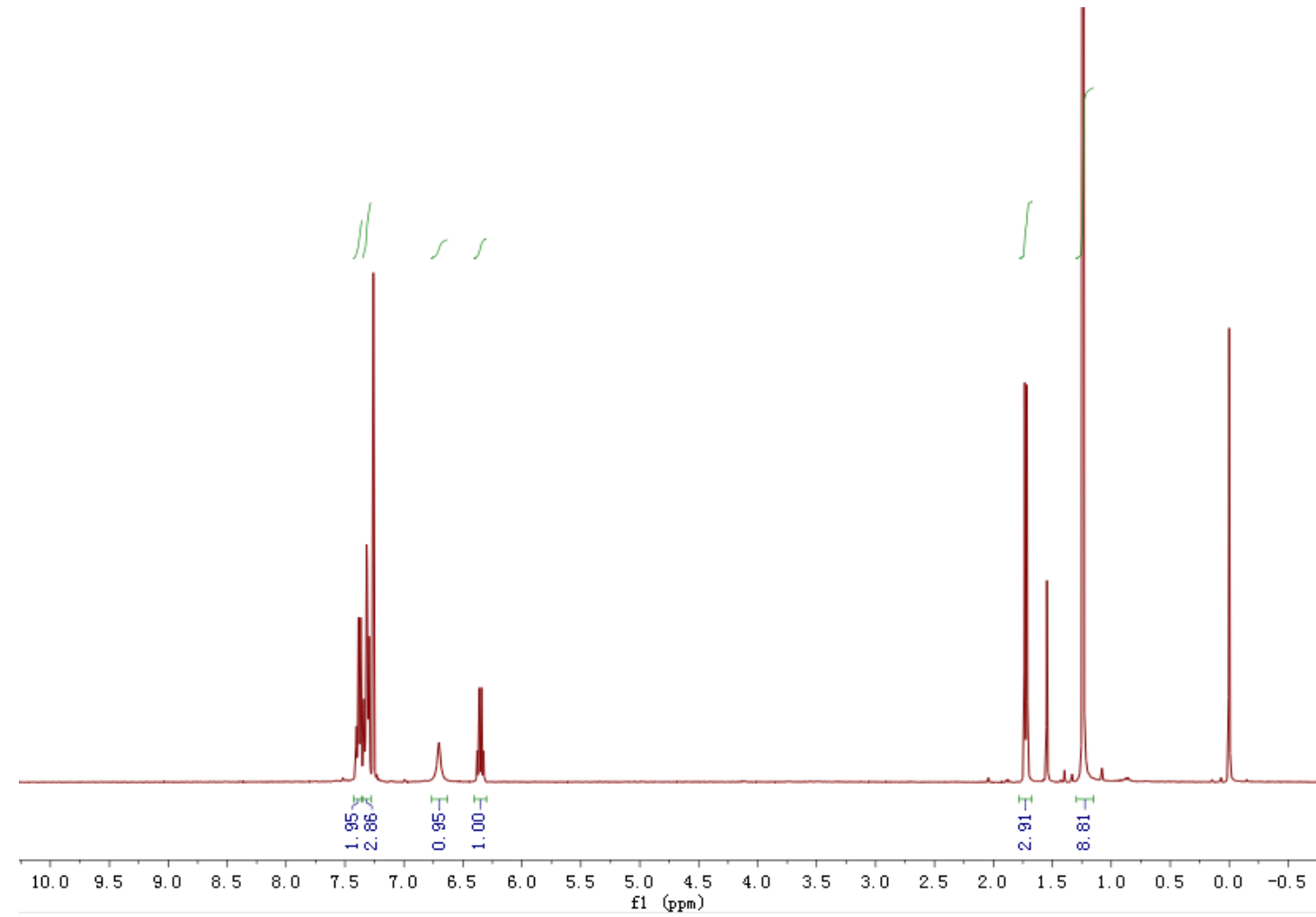

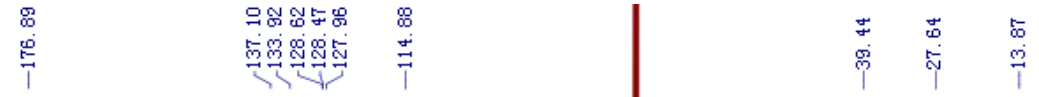

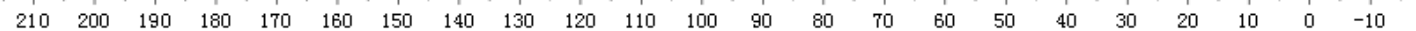


$\overbrace{\mathrm{H}}^{\mathrm{Ph}}$
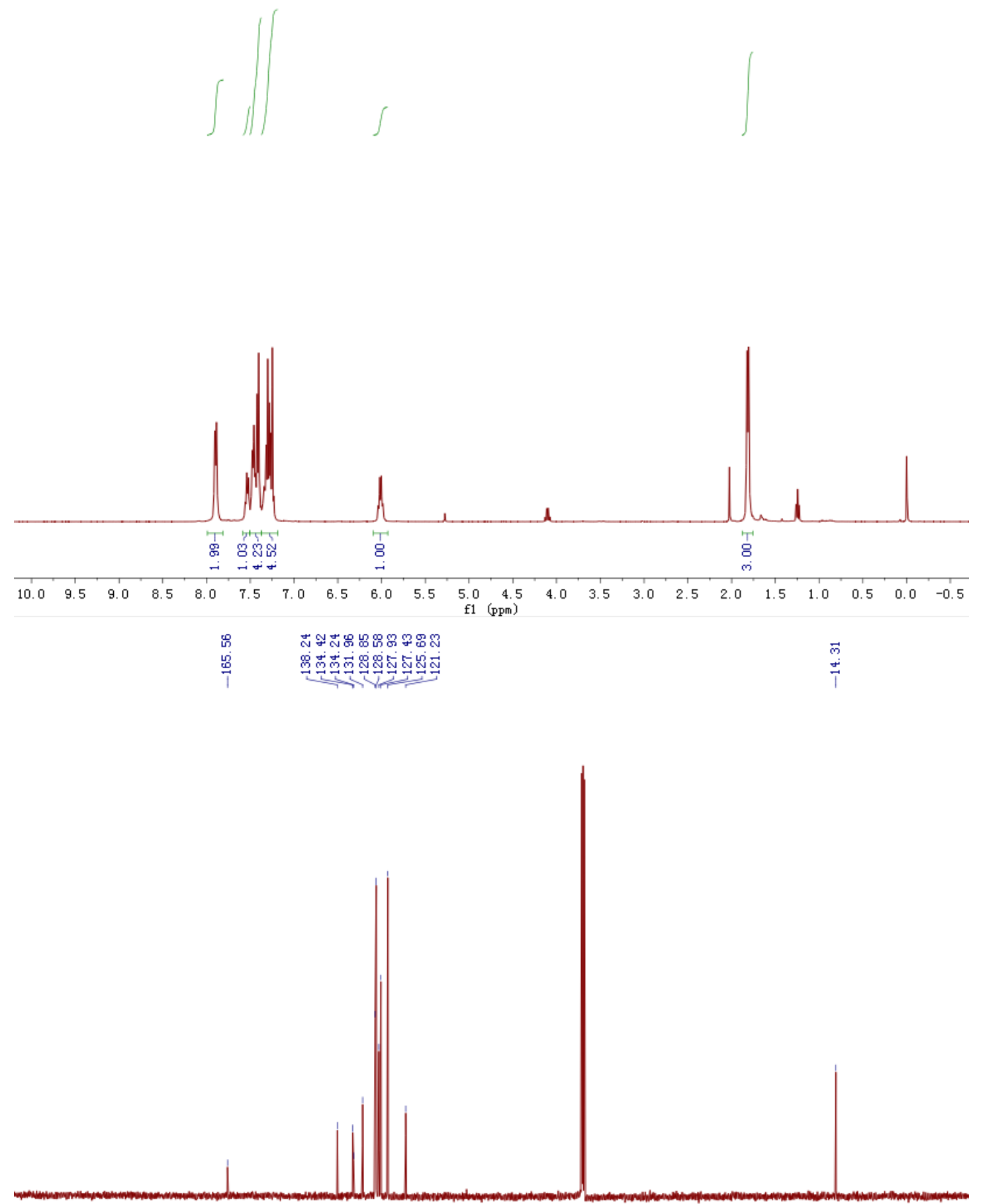

$\begin{array}{lllllllllllllllllllllll}210 & 200 & 190 & 180 & 170 & 160 & 150 & 140 & 130 & 120 & 110 & 100 & 90 & 80 & 70 & 60 & 50 & 40 & 30 & 20 & 10 & 0 & -10\end{array}$ f1 $(\mathrm{ppm})$ 

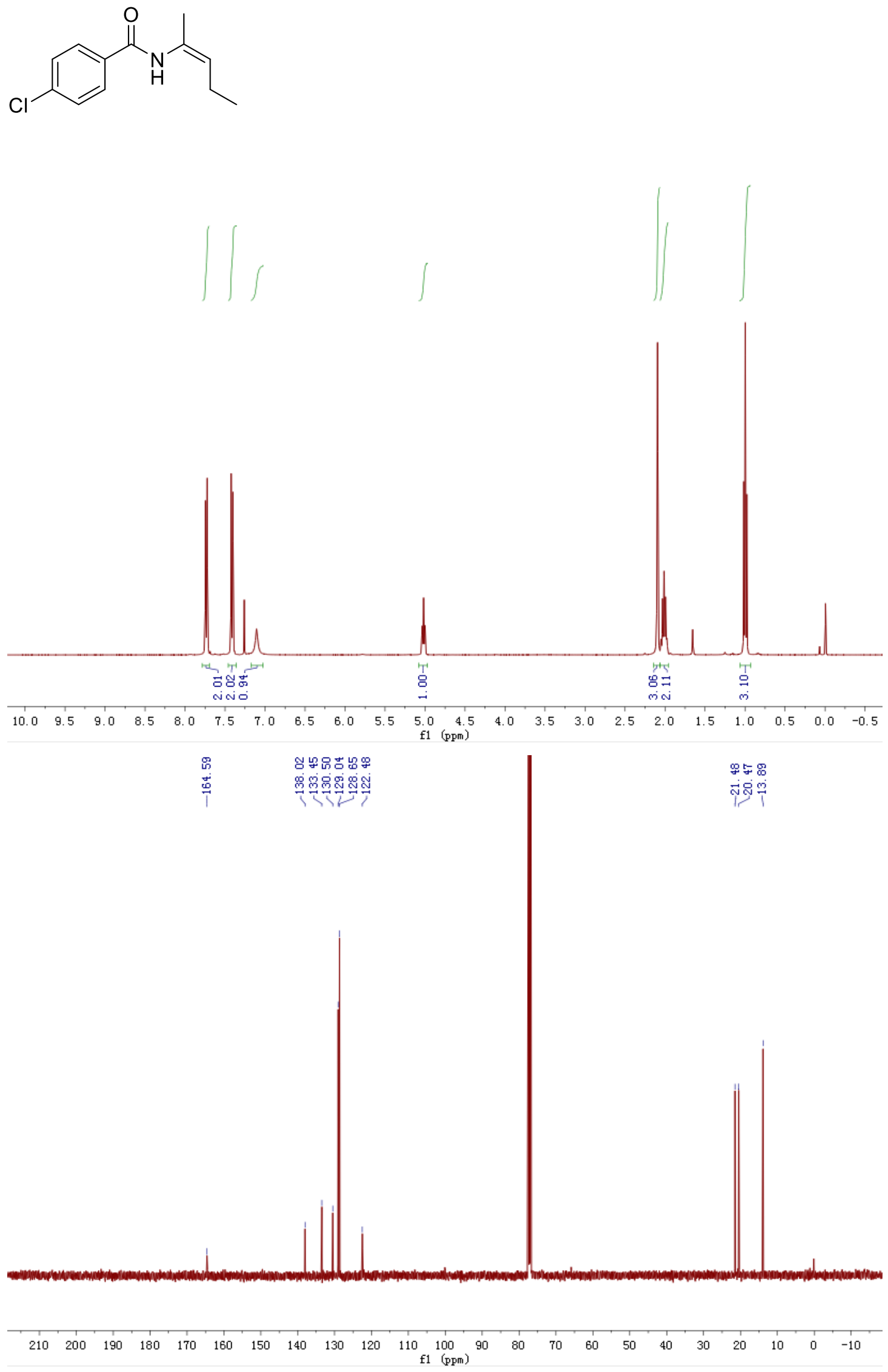

S185 
<smiles>C/C=C(\NC(=O)c1ccc(Cl)cc1)c1ccccc1</smiles>
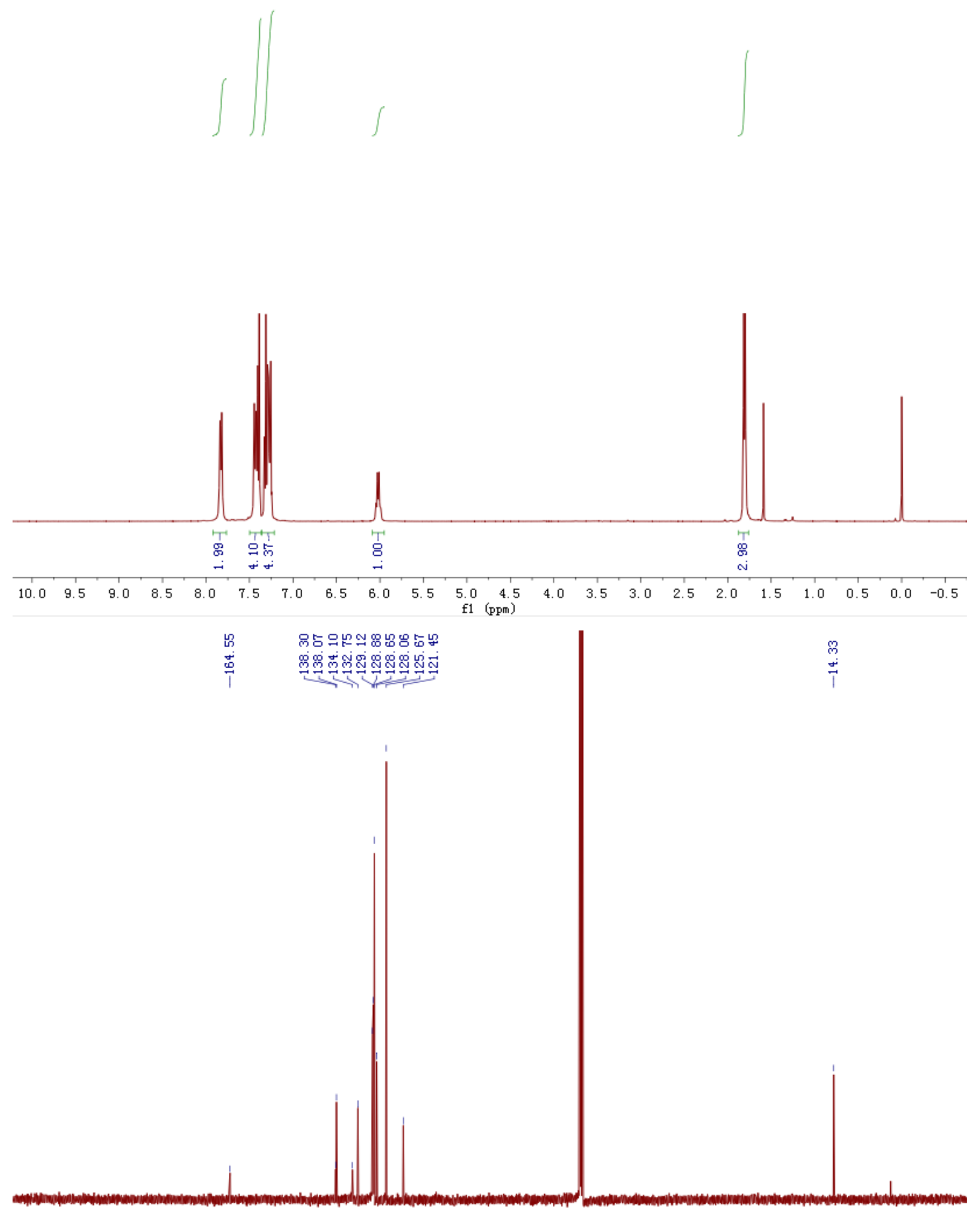

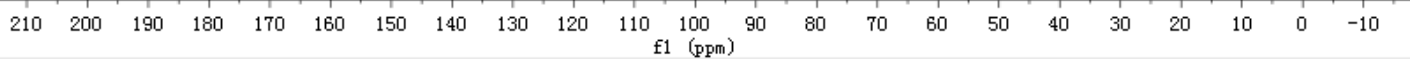



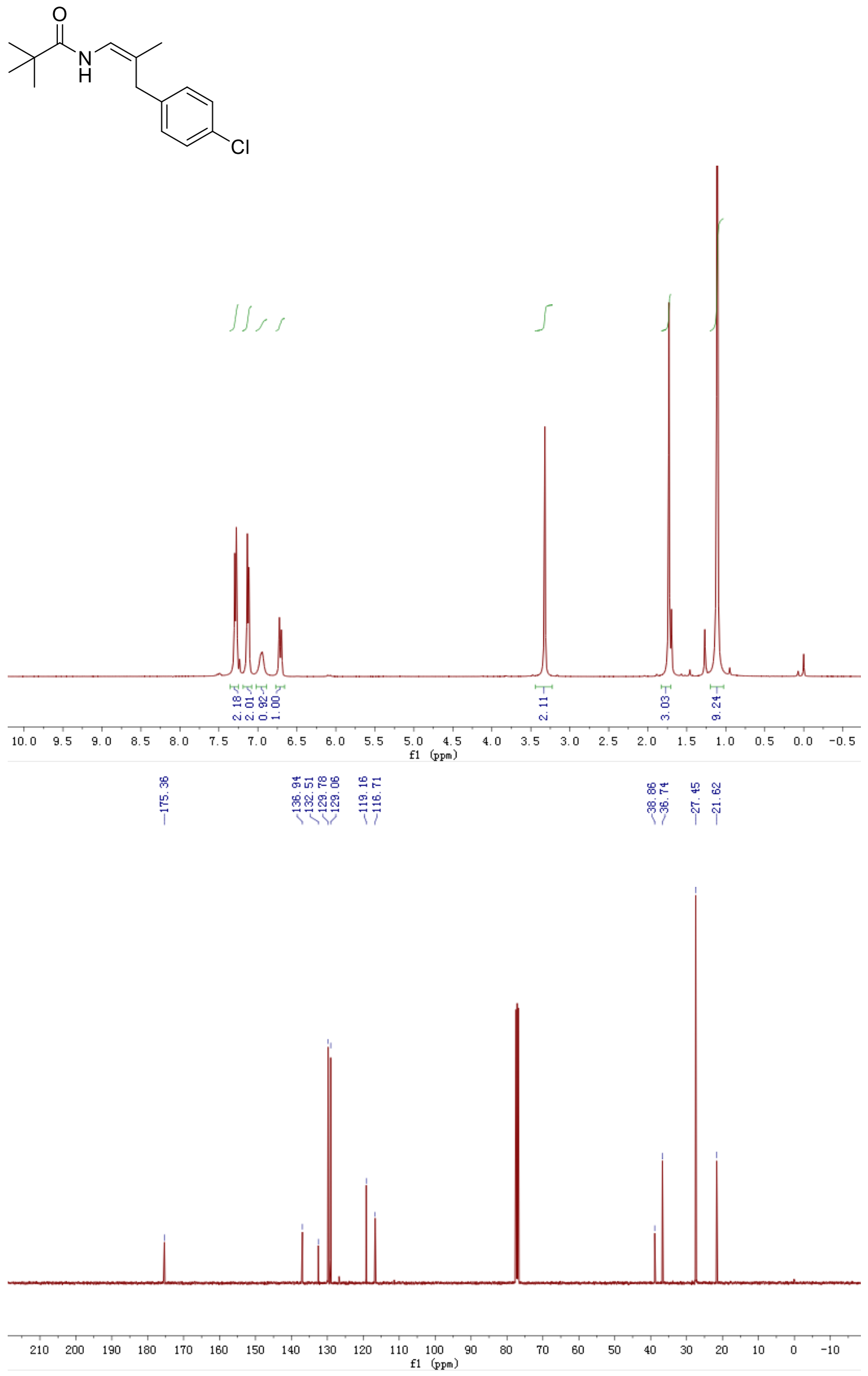

S187 
<smiles>CCCC(=CNC(=O)c1ccccc1)Cc1ccccc1</smiles>
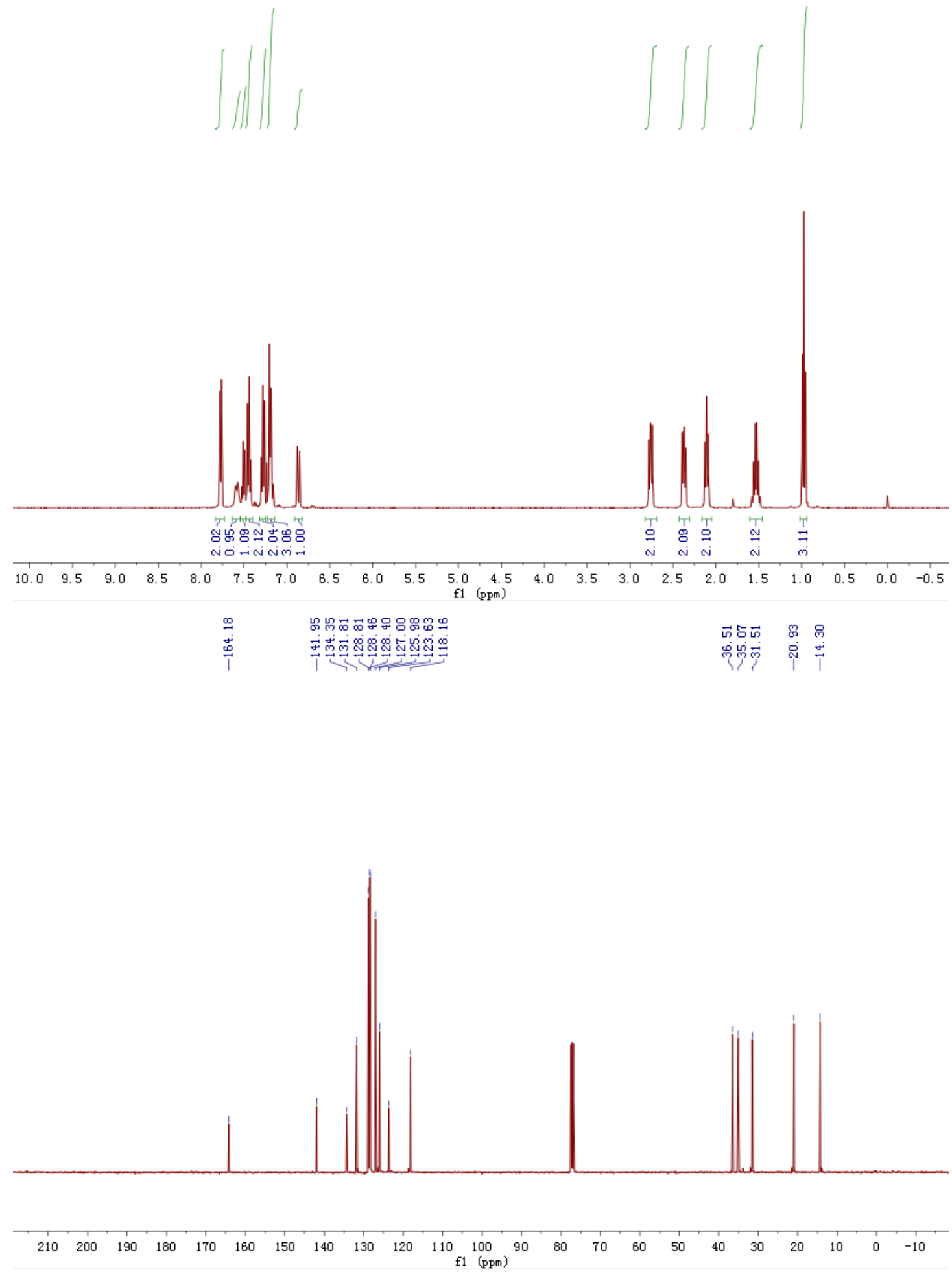


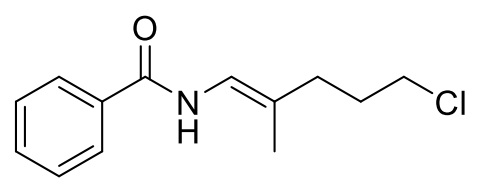

$\iint$
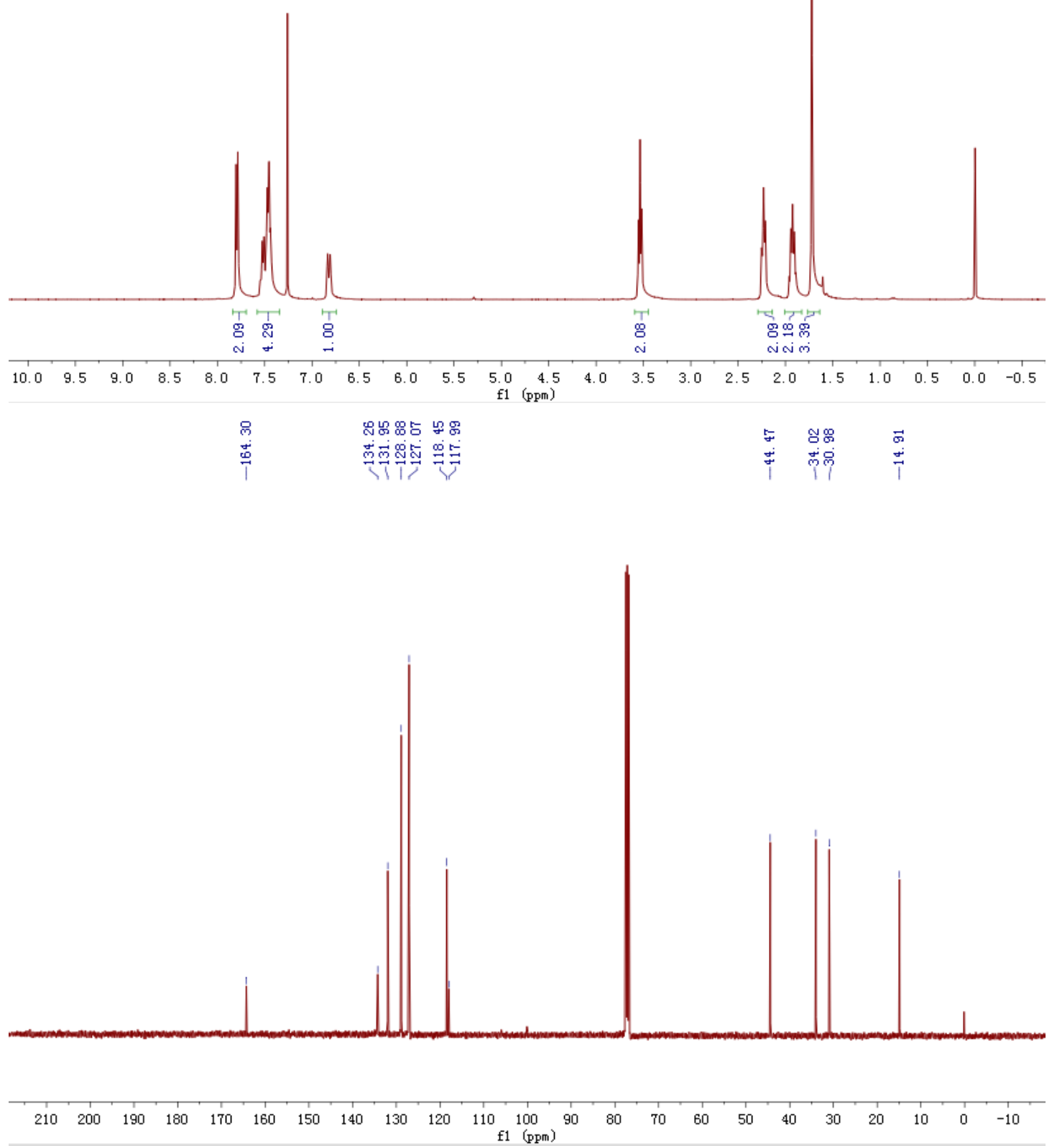

S189 


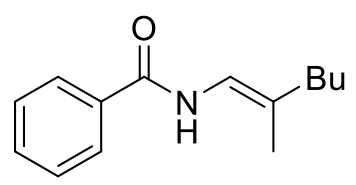

$\iint$
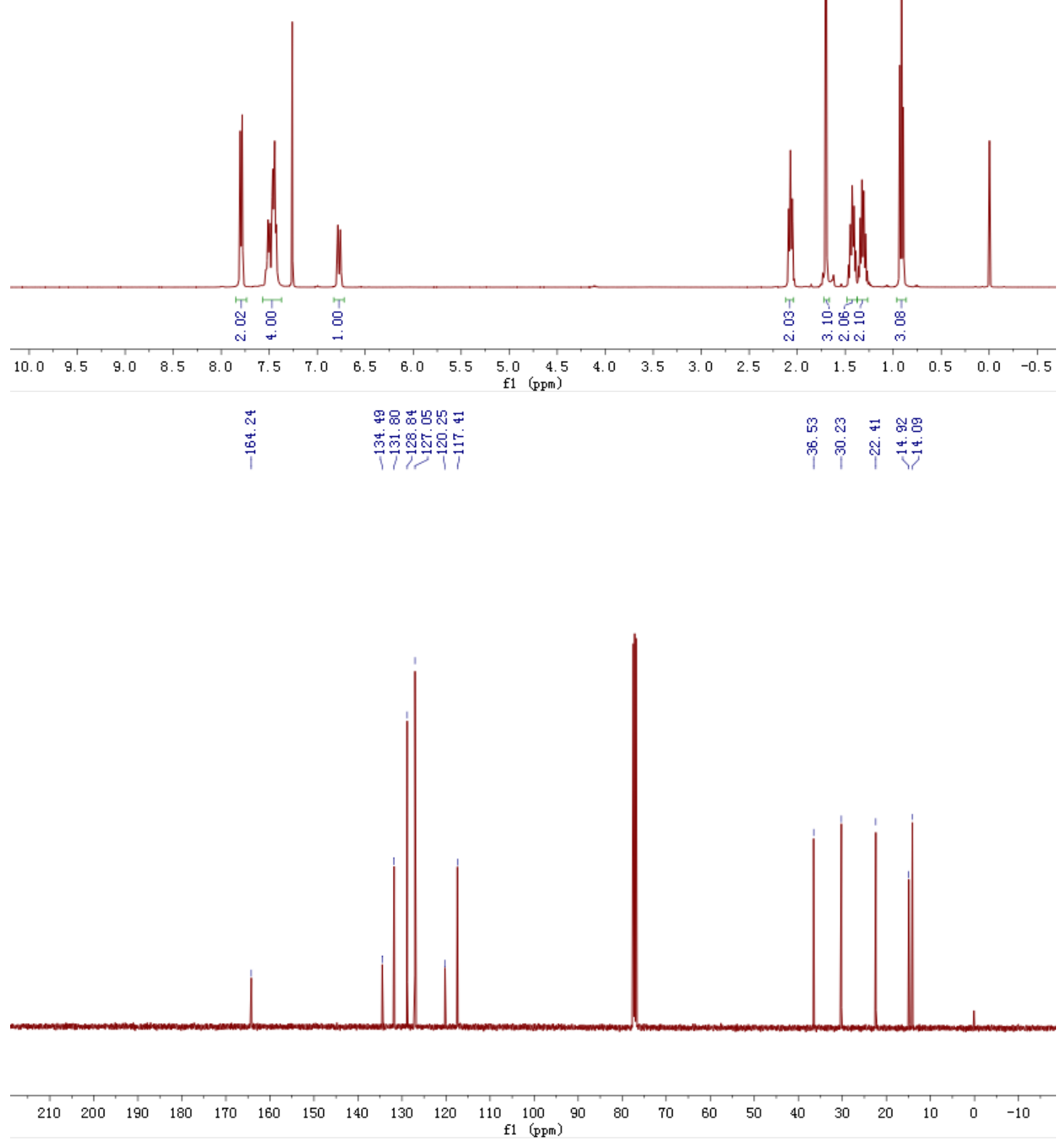

S190 

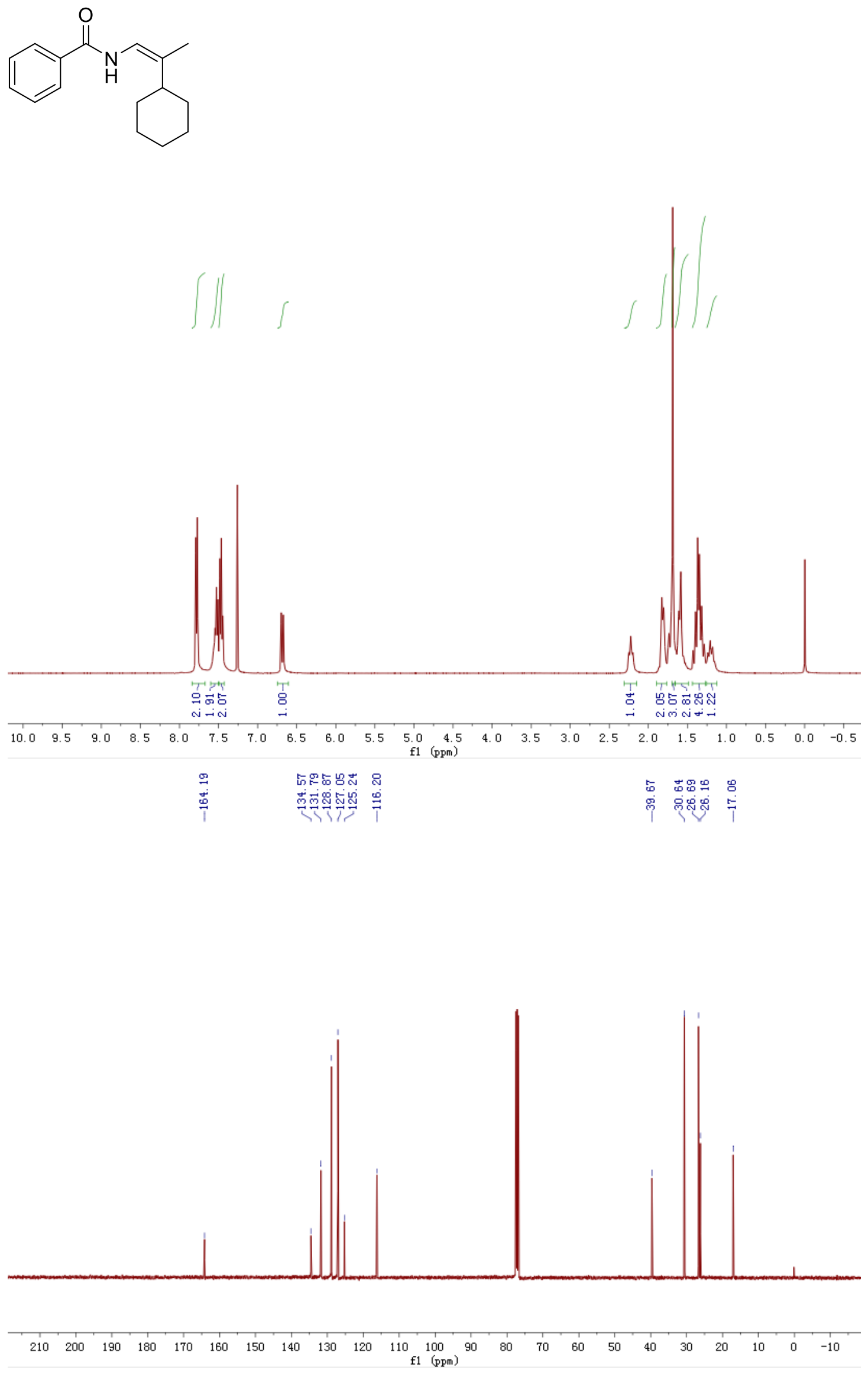

S191 
<smiles>O=C(NC=C1CCOCC1)c1ccccc1</smiles>
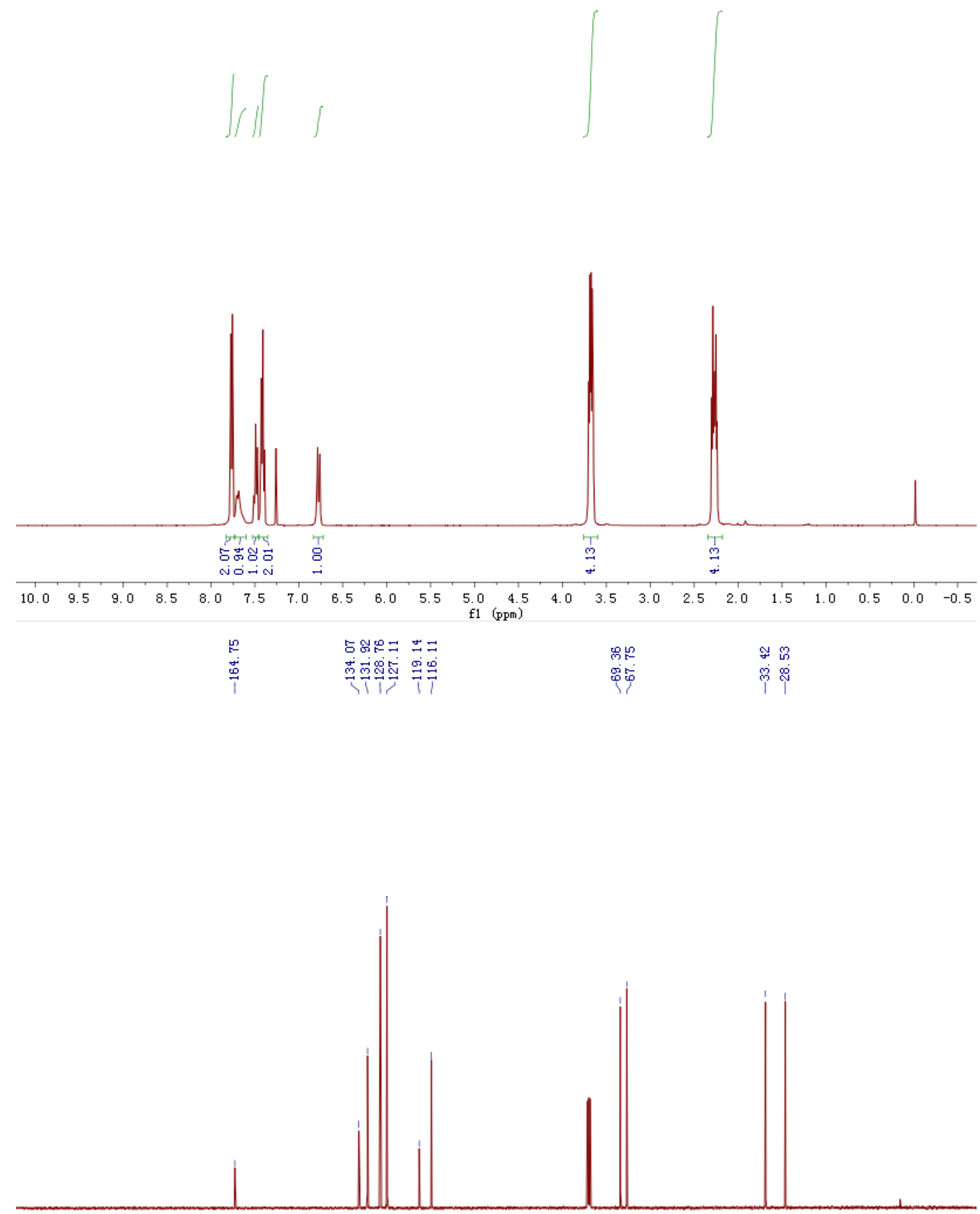

$\begin{array}{lllllllllllllllllllllll}210 & 200 & 190 & 180 & 170 & 160 & 150 & 140 & 130 & 120 & 110 & 100 & 90 & 80 & 70 & 60 & 50 & 40 & 30 & 20 & 10 & 0 & -10\end{array}$ 
<smiles>O=C(NC=C1CCCCC1)c1ccccc1</smiles>
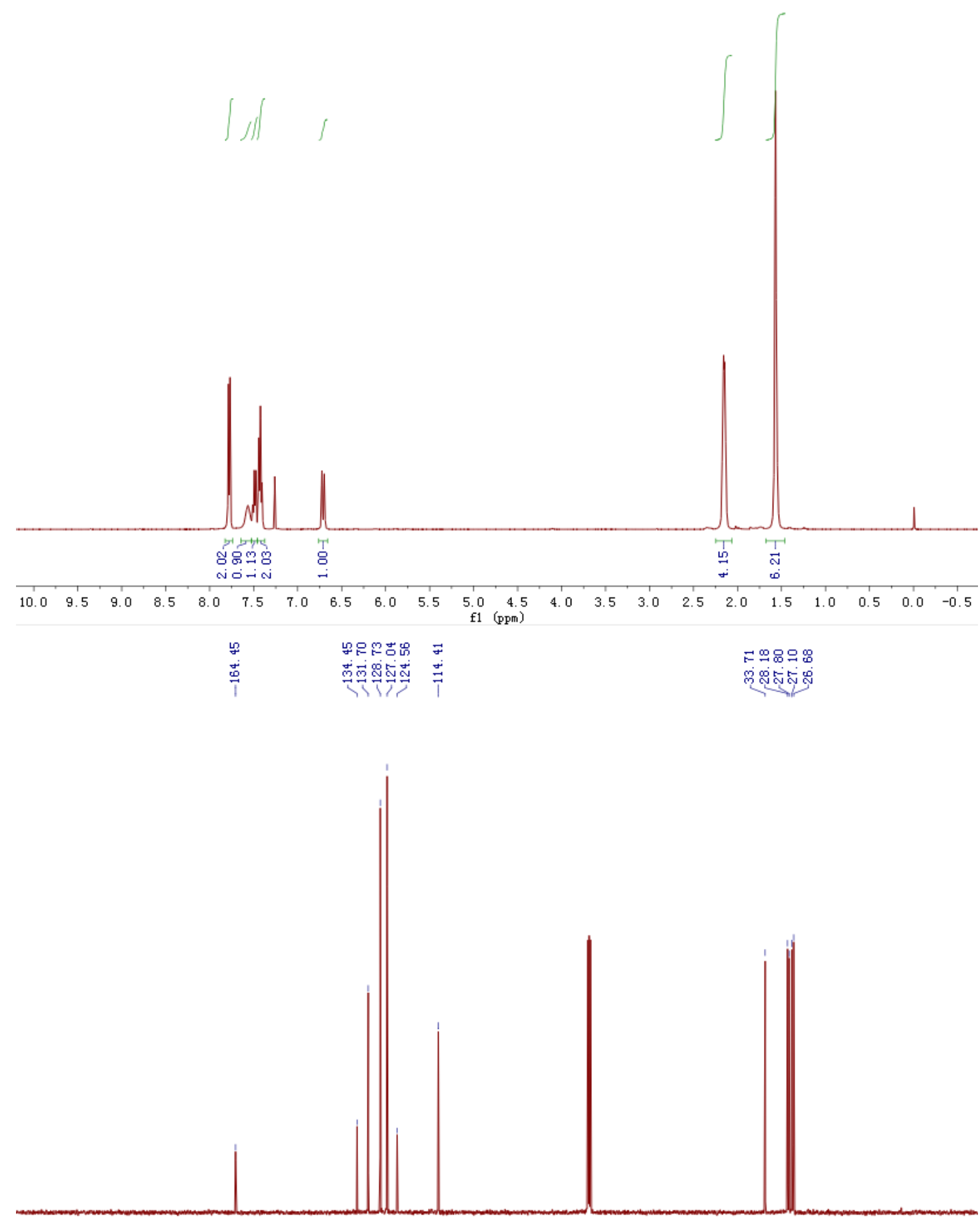

$\begin{array}{lllllllllllllllllllllllll}210 & 200 & 190 & 180 & 170 & 160 & 150 & 140 & 130 & 120 & 110 & 100 & 90 & 80 & 70 & 60 & 50 & 40 & 30 & 20 & 10 & 0 & -10\end{array}$ f1 (ppm) 

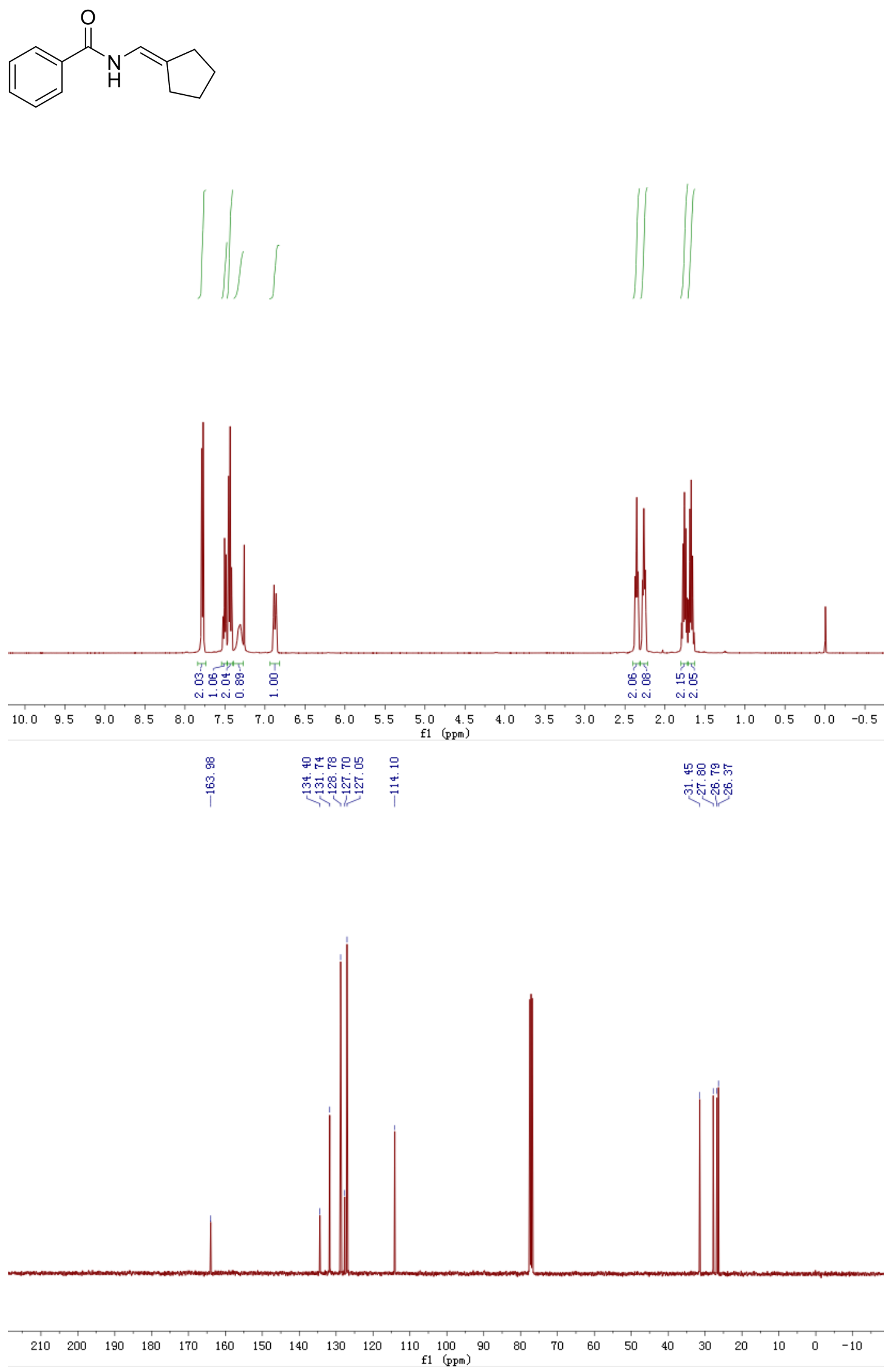

S194 
<smiles>CC(C)=CNC(=O)c1ccc(Cl)cc1</smiles>
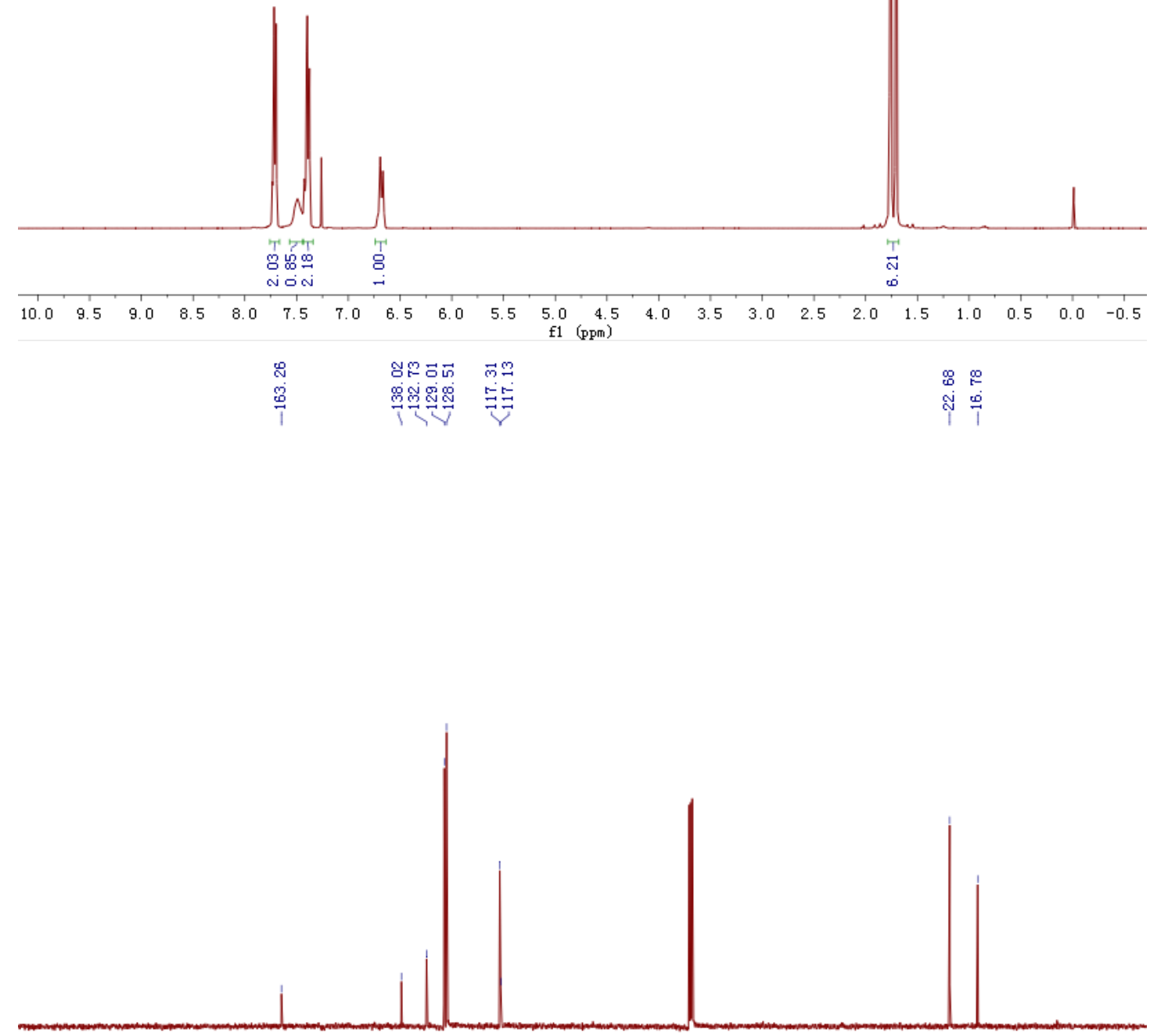

$\begin{array}{lllllllllllllllllllllllll}210 & 200 & 190 & 180 & 170 & 160 & 150 & 140 & 130 & 120 & 110 & 100 & 90 & 80 & 70 & 60 & 50 & 40 & 30 & 20 & 10 & 0 & -10\end{array}$ f1 (ppm) 
<smiles>C/C(=C/NC(=O)C(C)(C)C)CBr</smiles>
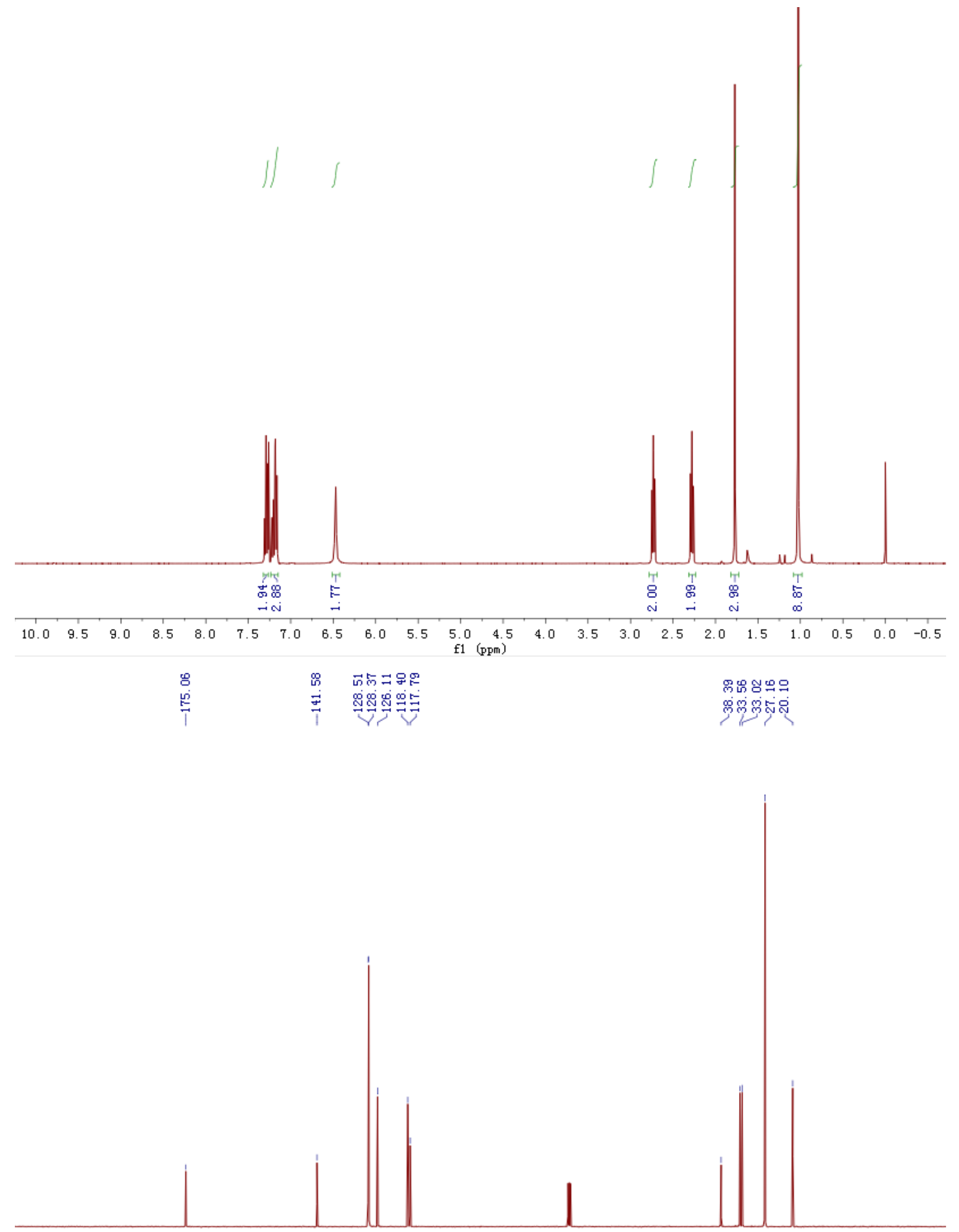

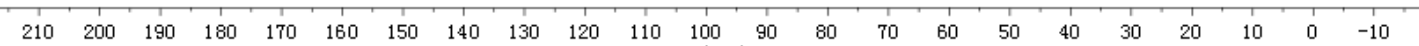


<smiles>CC(C)(C)C(=O)NC(CCCc1ccccc1)CCc1ccccc1</smiles>
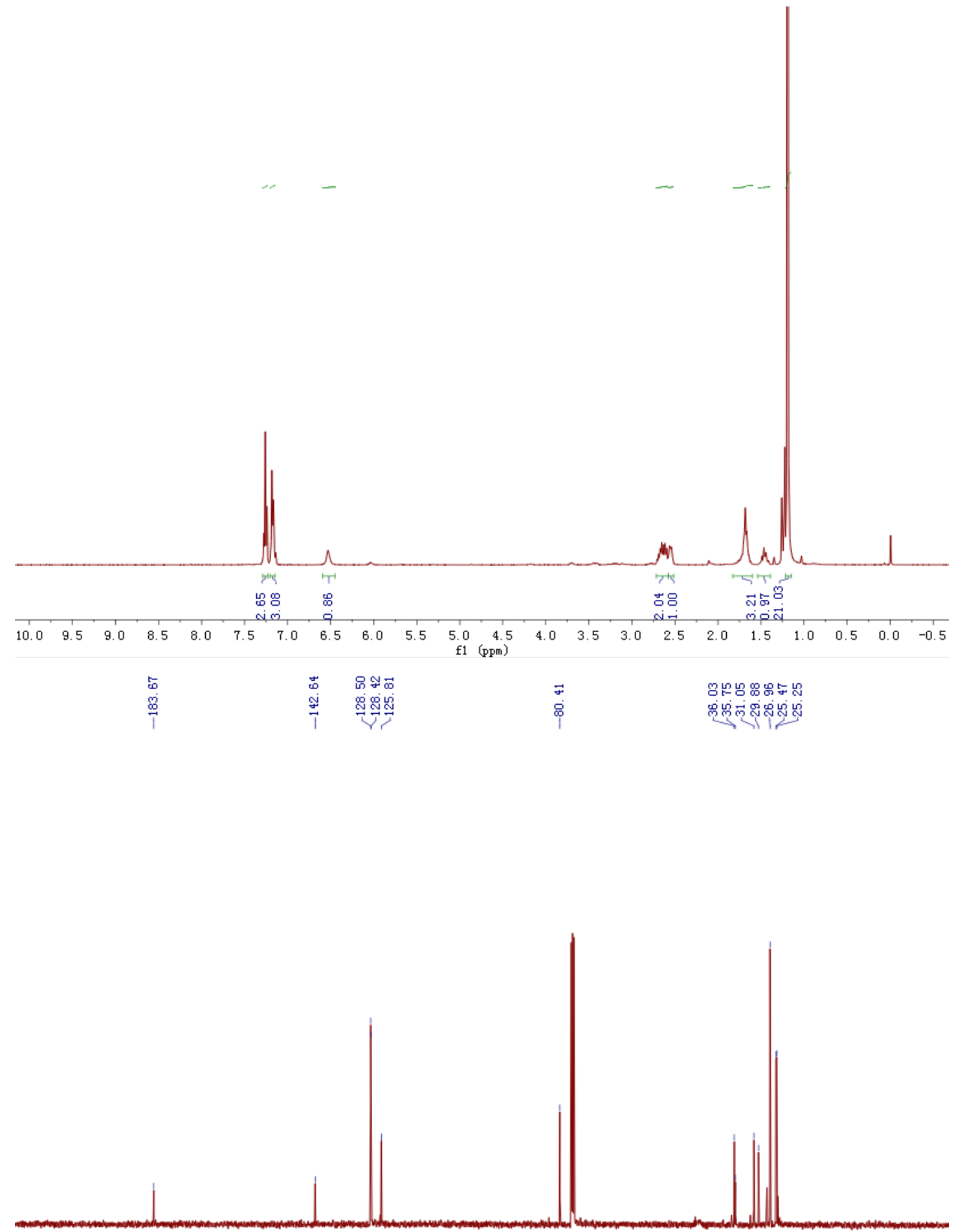

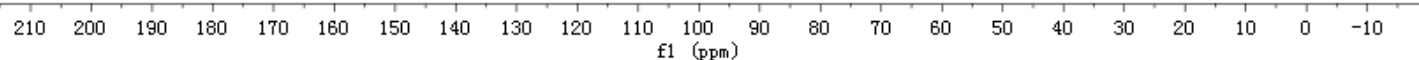


<smiles>CC(C)(C)C(=O)NCC(CCc1ccccc1)Cc1ccccc1</smiles>

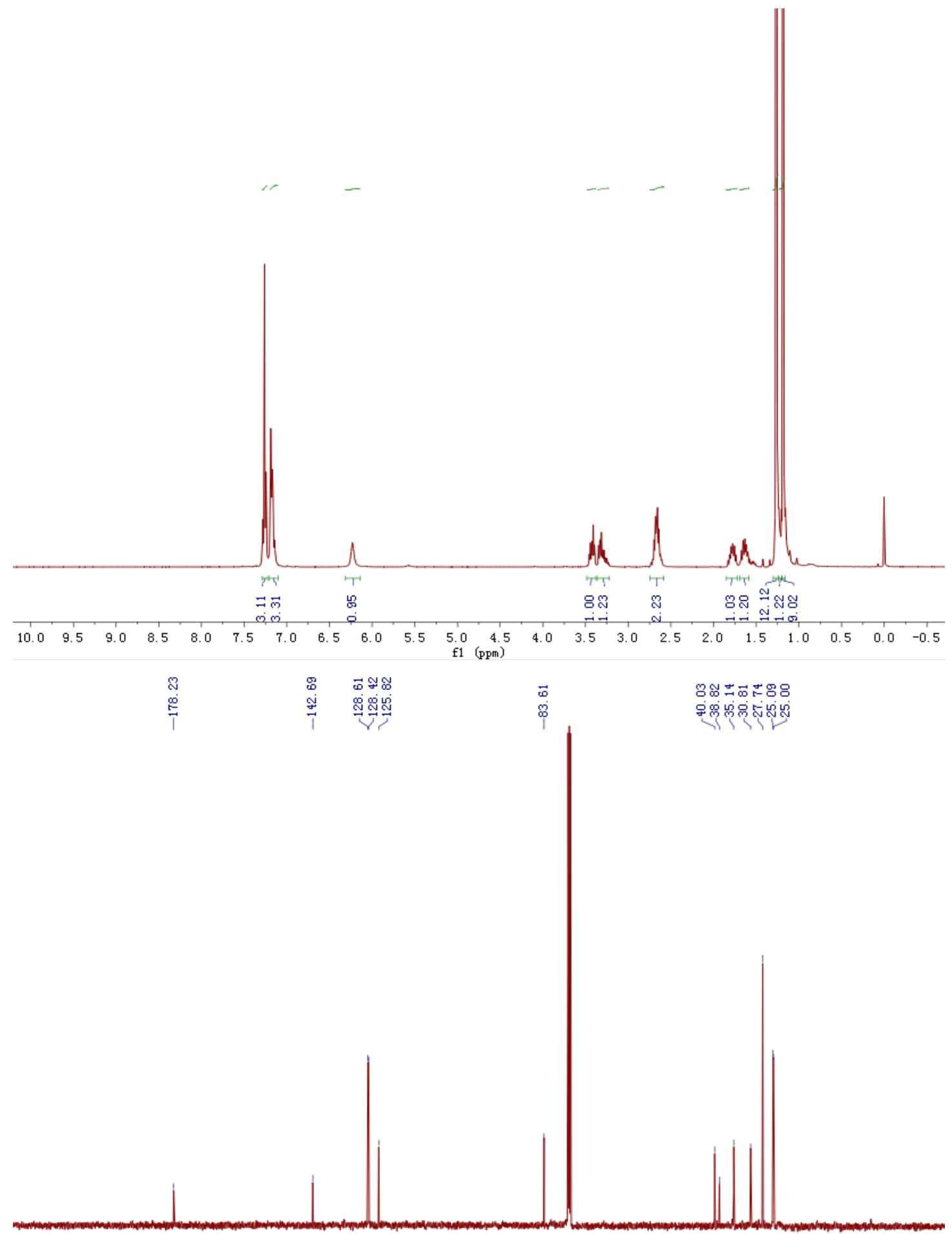

$\begin{array}{lllllllllllllllllllllllllll}210 & 200 & 190 & 180 & 170 & 160 & 150 & 140 & 130 & 120 & 110 & 100 & 90 & 80 & 70 & 60 & 50 & 40 & 30 & 20 & 10 & 0 & -10\end{array}$ f1 $(\mathrm{ppm})$ 
(N)

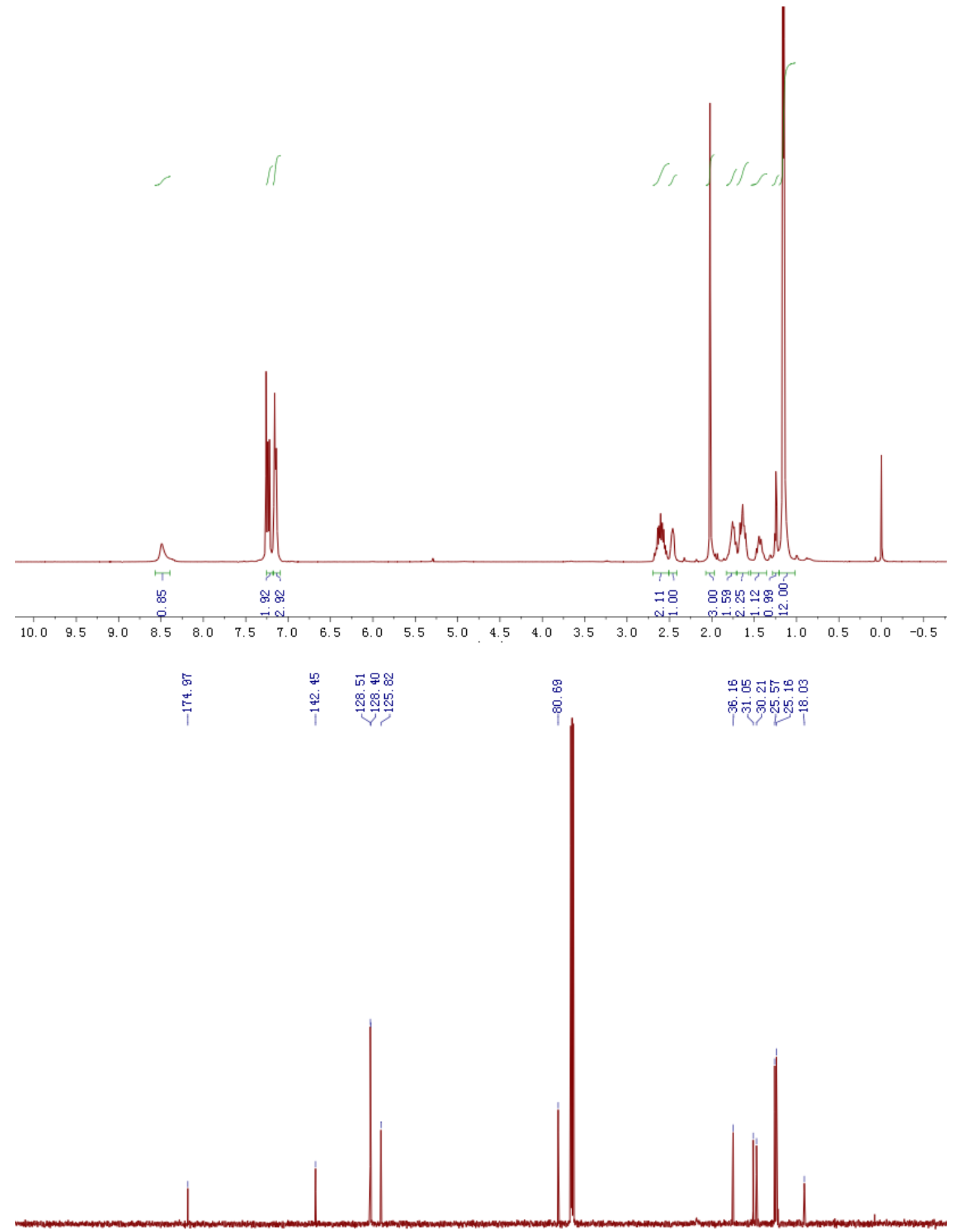

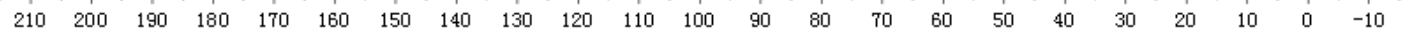


<smiles>CC(=O)NCC(CCc1ccccc1)Cc1ccccc1</smiles>

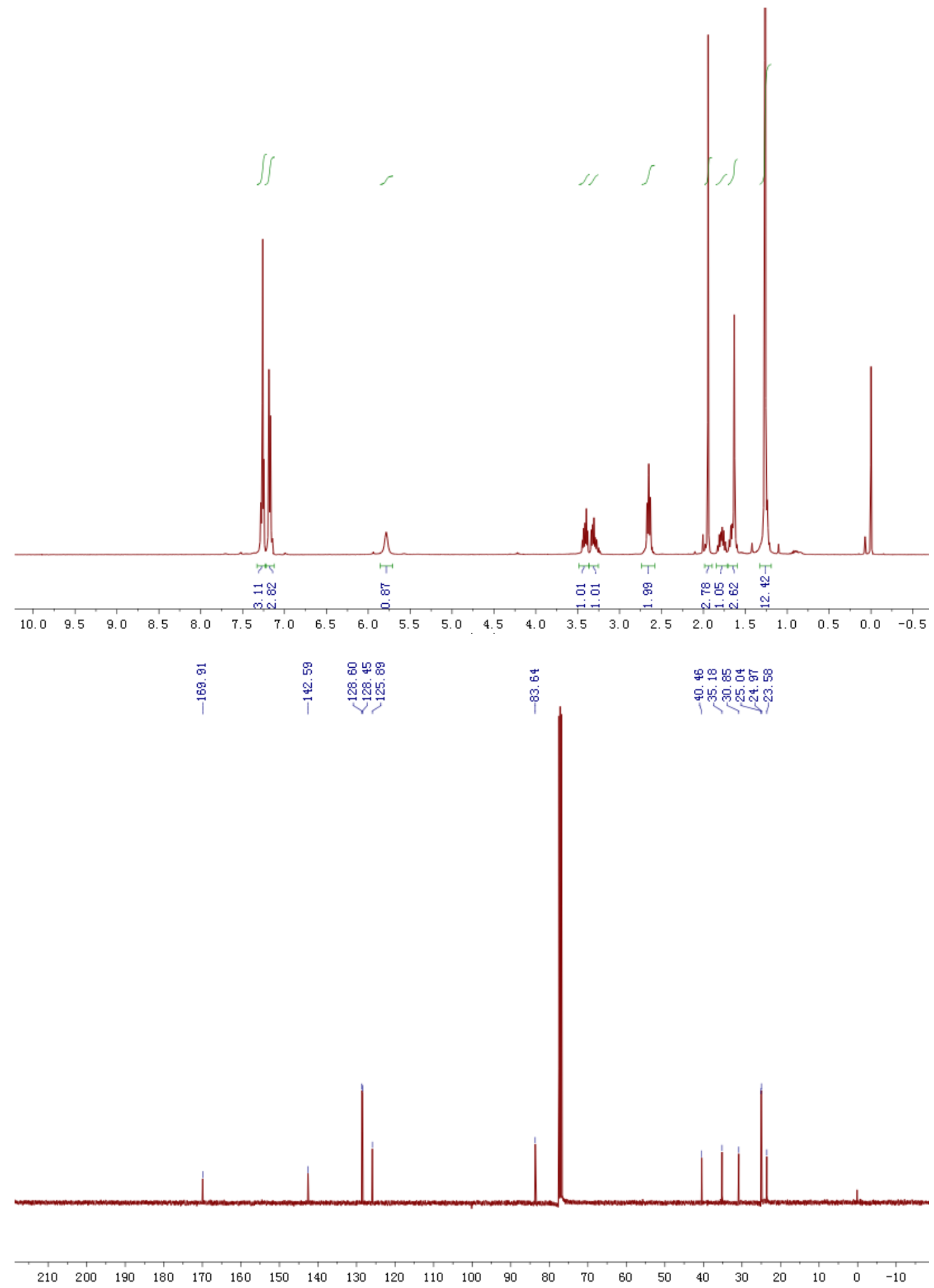


<smiles>CC(C)C(=O)NC(CCCc1ccccc1)Cc1ccccc1</smiles>

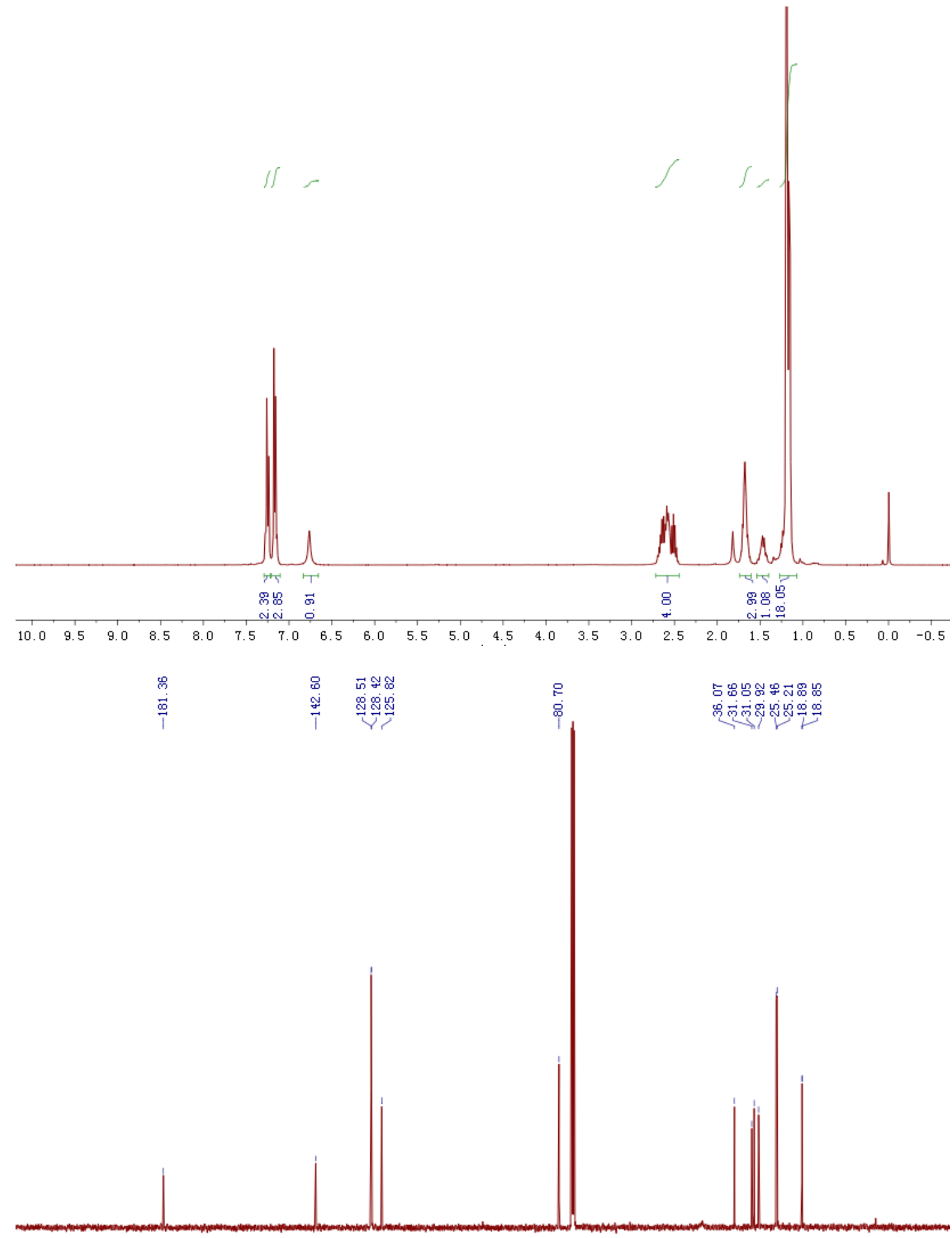

$\begin{array}{llllllllllllllllllllllll}210 & 200 & 190 & 180 & 170 & 160 & 150 & 140 & 130 & 120 & 110 & 100 & 90 & 80 & 70 & 60 & 50 & 40 & 30 & 20 & 10 & 0 & -10\end{array}$ 
<smiles>CC(C)C(=O)NCC(CCc1ccccc1)Cc1ccccc1</smiles>
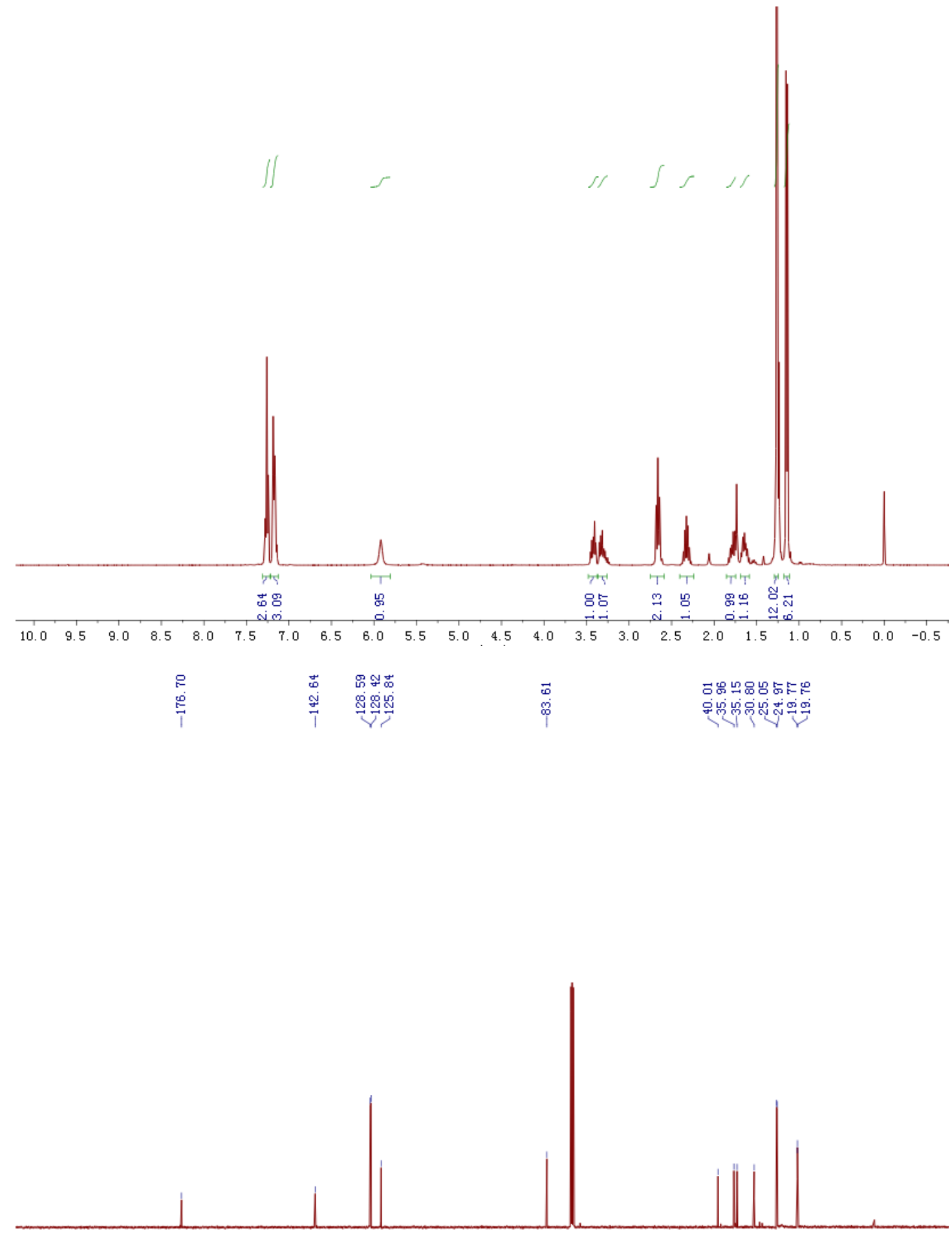

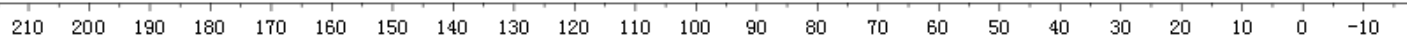


<smiles>O=C(NC(CCCc1ccccc1)Cc1ccccc1)c1ccccc1</smiles>

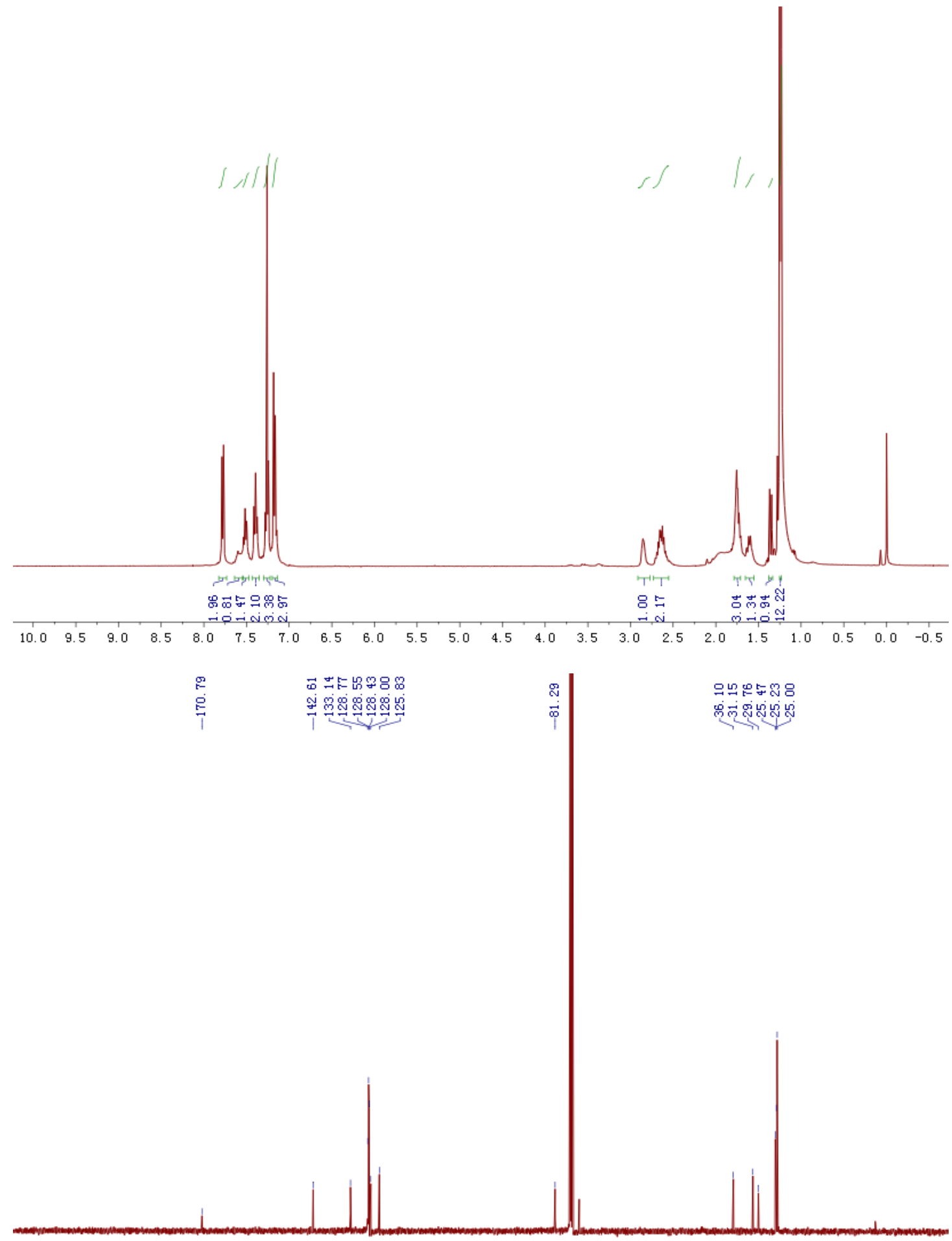

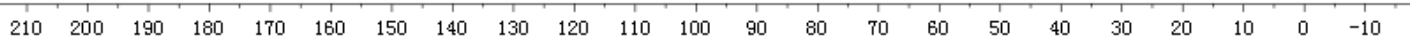


<smiles>O=C(NCC(CCc1ccccc1)Cc1ccccc1)c1ccccc1</smiles>

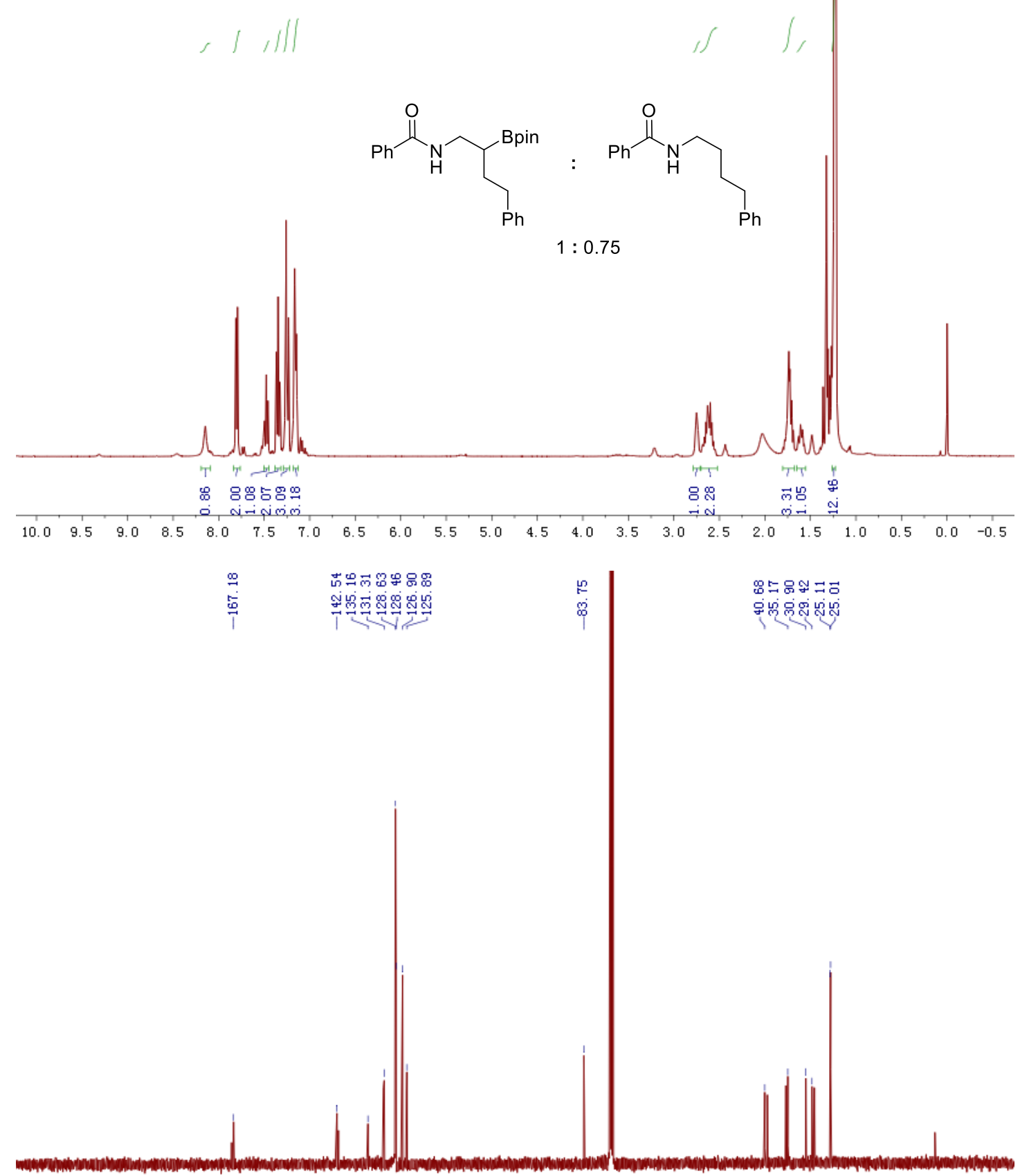

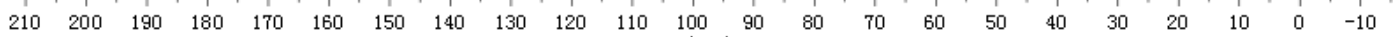


<smiles>CCC(Cc1ccccc1)NC(=O)C(C)(C)C</smiles>
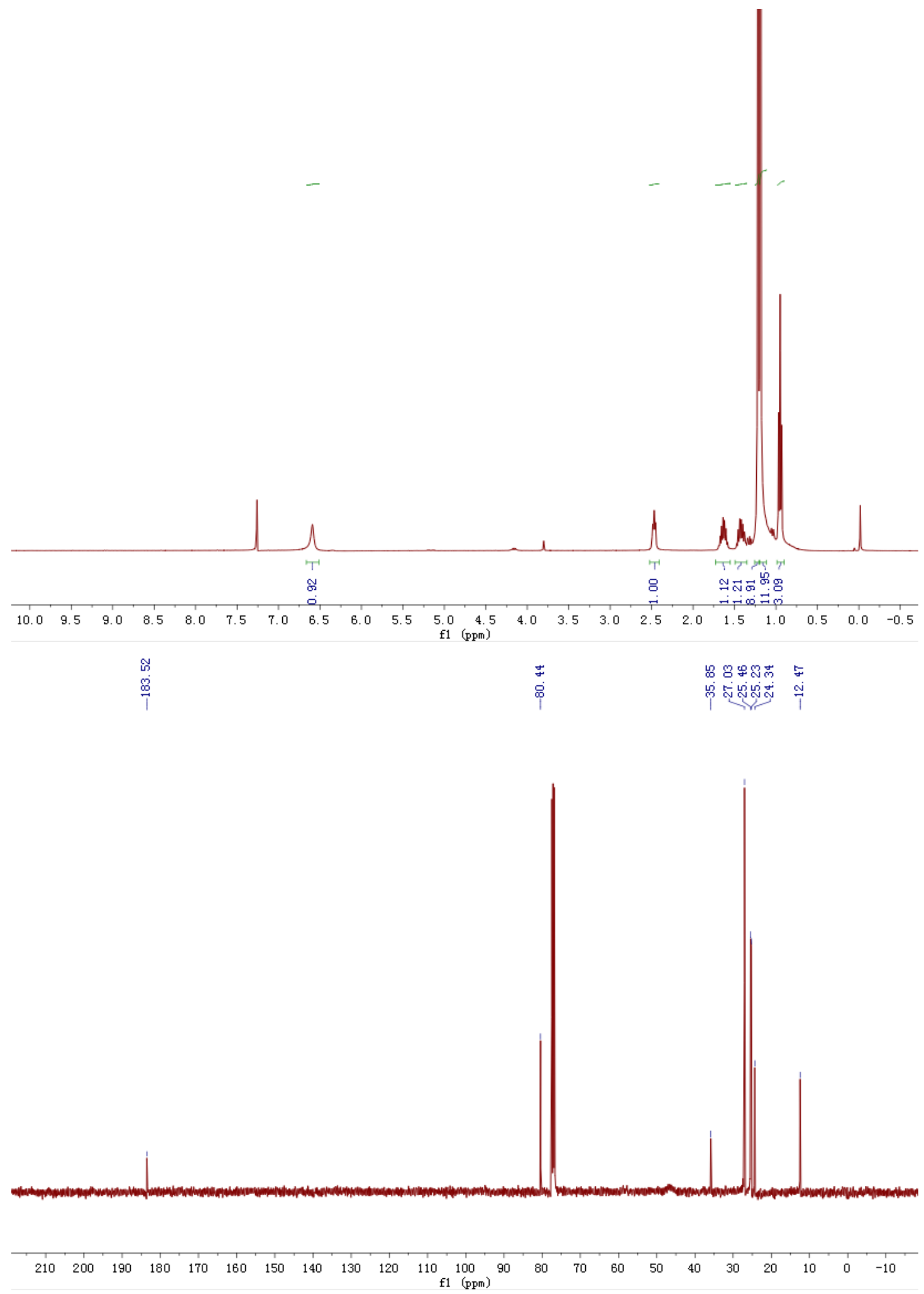

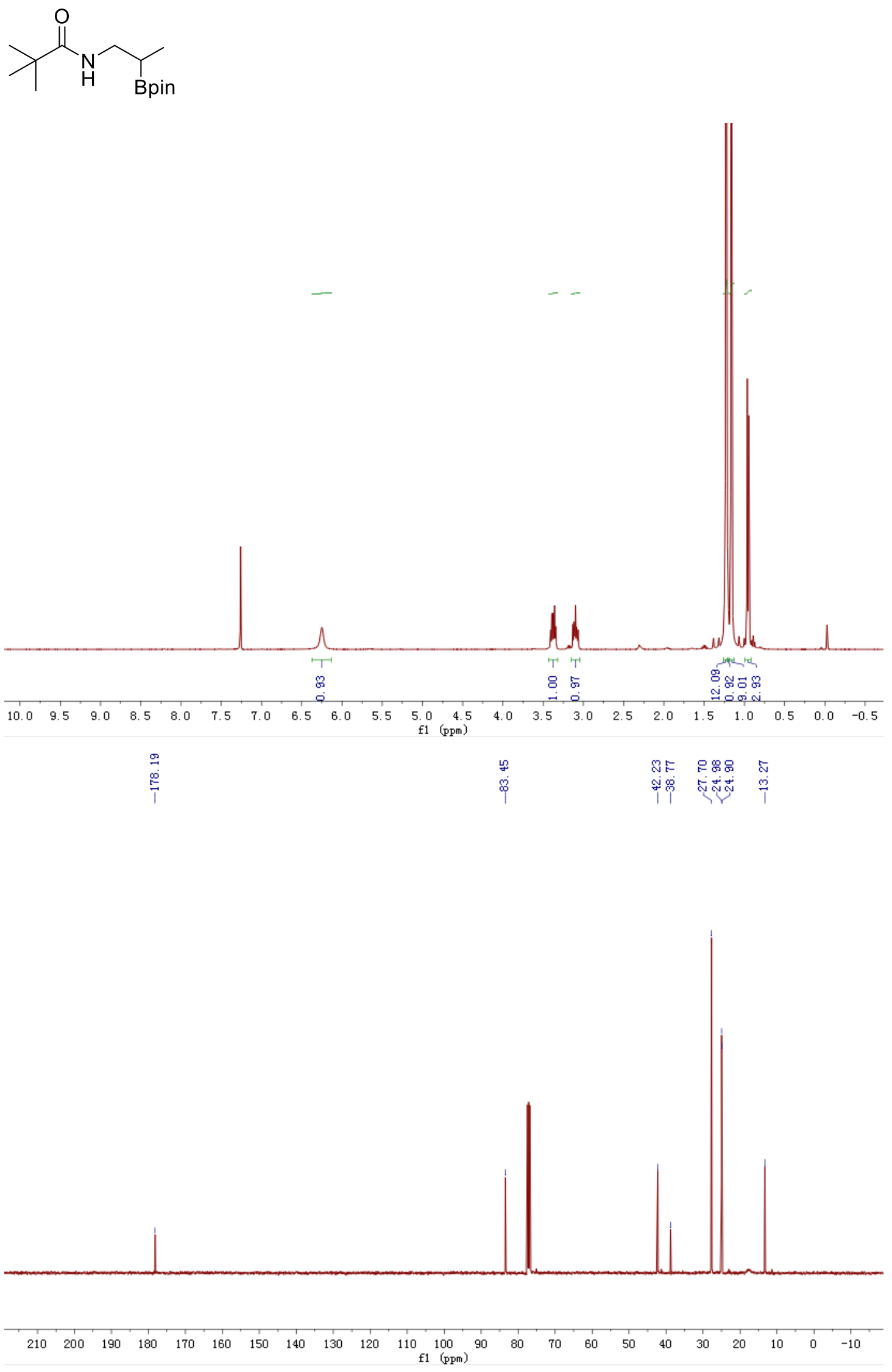

S206 
<smiles>CCCCCC(Cc1ccccc1)NC(=O)C(C)(C)C</smiles>
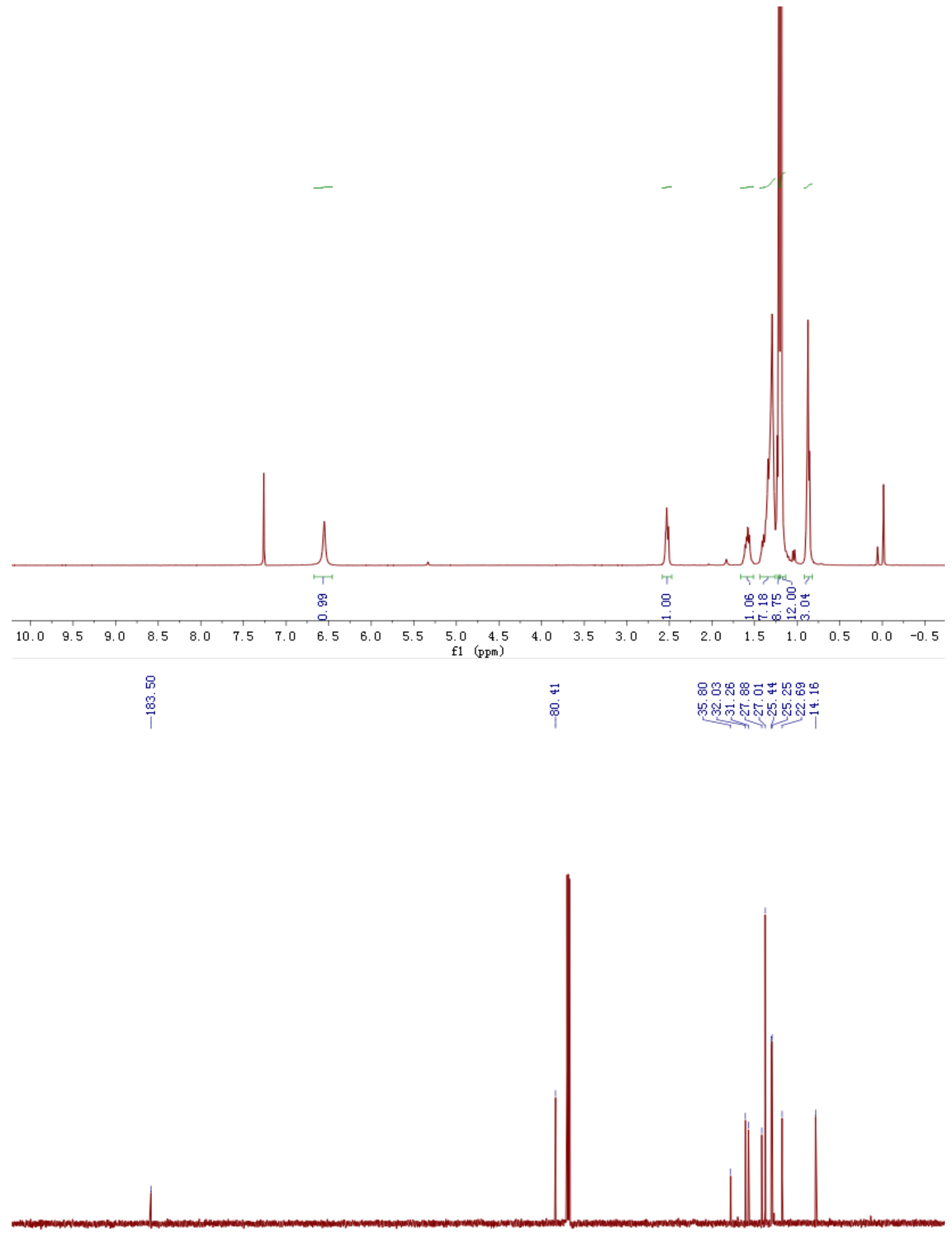

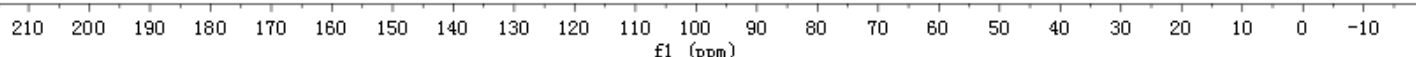


<smiles>CCCCC([Pb])CNC(=O)C(C)(C)C</smiles>
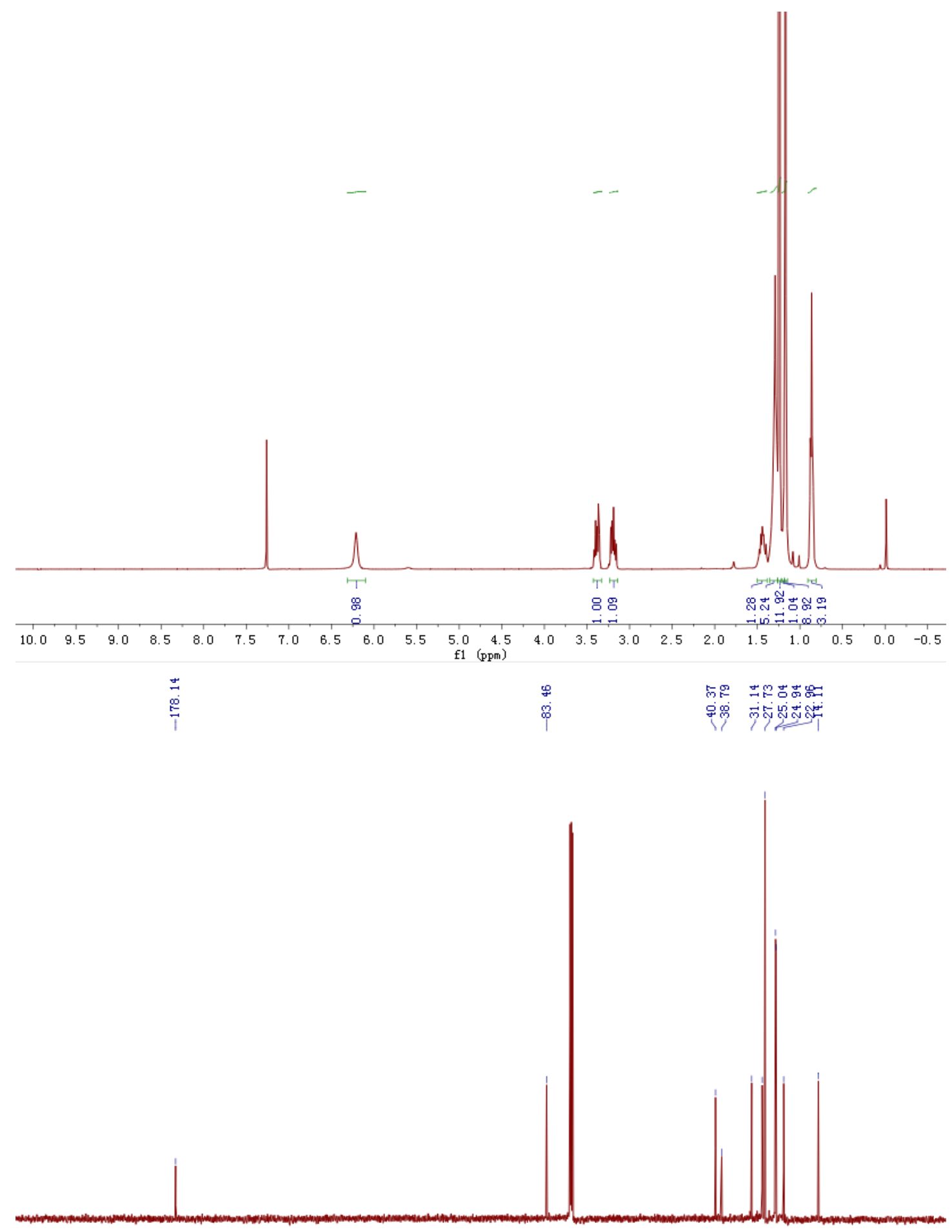

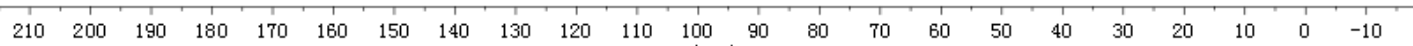
f1 $(\mathrm{ppm})$ 
<smiles>CC(C)CCCC(Cc1ccccc1)NC(=O)C(C)(C)C</smiles>

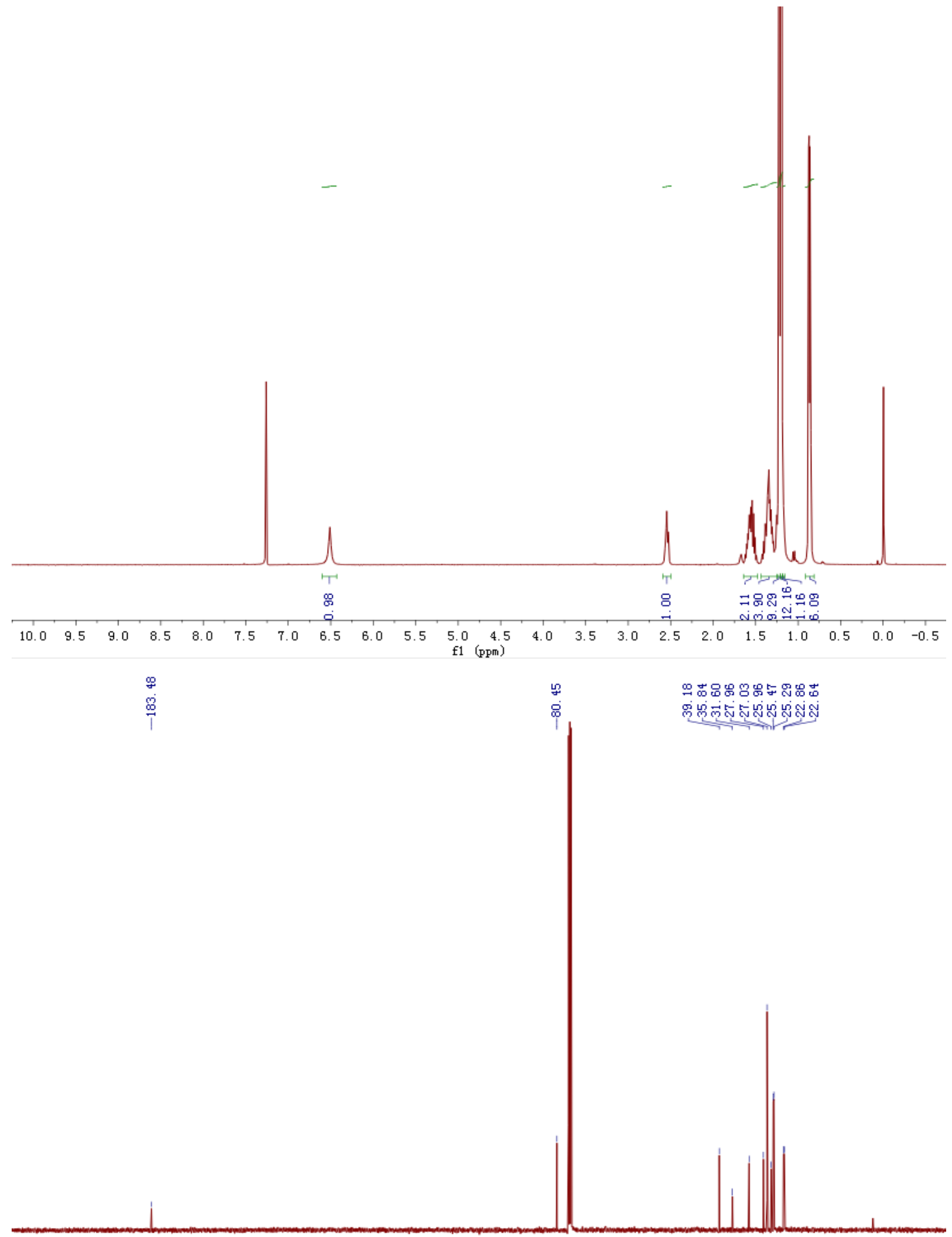

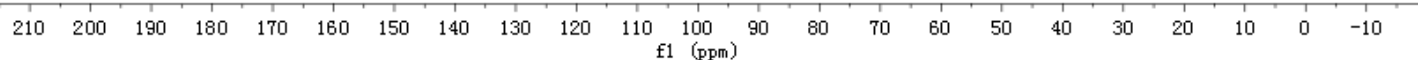


<smiles>CC(C)CCC([18OH])CNC(=O)C(C)(C)C</smiles>
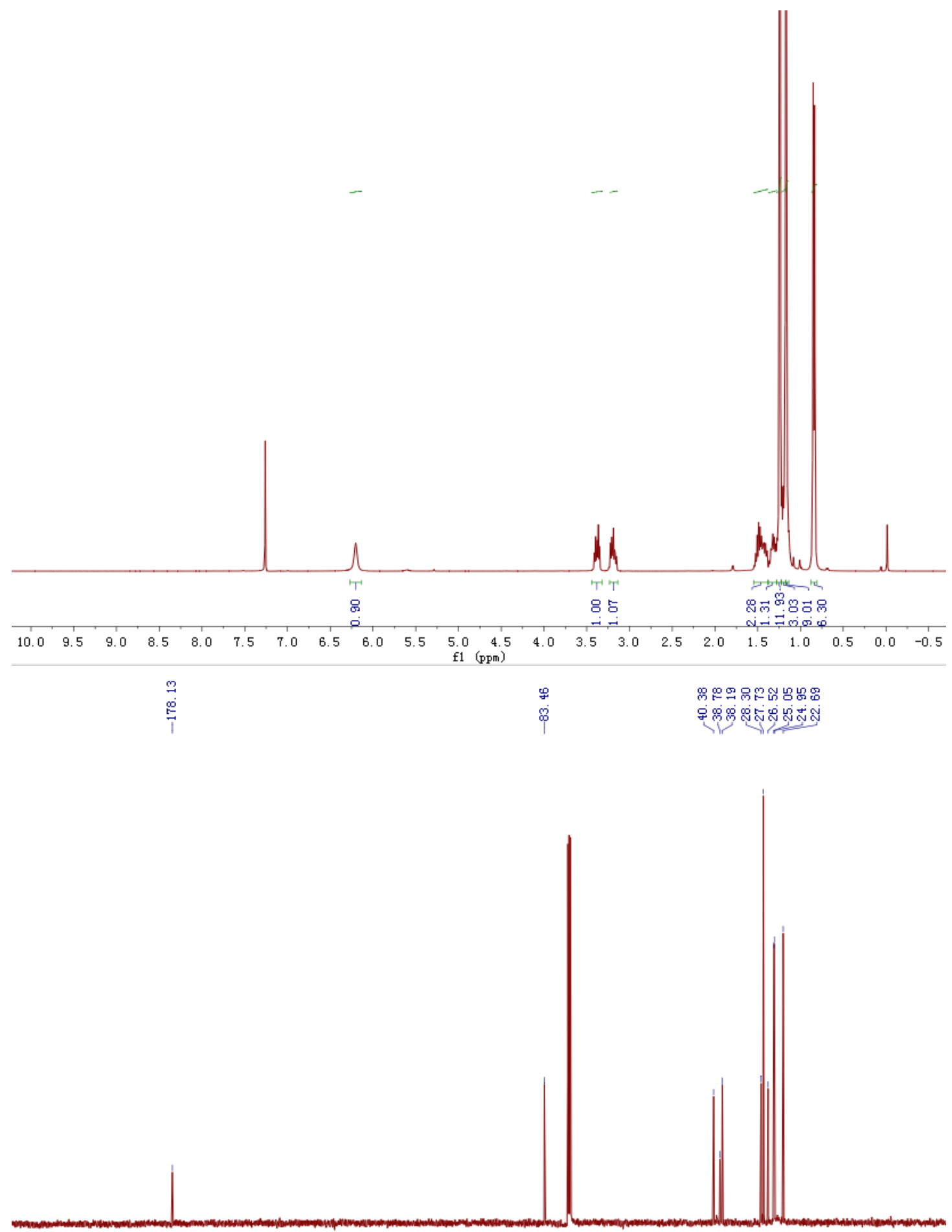

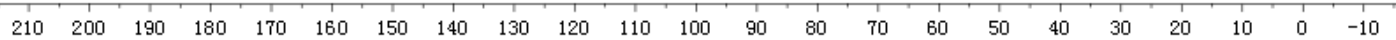
f1 (ppm) 
<smiles>CC(C)(C)C(=O)NC(CCCCCl)Cc1ccccc1</smiles>

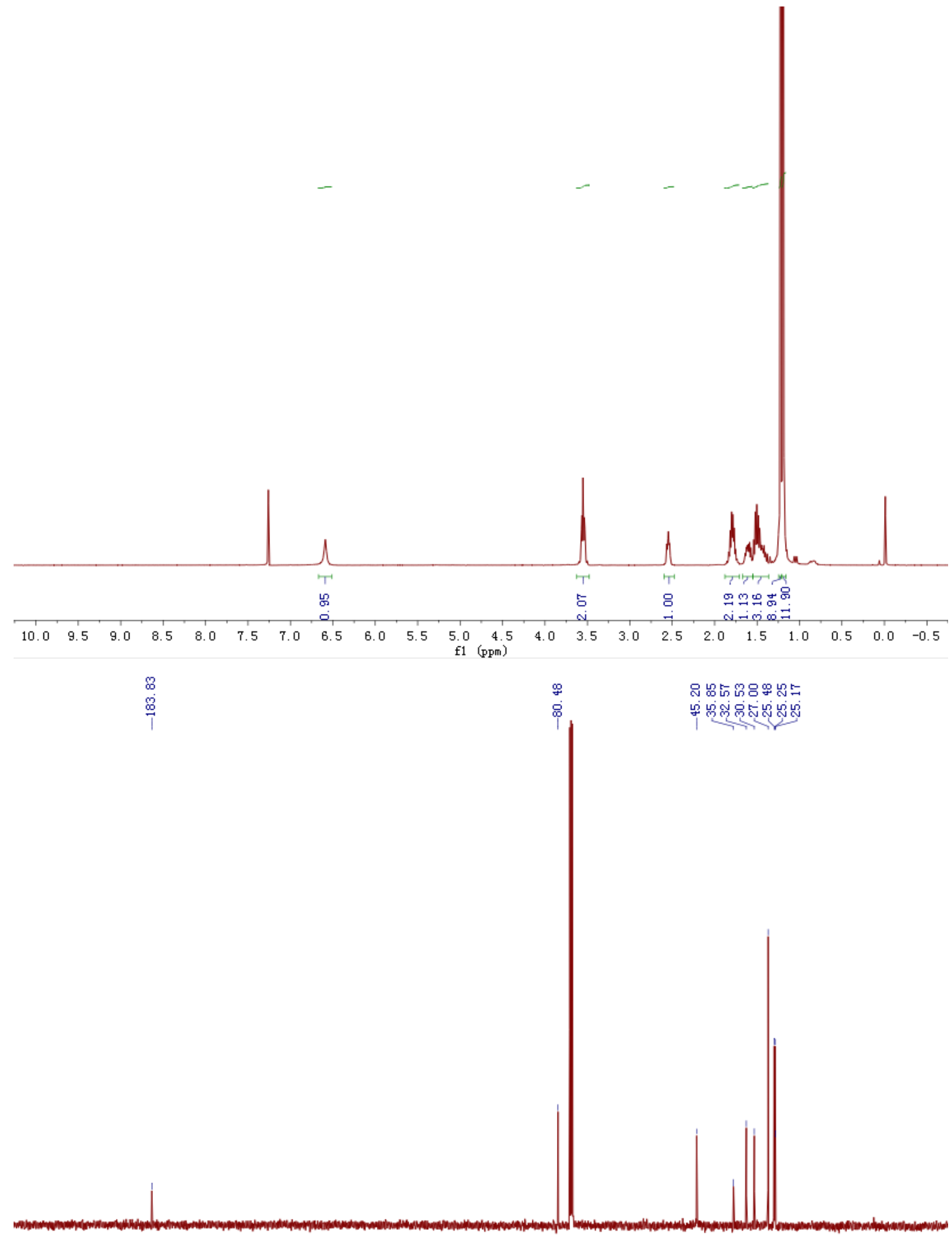

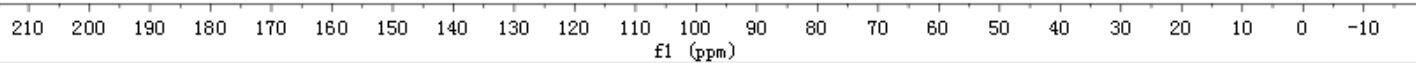


<smiles>CC(C)(C)C(=O)NCC([B]c1ccccc1)CCCCl</smiles>

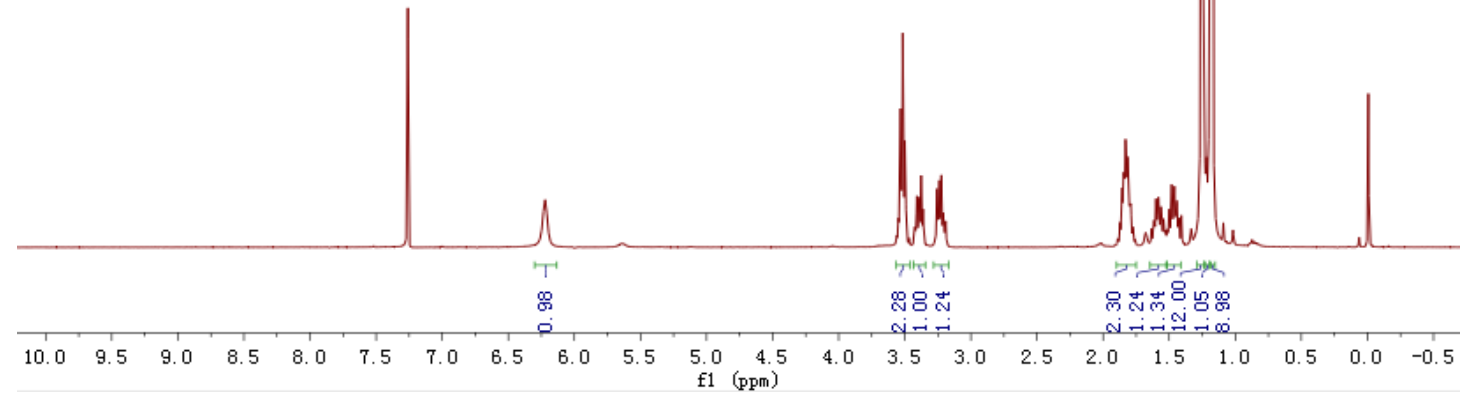

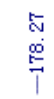

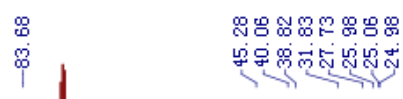

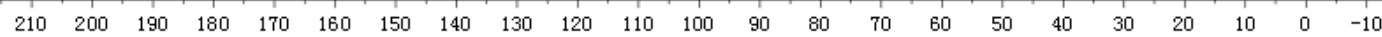
f1 $(\mathrm{ppm})$ 

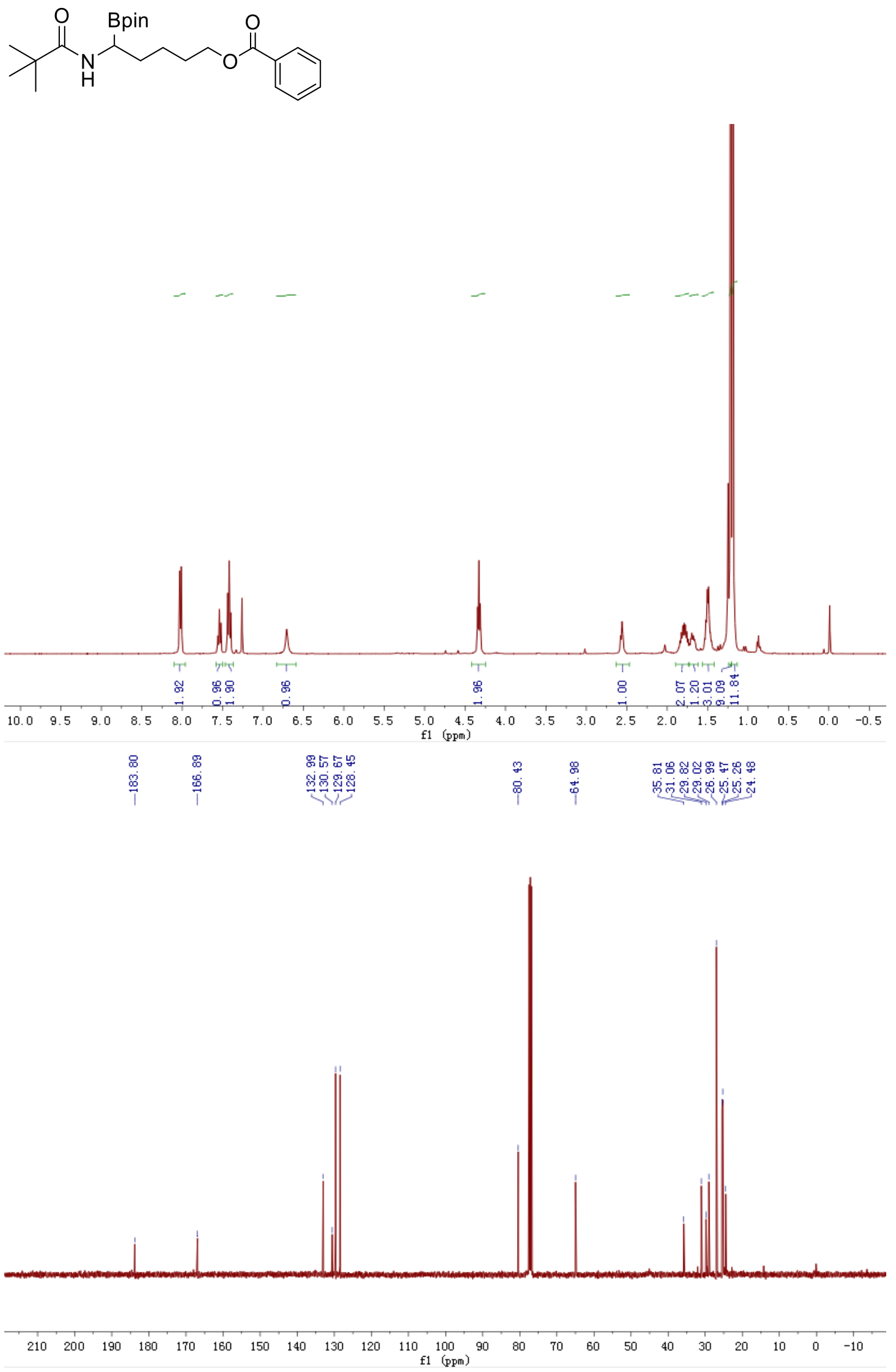

S213 

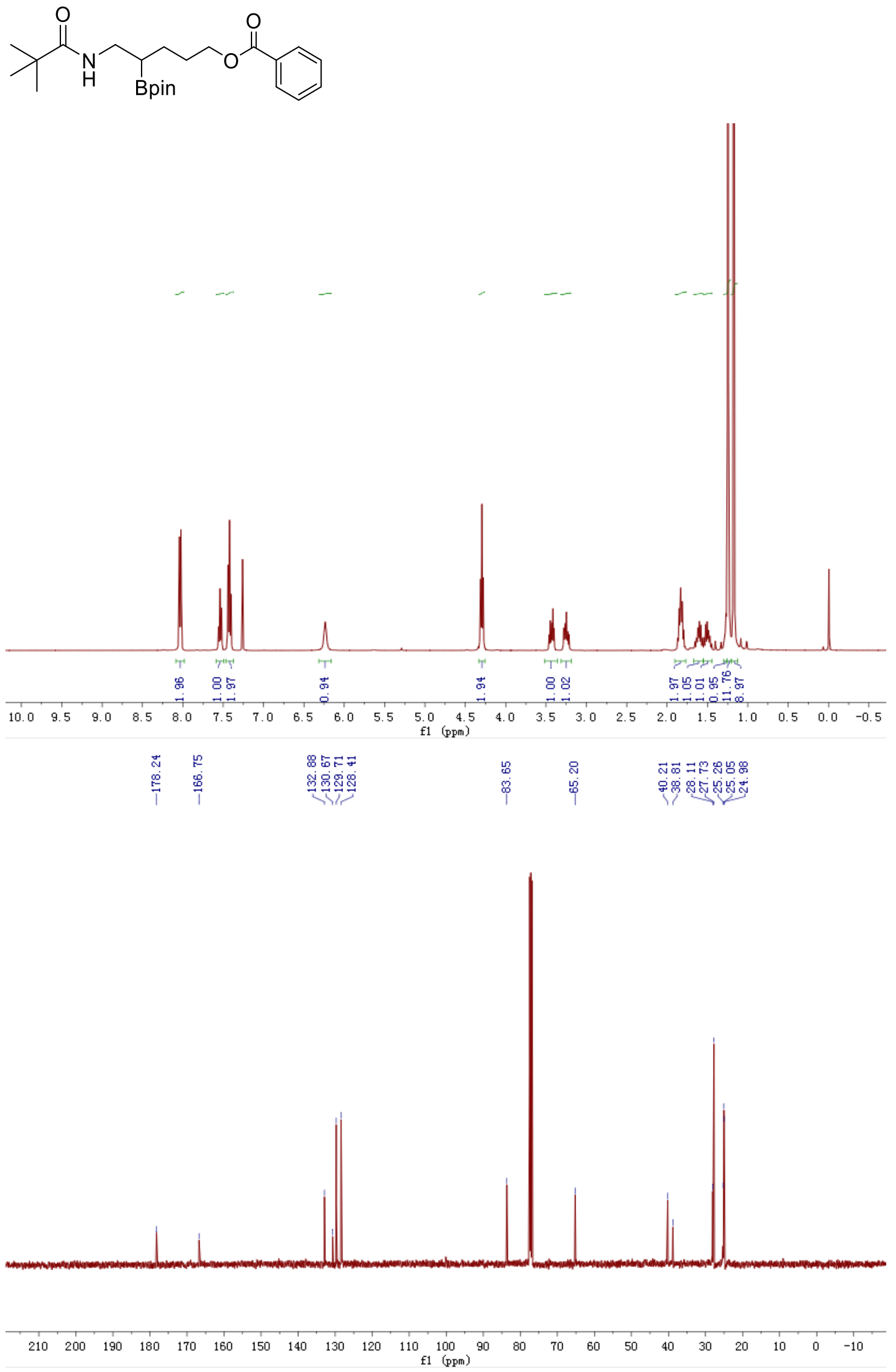

S214 
<smiles>CC(=O)OCCCCC(Cc1ccccc1)NC(=O)C(C)(C)C</smiles>
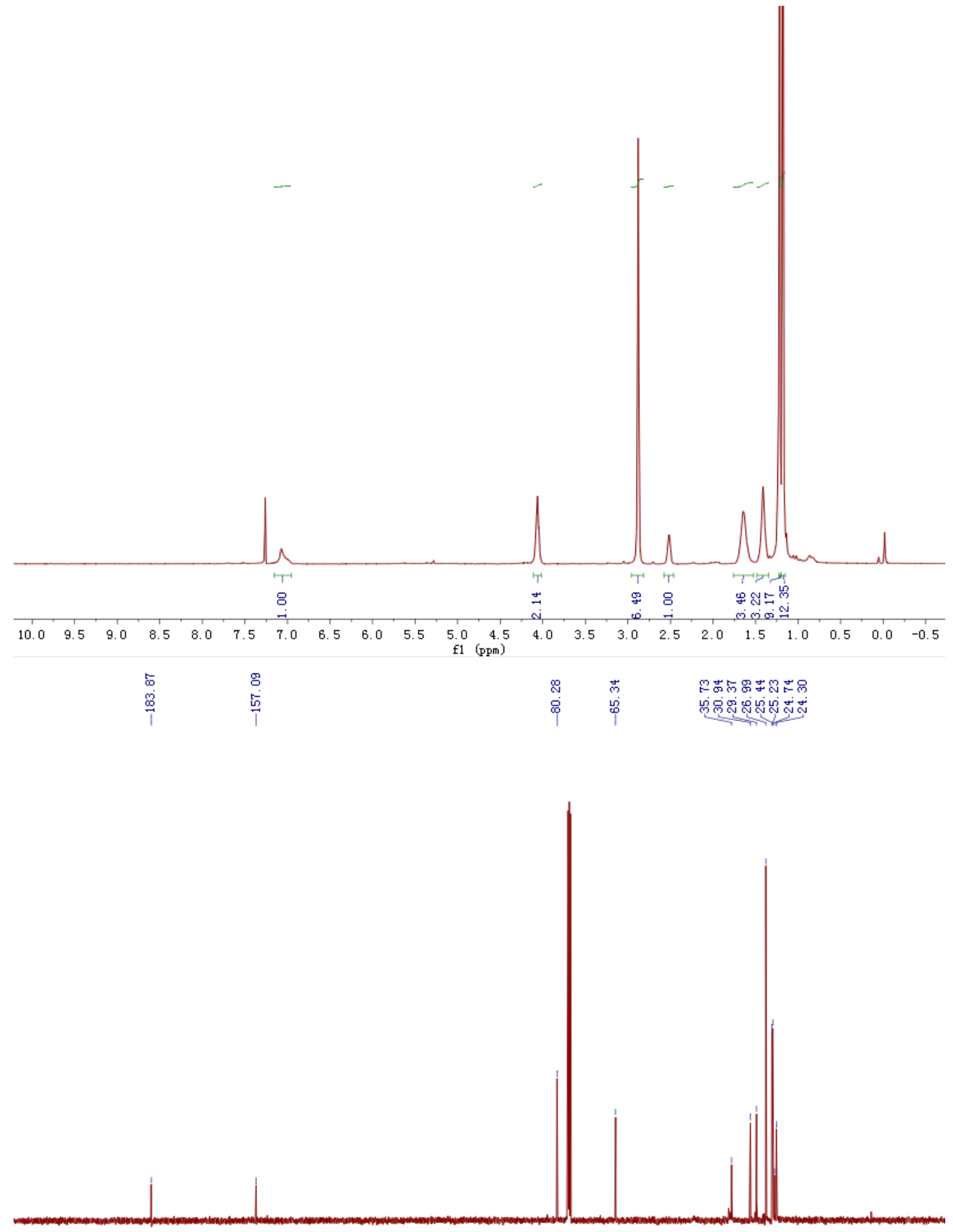

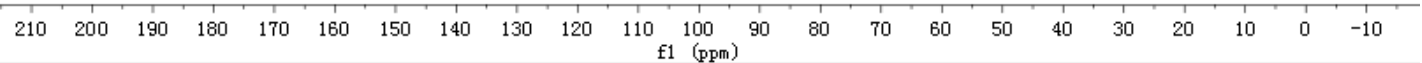



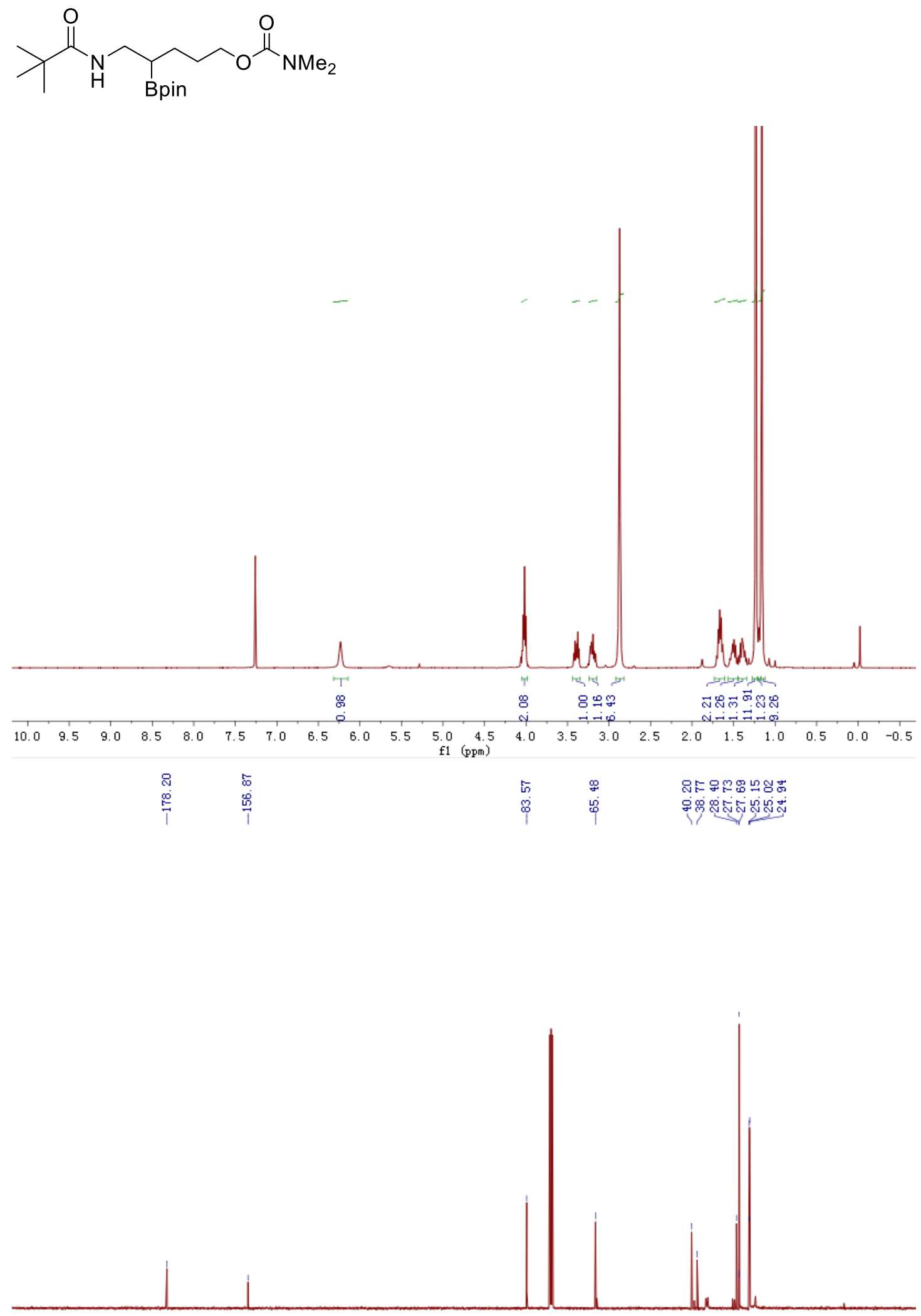

$\begin{array}{lllllllllllllllllllllllll}210 & 200 & 190 & 180 & 170 & 160 & 150 & 140 & 130 & 120 & 110 & 100 & 90 & 80 & 70 & 60 & 50 & 40 & 30 & 20 & 10 & 0 & -10\end{array}$ f1 (ppm) 
<smiles>CC(C)(C)C(=O)NC(CCCC[Se-])Cc1ccccc1</smiles>
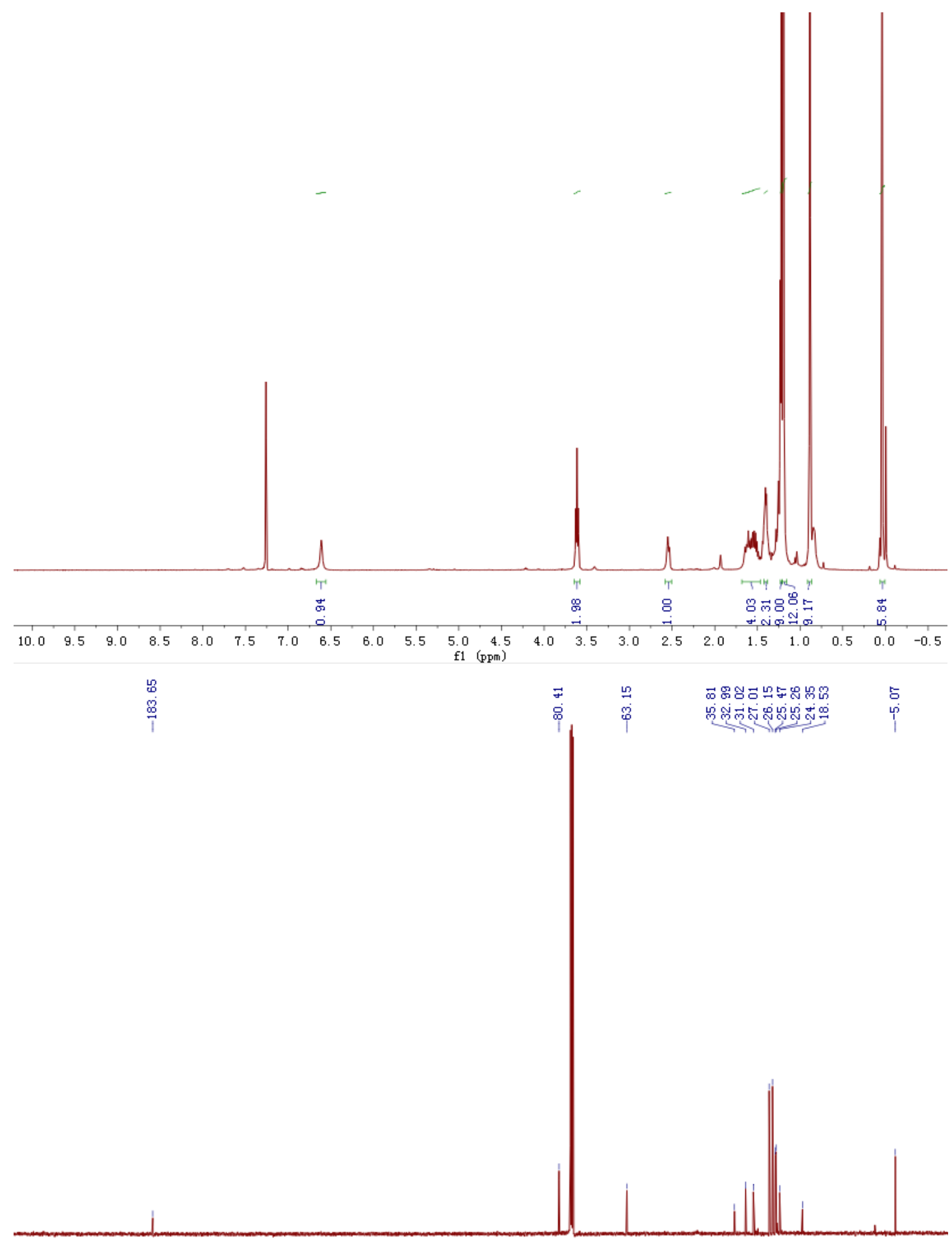

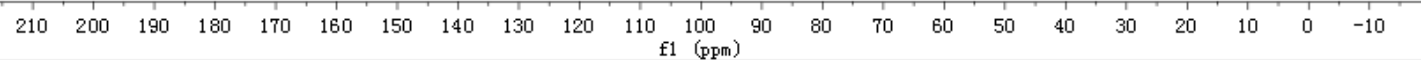


<smiles>CC(C)(C)C(=O)NCC([B]c1ccccc1)CCC[Se-](C)(C)C</smiles>
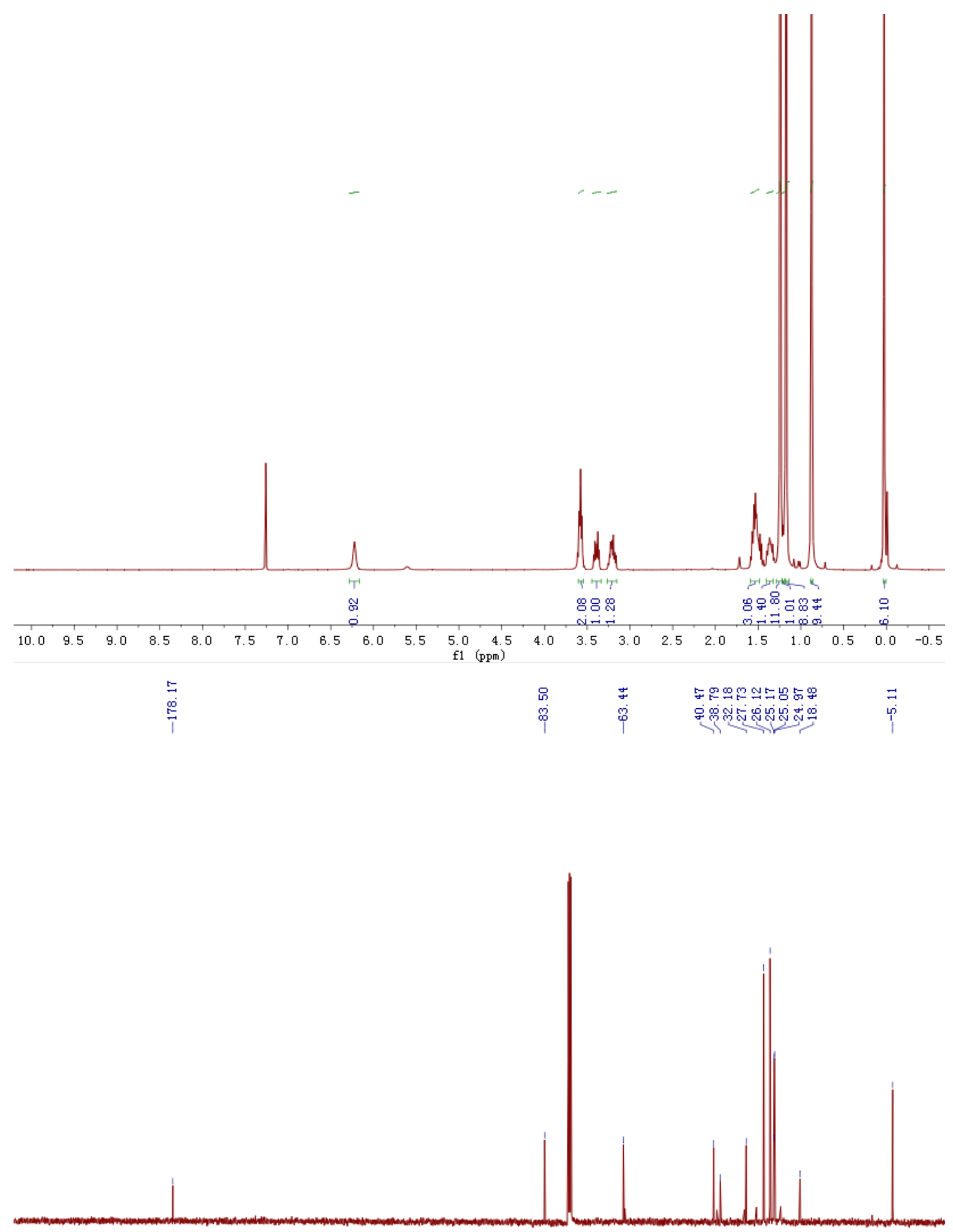

$\begin{array}{lllllllllllllllllllllll}210 & 200 & 190 & 180 & 170 & 160 & 150 & 140 & 130 & 120 & 110 & 100 & 90 & 80 & 70 & 60 & 50 & 40 & 30 & 20 & 10 & 0 & -10\end{array}$ f1 (ppm) 

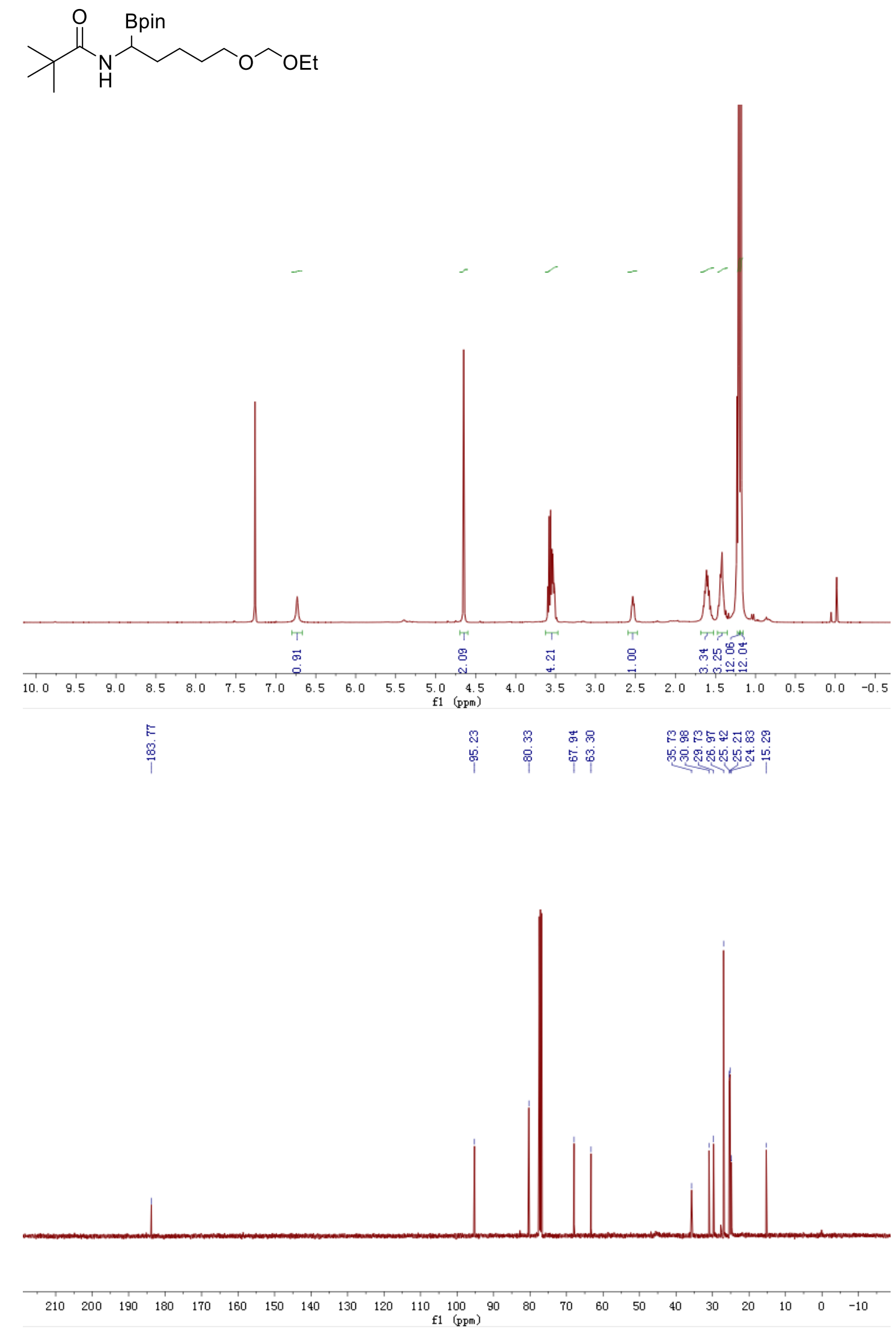
<smiles>CCOCOCCCC(CNC(=O)C(C)(C)C)Cc1ccccc1</smiles>
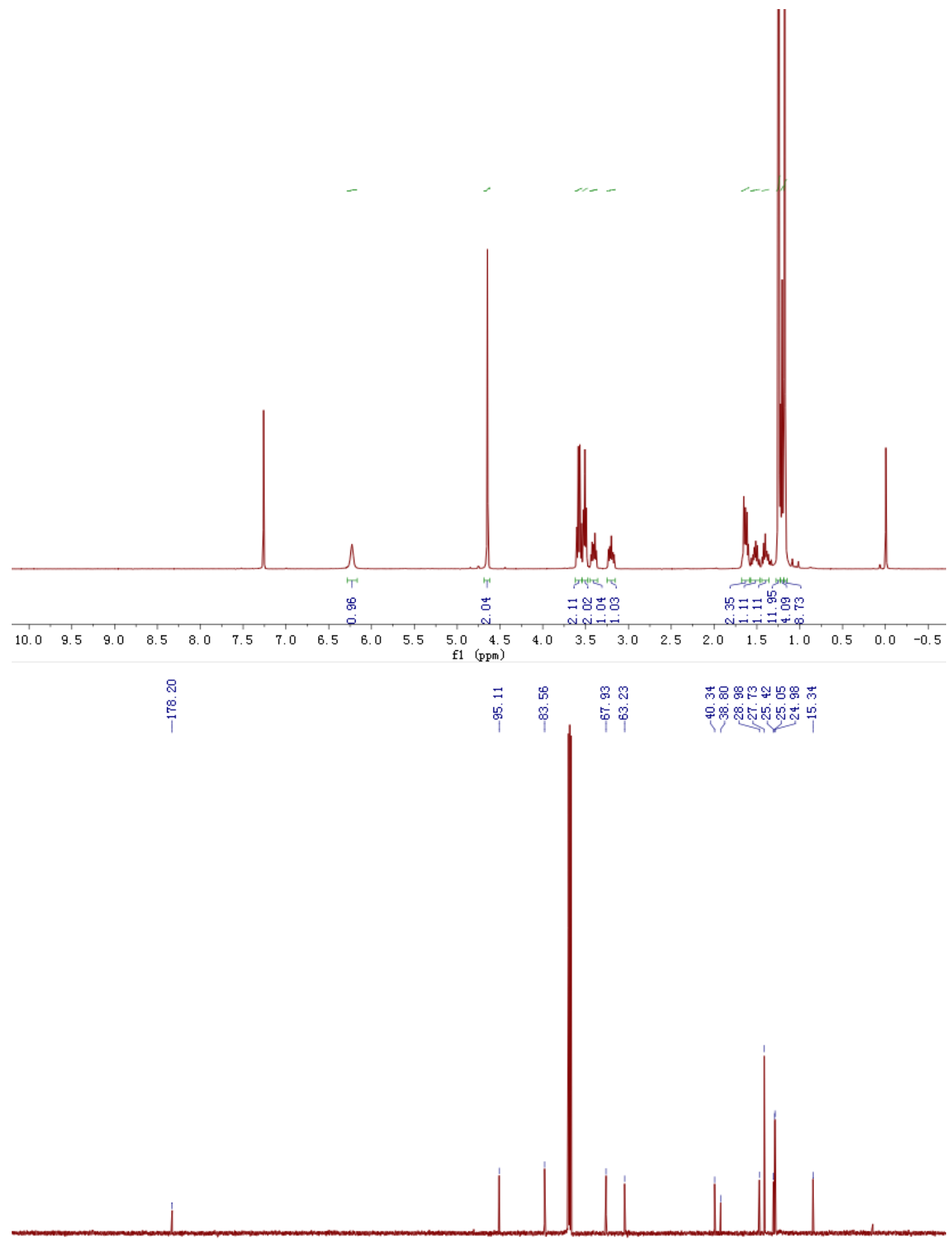

$\begin{array}{lllllllllllllllllllllllllll}210 & 200 & 190 & 180 & 170 & 160 & 150 & 140 & 130 & 120 & 110 & 100 & 90 & 80 & 70 & 60 & 50 & 40 & 30 & 20 & 10 & 0 & -10\end{array}$ f1 (ppm) 
<smiles>CC(C)(C)C(=O)NC(CCCCOCCc1ccccc1)Cc1ccccc1</smiles>

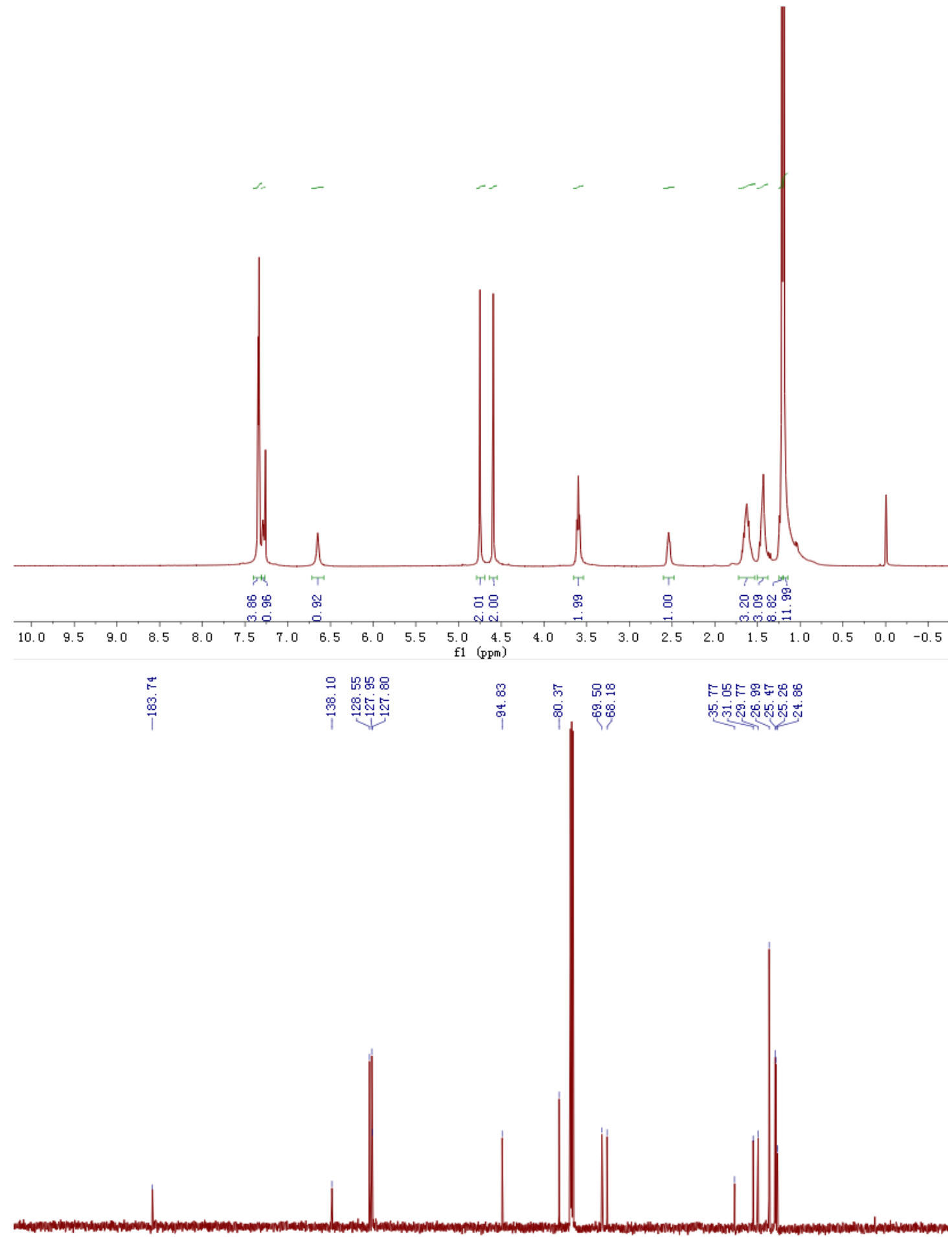

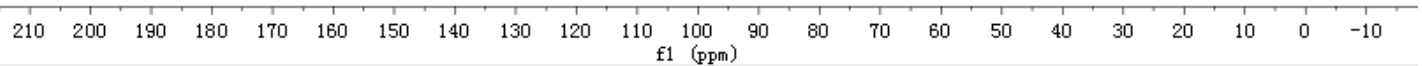



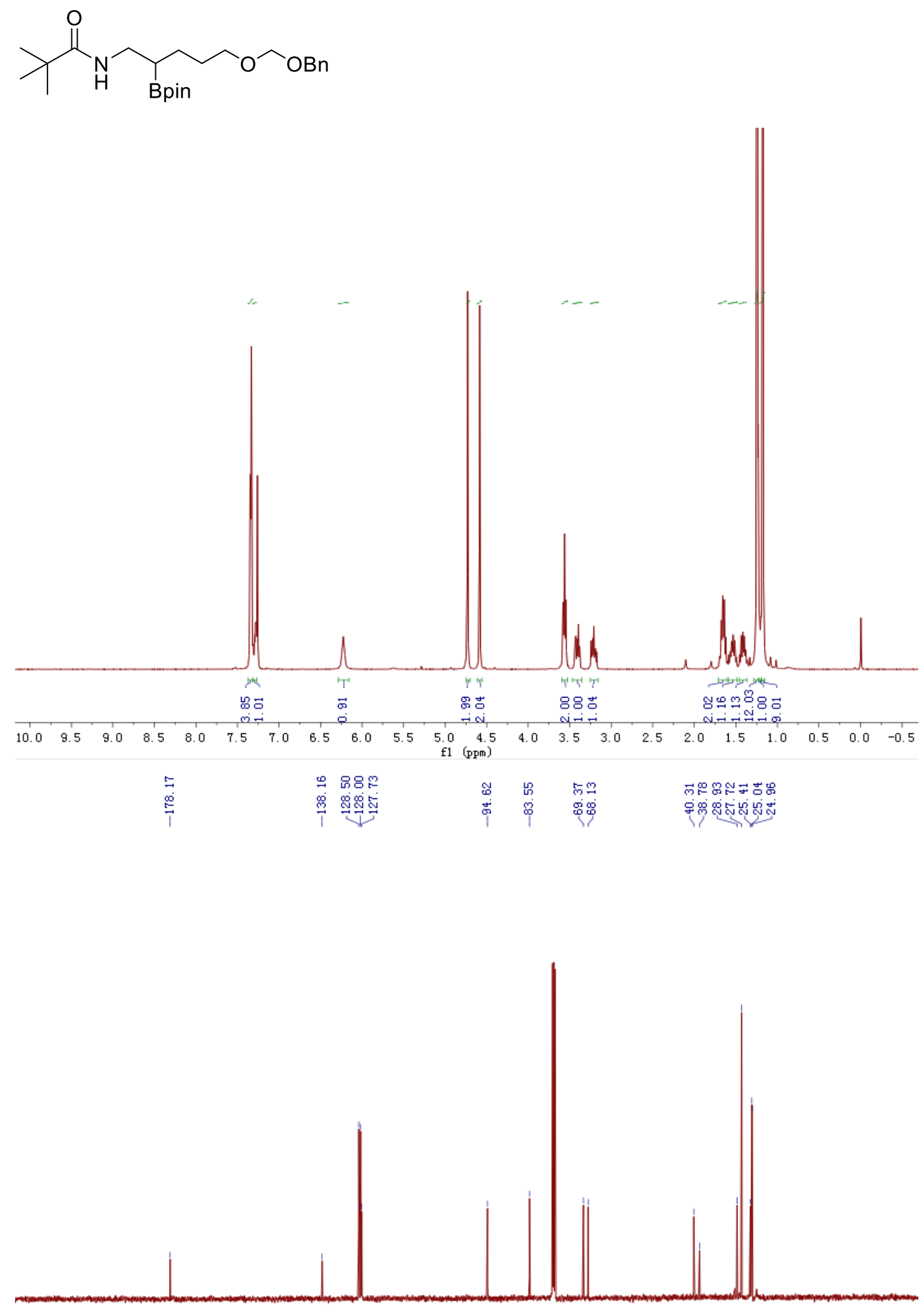

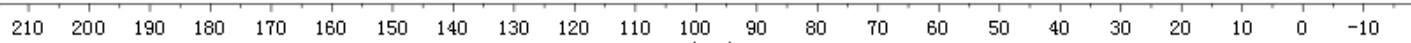
f1 (ppm) 

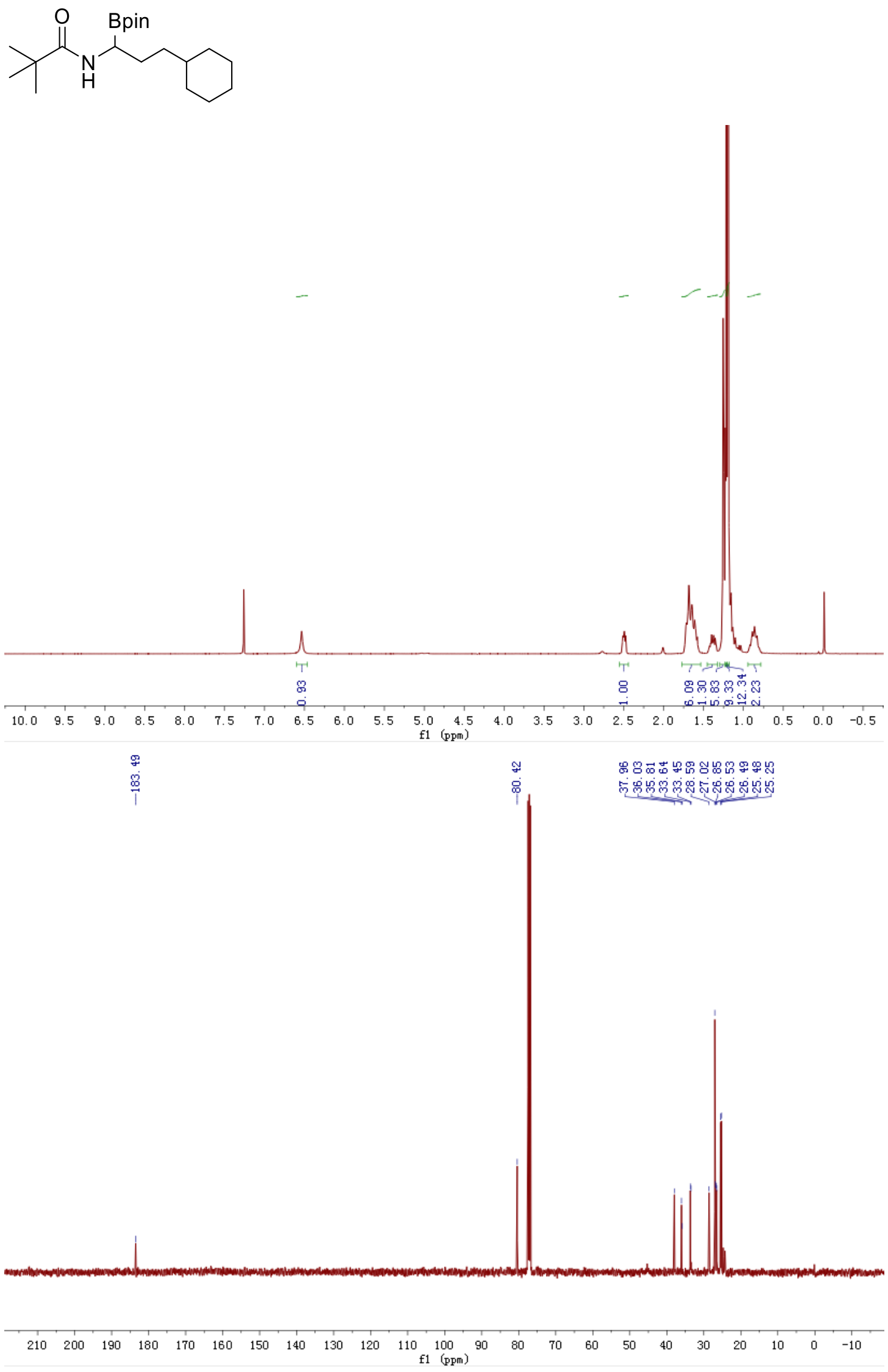

S223 
<smiles>CC(C)(C)C(=O)NCC([Pb])CC1CCCCC1</smiles>
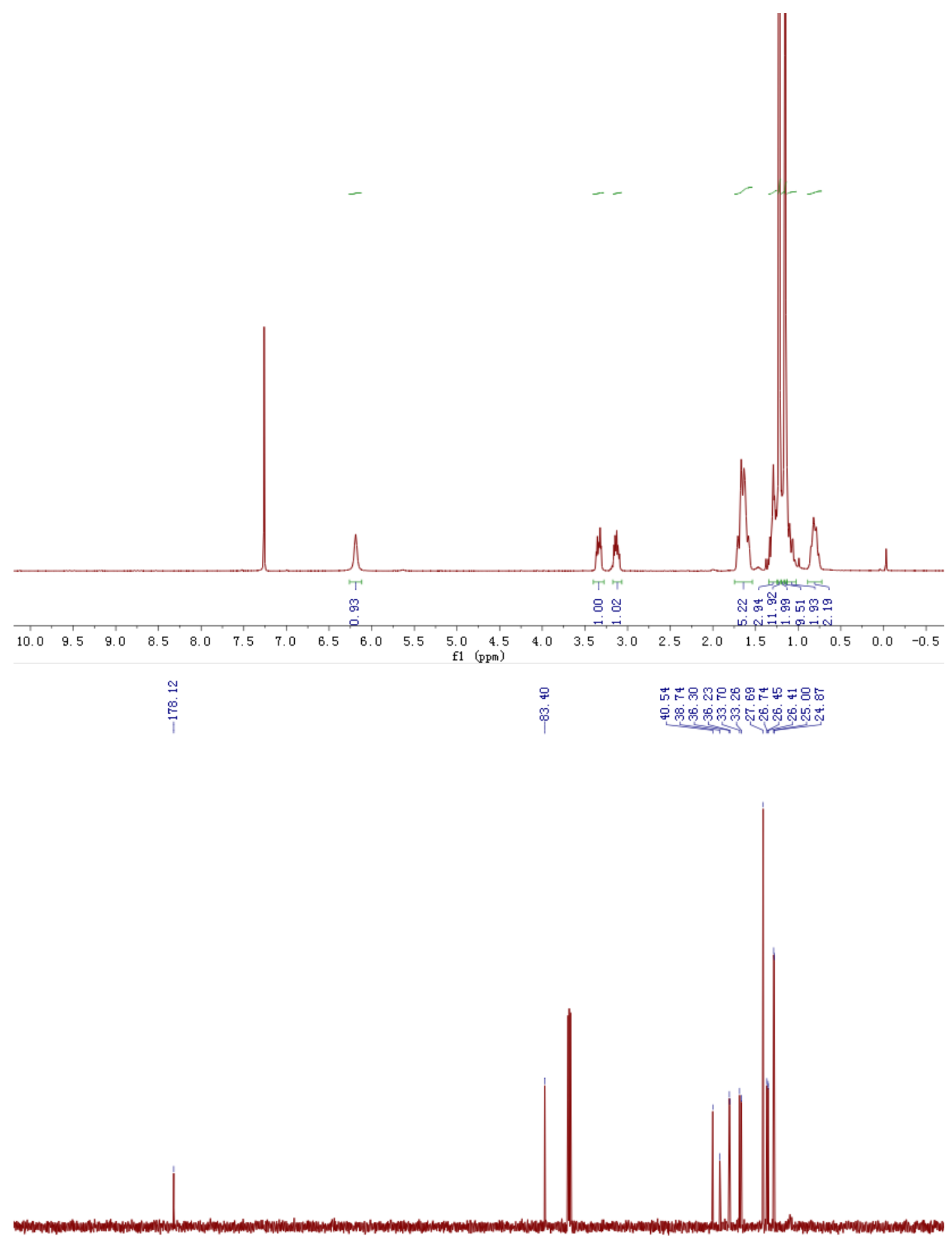

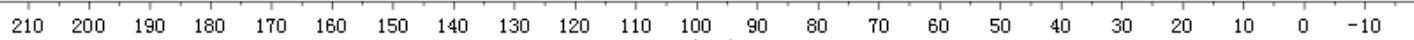
f1 (ppm) 
<smiles>CC(C)CC(Cc1ccccc1)NC(=O)C(C)(C)C</smiles>
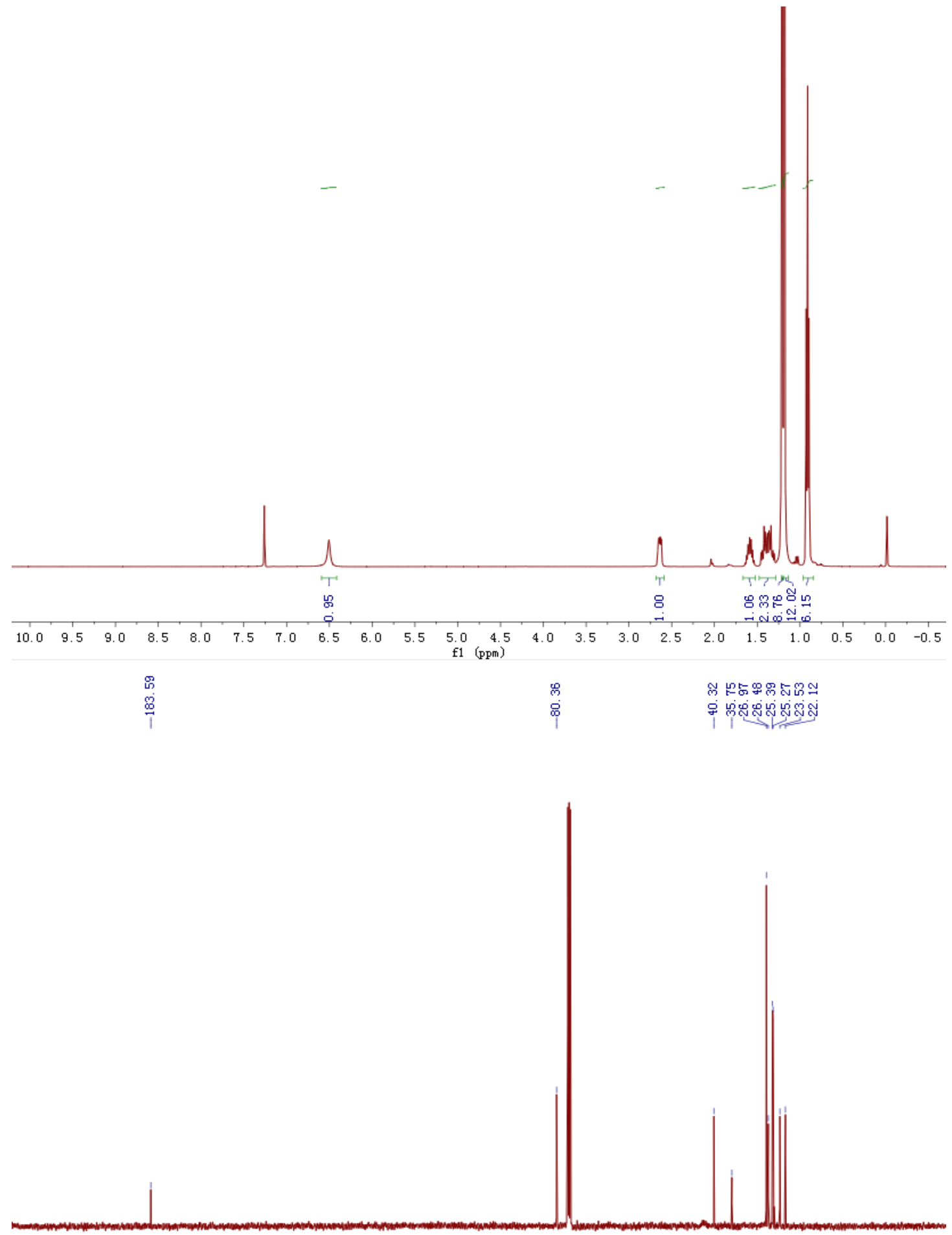

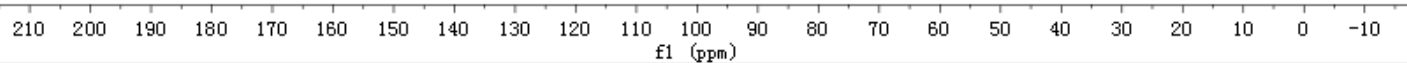


<smiles>CC(C)C(CNC(=O)C(C)(C)C)Cc1ccccc1</smiles>

:

$1: 1.4$

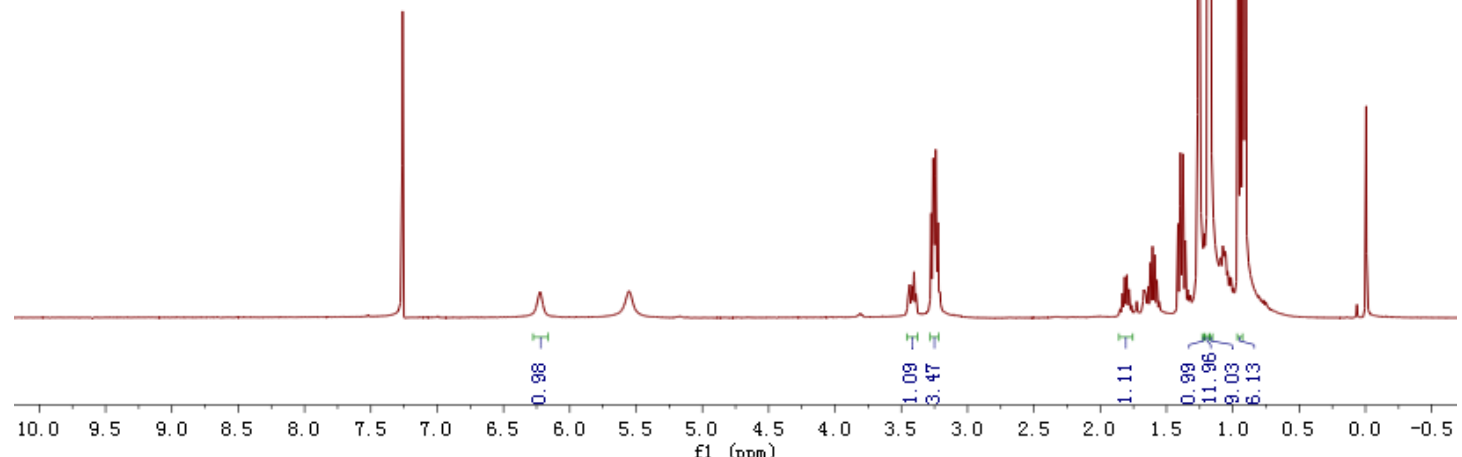

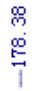

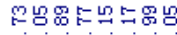

\%ับํํำ

$\begin{array}{llllllllllll}210 & 200 & 190 & 180 & 170 & 160 & 150 & 140 & 130 & 120 & 110 & 100\end{array}$ 
<smiles>CC(Cc1ccccc1)NC(=O)C(C)(C)C</smiles>
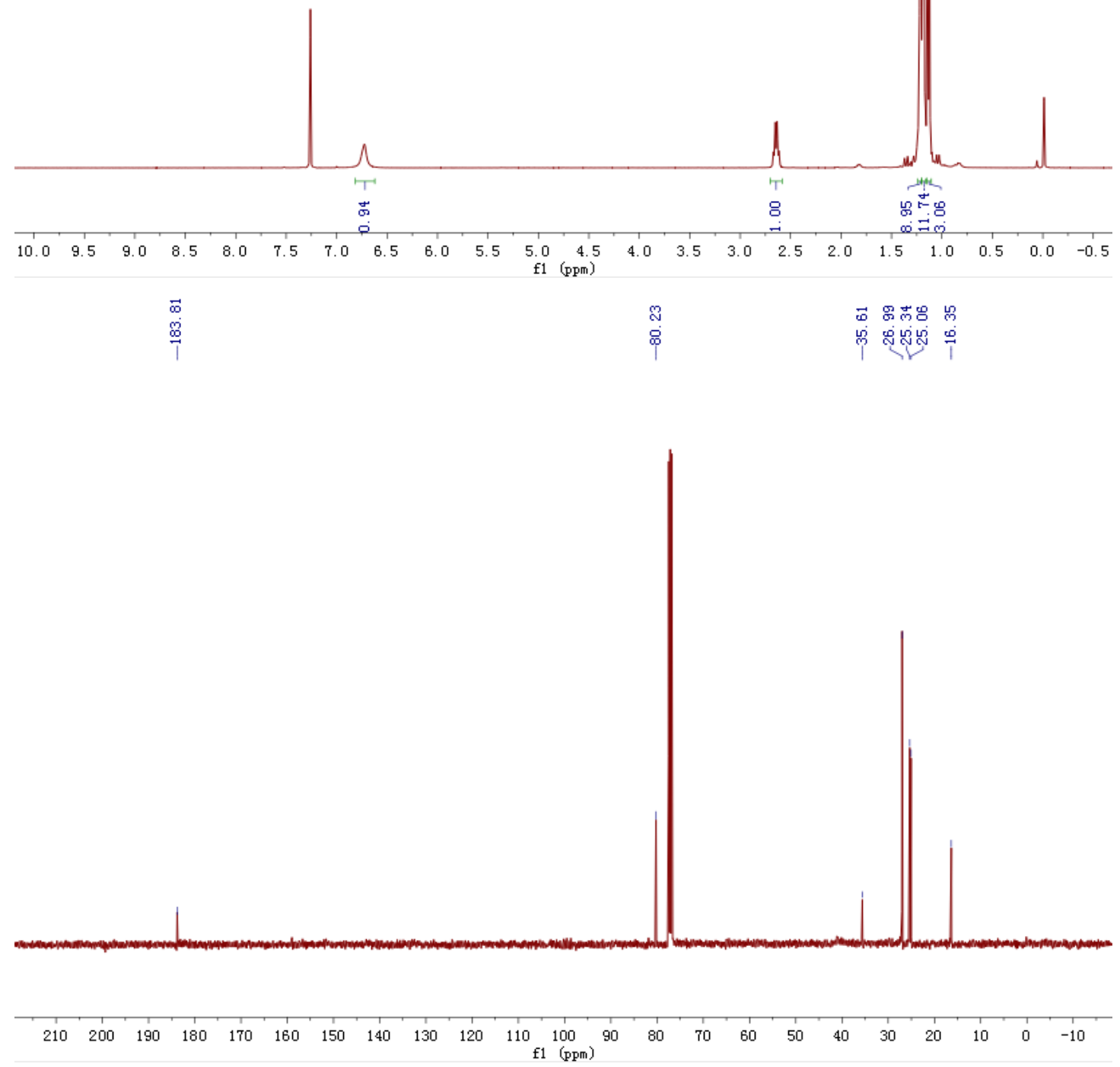
<smiles>CC(C)(C)C(=O)NCCCc1ccccc1</smiles>

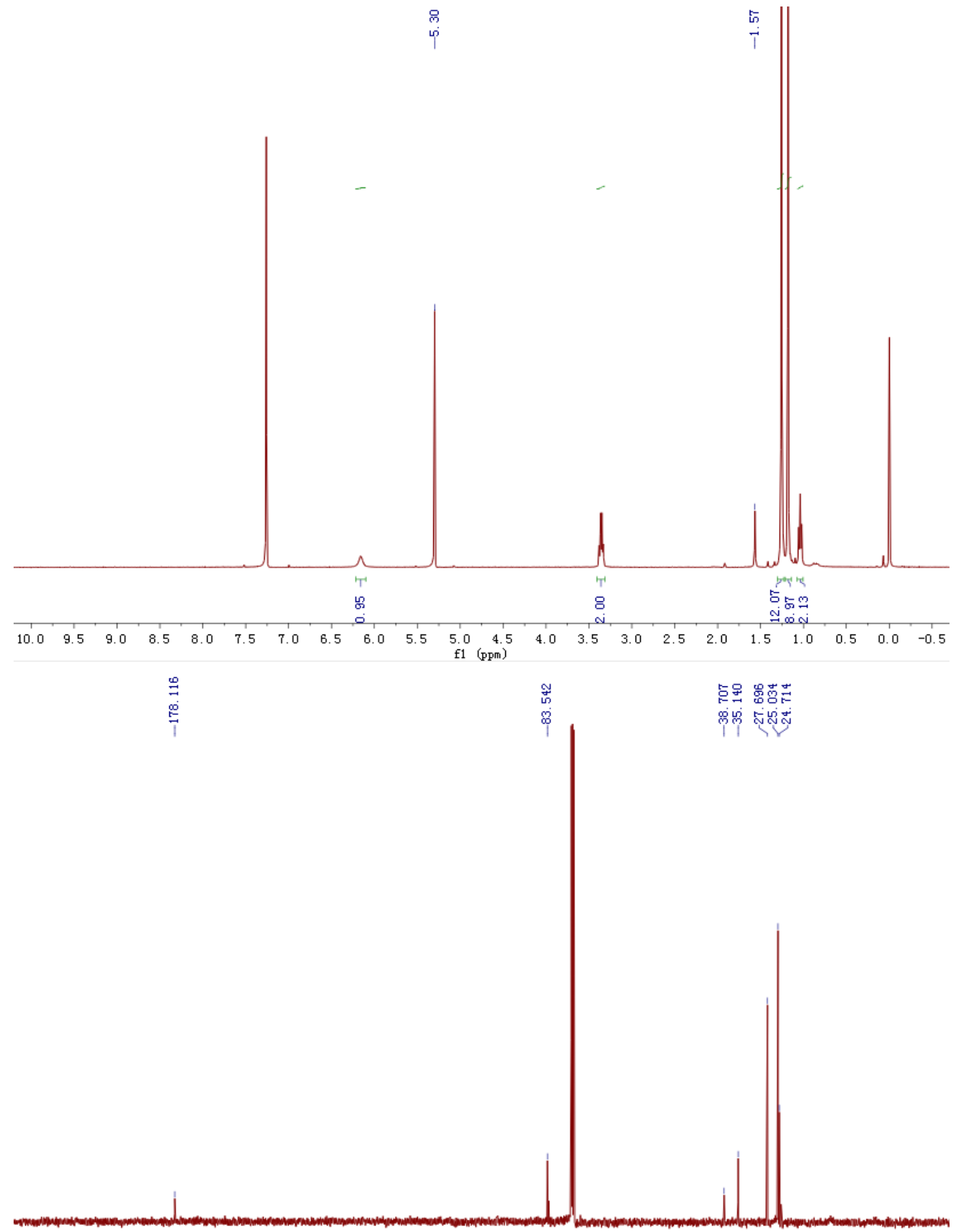

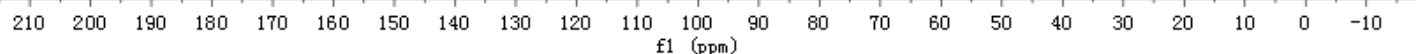


<smiles>C[C@H](Cc1ccccc1)C(Cc1ccccc1)NC(=O)C(C)(C)C</smiles>

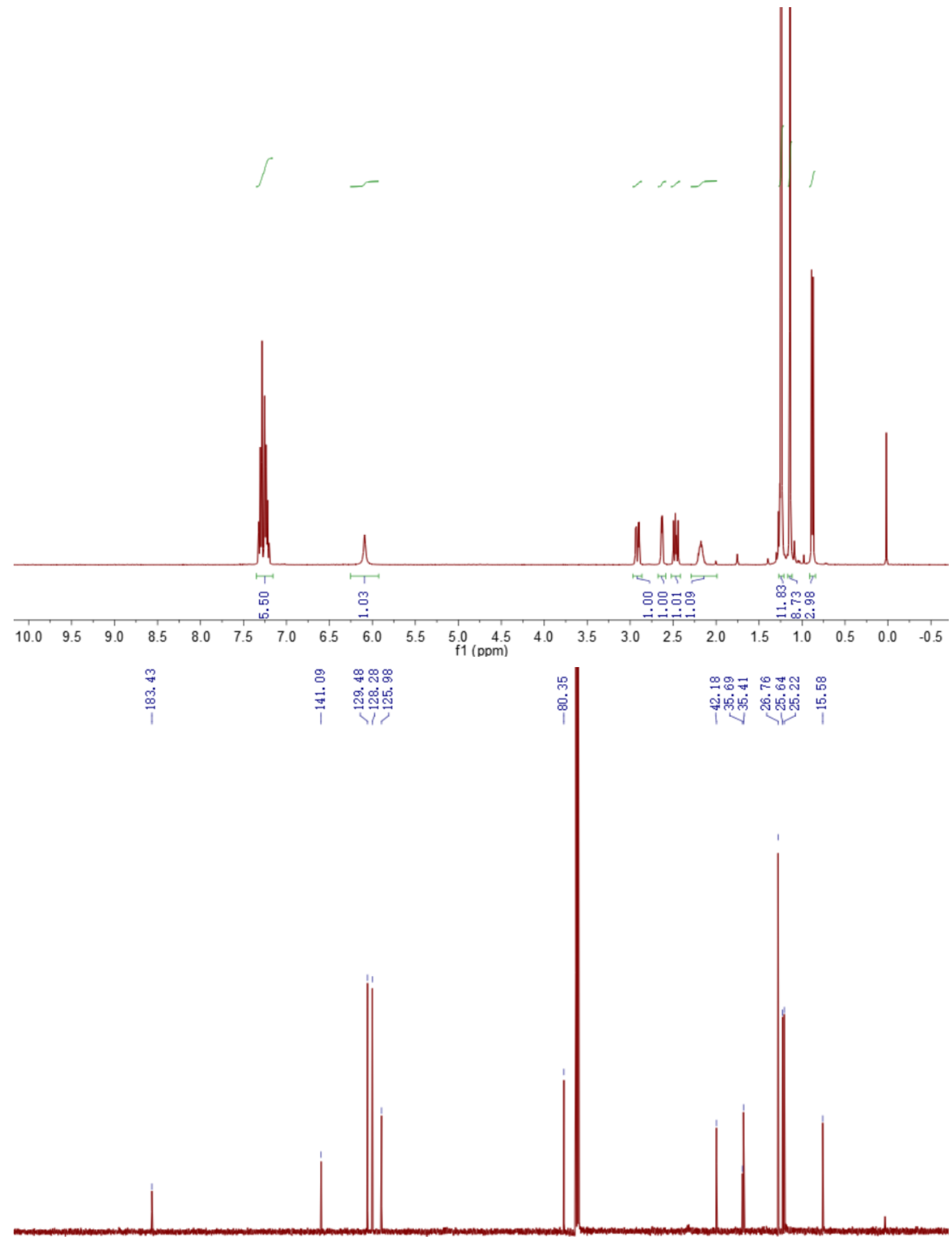

$\begin{array}{lllllllllllllllllllllll}210 & 200 & 190 & 180 & 170 & 160 & 150 & 140 & 130 & 120 & 110 & 100 & 90 & 80 & 70 & 60 & 50 & 40 & 30 & 20 & 10 & 0 & -10\end{array}$ 
<smiles>C[C@H](CCc1ccccc1)C(Cc1ccccc1)NC(=O)C(C)(C)C</smiles>
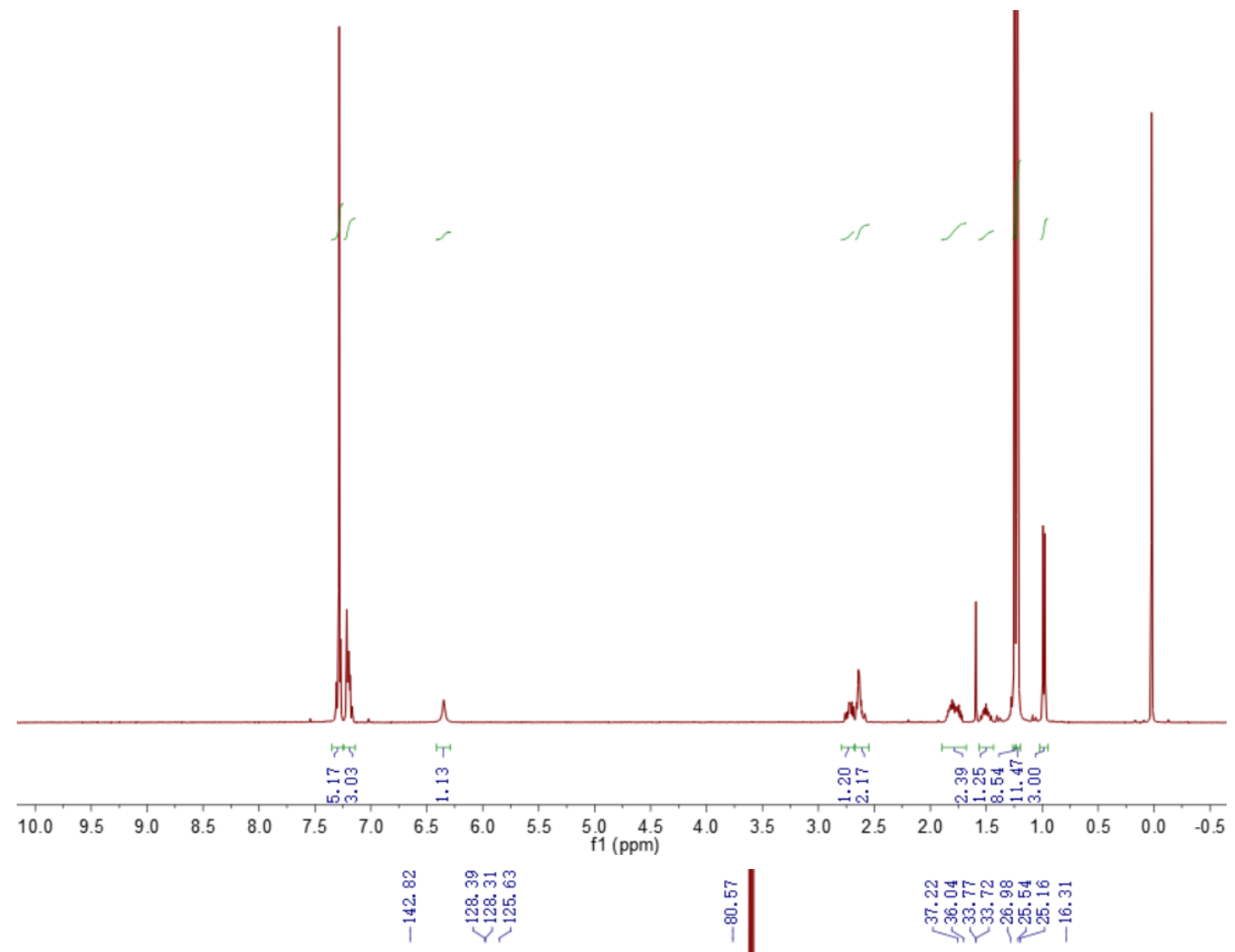

$\begin{array}{lllllllllllllllllllllll}210 & 200 & 190 & 180 & 170 & 160 & 150 & 140 & 130 & 120 & 110 & 100 & 90 & 80 & 70 & 60 & 50 & 40 & 30 & 20 & 10 & 0 & -10\end{array}$ 
<smiles>CCCC[C@H](C)C(Cc1ccccc1)NC(=O)C(C)(C)C</smiles>

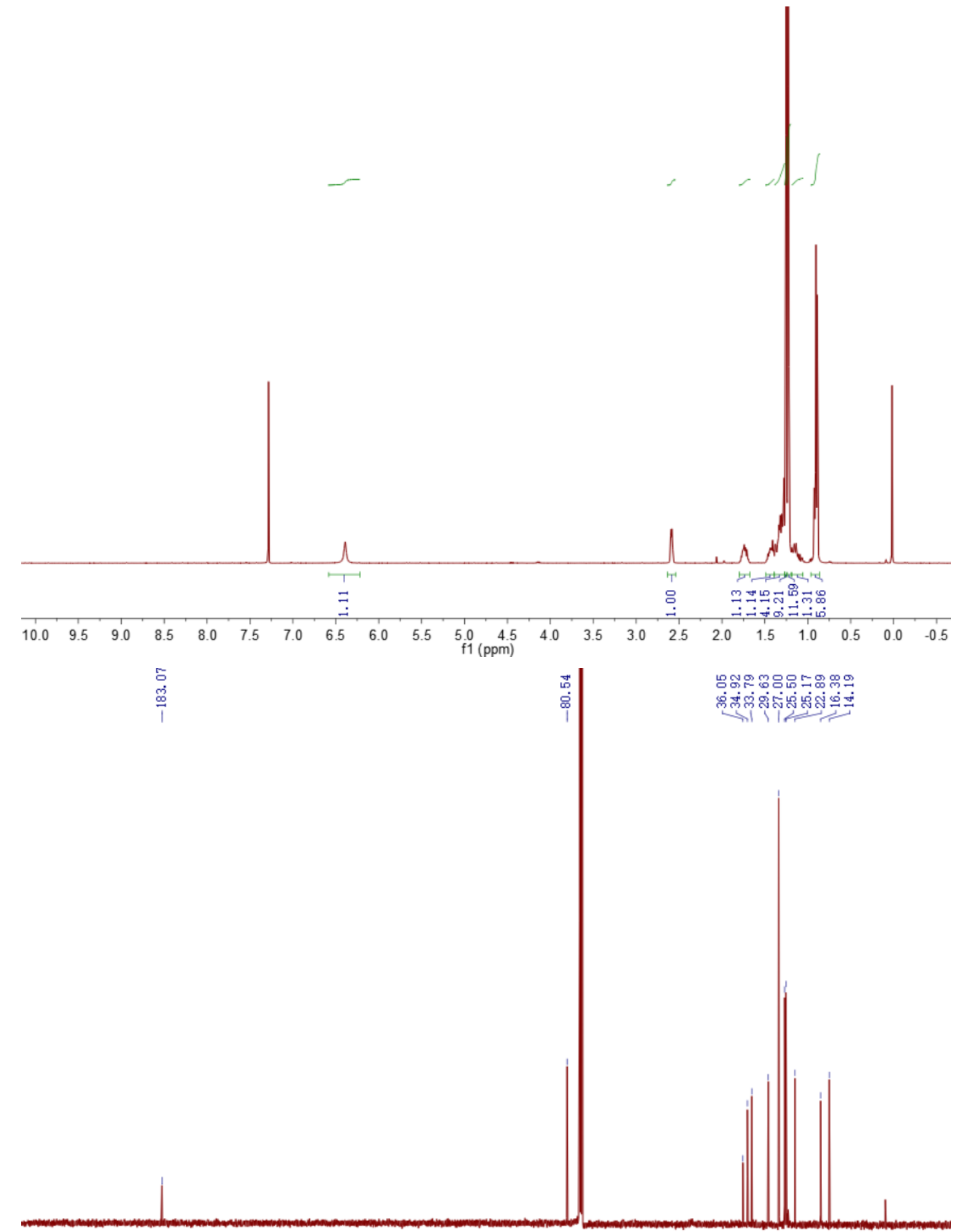

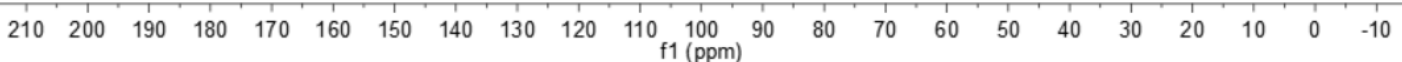


<smiles>CCCCCCCC[C@H](C)C(Cc1ccccc1)NC(=O)C(C)(C)C</smiles>

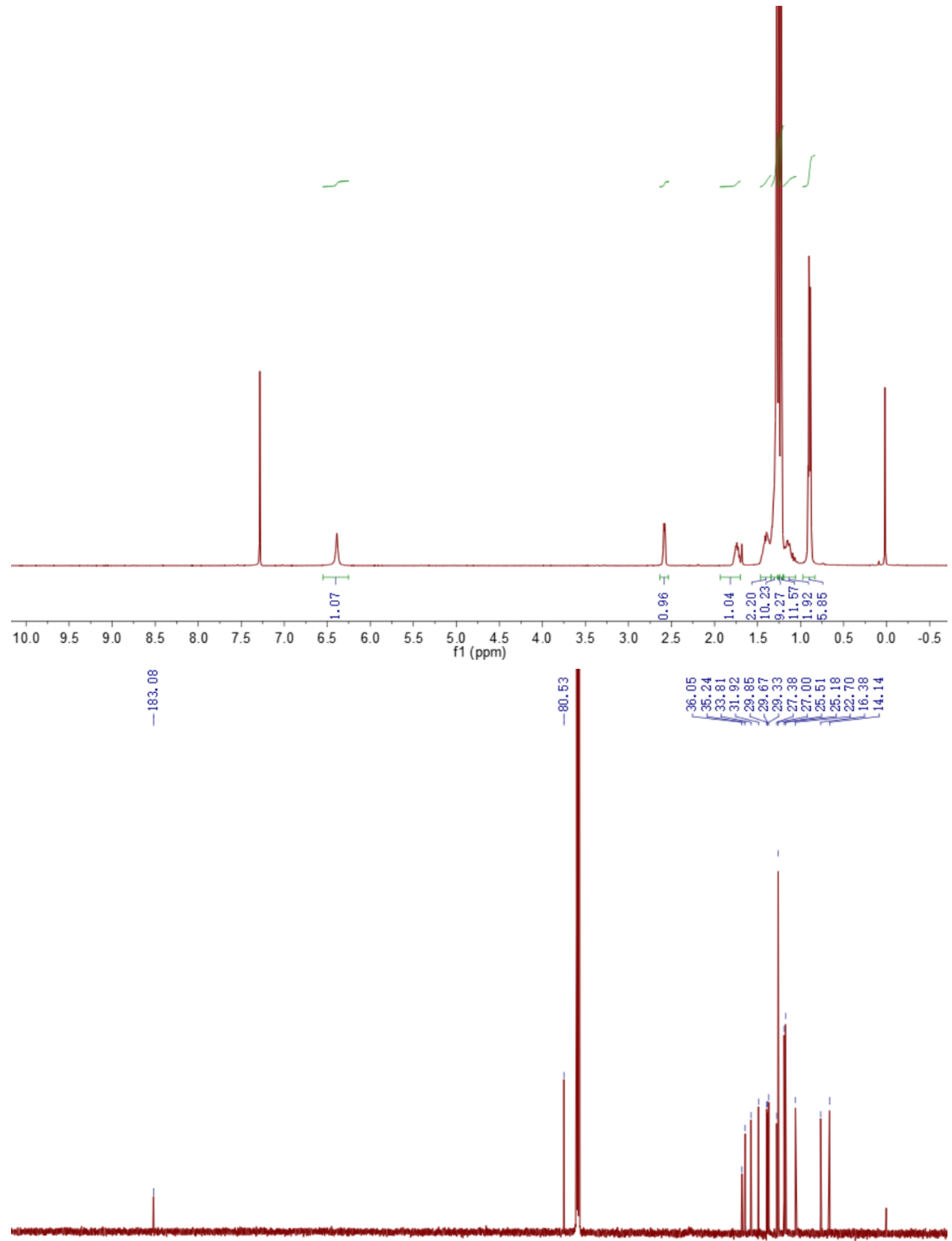

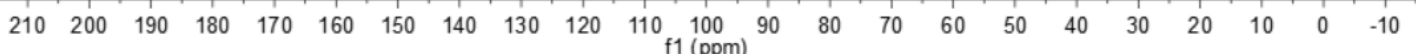


<smiles>CC(C)C(Cc1ccccc1)NC(=O)C(C)(C)C</smiles>

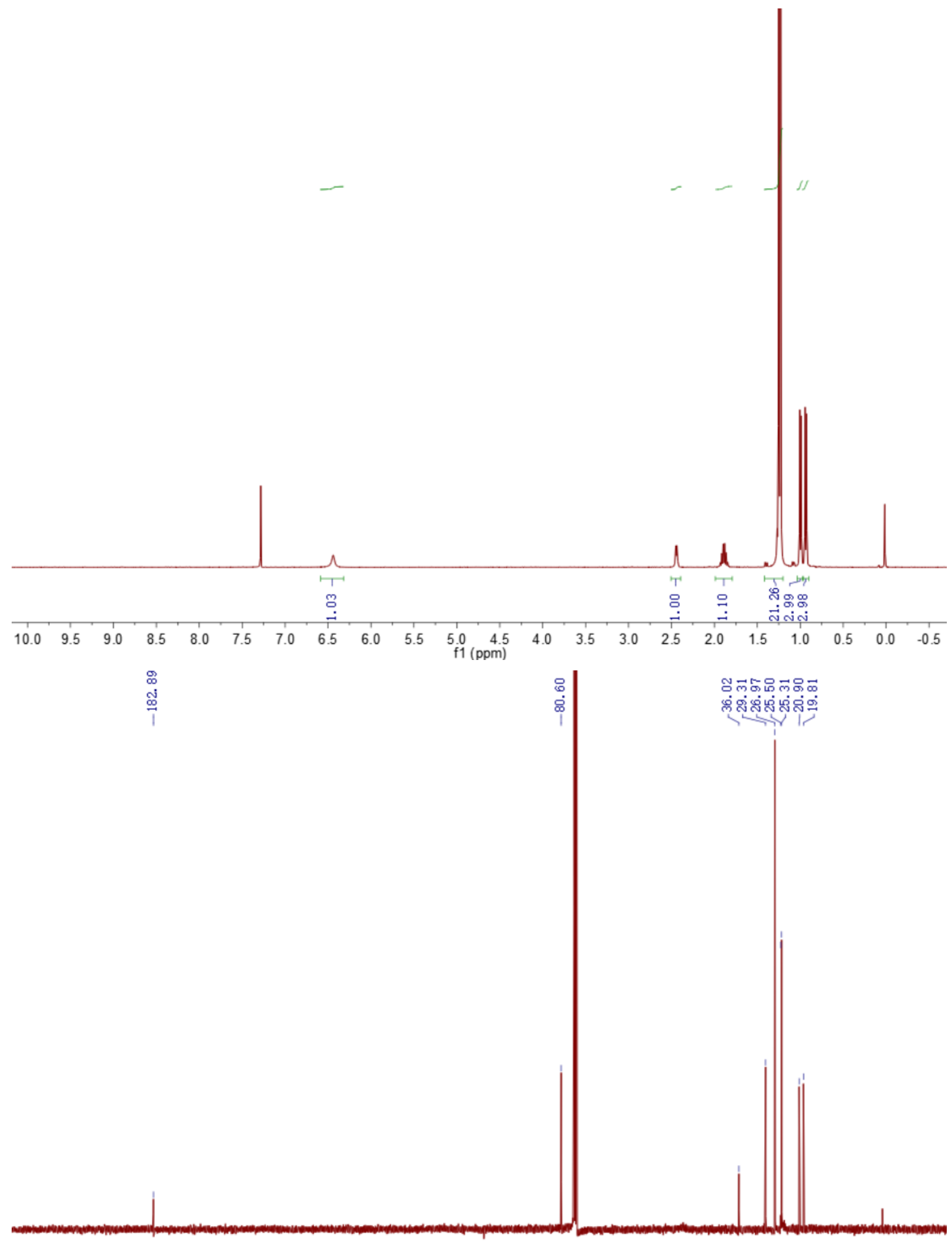

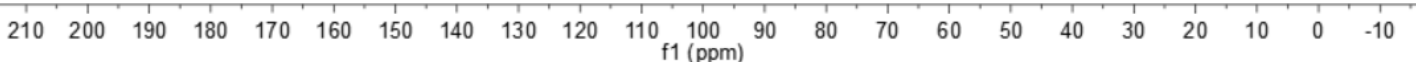



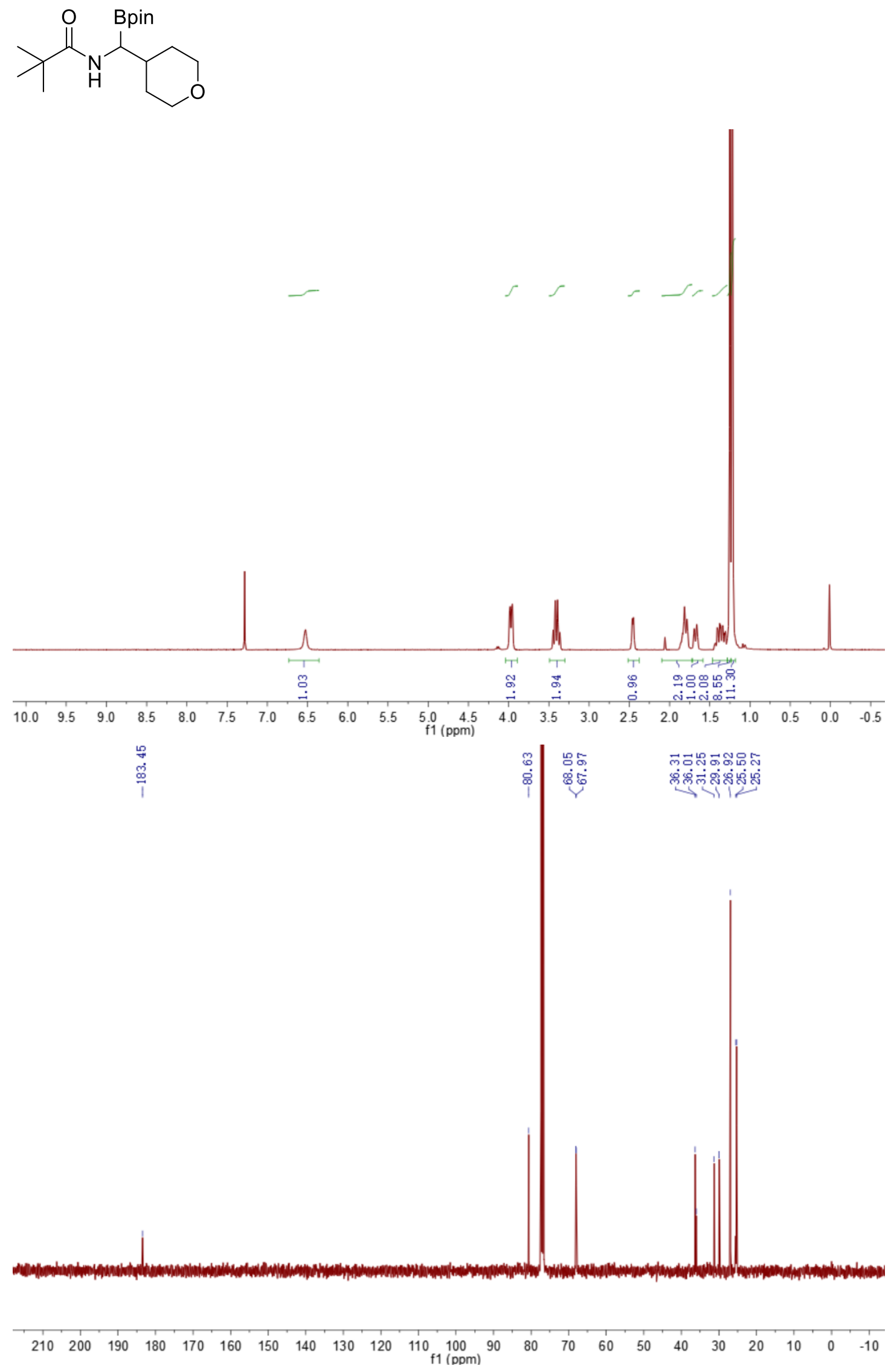

S234 
<smiles>CCC(Cc1ccccc1)[C@H](C)NC(=O)C(C)(C)C</smiles>
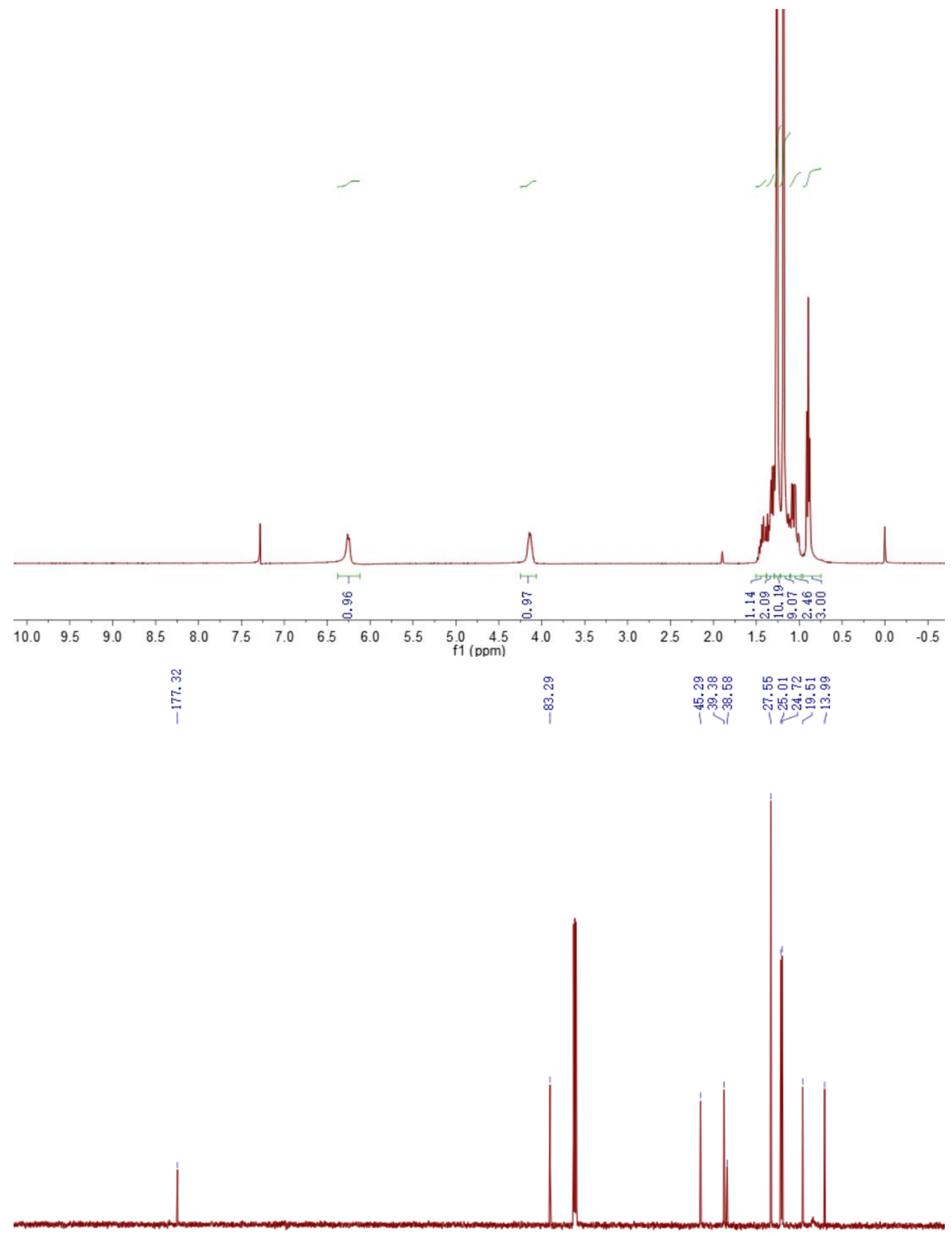

$\begin{array}{lllllllllllllllllllllll}210 & 200 & 190 & 180 & 170 & 160 & 150 & 140 & 130 & 120 & 110 & \begin{array}{c}100 \\ \mathrm{f} 1(\mathrm{ppm})\end{array} & 90 & 80 & 70 & 60 & 50 & 40 & 30 & 20 & 10 & 0 & -10\end{array}$ 
<smiles>CCCC([Pb])C(CC)NC(=O)C(C)(C)C</smiles>

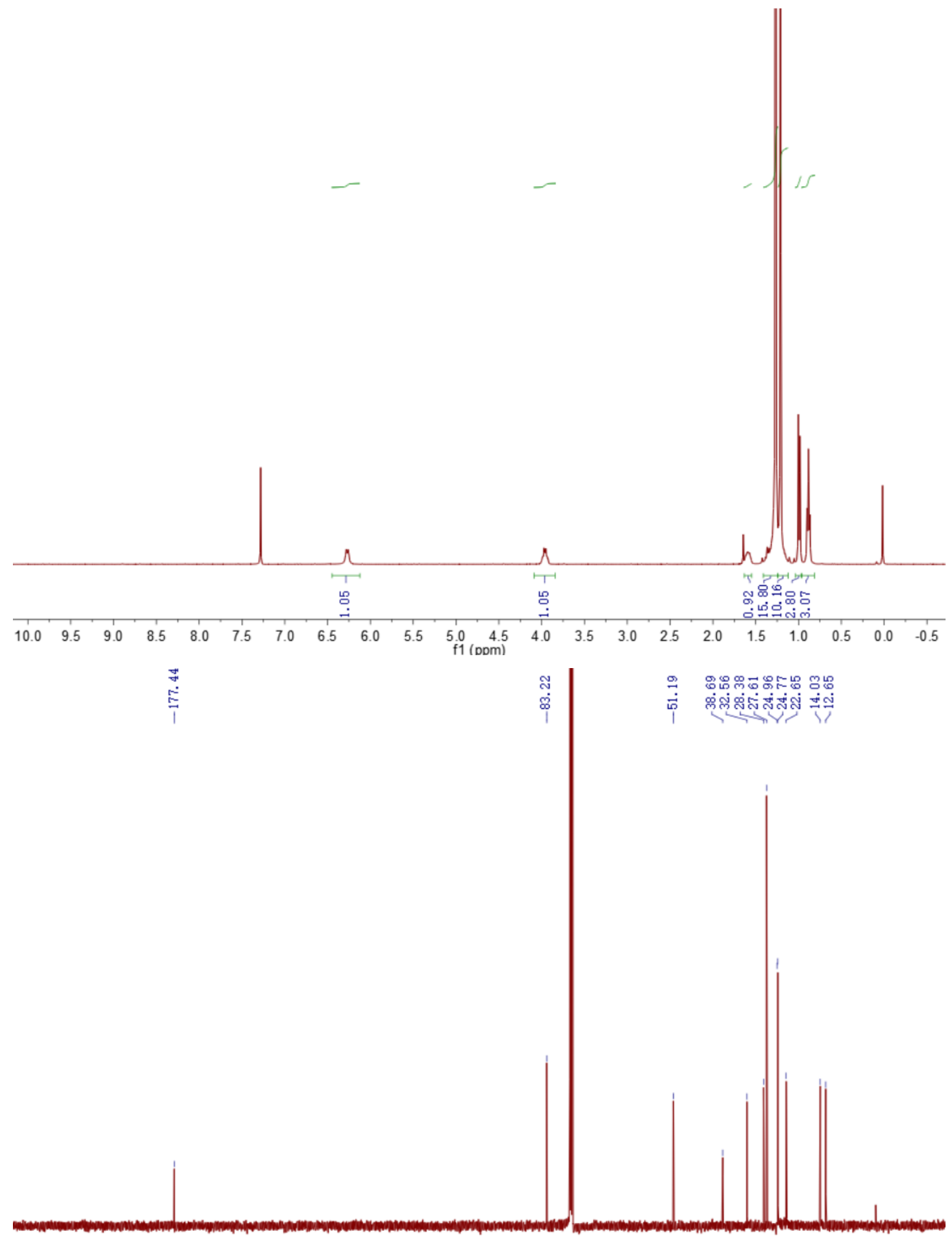

$\begin{array}{llllllllllllllllllllll}210 & 200 & 190 & 180 & 170 & 160 & 150 & 140 & 130 & 120 & 110 \begin{array}{c}100 \\ \mathrm{f} 1(\mathrm{ppm})\end{array} & 90 & 80 & 70 & 60 & 50 & 40 & 30 & 20 & 10 & 0 & -10\end{array}$ 
<smiles>CC(C[R8]c1ccccc1)NC(=O)C(C)(C)C</smiles>

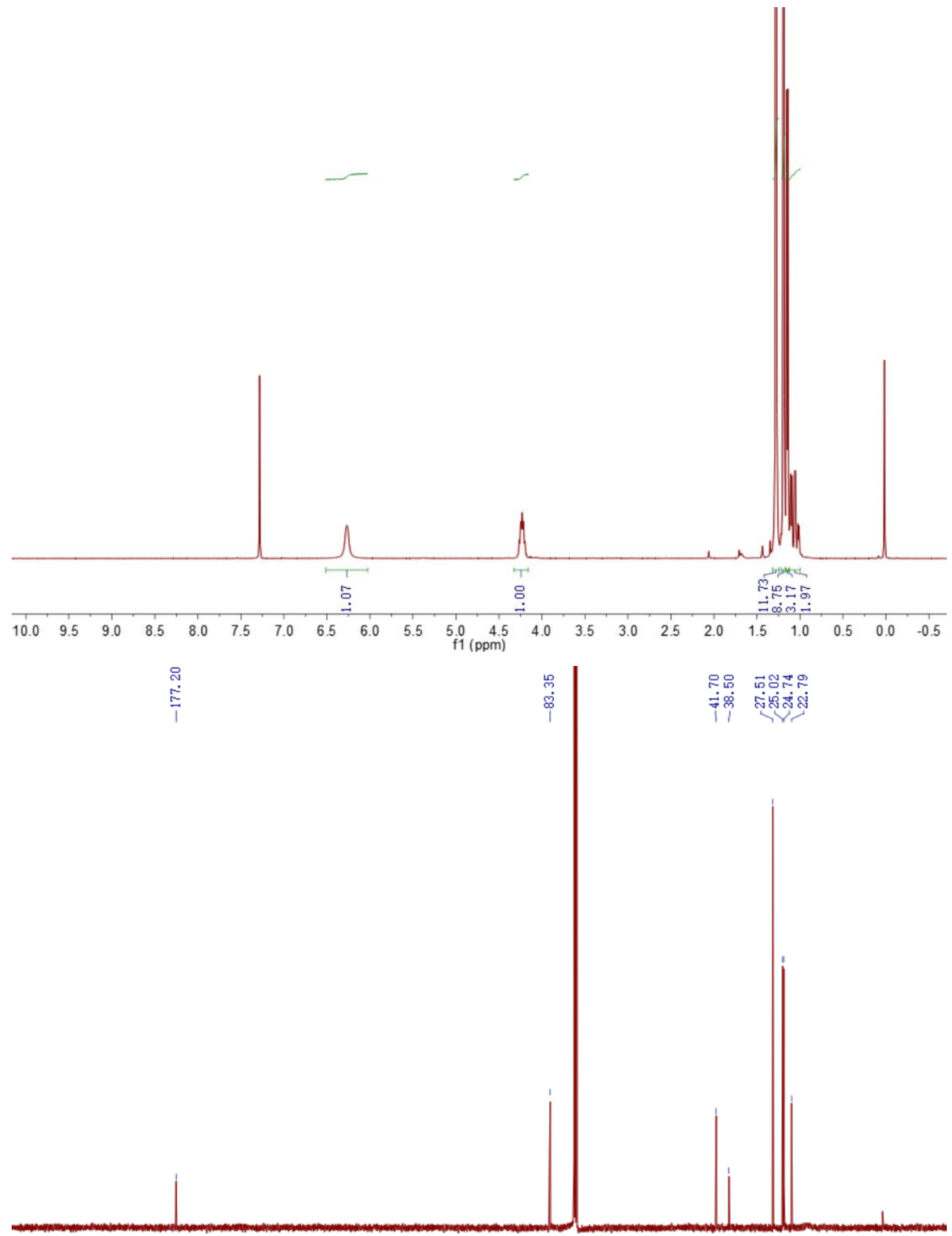

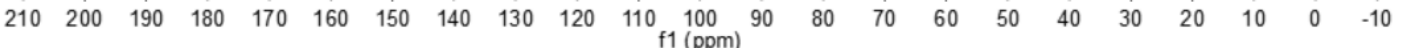




$$
\text { - }
$$


<smiles>CC(C)(C)C(=O)NCC(CCc1ccccc1)c1ccccc1</smiles>
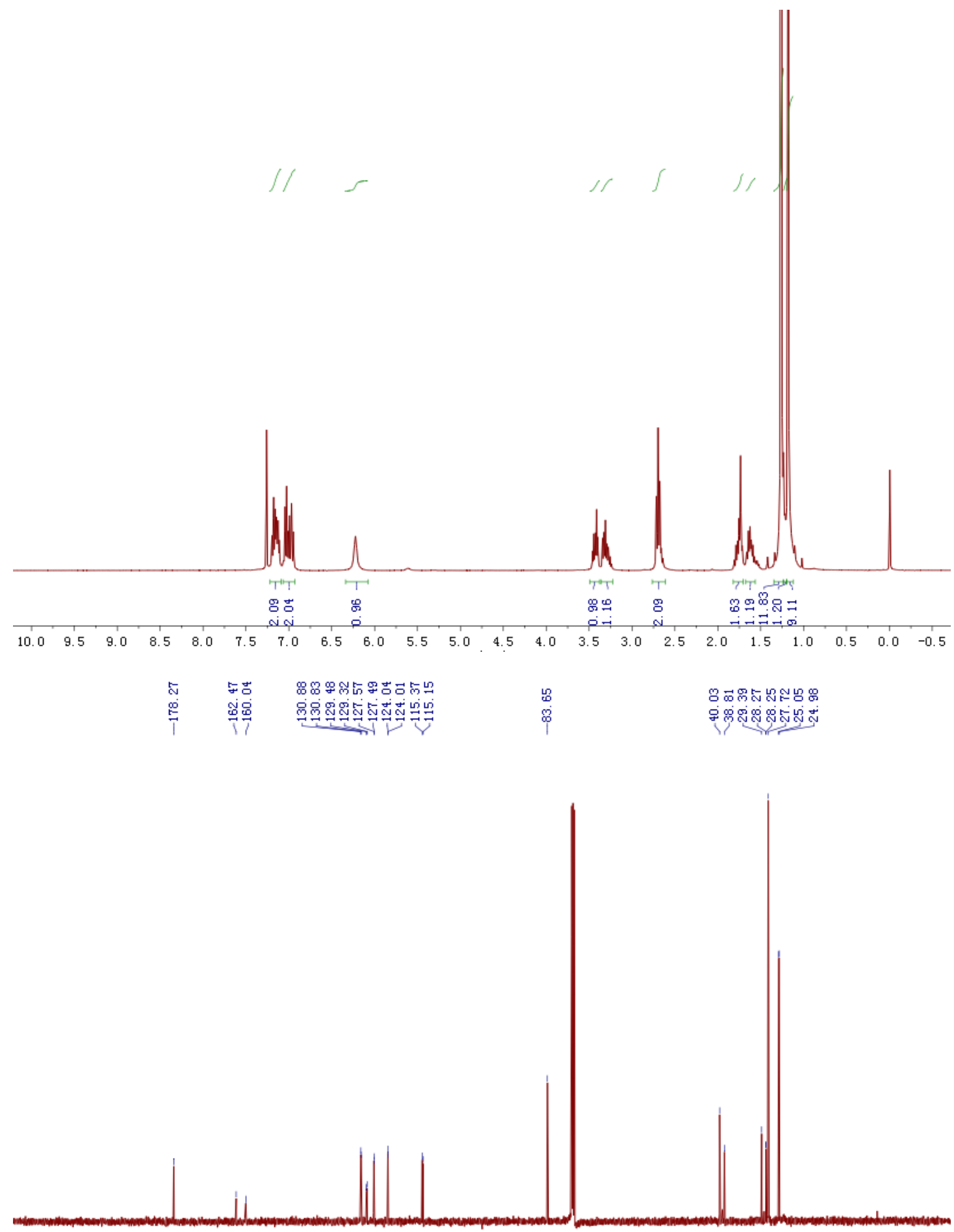

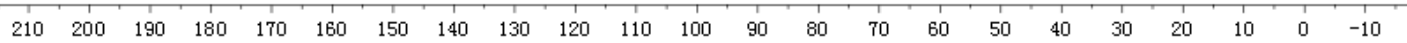


<smiles>CC(C)(C)C(=O)NC(CCCc1ccc(Cl)cc1)Cc1ccccc1</smiles>

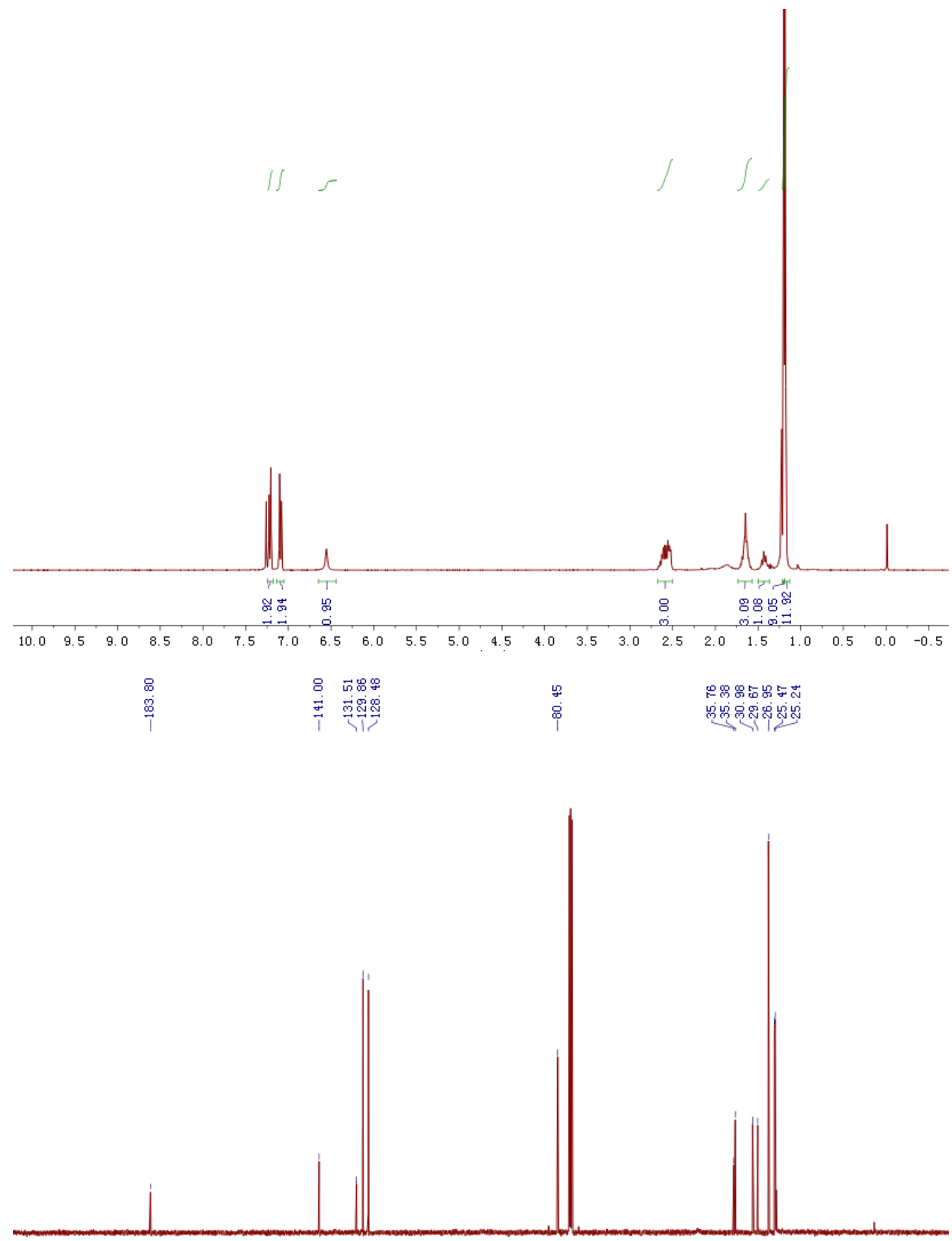

$\begin{array}{lllllllllllllllllllllll}210 & 200 & 190 & 180 & 170 & 160 & 150 & 140 & 130 & 120 & 110 & 100 & 90 & 80 & 70 & 60 & 50 & 40 & 30 & 20 & 10 & 0 & -10\end{array}$ 
<smiles>CC(C)(C)C(=O)NC[C@H](CCc1ccccc1)Cc1ccc(Cl)cc1</smiles>
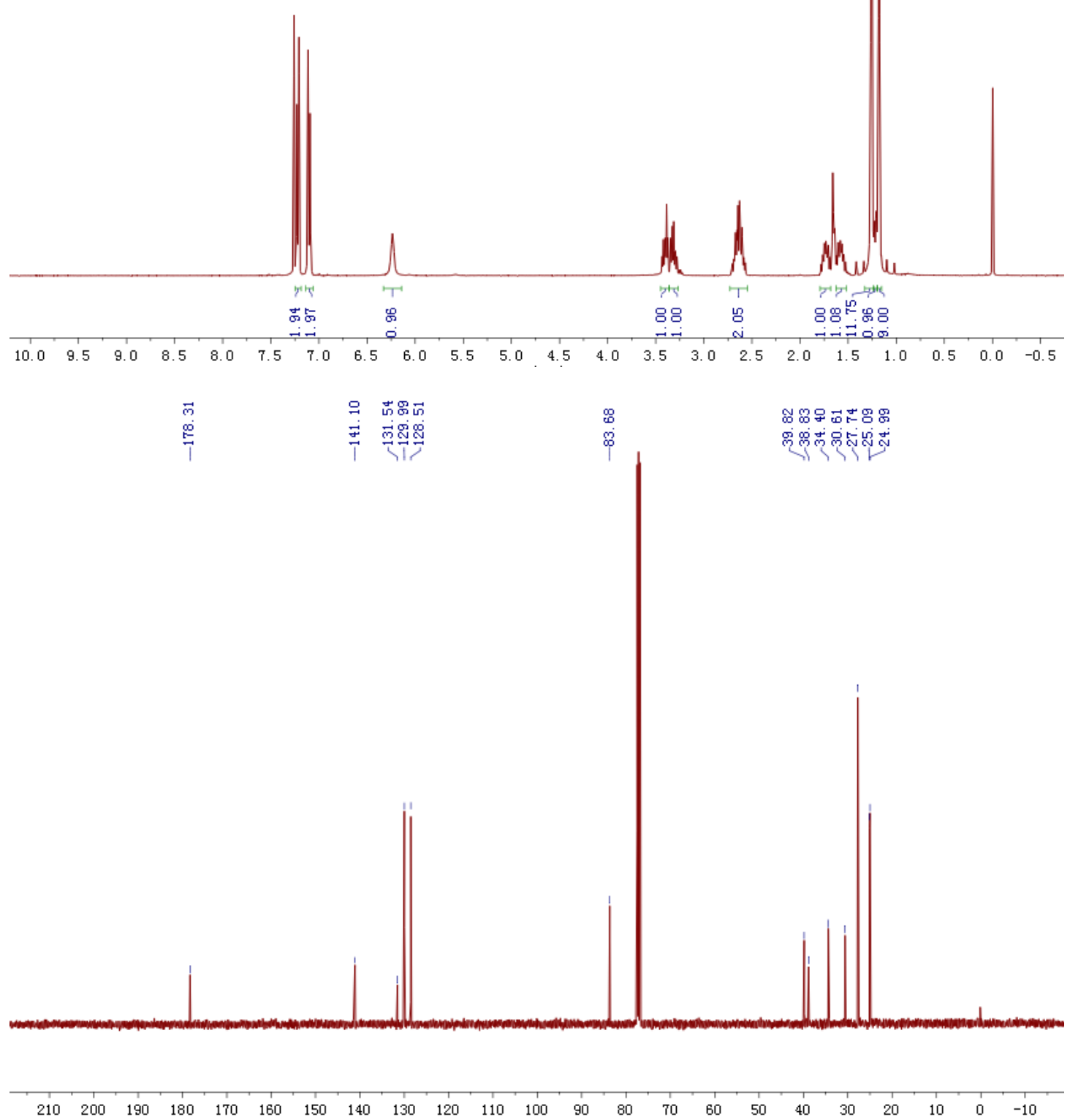

S241 

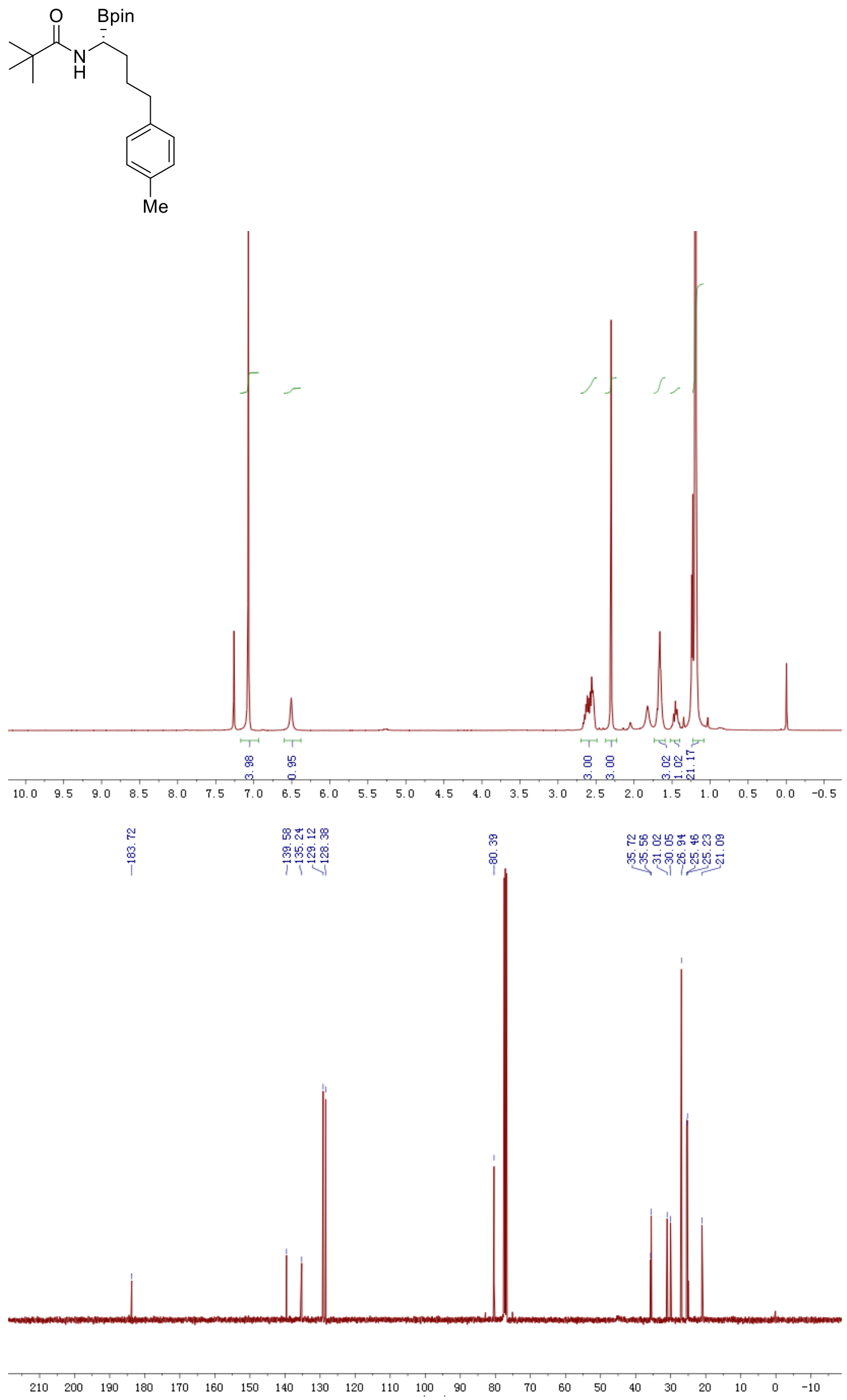

S242 
<smiles>Cc1ccc(CCC(CNC(=O)C(C)(C)C)Cc2ccccc2)cc1</smiles>
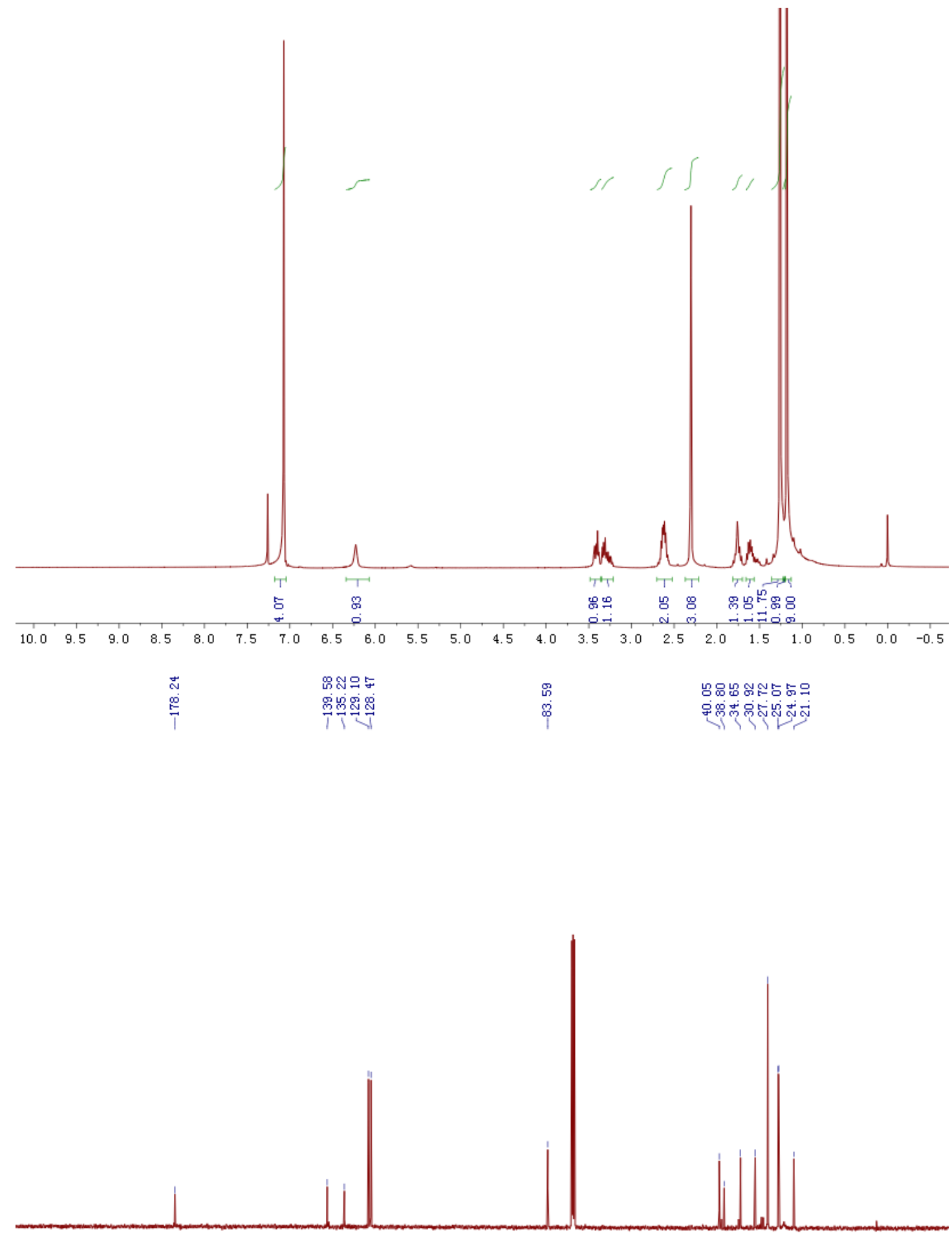

$\begin{array}{llllllllllllllllllllllll}210 & 200 & 190 & 180 & 170 & 160 & 150 & 140 & 130 & 120 & 110 & 100 & 90 & 80 & 70 & 60 & 50 & 40 & 30 & 20 & 10 & 0 & -10\end{array}$ 
<smiles>COc1ccc(CCCC(Br)c2ccccc2)cc1</smiles>
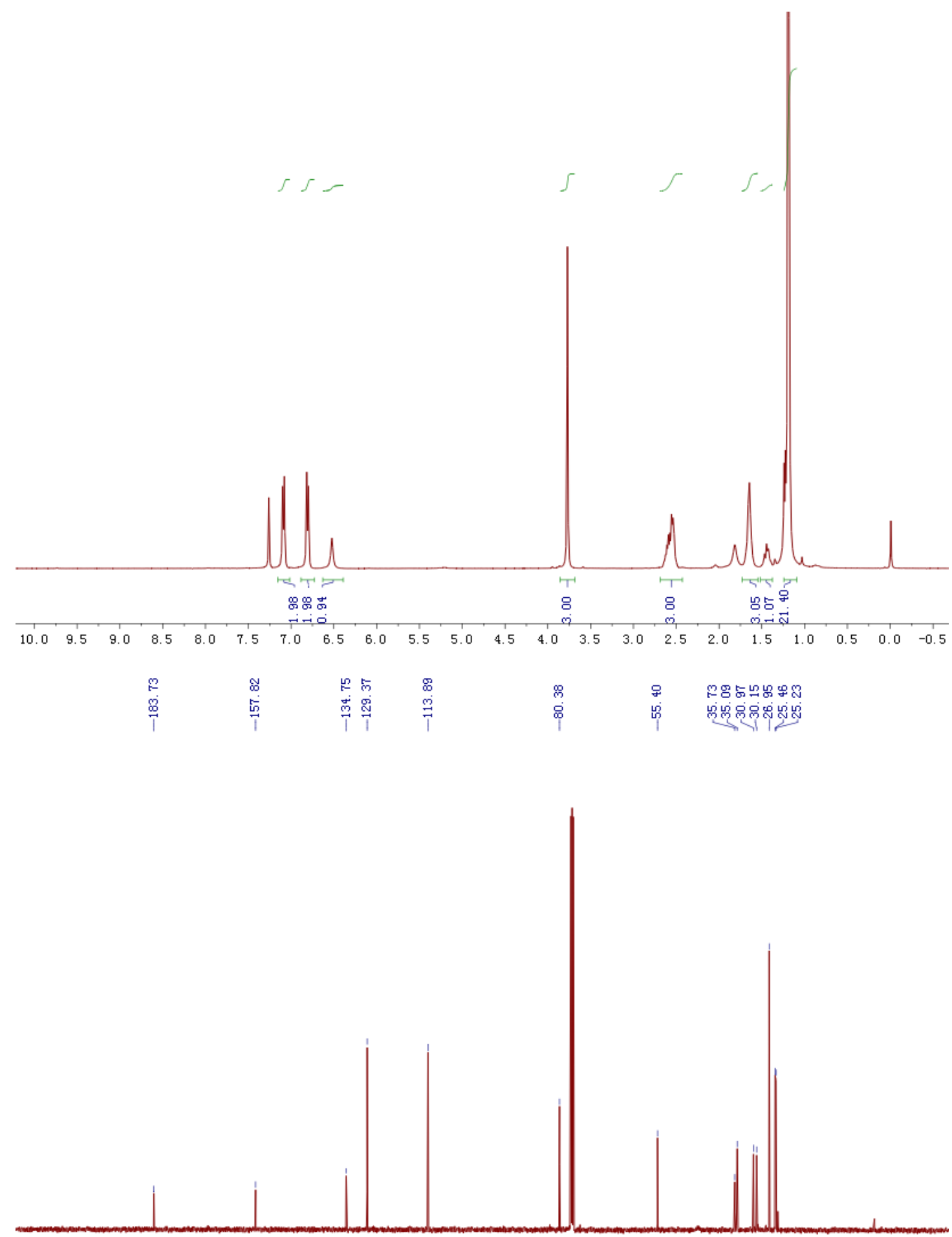

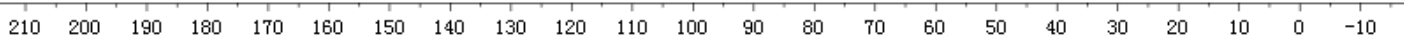



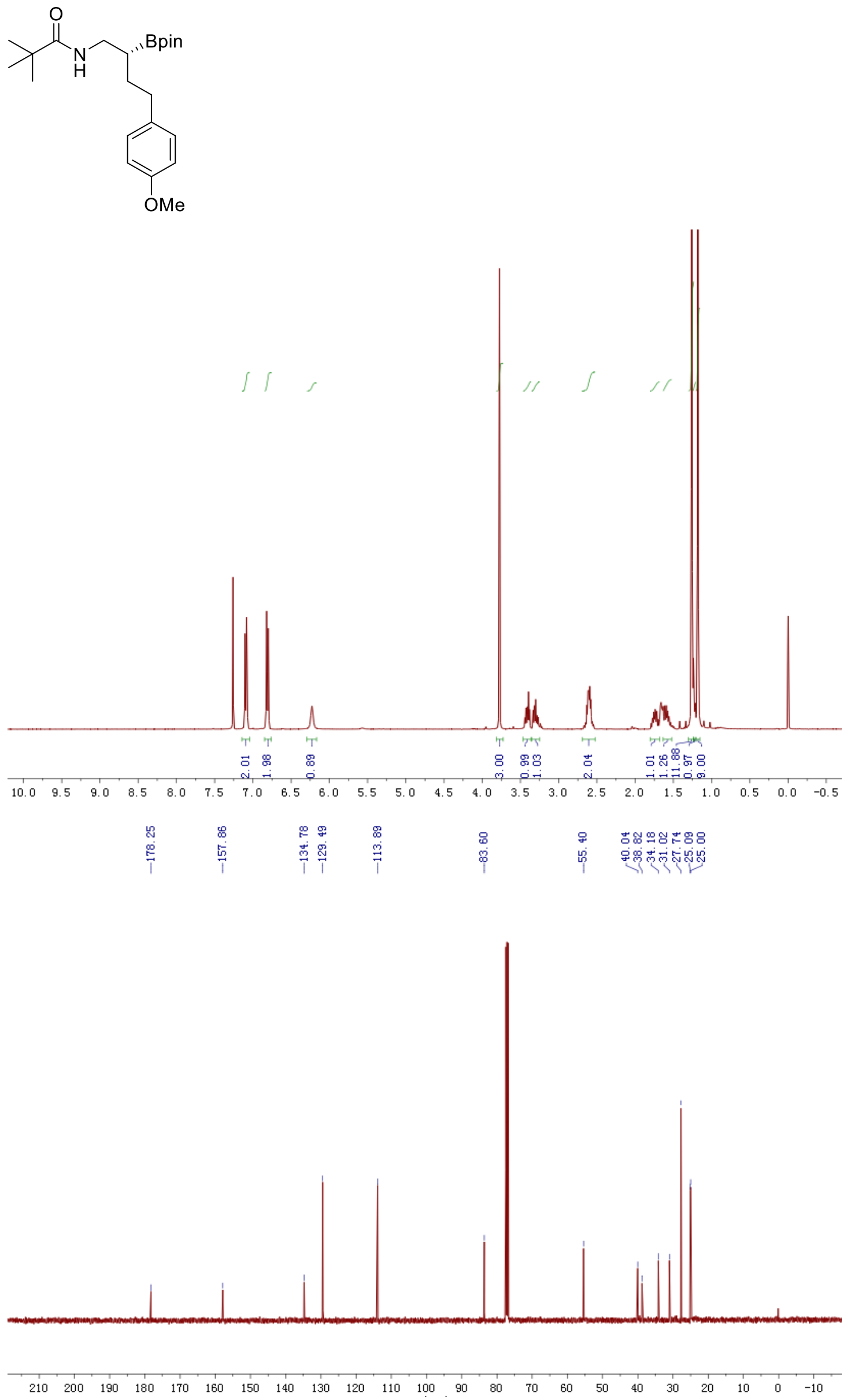

S245 
<smiles>COc1ccc(CCCC(Cc2ccccc2)NC(=O)C(C)(C)C)cc1</smiles>
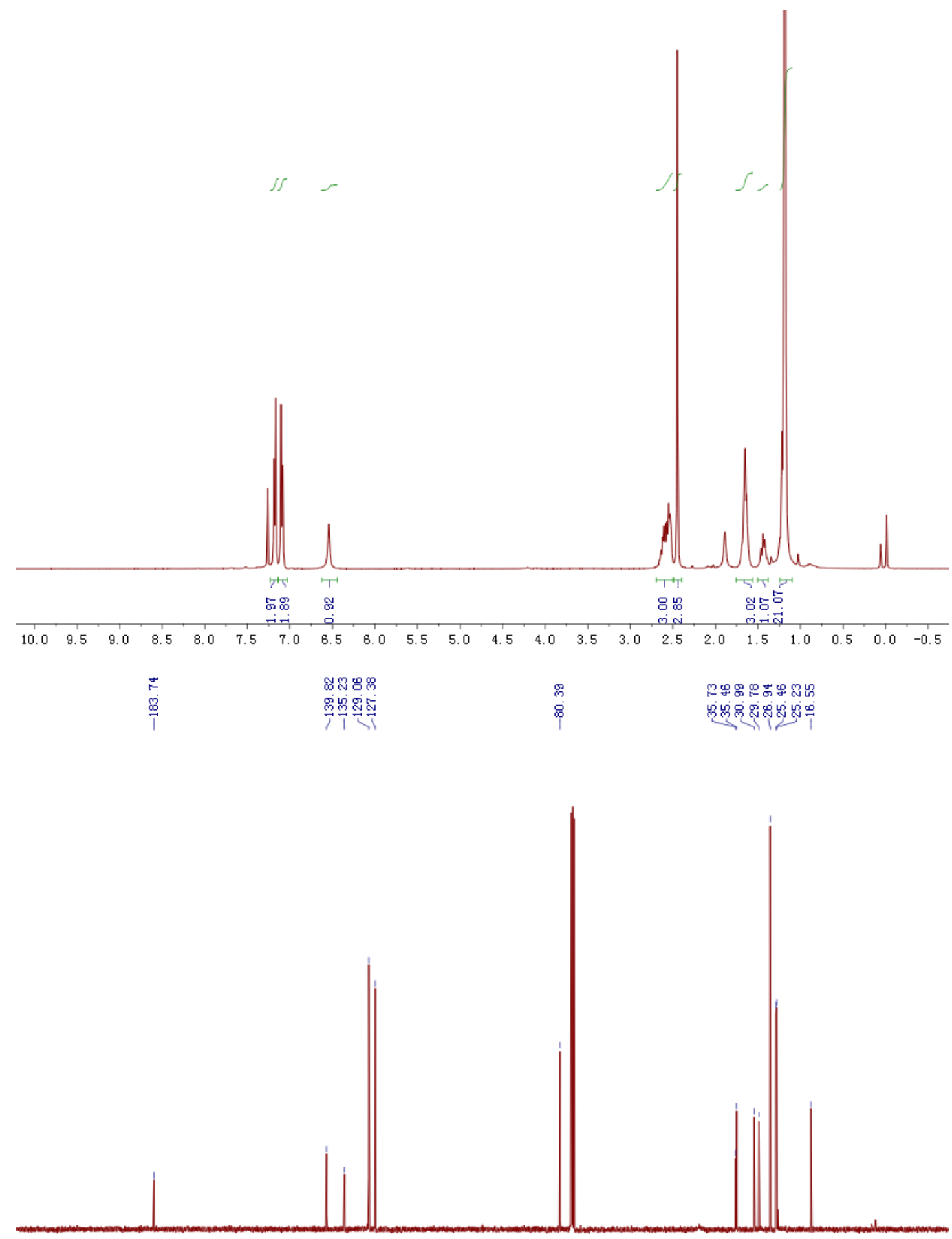

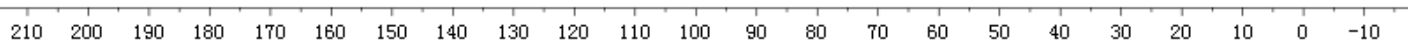


<smiles>COc1ccc(CCC(CNC(=O)C(C)(C)C)Cc2ccccc2)cc1</smiles>
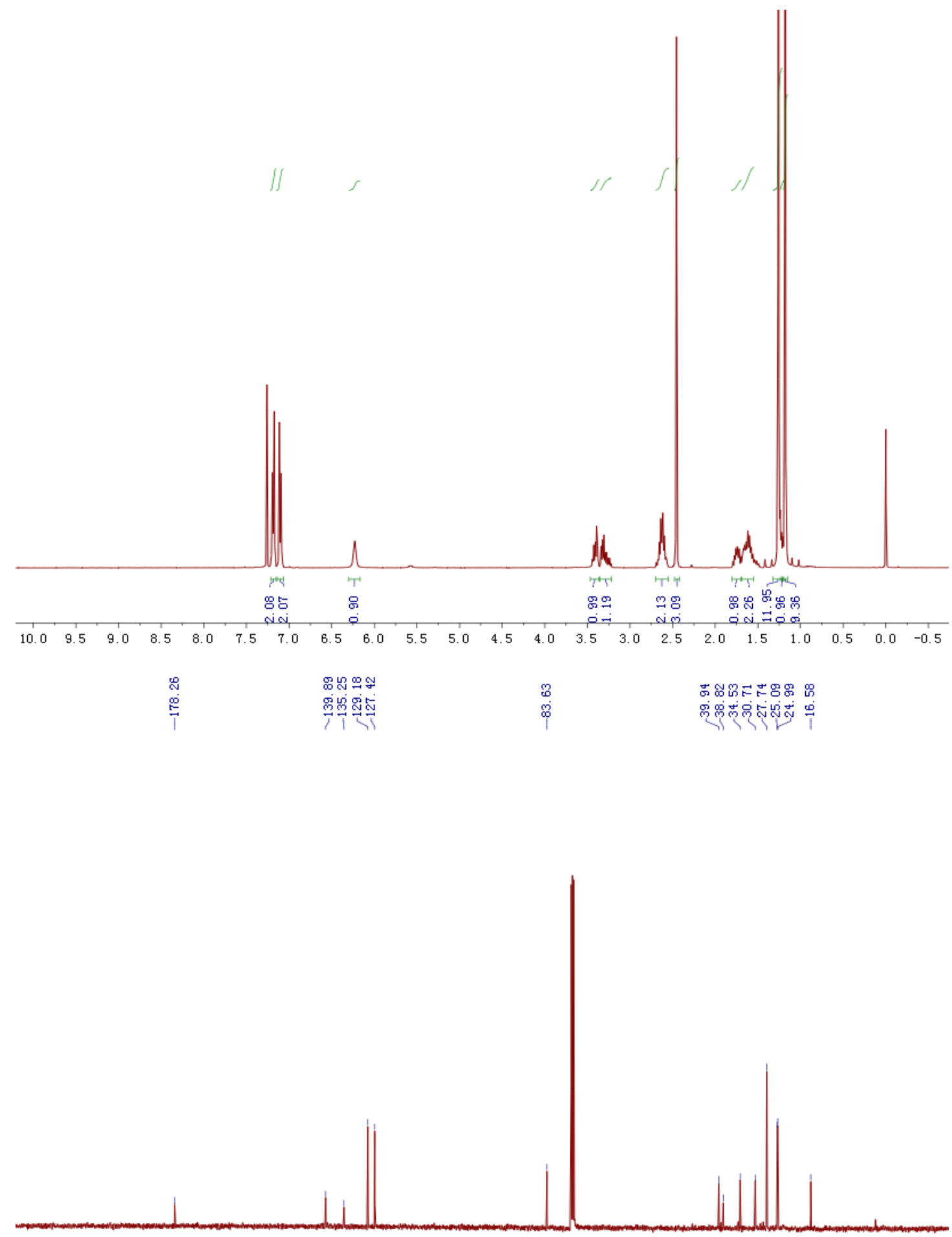

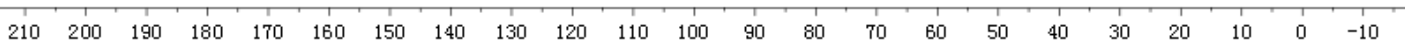



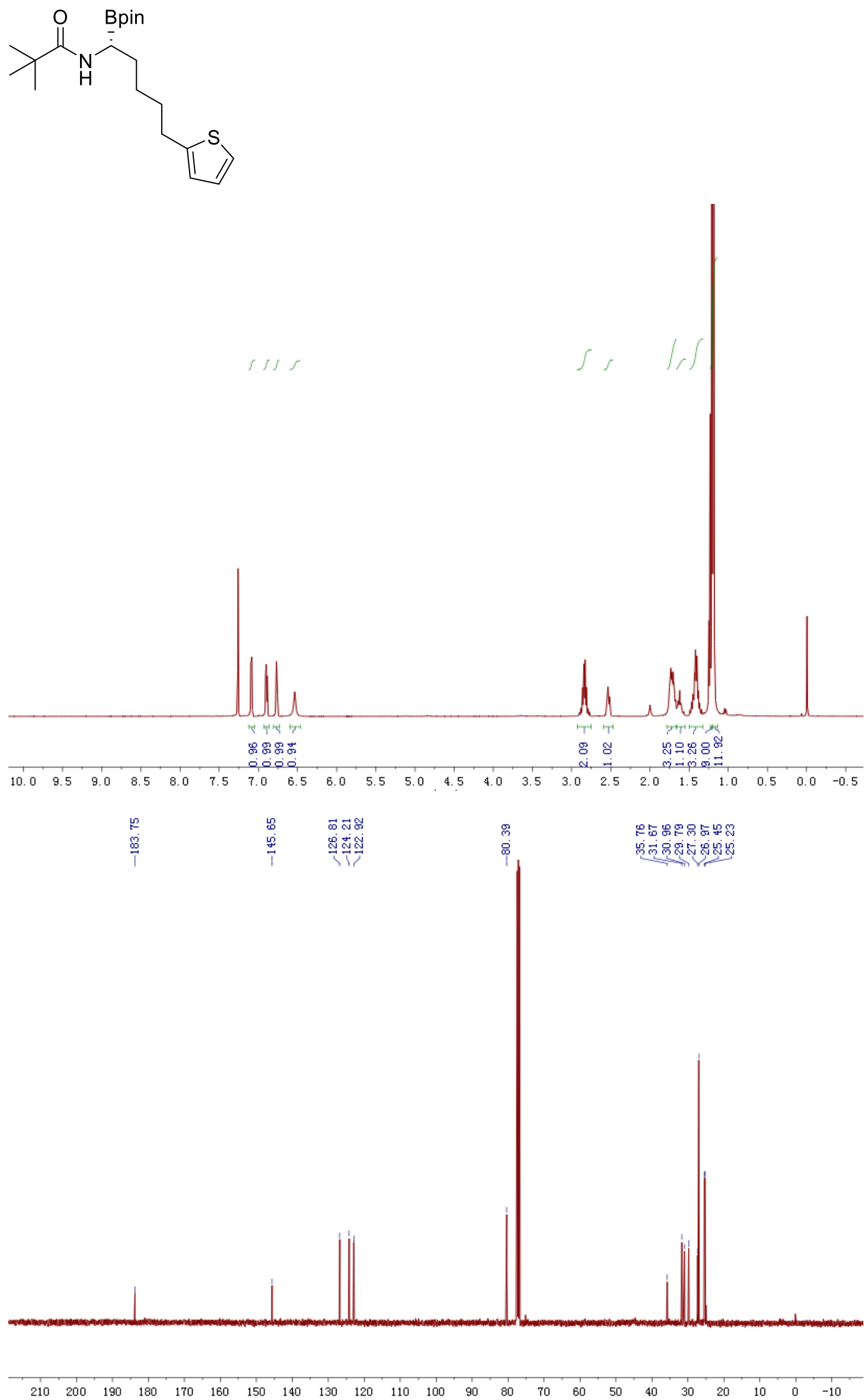

S248 

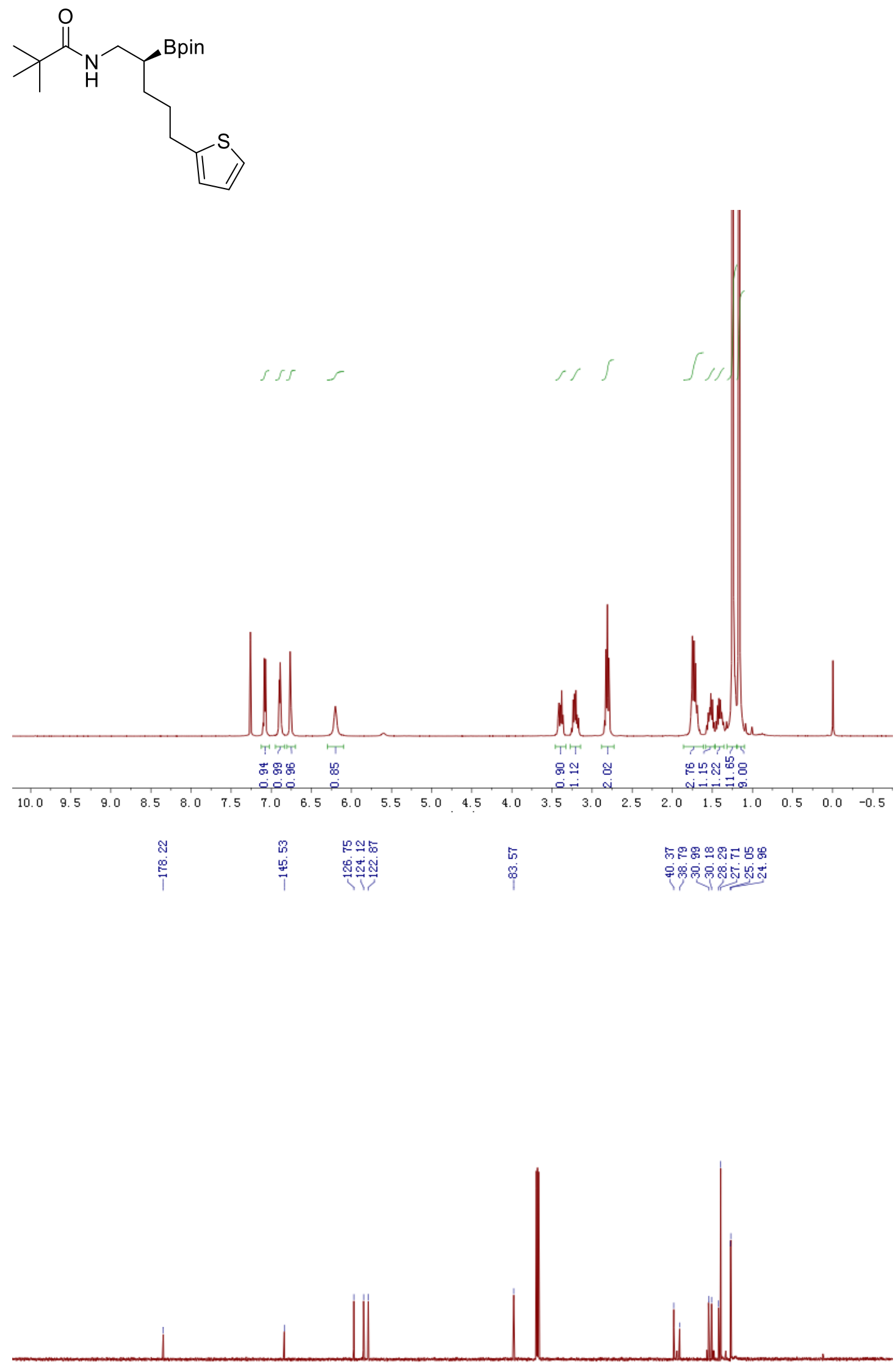

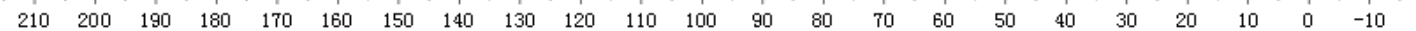



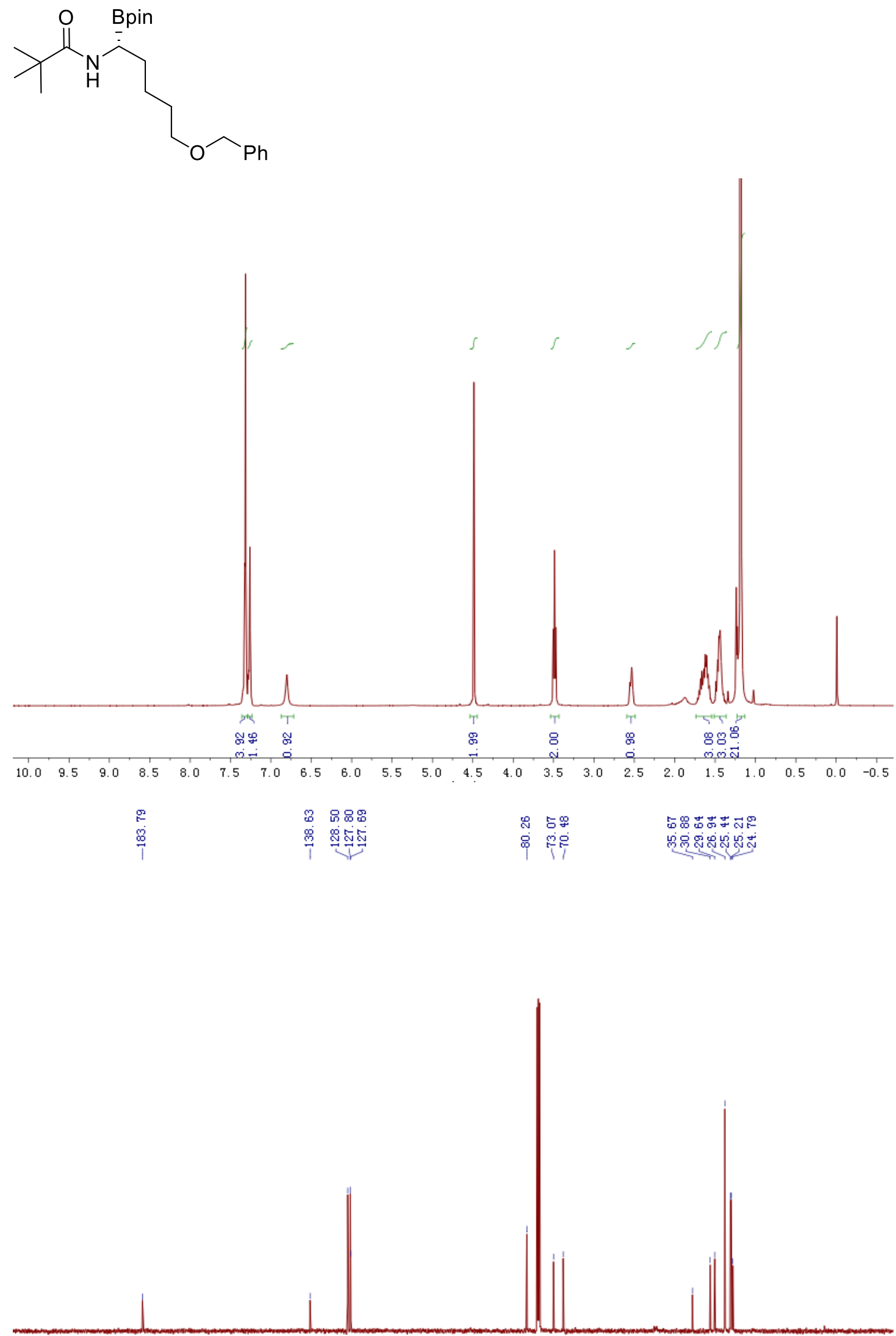

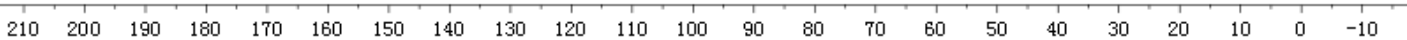


<smiles>CC(C)(C)C(=O)NC[C@H](CCCOCc1ccccc1)Cc1ccccc1</smiles>
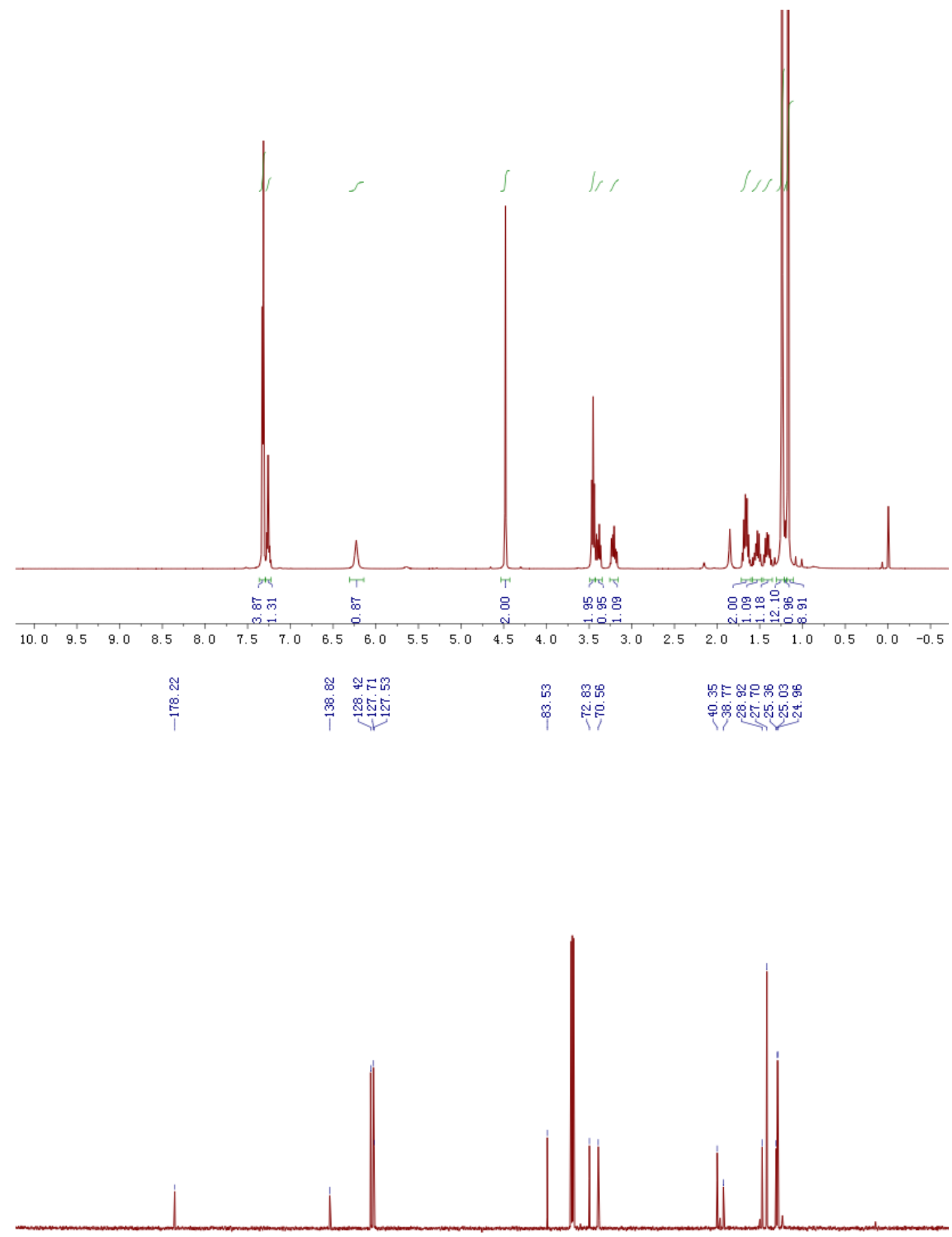

\begin{tabular}{lllllllllllllllllllllllll}
\hline & 10 & 200 & 190 & 180 & 170 & 160 & 150 & 140 & 130 & 120 & 110 & 100 & 90 & 80 & 70 & 60 & 50 & 40 & 30 & 20 & 10 & 0 & -10
\end{tabular} 
<smiles>CC([Pb])(CCBr)NC(=O)C(C)(C)C</smiles>
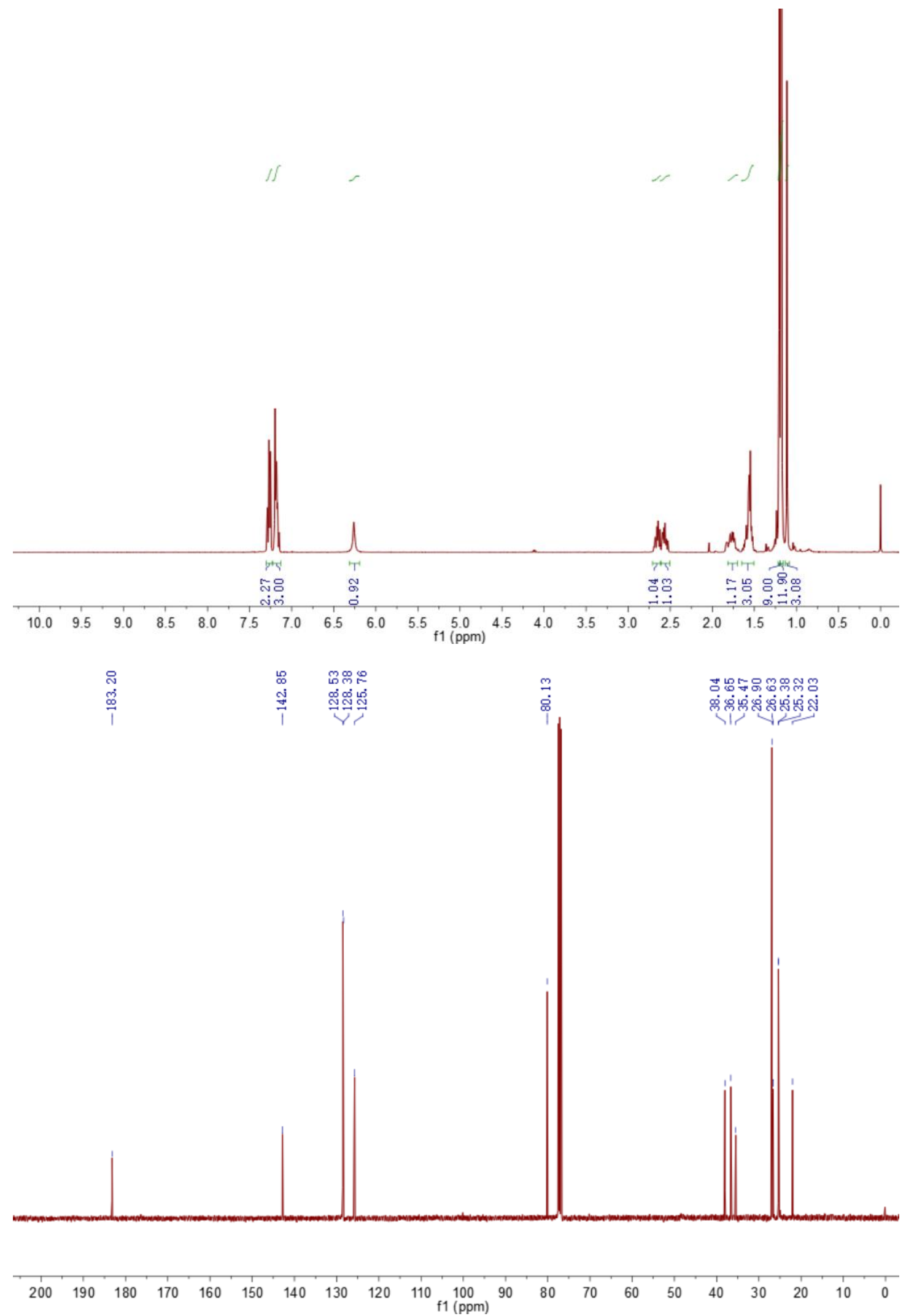
<smiles>CC[C@@H]([18OH])c1ccc(C(F)(F)F)cc1</smiles>
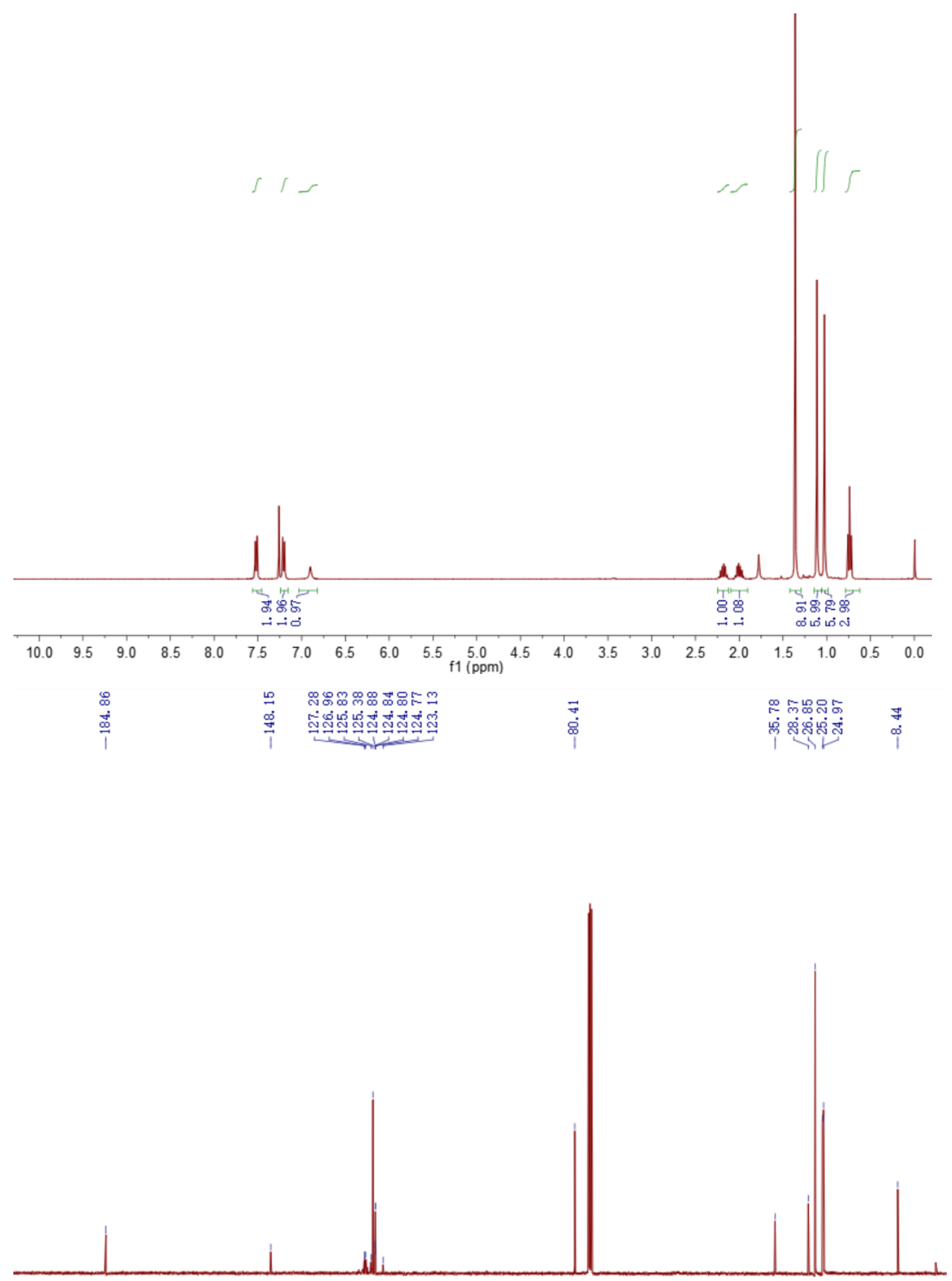

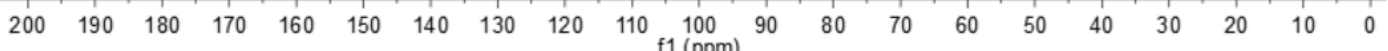


<smiles></smiles>

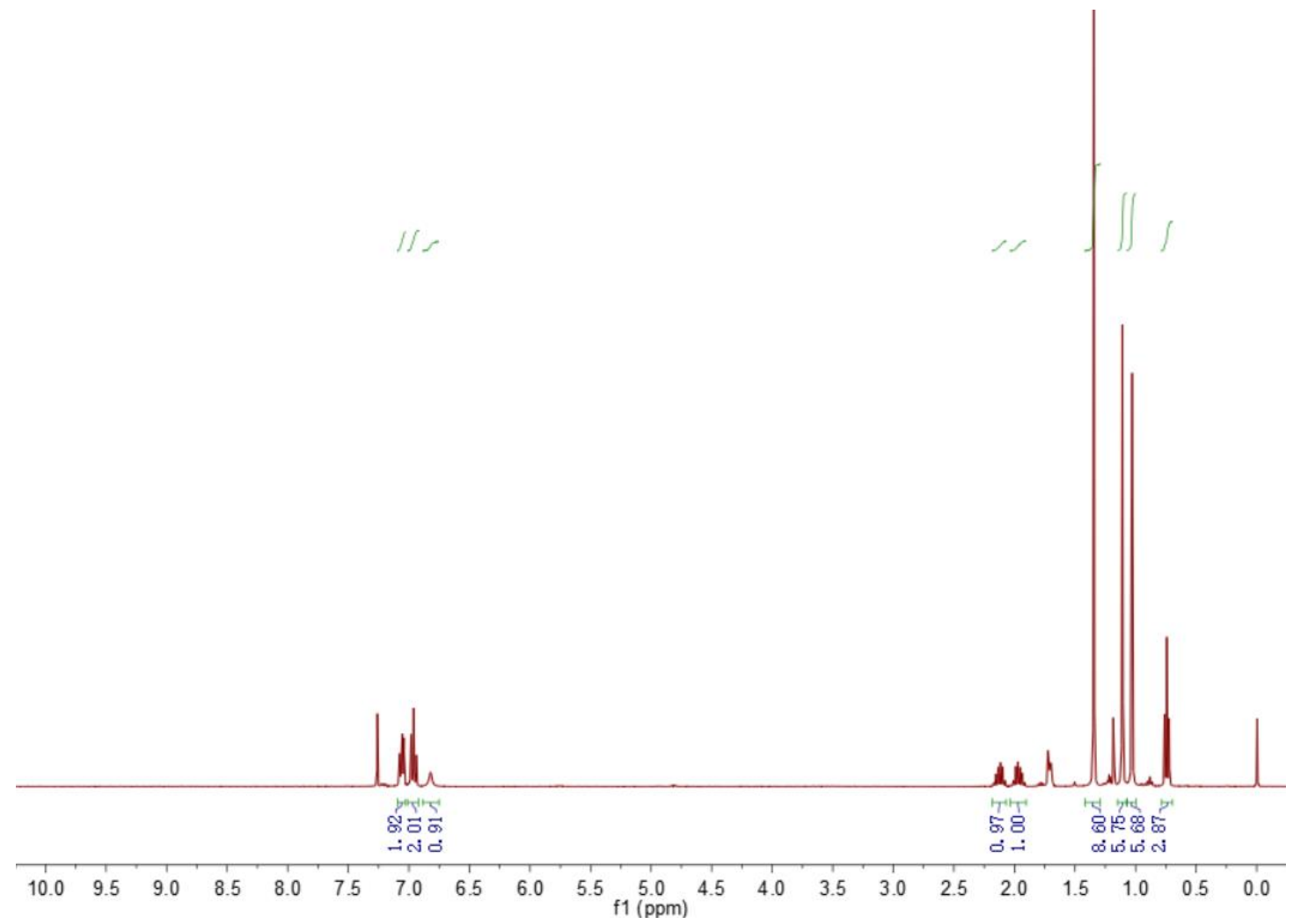

을

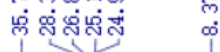


<smiles>CCCC([18CH])(CC)c1cccc(OC)c1</smiles>

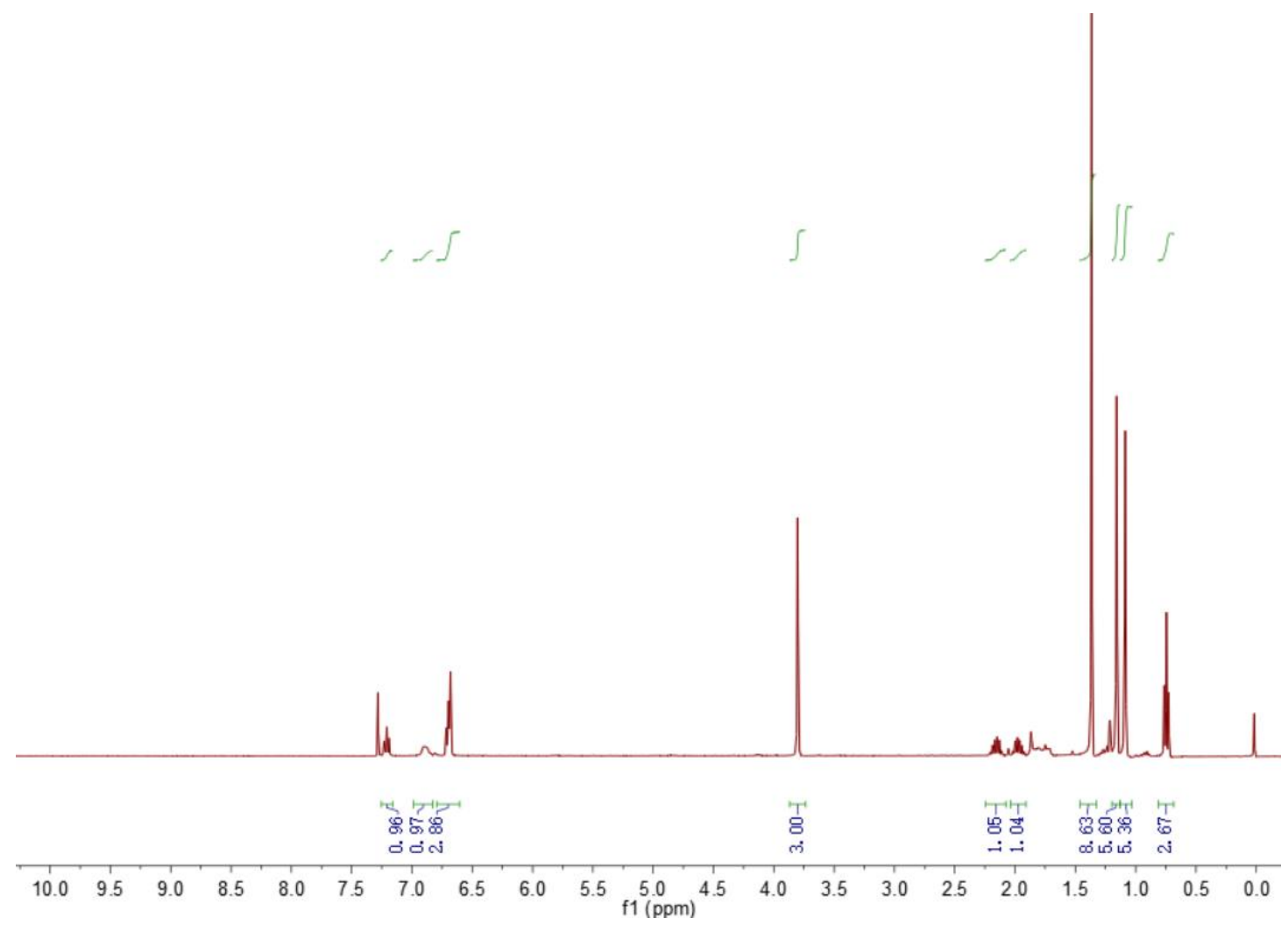

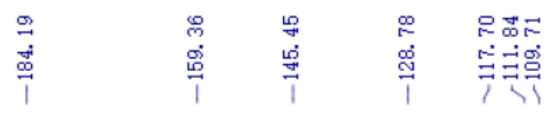

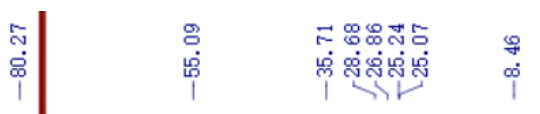

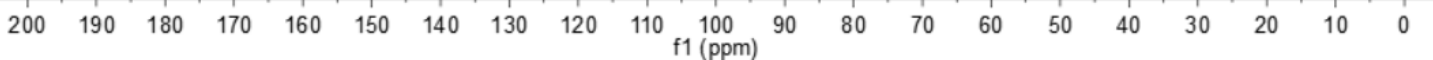


<smiles>CC[C@@H](C(=O)NC(=O)C(C)(C)C)c1ccccc1</smiles>

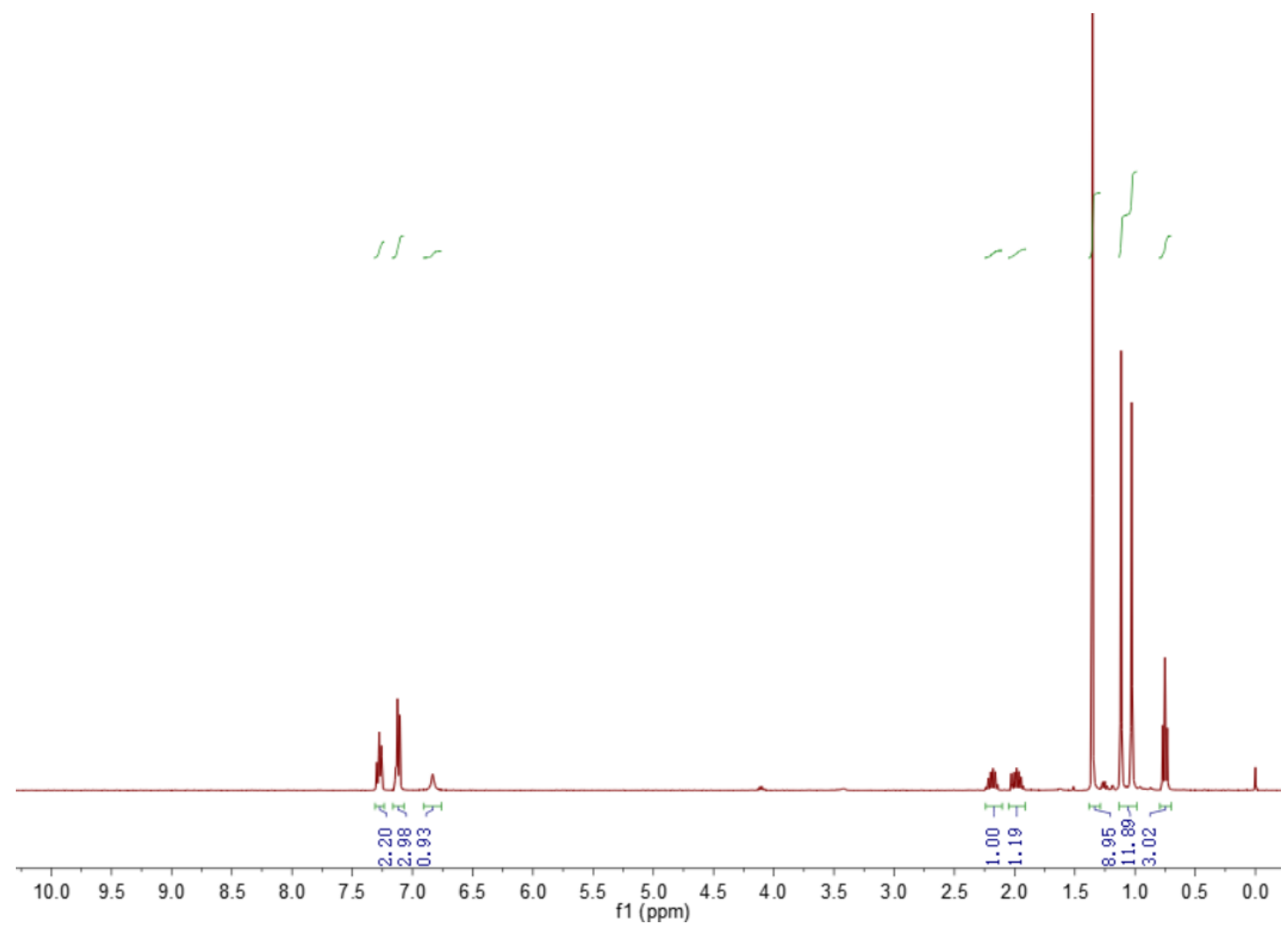

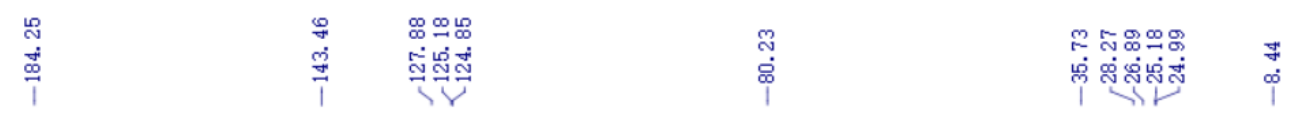

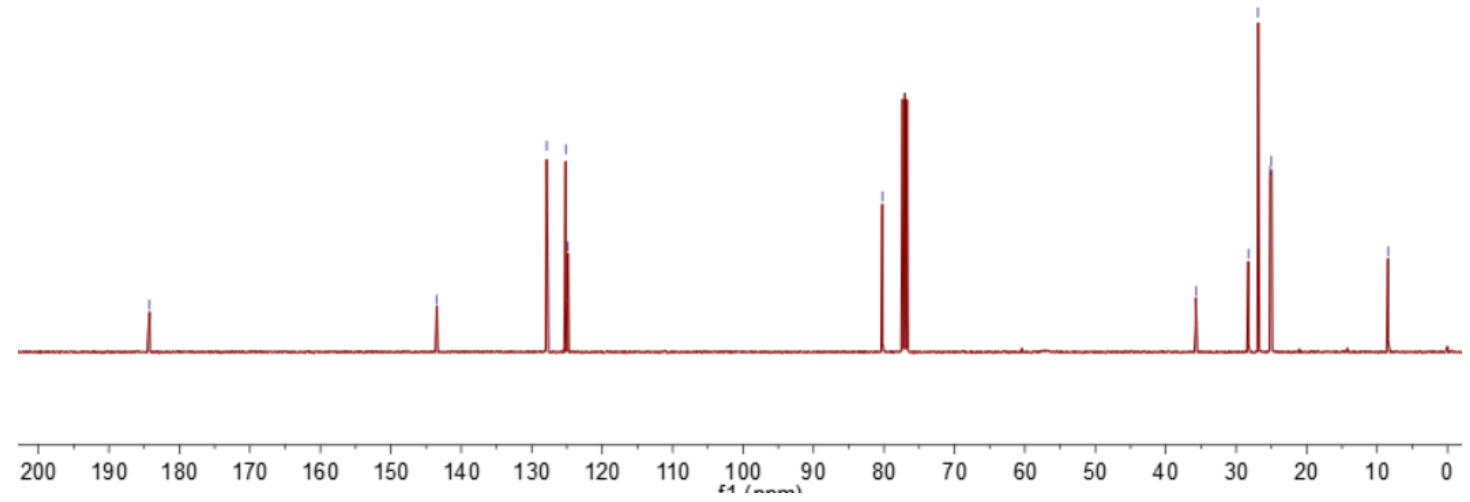


<smiles>CCC(Br)(Br)c1ccccc1</smiles>
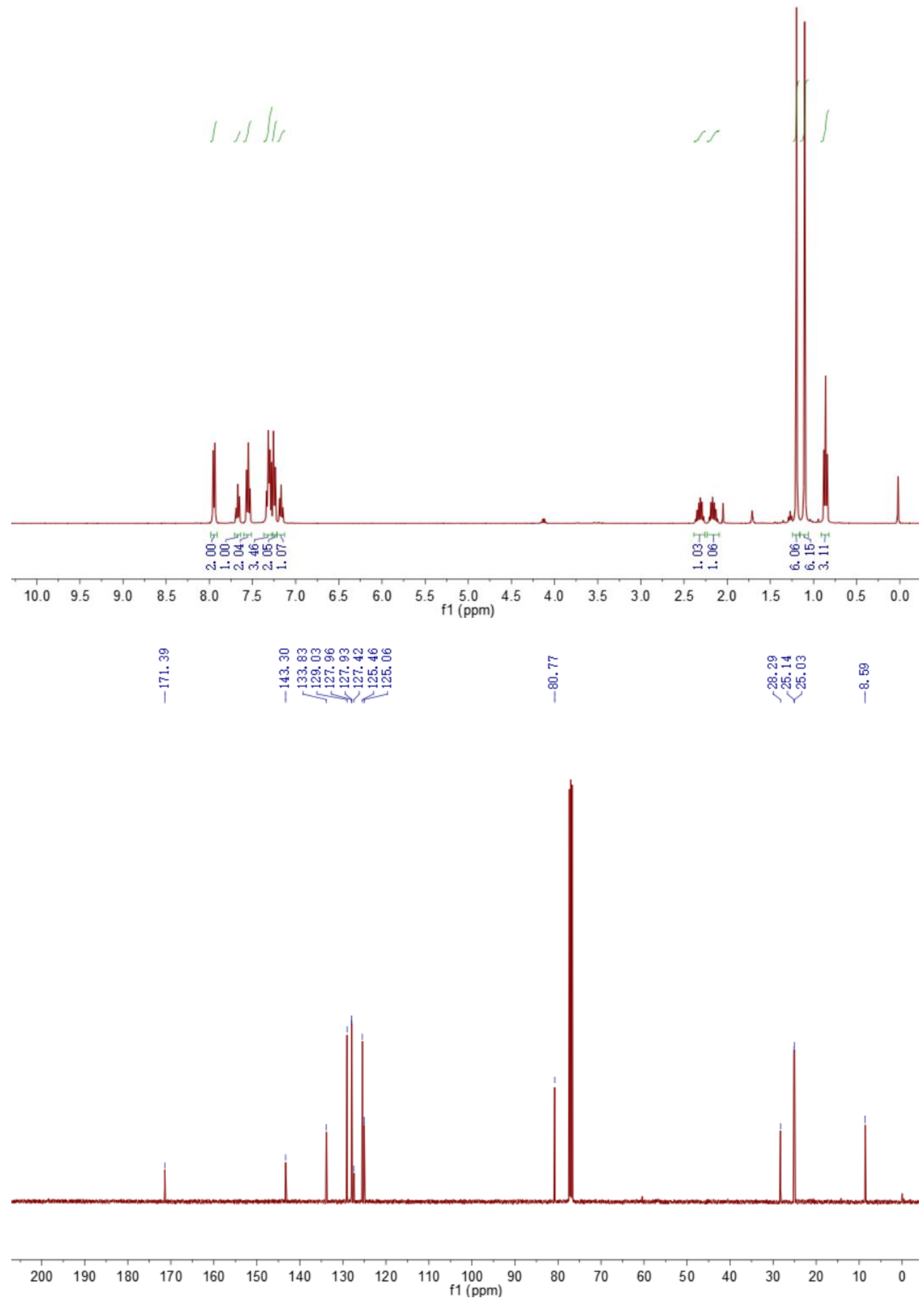
<smiles>CCC[C@](C)(Cc1ccccc1)NC(=O)c1ccc(Cl)cc1</smiles>
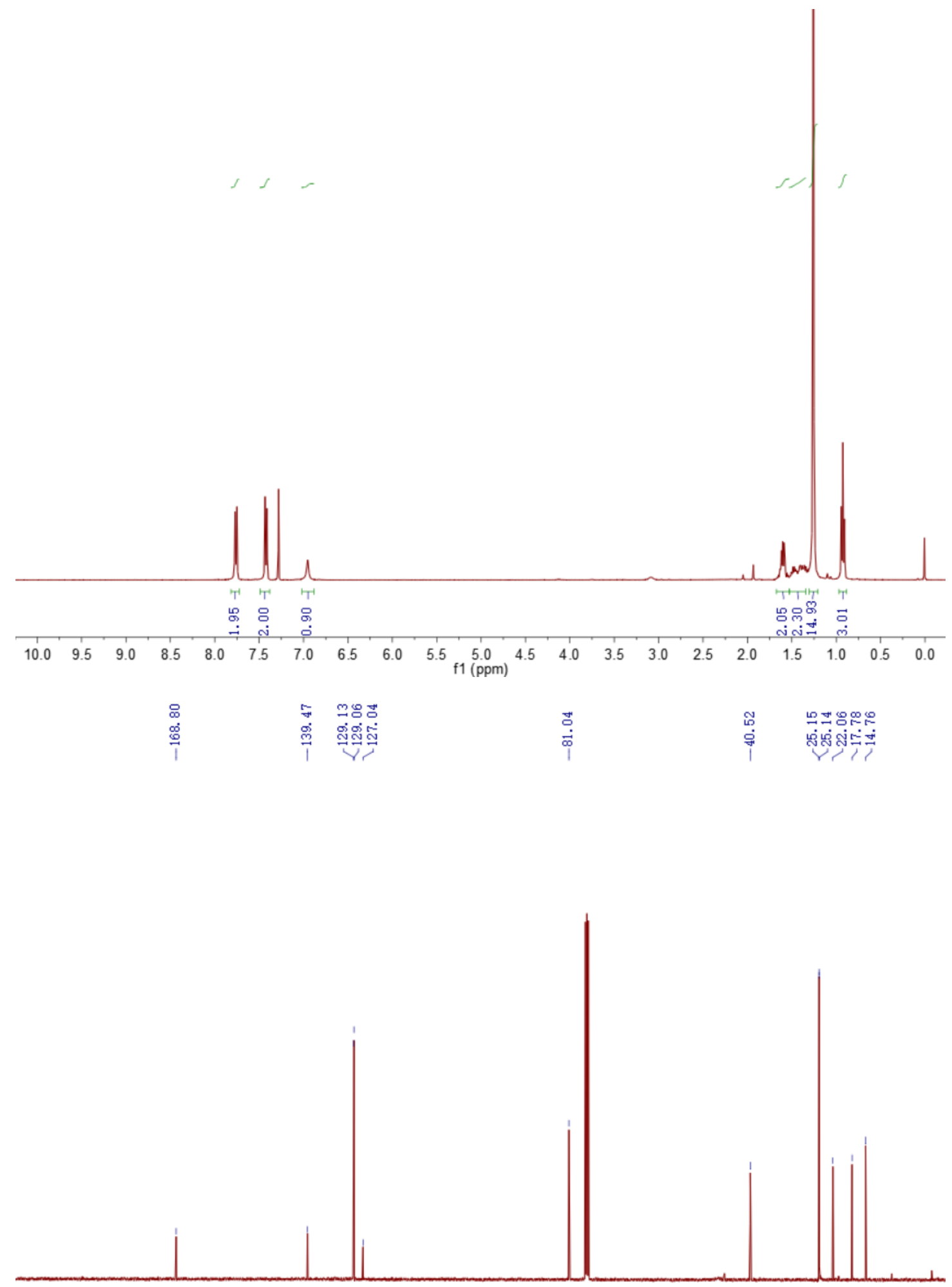

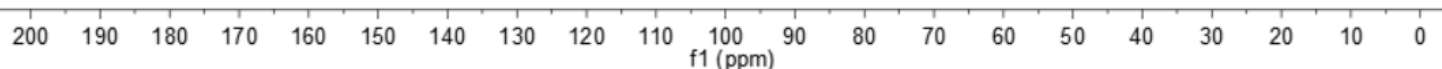


<smiles>CCC(Cc1ccccc1)(NC(=O)c1ccc(Cl)cc1)c1ccccc1</smiles>
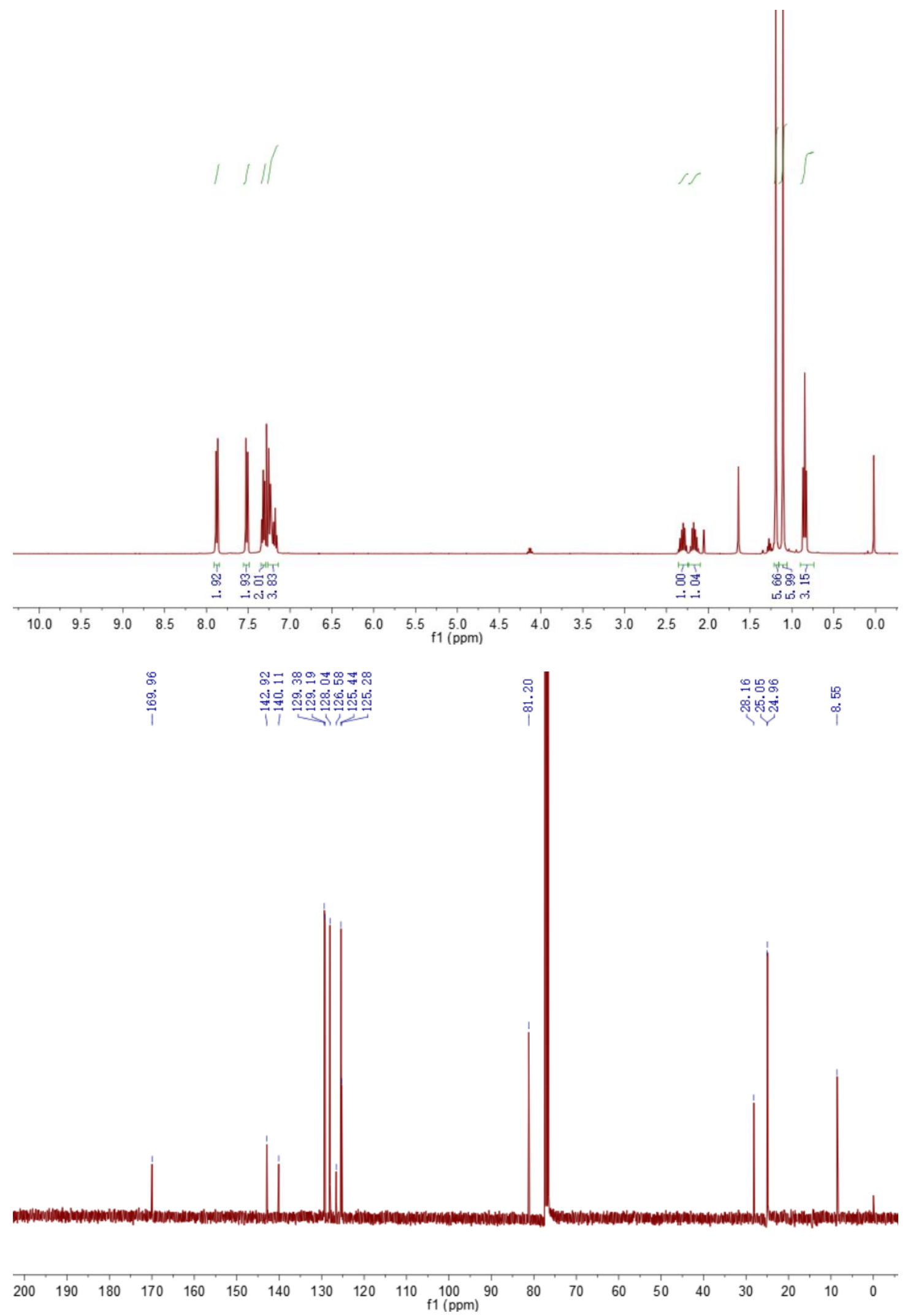
<smiles>[M]C(Cc1ccccc1)C(Cc1ccccc1)NC(=O)C(C)(C)C</smiles>
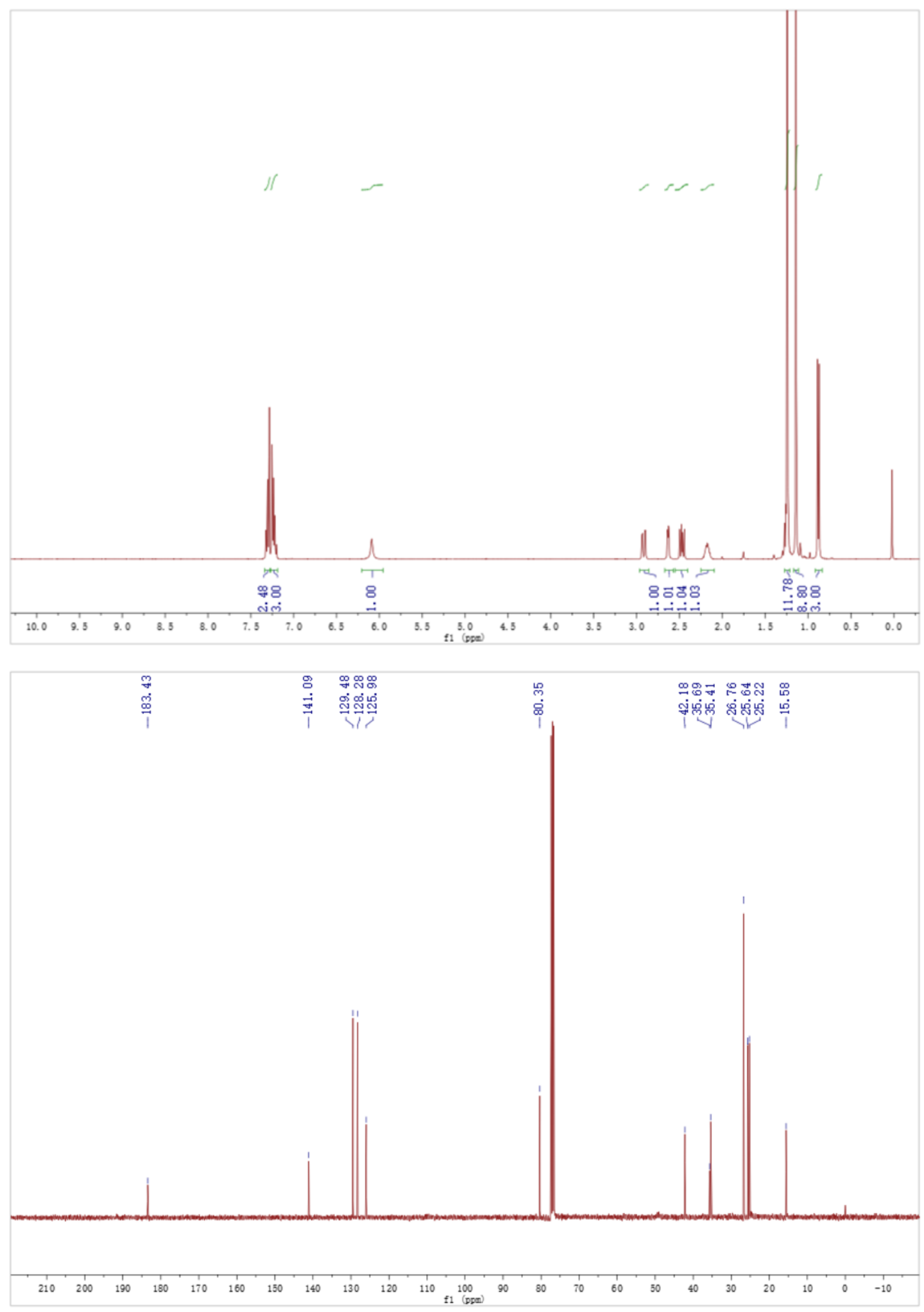

S260 
<smiles>CC(Cc1ccc(Cl)cc1)C(Cc1ccccc1)NC(=O)C(C)(C)C</smiles>
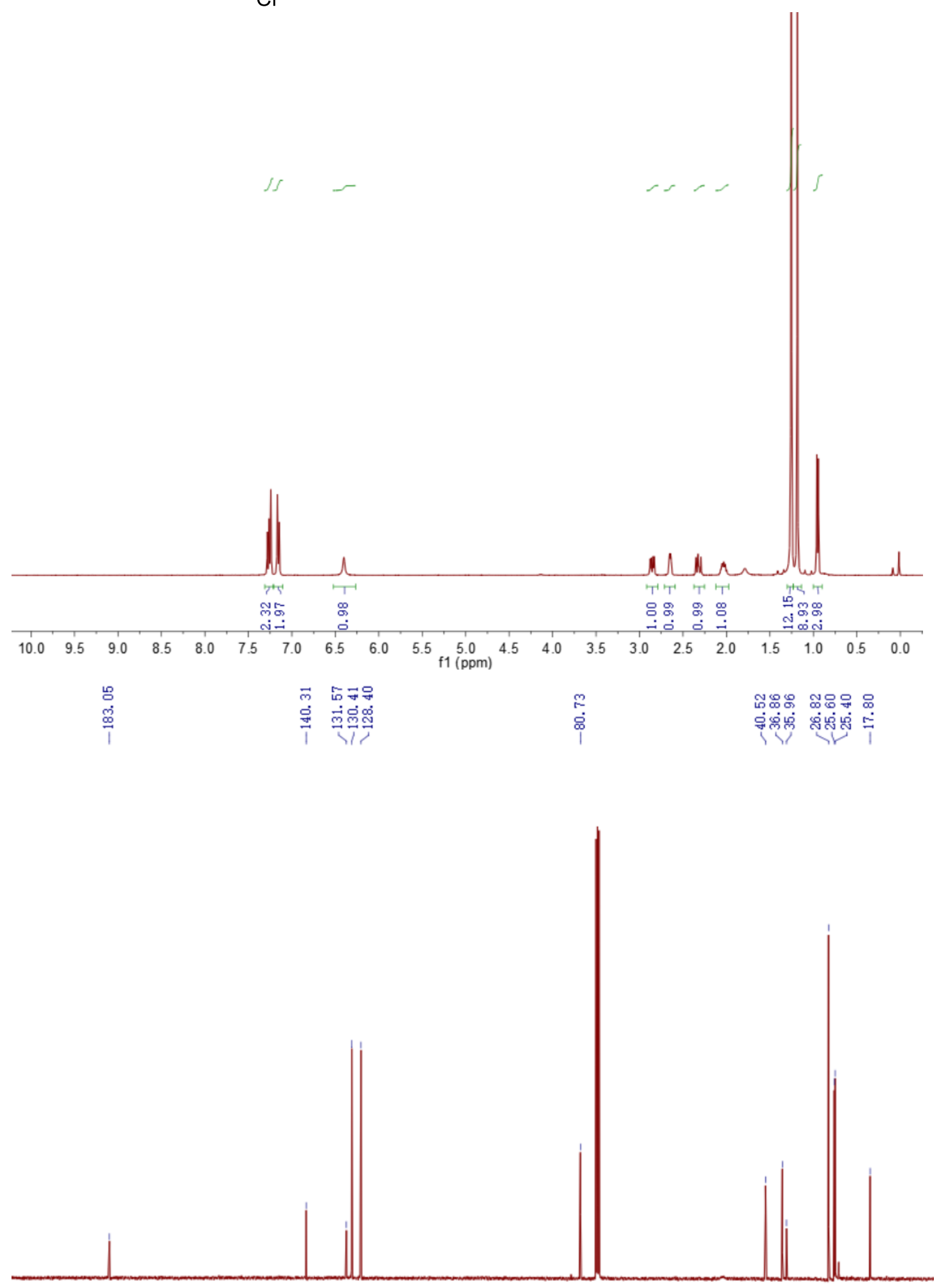

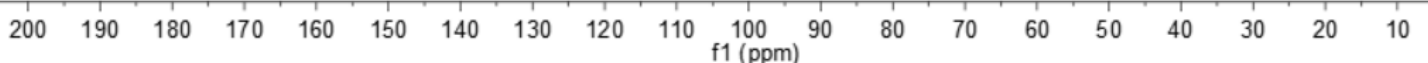


<smiles>C[C@H](CBr)[C@@H]([10CH2]c1ccccc1)NC(=O)C(C)(C)C</smiles>
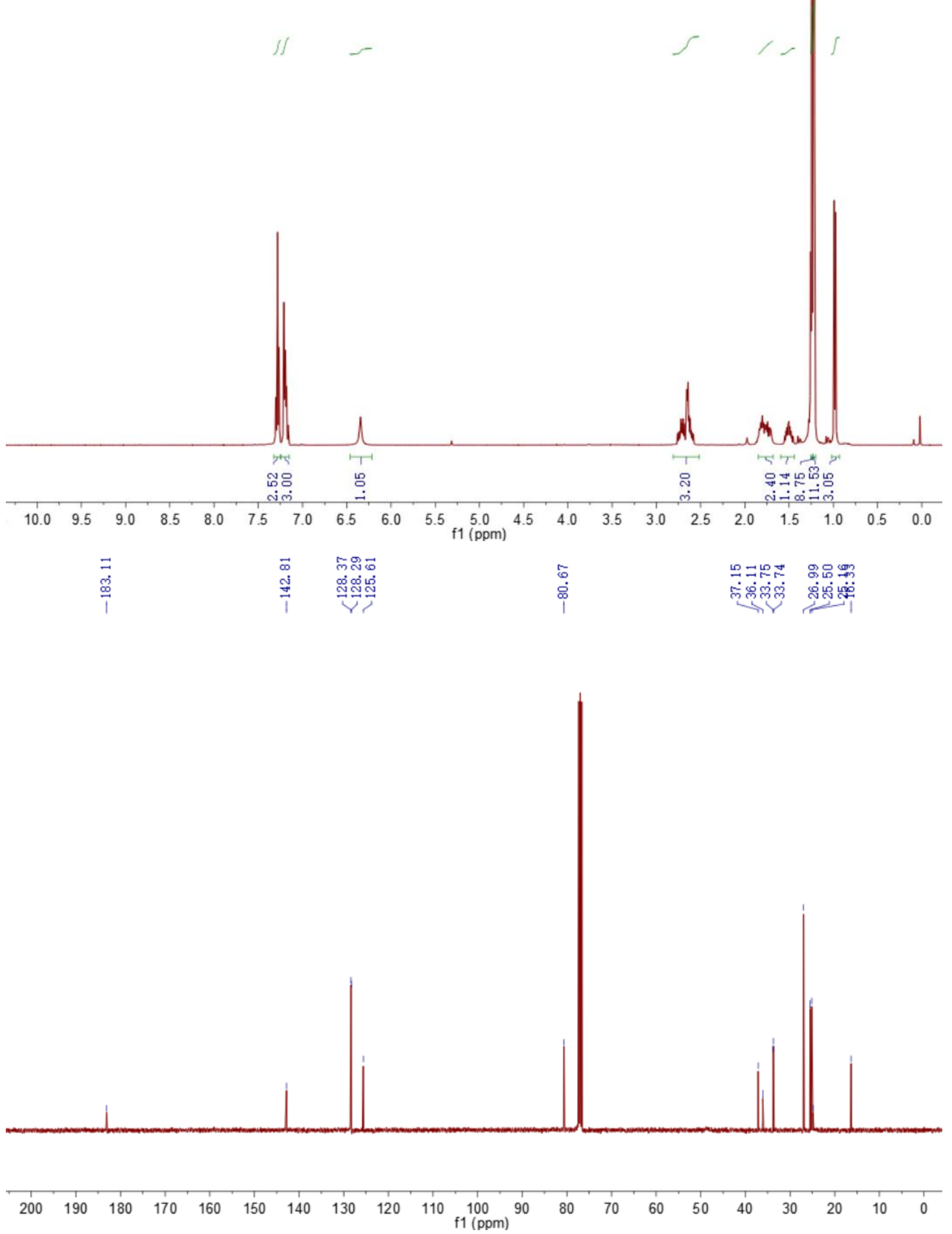

S262 
<smiles>O=C(NC(Cc1ccccc1)C(P)Cc1ccccc1)c1ccccc1</smiles>
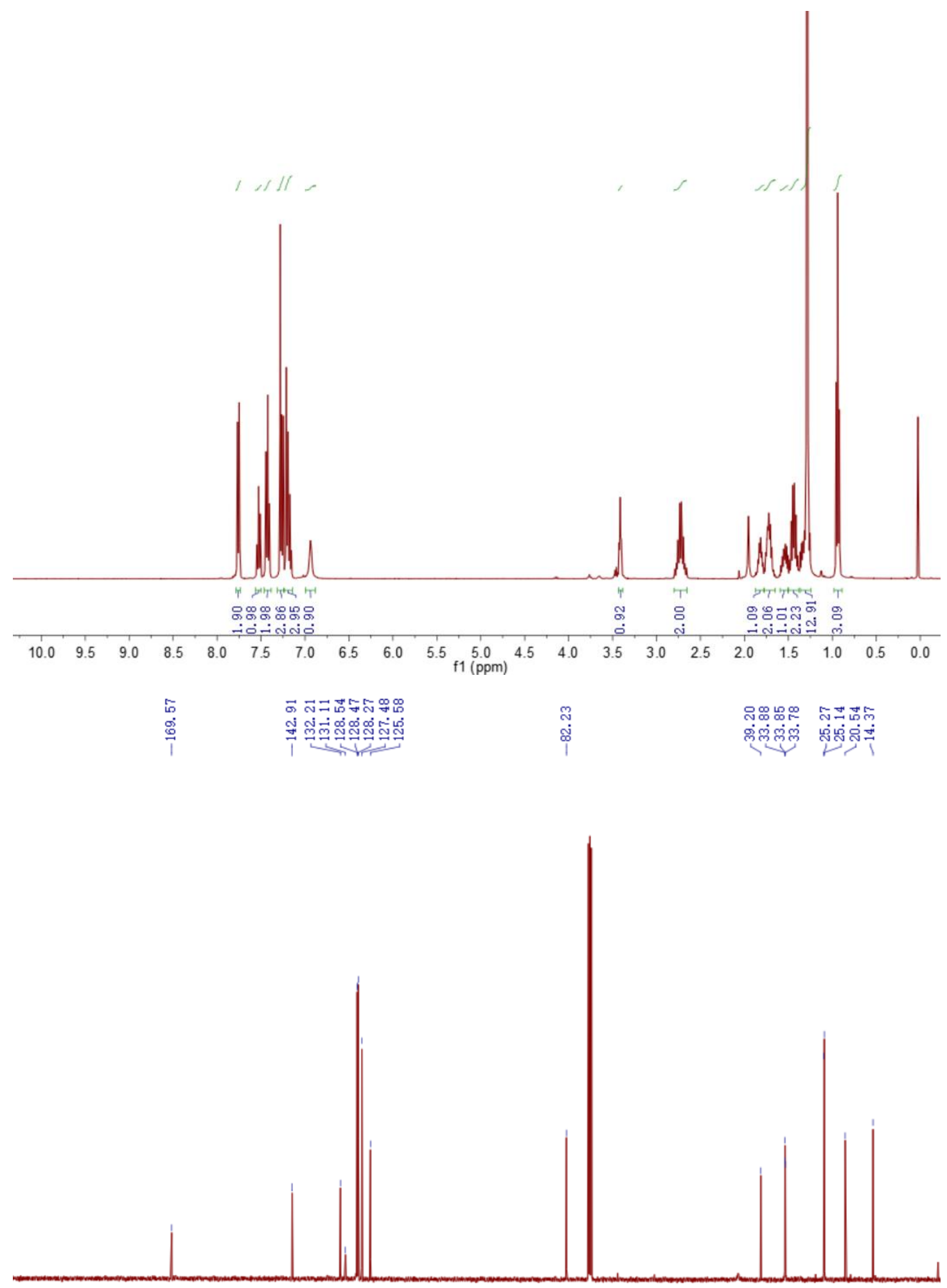

$\begin{array}{llllllllllllllllllllllllllllllll}200 & 190 & 180 & 170 & 160 & 150 & 140 & 130 & 120 & 110 & 100 & 90 & 80 & 70 & 60 & 50 & 40 & 30 & 20 & 10 & 0\end{array}$ 
<smiles>CC(CCCCl)C(Cc1ccccc1)NC(=O)c1ccccc1</smiles>
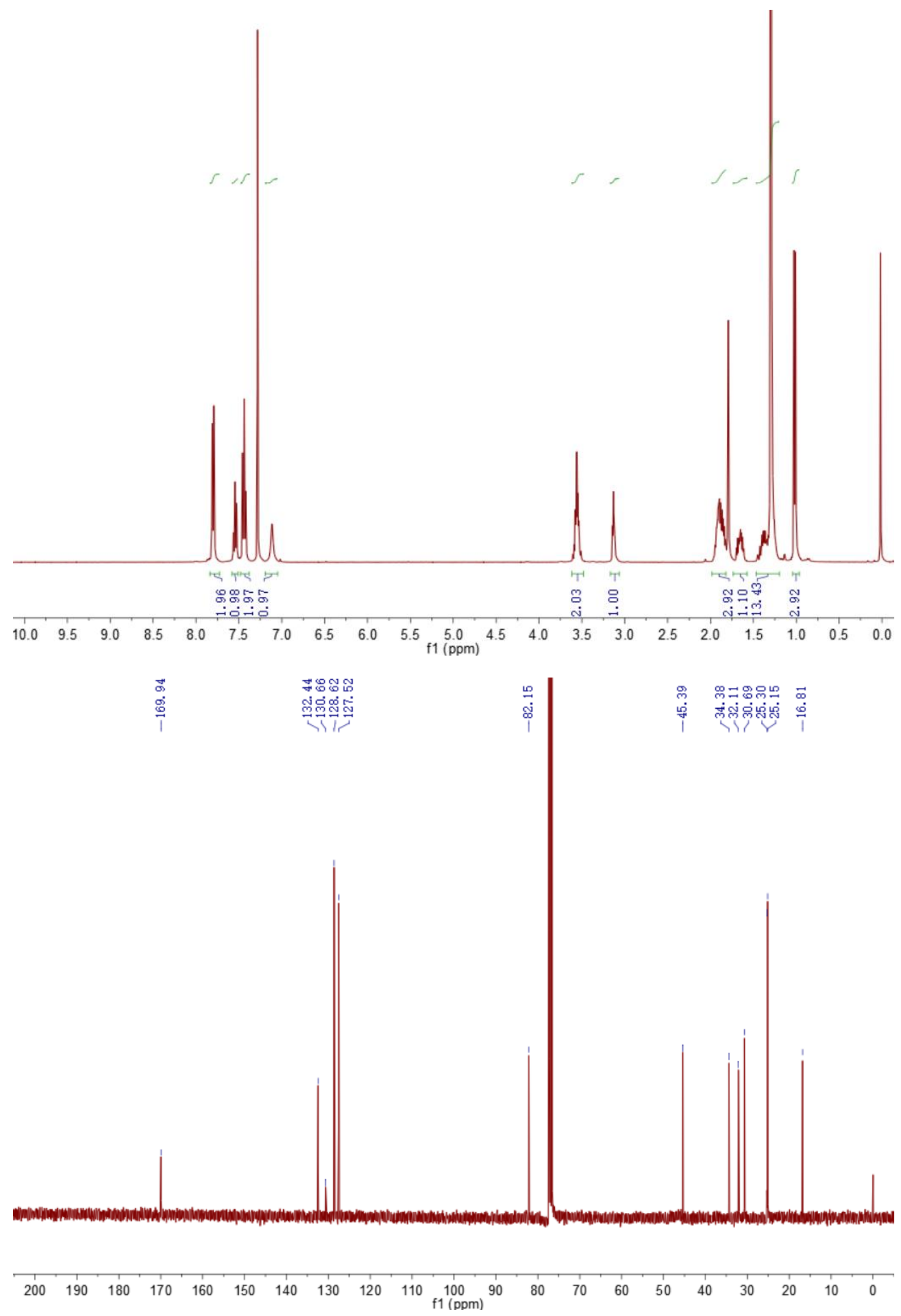
<smiles>[M]C(CCCC)C(Cc1ccccc1)NC(=O)c1ccccc1</smiles>
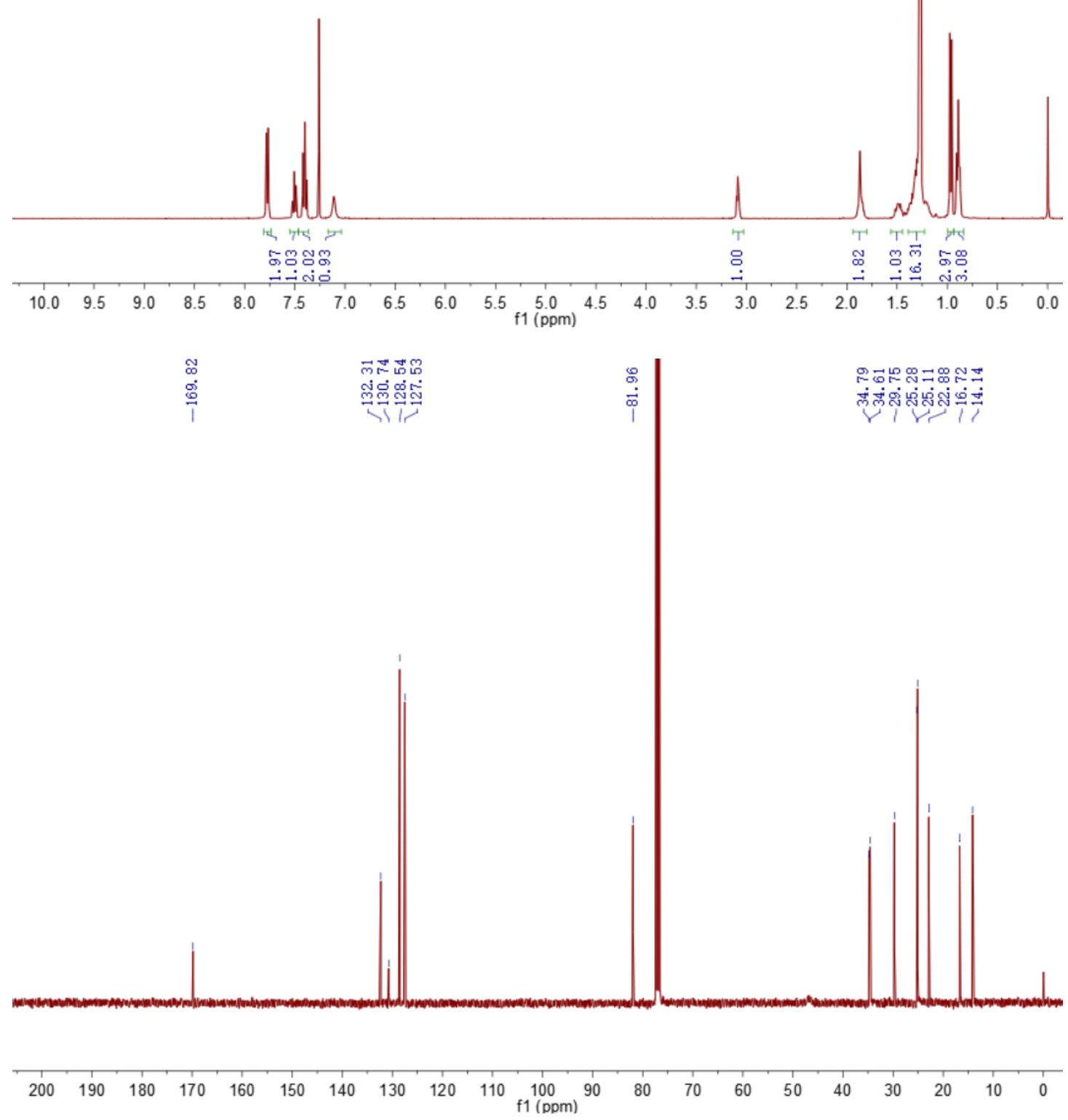
<smiles>CC(C1CCCCC1)C(Cc1ccccc1)NC(=O)c1ccccc1</smiles>

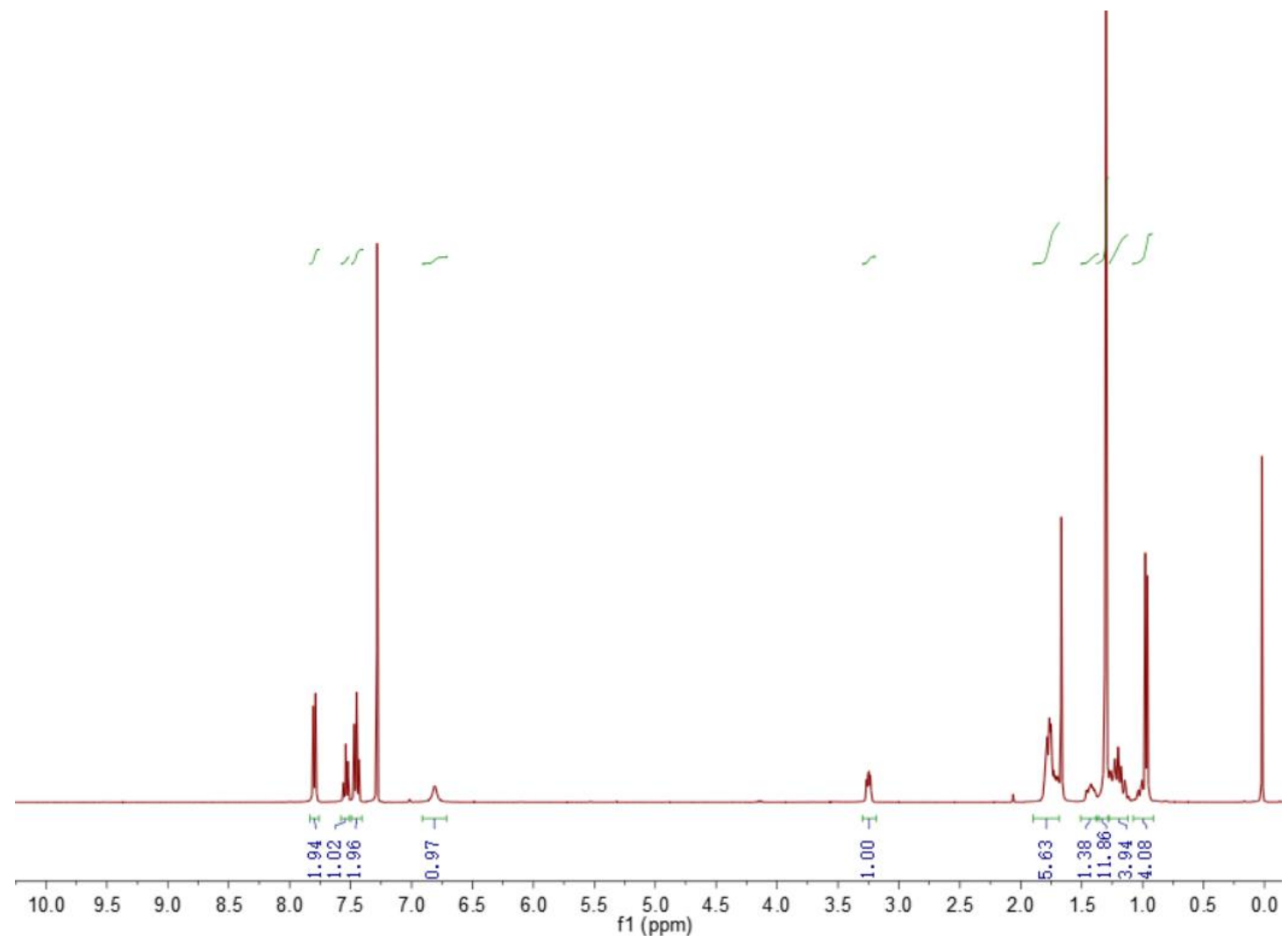

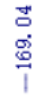

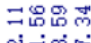

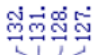

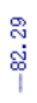

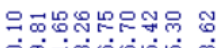
户்

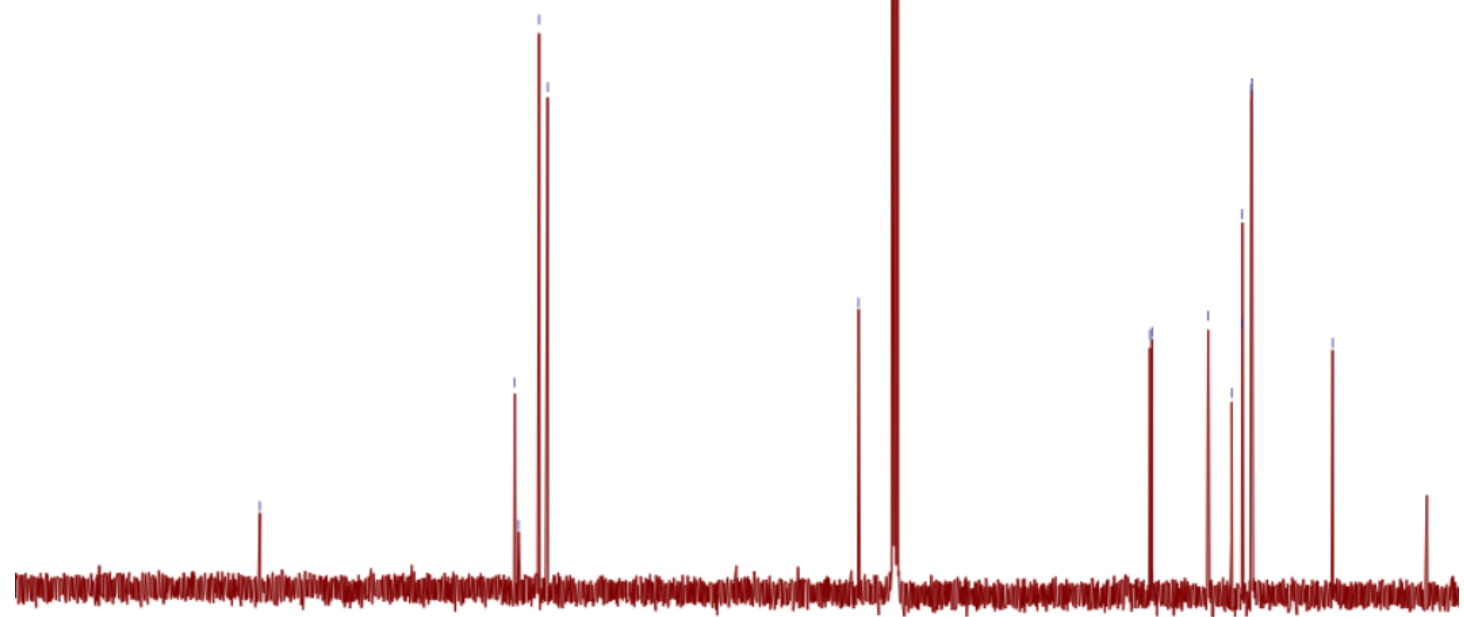

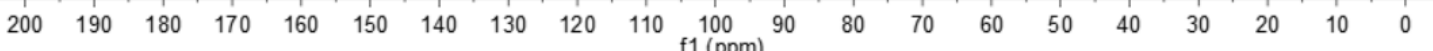


<smiles>O=C(NC(Cc1ccccc1)C1CCOCC1)c1ccccc1</smiles>
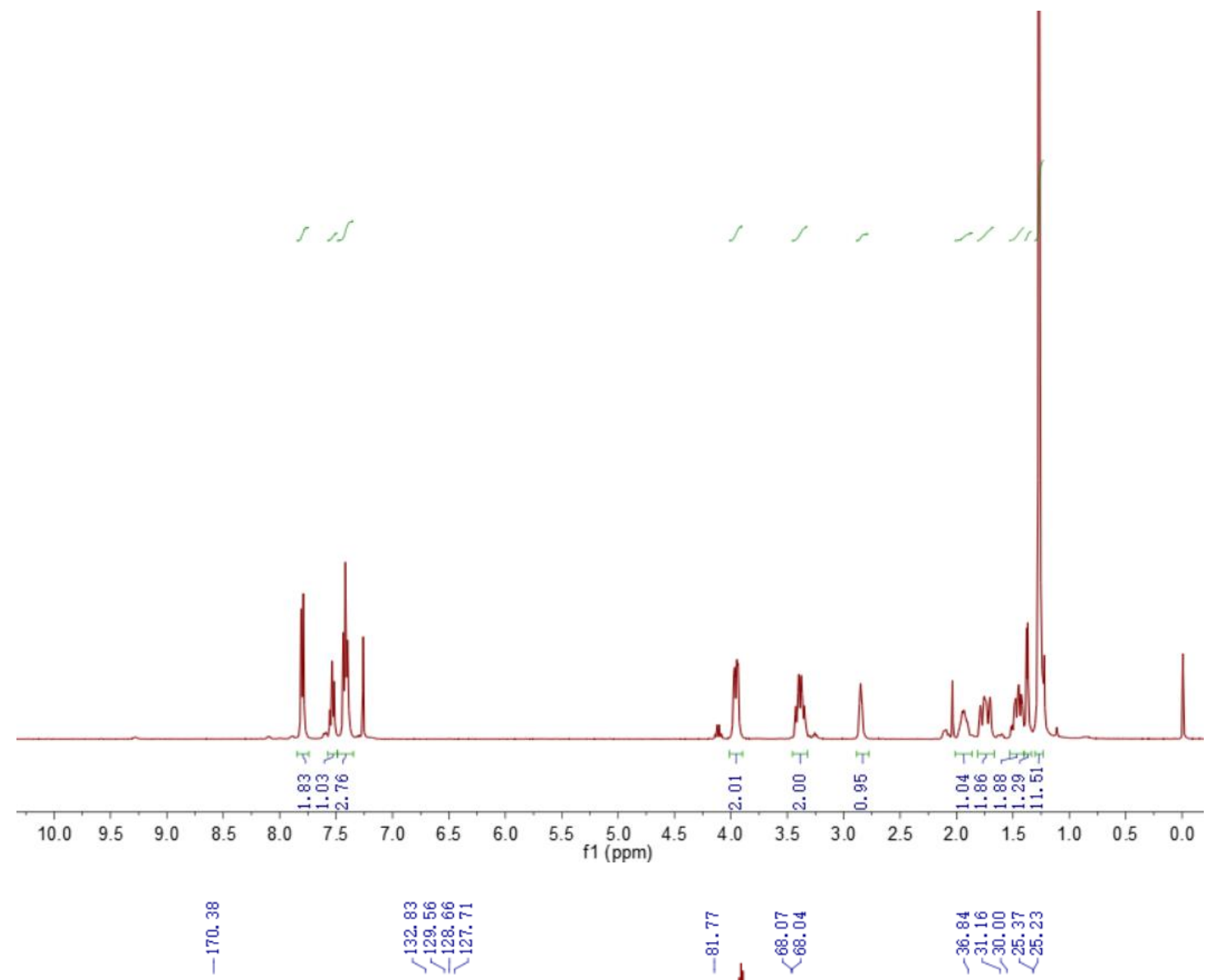

ڤ.

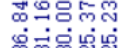

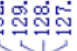

गुल

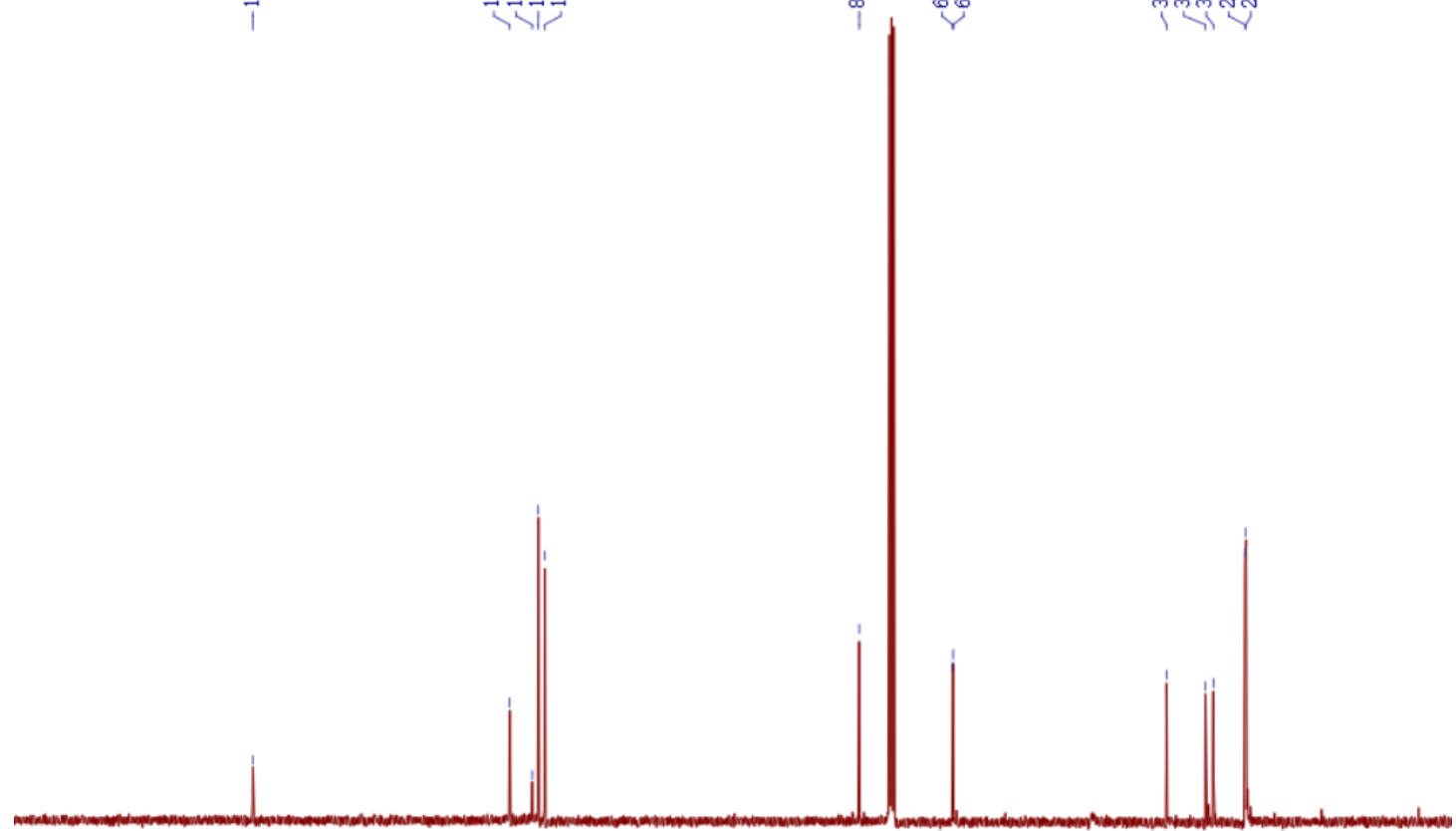

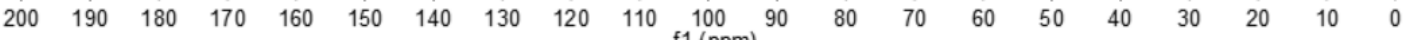


<smiles>O=C(NC([18CH]c1ccccc1)C1CCCCC1)c1ccccc1</smiles>
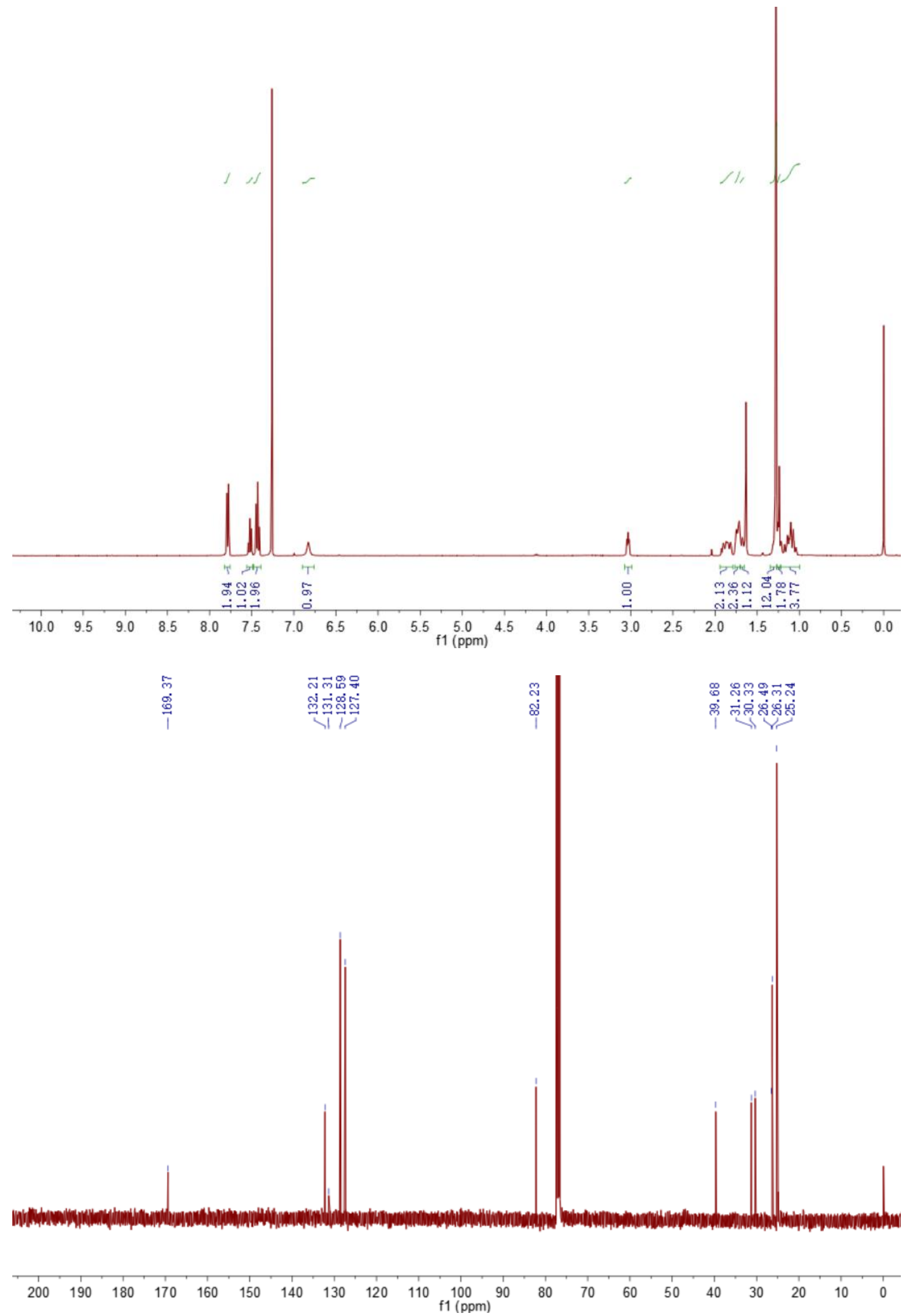
<smiles>O=C(NC(Cc1ccccc1)C1CCCC1)c1ccccc1</smiles>
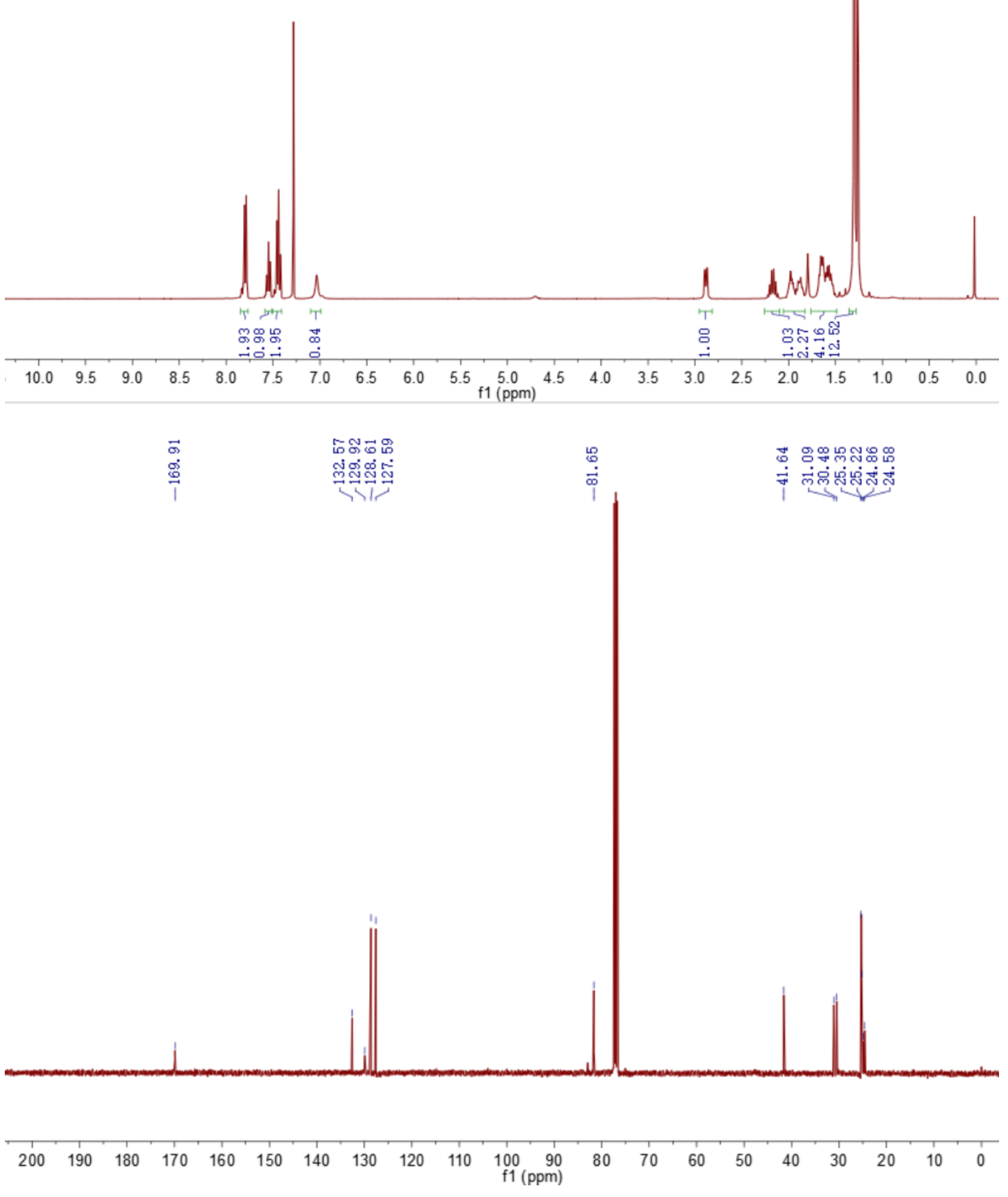

S269 
<smiles>CC(C)C(Cc1ccccc1)NC(=O)c1ccc(Cl)cc1</smiles>

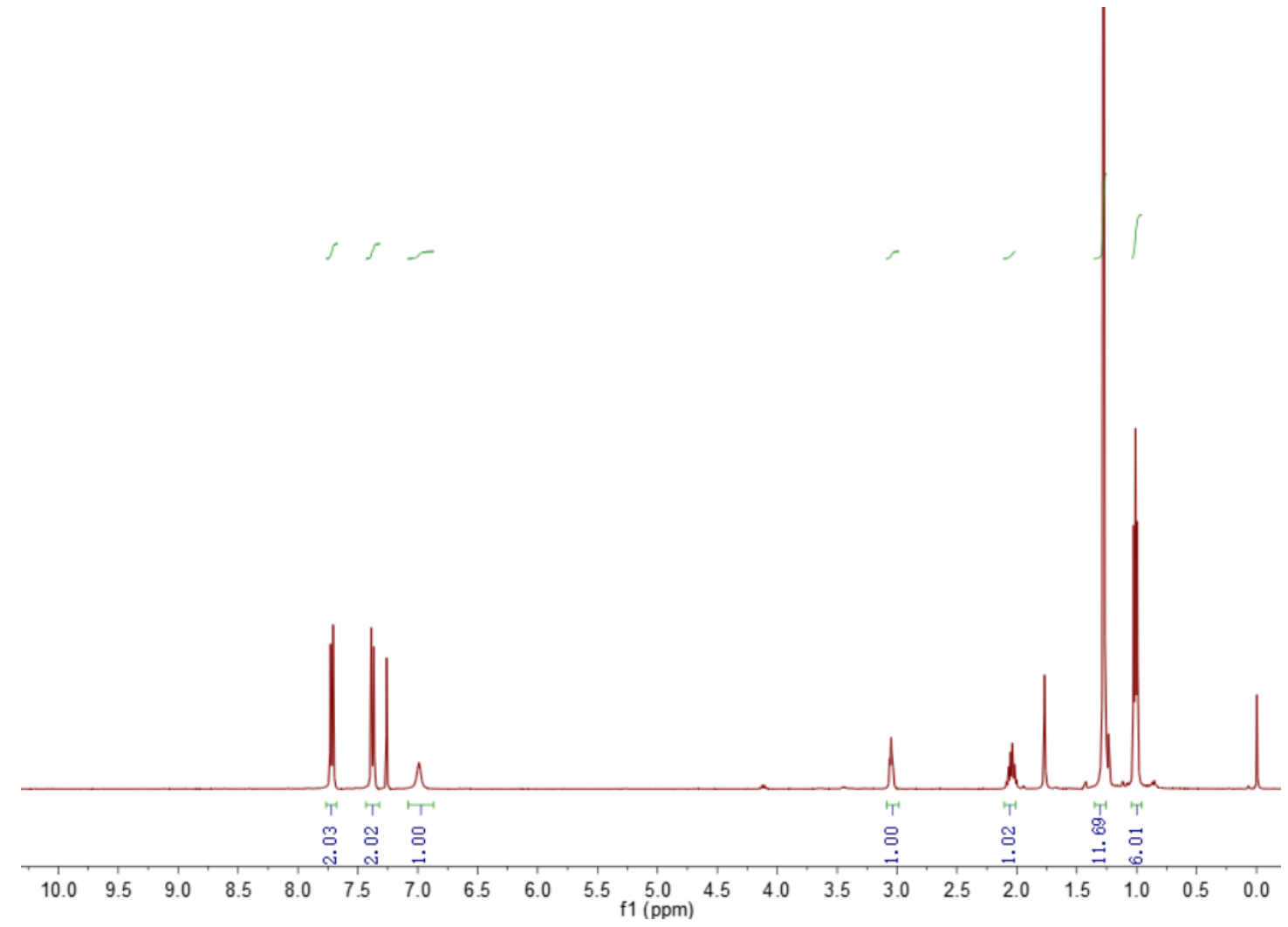

웅유. 웅

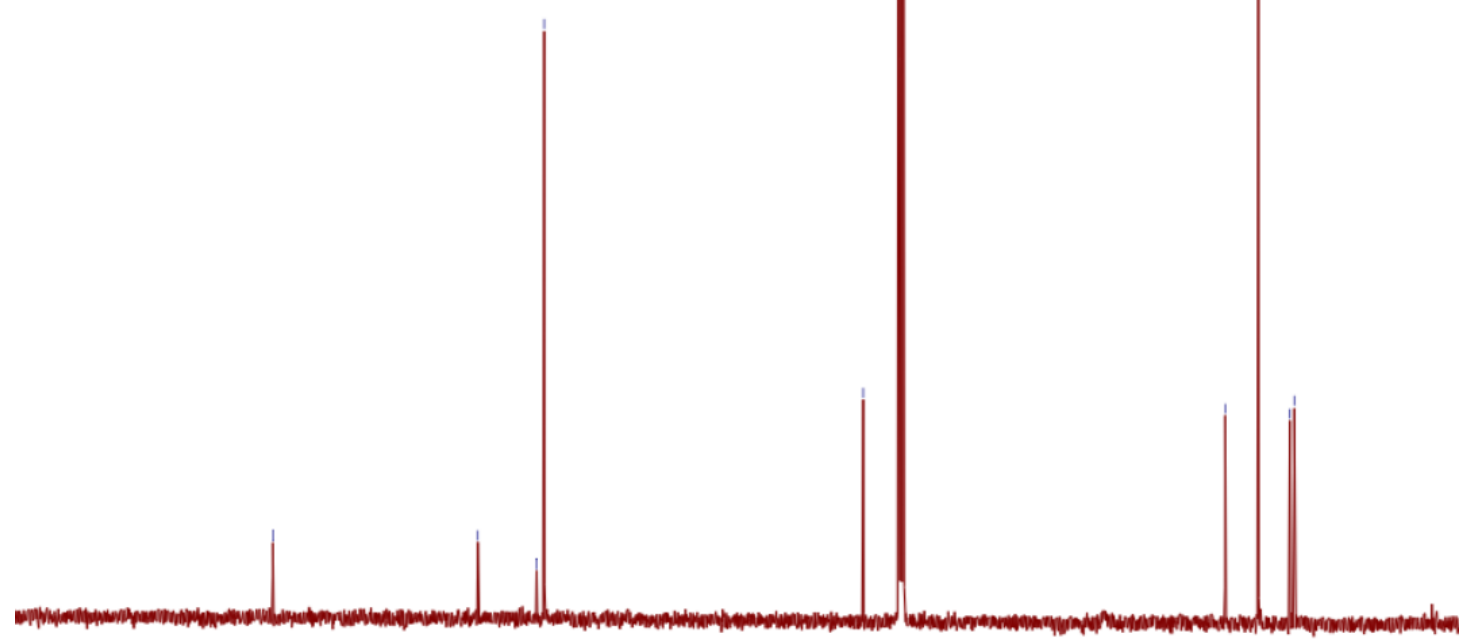

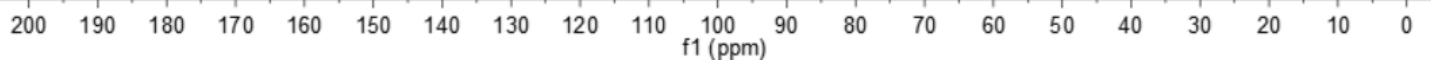


<smiles>CC(C)C(NC(=O)CC(C)(C)C)C(C)Cc1ccccc1</smiles>
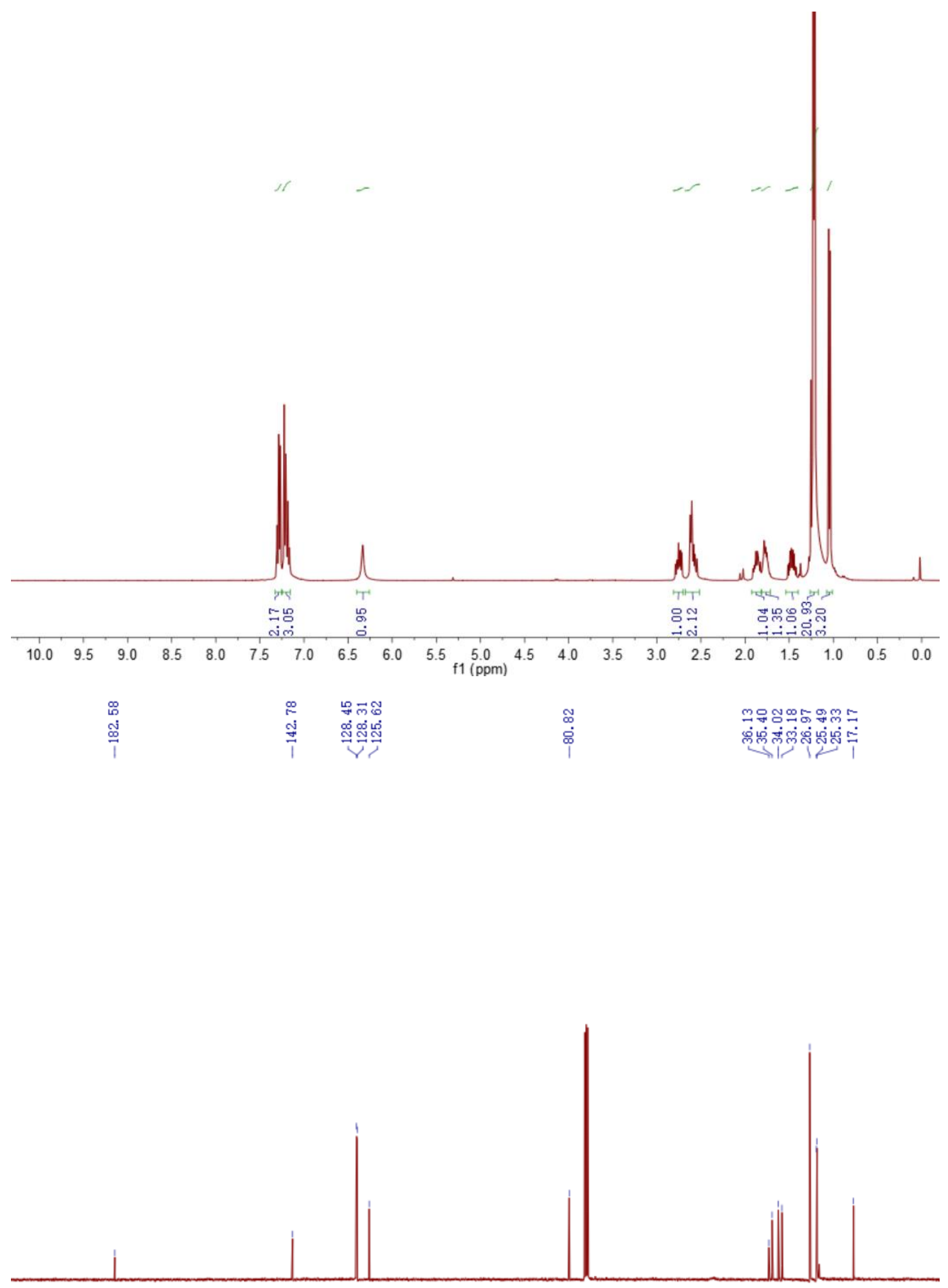

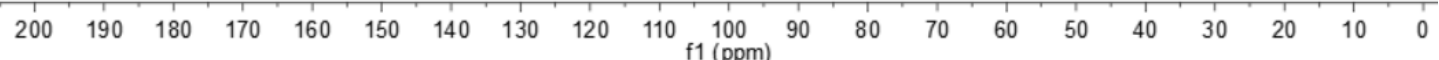


<smiles>CC(C)/C=C\NC(=O)C(Cc1ccccc1)NC(=O)OC(C)(C)C</smiles>

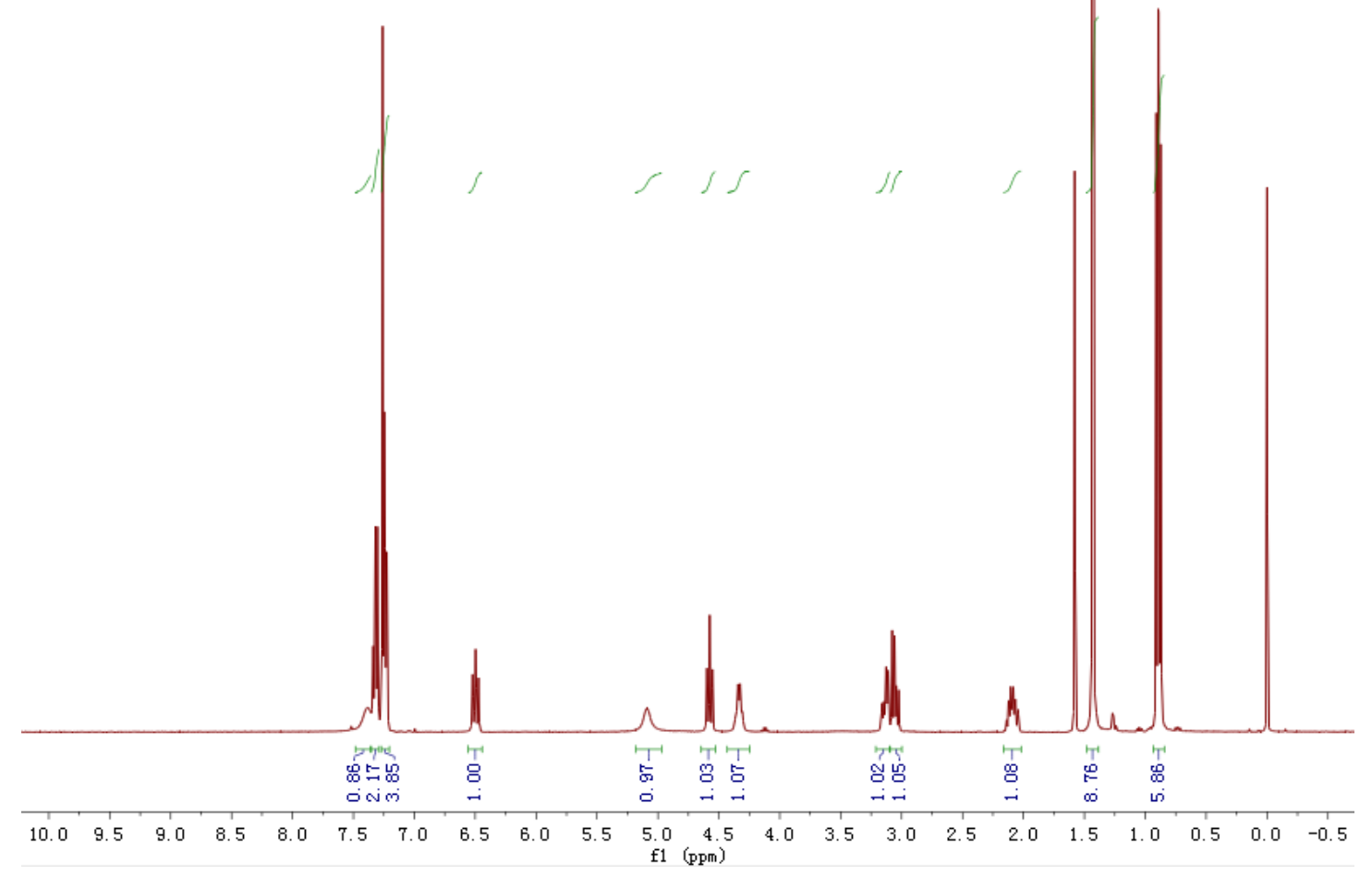


BochN
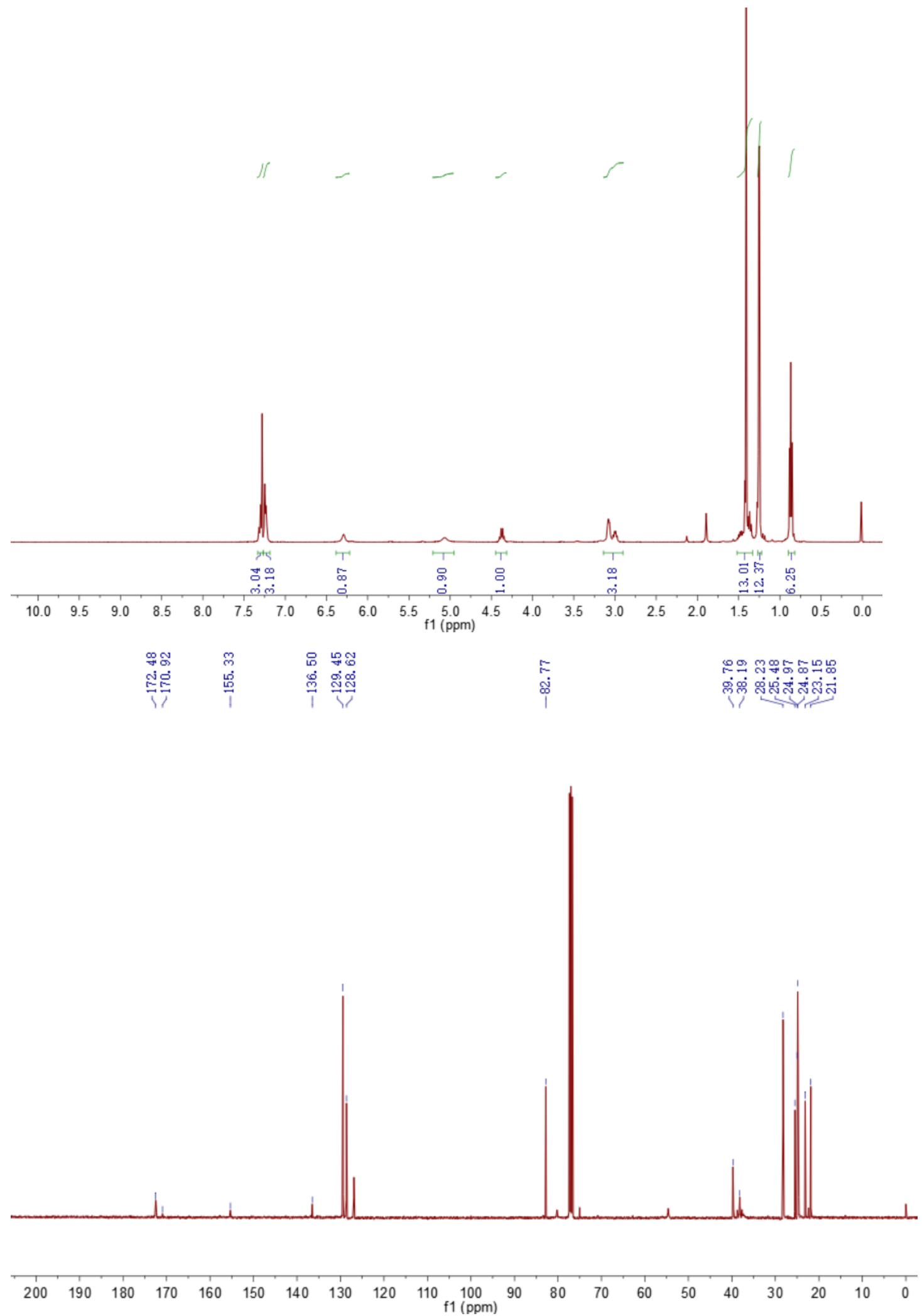

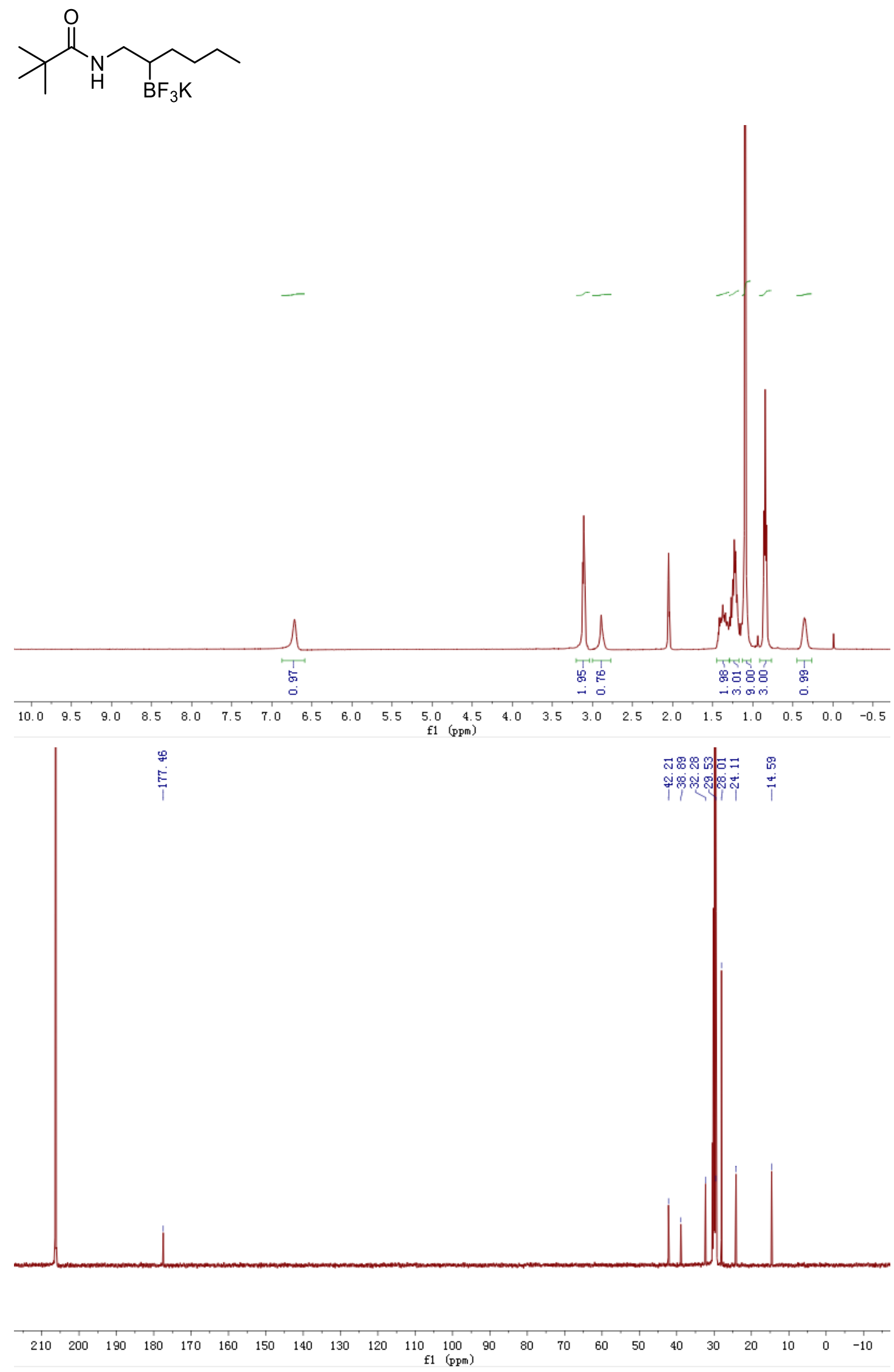

S274 
<smiles></smiles>

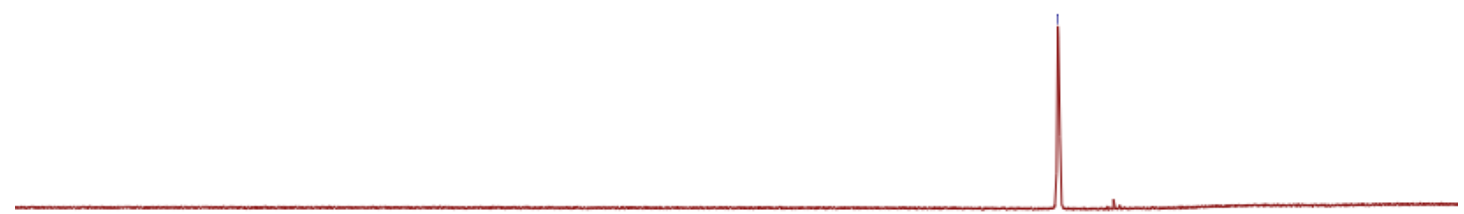

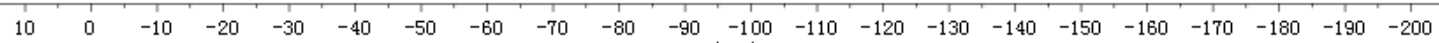
f1 (ppm) 
<smiles>CCCCC(O)CNC(=O)C(C)(C)C</smiles>

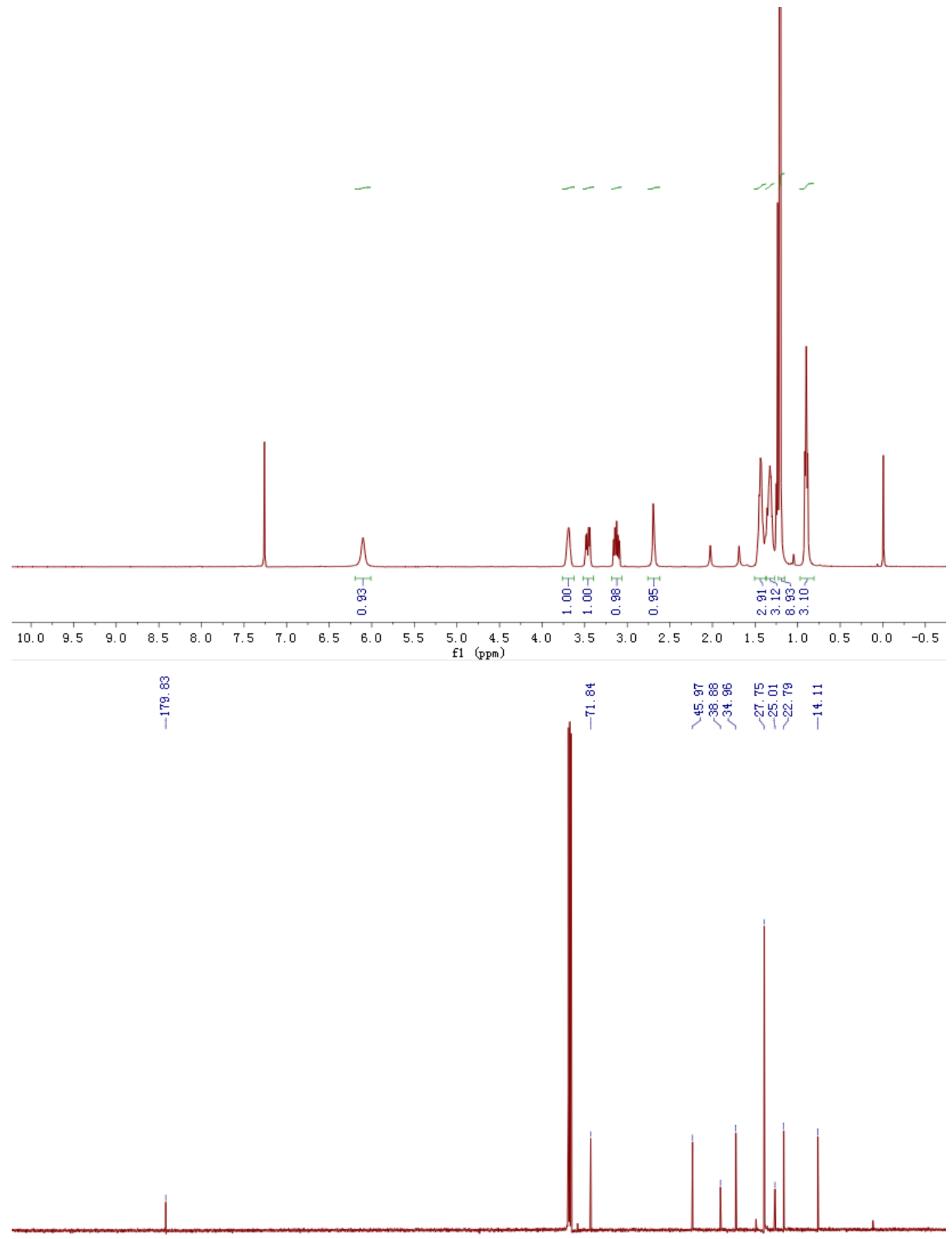

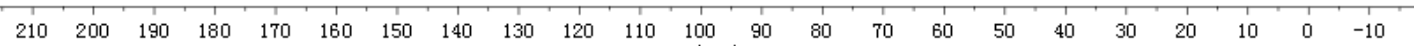


<smiles>C=CC(CCCC)CNC(=O)C(C)(C)C</smiles>
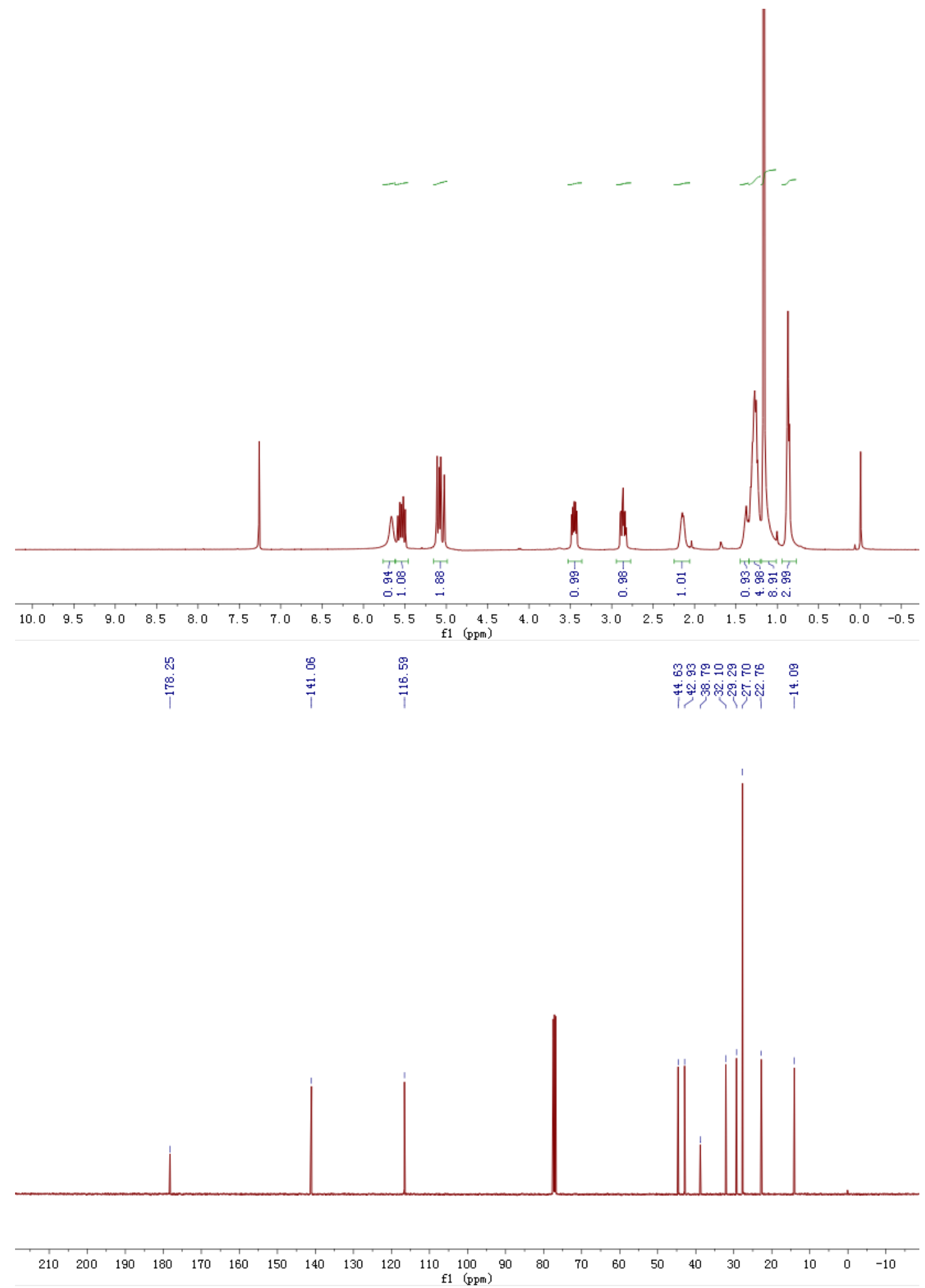
<smiles>CCCCC(CNC(=O)C(C)(C)C)c1cccs1</smiles>

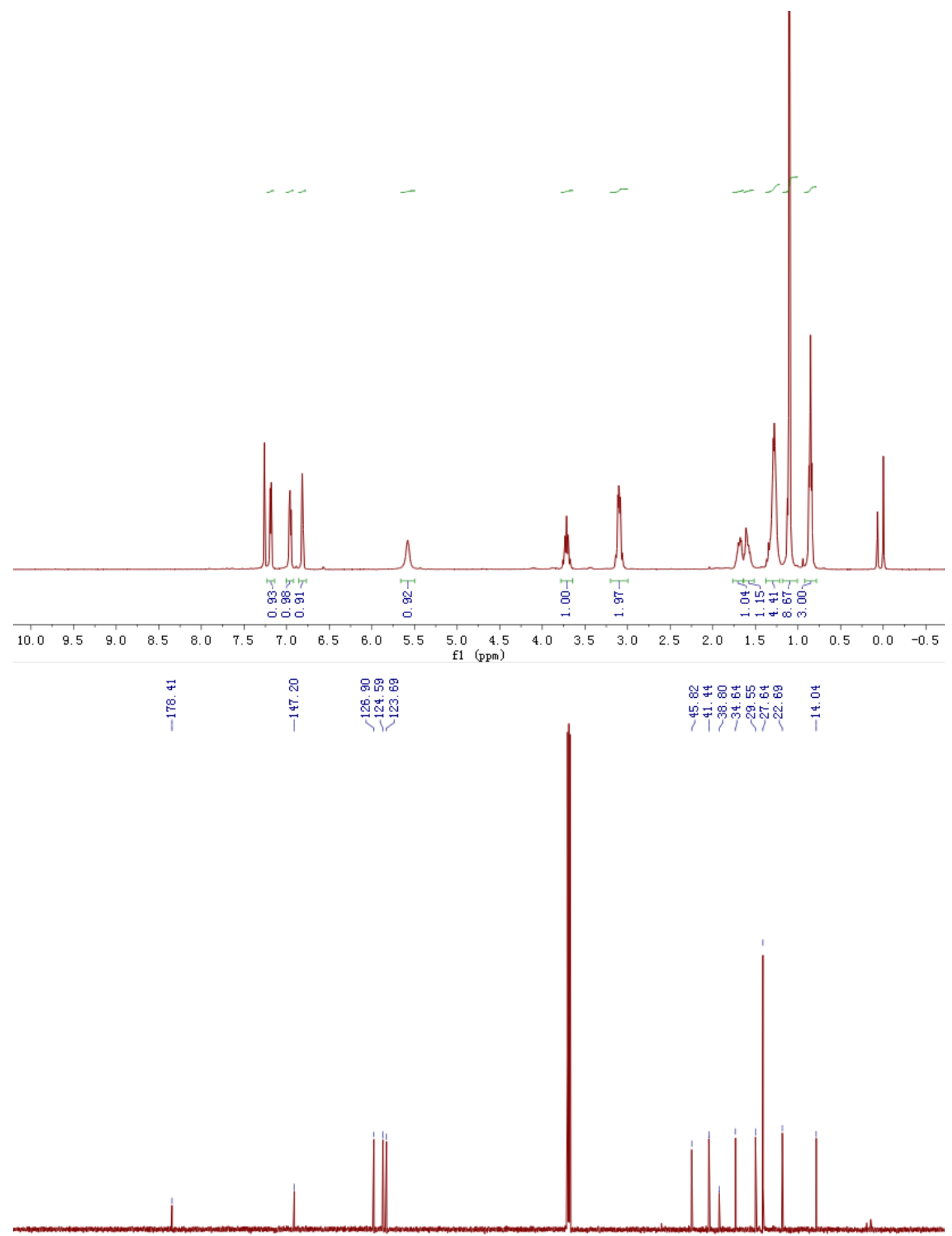

$\begin{array}{lllllllllllllllllllllllllll}210 & 200 & 190 & 180 & 170 & 160 & 150 & 140 & 130 & 120 & 110 & 100 & 90 & 80 & 70 & 60 & 50 & 40 & 30 & 20 & 10 & 0 & -10\end{array}$ f1 $(\mathrm{ppm})$ 
<smiles>CC(Cc1ccccc1)NC(=O)C(C)(C)C</smiles>

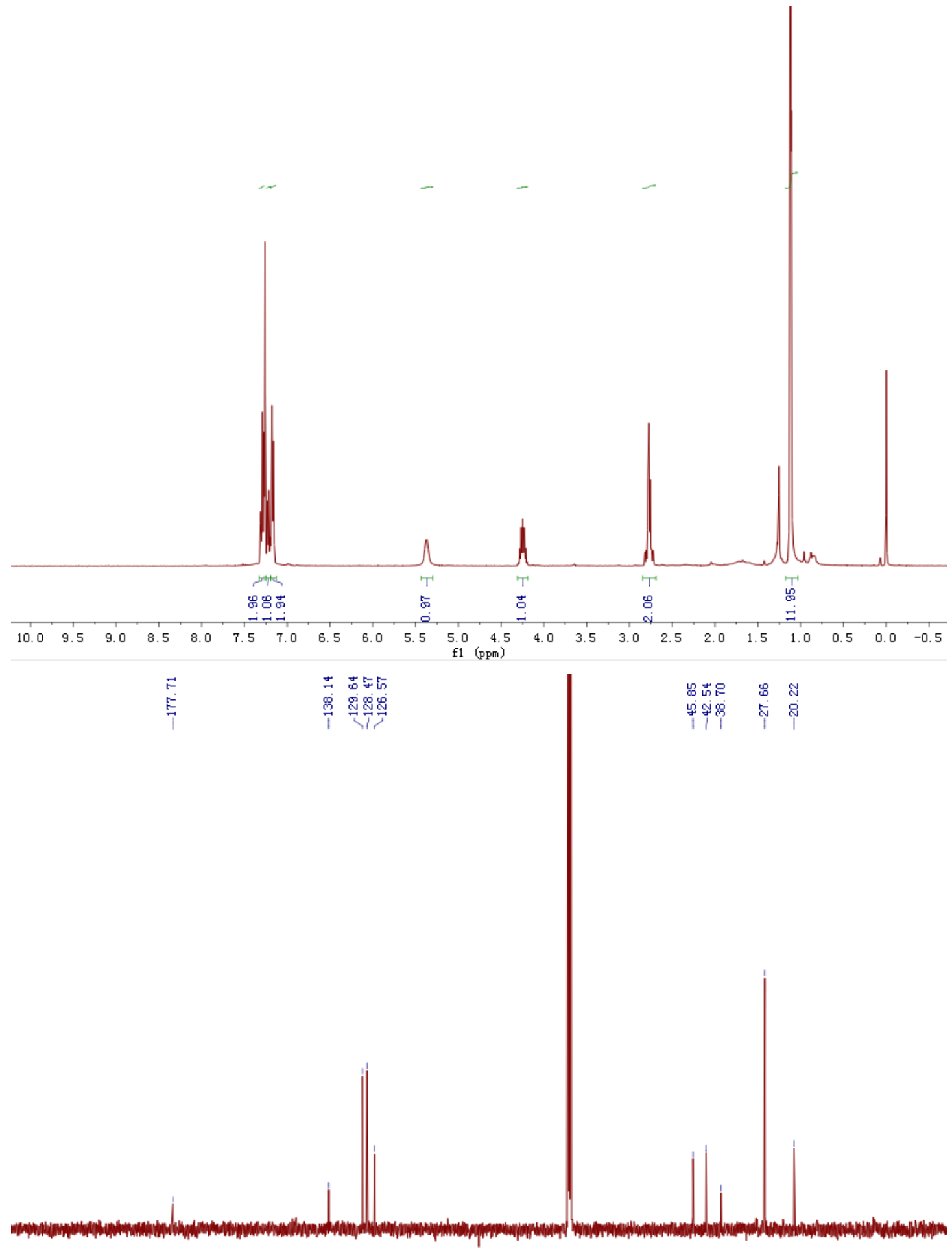

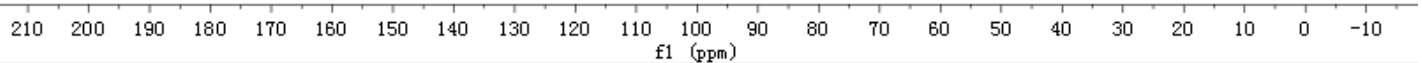



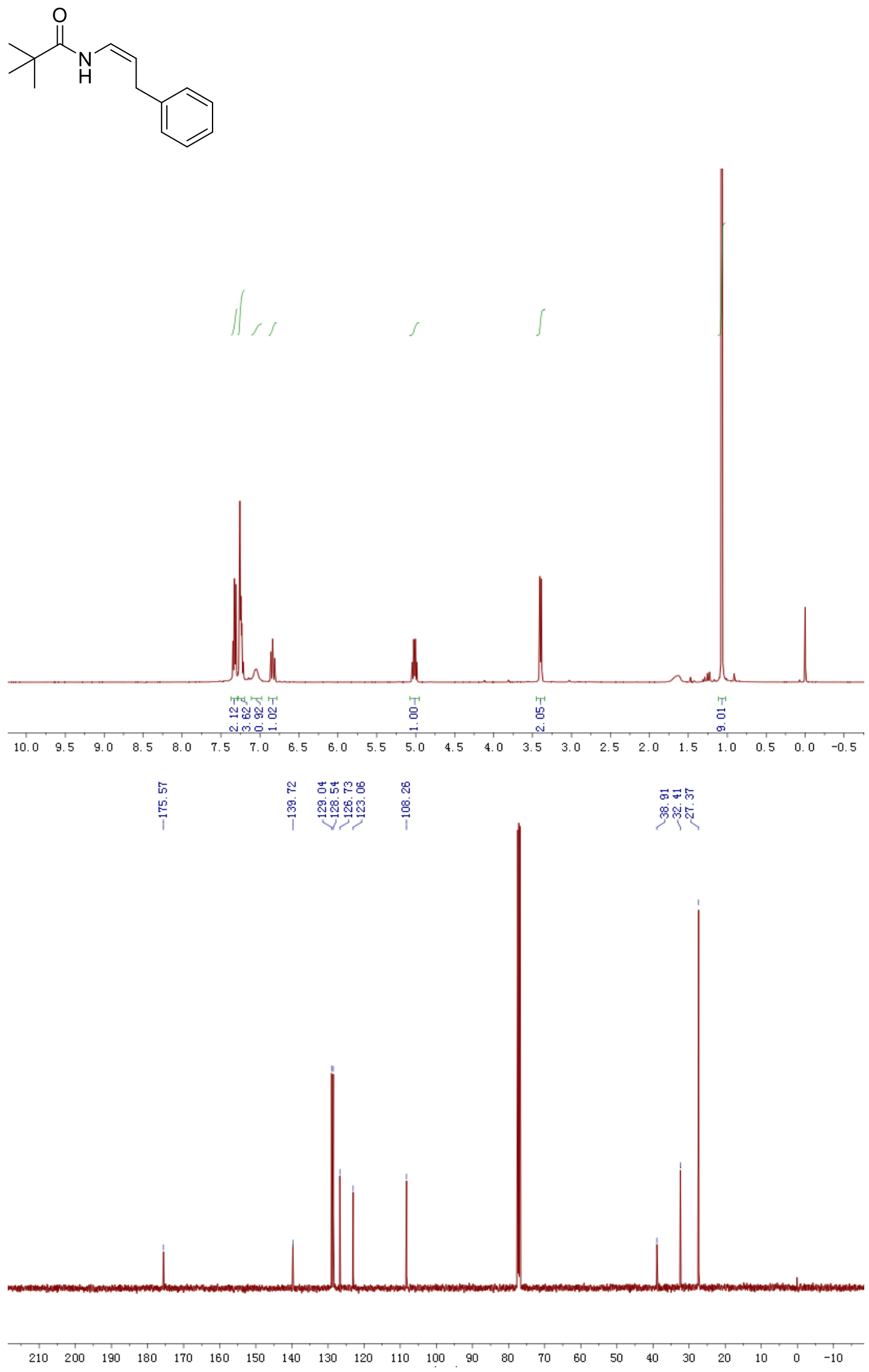

S280 
<smiles>CC(C)(C)C(=O)NC(CCc1ccccc1)Cc1ccccc1</smiles>
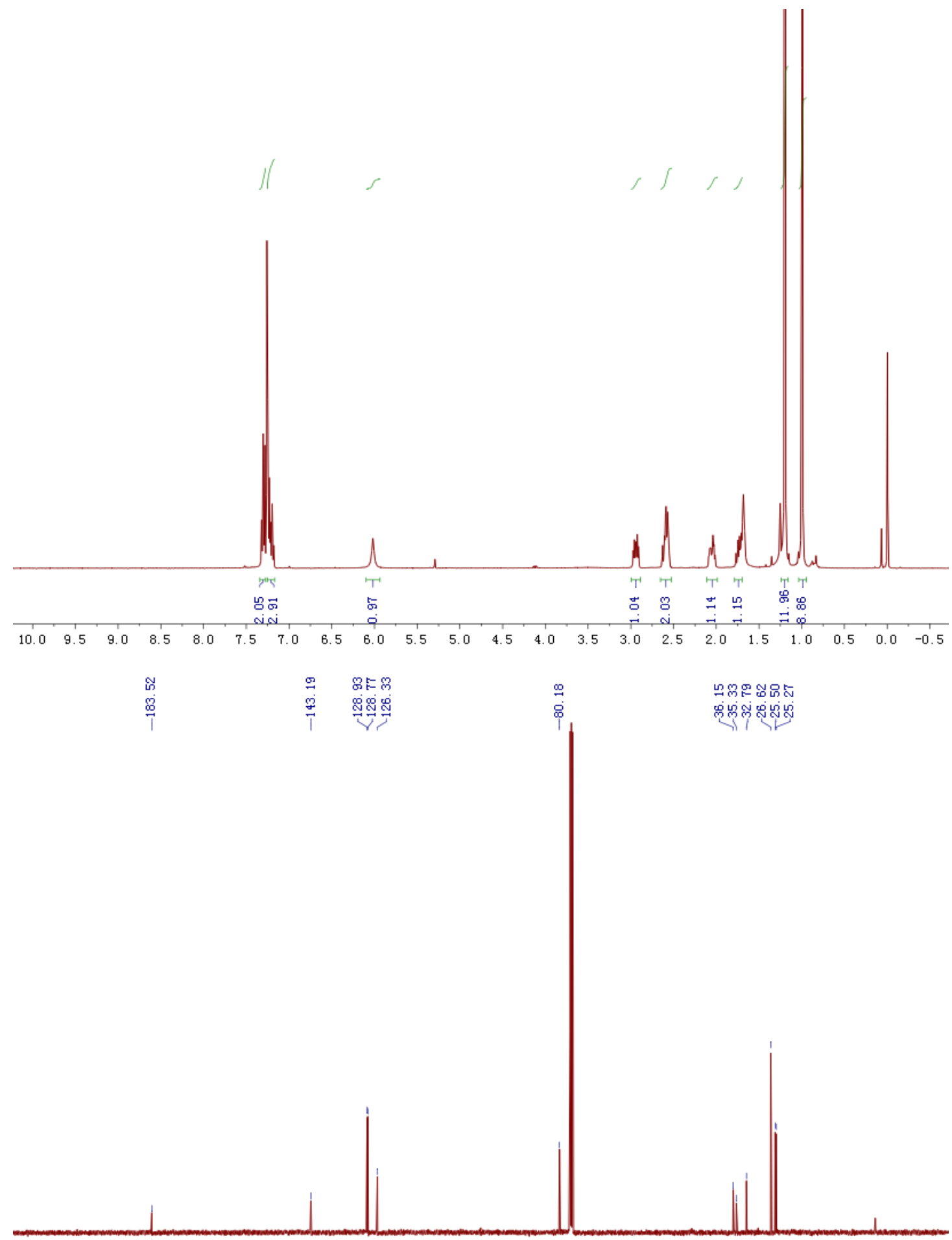

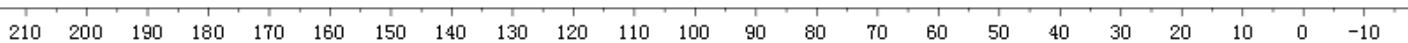


<smiles>C[C@H](O)CNC(=O)C(C)(C)C</smiles>
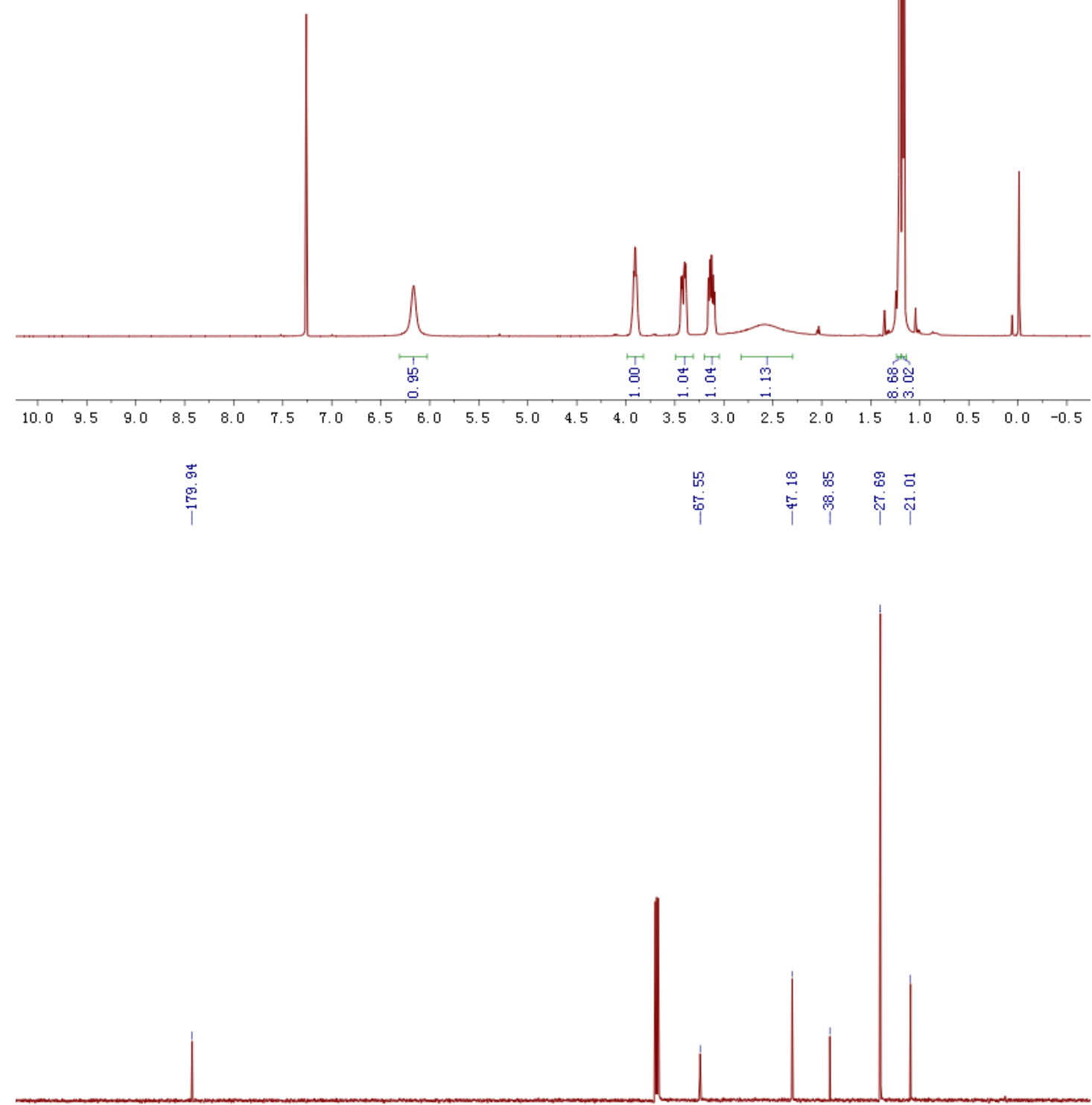

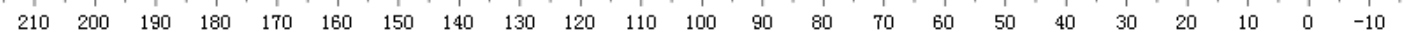

